\title{
The Growth of Naturally-Generated Small Fatigue Cracks in a Nickel-Base Single-Crystal Superalloy
}

\author{
by
}

Scott A. Yandt

M. Eng.

A thesis submitted to the Faculty of Graduate Studies and Research in partial fulfillment of the requirements for the degree of

\section{Doctor of Philosophy}

Department of Mechanical and Aerospace Engineering Ottawa-Carleton Institute for Mechanical and Aerospace Engineering

\author{
Carleton University \\ Ottawa, Ontario \\ April 2010 \\ (C) Copyright \\ 2010 S. Yandt
}




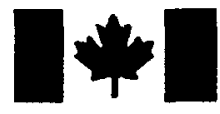

\author{
Library and Archives \\ Canada \\ Published Heritage \\ Branch \\ 395 Wellington Street \\ Ottawa ON K1A ON4 \\ Canada
}

Bibliotheque et

Archives Canada

Direction du

Patrimoine de l'édition

395, rue Wellington

Ottawa ON K1A ON4

Canada
Your file Votre reference
ISBN: 978-0-494-67897-8
Our file Notre reference
ISBN: $978-0-494-67897-8$
NOTICE:

The author has granted a nonexclusive license allowing Library and Archives Canada to reproduce, publish, archive, preserve, conserve, communicate to the public by telecommunication or on the Internet, loan, distribute and sell theses worldwide, for commercial or noncommercial purposes, in microform, paper, electronic and/or any other formats.

The author retains copyright ownership and moral rights in this thesis. Neither the thesis nor substantial extracts from it may be printed or otherwise reproduced without the author's permission.
AVIS:

L'auteur a accordé une licence non exclusive permettant à la Bibliothèque et Archives Canada de reproduire, publier, archiver, sauvegarder, conserver, transmettre au public par télécommunication ou par l'Internet, prêter, distribuer et vendre des thèses partout dans le monde, à des fins commerciales ou autres, sur support microforme, papier, électronique etlou autres formats.

L'auteur conserve la propriété du droit d'auteur et des droits moraux qui protège cette thèse. $\mathrm{Ni}$ la thèse ni des extraits substantiels de celle-ci ne doivent être imprimés ou autrement reproduits sans son autorisation.
In compliance with the Canadian Privacy Act some supporting forms may have been removed from this thesis.

While these forms may be included in the document page count, their removal does not represent any loss of content from the thesis.
Conformément à la loi canadienne sur la protection de la vie privée, quelques formulaires secondaires ont été enlevés de cette thèse.

Bien que ces formulaires aient inclus dans la pagination, il n'y aura aucun contenu manquant.

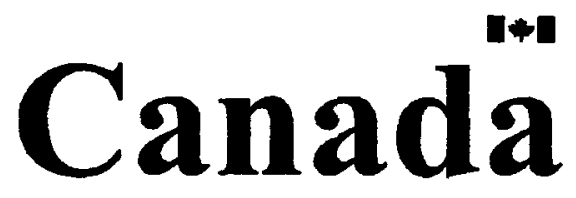




\begin{abstract}
An experimental and analytical study on the formation and growth small fatigue cracks embedded in a notch in single-crystal superalloy has been investigated. The experimental program consisted of 12 constant amplitude fatigue tests performed on single-edge notch (SEN) fatigue specimens oriented with the loading axis along [010] and with a notch factor of 2.7 . The fatigue tests concentrated on one temperature $\left(650^{\circ} \mathrm{C}\right)$ and loading condition with the secondary crystallographic orientation - the notch orientation - being the primary variable. Two secondary crystallographic orientations were considered in the present study, [101] and [100]. In the analytical study, the distribution of stresses and strains in the notch region and the stress-intensity factors and the elastic-plastic J-integral for Mode-I semi-elliptical surface cracks embedded at the notch root were investigated using the finite element method (FEM). The anisotropic material properties were shown to have a significant effect on both the stress and strain distribution in the notch region and the crack-tip parameters. The results of the experimental study have shown that fatigue cracks formation occurs via expansion of elliptical subsurface interdendritic pores located at high stress regions in the notch. Once the subsurface crack intersected the notch surface, subsequent crack growth occurred as semi-elliptical surface cracks. The secondary crystallographic orientation had a marked effect on crack-initiation life (the number of cycles to form a crack with a surface length of $760 \mu \mathrm{m}$ ) but no effect on small crack propagation behaviour. Crack initiation life predictions were made using a holistic lifing approach that considers the size, distribution and local stresses acting at the subsurface pores and utilizes the small fatigue crack growth data obtained from the experimental study. The predictions were found to agree reasonably well with the experimental test results and to account for the crack initiation life variability observed in the fatigue experiments.
\end{abstract}




\section{Acknowledgements}

I would like to express my sincere gratitude to my advisor, Dr. Jonathan Beddoes, for his continued guidance and advice while working on this project. I would also like to thank my thesis co-supervisor, Dr. Xijia Wu, for both his constructive advice and the many engaging discussions we've had regarding this research work.

I would also like to thank my colleague, Dr. Henry Saari, for his assistance with the Bridgman casting facility located at Carleton University. Many thanks to Dr. Richard Kearsey for his technical assistance with implementing the current focusing ACPD crack growth monitoring technique. The assistance of Dr. Dongyi Seo with microstructural and fractographic analysis is gratefully appreciated.

I would also like to thank the Natural Sciences and Engineering Research Council of Canada and the Ontario Graduate Scholarship Program for financial assistance provided during my brief tenure as a full-time student at Carleton University. 


\section{Table of Contents}

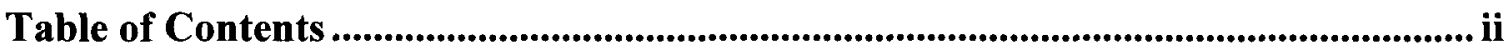

List of Figures..................................................................................................... vii

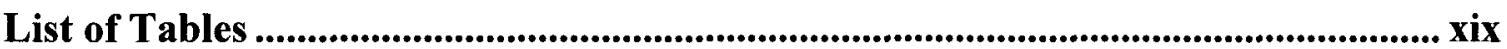

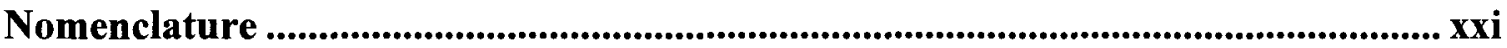

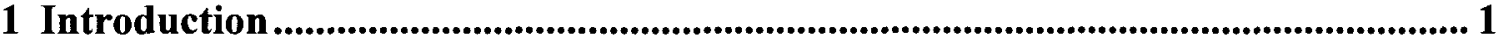

1.1 Research Background ................................................................... 1

1.2 Characteristics of Fatigue in Turbine Blades ............................................ 2

1.3 Life Prediction and Fatigue in Single-Crystal Superalloys............................ 4

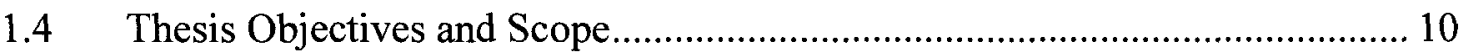

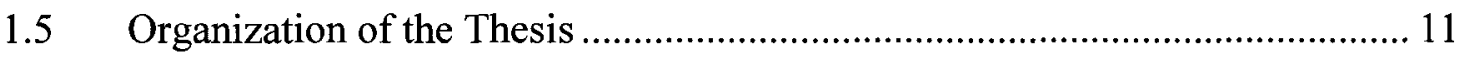

2 Literature Review: Single-Crystal Superalloys.......................................................... 17

2.1 Historical Development of Nickel-Base Superalloys ................................... 17

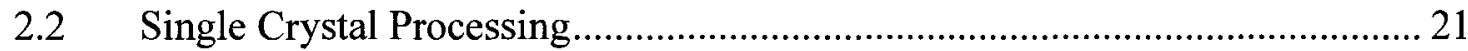

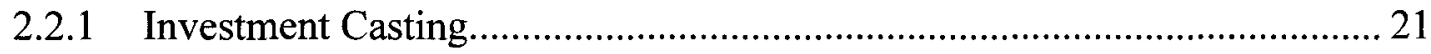

2.2.2 Directional Solidification Process.......................................................... 22

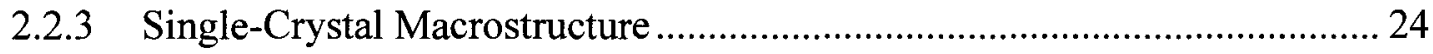

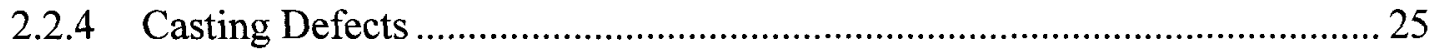

2.3 Single-Crystal Nickel-Base Superalloy Microstructure ................................ 28

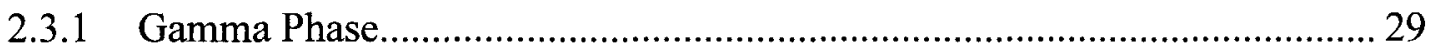

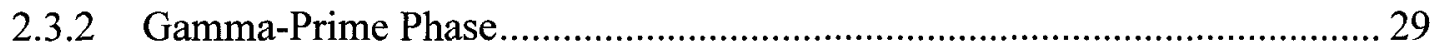

2.3.3 Topographically Close-Packed Phases .................................................... 31

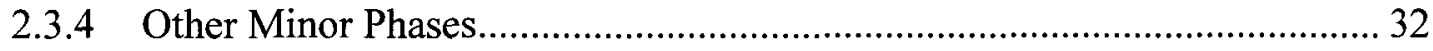

2.4 Single-Crystal Superalloy Metallurgy and Evolution ................................... 33

2.4.1 First-Generation Single-Crystal Superalloys .......................................... 33

2.4.2 Second-Generation Single-Crystal Superalloys...................................... 34

2.4.3 Third-Generation Single-Crystal Superalloys......................................... 35

2.4.4 Fourth-Generation Single-Crystal Superalloys....................................... 36

2.5 Deformation Mechanisms and Mechanical Properties of Nickel-Base Single-

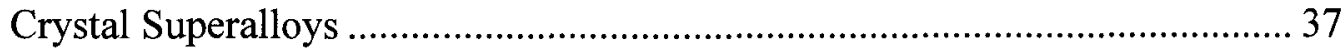

2.5.1 Elastic Properties of Single-Crystal Superalloys ................................... 38 
2.5.2 Inelastic Properties of Single-Crystal Superalloys ...................................... 39

3 Literature Review: Fatigue and Fracture Mechanics ................................................... 71

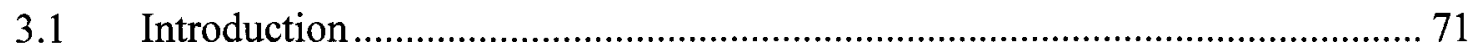

3.2 Review of Fracture Mechanics..................................................................... 73

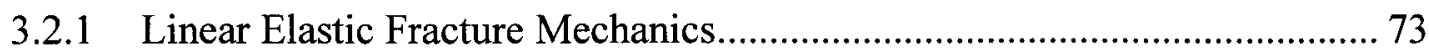

3.2.2 Elastic-Plastic Fracture Mechanics and the J-Integral .................................. 84

3.3 Numerical Methods for Fracture Mechanics Parameter Estimation ................. 87

3.4 Fatigue Crack Growth in Polycrystalline Materials.......................................... 90

3.4.1 Characterization of Fatigue Crack Growth .................................................... 90

3.4.2 Application of Linear Elastic Fracture Mechanics ....................................... 91

3.4.3 Application of Elastic-Plastic Fracture Mechanics....................................... 95

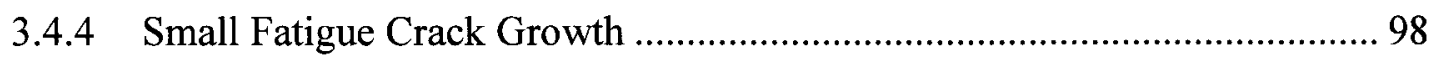

3.5 Fatigue Crack Formation and Propagation in Single-Crystal Superalloys .... 103

3.5.1 Crack Nucleation in Single-Crystal Superalloys ....................................... 103

3.5.2 Small Fatigue Crack Growth in Nickel-base Single-Crystal Superalloys .. 107

3.5.3 Mechanisms of Fatigue Crack Propagation in Single-Crystal Superalloys 108

3.5.4 Factors Influencing Small Crack Growth ................................................ 112

4 Literature Review: Single-Crystal Constitutive Models ........................................ 133

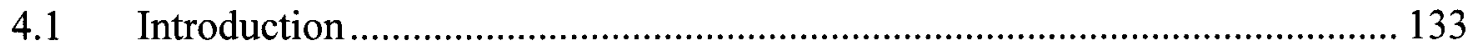

4.2 Nouailhas and Chaboche Phenomenological Constitutive Model for SingleCrystal Superalloys [225] .......................................................................... 135

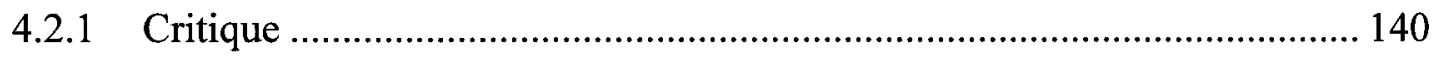

4.3 Han et al. Micro-phenomenological Constitutive Model for Single-Crystal Superalloys [226] .................................................................................. 142

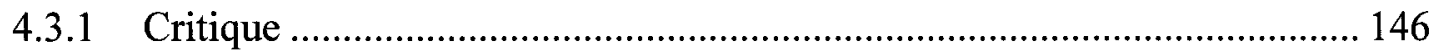

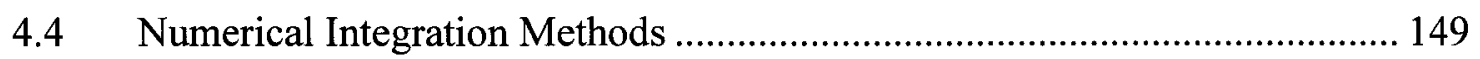

4.4.1 Numerical Integration Techniques............................................................ 149

5 Materials and Experimental Methods......................................................................... 156

5.1 Experimental Materials ........................................................................... 156

5.1.1 Alloy Selection and Procurement ............................................................. 156

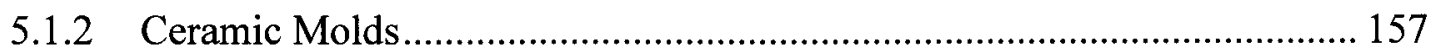

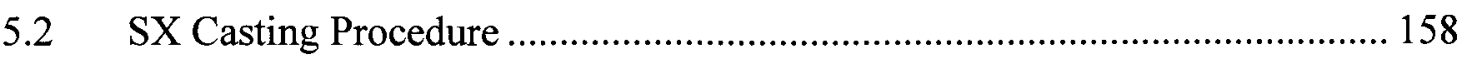

5.2.1 Carleton University DS Casting Facility .................................................. 158 
5.2.2 Seed Crystal Casting Process ................................................................. 159

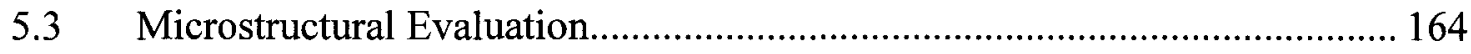

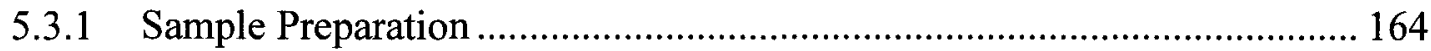

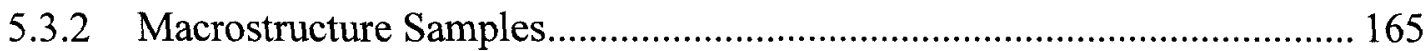

5.3.3 Microstructure Sample Preparation ……………..................................... 165

5.3.4 Optical and Scanning Electron Microscopy Equipment .............................. 165

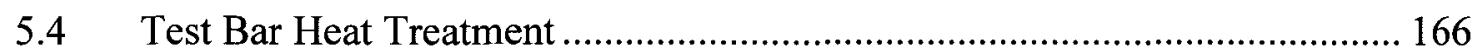

5.4.1 As-Cast Macro and Microstructure of Single-Crystal Test Bars ................. 166

5.4.2 Differential Scanning Calorimetry (DSC) Analysis ................................... 169

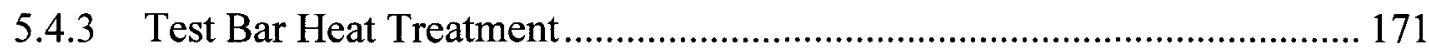

5.5 Constitutive Material Behaviour Testing ..................................................... 175

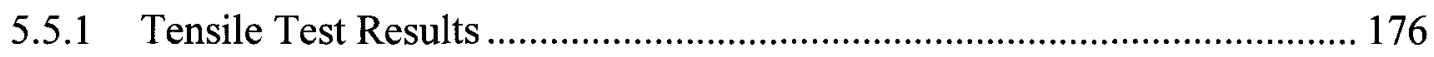

5.6 Small Fatigue Crack Growth Rate Testing ..................................................... 177

5.6.1 ACPD Crack Growth Monitoring System ………………………………... 179

5.6.2 ACPD Data Acquisition and Signal Analysis............................................. 181

5.6.3 ACPD Crack Length Calibration Tests......................................................... 183

5.6.4 Fatigue Data Analysis .......................................................................... 187

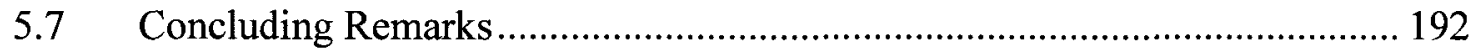

6 Finite Element Implementation of a Single-Crystal Constitutive Model.............. 224

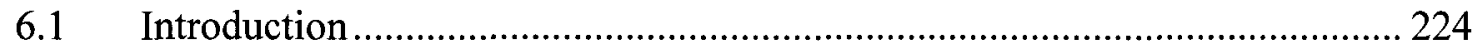

6.2 Single-Crystal Micro-Phenomenological Constitutive Model......................... 225

6.3 Numerical Implementation of the Constitutive Model .................................. 227

6.4 Consistent Tangent Stiffness Matrix ........................................................... 230

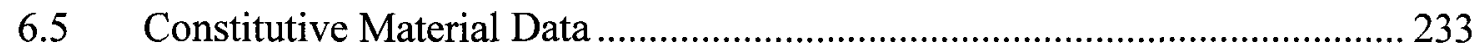

6.6 Identification of the Constitutive Material Parameters ................................... 234

6.6.1 Transformation of Stress and Strain Tensors............................................. 234

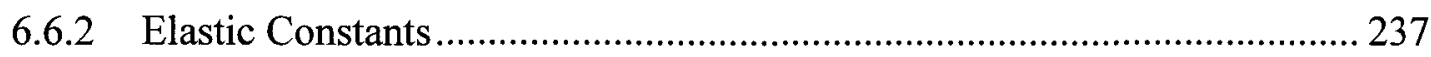

6.6.3 Inelastic Constitutive Material Parameters ............................................... 240

6.7 Comparison of Calculations to Experimental Data.......................................... 248

6.7.1 Monotonic Deformation Behaviour .......................................................... 248

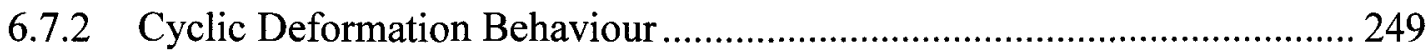

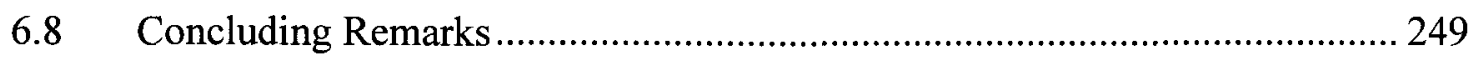

7 Finite Element Modeling .............................................................................................. 256 


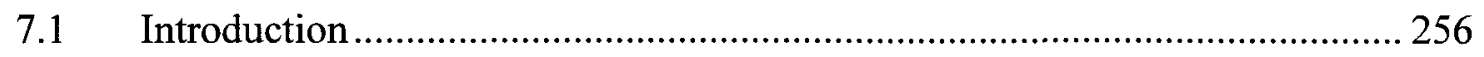

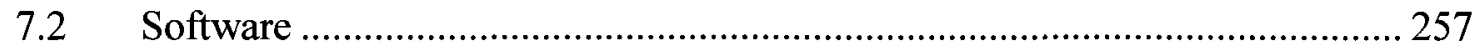

7.3 Geometry of the Specimen Modeled............................................................. 257

7.4 Finite Element Models for Notch root Stress and Strain Analysis ................. 258

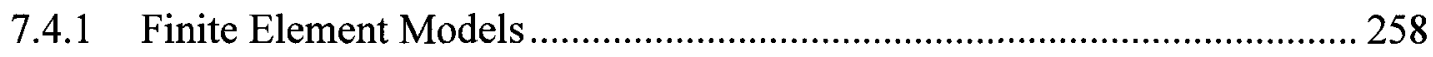

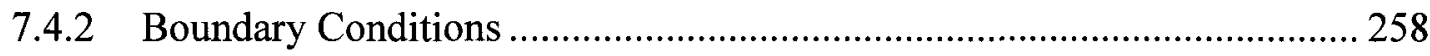

7.5 Finite Element Models for Crack Analysis .................................................... 259

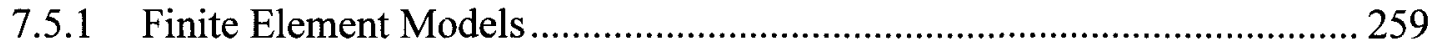

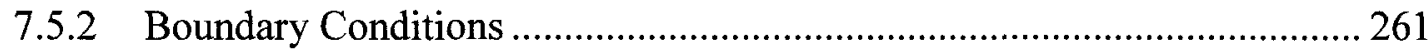

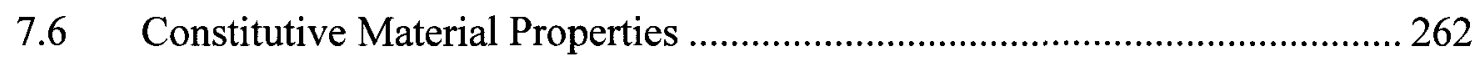

8 Finite Element Modeling Results and Discussion ...................................................... 274

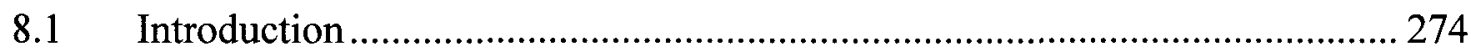

8.2 SEN Specimen Notch Fields Analysis ..................................................... 274

8.2.1 Linear Elastic Notch Root Analyses ........................................................ 274

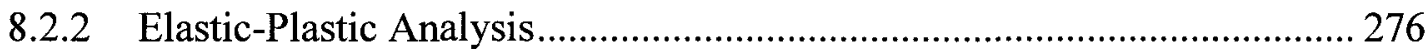

8.3 Analysis of SIF's for Semi-Elliptical Notch Root Cracks ........................... 281

8.3.1 Validation of SIF Calculations............................................................... 281

8.3.2 SIF's for Notch Root Semi-Elliptical Cracks .............................................. 283

8.4 Analysis of The J-Integral for Semi-Elliptical Notch Root Cracks ................ 287

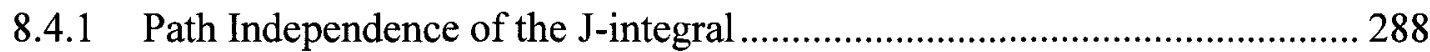

8.4.2 Elastic-Plastic J-Integral for Notch Root Semi-Elliptical Cracks............... 289

8.5 Relationships Between Crack-tip Parameters and Crack Geometry................ 294

8.5.1 Anisotropic Stress-Intensity Factors ....................................................... 295

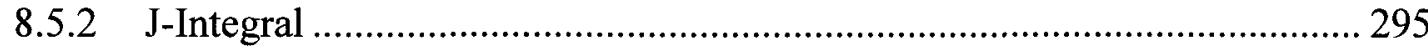

8.5.3 Polynomial Expressions for Crack Growth Rate Data Analysis ................ 296

8.6 Concluding Remarks ................................................................................. 297

8.6.1 Notch root Stress and Strain Fields Analysis............................................. 297

8.6.2 Fracture Mechanics Parameters for Notch Root Semi-Elliptical Cracks.... 298

9 Fractographic Analysis of Fatigue Crack Growth Rate Specimens - Results and

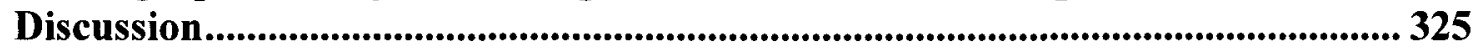

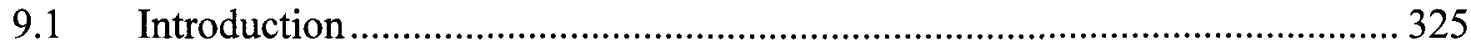

9.2 Fractographic Observations and Discussion .............................................. 325

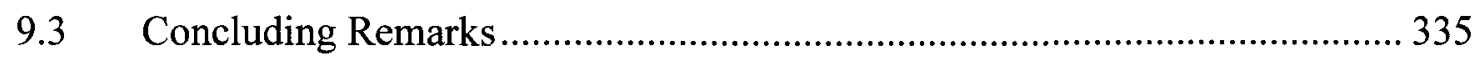


10 Fatigue Crack Growth Rate Test Results and Discussion............................ 347

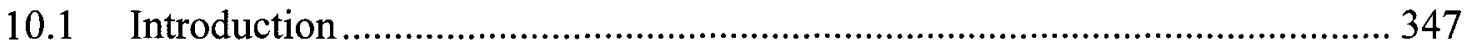

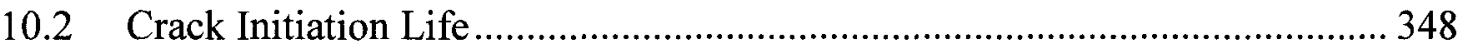

10.3 Small Fatigue Crack Growth Test Results and Discussion............................. 353

10.3.1 Application of Fracture Mechanics Parameters to FCGR Data.............. 353

10.3.2 Fatigue Crack Growth Rate Curves for Small Fatigue Cracks ................ 355

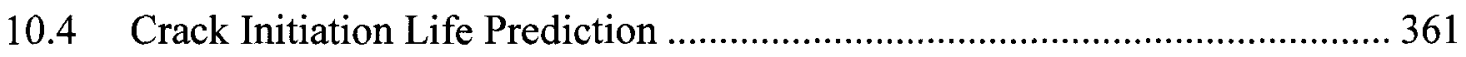

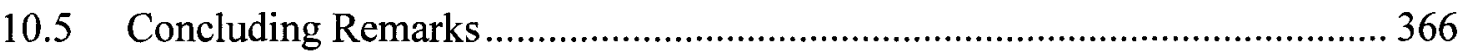

11 General Conclusions and Recommendations for Future Work ................. 378

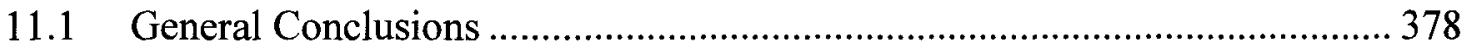

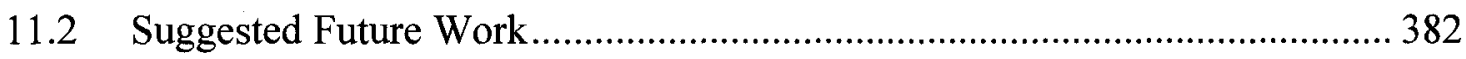

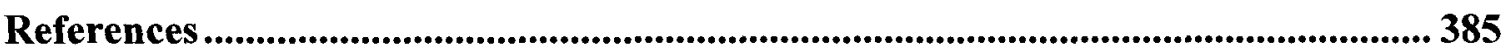

Appendix A: Micro-Phenomenological Constitutive Model Stress Update Algorithm

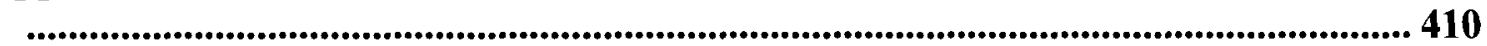




\section{List of Figures}

Figure 1.1 - Cutaway view of the Rolls-Royce Trent 900 turbofan engine used on the Airbus A380 family of aircraft adapted from [57] 13

Figure 1.2 - Cross section through a triple-spool gas turbine engine showing gas path pressure and temperature at various points in the engine [58].

Figure 1.3 - Single-crystal turbine blade design features. Note: Canadian dime is included in image to provide a sense of scale.

Figure 1.4 - (a) temperature and (b) stress distributions in an air-cooled turbine blade [59].

Figure 1.5 - Schematic cutaway view showing the internal turbine blade cooling arrangement used in first stage turbine blades [60]. 15

Figure 1.6 - Convention for defining crystal orientation of single-crystal turbine blade. 16 Figure 2.1 - Historical development of superalloys since 1940 and evolution of metal temperature capability[61]. 52

Figure 2.2 - Creep curves for conventional cast (PWA659) and directionally solidified (PWA664) Mar-M-200 tested at $1800^{\circ} \mathrm{F}$ and $20 \mathrm{ksi}$ [66]. Notice directionally solidified material has a substantially lower minimum creep rate and higher creep ductility than conventionally cast material.

Figure 2.3 - Creep curves for Mar-M-200 alloy with poly-crystalline (PC), columnar grain directionally solidified (CGDS) and single-crystal directionally solidified (SCDS) structure tested at $1800^{\circ} \mathrm{F}$ and $30 \mathrm{ksi}[80]$. 53

Figure 2.4 - Ceramic core and cutaway of turbine blade showing internal cooling features [80].

Figure 2.5 - Wax blade cluster pattern for casting SX turbine blades (adapted from

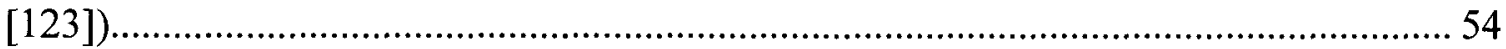

Figure 2.6 - Ceramic shell casting mold for single crystal turbine blades [123] ............ 55

Figure 2.7 - Transverse section of a conventionally cast superalloy bucket that was polished and etched to reveal the grain structure.

Figure 2.8 - Schematic illustration of an industrial Bridgman furnace for the production of DS and single-crystal turbine blades [124].......................................................... 56

Figure 2.9 - Directionally solidified turbine blade etched to reveal columnar grain structure (adapted from [58]). Note: Inset shows a magnified view of the DS grain structure.

Figure 2.10 - Grain selector designs used for single-crystal casting; a) helical grain selector and b) two turn selector (adapted from [80]). 57

Figure 2.11 - Schematic showing arrangement used for single-crystal part production using seed crystal (adapted from [80]). 58 
Figure 2.12 - As-cast dendritic structure observed in single-crystal superalloys; a) longitudinal section showing primary dendrite arms aligned with withdrawal direction and b) transverse section showing primary dendrite arm spacing (PDAS) and secondary and tertiary dendrite arms (adapted from [125]) .......................................................... 58

Figure 2.13 - Schematic diagram showing effect of solidification rate (growth rate) versus thermal gradient on microstructure [74]. 59

Figure 2.14 - Interdendritic casting microporosity in a single-crystal superalloy [126].. 59 Figure 2.15 - Freckle grain defect on the surface of a single-crystal cast bar [127]. Note: the solidification direction is from left to right. 60

Figure 2.16 - SEM micrograph showing the $\gamma-\gamma^{\prime}$ microstructure in the first generation single-crystal superalloy CMSX-2 after application of solutioning and aging heat treatments [86]. This $\gamma-\gamma^{\prime}$ microstructure common to all high $\mathrm{V}_{\mathrm{f}}$ single-crystal superalloys.

Figure 2.17 - FCC crystal structure of the $\gamma$-matrix phase [123]. Notice that aluminum and nickel, or other substitutional solid solution strengthening elements, can occupy any atomic site. 61

Figure 2.18 - Ordered $\mathrm{Ll}_{2}$ FCC crystal structure of the $\gamma^{\prime}$ phase [123]. Notice that only aluminum, or substitutional elements such as titanium or tantalum, may occupy the corner atomic sites; nickel (or substitutes) occupy the face center atomic sites. 61

Figure 2.19 - Creep strength of various nickel-base superalloys as a function of $\gamma^{\prime}$ volume fraction [128]. 62

Figure 2.20 - SEM micrograph showing heterogeneous as-cast $\gamma^{\prime}$ microstructure in the first generation single-crystal superalloy CMSX-2 [65].

Figure 2.21 - SEM micrographs showing TCP phases precipitated in RR2071; a) $\sigma$ phase, b) $\mu$-phase and c) P-phase (adapted from [129]). Note: the underlying microstructure consists of $\gamma$ and $\gamma^{\prime}$. 63

Figure 2.22 - Effective elastic modulus for PWA1480 as a function of orientation at room temperature [64]. 63

Figure 2.23 - Schematic representation of the FCC octahedral slip systems comprising 4 $\{111\}$ slip planes with each slip plane containing $3\langle\mathbf{1 1 0}\rangle$ slip directions. 64

Figure 2.24 - Schematic representation of the FCC octahedral slip systems comprising 3 $\{\mathbf{1 0 0}\}$ slip planes with each slip plane containing $2\langle\mathbf{1 1 0}\rangle$ slip directions. .........................65

Figure 2.25 - Schematic illustrating Schmid's law [130]................................................. 66

Figure 2.26 - Yield strength of PWA 1480 at $593^{\circ} \mathrm{C}$ versus orientation along the $<001>$ $<011>$ boundary of the standard stereographic triangle [87].

Figure 2.27 - Schematic diagram showing tension (T) and compression (C) asymmetry as a function of orientation [87]. 
Figure 2.28 - Tensile and compressive yield strength of PWA1480 as a function of

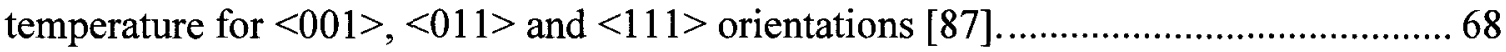

Figure 2.29 - Yield strength of PWA1480 as a function of temperature and strain rate [109] 68

Figure 2.30 - Schematic diagram showing a $a / 2\langle 110\rangle$ dislocation dissociated into two Shockley partial dislocations separated by a stacking fault (adapted from [131]). Note: colour of "atoms" indicate the relative atomic stacking positions. 69

Figure 2.31 - Monotonic stress-strain curve for [123] oriented Rene N4+ tensile sample tested at $760^{\circ} \mathrm{C}$ [132]. The tensile sample was subjected to double tensile test at $10^{-4} \mathrm{sec}^{-1}$ and $6 \times 10^{-4} \mathrm{sec}^{-1}$ to study recovery mechanisms. Notice the pronounced serrated yielding. 70

Figure 2.32 - First cycle stress-strain hysteresis loops for Rene N4 test samples with [001] [011] and [111] orientations tested at $760^{\circ} \mathrm{C}$ [100]. The tension-compression asymmetry is apparent for the [001] and [011] orientations. 70

Figure 3.1 - The three independent modes for crack opening [135]. (a) Mode I or tensile opening mode, (b) Mode II or in-plane shearing mode and (c) Mode III or anti-plane shearing mode. 119

Figure 3.2 - Schematic showing polar coordinate system at the crack-tip [133]. 119

Figure 3.3 - Schematic showing mechanically (or physically) small fatigue cracks and associated plastic zone sizes in (a) smooth and (b) notched fatigue specimens [49] .... 120

Figure 3.4 - Crystallographic slip sector boundaries for 2-D cracks; a) [001] loading axis with [100] crack growth direction and b) [001] loading axis with [110] crack growth direction. The Cartesian components of the stress tensor is constant in sectors A, B, C and D. The sector boundaries are denoted by rays with a given angle of theta where those associated with octahedral slip are denoted by solid rays and dashed rays for cubic slip [143] 120

Figure 3.5 - Nomenclature for a semi-elliptical surface crack in a 3-D body 121

Figure 3.6 - Fatigue crack growth mechanisms map for PWA1484 single-crystals [157]. Note: line denotes transition boundary between mixed-mode octahedral (crystallographic) crack growth and Mode I transprecipitate non-crystallographic growth (TPNC).

Figure 3.7 - Definition of the J-integral for a 2-D crack consisting of a closed contour $\Gamma$ originating from the lower crack surface which goes clockwise around the crack-tip to the upper crack surface[138].

Figure 3.8 - Typical crack growth behaviour obtained from laboratory specimens tested under constant stress amplitude loading [135]. 122

Figure 3.9 - Schematic illustrating the three different regions of stable crack propagation for long-fatigue cracks (adapted from [135]). 123

Figure 3.10 - Three mechanisms of fatigue crack closure: a) plasticity induced crack closure, b) oxide induced crack closure, and c) roughness induced crack closure. Adapted from Reference [185]. 123 
Figure 3.11 - Fatigue crack growth behaviour for Udimet 700 tested at $850 \mathrm{C}$ showing three region crack growth behaviour [192]. Note: solid line represents linear-elastic and dashed line represents elastic-plastic characterization of the fatigue crack growth behaviour. 124

Figure 3.12 - Schematic showing typical characteristics of small (or short) and long fatigue cracks [135].. 125

Figure 3.13 - Schematic of the Kitagawa-Takahashi curve showing the small (or short) and long crack growth regimes (after Miller and de los Rios [222]). 125

Figure 3.14 - Schematic of crack size versus life curve showing the four phase of fatigue crack formation and growth [8]. 126

Figure 3.15 - TEM micrograph showing persistent slip bands in $\langle 100>$-oriented singlecrystal superalloy tested at $700^{\circ} \mathrm{C}$ [206]. 126

Figure 3.16 - Schematic showing rough extrusion-intrusion surface topography caused by persistent slip bands [135]. 127

Figure 3.17 - Naturally nucleated surface fatigue crack sites in single-crystal superalloy CMSX-2, (a) surface connected shrinkage porosity and (b) surface connected eutectic $\gamma$ $\gamma^{\prime}$ nodule [45].

Figure 3.18 - Oxide spikes formed during high temperature fatigue in a nickel-base superalloy [223]. 128

Figure $3.19-\gamma^{\prime}$ depleted zone formed during high temperature fatigue in a nickel-base superalloy [223]. 128

Figure 3.20 - Small and long fatigue crack growth rates correlated with $\Delta \mathrm{K}$ for singlecrystal CMSX-2 tested at $950^{\circ} \mathrm{C}$ under creep-fatigue and continuous cycling conditions [45] 129

Figure 3.21 - Faceted crack growth on octahedral slip planes in single-crystal superalloy CMSX-2 tested at room temperature; a) profile view, and b) top view. Micrographs adapted from Reference [40]. 129

Figure 3.22 - High temperature fatigue crack growth along $\gamma-\gamma^{\prime}$ interface in the singlecrystal superalloy CMSX-2; a) profile view showing crack propagating along the $\gamma$ matrix channels, and b) fracture surface showing a propagating fatigue crack that is avoiding the $\gamma^{\prime}$ precipitates. a) adapted from Reference [45], and b) adapted from Reference [13]. 130

Figure 3.23 - Schematic defining the secondary orientation for a notched single-crystal fatigue specimen. Note: the direction denoted by the indices $<\mathrm{hkl}>$ represents the crystallographic direction perpendicular to the notch face.

130

Figure 3.24 - Scanning electron micrograph showing an oxidized fatigue crack in singlecrystal superalloy CMSX-2 [45]. 131

Figure 3.25 - Schematic of Stage II crack growth in vacuum [a, b, c] and in air [e, f, g] [69]. 131 
Figure 3.26 - Directionally coarsened $\gamma^{\prime}$ precipitates after IP and OP TMF testing: a) IP n-type (normal to applied stress) rafting, and b) OP p-type rafting (parallel to applied stress). Note the $\gamma^{\prime}$ phase appears dark in the micrographs. Adapted from Reference [221].

Figure 4.1 - Schematic definition of the back stress. The back stress, $\mathrm{X}$, causes a kinematic shift to the yield surface, $\Gamma(\mathrm{R})$, in deviatoric stress space [250]. 154

Figure 4.2 - Monotonic variation in yield strength of cubic single crystal materials predicted by the Hill yield function with $\mathrm{YS}_{[001]}=0.5 * \mathrm{YS}_{[11]}[233]$. 154

Figure 4.3 - Schematic illustrating the macroscopic definition of isotropic hardening. Isotropic hardening causes an expansion, $\mathrm{R}$, to the initial yield surface of radius $\sigma_{\mathrm{Y}}$. Note: The deformation response depicted in right half of the Figure shows the effect of isotropic hardening on reversed plastic loading [250]. 155

Figure 5.1 - PWA1484 test bars with large pores located near the top of the casting. .. 196

Figure 5.2 - Carleton University pilot scale Bridgman casting furnace. 196

Figure 5.3 - Schematic diagram showing major components of the Bridgeman casting furnace. 197

Figure 5.4 - Detailed view of Bridgman furnace cold zone. 198

Figure 5.5 - Detailed schematic of the single-crystal seed casting setup. 198

Figure 5.6 - Schematic showing the method used to mark the secondary orientation of the seed crystal test bar.

Figure 5.7 - Schematic showing the identification of the secondary orientation for the seed crystal from the longitudinal scribe lines added to the seed crystal test bar. 199

Figure 5.8 - Transverse section showing the dendrite structure in cube oriented SX PWA 1484 in the as-cast condition [262]. The arrows at the right indicate the crystal orientation with respect to the dendritic structure. 200

Figure 5.9 - Partial longitudinal cross section showing the seed crystal and new singlecrystal growth in a Bridgman cast single-crystal test bar. 201

Figure 5.10 - Composite optical micrograph showing a close up view of the longitudinal single-crystal dendritic macrostructure near the seed crystal. 202

Figure 5.11 - Optical micrograph showing the as-cast solidification structure in a transverse section cut from the middle of a single-crystal test bar. 203

Figure 5.12 - Backscatter electron micrograph showing the variation in $\gamma^{\prime}$ size within an individual primary dendrite core, the secondary dendrite arms and the interdendritic regions. 203

Figure 5.13 - Backscatter electron micrograph showing higher magnification view of the region indicated in Figure 5.12. The variation in $\gamma^{\prime}$ size between the primary dendrite core, secondary dendrite arms and the periphery of the dendritic structure is more apparent. 204 
Figure 5.14 - SEM micrograph showing the finer $\gamma^{\prime}$-phase located within the primary

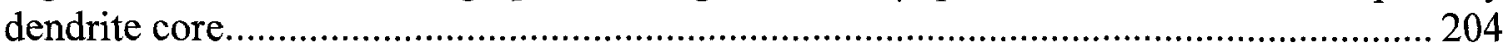

Figure 5.15 - SEM micrograph showing the larger $\gamma^{\prime}$-phase located between the dendrite core and interdendritic regions. 205

Figure 5.16 - SEM backscatter electron image showing the interdendritic constituents in as-cast PWA1484. 205

Figure 5.17 - DSC curves comparing PWA1484 material cast by PCC Airfoils to the Sophisticated Alloys version of PWA1484 after casting into single-crystal form in the Carleton University Bridgman casting facility. 206

Figure 5.18 - Composite BSE-SEM image showing the single-crystal macrostructure after heat treatment. 207

Figure 5.19 - SEM micrographs showing selected microstructural features after heat treatment; a) low magnification image showing residual $\gamma-\gamma^{\prime}$ eutectic phase and an shrinkage pore in the interdendritic regions, and $b$ ) higher magnification view showing $\gamma$ $\gamma^{\prime}$ eutectic phase.

208

Figure 5.20 - SEM micrographs showing $\gamma^{\prime}$-phase morphology after heat treatment; a) primary $\gamma^{\prime}$-phase, and b) secondary $\gamma^{\prime}$-phase.

Figure 5.21 - Experimental setup used for conducting strain-controlled constitutive material tests. 210

Figure 5.22 - Specimen geometry used for the constitutive material tests. Note: specimen dimensions are in inches. 210

Figure 5.23 - Constant total strain-rate engineering stress-strain tensile test curves for $<100>$ tensile axis Sophisticated Alloys PWA1484 alloy tested at $650^{\circ} \mathrm{C}$ 211

Figure 5.24 - Single-edge notch specimen geometry used for small fatigue crack growth characterization. Note: specimen dimensions are in inches. 212

Figure 5.25 - Schematic showing SEN specimen and details in the notch region for

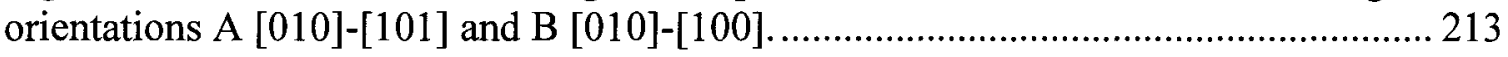

Figure 5.26 - General overview of mechanical test setup used for FCGR testing......... 214

Figure 5.27 - ACPD current and voltage probe arrangement. ...................................... 214

Figure 5.28 - Close-up view of the ACPD current and voltage probe arrangement ..... 215

Figure 5.29 - ACPD crack calibration specimen fracture surface. 215

Figure 5.30 - Recorded PD versus cycle data for an ACPD calibration test. Note: arrows indicate change from $\mathrm{R}=0.1$ to $\mathrm{R}=0.7$ loading to introduce a marker band................ 216

Figure 5.31 - Orientation A crack depth, $c$, versus PD change calibration curve.......... 217 Figure 5.32 - Orientation A half surface crack length, $a$, versus PD change calibration curve. 217

Figure 5.33 - Orientation A crack area versus PD change calibration curve................ 218 
Figure 5.34 - Orientation B crack depth, $c$, versus PD change calibration curve. 218

Figure 5.35 - Orientation B half surface crack length, $a$, versus PD change calibration curve. 219

Figure 5.36 - Orientation B crack area versus PD change calibration curve. 219

Figure 5.37 - Comparison of crack depth, $c$, versus PD change calibration curves for orientation $\mathrm{A}$ and $\mathrm{B}$ 220

Figure 5.38 - Orientation A crack aspect ratio, $a / c$, versus normalized crack depth, $c / W$. 220

Figure 5.39 - Orientation B crack aspect ratio, $a / c$, versus normalized crack depth, $c / W$. 221

Figure 5.40 - Comparison of crack aspect ratio calibration curves for orientations $\mathrm{A}$ and B. 221

Figure 5.41 - Comparison of crack area calibration curves for orientations A and B. .. 222

Figure 5.42 - Typical variation in working probe voltage versus cycles for a small fatigue crack growth rate test. 222

Figure 5.43 - Schematic showing method used to determine the onset PD voltage associated with fatigue crack growth. 223

Figure 6.1 - Engineering stress-strain curve for René N4 with [111] material orientation tested at $760^{\circ} \mathrm{C}$ using a double tensile test (total strain rates were $6.0 \times 10^{-4} \mathrm{sec}^{-1}$ and $\left.6.0 \times 10^{-4} \sec ^{-1}\right)$ [132]. 252

Figure 6.2 - Schematic showing the relationship between the material $\left(X^{\prime}, Y^{\prime}, Z^{\prime}\right)$ and global $(X, Y, Z)$ coordinate systems.

252

Figure 6.3 - Transformation from material cooridinate system $\left(X^{\prime}, Y^{\prime}, Z^{\prime}\right)$ and global cooridinate system $(X, Y, Z)$ with arbitrary miller indices [h $\mathrm{k} \mathrm{l}]$ along $Y$ axis using two rotations 253

Figure 6.4 - Schematic showing relationship between the cooridinate system transformation angles and the standard stereographic triangle. 253

Figure 6.5 - Comparison of predicted and experimental uniaxial tensile curves for loading along the $[010]$ crystallographic direction. 254

Figure 6.6 - Comparison of predicted and experimental uniaxial tensile curves for loading along the [111] crystallographic direction. Note: experimental data after Alden [132] 254

Figure 6.7 - Predicted first cycle stress-strain hysteresis loop for uniaxial loading along [010] crystallographic direction. 255

Figure 6.8 - Predicted first cycle stress-strain hysteresis loop for uniaxial loading along [111] crystallographic direction. 255

Figure 7.1 - Schematic of a notched rectangular bar with constant stress $\left(\sigma_{22}=\right.$ const.) or constant displacement $\left(\mathrm{u}_{2}=\right.$ const.) loading boundary conditions. 266 
Figure 7.2 - Schematic of a SEN specimen with threaded regions removed................ 267

Figure 7.3 - Finite element model for notch stress and strain field analysis................ 267

Figure 7.4 - Finite element mesh of the SEN specimen showing symmetry enforcing boundary conditions: a) $u_{3}=0$ on plane A-A (see Figure 7.2), and b) $u_{2}=0$ on plane B-B (see Figure 7.2). Note: highlighted nodes (in red) denote the nodes on which the constraints were applied. 268

Figure 7.5 - Finite element mesh of the SEN specimen showing the loading boundary conditions. A fixed $y$-displacement ( $\mathrm{u}_{2}=$ const.) was applied to the nodes highlighted in red. 269

Figure 7.6 - Finite element mesh showing the crack front defined by continuum brick elements. Note: the nodes defining the crack front are highlighted in red. 269

Figure 7.7 - Finite element mesh details for SEN specimens containing notch root semielliptical cracks: a) $c=200 \mu \mathrm{m}$ deep, $a / c=0.86$ and b) $c=1000 \mu \mathrm{m}, a / c=1.35$ cracks. Note nodes defining the crack front are highlighted in red. 270

Figure 7.8 - Schematic showing the conventional method for defining a contour by means of specifying a node set containing a single node (in the present case) for $\mathrm{J}$ integral calculations. Note: single crack front node is highlighted in red. 271

Figure 7.9 - Schematic showing contours automatically defined by ABAQUS for Jintegral calculations when multiple paths are requested (in this case 9 paths) [166]. Note: contour 1 is defined by the single node defining the crack front. 271

Figure 7.10 - Definition of the crack-front parametric angle, $\phi$, for a semi-elliptical crack. 272

Figure 7.11 - Finite element mesh of the SEN specimen showing y-direction $\left(\mathrm{u}_{2}=0\right)$ constraint applied to enforce the symmetry boundary condition. Note: nodes highlighted in red indicate the nodes that were constrained. 272

Figure 7.12 - Schematic definition of a local material orientation for orientation A SEN specimens. The local (material) coordinate system $X^{\prime}, Y^{\prime}, Z^{\prime}$ is defined by a rotation of the global coordinate system $X, Y, Z$ by an angle $\beta$ of $45^{\circ}$ about the global $Y$-axis. 273

Figure 8.1 - Contour plot showing the von Mises equivalent stress distribution at the notch root for isotropic linear elasticity. 303

Figure 8.2 - Contour plot showing the von Mises equivalent stress distribution at the notch root for orientation A with cubic anisotropic elastic properties. 303

Figure 8.3 - Contour plot showing the von Mises equivalent stress distribution at the notch root for orientation $\mathrm{B}$ with cubic anisotropic elastic properties. 304

Figure 8.4 - Calculated circumferential variation of von Mises equivalent stress distribution within the SEN specimen notch using linear elastic constitutive material behaviour. 304

Figure 8.5 - Finite element model of SEN specimen showing the paths used for extracting the stress distribution along the specimen thickness (z-direction) in the notch: a) isotropic and b) anisotropic cases. Note: red lines indicate the paths. 305 
Figure 8.6 - Calculated variation of the von Mises stress in the SEN specimen thickness direction using linear elastic constitutive material behaviour.................................... 306

Figure 8.7 - Maximum principal stress contour plot for orientation A. ....................... 306

Figure 8.8 - Maximum principal stress contour plot for orientation B. ...................... 307

Figure 8.9 - Calculated circumferential variation in the maximum principal stress. .... 307

Figure 8.10 - Maximum principal strain contour plot for orientation A..................... 308

Figure 8.11 - Maximum principal strain contour plot for orientation B........................ 308

Figure 8.12 - Equivalent plastic strain contour plot for orientation A. ....................... 309

Figure 8.13 - Equivalent plastic strain contour plot for orientation B. ....................... 309

Figure 8.14 - Equivalent plastic strain distribution within the SEN specimen notch.... 310

Figure 8.15 - Cumulative octahedral shear strain contour plot for orientation A......... 310

Figure 8.16 - Cumulative octahedral shear strain contour plot for orientation B........... 311

Figure 8.17 - Calculated circumferential variation in the cumulative octahedral shear

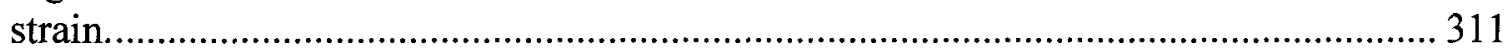

Figure 8.18 - Cumulative cubic shear strain contour plot for orientation A.................. 312

Figure 8.19 - Cumulative cubic shear strain contour plot for orientation B................. 312

Figure 8.20 - Calculated circumferential variation in the cumulative cubic shear strain.

Figure 8.21 - Convergence of the stress-intensity factors calculated along different crack front contours for $c=1.0 \mathrm{~mm} a / c=1.35$ notch root crack.

Figure 8.22 - Comparison of boundary correction factors for an $a / c=1.0, c=0.4 \mathrm{~mm}$ crack in the SEN specimen used in this investigation (ABAQUS), and results reported by Beres and Koul for a DEN specimen [269].

Figure 8.23 - Comparison of differences in boundary correction factors for an $a / c=1.0, c$ $=0.4 \mathrm{~mm}$ crack in the SEN specimen used in this investigation (ABAQUS), and results reported by Beres and Koul for a DEN specimen [269].

Figure 8.24 - Absolute values of the stress-intensity factor for the SEN specimen for $c=$ $0.2 \mathrm{~mm} a / c=0.86$ crack.

Figure 8.25 - Absolute values of the stress-intensity factor for the SEN specimen for $c=$ $1.0 \mathrm{~mm}$ a/c $=1.35 \mathrm{crack}$.

Figure 8.26 - Comparison of differences between the isotropic and anisotropic stressintensity factors for orientations $\mathrm{A}$ and $\mathrm{B}$ and $c=0.2 \mathrm{~mm} a / c=0.86 \mathrm{crack}$. 316

Figure 8.27 - Comparison of differences between the isotropic and anisotropic stressintensity factors for orientations $\mathrm{A}$ and $\mathrm{B}$ for $c=1.0 \mathrm{~mm} a / c=1.35 \mathrm{crack}$. 316

Figure 8.28 - Comparison of differences between orientations $\mathrm{A}$ and $\mathrm{B}$ anisotropic stressintensity factors for $c=0.2 \mathrm{~mm} a / c=0.86$ and $c=1.0 \mathrm{~mm} a / c=1.35$ crack geometries. 
Figure 8.29 - Path dependence of the J-integral evaluated from different crack front contours at the deepest part of the crack $\left(\phi=0^{\circ}\right)$, the notch surface $\left(\phi=90^{\circ}\right)$ and the crack front position where the calculated J-integral was highest $\left(\phi=67.75^{\circ}\right)$.

Figure 8.30 - Effect of crack-front mesh refinement on the J-integral for $c=1.0 \mathrm{~mm} a / c=$ 1.35 crack.

Figure 8.31 - J-integral variation along the crack front for $c=0.2 \mathrm{~mm} a / c=0.86$ crack. 318

Figure 8.32 - J-integral variation along the crack front for $c=1.0 \mathrm{~mm} \mathrm{a/c}=1.35$ crack.

Figure 8.33 - Comparison of differences between orientation $\mathrm{A}$ and $\mathrm{B}$ anisotropic elasticplastic J-integral values for $c=0.2 \mathrm{~mm} a / c=0.86$ and $c=1.0 \mathrm{~mm} \mathrm{a/c}=1.35$ crack geometries. 319

Figure 8.34 - Contour plot showing equivalent octahedral shear strain slip fields at the crack-tip ( $\phi=0^{\circ}$ crack-front position) for $c=0.2 \mathrm{~mm} \mathrm{a} / \mathrm{c}=0.86$ crack; a) orientation A and b) orientation B. Note: the approximate slip-sector angles are indicated on the contour plots 320

Figure 8.35 - Contour plot showing equivalent octahedral shear strain slip fields at the crack-tip $\left(\phi=90^{\circ}\right.$ crack-front position) for $c=0.2 \mathrm{~mm} a / c=0.86 \mathrm{crack}$; a) orientation A and b) orientation B. Note: the approximate slip-sector angles are indicated on the contour plots 321

Figure 8.36 - Contour plot showing equivalent cubic shear strain slip fields at the cracktip ( $\phi=0^{\circ}$ crack-front position) for $c=0.2 \mathrm{~mm} a / c=0.86 \mathrm{crack}$; a) orientation A and b) orientation $\mathrm{B}$. 322

Figure 8.37 - Contour plot showing equivalent cubic shear strain slip fields at the cracktip $\left(\phi=90^{\circ}\right.$ crack-front position) for $c=0.2 \mathrm{~mm} a / c=0.86 \mathrm{crack}$; a) orientation $\mathrm{A}$ and $\mathrm{b}$ ) orientation $\mathrm{B}$.

Figure 8.38 - Variation in the absolute anisotropic stress-intensity factor at the deepest part of the crack $\left(\phi=0^{\circ}\right)$ and the notch surface $\left(\phi=90^{\circ}\right)$ as function of crack depth for orientation $\mathrm{A}$ and $\mathrm{B}$. 324

Figure 8.39 - Variation in the J-integral at the deepest part of the crack $\left(\phi=0^{\circ}\right)$ and the notch surface $\left(\phi=90^{\circ}\right)$ as function of crack depth for orientation A and B. 324

Figure 9.1 - Back scatter electron micrographs showing representative crack paths on the notch surface; a) orientation A, b) orientation B. 338

Figure 9.2 - Scanning electron micrographs showing octahedral slip traces on the notch surface: a) orientation $\mathrm{A}$ and $\mathrm{b}$ ) orientation $\mathrm{B}$. 339

Figure 9.3 - Scanning electron micrographs showing secondary crack nucleation sites observed in the specimen notch: a) surface connected shrinkage porosity and b) subsurface nucleated crack breaking through the notch surface

Figure 9.4 - Schematic showing definition of parameters $\theta_{c}$ and $l_{c}$ used to characterize dominant fatigue crack position in the notch 341 
Figure 9.5 - Scanning electron micrographs showing representative fracture surfaces: a) single dominant fatigue crack in orientation $A$ fatigue specimen and b) specimen containing multiple (two) fatigue cracks (orientation B). Note: black arrow indicates the crack propagation direction and yellow arrows indicate crack nucleation sites. 342

Figure 9.6 - Secondary crack formation observed on fracture surfaces: a) $c \sim 200 \mu \mathrm{m}, \Delta \mathrm{K}$

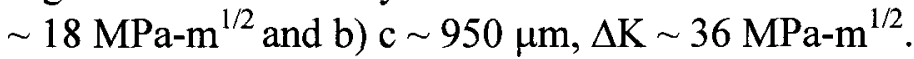
343

Figure 9.7 - Scanning electron micrographs showing typical crack nucleation sites: a) orientation $\mathrm{A}$ and $\mathrm{b}$ ) orientation $\mathrm{B}$. Note: black arrow indicates the crack propagation direction.

Figure 9.8 - Scanning electron micrographs showing representative details of orientation A SEN specimen fracture surface at different crack lengths. Note: arrow indicates the crack propagation direction.

345

Figure 9.9 Scanning electron micrographs showing representative details of orientation B SEN specimen fracture surface at different crack lengths. Note: arrow indicates the crack propagation direction. 346

Figure 10.1 - Stress range versus crack initation life for orientation A and B SEN fatigue specimens. 370

Figure 10.2 - Crack initiation life versus total microporosity area. 370

Figure 10.3 - Crack initiation life versus distance between subsurface micropore and the notch surface. 371

Figure 10.4 - Crack initation life as a function of the local maximum principal elastic/plastic stress acting at the subsurface pore associated with the dominant fatigue crack. 371

Figure 10.5 - Fatigue crack growth rate for orientation A SEN specimen as a function of the anisotropic stress-intensity factor range. Note: da/dN refers to crack growth rate at the notch surface and dc/dN is the crack growth rate at the deepest part of the crack. 372

Figure 10.6 - Fatigue crack growth rate for orientation B SEN specimen as a function of the anisotropic stress-intensity factor range. Note: $\mathrm{da} / \mathrm{dN}$ refers to crack growth rate at the notch surface and dc/dN is the crack growth rate at the deepest part of the crack. 372

Figure 10.7 - Fatigue crack growth rate for orientation A SEN specimen as a function of the anisotropic elastic-plastic J-integral. Note: da/dN refers to crack growth rate at the notch surface and dc/dN is the crack growth rate at the deepest part of the crack. 373

Figure 10.8 - Fatigue crack growth rate for orientation B SEN specimen as a function of the anisotropic elastic-plastic J-integral. Note: da/dN refers to crack growth rate at the notch surface and dc/dN is the crack growth rate at the deepest part of the crack. 373

Figure 10.9 - Composite of crack growth curves for orientation A SEN specimens. The mean crack growth rate is indicated by red circles and the error bars denote two standard deviations from mean. Note: $\Delta \mathrm{K}$ was obtained from anisotropic elastic analysis. 374 
Figure 10.10 - Composite of crack growth curves for orientation B SEN specimens. The mean crack growth rate is indicated by red circles and the error bars denote two standard deviations from mean. Note: $\Delta \mathrm{K}$ was obtained from anisotropic elastic analysis......... 374

Figure 10.11 - Variation of the fatigue crack growth rate as a function of the stressintensity factor range. Note: $\Delta \mathrm{K}$ was obtained from anisotropic elastic analysis.......... 375

Figure 10.12 - Comparison of mean crack growth rate curves for orientation A and B SEN specimens. The error bars denote two standard deviations from the mean crack growth rate for each orientation. Note: $\Delta \mathrm{K}$ was obtained from anisotropic elastic analysis. 375

Figure 10.13 - Schematic depicting LEFM life prediction methodology: a) embedded elliptical pore in SEN specimen, b) growth of embedded elliptical crack in strip with local stress range obtained from elastic/plastic FEM model and c) semi-elliptical crack in notch. 376

Figure 10.14 - Comparison of predicted fatigue lives for orientation A and B SEN specimens. 


\section{List of Tables}

Table 2.1 - Chemical compositions (in weight $\%$ ) for common first-generation singlecrystal superalloys.

Table 2.2 - Chemical compositions (in weight\%) for common second-generation singlecrystal superalloys.

Table 2.3 - Chemical compositions (in weight\%) for common third-generation singlecrystal superalloys.

Table 2.4 - Chemical compositions (in weight\%) for common fourth-generation singlecrystal superalloys.

Table 4.1 - Slip plane normal and slip directions for the 12 FCC octahedral slip systems. 153

Table 4.2 - Slip plane normal and slip directions for 6 FCC cubic slip systems. 153

Table 5.1 - Target composition range for PWA1484 and the actual composition of the master alloy material (heat \#01091032A) received from Sophisticated Alloys. 195

Table 5.2 - ACPD power law calibration curve fitting parameters............................... 195

Table 5.3 - ACPD Crack aspect ratio calibration curve fitting parameters..................... 195

Table 6.1 - Elastic stiffness constants and compliance coefficients (in Voigt notation) determined from the [010] and [111] tensile curves................................................ 251

Table 6.2 - Inelastic material parameters for octahedral and cubic slip........................ 251

Table 7.1 - Summary of SEN specimen dimensions from Figure 5.24 ....................... 264

Table 7.2 - Crack sizes for the finite element models used to estimate the elastic and

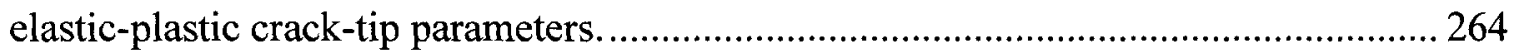

Table 7.3 - Elastic material properties used in the isotropic linear elastic FEM calculations. 265

Table 8.1 - Linear elastic stress concentration factors and location of maximum von Mises equivalent stress. 301

Table 8.2 - Finite element model parameters for validation of extracted SIF's from ABAQUS/Standard using non-singular crack-tip elements. 301

Table 8.3 - Calculated anisotropic stress-intensity factors for a crack depth range of 0.2 to $1.0 \mathrm{~mm}$ for orientations $\mathrm{A}$ and $\mathrm{B}$.

Table 8.4 - J-integral for a crack depth range of 0.2 to $1.0 \mathrm{~mm}$ for orientations A and B based on the micro-phenomenological constitutive model. 302

Table 8.5 - Fourth order polynomial coefficients for computation of the stress-intensity factors and J-integral at the deepest part of the crack $\left(\phi=0^{\circ}\right)$ and the notch surface $(\phi=$ $90^{\circ}$ ). Note: In equations 8.6 and 8.7 the force must be specified in $\mathrm{N}$ and geometrical parameters must be in $\mathrm{mm}$ to return the correct values for $\Delta \mathrm{K}$ and the J-integral. ........ 302 
Table 9.1 - Crack positions in the specimen notch. Note: $\theta_{\mathrm{c}}$ represents the angular position from the minimum specimen cross section (the notch root) and $l_{c}$ represents the distance from the center line of the specimen to the mid-point of the surface crack. .... 337

Table 9.2 - Size of subsurface pores that nucleated fatigue cracks and their proximity to the SEN specimen notch surface. Note: the subscripts 1 and 2 denote the primary and secondary crack nucleation sites.................................................................................... 337

Table 10.1 - Crack initation lives for each fatigue specimen......................................... 368

Table 10.2 - Paris law parameters for the mean and upper and lower bound fatigue crack growth rate curves. 368

Table 10.3 - Comparison of predicted, $\mathrm{N}_{\mathrm{p}}$, and actual, $\mathrm{N}_{\mathrm{a}}$, crack initation lives. Note: number in parenthses indicates the life spent as a subsurface fatigue crack and asterisk indicates subsurface life for the dominant subsurface crack. 369 


\section{Nomenclature}

General Notation

$$
\begin{aligned}
\left.\int\right]^{T} & : \text { matrix transpose } \\
{[]^{-1} } & : \text { matrix inverse } \\
: & : \text { tensor product contracted twice } \\
\otimes & : \text { tensor product } \\
\dot{\omega} & : \text { time derivative of } \omega \\
\Delta \omega & : \text { increment of } \omega, \text { or range of } \omega \\
\partial / \partial \omega & : \text { partial derivative } \\
d / d \omega & : \text { total derivative }
\end{aligned}
$$

Specific Variables or Functions

$10^{4 b}$ : fatigue crack growth rate ratio

a : crack size or half surface crack length

$a_{y}$ : : gamma prime phase lattice parameter

$a_{y}$ : gamma phase lattice parameter

$a_{i j}:$ second-order elastic compliance tensor in concise tensor notation

$\frac{a}{c}, \frac{c}{a}:$ crack aspect ratio

$A^{\prime} \quad$ : pre-exponential constant

$A$ : tensor transformation matrix

ACPD : alternating current potential drop
APB : anti-phase boundary
$\alpha \quad$ : primary crystallographic orientation 
$\alpha_{g} \quad$ : stress-intensity factor geometry parameter

$b \quad$ : standard deviation from mean fatigue crack growth rate

$b_{c} \quad$ isotropic hardening rate constant for cubic slip

$b_{o} \quad$ : isotropic hardening rate constant for octahedral slip

$B$ : pre-logarithmic energy matrix

BSE : back scatter electron

$\beta$ : secondary crystallographic orientation

c : crack size or crack depth

$c_{c} \quad$ : kinematic hardening modulus for cubic slip

$c_{o}$ : kinematic hardening modulus for octahedral slip

$c^{[i]^{\prime}} \quad$ : kinematic hardening modulus regression coefficient $(i=1,2)$

$C$ : Paris law intercept

C. $C_{i j k l} \quad$ : fourth-order elastic stiffness tensor

$C_{i}$ : polynomial coefficient

$C_{1}$ : PD calibration curve power law constant

CC : conventionally cast

$\mathrm{CO}_{2}$ : carbon dioxide

CRSS : critical resolved shear stress

$\mathrm{C}(\mathrm{T})$ : compact tension

$\chi \quad$ : second-order back stress tensor

$\frac{d a}{d N}, \frac{d c}{d N}$ : fatigue crack growth rate

$d_{c} \quad$ : kinematic hardening recall term for cubic slip 
$d_{o}$ : kinematic hardening recall term for octahedral slip

$d_{p} \quad$ : subsurface pore depth from notch surface

$d^{[1]^{\prime}}$ : kinematic hardening recall term regression coefficient

DEN : double-edge notch

DS : directionally solidified

DSC : differential scanning calorimetry

$\delta$ : lattice misfit parameter

$\delta_{i j} \quad:$ Kronecker delta

$E \quad$ : elastic modulus (isotropic material)

$E^{\prime} \quad$ : constraint corrected elastic modulus

$E_{[010]}:$ apparent elastic modulus for [010] crystallographic direction

$E_{[h k l]}:$ apparent elastic modulus for [hkl] crystallographic direction

EBSD : electron backscatter diffraction

EDS : energy dispersive spectroscopy

EPFM : elastic-plastic fracture mechanics

$\varepsilon, \varepsilon_{i j} \quad:$ second-order total strain tensor

$\varepsilon^{e l} \quad$ : second-order elastic strain tensor

$\varepsilon^{e q} \quad$ : cumulative inelastic strain

$\varepsilon_{[h k l]} \quad$ : uniaxial strain along [hkl] crystallographic direction

$\varepsilon_{[010]}^{i n}:$ uniaxial inelastic strain along [010] crystallographic direction

$\varepsilon_{[111]}^{i n}:$ uniaxial inelastic strain along [111] crystallographic direction $\varepsilon^{i n}:$ second-order inelastic strain tensor 


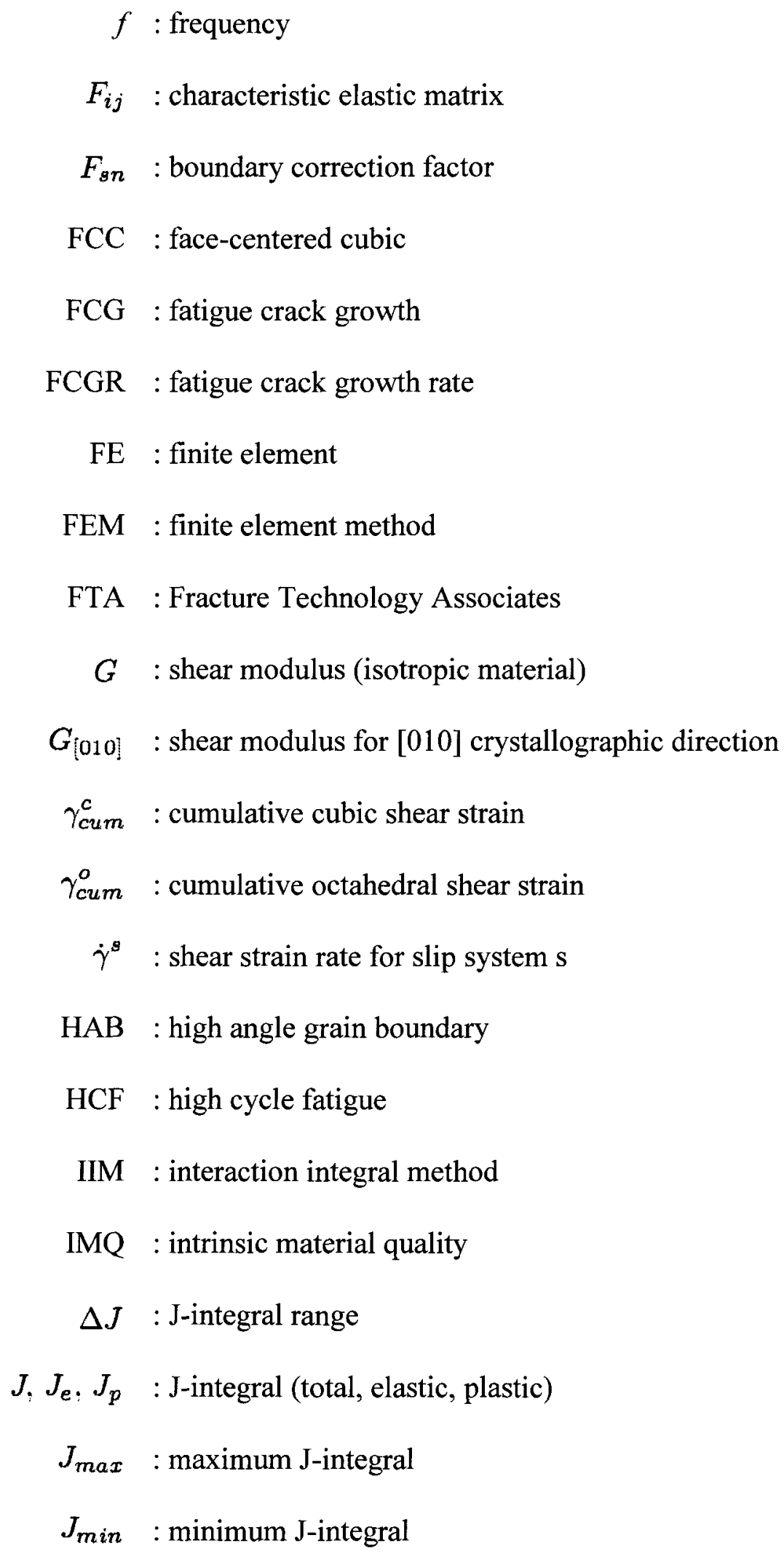


$J_{\text {int }}^{\alpha} \quad$ : J-integral interaction component mode ( $\alpha$ ) I, II, or III

$J_{\text {tot }}^{\alpha} \quad$ : J-integral total component mode ( $\alpha$ ) I, II, or III

$J_{a u x}^{\alpha} \quad$ : J-integral auxiliary component mode ( $\left.\alpha\right)$ I, II, or III

$k^{\prime}$ : critical resolved shear stress regression coefficient

$k_{c} \quad$ : critical resolved shear stress for cubic slip

$k_{o}:$ critical resolved shear stress for octahedral slip

$K \quad$ : stress-intensity factor, scalar drag stress

$K_{c} \quad$ : cubic slip drag stress

$K_{f} \quad$ : stress concentration factor

$K_{o} \quad$ : octahedral slip drag stress

$\Delta K \quad$ : stress-intensity factor range

$\Delta K_{\text {th }}:$ threshold stress-intensity factor

$K_{\mathrm{C}} \quad$ : critical stress-intensity

$K_{I}, K_{I I}, K_{I I I}$ : stress-intensity factors (mode I, II and III)

$K_{I C} \quad:$ Mode I critical stress-intensity

$K_{m a x}:$ maximum stress-intensity factor

$K_{\min }:$ minimum stress-intensity factor

$K_{O C T}:$ octahedral stress intensity factor on a slip system

$K_{o p} \quad$ : crack opening stress-intensity factor

$K_{r n s}:$ resolved normal stress intensity factor on a slip system

$K_{r s s} \quad$ : resolved shear stress intensity factor on a slip system

$l_{c}:$ crack position from specimen centerline 
$l_{\mathrm{i}} \quad$ : unit slip direction vector

$l_{i}^{s}:$ unit slip direction vector for slip system $\mathrm{s}$

$L_{1} \quad$ : PD calibration curve linear fit coefficient

$L_{2} \quad$ : PD calibration curve linear fit coefficient

LAB : low angle grain boundary

LEFM : linear-elastic fracture mechanics

LCF : low cycle fatigue

LOESS : locally weighted scatter smoothing

$m$ : Paris law slope

$m_{\mathrm{ij}} \quad$ : generalized Schmid factor

$m^{s}$ : generalized Schmid factor for slip system $\mathrm{s}$

$M$ : fourth-order anisotropic yield tensor

$M \quad$ : mean fatigue crack growth rate

$\mu_{i} \quad$ : roots of characteristic equation

$n$ : viscosity exponent

$\boldsymbol{n}$ : flow direction

$n_{1} \quad$ : PD calibration curve power law exponent

$n_{c} \quad$ viscosity exponent for cubic slip

$n_{i} \quad$ : outward normal vector, unit slip plane normal vector

$n_{i}^{s} \quad$ : unit slip plane normal vector for slip plane s

$n_{o} \quad$ : viscosity exponent for octahedral slip

$\boldsymbol{N}$ : anisotropic kinematic hardening tensor 
NDI : non-destructive inspection

$\mathrm{NO}_{\mathrm{x}}$ : oxides of Nitrogen

N-R : Newton-Raphson

NRC-IAR : National Research Council - Institute for Aerospace Research

$\nu \quad:$ Poisson ratio

$\nu_{[010]}:$ Poisson ratio for [010] crystallographic direction

$\Omega \quad$ : viscoplastic potential function

$\dot{p} \quad$ : cumulative inelastic strain rate

$P \quad:$ load

$P_{\max } \quad$ : maximum load

PD : potential drop

$P D_{0} \quad$ : initial potential drop

$P D_{N}$ : potential drop for cycle $\mathrm{N}$

PDAS : primary dendrite arm spacing

PSB : persistent slip band

P\&WA : Pratt \& Whitney Aircraft

$\phi:$ crack front parametric angle

$\psi \quad$ : material orientation angle

$q_{c} \quad$ : isotropic hardening constant for cubic slip

$q_{o}$ : isotropic hardening constant for octahedral slip

$Q$ : elliptical crack shape parameter, activation energy

$Q \quad$ : anisotropic kinematic hardening recall tensor 
$Q_{i j} \quad$ : coordinate transformation tensor

$r \quad:$ distance from crack tip

$r_{o}^{s} \quad$ : isotropic hardening state variable for octahedral slip system $\mathrm{s}$

$r_{c}^{s}$ : isotropic hardening state variable for cubic slip system $\mathrm{s}$

$r_{p} \quad$ : crack tip plastic zone size

$R \quad$ : stress ratio, universal gas constant, notch radius

$[R] \quad:$ Reuter matrix

rpm : revolutions per minute

Re : real component of a complex number

RSS : resolved shear stress

$\boldsymbol{s}, s_{i j}$ : second-order stress deviator tensor

$\boldsymbol{S}$ : fourth-order compliance tensor

SEM : scanning electron microscope

SEN : single-edge notch

SFE : stacking fault energy

SIF : stress-intensity factor

SII : safe inspection interval

$\sigma_{0} \quad$ : far-field stress

$\sigma_{[010]}$ : uniaxial stress along [010] crystallographic direction

$\sigma_{[111]}$ : uniaxial stress along [111] crystallographic direction

$\sigma_{[h k l]}$ : uniaxial stress along [hkl] crystallographic direction

$\sigma_{i j}, \boldsymbol{\sigma}:$ second-order stress tensor 


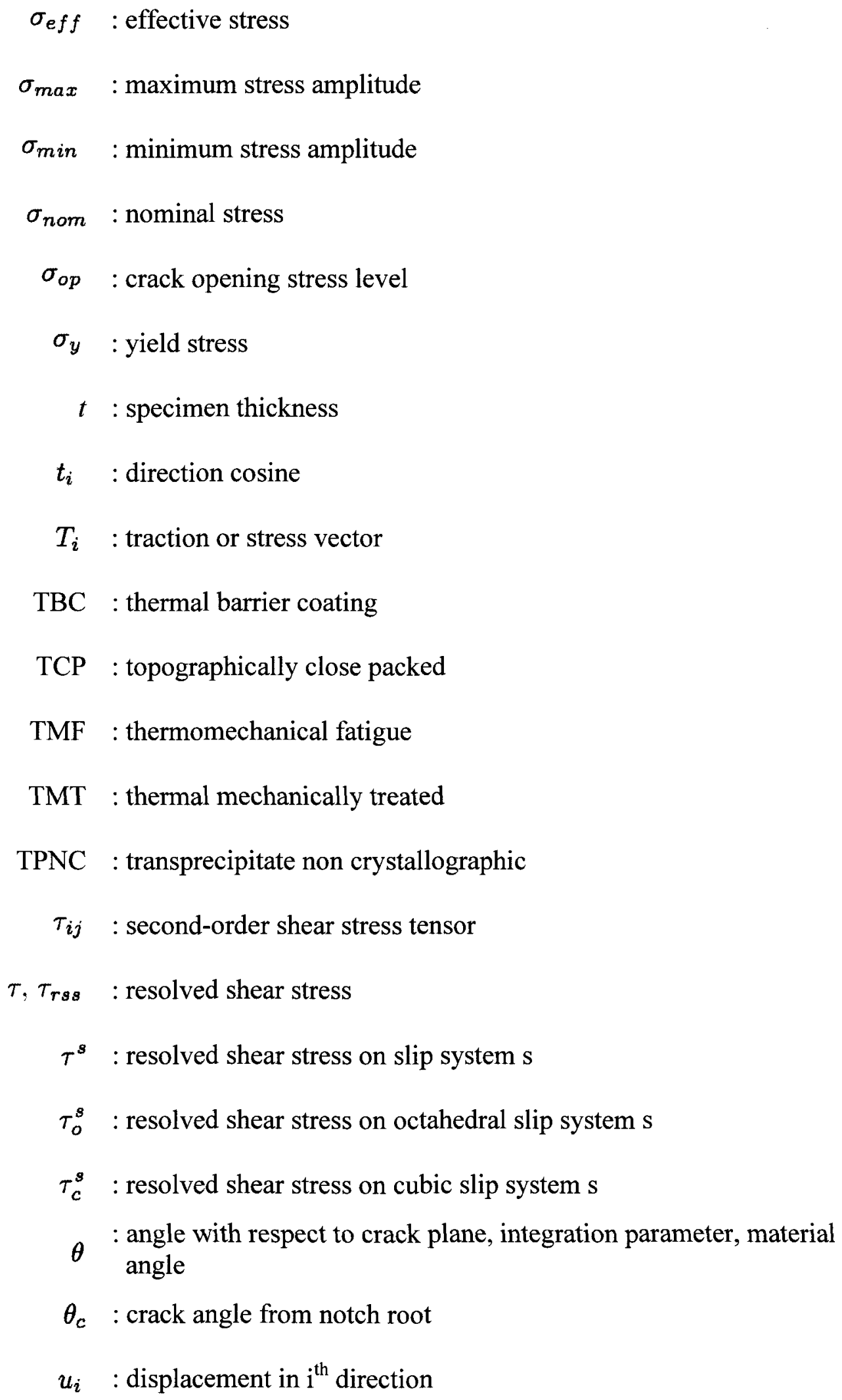




$$
\begin{aligned}
\mathrm{V}_{\mathrm{f}} & : \text { volume fraction } \\
V_{\mathrm{ref0}} & : \text { initial PD reference voltage } \\
V_{\mathrm{ref}} & : \text { instantaneous PD reference voltage } \\
\mathrm{VCE} & : \text { virtual crack extension } \\
\Delta W & : \text { total strain energy density range } \\
W & : \text { total strain energy density, width of finite fatigue specimen } \\
x_{c}^{s} & : \text { back stress state variable for cubic slip system s } \\
x_{i}, X_{i} & : \text { Cartesian coordinate axis } \\
x_{o}^{s} & : \text { back stress state variable for octahedral slip system s } \\
Y\left(\frac{a}{W}\right) & : \text { finite geometry correction factor }
\end{aligned}
$$




\section{Introduction}

\subsection{Research Background}

Driven by the need to meet increasingly strict legislative requirements for acoustic, $\mathrm{NO}_{\mathrm{x}}$ and $\mathrm{CO}_{2}$ emissions, civil aircraft engine manufactures have had to make fundamental changes to civil gas turbine propulsion system designs. As indicated in Figure 1.1 these changes include, among others, a larger diameter fan to achieve a higher bypass ratio and propulsive efficiency, and a smaller engine "core" with increased pressure ratio and turbine inlet temperature to achieve a higher thermal efficiency. As a result, components in gas turbine engines operate at higher stress and temperature regimes, which places more stringent demands on materials selection to meet performance and durability requirements. The problem of materials selection can be better appreciated by considering the relative temperature and pressure distribution inside a civil turbofan engine, as shown in Figure 1.2. Referring to this figure, it can be observed that the first stage turbine blades operate in an extremely hostile environment comprising of high rotation speeds $(>12,000 \mathrm{rpm})$, high temperatures and erosive and corrosive byproducts of combustion. Consequently, it is the materials used to manufacture the first stage turbine blades which restrict additional increases to the turbine inlet temperature needed to increase thermal efficiency and limits the durability of the engine.

The first stage turbine blades and vanes in gas turbine engines are increasingly manufactured from single-crystal nickel-base superalloys. The nickel-base single-crystal superalloys represent a special group of alloys that utilize directional solidification and have highly optimized alloy chemistries to maximize high temperature mechanical properties. These alloys are primarily used in applications requiring the higher creep, fatigue, and oxidation resistance than provided by directionally solidified, conventionally 
cast or wrought nickel-base superalloys. Since their introduction to gas turbine engines in the 1980's, single-crystal superalloy development has primarily focused on increasing creep resistance by adjusting alloy chemistry to reduce bulk diffusion kinetics, reduce microsegregation and improve phase stability. While single-crystal superalloy alloy design continues to focus on enhancing creep resistance, the fatigue properties of the single-crystal superalloys have not been subject to the same degree of scrutiny. Although it has been demonstrated that alloy chemistry changes that impart higher creep resistance result in more fatigue tolerant materials [1], instances of fatigue failure in single-crystal components are becoming more common [1] and it is the leading cause of failure for hot section components in military aircraft engines [2]. Consequently, recent research has been more closely examining fatigue failure mechanisms in single-crystal superalloys for the purpose of developing life prediction models to estimate the component life and to develop more robust component designs.

\subsection{Characteristics of Fatigue in Turbine Blades}

As shown in Figure 1.3, turbine blades consist of an airfoil section and a serrated region, referred to as the blade root, which provides a mechanical coupling between the blade and rotating disc assembly. In between the airfoil section and the blade root is a platform which functions to prevent hot gas from entering the space between turbine discs, and to provide vibration damping. The airfoil section of the first-stage turbine blades in modern gas turbine designs can be continuously exposed to gas temperatures up to $1700^{\circ} \mathrm{C}$, but blade cooling and thermal barrier coatings are used to reduce the bulk metal temperature to less than $1100^{\circ} \mathrm{C}$. The temperature in the blade root region is primarily limited by the temperature capability of the disc material, and as a result it is limited to 550 to $760^{\circ} \mathrm{C}$. As indicated in Figure 1.4, the stresses induced by centrifugal, gas bending and thermal 
loads are relatively lower in the airfoil section than the blade root. Because the blade airfoil and root sections are exposed to different temperature and stress regimes, further consideration should be given to the nature of fatigue failures in the blade root and airfoil sections.

The first stage turbine blades in modern gas turbine engines are hollow thin walled structures containing serpentine cooling passages and film cooling holes, as shown in Figure 1.5 , through which a portion of the compressor discharge airflow is directed to lower the bulk metal temperature. In addition to introducing stress-raising features to the component geometry, the application of continuous blade cooling causes substantial and localized temperature gradients. Therefore, engine designers must not only consider the mechanical loads imposed by centrifugal forces and the airfoils pressure distribution, but also the thermal stresses induced by these temperature gradients. During the aircraft's mission, the thermal and mechanical loads in the airfoil section change due to temperature and rotational speed excursions contributing to fatigue damage. The fatigue damage that occurs under such conditions is called thermomechanical fatigue (TMF), and it is the primary cause for fatigue failures in the airfoil section in engines subjected to repeated start-stop cycles [3].

The turbine blade root consists of one or more serrated teeth that provide a mechanical coupling between the turbine blade and disc. Fatigue damage in the blade root occurs by several different mechanisms and is aided by the stress-raising features of the blade root. The first fatigue failure mechanism to consider is high-cycle fatigue, which is cased by high-frequency vibrations that contribute a small alternation stress component that is superimposed with the large mean stress component arising from centrifugal forces on the component. In addition to high-cycle fatigue (HCF), engine start-up and shut-down 
cycles and transient engine operation cause large fluctuations in stress amplitude that contribute to so-called low-cycle fatigue (LCF) damage. The last fatigue failure mechanism, called fretting fatigue, involves the high contact stresses generated at the interfaces between the blade and disc serrations and the relative motions that occur between these contact surfaces during transient engine operation. The subject of lowcycle fatigue and prediction of fatigue life in the blade root notch features are of significant concern to designers for two reasons. First, single-crystal superalloy microstructures and chemistry were developed to optimize creep, fatigue and oxidation resistance in the airfoil section and not the blade root. Secondly, since life prediction methodologies that consider fatigue in notched bodies has been primarily developed for equiaxed grained isotropic materials, it is unclear what the effects are of the anisotropic properties on notch fatigue resistance in single-crystal superalloys. This later point will be considered further in the following section.

\subsection{Life Prediction and Fatigue in Single-Crystal Superalloys}

Life prediction of gas turbine hot section components fabricated from single-crystal superalloys is a major engineering challenge since many factors must be considered including [4]:

1. Component geometry

2. Component temperature distributions

3. Mechanical loading

4. Material property variation with temperature

5. Material orientation

In addition to these factors, single-crystal superalloys exhibit complex elastic-plastic anisotropy, and therefore load and material orientation effects must be considered in the analysis. The deformation and fatigue failure mechanisms of single-crystal superalloys 
are notably different than poly-crystalline materials and depend on loading frequency, temperature and crystallographic orientation. Extensive material testing is necessary to adequately characterize and establish the effects of these factors on the creep and fatigue properties for single-crystal materials. In cases where inelastic material behaviour is the primary design consideration, sophisticated elastic-plastic constitutive material laws are also needed to estimate component stresses and strains, which are inputs to life prediction algorithms.

In single-crystal turbine blades the material orientation is specified by two angles, alpha $(\alpha)$ and beta $(\beta)$, as shown schematically in Figure 1.6. Alpha represents the angle between the $\langle 100\rangle$ crystal directions and the airfoil stacking line and specifies the primary orientation of blade. The primary orientation is carefully controlled during the production process to ensure that it is closely aligned with the $\langle 100\rangle$ crystal direction as this orientation has been shown to give the best mechanical properties in single-crystal superalloys [5]. Due to variability in the manufacturing process deviations of up to $10^{\circ}$, i.e. $\alpha=10^{\circ}$, are permitted in single-crystal turbine blades. The second angle, $\beta$, specifies the angle between a plane parallel to the serrated blade root and the $\langle 100\rangle$ crystal directions, and represents the secondary orientation of the turbine blade. The control of secondary orientation is not subject to the same scrutiny as the primary orientation. In many cases the secondary orientation is neither controlled nor specified and therefore is a random variable in the manufacturing process [6]. Because of material anisotropy effects on the material constitutive deformation response, the primary and secondary orientation must be considered in the analysis and life-prediction of single-crystal turbine blades. 
At blade root temperatures inclusions such as carbides and/or borides and intrinsic material quality (IMQ) defects such as porosity play a dominant role in fatigue crack formation in single-crystal superalloys [7]. Their occurrence depends on many factors including superalloy composition, casting process parameters, application of heat treatments and subsequent processing steps such as hot isostatic pressing. Incompatibility of deformation between inclusions and the local stress-raising effect of porosity can lead to local plastic deformation even when the applied loading is significantly lower than the macroscopic yield strength of the material. Under cyclic loading this local plasticity can lead to the formation of fatigue cracks. If sufficient driving force is available the fatigue cracks can continue to propagate leading to component failure. A rigorous understanding of the role of these material discontinuities on crack formation and propagation are essential to accurate component life prediction.

In general, two life prediction methodologies have been established for minimizing the occurrence of gas turbine component failures, safe-life and damage tolerance. In the safelife approach, the fatigue life is defined as the number of cycles to form an engineering crack (usually $760 \mu \mathrm{m}$ ) with a 1 in 1000 probability of occurrence [8]. The fatigue life correlations are derived from the macroscopic stress or strain. Additional factors that affect the fatigue life such as the macroscopic stress concentrations, multiaxial stress or strain states, material anisotropy, etc. are treated empirically. The effect of material discontinuities are not explicitly considered in the safe-life approach although it is thought that the effects of these defects are accounted for by design factors and using the statistical lower bound to define the fatigue life [8].

The damage tolerance approach recognizes that initial flaws exist in the form of cracks before the component enters into the service. Therefore, crack propagation resistance is 
one of the primary concerns for the component to endure the expected service cycles [9]. In practice, it is assumed that fatigue crack propagation occurs immediately from discontinuities, i.e. there is no incubation period to form a fatigue crack. Damage tolerant life assessments are undertaken using fatigue crack propagation data in association with fracture mechanics analyses to ensure that fatigue cracks will not grow to such a size as to result in catastrophic failure, which has to be assured by periodic inspections.

More recently, a new lifing paradigm - the holistic structural integrity concept—has been proposed [10] to unify the safe-life and damage tolerance concepts by considering the continuous evolution from crack nucleation to small and large fatigue cracks. The advantage is to render a physics-based description of the material discontinuity to ensure safe extension of the components useful life. This approach is adopted in the present investigation.

Numerous researchers have investigated the effects of crystallographic orientation, temperature, loading frequency, microstructure, and environmental effects on fatigue crack propagation behaviour of long fatigue cracks in single-crystal superalloys [11-30]. From these studies it has been established that fatigue cracks can propagate by noncrystallographic or crystallographic mechanisms depending on the temperature, loading frequency, material orientation and applied stresses. The subject of small (or short) fatigue crack growth in materials is also of significant academic and practical interest because the fatigue crack nucleation and small crack propagation phases of crack formation constitute the majority of the fatigue life in highly stressed components [8]. In fact it has been established from experiments that growth of fatigue cracks less than 300 $\mu \mathrm{m}$ in length can constitute $60-80 \%$ of fatigue life for naturally-generated fatigue cracks in single-crystal superalloys [31, 32]. From an applications viewpoint, turbine blades are 
highly stressed and physically small, and therefore one can expect that the critical crack sizes that result in catastrophic failure will be much smaller than those considered for long crack growth.

There have been a number of experimental studies in the literature concerning the fatigue properties of small or short fatigue cracks in single-crystal superalloys obtained from uniaxial fatigue specimens with the loading direction aligned with the $\langle 100\rangle$ crystal direction [26, 33-47]. In general, it has been reported at blade attachment temperatures that fatigue crack growth tends to occur macroscopically on $\{001\}$ planes normal to the loading direction, i.e. Mode I or Stage II non-crystallographic crack growth. Transitions from Stage II to Stage I $\{111\}$ crystallographic crack growth occurs at higher stress intensities and results in the formation of localized or extended $\{111\}$ crystallographic facets. For naturally-generated fatigue cracks in a notch root, fatigue cracks were reported to form at surface connected and subsurface interdendritic shrinkage porosity [48]. The incubation phase associated with the formation of propagating small fatigue cracks in a notch root is negligible, and the majority of life is spent propagating small fatigue cracks from these initial material discontinuities [33]. Considerable variability in fatigue lives is observed in smooth and notched fatigue specimens, which is generally thought to be associated with the size and distribution of these initial material discontinuities.

A number of researchers have also investigated the effect of secondary crystallographic orientation on the propagation behaviour of small fatigue cracks in fatigue specimens with unaxially loaded along the $\langle 001\rangle$ crystal direction. Some researchers $[33,34,40]$ have reported that secondary crystallographic orientation has a significant effect on small crack growth behaviour, while other researchers have concluded that no effect was 
apparent $[36,47]$. In studies that have reported secondary orientation effects on the fatigue crack propagation behaviour of small fatigue cracks, crack growth rates were highest in specimens with a $\langle 100\rangle$ secondary crystallographic orientation.

Researchers studying small crack growth in single-crystal superalloys have predominantly focused on physically short through-thickness fatigue cracks formed by various fatigue pre-cracking and load shedding procedures. Small fatigue cracks generated by these techniques have prior deformation history contributing to plasticity and/or roughness induced crack closure. One reason that small fatigue cracks are believed to behave anomalously with respect to long fatigue cracks is that crack closure effects take time to develop for naturally-generated small fatigue cracks [49-51]. On the other hand, small fatigue cracks generated using load shedding procedures may be considered physically short, but in reality they are distinctly different than naturally-generated small fatigue cracks since the crack closure levels develop differently. The behaviour of naturally-generated small fatigue cracks is considered in this investigation.

Fatigue crack growth rate data for small and long fatigue cracks are usually presented as crack growth rate as function of the stress-intensity factor range $(\Delta K)$ established from linear-elastic fracture mechanics (LEFM). Some researchers [31, 33, 34, 38, 41, 47, 52] have explicitly assumed that isotropic elastic stress intensity factors could be used to characterize short crack growth behaviour in single-crystal superalloys. Chan and Cruise [53] have performed numerical calculations, assuming isotropic and anisotropic elasticity to estimate $K$, that indeed show that this assumption is valid for long cracks in compact tension specimens that are inclined less than $30^{\circ}$ to a plane normal to the applied loading. Tinga $[54,55]$ has recently presented finite element results for thumbnail (surface) cracks in corner crack specimens, assuming isotropic and anisotropic elasticity, that show elastic 
anisotropy has a significant effect on $K$ estimates. His results suggest elastic anisotropy must be taken into consideration for naturally-generated small fatigue cracks in singlecrystal superalloys, which can be considered a surface crack problem. The application of elastic-plastic fracture mechanics (EPFM), specifically the J-integral, to small cracks emanating from notches in single-crystal superalloys was recently considered by Zhao and co-workers [56]. However, the application of EPFM to characterize small crack growth in single-crystal superalloys has not been demonstrated. This warrants further investigation specifically in regard to naturally-generated fatigue cracks from macroscopic notches, such as those that occur in the highly-stressed attachment region of single-crystal turbine blades.

\subsection{Thesis Objectives and Scope}

This thesis is part of a collaborative research project established between Carleton University and the National Research Council of Canada Institute for Aerospace Research (NRC-IAR). The primary goal of this research is to investigate the notched fatigue behaviour of single-crystal superalloys and use the lessons learned to develop and improve life prediction methods for single-crystal superalloy components. With this in mind, the main objectives of this investigation include:

1. To identify the critical factors controlling the notch fatigue life of single-crystal superalloys.

2. To investigate the effect of secondary crystallographic orientation on the growth of small fatigue cracks and characterize their behaviour by conducting fatigue crack growth rate tests on a representative single-crystal superalloy used to manufacture turbine blades. 
3. To investigate the application of LEFM and EPFM parameters to characterize the small crack growth behaviour for notch root fatigue cracks.

\subsection{Organization of the Thesis}

This thesis consists of eleven chapters. In Chapter 1 sections 1.1, 1.2, 1.3 and 1.4 provide an introduction to present research. An overview of single-crystal casting technology, single-crystal superalloys and their microstructural features, and a review of mechanical properties and deformation mechanisms in single-crystal superalloys are discussed in Chapter 2. In Chapter 3 the basic theoretical background on LEFM and EPFM, the application of finite element methods (FEM) to fatigue and fracture problems, the small crack problem, and the characteristics and factors affecting small crack growth in singlecrystal superalloys are discussed. The theoretical framework for elastic-plastic constitutive models based on continuum and micro-mechanical crystallographic formulations for single-crystal materials and numerical solution procedures is reviewed in Chapter 4 . The experimental details, including single-crystal alloy selection, the singlecrystal casting process, details of the fatigue crack growth rate tests, the alternating current potential drop (ACPD) system and calibration procedures, and constitutive material tests are described in Chapter 5. In Chapter 6 the single-crystal constitutive model used for conducting elastic-plastic FEM simulations and its implementation within the framework of the finite element method is discussed. The FEM modeling procedures used to conduct linear elastic and elastic-plastic notch root stress and strain fields analysis, and to estimate the stress-intensity factor and J-integral for small fatigue cracks is described in Chapter 7. The FEM results for the notch root fields analysis and fracture mechanics parameter calculations, including the effects of constitutive material behaviour and anisotropy are presented and discussed in Chapter 8. In Chapter 9 the fractographic results for the small fatigue crack experiments performed in this investigation are 
presented and discussed. A detailed study of the factors contributing to variability in crack initiation lives, the propagation behaviour of naturally-generated small fatigue cracks and a LEFM lifing approach for estimating crack initiation life for the fatigue specimens is presented and discussed in Chapter 10. Finally, from the results presented in Chapters 8,9 and 10 conclusions and lessons learned from this research and recommendations for future work are presented in Chapter 11. 


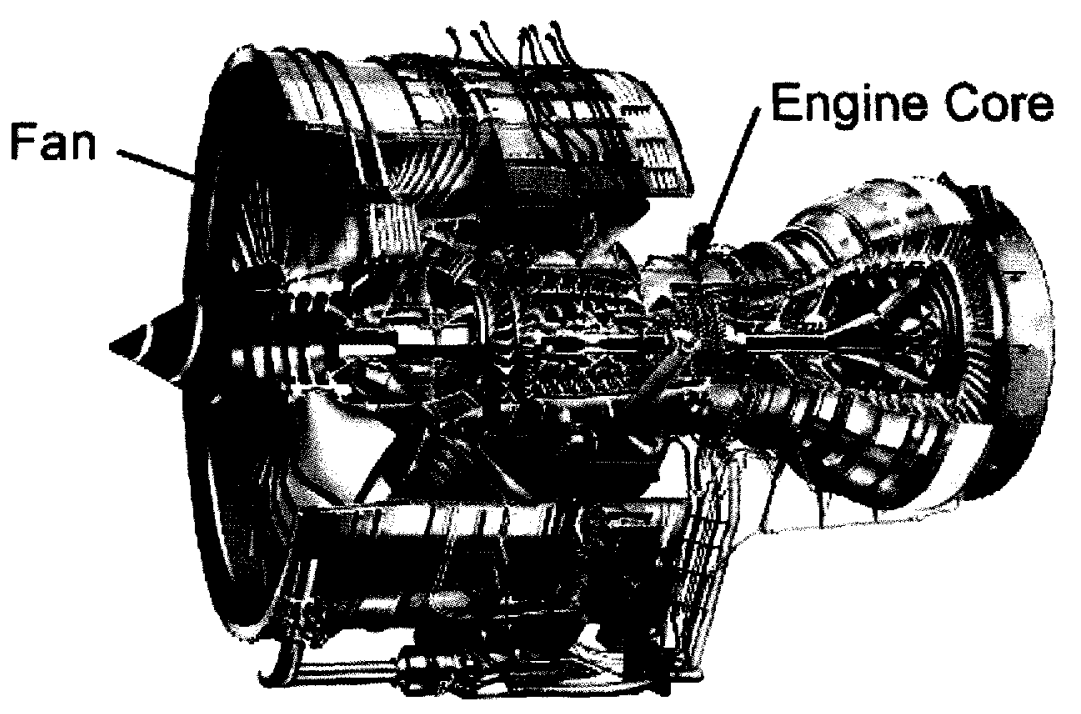

Figure 1.1 - Cutaway view of the Rolls-Royce Trent 900 turbofan engine used on the Airbus A380 family of aircraft adapted from [57].

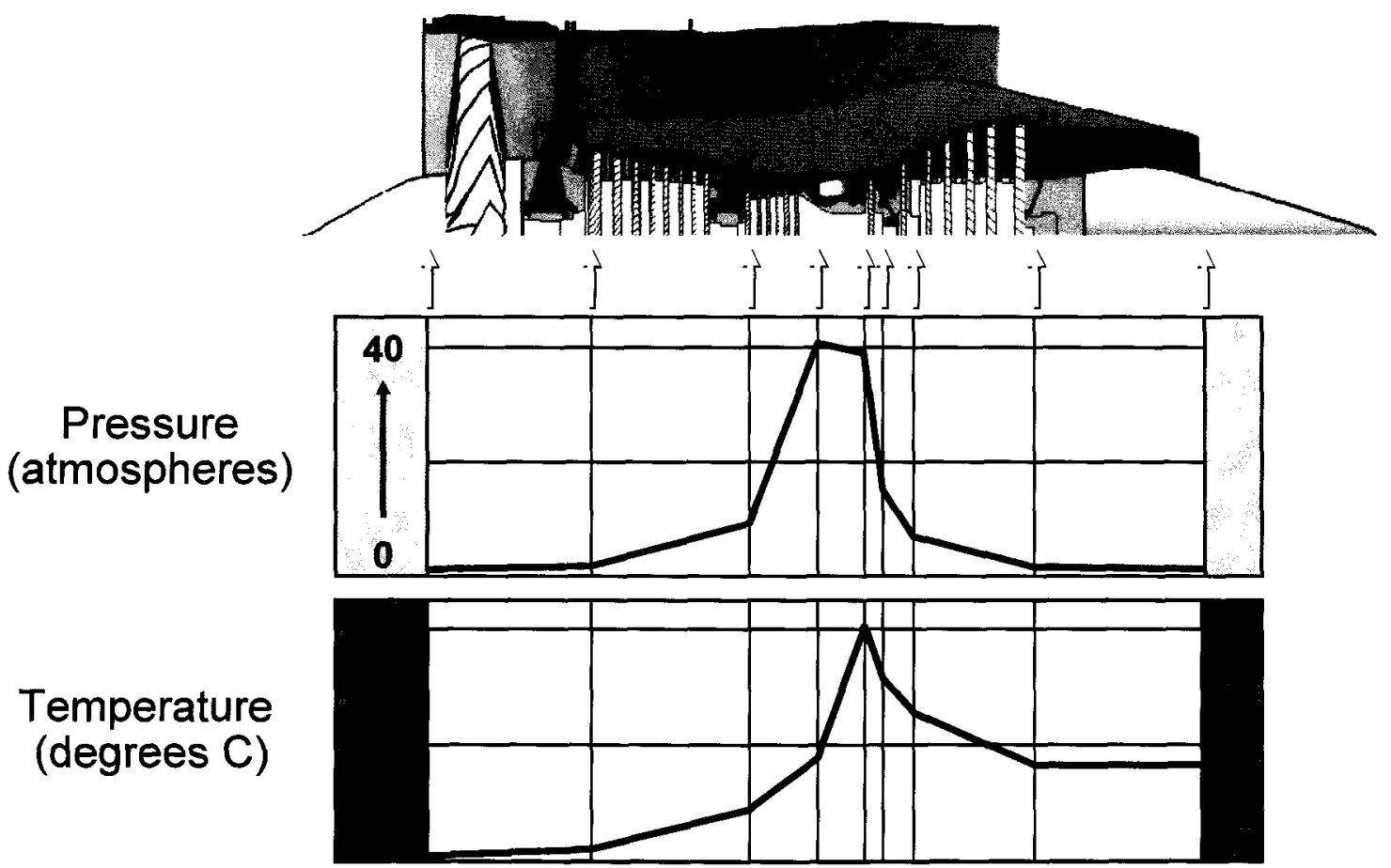

Figure 1.2 - Cross section through a triple-spool gas turbine engine showing gas path pressure and temperature at various points in the engine [58]. 


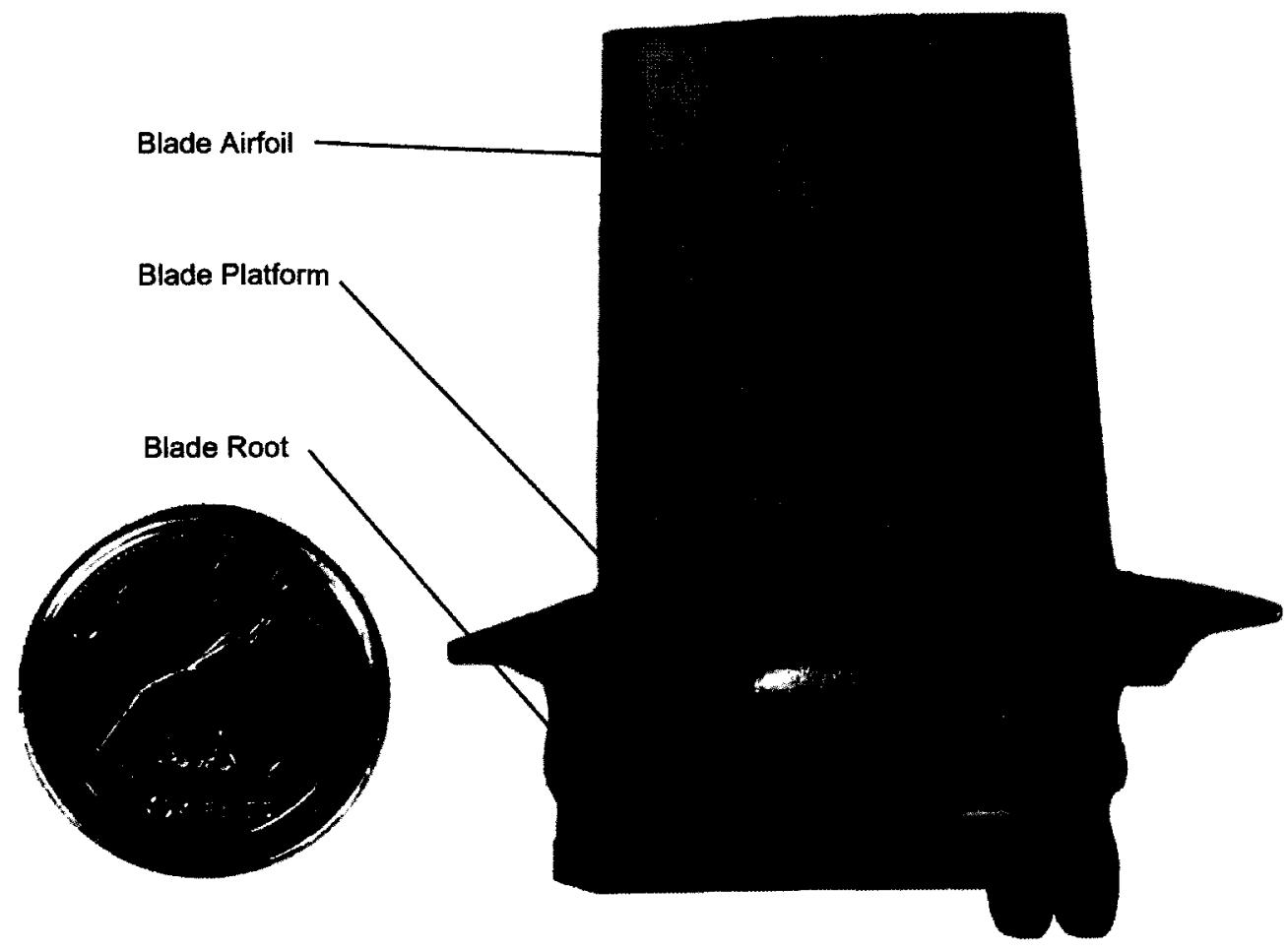

Figure 1.3 - Single-crystal turbine blade design features. Note: Canadian dime is included in image to provide a sense of scale.

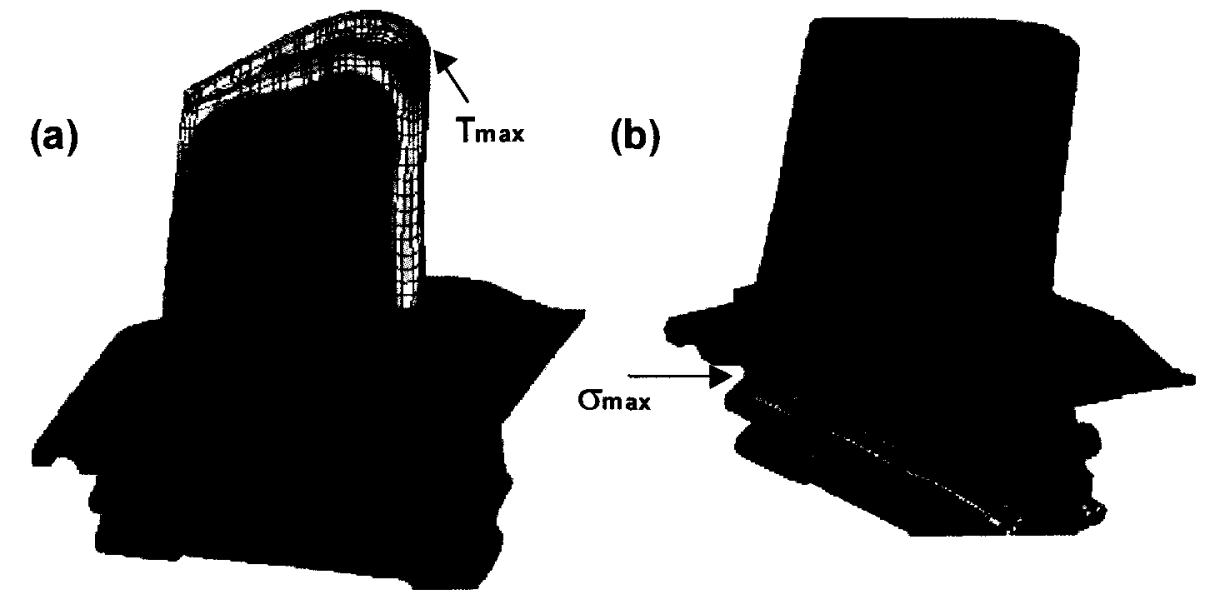

Figure 1.4 - (a) temperature and (b) stress distributions in an air-cooled turbine blade [59]. 


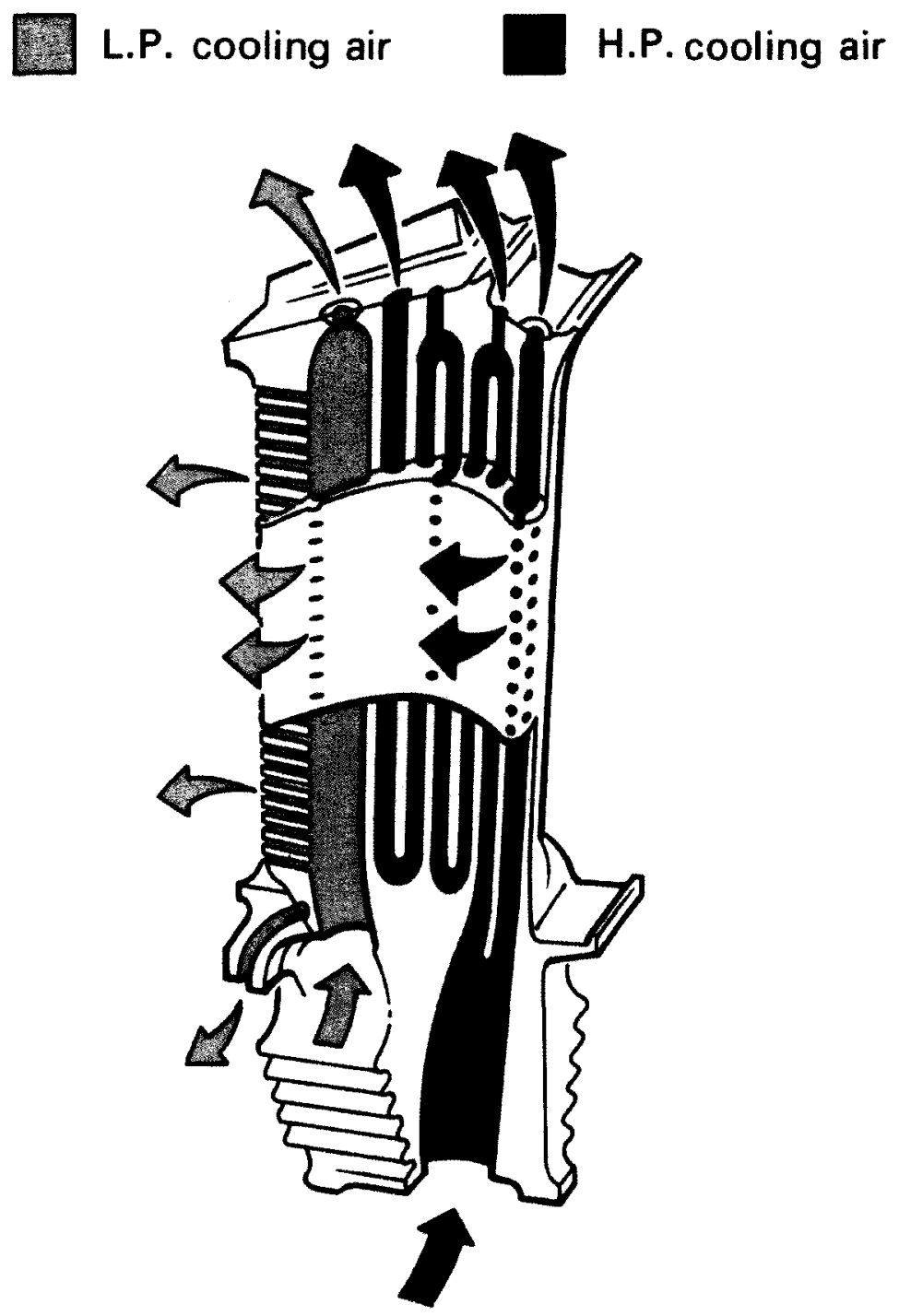

Figure 1.5 - Schematic cutaway view showing the internal turbine blade cooling arrangement used in first stage turbine blades [60]. 


\section{Airfoil Stacking Axis}

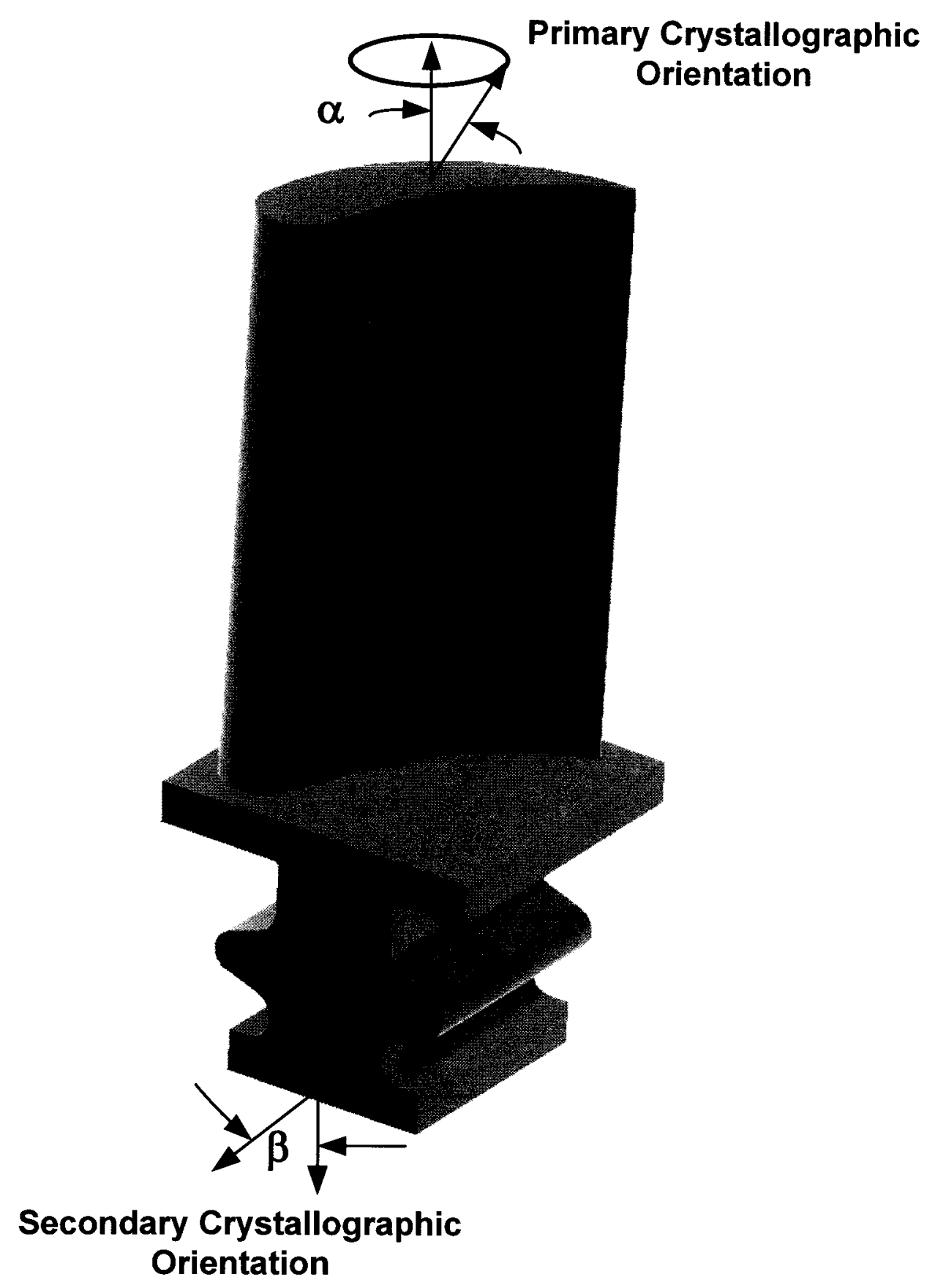

Figure 1.6 - Convention for defining crystal orientation of single-crystal turbine blade. 


\section{Literature Review: Single-Crystal Superalloys}

The superalloys comprise a group of nickel and cobalt-base alloys that exhibit exceptional mechanical properties and environmental resistance at temperatures exceeding $600^{\circ} \mathrm{C}$ and up to $85 \%$ of their absolute melting temperature $\left(\sim 1150^{\circ} \mathrm{C}\right)$. Due to abundance, high solubility for transition metals, and extensive capacity to be strengthened by precipitation hardening, nickel-base superalloys have supplanted cobaltbase superalloys for the majority of high temperature applications. During the past 60 years the temperature capability of nickel base superalloys have increased substantially, largely as a result exotic alloying additions and advanced processing, and through intensive research to understand the effect of alloy chemistry on physical and mechanical properties of the these alloys. Single-crystal nickel-base superalloys represent the stateof-the-art in nickel-base alloy design and processing practices. These alloys afford the gas turbine designer with the best combination of damage tolerance, oxidation, fatigue, and creep resistance of any material presently available for gas turbine blade and vane applications. This chapter discusses the historical development of superalloys, the microstructure and metallurgy of single-crystal superalloys and the single-crystal casting process. In addition, the role of microstructure on mechanical properties, and physical phenomenon responsible for the high temperature mechanical behavior of single-crystal superalloys are also discussed.

\subsection{Historical Development of Nickel-Base Superalloys}

Nickel base superalloy development has been a subject of intense research and development efforts worldwide since 1940's. The efforts of this research have culminated in numerous breakthroughs in alloy composition and processing that have resulted in 
substantial improvements in material capability and gas turbine engine performance. A chronological summary of alloy and processing (metallurgical and processing accomplishments) breakthroughs and material temperature capability, measured in terms of maximum temperature for $1000 \mathrm{hr}$ creep rupture life at $137 \mathrm{MPa}$, is shown in Figure 2.1. During the period 1940 to 1960 nickel base superalloys were mainly utilized in wrought form, and then machined to the required part geometry. Two factors contributed to the temperature capability in wrought alloys; 1) development of alloys with increased additions of solid solution and precipitation strengthening elements, and 2) the development vacuum induction melting to increase alloy cleanliness and to reduce oxidation of highly reactive elements $[61,62]$. Further advancements in wrought alloy metal temperature capability obtained by alloying, while maintaining acceptable hot workability and ductility, were exhausted by the early 1960 's.

In the mid 1950's (see Figure 2.1) precision investment casting processes were developed for manufacturing gas turbine airfoils with increasingly complex shapes. Cast alloys compositions are less restrictive than wrought alloys [63], and therefore solid solution and precipitation strengthening elements were employed more liberally resulting in the development of a number of cast alloys with higher creep strength capability than the strongest wrought alloys. As cast alloys were increasingly used for gas turbine blades it became apparent that the most common mode of failure of conventionally cast (CC) alloys involved intergranular fracture along grain boundaries oriented normal to the airfoil stacking axis (transverse grain boundaries) due to creep and/or thermal fatigue [61, 64-67]. Equally troubling, cast superalloys also exhibited limited ductility at intermediate temperatures, which was exacerbated by microporosity-a type of casting defect-in high stress regions, such as at the blade root [66]. Further increases in temperature capability and ductility were achieved by improving the casting process to produce 
columnar grain structures, which eliminated transverse grain boundaries altogether. This so-called directional solidification (DS) process was developed by VerSnyder and coworkers [66-68] at Pratt and Whitney Aircraft (P\&WA) during the 1960's (see Figure 2.1). Directional solidification processing resulted in an immediate improvement in creep resistance and creep ductility, as shown in Figure 2.2.

The improvement in creep rupture and thermal fatigue resistance and other mechanical properties exhibited by DS superalloys cannot solely be attributed to the removal of transverse grain boundaries. During casting the columnar grains of the DS structure take on a preferred crystallographic orientation, which for face-centered-cubic (FCC) metals such as nickel base superalloys, is aligned with the $\langle 001\rangle$ crystal direction. It is perhaps a fortunate coincidence that $\langle 001\rangle$ orientation in FCC metals exhibits a lower elastic modulus than all other crystallographic orientations [64]. This property of DS structures is particularly beneficial for improving thermal fatigue resistance since thermal stresses arising from constrained thermal expansion along the airfoil stacking line are smaller for $\langle 001\rangle$ than other crystal orientations.

In addition, improvements in oxidation resistance were immediately realized in DS materials since transverse grain boundaries facilitate diffusion, and therefore oxidize preferentially to act as incipient crack nucleation sites [69]. In spite of the substantial improvements offered by DS superalloys in comparison to conventional cast structures, the full mechanical property advantage of DS superalloys could not be realized due to extensive segregation and the presence of grain boundary strengthening elements, both of which reduce the alloys incipient melting temperature below the precipitate solvus temperature. It was therefore impossible to fully solution the coarse as-cast precipitate 
microstructure and to fully homogenize DS superalloys, which was essential to obtain the best combination of mechanical strength and creep resistance [70]. The solution to the forgoing problems with DS alloys was to develop direction solidification processing technology in which all grain boundaries and associated grain boundary strengthening elements were eliminated. The resulting products from this process were referred to as single-crystals since they consisted of a single grain with $\langle 001\rangle$ texture. A production process which made large-scale production single crystal blades and vanes economically feasible was developed in parallel with DS processing technology by VerSnyder and coworkers $[68,71]$ at $\mathrm{P} \& W A$ during the later part of the 1960's (Figure 2.1).

Initial single-crystal process development research predominantly made use of existing conventional cast superalloys, such as MAR-M200 $[68,71]$. It was soon realized that single-crystal processing offered no substantial improvements in creep strength or thermal fatigue resistance over DS processing when making use of existing alloy compositions [71]. New alloys were developed during the 1970's, still largely based on the Mar-M200 composition, to exploit the benefits of single-crystal processing. Pratt and Whitney was the first gas turbine OEM to both, develop an alloy specifically for singlecrystal parts and to put single-crystal turbine blades into service in civil and military gas turbine engines during the early 1980 's $[61,71]$. The improvement in creep properties with single-crystal alloys was substantial in comparison to DS and CC superalloys, as shown in Figure 2.3. Since the 1980's the metal temperature capability of single-crystal superalloys has improved by approximately $150^{\circ} \mathrm{F}$ due to a better understanding of the effect of alloy composition on physical and mechanical properties (Figure 2.1). Given the importance of the DS casting process to modern gas turbine materials, further discussion of the DS and single-crystal casting process is warranted. This is the subject of the following section. 


\subsection{Single Crystal Processing}

As discussed in the last Chapter, single-crystal turbine blades employ elaborate internal cooling features to reduce metal temperatures. They are manufactured in complex shapes to maximize aerodynamic efficiency and can have substantial and abrupt changes in cross section to accommodate features to reduce aerodynamic losses. To accurately reproduce the intricate features utilized in modern gas turbine blade and vane designs, the investment casting process is used. The following sections discuss the investment casting process, the method by which directional grain structures produced, and casting defects.

\subsubsection{Investment Casting}

All investment cast superalloys are produced using the same basic investment casting process $[63,72]$. First, a wax pattern that is an exact replica of the part is produced by injection molding. For internally cooled turbine airfoils, a ceramic core (see example in Figure 2.4) is inserted into to the pattern die cavity before injecting the wax. To increase productivity and the economics when casting smaller parts, a number of wax patterns are assembled together to form a cluster mold. Additional features, such as the sprue, downgates, risers, the starter block, and grain selectors (for single-crystal castings) are added manually to complete the cluster mold assembly. An assembled cluster mold illustrating these features is shown in Figure 2.5. After the wax pattern is completed, the assembly is dipped into ceramic slurry, and then showered in larger granules of ceramic material, referred to as stucco, to rapidly build-up the mold shell thickness and to give the shell mechanical strength. This process of dipping and stuccoing may be repeated numerous times to build-up sufficient wall thickness so as to ensure mold integrity during the casting process. After drying, the casting mold is heated to remove the wax pattern and then fired at high temperature to strengthen the mold. A completed casting mold assembly for production of single-crystal turbine blades is shown in Figure 2.6. 


\subsubsection{Directional Solidification Process}

In conventional castings a metal charge is melted under vacuum, poured into a preheated casting mold and allowed to cool. Cooling takes place by radiation from the mold surface resulting in radial temperature gradients, and therefore solidification occurs progressively from the mold wall to the center of the cast part. The resulting microstructure consists of columnar grains nucleated from the mold wall and equiaxed grains near the center of the cast part similar to that shown in Figure 2.7. The casting process for DS components is substantially different than that used for producing conventional castings. For directional solidification to occur the following two conditions must be satisfied [67]:

1. heat flow from the casting must be unidirectional which results in a solid-liquid interface at the growing grains that moves in one direction and,

2. the mold temperature ahead of the solid-liquid interface must remain above the alloy liquidus temperature so as to ensure that no misoriented grains nucleate from the mold wall.

Both of these conditions can be satisfied by variety of solidification techniques; however, for mass production of DS and single-crystal parts derivatives of the Bridgman casting process are most commonly used.

The Bridgman casting process is shown schematically in Figure 2.8. A Bridgman casting furnace consists of two major components; a vacuum containment vessel that is divided into two chambers and water cooled chill plate with motorized ram for casting mold withdrawal at a controlled rate. Ancillary equipment used for heating and maintaining the vacuum environment in the casting furnace is similar to that used in production of conventional castings. The upper chamber of the vacuum containment vessel is heated either by induction coil with susceptor or radiation heating elements to melt the 
superalloy and maintain the mold temperature above the alloys liquidus temperature. Some commercial furnaces utilize separate heating systems for the crucible holding the superalloy charge and the casting mold (see Figure 2.8). The lower chamber is maintained at a temperature substantially lower than the alloys liquidus temperature by circulating cooling water though a water jacket in the vacuum containment vessel. A baffle is used to separate the heated upper chamber from the lower chamber and facilitates maintaining a high temperature gradient at the solid-liquid metal interface in the cast part [64].

The function of the water-cooled chilled plate is to extract heat from the casting and to provide the temperature gradient needed to initiate directional solidification. When the molten metal is poured into the ceramic shell it immediately solidifies upon contact with the chill plate forming a layer of equiaxed grains. Subsequent grain growth preferentially occurs in grains most closely aligned with the $\langle 001\rangle$ crystal direction resulting in a columnar array of grains with $\langle 001\rangle$ texture similar to that shown in Figure 2.9. Solidification of the molten alloy proceeds in a directional manner as a result of the longitudinal temperature gradient between the heated furnace chamber and the chill plate. Since the chill plate heat removal capacity by conduction is restricted though the solidified material in the heated furnace chamber [64], the entire mold assembly and chill plate are withdrawn from the heated furnace into the unheated lower chamber at a controlled velocity to maintain the longitudinal temperature gradient. As the mold is withdrawn from the heated upper chamber, the heat removal from casting occurs predominantly by radiation to the cooled walls of the lower chamber.

Based on the forgoing description of the Bridgman casting process it is clear that the required conditions for columnar grain growth are satisfied; however, the resulting cast 
product using the process as described will only provide a DS part. Therefore, one additional element is needed to ensure that only a single columnar grain with correct orientation enters the mold cavity to produce a single-crystal part. Two common methods to ensure that the resulting part consists of a single grain are to employ a grain selector or seed crystal. The grain selector is an element added to the bottom of the casting mold whose function is to filter out DS grains growing from the chill plate so that only a single grain with $\langle 001\rangle$ orientation enters the mold cavity. Two commonly used grain selector designs are shown in Figure 2.10. It may be noted that while the grain selector may ensure that the primary orientation of the finished single-crystal part is approximately aligned with the $\langle 001\rangle$ orientation, the transverse crystallographic orientation of the finished part is uncontrolled.

When a seed is used to initiate single-crystal solidification it functions as a template controlling the orientation of the molten alloy as it solidifies. A schematic showing the placement of the seed crystal in the casting mold is shown in Figure 2.11. Notice that the seed crystal is placed directly on the chill plate to prevent the seed from melting entirely during mold preheating and when the molten alloy is poured. In contrast to the grain selection method, when a seed crystal is used to produce single-crystal parts both the primary and secondary orientation of the part may be controlled by the primary orientation of the seed crystal and the placement of the seed in relation to the final part.

\subsubsection{Single-Crystal Macrostructure}

Solidification rates in the Bridgman process are such that the alloy solidifies with a dendritic form, as shown in Figure 2.12. The macrostructure of single-crystal cast parts can thus be characterized by parallel, uninterrupted primary dendrites, which span the entire length of casting in the direction of solidification. Also shown in Figure 2.12 are 
secondary and tertiary dendrite arms, which are orthogonal to the primary and secondary dendrite arms, respectively. The dendrites in single-crystal superalloys are aligned with $\langle 001\rangle$ as a consequence of higher solidification rates along the $\langle 001\rangle$ crystal directions in cubic materials $[61,64]$. The primary dendrite arm spacing (PDAS) indicated in Figure 2.12 has some special significance in cast parts as the PDAS provides an indication of the extent of chemical microsegregation and is a record of the solidification conditions during processing [73]. A large PDAS is indicative of extensive microsegregation that necessitates extended, multi-step, homogenization and solution heat treatment cycles to avoid incipient melting. In terms of processing, the PDAS is primarily a function of two casting parameters; the gradient and the solidification rate. As shown schematically in Figure 2.13, rigorous control of casting parameters, especially the temperature gradient, are essential to maintain directional solidification and to avoid nucleation and growth of equiaxed grain defects.

The interdendritic regions (see Figure 2.12) are occupied with the last chemical constituents to solidify in the cast part. Usually, these interdendritic constituents have a eutectic composition, and therefore the interdendritic regions directly affect the ability to solution heat-treat the part. In addition to the interdendritic regions affecting the capacity for solution heat treating the single-crystal material, they can host casting defects such shrinkage porosity, as shown in Figure 2.14, which can be detrimental mechanical properties.

\subsubsection{Casting Defects}

The latest single-crystal turbine blade designs that employ a hollow, thin wall design with sophisticated cooling features, and large transitions in cross section to accommodate features such as the blade platform and tip shrouds, present a formidable challenge to 
mass produce without casting defects. One common problem that can occur during production of the wax pattern for hollow airfoils is that the ceramic core can shift or bow during the wax injection, resulting in part with unacceptable variations in wall thickness [63]. Another common cause for rejection of single-crystal castings are the result of grain defects, such as freckle chains, high angle grain boundaries and recrystallization. In addition, single-crystal parts may be rejected due to strict limits placed on how much the primary orientation may deviate from $\langle 001\rangle$; variations of up to $10^{\circ}$ are generally accepted. Single-crystal parts are also susceptible to other casting defects that are common in $\mathrm{CC}$ casting processes, such as shrinkage and gas porosity, and non-metallic inclusions due to ceramic particles from the casting mold or melting crucible.

As noted earlier (see Section 1.2.3), casting process parameters for single-crystal parts (DS parts too) are carefully controlled to prevent the formation of equiaxed grains that would affect structural integrity of the component during service. In spite of these efforts thicker sections in single-crystal parts, such as the blade root and platform areas, are especially susceptible to the formation of equiaxed grains because the large changes in cross-sectional area strongly affect the shape of the solid-liquid interface during casting. One common type of grain defect that occurs in these regions on cast single-crystal parts is referred to as freckles. Freckles are long chains of equiaxed grains that form parallel to the solidification direction, as shown in Figure 2.15. It is believed that freckles form as a result of density variations caused by chemical heterogeneity in the mushy zone- the two phase region at the solid-liquid interface containing both solid and liquid-that can force low density solute lean liquid metal to the top of the solid-liquid interface breaking dendrite tips $[61,64,68,74-76]$. The broken dendrite tips then function as grain nucleation sites causing freckles to form. Since the driving force is mainly due to chemical heterogeneity, alloys that exhibit a high degree of segregation during 
solidification, such as the latest single-crystal blade alloys that employ large amounts of refractory elements, have a higher propensity for forming freckles [75].

Another type of defect observed in single-crystal parts is the formation of stray grains that have an orientation substantially different from that of the part. This type of defect is commonly referred to as a high angle grain boundary (HAB). A HAB can form when the grain selector fails to ensure that a single grain enters the casting mold or as a result of grain nucleation on the mold wall where large changes in part cross-section are encountered [61]. Single-crystal parts have little tolerance to the presence of HAB's due to the absence of any grain boundary strengthening elements, and therefore parts containing such defects are rejected. It may be noted that in single-crystal parts it's quite common to observe that parts of the solidified structure have small orientation variations (less than $10^{\circ}$ ) having tilt character, i.e. one grain is rotated with respected to the other about an axis parallel to the boundary. This is referred to as low angle grain boundary (LAB). While HAB defects in single-crystal parts are totally unacceptable, LAB's with a misorienation of less than $10^{\circ}$ in finished parts are usually tolerated $[64,77,78]$.

Recrystallization is another mechanism by which equiaxed grains can form in singlecrystal parts. Unlike freckles, which are equiaxed grains that form during solidification process, the driving force for recrystallization is residual stresses from the casting process or cold work imparted during finishing operations, coupled with thermal exposure at elevated temperatures $[61,64]$. Should there be sufficient localized residual stress, recrystallization can occur during solution heat treatment operations carried out on the finished part, since there are few precipitates present to impede grain growth [64]. 
Shrinkage porosity (see Figure 2.14) occurs in cast structures as a result of the volume change associated with the change from a liquid to a solid. Although shrinkage porosity may be found in all cast superalloys, the occurrence of such defects in DS parts tends to be substantially less than that in CC parts, which lack a large head of liquid metal present in DS casting processes to feed shrinkage regions [64]. Gas porosity occurs when the concentration of gasses in the molten metal, such as hydrogen and nitrogen, exceed their solubility limit in the molten alloy as it solidifies forming gas bubbles $[68,79]$. Since the gas bubbles have lower density than the molten alloy they tend to be swept upwards by the solid-liquid interface, and therefore the occurrence of gas porosity defects in singlecrystal parts is usually limited to the feeders (risers), which are removed from the cast part. The casting mold and crucible used for alloy melting are manufactured from ceramics, and constitute a potential source for non-metallic inclusions to be swept into casting mold when poring commences. Typically cast parts are subjected to a nondestructive inspections using radiographic equipment [61, 64] after casting, which normally catch parts containing non-metallic inclusion defects.

\subsection{Single-Crystal Nickel-Base Superalloy Microstructure}

Single-crystal nickel-base superalloys are essentially two-phase materials consisting of a solid solution strengthened authentic matrix phase, referred to as gamma $(\gamma)$, and a high volume fraction $\left(V_{f}\right)$ of ordered precipitates called gamma prime $\left(\gamma^{\prime}\right)$. An example of the two phase $\gamma-\gamma^{\prime}$ microstructure of single-crystal blade alloys is shown in Figure 2.16. Other phases, such as carbides and borides may also be present in the in $\gamma$-matrix in limited quantities, since these elements are not usually intentionally added to most singlecrystal alloy compositions. Some single-crystal alloy compositions, especially those that contain substantial additions of refractory elements, are susceptible to the precipitation of 
topographically close-packed phases (TCP) during exposure to service conditions. Further details on selected phases present in single-crystal superalloys are discussed in the following sections.

\subsubsection{Gamma Phase}

The $\gamma$ phase comprises the continuous matrix phase in nickel-base superalloys which contains all secondary phases. It primarily consists of FCC nickel, as shown in Figure 2.17 , with other chemical additions that promote solid solution strengthening. The primary mechanism of solid solution strengthening is due to addition of chemical elements with a similar atomic size to nickel, which substitute for nickel atoms in the $\gamma$ phase and distort the crystal lattice. These substitutional species also play a role in lowering the stacking fault energy of nickel $[65,70,80,81]$, which impedes dislocation cross-slip in the $\gamma$ phase. Refractory elements, such as tungsten and molybdenum, provide additional strengthening by increasing the cohesive strength of atomic bonds in the $\gamma$ phase [65] and also reduce bulk diffusion rates at high temperatures $[65,70,82]$. Although solid solution strengthening provides some enhancement to the mechanical properties of nickel-base superalloys, it is the $\gamma^{\prime}$ phase that is primarily responsible for the substantial strength exhibited by superalloys at high temperatures.

\subsubsection{Gamma-Prime Phase}

Although nickel has a high solubility for many of the transition metals it can form intermetallic phases when chemical species with substantially different electonegativities than nickel are added. The most important of these intermetallic phases for nickel-base superalloys is $\gamma^{\prime}$, which precipitates as a coherent phase in the $\gamma$-matrix. Gamma prime is an ordered intermetallic phase with FCC $\left(\mathrm{L1}_{2}\right)$ crystal structure and $\mathrm{A}_{3} \mathrm{~B}$ stoichiometry. In the $\gamma^{\prime}$-phase A represents nickel, although nickel may be substituted with cobalt; B 
represents aluminum, although elements that are commonly added to nickel-base superalloys, such as titanium, tantalum, hafnium and niobium, may substitute for aluminum. The general atomic arrangement for these elements within the $\gamma^{\prime}$-phases FCC crystal lattice is shown in Figure 2.18.

A high volume fraction of $\gamma^{\prime}$-phase can be accommodated in $\gamma$ because its lattice parameter-the smallest interatomic distance in the FCC unit cell—closely matches that of the $\gamma$-matrix. The size mismatch between the $\gamma^{\prime}$ precipitates $\gamma$-matrix is typically specified by the misfit parameter, $\delta$, defined as:

$$
\delta=\frac{2\left(a_{\gamma^{\prime}}-a_{\gamma}\right)}{\left(a_{\gamma^{\prime}}+a_{\gamma}\right)}
$$

where $a_{\gamma}$, and $a_{y}$ are the lattice parameters for the $\gamma^{\prime}$ and $\gamma$-phases, respectively. The misfit parameter $(\delta)$ depends on alloy composition, and ultimately determines the morphology of the $\gamma^{\prime}$ precipitates. A small $\gamma-\gamma^{\prime}$ misfit parameter $(0.5 \%)$ results in spherical precipitates, while larger lattice misfit (0.5-1.5\%) results in $\gamma^{\prime}$ precipitates with a cuboidal or plate morphology $[70,80]$, which is typical of many single crystal nickel-base superalloys.

In nickel-base superalloys the two main factors that affect the strengthening conferred by the $\gamma^{\prime}$-phase are the volume fraction $\left(\mathrm{V}_{\mathrm{f}}\right)$ and the size of the precipitates. The $\gamma^{\prime} \mathrm{V}_{\mathrm{f}}$ is controlled by the amount of aluminum, titanium, tantalum, and other $\gamma^{\prime}$ forming constituents contained in the alloy. Most commercial single-crystal superalloys contain a $\gamma^{\prime}$ precipitate $\mathrm{V}_{\mathrm{f}}$ of approximately $60-80 \%[61,64,65]$. As shown in Figure 2.19 , the creep resistance of SX superalloys increases nearly monotonically with $\gamma^{\prime} V_{f}$. At first glance this suggests that increasing the $\gamma^{\prime} V_{f}$ further would be beneficial and provide 
additional strengthening. Although it is physically possible to develop alloy designs with higher $\gamma^{\prime} \mathrm{V}_{\mathrm{f}}$, such alloys would have substantially lower strength [65] than current alloy designs because of the low strength exhibited by $\gamma^{\prime}$ at low temperatures [83]. A higher $\gamma^{\prime}$ $V_{f}$ would also result in a precipitous drop in creep resistance because of the crucial role that the $\gamma-\gamma^{\prime}$ interface plays on conferring creep resistance [61]. Furthermore, loss of $\gamma$ matrix due to higher $\gamma^{\prime} V_{f}$ increases the propensity to form deleterious topographically close-packed phases (TCP) due to the higher concentration of TCP forming elements that partition preferentially to the $\gamma$-matrix [82].

Many mechanical properties in single-crystal superalloys, such as yield strength, creep rupture resistance, low-cycle and thermomechanical fatigue performance are strongly influenced by the size of the $\gamma^{\prime}$ precipitates. Experimental evaluations of single-crystal superalloy suggest that the optimum $\gamma^{\prime}$ size to achieve a balance in these mechanical properties is $0.3-0.5 \mu \mathrm{m}$ [84-87]. In the as-cast state single crystal superalloys contain much larger $\gamma^{\prime}$ precipitates than this optimum size, as shown in Figure 2.20. Furthermore, the interdendritic regions usually contain massive primary $\gamma^{\prime}$ as one of the constituents of the interdendritic eutectic structure. The massive interdendritic $\gamma^{\prime}$-phase has been associated with initiating cleavage fracture in single crystal superalloys $[65,88]$, and therefore is very detrimental to mechanical properties. However, with carefully developed heat treatments it is possible to fully solution the coarse as-cast precipitate structure, to substantially reduce the interdendritic eutectic $\gamma-\gamma^{\prime}$ phase, and re-precipitate the $\gamma^{\prime}$-phase within the optimal size range.

\subsubsection{Topographically Close-Packed Phases}

Topographically close-packed phases (TCP) are intermetallic precipitates not normally present in single-crystal superalloys after processing and application of standard heat 
treatments. In alloys that are susceptible to TCP formation, their occurrence is usually the result of extended service exposure $[61,70]$. The precipitation of TCP can be attributed to excessive concentrations of chromium, and refractory elements, such as rhenium, tungsten, and molybdenum in the $\gamma$-matrix. Topographically close-packed phases are usually described as having a chemical stoichiometry of the form $A_{x} B_{y}$, where $A$ and $B$ both represent transitions metals, such that A and B usually elements that occupy periods towards the left and right side, respectively, of the transition metals [61]. Common TCP phases found to precipitate in SX superalloys are sigma $(\sigma), \mathrm{mu}(\mu)$ and P-phase, as shown in Figure 2.21. The main feature differentiating sigma, mu and P-type TCP precipitates is chemical stoichiometry and the crystal structure. Of the common TCP precipitates, it is sigma phase that is usually considered to be the most deleterious because of its brittle nature and tendency to have an elongated plate or needle like morphology, which can facilitate fatigue crack initiation $[65,70,89]$. In general, TCP precipitation is undesirable because it ties up solid solution strengthening elements [84, 89], and therefore reduces the high-temperature strength and creep resistance of the $\gamma$ matrix.

\subsubsection{Other Minor Phases}

Other minor phases such as carbides and borides, in alloys containing sufficient quantities of these chemical species, also tend to form in the interdentritic regions. These elements have been intentionally added to some single-crystal alloys to improve casting yields by increasing tolerance to low-angle grain boundary defects. This will be discussed further among other developments in single-crystal alloy chemistry in the following section. 


\subsection{Single-Crystal Superalloy Metallurgy and Evolution}

\subsubsection{First-Generation Single-Crystal Superalloys}

As noted earlier (see Section 2.1), the first single crystal nickel-base superalloys were derived from nickel-base superalloys such as MAR-M200, which were primarily designed for conventional casting techniques. During the 1970's initial work was undertaken to develop alloys that specifically exploit the benefits of single crystal processing by eliminating carbon, boron, zirconium and hafnium, which were primarily added to improve the strength, chemistry and grain boundary morphology of CC and DS superalloy components [71]. The advantage of removing these elements, which are solidus temperature depressants, is to increase the incipient melting temperature and thus make it possible, with appropriately designed heat treatments, to homogenize the cast microstructure and fully solution the $\gamma^{\prime}$ phase for better control of $\gamma^{\prime}$ size and morphology $[71,82,88,90,91]$. Other alloy chemistry changes to adjust for SX processing include the addition of substantial amounts of tantalum. The resulting single-crystal alloy compositions derived during this pioneering work are referred to as the first-generation single crystal nickel-base superalloys. These alloys afforded a $20-50^{\circ} \mathrm{C}$ improvement in metal temperature capability over the strongest DS alloys available $[71,77,90,92,93]$. A table of several commonly used first generation single-crystal alloys is presented in Table 2.1 .

Referring to Table 2.1, the main alloying elements of first-generation single-crystal superalloys consist of chromium, cobalt, molybdenum, tungsten, aluminum, titanium and niobium. While some of the elements have been added to fulfil a specific purpose, most elements in single-crystal superalloy compositions have been added to fulfil a variety of roles. Elements such as chromium, molybdenum, niobium and tungsten mainly partition 
to the $\gamma$-matrix to promote solid solution strengthening. Cobalt is similar in size and electronegativity to nickel, and therefore can substitute for nickel in the $\gamma$-matrix or in $\gamma^{\prime}$. The main effect of cobalt is to decrease the stacking fault energy of nickel, increase the solubility of $\gamma$ 'and to raise the alloy solidus temperature [65]. Titanium and aluminum are the main chemical constituents responsible for formation of the $\gamma^{\prime}$ precipitate phase. Tantalum and niobium also participate as $\gamma^{\prime}$ forming agents, where they play a fundamental role in strengthening the precipitates by substituting for aluminum or titanium. Tantalum also raises alloy solidus temperature [65], significantly reduces the propensity for freckle formation in single-crystal parts $[65,71,92]$ and facilitates alumina $\left(\mathrm{Al}_{2} \mathrm{O}_{3}\right)$ formation to enhance oxidation resistance $[82,92]$. As noted earlier (see 2.3.1), the refractory elements such as molybdenum, tungsten, tantalum and niobium also fulfil a secondary role by reducing the bulk diffusion rate of $\gamma^{\prime}$ forming elements in the $\gamma$-matrix. This reduces the $\gamma^{\prime}$ prime coarsening kinetics and the rate of diffusion controlled creep mechanisms such as climb or thermally activated cross-slip, thus reducing creep kinetics $[82,90]$. Both aluminum and chromium have secondary roles, in which they promote the formation of a protective oxide layer $\left(\mathrm{Al}_{2} \mathrm{O}_{3}\right.$, and $\mathrm{Cr}_{2} \mathrm{O}_{3}$, respectively), which protects the alloy against further oxidation.

\subsubsection{Second-Generation Single-Crystal Superalloys}

Based on the success of the first generation single-crystal superalloy compositions, alloy designers continued the development work during the 1980's on single-crystal nickelbase superalloys that offered substantial improvements in metal temperature capability. The resulting alloys derived form this intensive alloy development effort afforded a metal temperature capability improvement of $30-35^{\circ} \mathrm{C}$ over the first-generation alloys, particularly at high-temperatures $\left(\mathrm{T}>950^{\circ} \mathrm{C}\right)[82,84,90,92]$, and therefore were referred to as second-generation single-crystal alloys. The compositions of commercially 
important second-generation single-crystal superalloys are summarized in Table 2.2. Second-generation single-crystal alloys can be distinguished from the first-generation alloys because they contain $3 \mathrm{wt} . \%$ rhenium and a higher total refractory element content $(\mathrm{W}+\mathrm{Ta}+\mathrm{Mo}+\mathrm{Re}+\mathrm{Nb})$. Rhenium partitions mainly to the $\gamma$-matrix and has been shown to be beneficial to SX superalloys by substantially reducing $\gamma^{\prime}$ coarsening kinetics, and increasing $\gamma-\gamma^{\prime}$ misfit $[82,85,88]$. Rhenium has also been found to form clusters in the $\gamma-$ matrix, thereby providing more potent solid solution strengthening $[82,85,88]$. In spite of these benefits, the addition of rhenium and higher total refractory content makes second-generation alloys more susceptible to forming TCP phases, and therefore alloy designers have reduced chromium content significantly from that in the first generation single-crystal alloys (from approximately 9 wt.\% to approximately 6 wt.\%) to compensate $[82,90]$. Although chromium plays a crucial role in conferring hot corrosion resistance, the reduction of chromium is usually compensated for by the application of protective coatings. It may also be noted that second-generation single-crystal superalloys contain significantly more cobalt than the first generation alloys to exploit the benefits of cobalt noted earlier (see 2.4.1).

\subsubsection{Third-Generation Single-Crystal Superalloys}

By the mid 1990's, further single-crystal alloy development work paid off, as thirdgeneration single-crystal superalloys were developed which could afford the gas turbine designer with a $30^{\circ} \mathrm{C}$ improvement in the metal temperature capability over the secondgeneration superalloys $[88,92,94]$. The composition of common third-generation singlecrystal superalloys are summarized in Table 2.3. Third generation single-crystal superalloys can be distinguished from the second-generation alloys by the increased rhenium (up to $6 \mathrm{wt} \%$ ) and total refractory element content. The chromium content in the third-generation alloys were decreased as compared to second-generation alloys to reduce 
the tendency to promote TCP formation. One notable feature about René N6 (see Table 2.3 ) is that its alloy designers chose to employ small additions of carbon and hafnium, both of which are carbide formers and normally associated with DS alloy compositions. These elements were added to increase casting yields by suppressing freckle formation (a common problem in high $\mathrm{Re}$ and high refractory content alloys $[75,76,82])$ and to increase tolerance of LAB's $[82,88,94]$. Although progress was made to increase metal temperature capability in third generation single-crystal superalloys, this gain came at the expense of higher alloy density, a much smaller heat treatment window (the difference between the $\gamma^{\prime}$ solvus and incipient melting temperature) and the requirement for substantially longer homogenization heat treatment cycles to reduce segregation.

\subsubsection{Fourth-Generation Single-Crystal Superalloys}

Development of the fourth-generation single-crystal superalloys started during the late 1990's. Although these alloys are still under evaluation, a list of alloys considered to be the fourth-generation single-crystal superalloys are presented in Table 2.4. The fourthgeneration single-crystal superalloys are characterized (in some cases) by partial substitution of rhenium with ruthenium, and higher total refractory contents. In spite of the higher total refractory content in these alloys, they exhibit improved phase stability in comparison to the third-generation superalloys [95-97], lower density [95, 98] and improved creep resistance, which translates into higher temperature capability $[96,97]$. It is the addition of ruthenium that is mainly responsible for the remarkable improvement in properties exhibited by the fourth-generation single-crystal superalloys. The lower density of the fourth-generation alloys can be attributed to the fact that ruthenium has approximately half the density of rhenium. The exact mechanisms by which ruthenium contributes to the improvements in phase stability is still unclear, which is a subject of ongoing research; however, some researchers have noted that ruthenium causes 
refractory elements to partition more strongly to $\gamma^{\prime}$ rather than to the $\gamma$-matrix [97]. This so-called reversed partitioning reduces the amount of solid solution elements in the $\gamma$ matrix and may explain why fourth-generation alloys can accommodate higher refractory element content without exhibiting the phase stability issues common to the thirdgeneration single-crystal superalloys.

During the past 30 years the temperature capability of single-crystal superalloys has been increased by $100^{\circ} \mathrm{C}$ as a result of rigorous optimization of alloy compositions. However, to translate the material temperature capability afforded by the latest single-crystal alloys into benefits for real gas turbine applications requires a fundamental understanding of the deformation mechanisms and their mechanical properties. This will be elaborated in the following section, and it will be the core discussion of this thesis.

\subsection{Deformation Mechanisms and Mechanical Properties of Nickel- Base Single-Crystal Superalloys}

All crystalline materials exhibit anisotropic properties whether in single-crystal or polycrystalline form. The anisotropic properties of crystalline materials originate from the periodic atomic arrangement and variations in atomic bonding strength within the crystal lattice. Polycrystalline materials usually exhibit isotropic material properties because they consist of a large aggregate of grains with random crystallographic orientations which tend to average out the anisotropy of individual grains at the macroscale. On the other hand, single-crystal materials tend to exhibit large variations in elastic and inelastic material properties, which depend on the relative orientation between materials crystalline structure and applied mechanical loads. These anisotropic effects must be taken into consideration when carrying out structural analysis calculations on 
single-crystal components. In this section, the anisotropic elastic properties and physical factors that contribute to the inelastic anisotropy observed in single-crystal superalloys are discussed.

\subsubsection{Elastic Properties of Single-Crystal Superalloys}

Elastic deformation in a material is a reversible process because it involves the stretching of the atomic bonds in a crystal lattice without causing any permanent change in the atomic arrangement. The stress-strain relationship can be given by Hooke's law, as in the following tensor form for general anisotropy:

$$
\sigma=C: \varepsilon^{e l}
$$

where, $\sigma$ is the stress tensor, $\varepsilon^{e l}$ is the elastic strain tensor and $C$ is a fourth-order tensor of elastic constants for a material. By taking into consideration various tensor and material symmetries, the number of independent elastic constants can be substantially reduced. For cubic materials only three independent elastic constants are required to describe the anisotropic elastic properties, specifically:

$$
\begin{aligned}
& C_{1111}=C_{2222}=C_{3333} \rightarrow C_{11} \\
& C_{1122}=C_{1133}=C_{2233} \rightarrow C_{12} \\
& C_{1212}=C_{1313}=C_{2323} \rightarrow C_{44}
\end{aligned}
$$

where the quantities to the right represent the three independent elastic constants in concise (Voigt) tensor notation. The values for the remaining elastic constants excluded from equation (2.3) are zero. The three independent elastic constants for single-crystal superalloys are invariably temperature dependent, but are generally considered to be insensitive to alloy composition [64].

In mechanical testing, it is usually the effective elastic modulus that is measured from the elastic behaviour of single-crystal superalloys. The effective elastic modulus represents 
the stiffness of an anisotropic material along a specific crystallographic direction when subjected to uniaxial loading. For single-crystal superalloys, the effective elastic modulus as a function of crystallographic orientation can be conveniently plotted on the standard stereographic triangle (a form of map that represents all crystallographic orientations), as shown in Figure 2.22. Single-crystal superalloys exhibit considerable elastic anisotropy; the effective elastic modulus is the lowest in $\langle 001\rangle$ directions and nearly tripled near $\langle 111\rangle$

Gas turbine engines are operated with loads sufficiently low to ensure that the majority of the components behave elastically, However, unavoidable features, such as bolt holes, blade fixing slots, etc., where the local geometry change present stress-raisers, localized inelastic deformation can occur in gas turbine components. Therefore, it is essential to understand the inelastic properties of single-crystal superalloys as well.

\subsubsection{Inelastic Properties of Single-Crystal Superalloys}

In contrast to elastic deformation, inelastic deformation in materials is an irreversible process. It involves breaking and rearrangement of atomic bonds in the crystal lattice. The mechanisms by which inelastic deformation occurs in ductile materials are movements of dislocations as a result of applied shear stresses. In the following sections, the factors contributing to the anisotropic inelastic deformation behaviour of singlecrystal superalloys are discussed.

\subsubsection{Slip Systems and the Critical Resolved Shear Stress}

The movement of dislocations through a crystal lattice is not arbitrary; it is energetically favorable for dislocation mobility to be restricted to specific crystallographic planes and along specific crystallographic directions that have the highest areal and linear atomic 
packing density, respectively. In FCC materials, such as single-crystal superalloys, the octahedral $\{111\}\{110\rangle$ slip systems are therefore the most preferred. At higher temperatures, when the atomic thermal energies are high, other slip systems can be activated in single-crystal superalloys, such as the primary cubic $\{100\}\langle 110\rangle[87,99-106]$ and the secondary octahedral $\{111\}\{112\rangle$ slip systems [61]. The secondary octahedral $\{111\}\{112\rangle$ slip systems are observed to be active under very specific conditions during creep deformation [61], and will not be discussed further. The 12 primary octahedral and 6 primary cube slip systems are shown schematically in Figures 2.23 and 2.24 , respectively.

Inelastic deformation can occur on one or more of the octahedral and/or cubic slipsystems. Slip system activity, i.e. the slip systems that experience inelastic deformation, usually have a threshold, which is the critical resolved shear stress (CRSS). Physically, the CRSS represents the shear stress required to cause a dislocation to glide in slip plane along a particular slip direction. One may determine which of the 12 octahedral and 6 cubic slip systems are active by comparing the CRSS and the resolved shear stress (RSS) on each slip system using Schmid's law. Schmid's law in tensor form is given by:

$$
\begin{gathered}
\tau=\boldsymbol{m}: \boldsymbol{\sigma} \\
m_{i j}=\frac{1}{2}\left(n_{i} l_{j}+n_{j} l_{i}\right)
\end{gathered}
$$

where $l_{i}$ is the slip direction unit vector and $n_{i}$ is the slip plane normal unit vector. A uniaxial illustration of Schmid's law is shown in Figure 2.25. From, Schmid's law one may infer that yield anisotropy of single-crystals is only a function of the geometry. 
However, in single-crystal superalloys additional yield anisotropy is observed that cannot be explained solely by Schmid's law. This is the subject of the following section.

\subsubsection{Temperature and Orientation Dependence of Yield in Single-Crystal Superalloys}

The inelastic response of single-crystal superalloys is significantly different from that of disordered FCC materials. Experimental results show that the yield strength (related to the CRSS through the Schmid factor) depends on material orientation and the direction (tension or compression) of initial loading. For example, the variation in tensile and compressive yield stress for the first generation single-crystal superalloy PWA1480 as a function of orientation along the [001]-[011] boundary of the standard stereographic triangle is shown in Figure 2.26. It is apparent that the [001] orientation has a higher yield strength in tension than in compression; the opposite is the case for the [011] orientation. Experimental results also indicate that the tension-compression anisotropy becomes insignificant for crystal orientations near [111] (see Figure 2.27), which deform by primary cube slip [87, 99-106]. This later finding suggests that the tension-compression anisotropy is a property of orientations that deform by primary octahedral slip. Another striking feature shown in Figure 2.26, if one considers only the tensile yield strength, is that the [001] orientation possesses a substantially higher yield strength than [011]. This later observation is significant because [001] and [011] both have identical Schmid factors, and therefore these two orientations should have identical yield strength if the anisotropy can be rationalized solely by Schmid's law. Clearly this is not the case.

Numerous researchers have indicated that in addition to material orientation, yield behaviour in single-crystal superalloys is significantly affected by temperature $[87,99$, $101,102,104-110]$. Yield strength is reported to increase with temperature until a plateau 
is reached at some critical temperature between $700-800^{\circ} \mathrm{C}$. The yield strength below the critical temperature is insensitive to strain rate. At higher temperatures, i.e. above the critical temperature, the tension-compression anisotropy and yield strength drop precipitously with increasing temperature, as shown in Figure 2.28. The yield behaviour is also observed to be increasingly strain rate-dependent as temperatures are increased, as shown in Figure 2.29.

The complex tension-compression anisotropy and the temperature dependence of the yield strength in single-crystal superalloys can be explained by considering microstructural factors and the deformation mechanisms observed in these materials. Unlike disordered FCC single-crystals, the microstructure of single-crystal nickel-base superalloys contains a high volume fraction of ordered $\gamma^{\prime}$ precipitates, with which mobile dislocations interact. Detailed studies of the dislocation structures in single-crystal superalloys indeed suggest that such interactions do occur. Several researchers $[101,102$, $108,109]$ have reported that at temperatures below the critical temperature, that shearing of the $\gamma^{\prime}$ precipitates by dislocations is the dominant deformation mechanism. At higher temperatures, i.e. above the critical temperature, deformation is predominantly limited to dislocation activity in the $\gamma$-matrix, although some shearing of the $\gamma^{\prime}$ phase has been reported by Milligan [109] for PWA1480 tested at high strain rates at $927^{\circ} \mathrm{C}$. These finding suggests that shearing of $\gamma^{\prime}$-phase plays an important role in the anisotropic yield behaviour observed in single-crystal superalloys, at least up to the critical temperature. It also follows that another deformation mechanism other than $\gamma^{\prime}$ shearing must be operative at temperatures above the critical temperature.

Several mechanistic models have been proposed to explain the anomalous yield behaviour observed in $\mathrm{L}_{2}$ ordered structures, such as $\gamma^{\prime}$, including the Takeuchi and 
Karamoto model [111], the Paidar-Pope-Vitek (PPV) model [112], and a more recent model proposed by Hirsch [113]. A cursory discussion of some elements of these models is warranted to explain the physical deformation processes involved in shearing of the $\gamma^{\prime}$ precipitates and the relationship between these deformation processes and the anomalous yield behaviour observed in single-crystal superalloys. The more ambitious reader should refer to the work of Hirsch [113] and Paidar et al. [112] for a thorough discussion of the dislocation mechanisms responsible for the anomalous yield behaviour exhibited by $\mathrm{L}_{2}$ ordered materials.

One common element between the aforementioned models is that it is assumed that $\gamma^{\prime}$ shearing occurs on the octahedral slip planes as a result of pair of $a / 2\langle 110\rangle$ dislocations, which are collectively referred to as a superdislocation. A pair of dislocations is involved in the $\gamma^{\prime}$ shearing process since the passage of a single $a / 2\langle 110\rangle$ dislocation creates an anti-phase boundary (APB) in which the ordered atomic arrangement on slip plane is destroyed. The passage of a second $a / 2\langle 110\rangle$ dislocation reduces the energy associated with the APB by restoring the ordered atomic arrangement. The two dislocations that constitute a superdislocation are separated by APB because of mutually repulsive forces between the two dislocations.

Recall that slip in superalloys has been observed to occur on both the cube and octahedral slip planes. Since both the cube and octahedral slip planes share common slip directions, screw dislocations can cross-slip from the primary octahedral to the primary cube slip planes. The APB energy is anisotropic in $\mathrm{L}_{2}$ ordered structures, being higher on an octahedral slip plane than an APB created on a cube slip plane due to the more numerous bonding violations involved in the APB on an octahedral slip plane. Therefore, it is 
energetically favorable for the leading dislocation to cross-slip from the octahedral to the cube slip plane (a process called cube cross-slip) to reduce the APB energy. However, this cross-slip event comes at the expense of rendering the entire superdislocation sessile by a locking mechanism $[112,113]$, which also inhibits the mobility of other superdislocations shearing the precipitate. Since cross-slip is a thermally activated process, i.e. more cross-slip events can occur at higher temperatures, the cube cross-slip superdislocation pinning mechanism partially explains the increase in yield strength observed in single-crystal superalloys. An explanation of the tension-compression anisotropy observed in single-crystal superalloys requires consideration of another property of dislocations in FCC materials.

Dislocations of the type $a / 2\langle 110\rangle$ on the octahedral planes in FCC materials including $\gamma^{\prime}$, dissociate into two partial dislocations whose displacement vectors sum up to give the same $a / 2\langle 110\rangle$ displacement of a full dislocation. These partial dislocations (called Shockley partial dislocations) are separated by a stacking fault where the regular atomic arrangement of the FCC lattice is disrupted forming an extended dislocation arrangement, as shown in Figure 2.30. The core-width, i.e. the width of the stacking fault, is the result of the repulsive forces between the partial dislocations and the stacking fault energy (SFE), which depends on alloy composition. The significance of the extended dislocation arrangement is that in order for cross-slip of a screw dislocation to occur, such as from the octahedral to the cube slip plane as described earlier, the two partial dislocations must constrict to form a full dislocation so that the slip vector lies in the cross-slip plane. The constriction process requires some additional energy, which is supplied by an externally applied stress. Depending on which slip system is active and the sign of the resolved shear stress in relation to the partial dislocation slip directions, the constriction process 
may be aided or impeded. It is this so-called "core-width effect" that is believed to contribute to the tension-compression anisotropy observed in single-crystal superalloys $[108,109,114,115]$.

The thermally activated cube cross-slip mechanism and core-width effect can explain some of the anisotropy effects observed in single-crystal superalloys at low-temperatures where precipitate shearing is the dominant deformation mechanism. However, as noted earlier, deformation structures at higher temperatures do not exhibit significant precipitate shearing nor do they exhibit significant tension-compression anisotropy and deformation is mainly restricted to the $\gamma$-matrix between the precipitates. At higher temperatures, i.e. those above the critical temperature, dislocations in $\gamma$ can cross-slip (discussed earlier) or climb to circumvent obstacles, such as the $\gamma^{\prime}$ precipitates. Dislocation climb is a thermally activated process in which segment of an edge dislocation avoids an obstacle by diffusion of atoms or vacancies to the dislocation core. It may be noted that if strain rates are sufficiently high, that cross-slip and/or climb of dislocations in the softer $\gamma$-matrix may be insufficient to avoid dislocations shearing the $\gamma^{\prime}$ precipitates, which would be observed as an increase in flow stress. This latter point can explain the rate dependency of the yield stress observed at high temperatures in single-crystal superalloys.

In addition to the interaction between dislocations and the microstructure discussed thus far, changes to the microstructure and the effect of such changes on the flow strength have not been considered. At elevated temperatures partial dissolutioning of the $\gamma^{\prime}$-phase may occur resulting in a reduction in the $\gamma^{\prime} \mathrm{V}_{\mathrm{f}}$. As noted earlier, the $\gamma^{\prime} \mathrm{V}_{\mathrm{f}}$ is important for conferring strength to single-crystal superalloys, and therefore some portion of the 
reduction in yield strength observed at high temperatures may be attributed to this phenomenon.

While it is important to consider the deformation mechanisms that contribute to initiating plastic flow in single-crystal superalloys, it is also important to consider what occurs after yielding commences. The behaviour of single-crystal superalloys under tensile and cyclic loading are discussed in the following sections.

\subsubsection{Monotonic Deformation Behaviour of the Single-Crystal Superalloys}

During uniaxial tensile or compressive loading, the deformation mechanisms in singlecrystal superalloys depend on temperature, strain rate, strain level and stress state [116]. The temperature and strain rate dependence of the flow stress and deformation mechanisms has been extensively investigated in single-crystal nickel-base superalloys $[87,99,101,104,106,107,109]$. Results from these investigations indicate that the

temperature, orientation and strain rate dependence of deformation mechanisms in these alloys can be summarized into three distinct temperature regimes:

1. Low temperatures $\left(\mathbf{T}<\mathbf{7 6 0}^{\circ} \mathbf{C}\right)$ : The dominant deformation mechanism is $\gamma^{\prime}$ shearing. For orientations that exhibit primary octahedral slip, slip occurs on discrete octahedral slip planes with the highest CRSS forming heterogeneous slip bands except in [001] single-crystals which has a maximum number of available slip systems and hence exhibit more homogenous slip. Orientations near [111] deform by primary cube slip on discrete cube slip planes with the highest CRSS and also exhibit heterogeneous slip behaviour. Single-slip orientations, i.e. orientations away from [001] and [111] have pronounced serrated yield behaviour (Figure 2.31) as a result of heterogeneous slip and little post-yield hardening. Multiple-slip orientations, such as [001] and [111], have smooth tensile curves 
and exhibit significant post-yield hardening. No significant strain rate dependence is observed below $760^{\circ} \mathrm{C}$.

2. Intermediate temperatures $\left(760<\mathbf{T}<\mathbf{9 0 0}^{\circ} \mathrm{C}\right)$ : In the intermediate temperature regime the flow-stress is strongly rate dependent; however, the rate dependence increases with increasing temperature. The deformation structures are homogeneous, tensile curves are smooth and a rate dependent transition from $\gamma^{\prime}$ shearing to $\gamma^{\prime}$ bypass is observed. Orientations near [111] deform by primary cube slip and other orientations deform by octahedral slip. All orientations exhibit work hardening that increases with increasing temperature.

3. High temperatures $\left(\mathbf{T}>\mathbf{9 0 0}^{\circ} \mathrm{C}\right)$ : The dominant deformation mechanism is cross-slip induced $\gamma$ ' bypass. All orientations exhibit homogenous slip and smooth tensile curves. The dominant slip systems contributing to inelastic deformation, the marked strain rate dependence, and work hardening behaviour are similar to that observed in the intermediate temperature regime.

Based on the forgoing summary of the temperature, orientation and strain rate dependence of the monotonic deformation mechanisms several points should be noted. First, multiple-slip orientations, i.e. orientations near [001] and [111], exhibit higher work hardening rates than orientations that have one or few preferred slip system (single-slip orientations). This observation can be rationalized if one considers that in instances where multiple slip systems are active, that dislocation mobility can be impeded by the interaction of dislocations on intersecting slip systems. The second point to be noted is that the work hardening behaviour is anisotropic in single-crystal superalloys, being highest for [001] oriented single-crystals and lower for other orientations. The last point to consider is the diminished serrated (jerky) yield phenomenon and the increased work 
hardening behaviour exhibited at higher temperatures. Presumably, this behaviour can be attributed to increased homogeneity of the deformation and the higher propensity for dislocation interactions on intersecting slip systems. One additional point worth mentioning is latent hardening. Latent hardening occurs when a single-crystal sample with a single-slip orientation is deformed plastically to a certain amount of inelastic strain under uniaxial loading. When the same single-crystal sample is subsequently loaded (uniaxially) such that single-slip occurs on a different (latent) slip system than during the previous inelastic straining, the flow stress would be higher. The higher flow stress observed during the second loading period is believed to be due to "latent hardening" arising from the deformed structure generated during the initial inelastic straining. Latent hardening effects have not been reported in single-crystal superalloys presumably because the strengthening effect of the $\gamma^{\prime}$ precipitates is substantially higher than that generated by the deformation structure [115].

\subsubsection{Cyclic Deformation Behaviour of the Single-Crystal Superalloys}

When single crystals with different crystallographic orientations are fatigued under strain control within a fixed strain range, the elastic anisotropy can have a significant impact on the resulting stress and inelastic strain ranges. Consider for example that two orientations have the same yield strength; the orientation with a higher elastic modulus will yield at a smaller total strain than the low-modulus orientation. Therefore, the high modulus orientation will exhibit a larger inelastic strain range than an orientation with a lower modulus when cycled within the same fixed strain limits. This is in fact is confirmed by low-cycle fatigue (LCF) and thermomechanical fatigue (TMF) tests conducted on singlecrystals with different crystallographic orientations $[32,100,103]$. The variation in elastic modulus with crystal orientation is frequently used to explain the orientation 
dependence of fatigue life in single crystal and directionally solidified nickel-base superalloys $[117,118]$.

In addition to effects of elastic anisotropy on cyclic stress/strain response, temperature has a significant influence on cyclic deformation behaviour. In many respects, the temperature dependence of the cyclic material response and the dominant slip systems contributing to inelastic deformation are similar to what is observed during monotonic deformation (see Section 2.5.2.3). Depending on whether a single crystal is oriented for multiple-slip or single-slip, the following material response has been reported during strain-controlled cyclic deformation $[100,103,108,116,119]$ :

1. Low Temperatures $\left(\mathbf{T}<\mathbf{7 6 0}^{\circ} \mathrm{C}\right)$ : Single crystals having multiple-slip orientations exhibit greater cyclic hardening, higher stress ranges and shorter cyclic lives than single-slip orientations. Regardless of orientation, cyclic hardening occurs rapidly to reach a stabilized stress range due to a precipitate shearing process. Discrete deformation structures are observed regardless of single-crystal orientation.

2. Intermediate Temperatures $\left(\mathbf{7 6 0}<\mathbf{T}<\sim 900^{\circ} \mathrm{C}\right)$ : At intermediate temperatures, there is a strain-rate dependent transition from cyclic hardening to cyclic softening. This is due to a gradual transition from low-temperature precipitate shearing to high-temperature precipitate bypass mechanisms. In multiple-slip single-crystals, the deformation structures in this temperature regime are increasingly homogeneous, depending on the strain rate.

3. High Temperatures $\left(\mathbf{T}>\mathbf{9 0 0}^{\circ} \mathbf{C}\right)$ : At high-temperatures softening is observed during cyclic loading conditions regardless of strain rate and crystal orientation. Cyclic softening occurs due to precipitate coarsening and/or dissolution of 
hyperfine $\gamma^{\prime}$ present in alloys having a bimodal $\gamma^{\prime}$ size distribution. The deformation structures observed after high temperature cyclic loading are homogeneous.

It addition to orientation and strain-rate dependent behaviour noted above, it may also be noted that during fully-reversed strain-controlled LCF tests that the tension-compression yield asymmetry is observed at low-temperatures as shown in Figure 2.32. 
Table 2.1 - Chemical compositions (in weight \%) for common first-generation single-crystal superalloys.

\begin{tabular}{|l|c|c|c|c|c|c|c|c|c|c|c|}
\hline Alloy & $\mathrm{Cr}$ & $\mathrm{Co}$ & $\mathrm{Mo}$ & $\mathrm{W}$ & $\mathrm{Al}$ & $\mathrm{Ti}$ & $\mathrm{Ta}$ & $\mathrm{Nb}$ & Other & $\mathrm{Ni}$ & Ref. \\
\hline CMSX-2 & 8 & 4.6 & 0.6 & 8 & 5.6 & 1 & 6 & - & - & Bal. & {$[120]$} \\
\hline CMSX-6 & 9.8 & 5 & 3 & - & 4.8 & 4.7 & 2 & - & $0.1 \mathrm{Hf}$ & Bal. & {$[121]$} \\
\hline PWA 1480 & 10 & 5 & - & 4 & 5 & 1.5 & 12 & - & - & Bal. & {$[71]$} \\
\hline René N4 & 9 & 8 & 2 & 6 & 3.7 & 4.2 & 4 & 0.5 & - & Bal. & {$[77]$} \\
\hline RR2000 & 10 & 15 & 3 & - & 5.5 & 4 & - & - & $1 \mathrm{~V}$ & Bal. & {$[93]$} \\
\hline SRR99 & 8 & 5 & - & 10 & 5.5 & 4 & 3 & - & - & Bal. & {$[93]$} \\
\hline
\end{tabular}

Table 2.2 - Chemical compositions (in weight\%) for common second-generation singlecrystal superalloys.

\begin{tabular}{|l|c|c|c|c|c|c|c|c|c|c|c|}
\hline Alloy & $\mathrm{Cr}$ & $\mathrm{Co}$ & $\mathrm{Mo}$ & $\mathrm{Re}$ & $\mathrm{W}$ & $\mathrm{Al}$ & $\mathrm{Ti}$ & $\mathrm{Ta}$ & $\mathrm{Hf}$ & $\mathrm{Ni}$ & Ref. \\
\hline CMSX-4 & 6.5 & 9 & 0.6 & 3 & 6 & 5.6 & 1 & 6.5 & 0.1 & Bal. & {$[85]$} \\
\hline PWA 1484 & 5 & 10 & 2 & 3 & 6 & 5.6 & - & 8.7 & 0.1 & Bal. & {$[84]$} \\
\hline René N5 & 7 & 8 & 2 & 3 & 5 & 6.2 & - & 7 & 0.2 & Bal. & {$[122]$} \\
\hline
\end{tabular}

Table 2.3 - Chemical compositions (in weight\%) for common third-generation singlecrystal superalloys.

\begin{tabular}{|l|c|c|c|c|c|c|c|c|c|c|c|c|c|}
\hline Alloy & $\mathrm{Cr}$ & $\mathrm{Co}$ & $\mathrm{Mo}$ & $\mathrm{Re}$ & $\mathrm{W}$ & $\mathrm{Al}$ & $\mathrm{Ti}$ & $\mathrm{Ta}$ & $\mathrm{Nb}$ & $\mathrm{Hf}$ & Other & $\mathrm{Ni}$ & Ref. \\
\hline CMSX-10 & 2 & 3 & 0.4 & 6 & 5 & 5.7 & 0.2 & 8 & 0.1 & 0.03 & - & Bal. & {$[92]$} \\
\hline René N6 & 4.2 & 12.5 & 1.4 & 5.4 & 6 & 5.75 & - & 7.2 & - & 0.15 & $\begin{array}{c}0.05 \mathrm{C} \\
0.004 \mathrm{~B}\end{array}$ & Bal. & {$[94]$} \\
& & & & & & & & & & & \\
& & & & & & & & & & & $0.01 \mathrm{Y}$ & & \\
\hline
\end{tabular}

Table 2.4 - Chemical compositions (in weight \%) for common fourth-generation singlecrystal superalloys.

\begin{tabular}{|c|c|c|c|c|c|c|c|c|c|c|c|c|c|}
\hline Alloy & $\mathrm{Cr}$ & $\mathrm{Co}$ & Mo & $\mathrm{Re}$ & $\mathrm{Ru}$ & W & $\mathrm{Al}$ & $\mathrm{Ti}$ & $\mathrm{Ta}$ & Hf & Other & $\mathrm{Ni}$ & Ref. \\
\hline MC-NG & 4 & $<0.2$ & 1 & 4 & 4 & 5 & 6 & 0.5 & 5 & 0.1 & - & Bal. & {$[98]$} \\
\hline $\begin{array}{l}\text { MX4/ } \\
\text { PWA1497 }\end{array}$ & 2 & 16.5 & 2.0 & 5.95 & 3 & 6 & 5.55 & - & 8.25 & 0.15 & $\begin{array}{c}0.03 \mathrm{C} \\
0.004 \mathrm{~B} \\
0.01 \mathrm{Y}\end{array}$ & Bal. & {$[97]$} \\
\hline TMS-138 & 2.8 & 5.8 & 2.9 & 5.1 & 1.9 & 6.1 & 5.8 & - & 5.6 & 0.05 & - & Bal. & {$[96]$} \\
\hline TMS-162 & 2.9 & 5.8 & 3.9 & 4.9 & 6 & 5.8 & 5.8 & - & 5.6 & 0.09 & - & Bal. & {$[96]$} \\
\hline
\end{tabular}




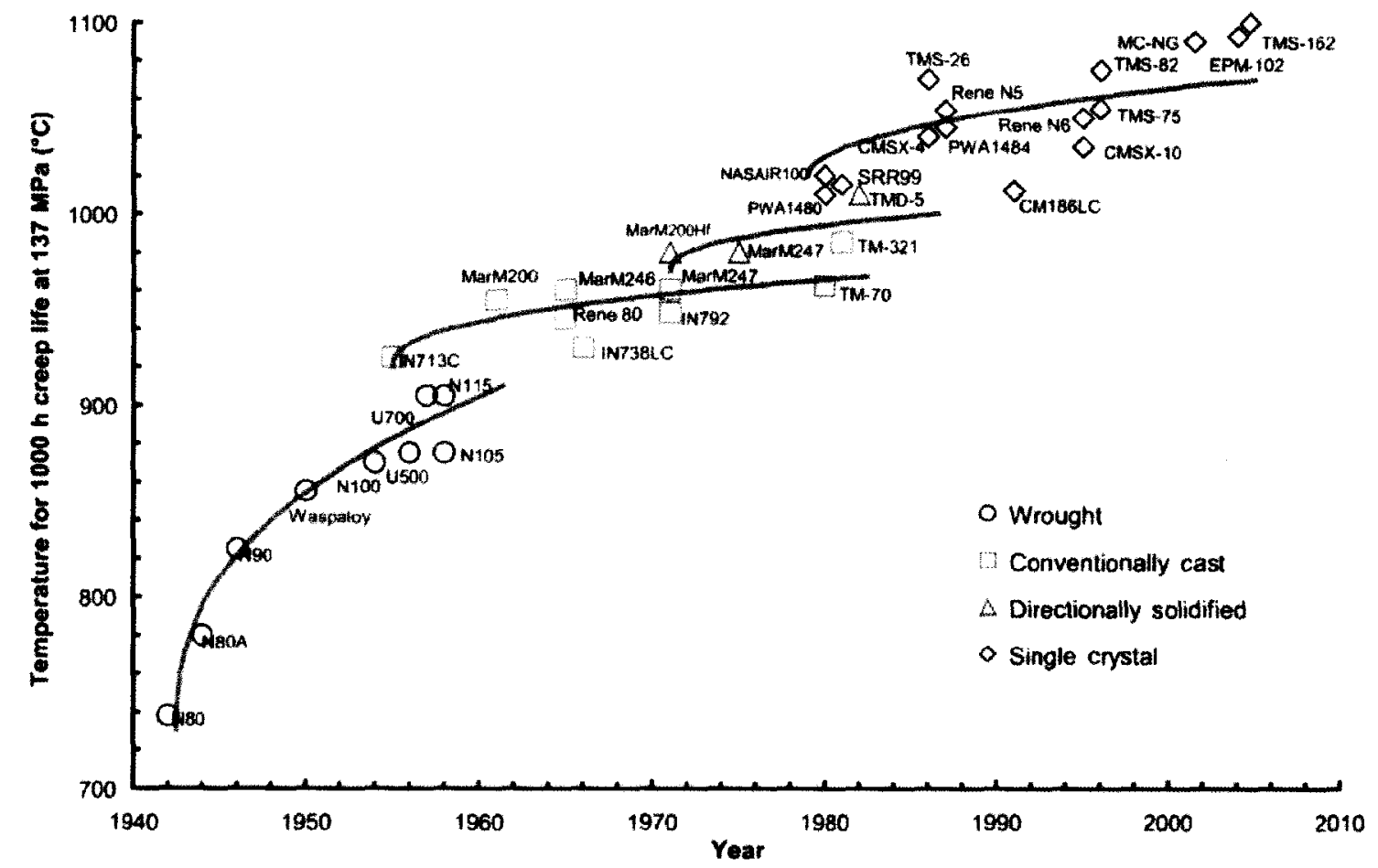

Figure 2.1 - Historical development of superalloys since 1940 and evolution of metal temperature capability[61].

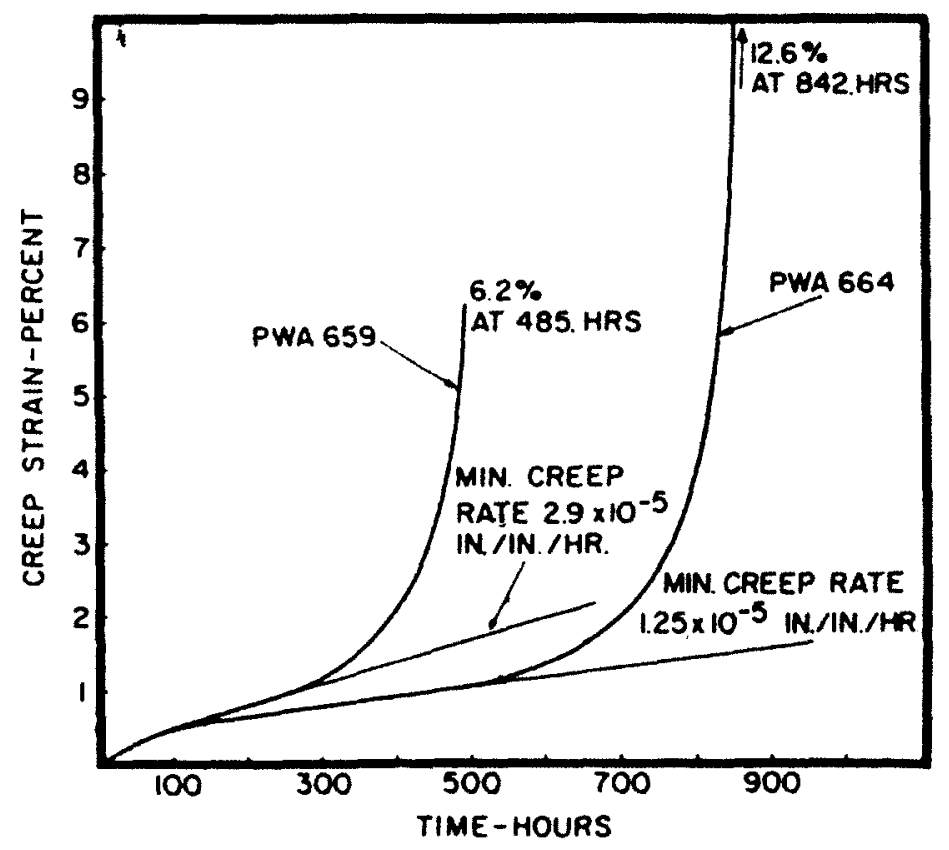

Figure 2.2 - Creep curves for conventional cast (PWA659) and directionally solidified (PWA664) Mar-M-200 tested at $1800^{\circ} \mathrm{F}$ and $20 \mathrm{ksi}$ [66]. Notice directionally solidified material has a substantially lower minimum creep rate and higher creep ductility than conventionally cast material. 


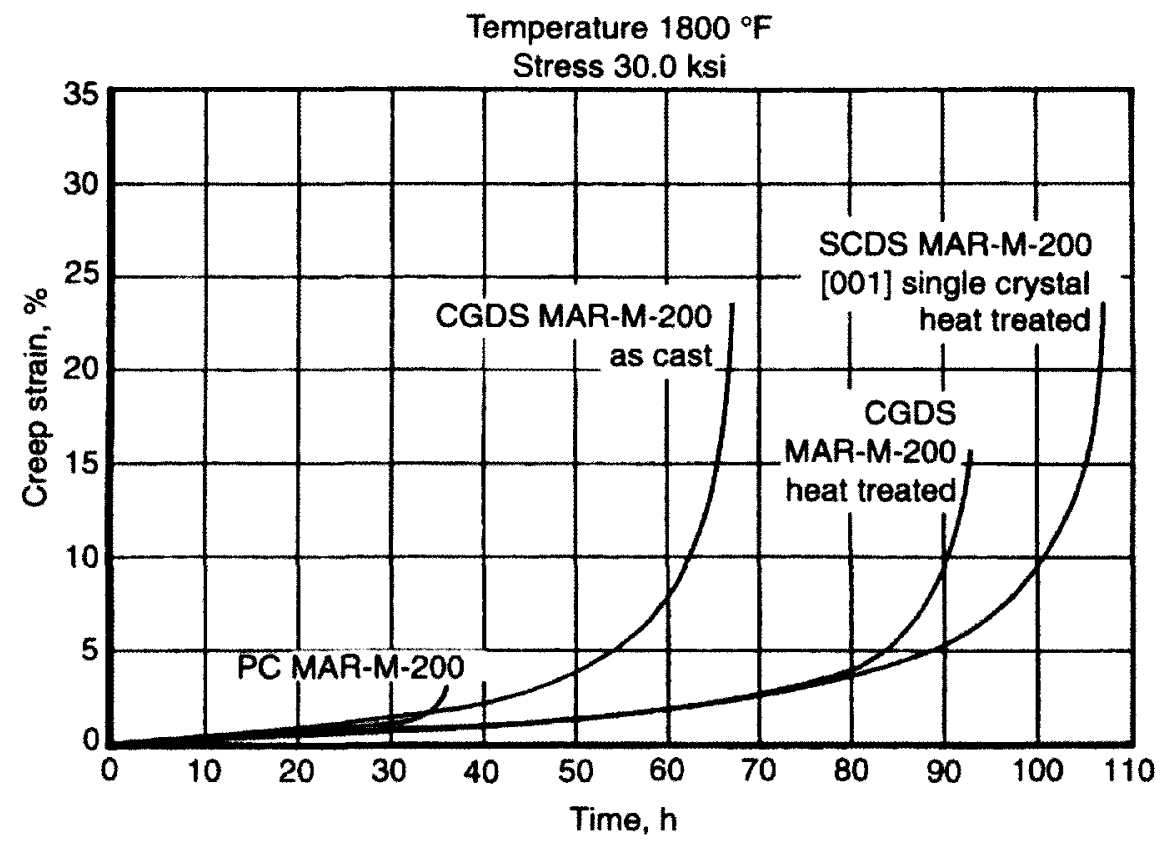

Figure 2.3 - Creep curves for Mar-M-200 alloy with poly-crystalline (PC), columnar grain directionally solidified (CGDS) and single-crystal directionally solidified (SCDS) structure tested at ${ }^{1800}{ }^{\circ} \mathrm{F}$ and $30 \mathrm{ksi}[80]$.

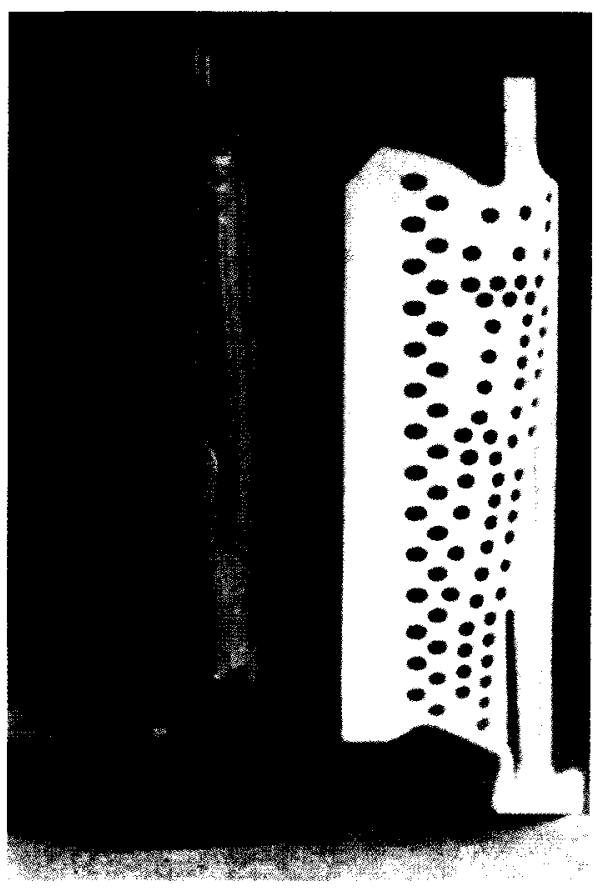

Figure 2.4 - Ceramic core and cutaway of turbine blade showing internal cooling features [80]. 


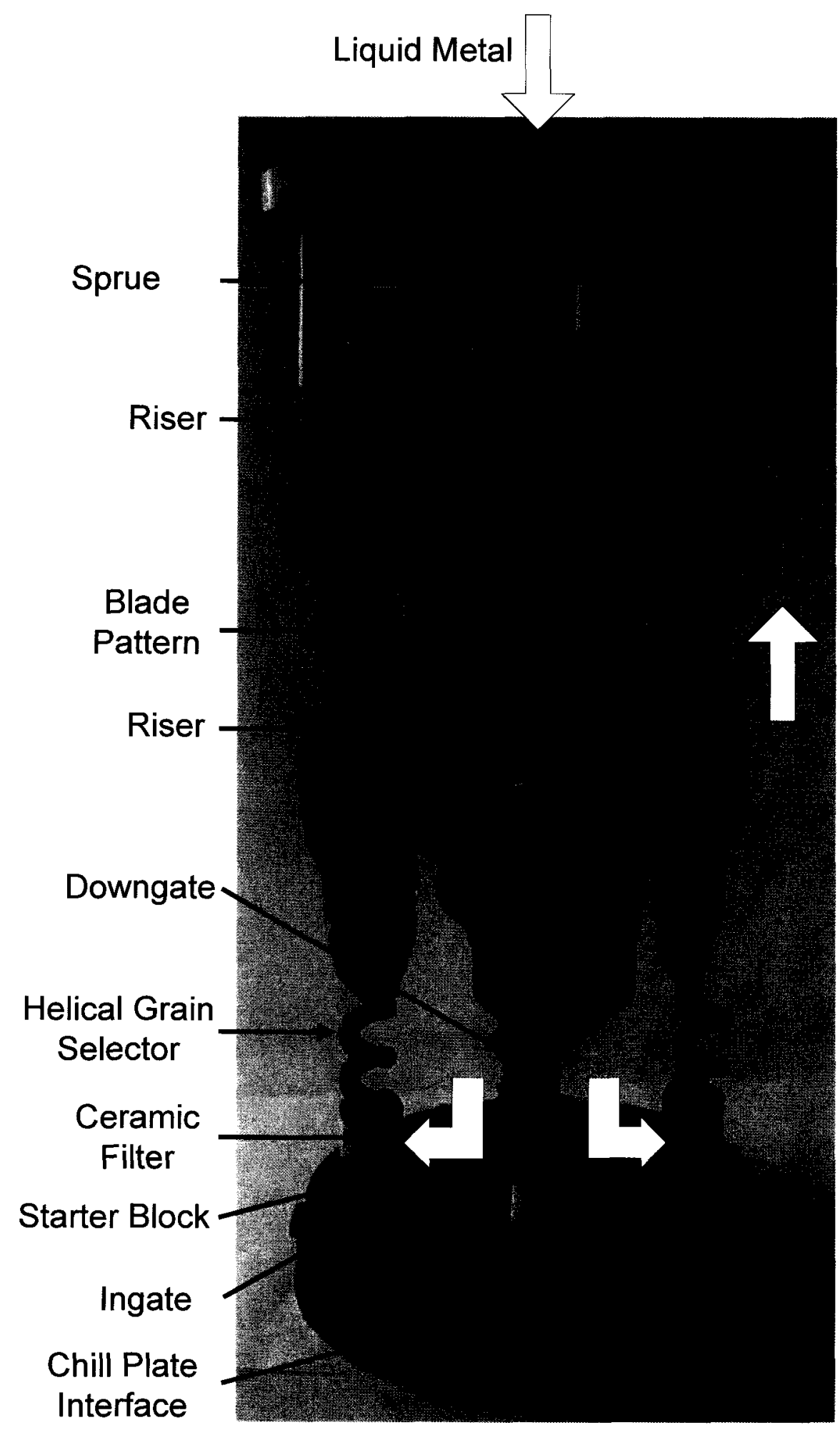

Figure 2.5 - Wax blade cluster pattern for casting SX turbine blades (adapted from [123]). 


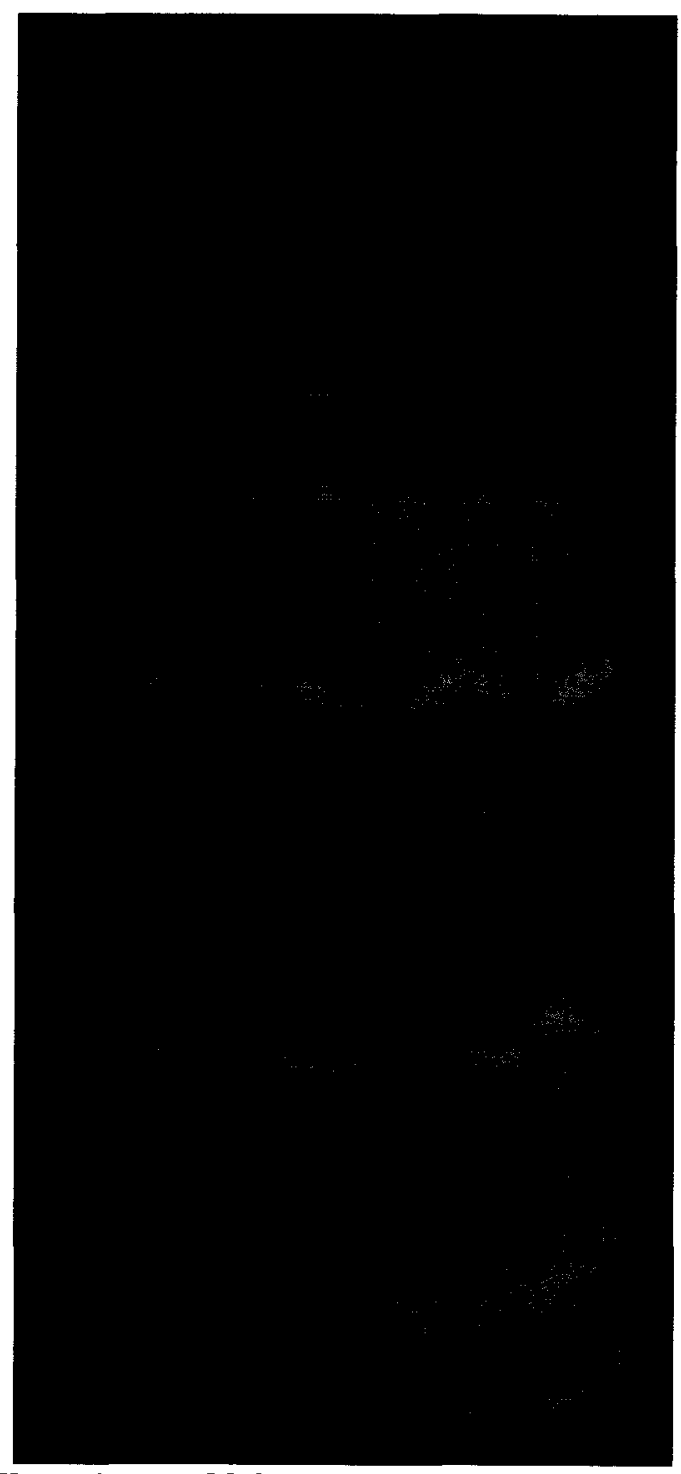

Figure 2.6 - Ceramic shell casting mold for single crystal turbine blades [123].

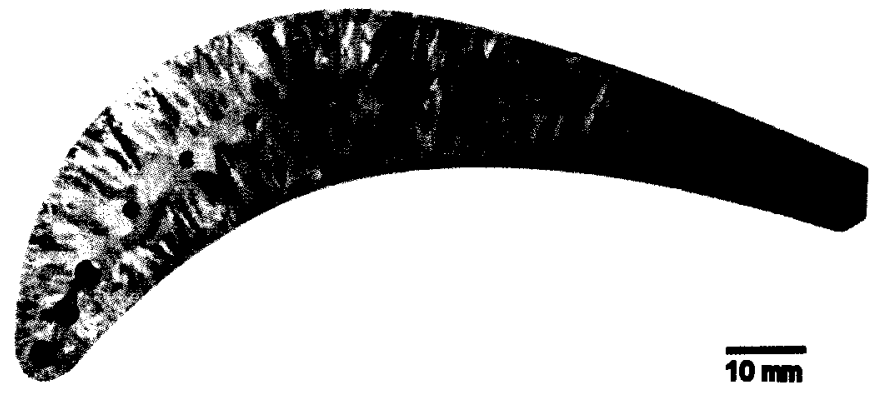

Figure 2.7 - Transverse section of a conventionally cast superalloy bucket that was polished and etched to reveal the grain structure. 


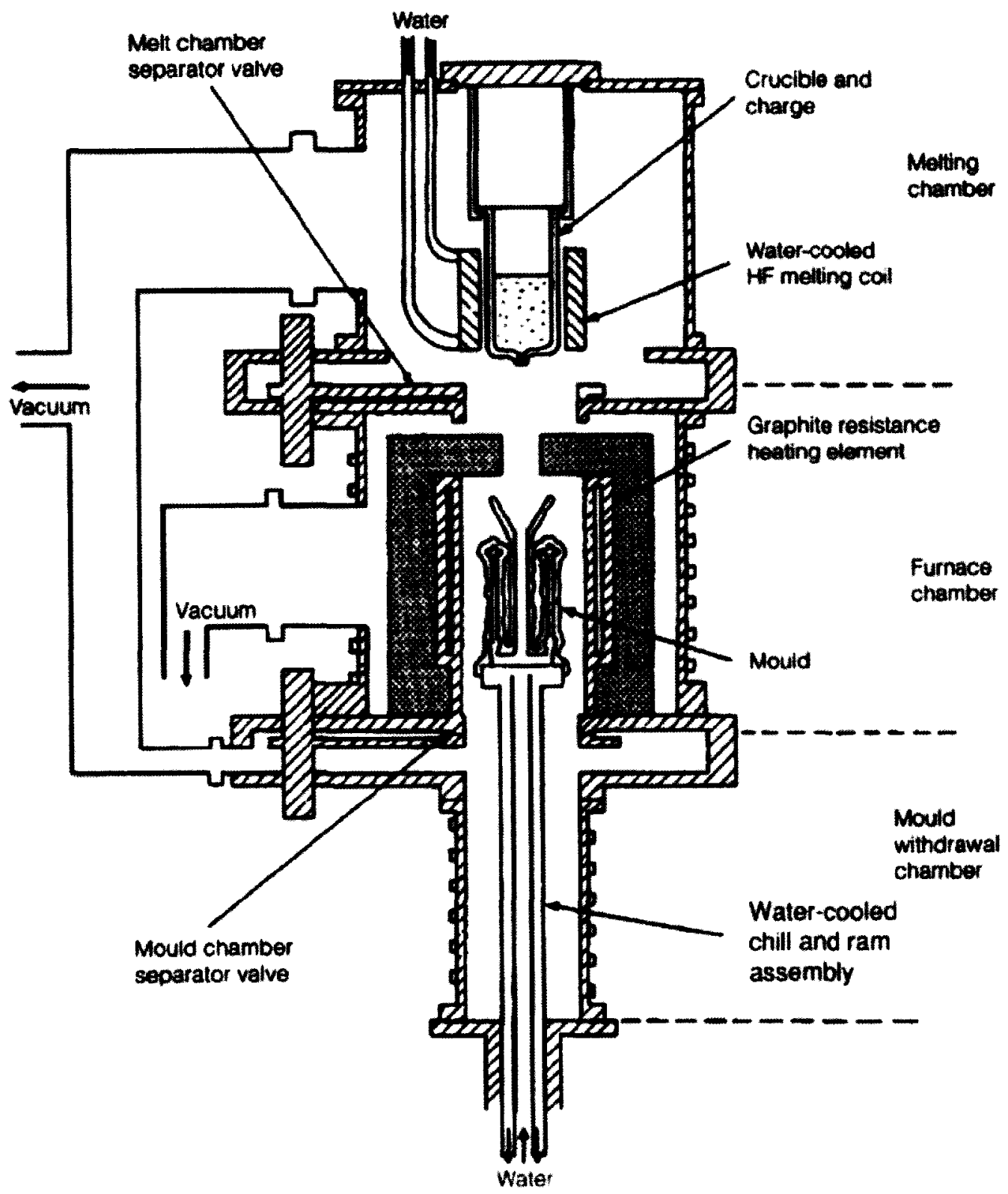

Figure 2.8 - Schematic illustration of an industrial Bridgman furnace for the production of DS and single-crystal turbine blades [124]. 


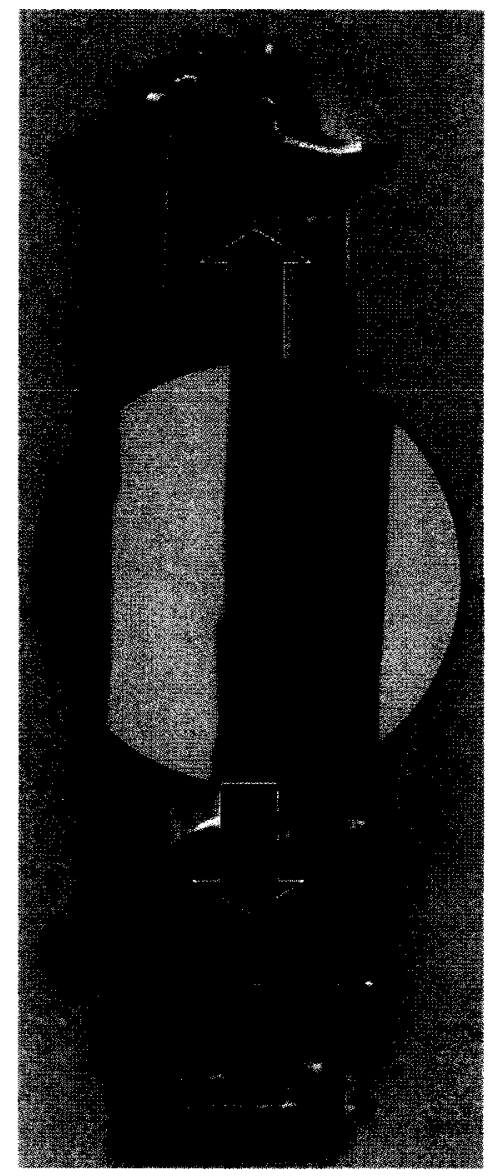

Figure 2.9 - Directionally solidified turbine blade etched to reveal columnar grain structure (adapted from [58]). Note: Inset shows a magnified view of the DS grain structure.

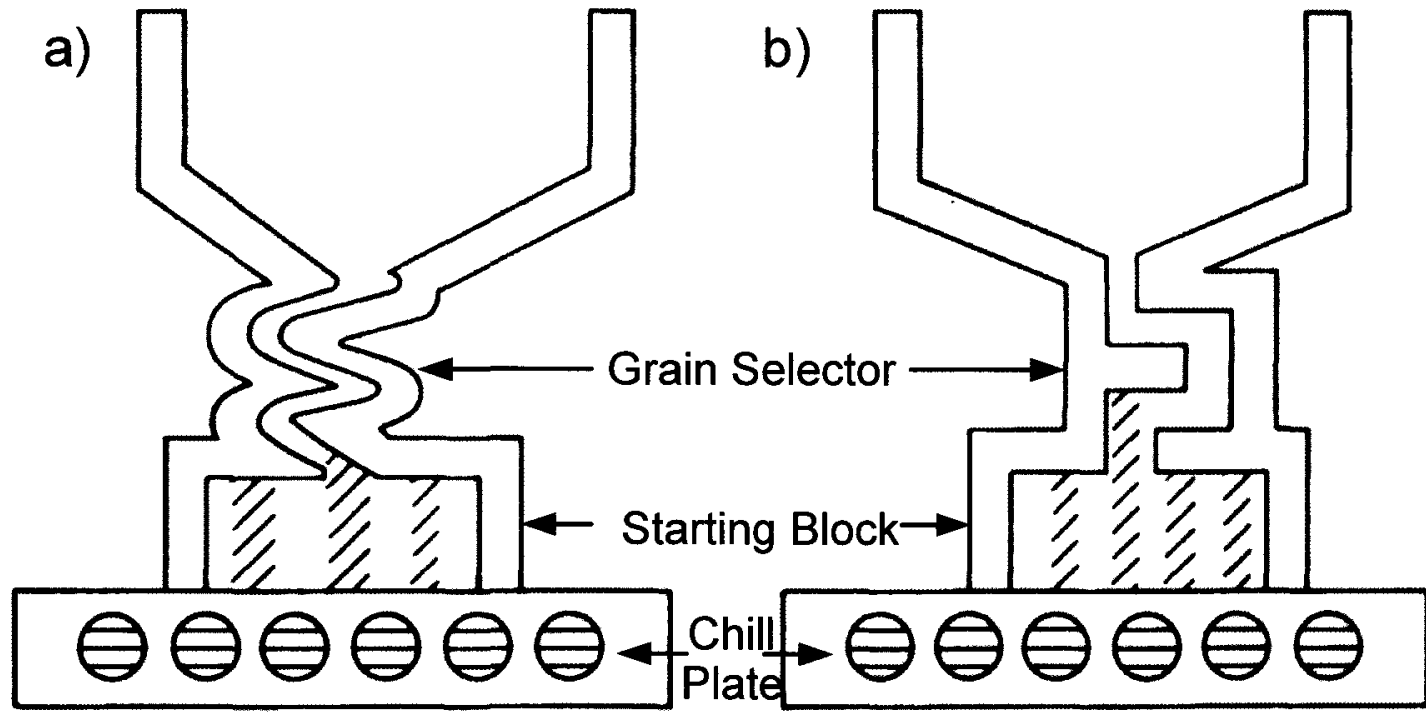

Figure 2.10 - Grain selector designs used for single-crystal casting; a) helical grain selector and b) two turn selector (adapted from [80]). 


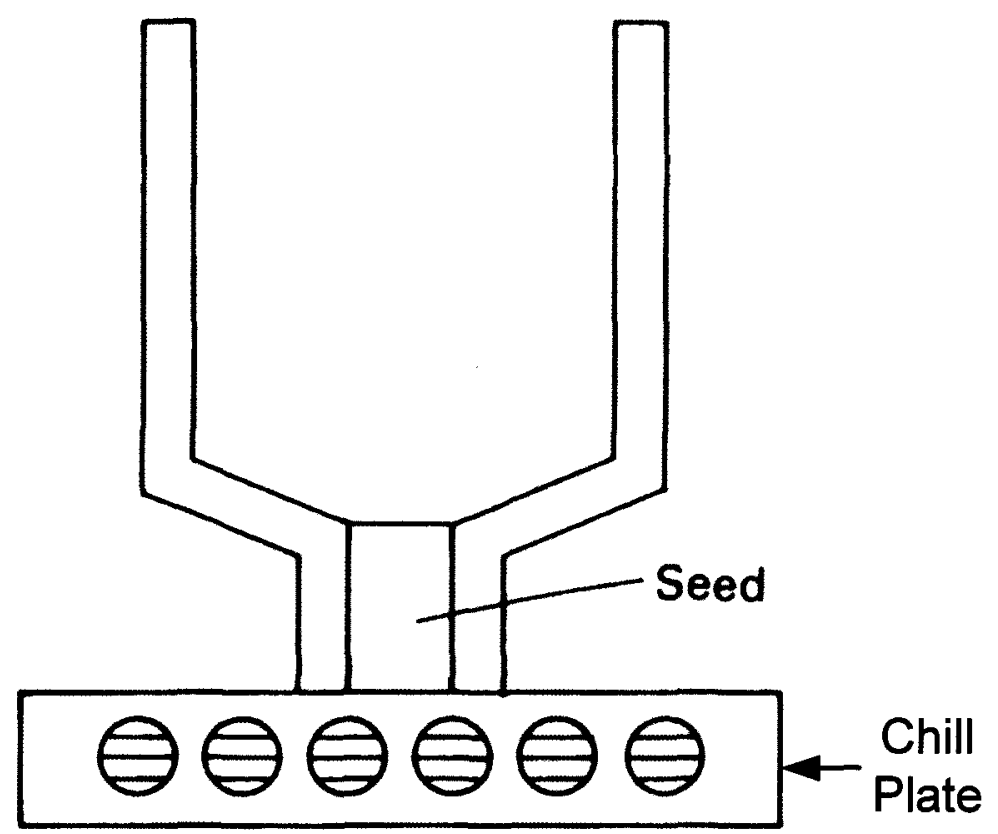

Figure 2.11 - Schematic showing arrangement used for single-crystal part production using seed crystal (adapted from [80]).
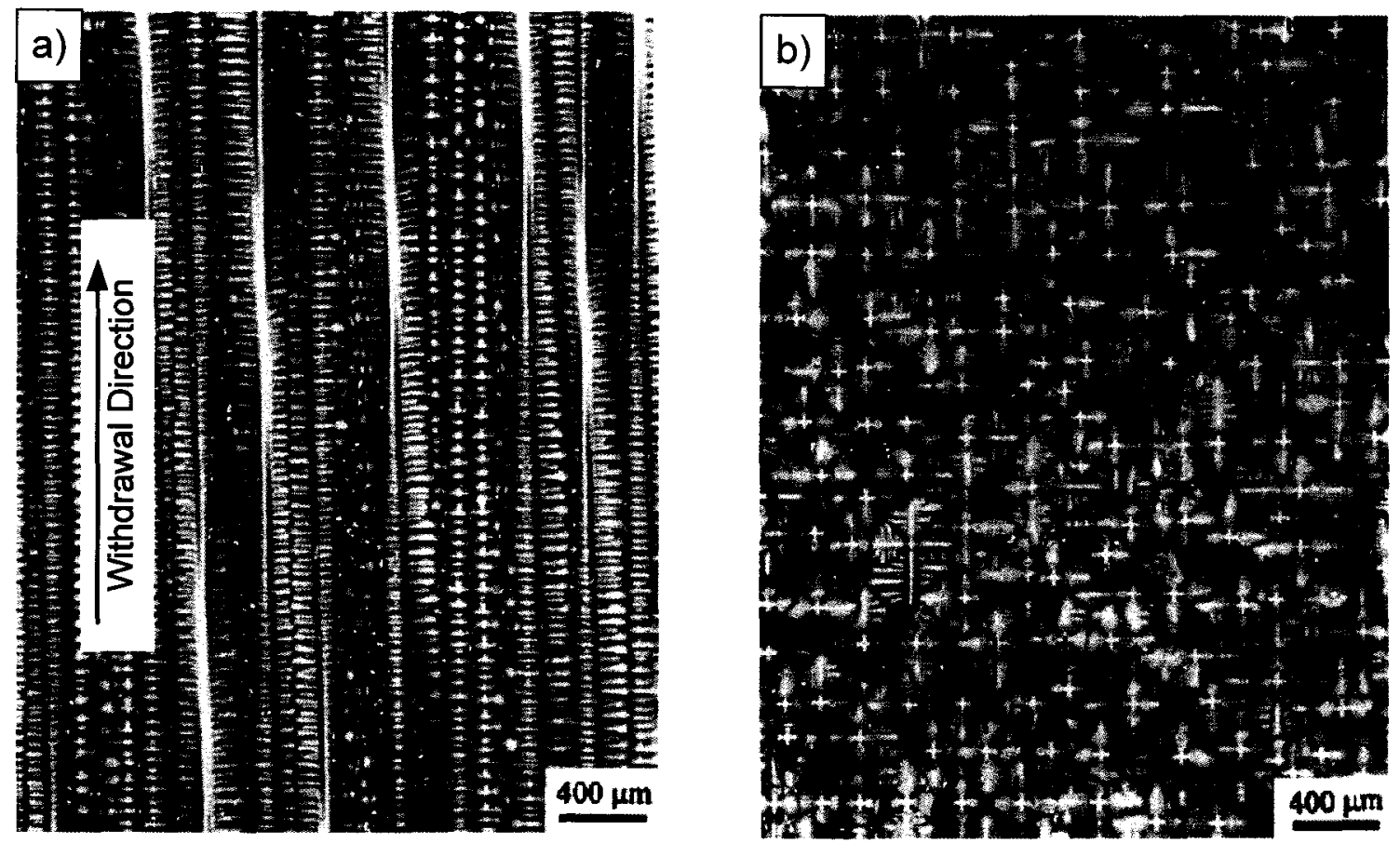

Figure 2.12 - As-cast dendritic structure observed in single-crystal superalloys; a) longitudinal section showing primary dendrite arms aligned with withdrawal direction and b) transverse section showing primary dendrite arm spacing (PDAS) and secondary and tertiary dendrite arms (adapted from [125]). 


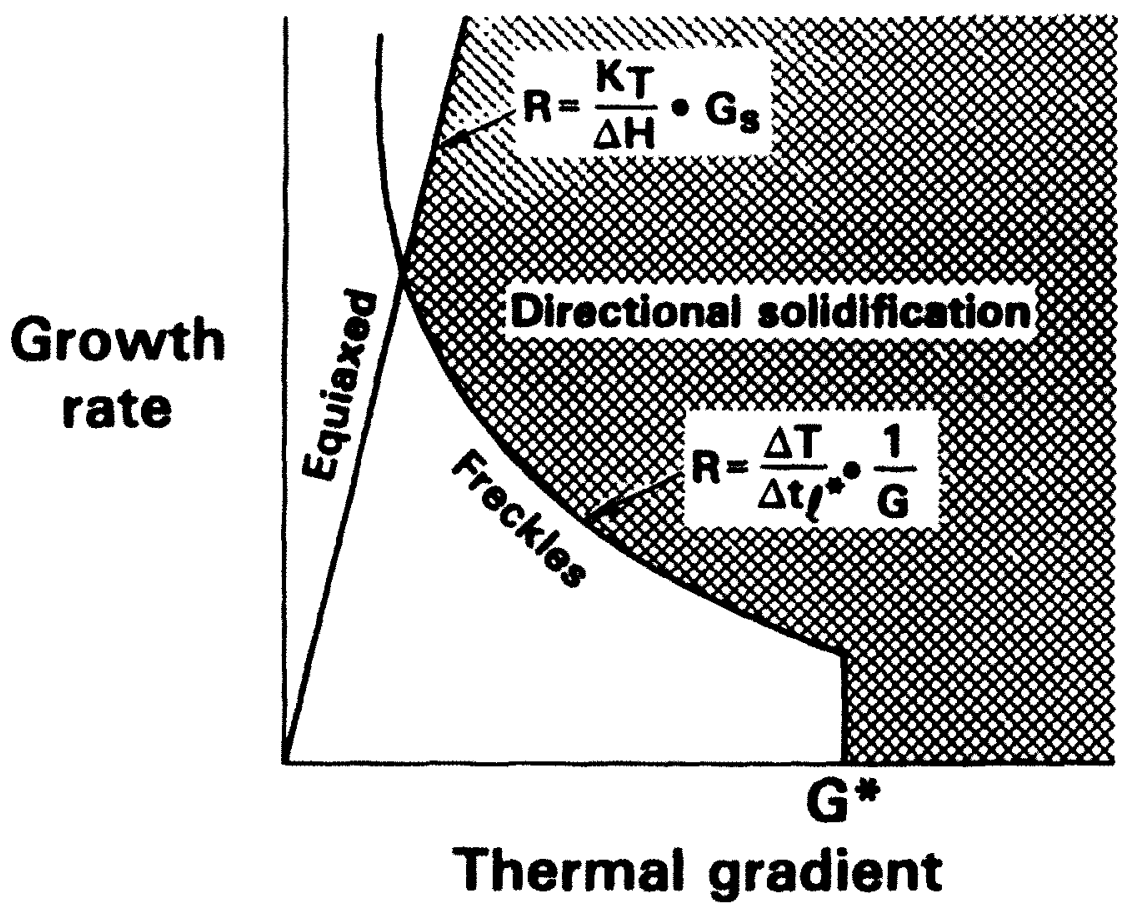

Figure 2.13 - Schematic diagram showing effect of solidification rate (growth rate) versus thermal gradient on microstructure [74].

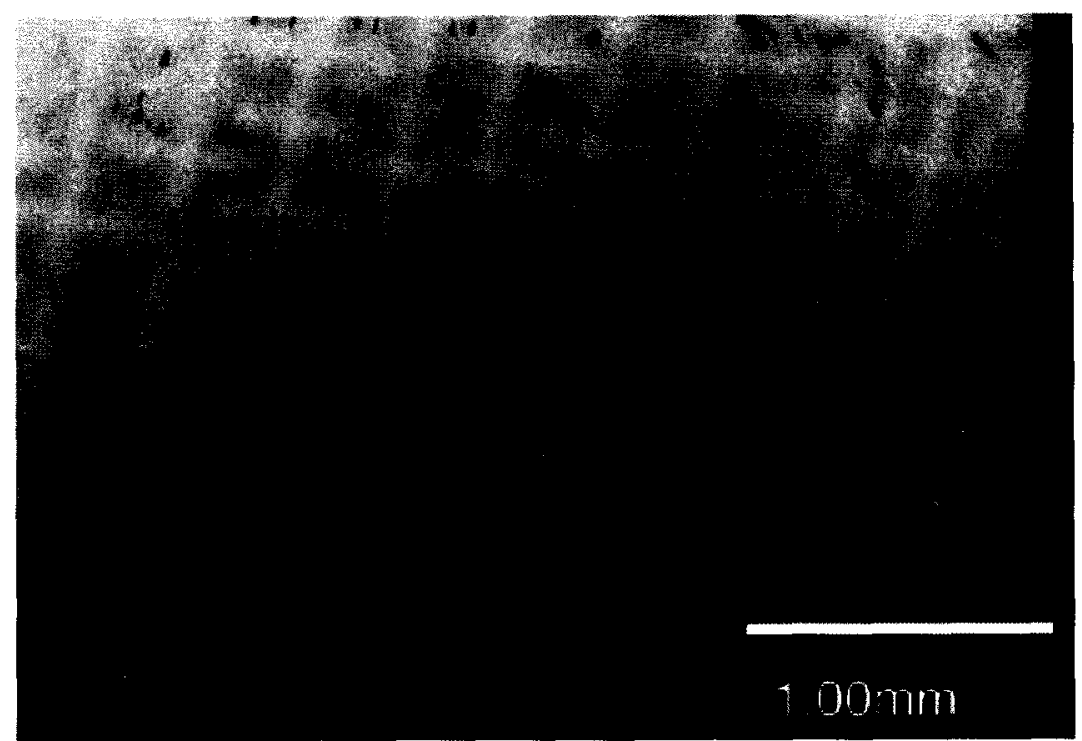

Figure 2.14 - Interdendritic casting microporosity in a single-crystal superalloy [126]. 
Figure 2.15 - Freckle grain defect on the surface of a single-crystal cast bar [127]. Note: the solidification direction is from left to right.

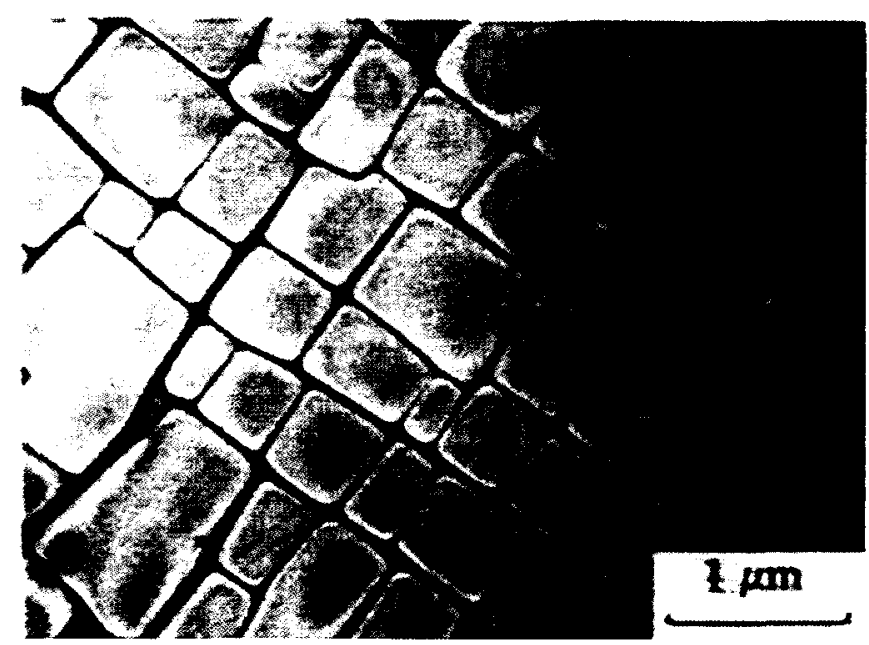

Figure 2.16 - SEM micrograph showing the $\gamma$ - $\gamma^{\prime}$ microstructure in the first generation single-crystal superalloy CMSX-2 after application of solutioning and aging heat treatments [86]. This $\gamma$ - $\gamma$ ' microstructure common to all high $V_{f}$ single-crystal superalloys. 


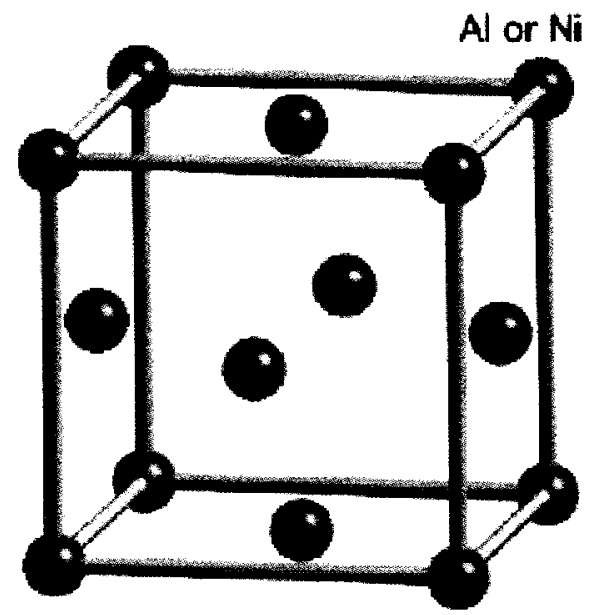

Figure 2.17 - FCC crystal structure of the $\gamma$-matrix phase [123]. Notice that aluminum and nickel, or other substitutional solid solution strengthening elements, can occupy any atomic site.

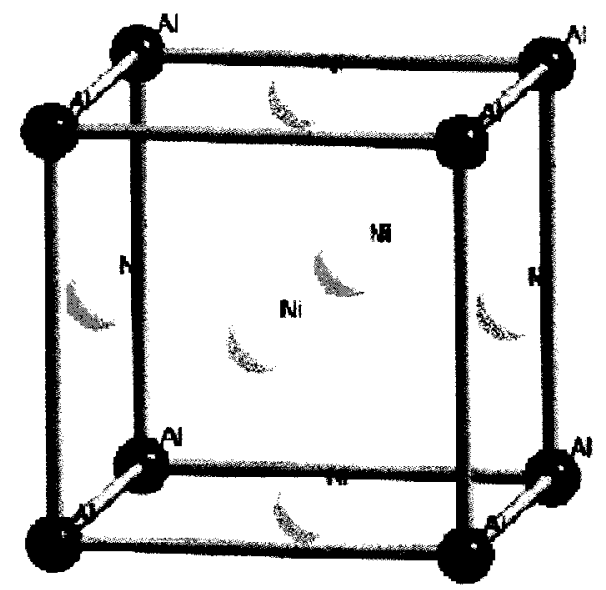

Figure 2.18 - Ordered $\mathrm{L1}_{2}$ FCC crystal structure of the $\gamma^{\prime}$ phase [123]. Notice that only aluminum, or substitutional elements such as titanium or tantalum, may occupy the corner atomic sites; nickel (or substitutes) occupy the face center atomic sites. 


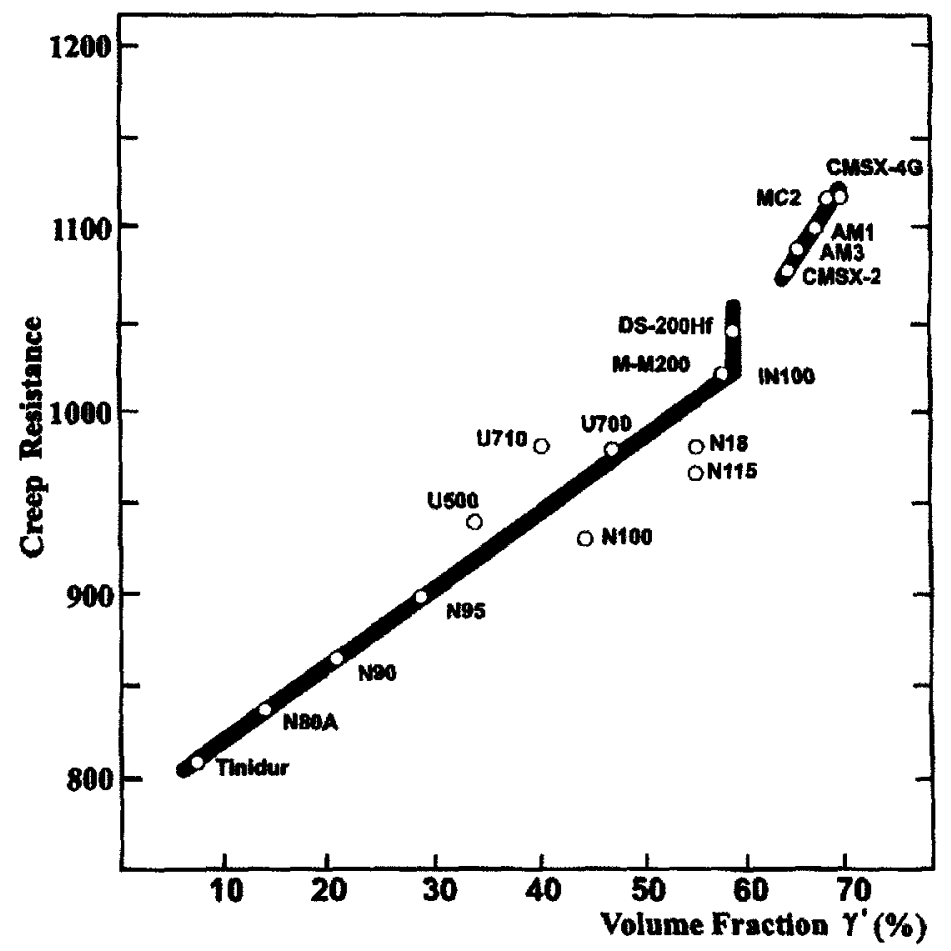

Figure 2.19 - Creep strength of various nickel-base superalloys as a function of $\gamma^{\prime}$ volume fraction [128].

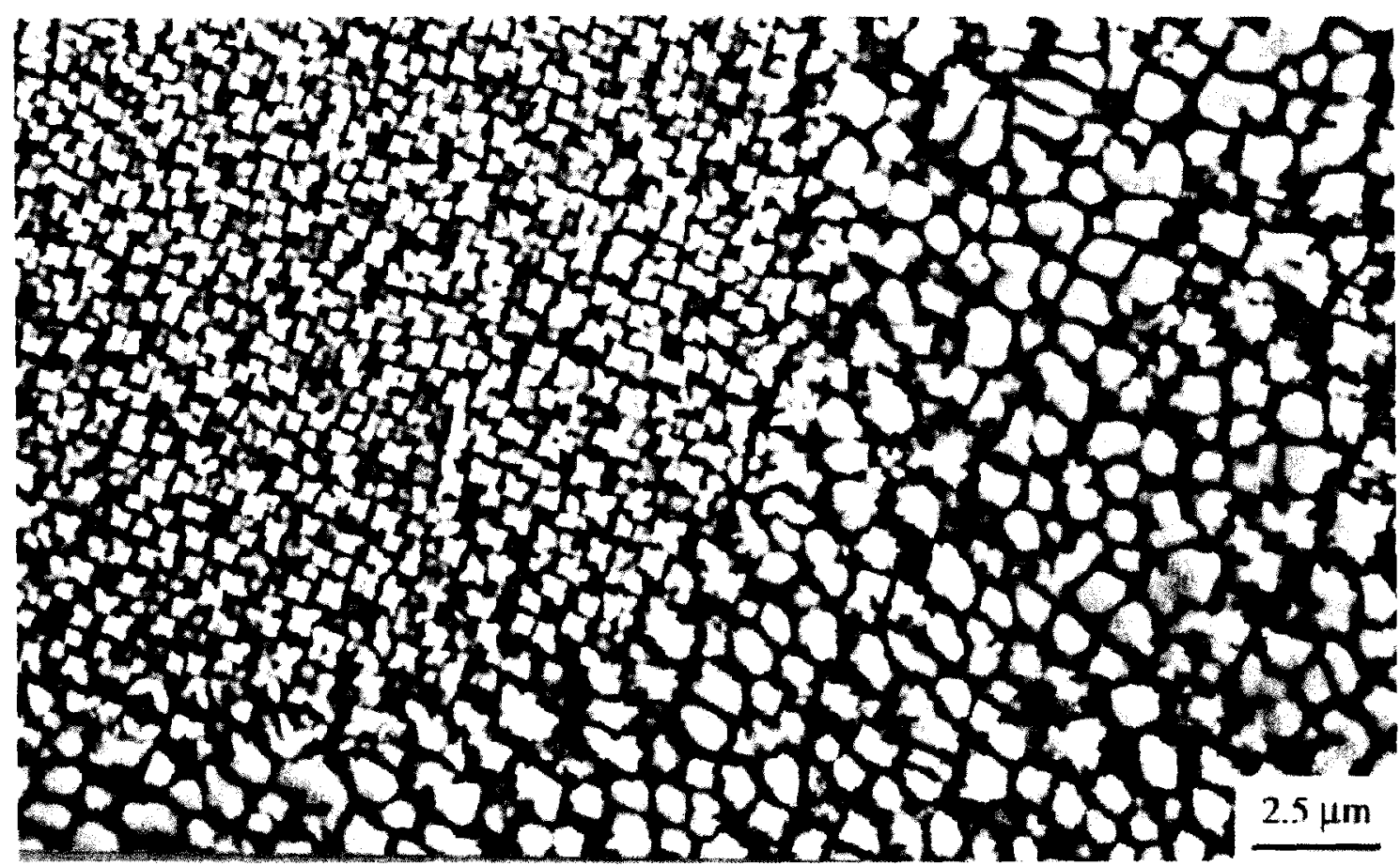

Figure 2.20 - SEM micrograph showing heterogeneous as-cast $\gamma^{\prime}$ microstructure in the first generation single-crystal superalloy CMSX-2 [65]. 

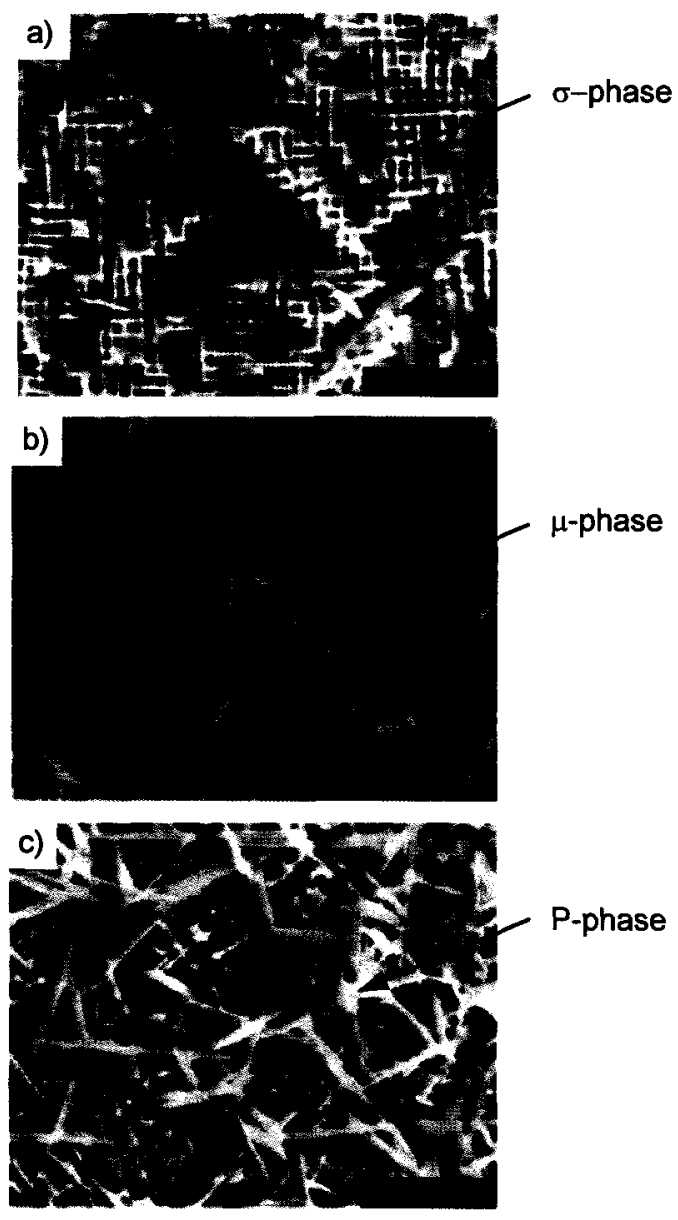

Figure 2.21 - SEM micrographs showing TCP phases precipitated in RR2071; a) $\sigma$-phase, b) $\mu$-phase and c) P-phase (adapted from [129]). Note: the underlying microstructure consists of $\gamma$ and $\gamma^{\prime}$.

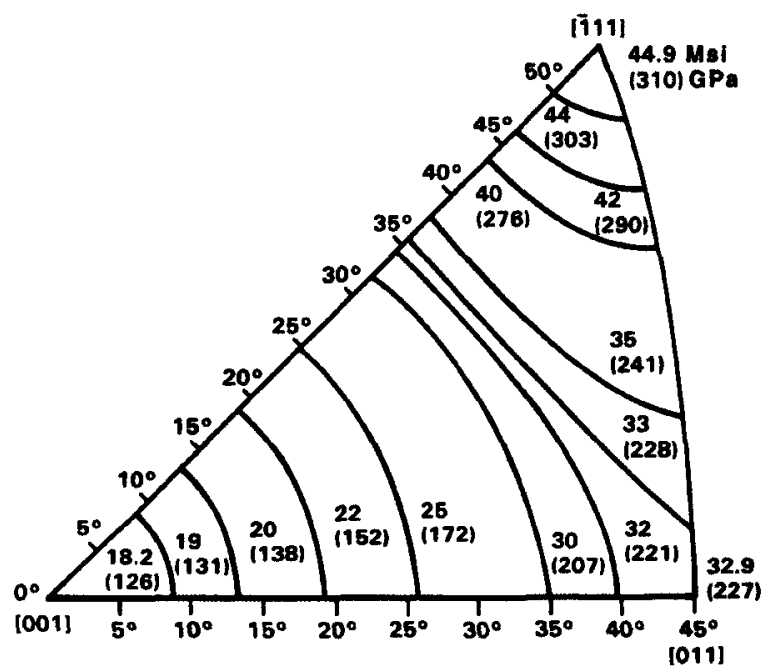

Figure 2.22 - Effective elastic modulus for PWA1480 as a function of orientation at room temperature [64]. 
Plane 1

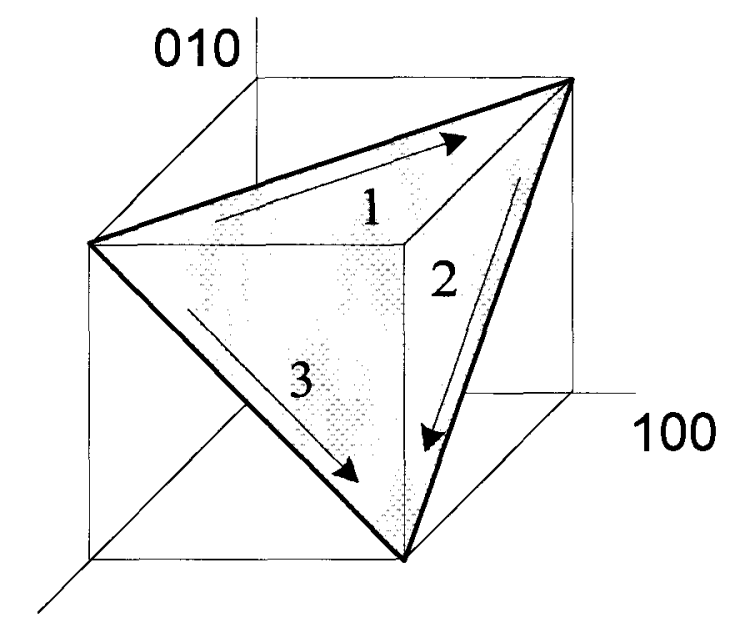

001
Plane 2

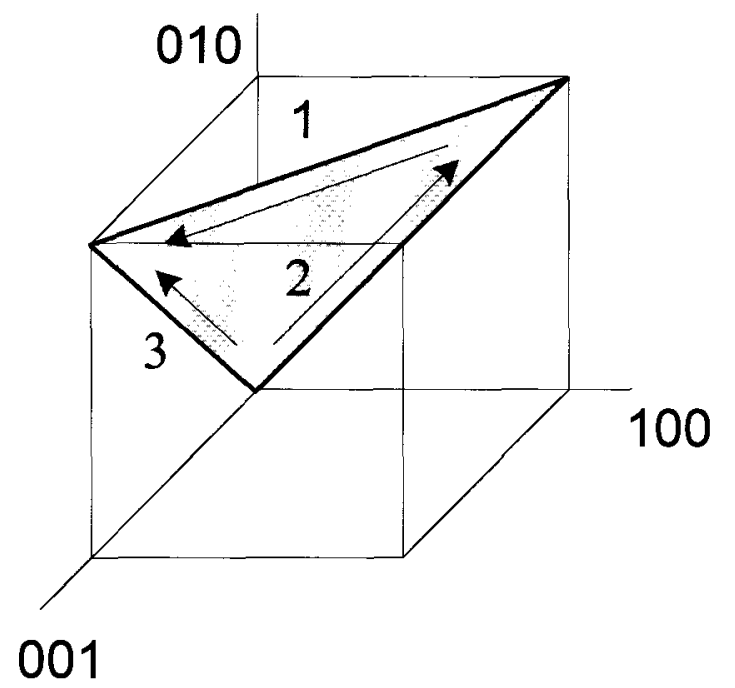

Plane 3

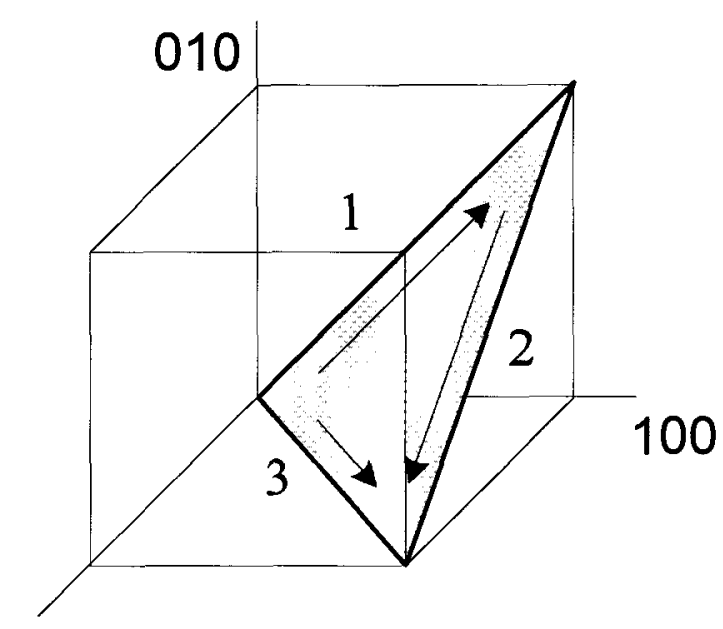

001
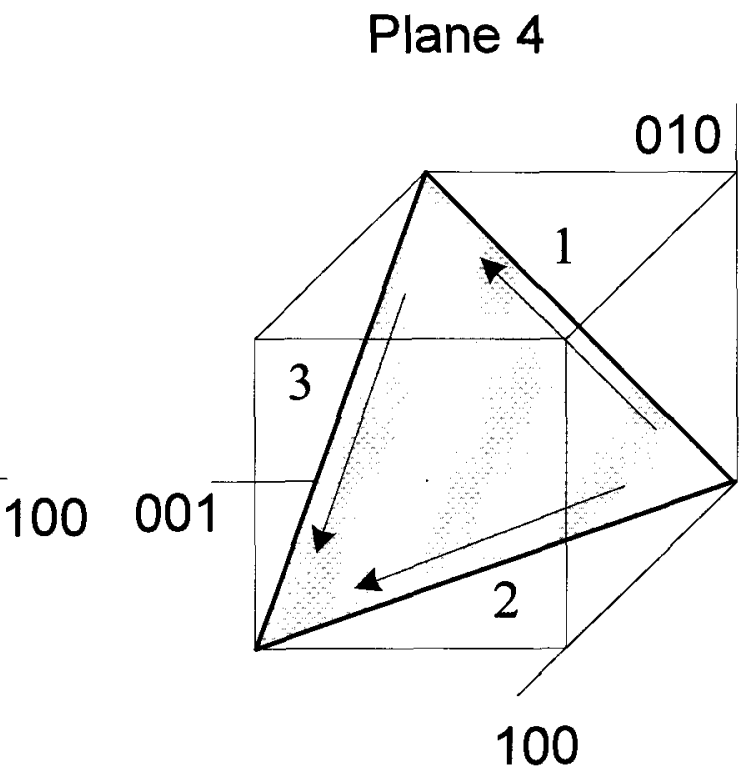

Figure 2.23 - Schematic representation of the FCC octahedral slip systems comprising 4 $\{111\}$ slip planes with each slip plane containing $3\langle 110\rangle$ slip directions. 
Plane 1

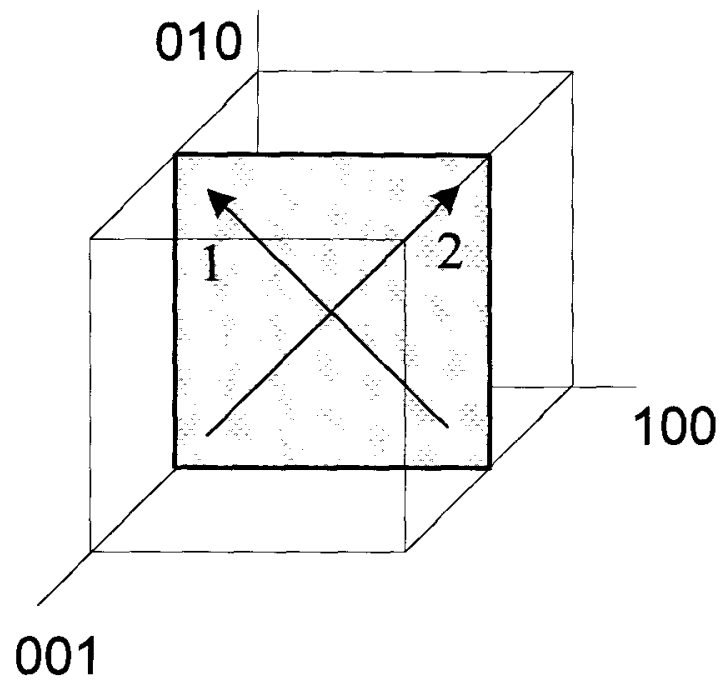

Plane 2

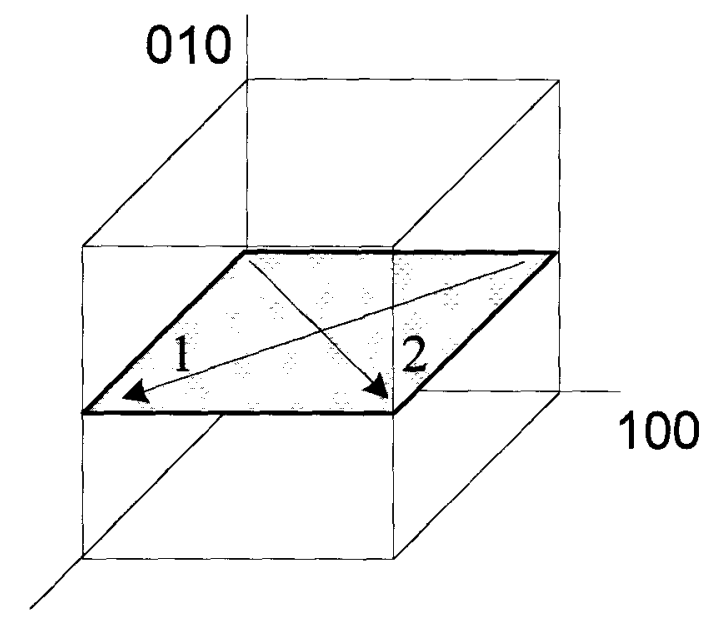

001

Plane 3

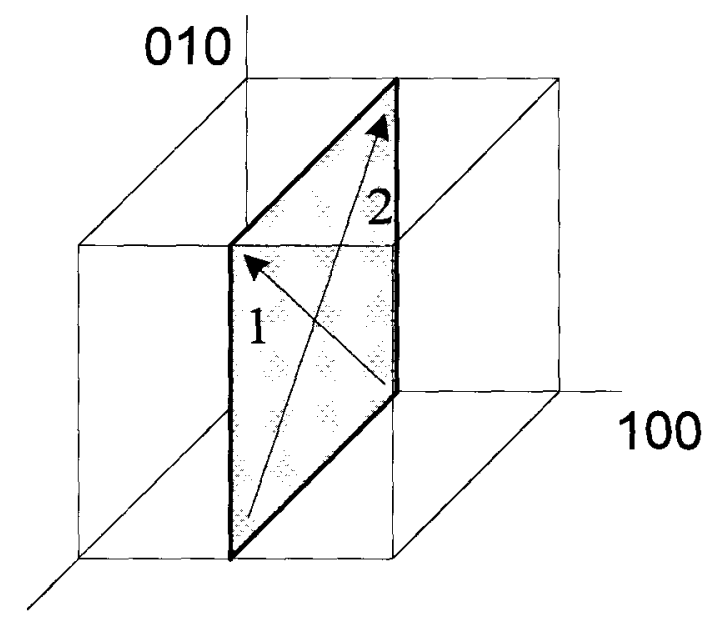

001

Figure 2.24 - Schematic representation of the FCC octahedral slip systems comprising 3 $\{100\}$ slip planes with each slip plane containing $2\langle 110\rangle$ slip directions. 


$$
H
$$




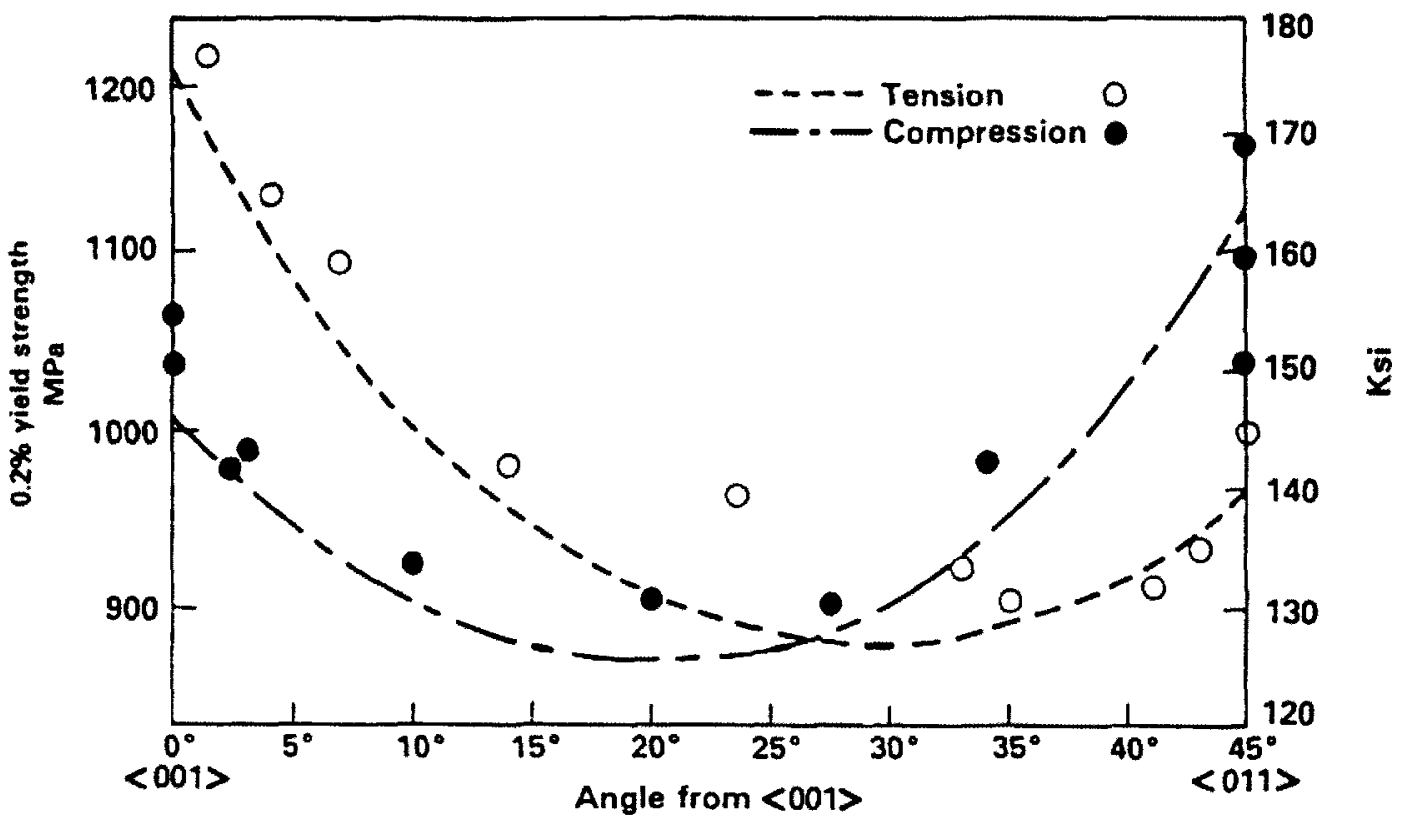

Figure 2.26 - Yield strength of PWA1480 at $593^{\circ} \mathrm{C}$ versus orientation along the $<001>-$ $<011>$ boundary of the standard stereographic triangle [87].

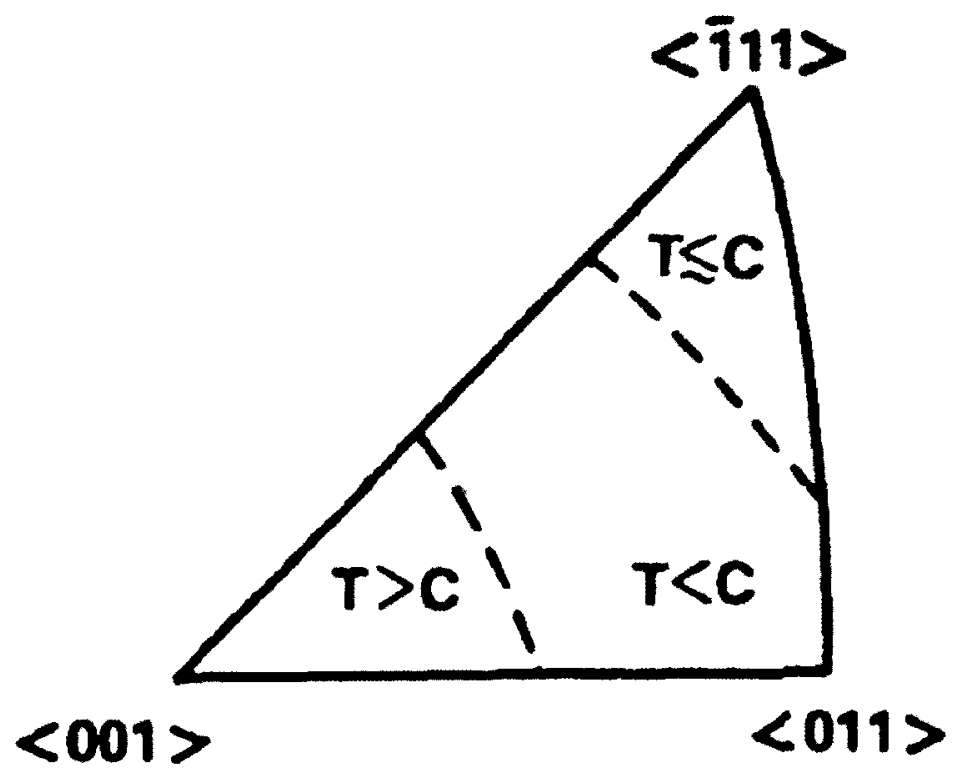

Figure 2.27 - Schematic diagram showing tension (T) and compression (C) asymmetry as a function of orientation [87]. 

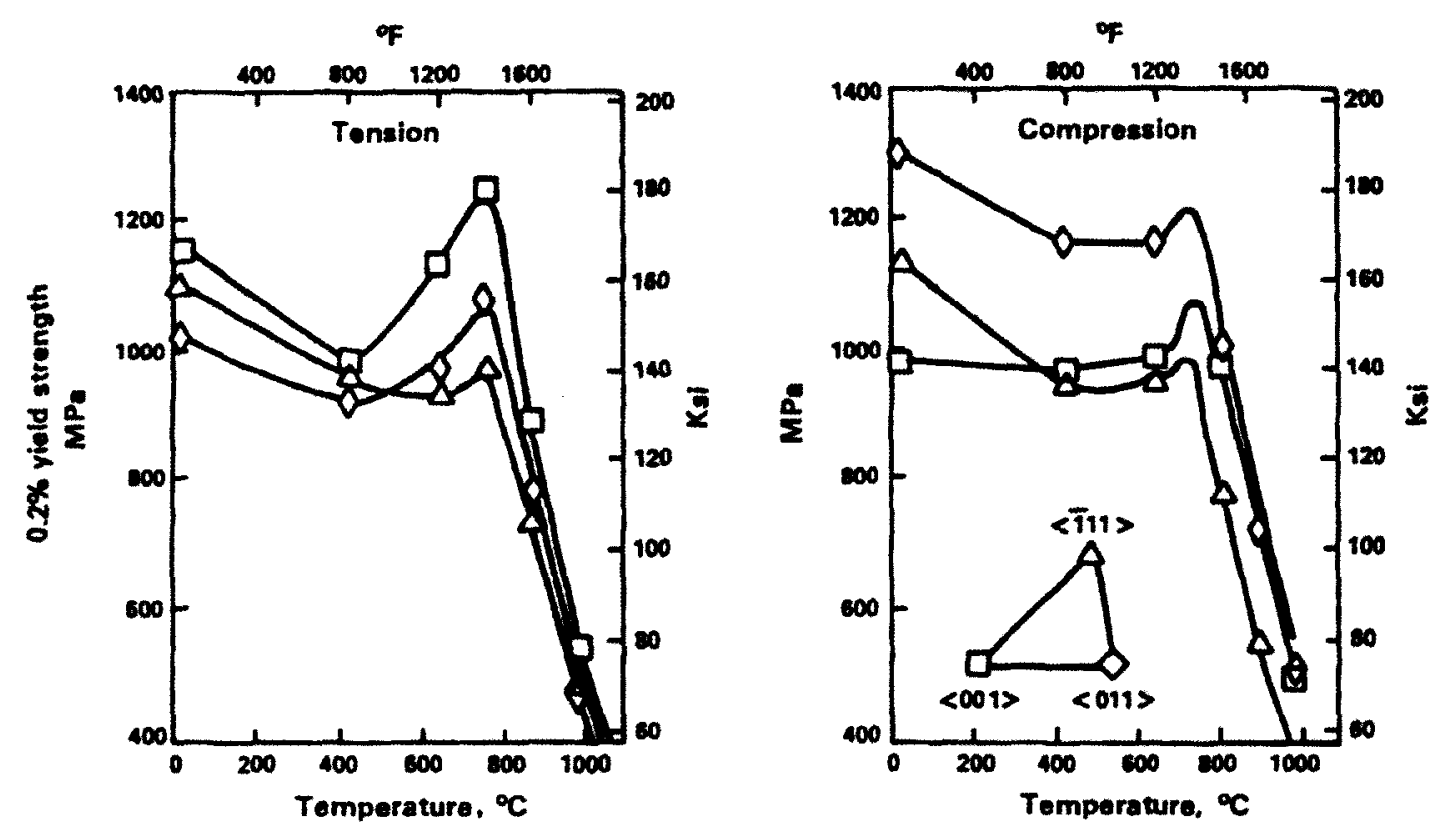

Figure 2.28 - Tensile and compressive yield strength of PWA1480 as a function of temperature for $<001>,<011>$ and $<111>$ orientations [87].

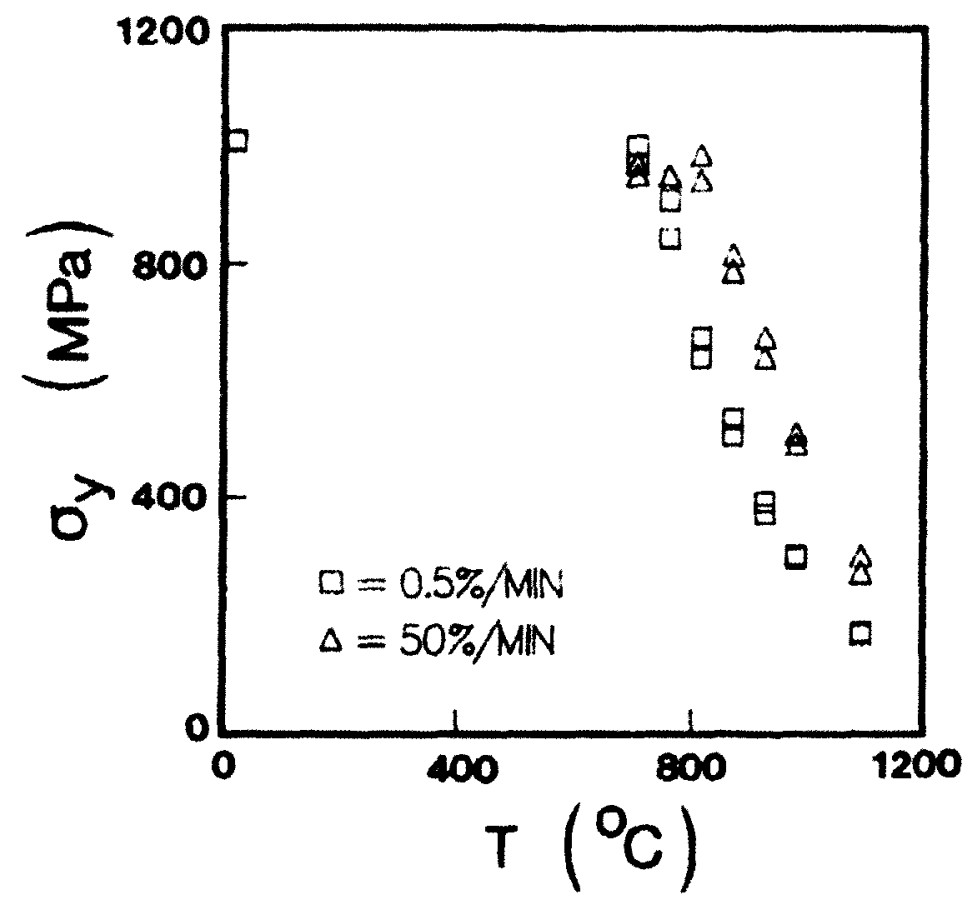

Figure 2.29 - Yield strength of PWA1480 as a function of temperature and strain rate [109]. 


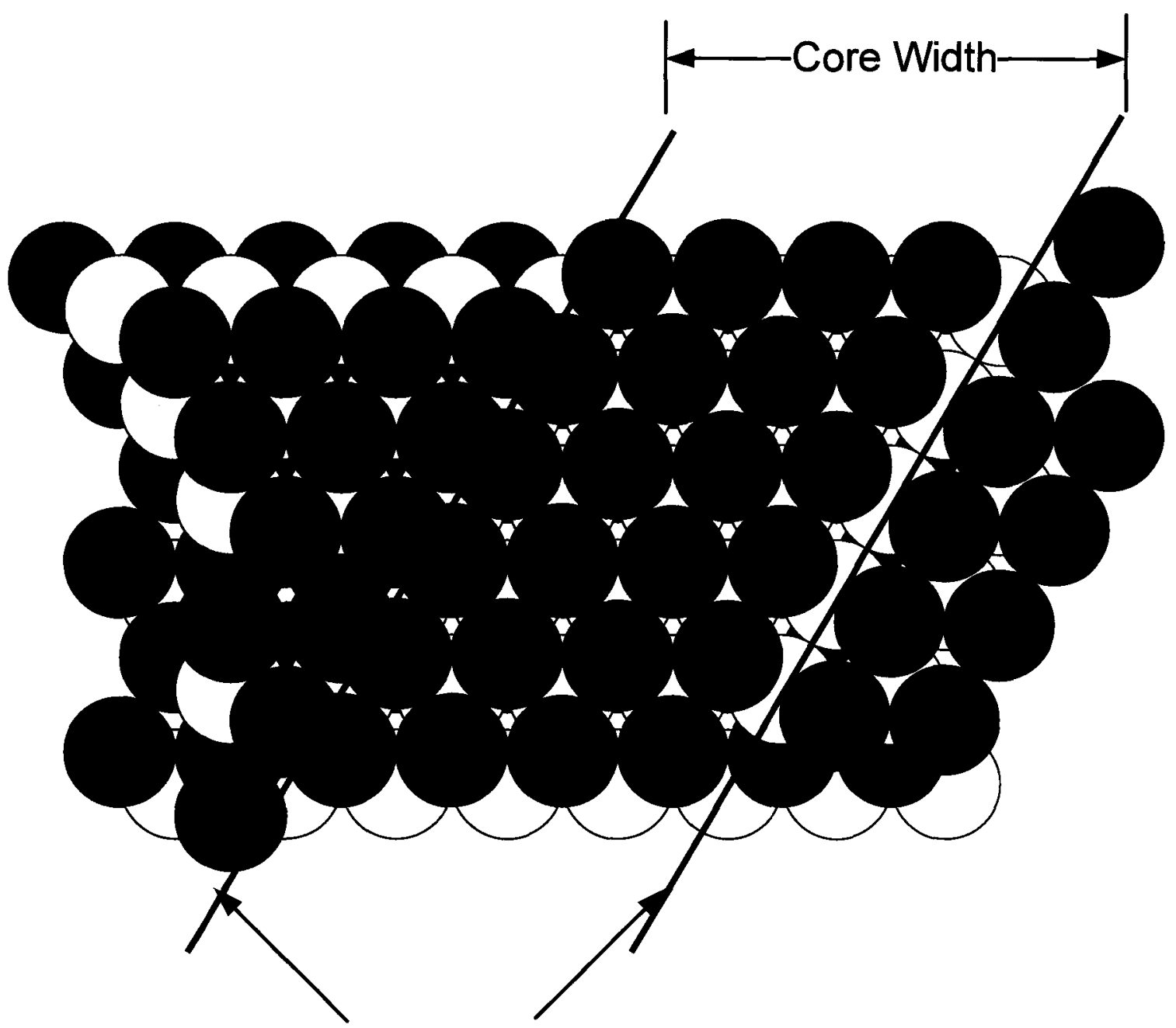

\section{Shockley Partial}

Dislocations

Figure 2.30 - Schematic diagram showing a $a / 2\langle 110\rangle$ dislocation dissociated into two Shockley partial dislocations separated by a stacking fault (adapted from [131]). Note: colour of "atoms" indicate the relative atomic stacking positions. 


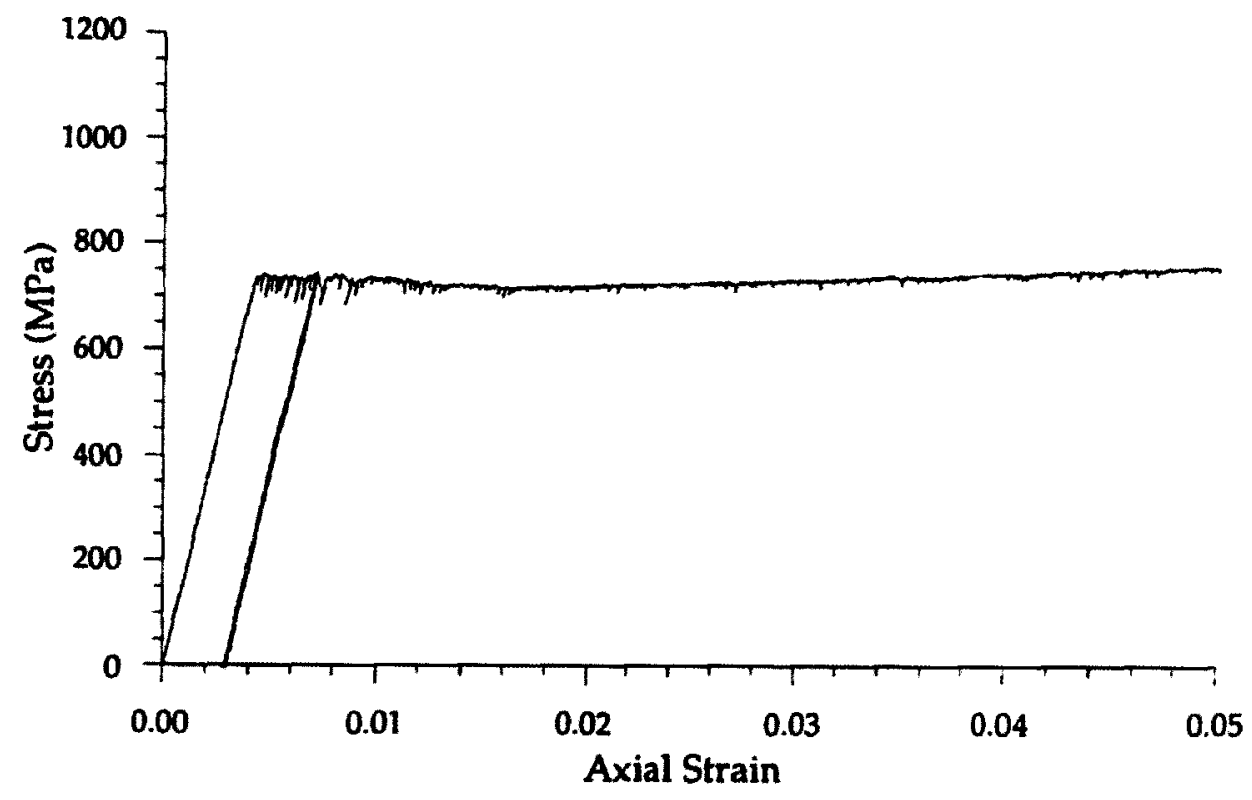

Figure 2.31 - Monotonic stress-strain curve for [123] oriented Rene N4+ tensile sample tested at $760^{\circ} \mathrm{C}[132]$. The tensile sample was subjected to double tensile test at $10^{-4} \mathrm{sec}^{-1}$ and $6 \times 10^{-4} \mathrm{sec}^{-1}$ to study recovery mechanisms. Notice the pronounced serrated yielding.
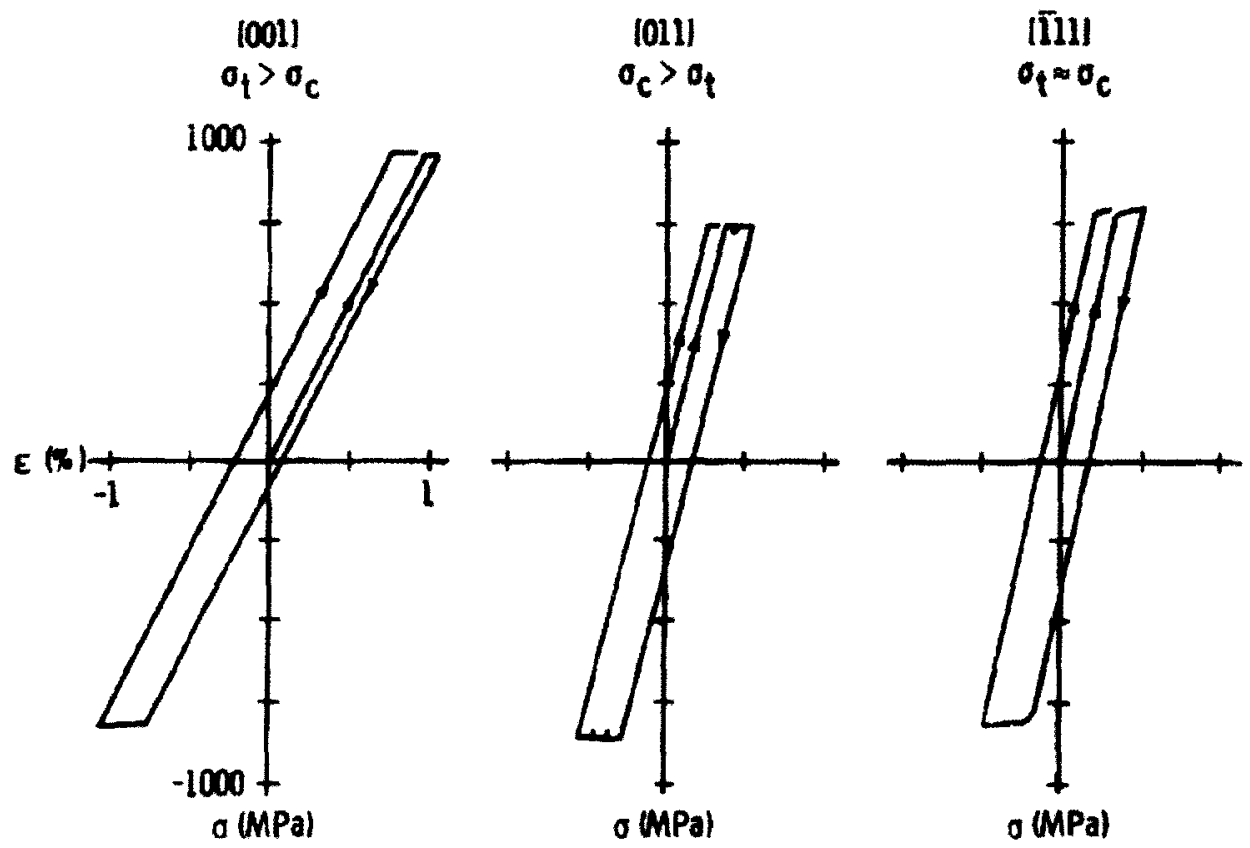

Figure 2.32 - First cycle stress-strain hysteresis loops for Rene N4 test samples with [001] [011] and [111] orientations tested at $760^{\circ} \mathrm{C}[100]$. The tension-compression asymmetry is apparent for the [001] and [011] orientations. 


\section{Literature Review: Fatigue and Fracture Mechanics}

\subsection{Introduction}

In addition to requirements for high-temperature strength and creep resistance, singlecrystal superalloys must have significant resistance to fatigue in order to fulfill their function. Fatigue is a term used to describe the cumulative damage that occurs in ductile materials subjected to cyclic loads. In gas turbine engines, cyclic loads can arise from centrifugal forces on rotating parts, vibrations or resonance, variations in temperature, or any combination of these loading sources. Fatigue is an insidious fracture process where damage is accumulated even when the nominal stresses resulting from the cyclic loads are substantially lower than the macroscopic yield strength of the material [133]. This is because fatigue damage often occurs locally at microstructural defects/discontinuities or manufacturing/service-induced flaws as these features can raise the local stress to exceed the critical resolved shear stress to cause crystallographic slip before the entire body experiences gross plasticity (yielding).

The fatigue life of a gas turbine component has been traditionally established using a socalled safe-life approach based on fatigue crack initiation criteria. The safe-life is usually defined by the number of cycles for a $0.76 \mathrm{~mm}(1 / 32 ")$ surface crack to initiate with a 1 in 1000 probability of occurrence [134]. The crack initiation life is primarily determined from fatigue data obtained from stress or strain controlled fatigue tests on smooth and notched laboratory specimens, which would then be further verified through component testing. The fatigue properties of single-crystal superalloys are often characterized by uniaxial fatigue tests on smooth fatigue specimens of selected orientations such as $\langle 100\rangle$ and $\langle 111\rangle$ crystal orientations. These two orientations exhibit the largest variation in 
mechanical properties [6]. The prediction of crack initiation life is usually made from laboratory fatigue test data using the Basquin or Manson-Coffin equations and variations of the Goodman equation when mean stresses are non-zero. Alternative fatigue crack initiation life criterion have also been considered for single-crystal superalloys as discussed in Reference [6]. The effects of dwell periods, mean stress (or strain), temperature, notches, surface conditions and environment on the fatigue life are usually assessed empirically $[8,9]$. The empirical safe-life approach does not explicitly recognize the role of material, manufacturing or service induced discontinuities on fatigue resistance of components. It is anticipated that such effects are accounted for by using the statically determined lower bound crack initiation life and the application of correction factors to deal with deviations in behaviour [8].

Another approach to deal with component lifing is the damage tolerance approach. The damage tolerance approach recognizes that initial flaws exist in the form of cracks before the component entering into the service, and therefore crack propagation resistance is one of the primary concerns for the component to endure the expected service cycles [9]. In practice, it is assumed that fatigue crack propagation occurs immediately from discontinuities, i.e. the crack nucleation phase is disregarded. Damage tolerant life assessments are undertaken using fatigue crack propagation data in association with fracture mechanics analyses to ensure that fatigue cracks will not grow to such a size as to result in catastrophic failure, which has to be assured by periodic inspections. The safe inspection interval (SII) is actually the crack propagation life. To characterize the material's fatigue crack growth resistance, the crack propagation data are obtained from laboratory tests on standardized fracture mechanics specimens, e.g., compact tension specimens, tested under the presumed service loading conditions and the data are fitted with a crack-growth law that describes the crack propagation rate as a function of the 
controlling fracture mechanics parameter. The damage tolerance life or SII of the component is then estimated by integrating the crack growth law from an initial flaw size defined by the minimum non-destructive inspection (NDI) limit to a final crack size that would result in dysfunction of the component.

More recently, a new lifing paradigm — the holistic structural integrity concept—has been proposed [10] to unify the safe-life and damage tolerance concepts by considering the continuous evolution from crack nucleation to small and large fatigue cracks. The advantage is to render a physics-based description of the material discontinuity to ensure safe extension of the components useful life. This study attempts to follow the holistic lifing approach. Therefore, in this chapter, the broad subjects of fracture mechanics, from LEFM to EPFM, and crack formation processes including crack nucleation, small crack growth and long crack growth, are reviewed with particular emphasis on phenomena related to single-crystal superalloys. The development of fracture mechanics for singlecrystal superalloys will be discussed as well as the use of computational methods to estimate fracture mechanics crack tip parameters. The application of fracture mechanics to fatigue crack propagation will be considered further when discussing the fatigue crack formation and propagation processes in single-crystal superalloys.

\subsection{Review of Fracture Mechanics}

\subsubsection{Linear Elastic Fracture Mechanics}

Linear elastic fracture mechanics is predicated on the existence of a single parameter, the stress-intensity factor (SIF or $K$ ), which characterizes the crack-tip elastic stress and displacement fields in terms of the applied loads, boundary conditions, and component and crack geometry. In theory, the stress-intensity factor provides a unique measure of 
the state stress or crack tip displacements at the crack tip when the fracture problem being considered conforms with the following conditions [135]:

1. the flaw exists in a homogenous elastic continuum, and;

2. the crack tip stress and displacement fields can be characterized based on smallscale yielding assumptions.

Fracture mechanics generally deals with cracks in three distinct opening modes: Mode I (under symmetric loading), Mode II (under skew-symmetric loading) and Mode III (under anti-plane shear loading), as shown in Figure 3.1. Taking the plain-strain Mode I problem for example, in a polar coordinate system with its origin located at the crack-tip as shown in Figure 3.2, for isotropic linear elastic materials, the crack-tip stress field can be expressed as [135]:

$$
\left\{\begin{array}{l}
\sigma_{x x} \\
\sigma_{y y} \\
\sigma_{x y}
\end{array}\right\}=\frac{K_{I}}{\sqrt{2 \pi r}}\left\{\begin{array}{l}
1-\sin \frac{\theta}{2} \sin \frac{3 \theta}{2} \\
1+\sin \frac{\theta}{2} \sin \frac{3 \theta}{2} \\
\sin \frac{\theta}{2} \sin \frac{3 \theta}{2}
\end{array}\right\}
$$

The stresses $\sigma_{i j}$ are shown to depend inversely on the square root of $\mathrm{r}$ - the distance from the crack tip — and their spacial distribution are described by some angular function of $\theta$. In an anisotropic material, however, the Mode I crack-tip stress field is described by [136]:

$$
\left\{\begin{array}{l}
\sigma_{x x} \\
\sigma_{y y} \\
\sigma_{x y}
\end{array}\right\}=\frac{K_{I}}{\sqrt{2 \pi r}} \operatorname{Re}\left\{\begin{array}{l}
\frac{\mu_{1} \mu_{2}}{\mu_{1}-\mu_{2}}\left(\frac{\mu_{2}}{\sqrt{\cos \theta+\mu_{2} \sin \theta}-\frac{\mu_{1}}{\sqrt{\cos \theta+\mu_{1} \sin \theta}}}\right) \\
\frac{1}{\mu_{1}-\mu_{2}}\left(\frac{\mu_{1}}{\sqrt{\cos \theta+\mu_{2} \sin \theta}-\frac{\mu_{2}}{\sqrt{\cos \theta+\mu_{1} \sin \theta}}}\right) \\
\frac{\mu_{1} \mu_{2}}{\mu_{1}-\mu_{2}}\left(\frac{1}{\sqrt{\cos \theta+\mu_{1} \sin \theta}-\frac{2}{\sqrt{\cos \theta+\mu_{2} \sin \theta}}}\right)
\end{array}\right\}
$$


where the complex roots, $\mu_{i}$, are solved from the characteristic equation $[54,55]$,

$$
a_{11} \mu^{4}-2 a_{16} \mu^{3}+\left(2 a_{12}+a_{66}\right) \mu^{2}-2 a_{26} \mu+a_{22}=0
$$

where $a_{i j}$ represent the anisotropic compliance coefficients (in Voigt notation).

Strictly speaking the solution for the crack-tip stress fields in equations 3.1 and 3.2 are only valid when the contribution from the higher order terms (not shown) is insignificant. This condition is satisfied as the distance from the crack tip, $r$, approaches zero [137, 138]. Mathematically, this implies that stresses at the crack-tip approach infinity, which is clearly not the case in ductile materials. In reality, the crack-tip stresses remain finite because localized yielding occurs when the crack-tip stresses exceed the materials yield strength. This results in the formation of plastic zone near the crack-tip in which the uniqueness of the near-tip solutions lose their validity [135]. Nonetheless, when the plastic zone satisfies the small-scale yielding condition, the stress intensity factor still uniquely characterizes the fatigue crack growth properties as will be discussed later.

For the stress-intensity factor to uniquely characterize the fatigue crack-tip fields, the $K$ dominance must be warranted [135]. This is primarily controlled by characteristic microstructural dimensions, such as the grain size and the crack-tip plastic zone size. Specific limits on these quantities are imposed by the fundamental assumptions from which LEFM was derived. For example, in polycrystalline materials the crack size must be larger than several grain diameters in order to consider the crack to exist in a homogenous elastic continuum (provided that small-scale yielding conditions are satisfied). To ensure that small-scale yielding conditions are satisfied, the crack-tip 
plastic zone size, $r_{p}$, must be small compared to the in-plane dimensions of the body, namely the crack length $(a)$ and un-cracked ligament depth $(W-a)$, in which the flaw resides [139]. For cracks in an isotropic elastic body, the plastic zone size is a function of the stress-intensity factor, the yield strength, and the constraint conditions imposed at the crack-tip. An estimate of the monotonic plastic zone size for a plane crack in an isotropic elastic material under Mode I loading can be obtained from [133, 135]:

$$
\begin{aligned}
& r_{p}=\frac{1}{3 \pi}\left(\frac{K_{I}}{\sigma_{y}}\right)^{2}, \text { for plane strain } \\
& r_{p}=\frac{1}{\pi}\left(\frac{K_{I}}{\sigma_{y}}\right)^{2}, \text { for plane stress. }
\end{aligned}
$$

where $\sigma_{y}$ represents the yield strength of the material. A small-scale yielding condition is said to exist when [140],

$$
r_{p} \ll \frac{1}{15}[a,(W-a)]
$$

Unlike polycrystalline materials which exhibit a uniform crack-tip plastic zone under small-scale yielding conditions and when a small fatigue crack exceeds microstructural dimensions, as depicted in Figure 3.3a, single-crystal superalloys exhibit a different crack-tip plastic zone shape. Rice [141] was first to demonstrate that crack-tip stress and deformation fields in single-crystals is locally constant within specific angular sectors, as shown schematically in Figure 3.4, using an asymptotic solution for an elastic perfectly plastic single-crystal under plane-strain conditions. These angular sectors correspond to specific slip-systems and can be physically interpreted as strain localization bands in 
which plastic strain is highly concentrated. The existence of these localization angular sectors of plastic deformation has been shown to exist in the deformed structures of single-crystal materials by Kysar and Briant [142] and Flouriot and coworkers [143] using the electron back-scatter diffraction (EBSD) technique. Flouriot and coworkers [143] and Marchal et al. [144] have conducted finite element simulations incorporating both the octahedral and cubic slip systems for single-crystal superalloys, which show that crack-tip plasticity is mainly concentrated in specific slip sectors resembling what Rice [141] predicted. Furthermore, they also found that the angular position of the slip-sectors and magnitude of plastic shear strain depended on the loading axis and crack orientation relative to the crystallographic directions under Mode I loading conditions. Interestingly, for anisotropic materials under small-scale-yielding conditions in Mode I dominance, equation 3.2 also holds true for the strip-yielding condition when the gross yield strength is replaced with the friction resistance to slip in the particular direction [145].

The solutions of the stress-intensity factors for engineering problems depend on the loads, the boundary conditions, and the crack and body geometry. Analytical closedform solutions derived from elasticity theory exist only for a small number of thruthickness crack (plane crack) $[133,137,138]$ and elliptical crack (surface crack) $[133$, $137,138]$ problems. These solutions are generally represented by,

$$
K_{I}=Y\left(\frac{a}{W}\right) \sigma_{0} \sqrt{\pi a}
$$

for thru-thickness cracks under Mode I loading, and; 


$$
K_{I}=Y\left(\frac{a}{W}\right) \sigma_{0} \sqrt{\frac{\pi a}{Q}}
$$

for semi-elliptical surface cracks subject to Mode I loading. The non-dimensional function $Y(a / W)$ in equations (3.6) and (3.7) is to correct the stress-intensity factor for effects of free surface and finite geometry, the stress distribution, and the boundary conditions, $W$ is a characteristic dimension of the cracked body, $\sigma_{0}$ is the nominal (farfield) stress and $a$ represents the crack size. The additional term, $Q$, in equation (3.7) is the elliptical shape factor, which can be approximated by the following empirical expressions $[146,147]$ :

$$
\begin{aligned}
& Q=1+1.464\left(\frac{a}{c}\right)^{1.65} \text { for } \frac{a}{c} \leq 1 \\
& Q=1+1.464\left(\frac{c}{a}\right)^{1.65} \text { for } \frac{a}{c}>1
\end{aligned}
$$

where $a$ is the semi surface crack length as defined by the three-dimensional (3-D) crack nomenclature shown in Figure 3.5. In 3-D crack problems, the values for the stressintensity factor change along the crack front as a function of the parametric angle, $\phi$, defined in Figure 3.5. The stress-intensity factors at the surface $\left(\phi=90^{\circ}\right)$ and the deepest part $\left(\phi=0^{\circ}\right)$ of semi-elliptical cracks are generally considered the most important in surface crack problems.

Because of the complexities associated with calculating stress-intensity factors for surface cracks in 3-D bodies, exact solutions for laboratory specimens are generally unavailable. Instead, a number of empirical equations have been proposed to provide 
approximate solutions. The most widely used empirical stress intensity factor solution for semi-elliptical surface cracks in finite elastic plates is that proposed by Newman and Raju [148]. The solution was developed based on stress-intensity factors extracted from 3-D finite element calculations $[149,150]$ and is stated in a series of equations. The equations can be used to calculate the stress-intensity factors as functions of the parametric angle, surface crack length and crack depth, and plate width and thickness, for tension and bending loads and crack aspect ratios $(a / c) \leq 1$. In later work, Fett [151] proposed modifications to the original Newman and Raju model to extend its capabilities to estimate stress-intensity factors for crack aspect ratios in the range of $1-2$.

Of more practical importance to the present research work are the empirical solutions for stress-intensity factors at the center of a semi-circular notch. A number of empirical equations have been proposed for calculating stress-intensity factors for thru-thickness and surface cracks. Fett [152], Kujawski [153], Newman [146], Schijve [154] and Yamamoto [155] among others have developed approximate analytical expressions for the stress-intensity factors for thru-thickness cracks emanating from the semi-circular and semi-elliptical notch roots. For 3-D cases involving surface cracks, Newman [146] has presented a series of empirical equations for calculating stress-intensity factors for semielliptical cracks in double-edge notch (DEN) laboratory specimens. These equations were developed based on 3-D finite-element calculations $[147,156]$ performed on the standard DEN specimen geometry adopted by the Advisory Group for Aerospace Research and Development (AGARD). Unfortunately, this solution cannot be used universally for all notch surface crack problems because it was derived specifically for the $r / W=1 / 16$ (see Figure 3.5 for nomenclature) AGARD specimen design. 
The existing stress-intensity factor solutions for surface cracks in plates and notched specimens were developed for isotropic elastic materials and Mode I loading conditions. Application of these solutions to cracks in anisotropic materials, such as single-crystal superalloys, was at first questionable for two reasons. First, the role of elastic anisotropy could not be dismissed as having a second-order effect on the stress-intensity factors because the apparent elastic modulus values could vary by a factor of approximately three in single-crystal superalloys (see the previous Chapter). Second, depending on the temperature, the stress-state and other factors, cracks in single-crystal superalloys may exist on specific crystallographic planes. The crack plane in such cases would be inclined to the applied stress and therefore the displacements at the crack-tip would consist of Mode I, II and III components.

Because of these complicating factors, stress-intensity factor solutions for anisotropic materials are not readily available. It has been assumed by some researchers $[24,28,29$, 52] that differences between the isotropic and anisotropic stress-intensity factors are negligible for specific crack problems. Other researchers have conducted numerical simulations to investigate the effect of elastic anisotropy on the stress-intensity factors for thru-thickness $[24,53,157]$ and thumbnail cracks $[54,55,158,159]$.

Chan and Cruise [53] used the boundary integral equation method to estimate stressintensity factors for a thru-thickness cracks on a plane normal to the applied load and several different inclined crack plane configurations in compact tension, $\mathrm{C}(\mathrm{T})$, specimens. The elastic properties for Mar-M200 single-crystal superalloy was used in the analyses. Among their findings, they showed that the standard stress-intensity factor solutions for C(T) specimens (e.g. ASTM E647 [160]) could be used for both isotropic and anisotropic materials when the crack plane inclinations were less than $30^{\circ}$ to the 
loading axis so long as the projected crack length was used in the stress-intensity factor calculations. The projected crack length in this case corresponded to the length of the inclined crack when projected onto a plane normal to the applied loading.

For 3-D bodies, Tinga [54, 55] has considered the effect of elastic anisotropy on stressintensity factors for thumbnail cracks in a corner crack fatigue specimen. In this investigation, the stress-intensity factors were determined from 3-D finite element simulations that considered two crack plane orientations, namely, a corner crack on a plane normal to the applied load and a corner crack on a plane inclined at $45^{\circ}$ to the applied load, for various crack depth to ligament ratios $(a / t)$ assuming $a / c=1$, and using the elastic properties for the single-crystal alloy CMSX-4. The simulation results for the normal crack orientation showed that anisotropic Mode I stress-intensity factors were approximately two times higher than those calculated assuming isotropic elasticity. The simulation results for the inclined crack orientation showed that the stress-intensity factors consisted of Mode I, II and III components and the degree of mixed modality at the crack front was a function of the parametric angle and $a / t$. Tinga also showed that the projected crack length approximation used in Chan and Cruise's investigation [53] over predicted the effective stress-intensity factors, $K_{\text {eff }}\left(\right.$ where $K_{\text {eff }}=\sqrt{K_{I}^{2}+K_{I I}^{2}+K_{I I I}^{2}}$ ), for the inclined crack orientation.

For the convience of characterizing cracks on specific crystallographic planes in singlecrystal materials, the resolved shear stress-intensity factor was first proposed by Chen and Liu [161]. They have also shown that this parameter could be used for predicting the crack propagation planes and directions in single-crystals. The resolved shear stressintensity factor is defined as follows: 


$$
K_{r s s}=\lim _{r \rightarrow 0} \tau_{r s s} \sqrt{2 \pi r}
$$

where $\tau_{r s s}$ is the resolved shear stress obtained by projecting the components of the stress tensor (in the material coordinate frame) onto specific slip planes using Schmid's law. Evaluation of the resolved shear stress-intensity factor involves reconstructing the singular crack-tip fields, resolving the stress components onto all of the octahedral $\{111\}$ slip planes and slip directions ahead of the crack-tip, and finally extrapolating the results to the crack-tip, i.e. $r=0$. Presumably, the slip system(s) on which resolved shear stress-intensity factor is highest is the direction that a crystallographic crack will propagate. There has been some success reported using this criterion to predict the crack propagation planes for Mode I loading [28], but this criterion has also been shown to be inadequate when considering cracks propagating under mixed-mode loading conditions $[24,52]$.

The resolved shear stress-intensity factor considers only the shear opening component (i.e. Mode II component) on a particular slip system and therefore it is implicitly assumed that crystallographic crack propagation occurs by a shear decohesion mechanism. However, experimental observations [162] indicate that normal stresses, i.e. Mode I component of stress acting on a slip system, are also involved in the propagation of crystallographic cracks in nickel-base superalloys. Having recognized this limitation of the resolved shear stress-intensity factor, Telesman and Ghosn [29] have proposed an octahedral crack-tip parameter that considers both the resolved shear stresses and normal stresses acting on a particular slip system. The octahedral crack-tip parameter is defined as: 


$$
K_{O C T}=\sqrt{K_{r s s}^{2}+K_{r n s}^{2}}
$$

where $K_{r n s}$ represents the resolved shear stress acting normal to the slip system(s) under consideration. The resolved shear stress normal to the slip system is defined through expression that is analogous to equation 3.9. Reed and co-workers [52] have conducted a detailed analysis on the application of $K_{r s s}$ and $K_{r n s}$ for predicting crack paths in singlecrystal superalloys subjected to Mode I and mixed mode loading conditions. Among their findings they reported excellent predictions of octahedral crack paths by considering slip systems on which $K_{O C T}$ was a maximum and the ratio $K_{r s s} / K_{r m s}$ exceeded a minimum value.

In spite of the success in using $K_{O C T}, K_{r s s}$ and $K_{m s}$ as crack tip parameters in fracture and fatigue problems, they are ineffective for identifying the conditions under which crystallographic and/or transitions between Mode I or crystallographic propagation will occur in single-crystal materials. Leverant and Gell [163] were first to propose that the cyclic frequency and temperature dependence of the Mode I to crystallographic transition could be described by Arrhenius rate kinetics. This concept was later extended by Gallagher and coworkers [157] to incorporate stress-state dependence by including the stress-intensity factor in the formulation. The model proposed by Gallagher et al. [157] to predict the Mode I to crystallographic crack growth transition is given by the relation:

$$
f \Delta K=A^{\prime} \exp \left(\frac{-Q}{R T}\right)
$$


where $f$ is the cyclic frequency, $\Delta K$ is the stress-intensity factor range, $A$ ' is the preexponential coefficient, $Q$ is the apparent activation energy, $R$ is the universal gas constant and $T$ is the absolute temperature. Gallagher et al. [157] used experimental data and equation 3.11 as the basis for developing a fatigue crack growth mechanisms map for PWA1484 single-crystals, as shown in Figure 3.6. Interestingly, the activation energy associated with the Mode I to crystallographic fracture morphology transition corresponds with the activation energy associated with the $\gamma^{\prime}$ shearing deformation mechanism [109].

Not all cracks can be characterized by stress-intensity factor or resolved shear stressintensity factors because the small scale yielding condition is violated due to elasticplastic loading conditions. In such cases EPFM parameters must be utilized in the analysis of cracks. This will be discussed further in the following section.

\subsubsection{Elastic-Plastic Fracture Mechanics and the J-Integral}

In some fracture problems the stress-state ahead of the crack-tip is no longer uniquely characterized by the linear-elastic fracture mechanics parameter, $K$, because the extent of plasticity at the crack-tip becomes appreciable. Such cases are encountered when the length of a crack is short in comparison to the plastic zone size, when the bulk material in which a crack is embedded undergoes plastic deformation, or when a crack is present in a locally plastically deformed region such as a notch root. For these problems elasticplastic fracture mechanics parameters, such as the J-Integral, is thought to be more appropriate to characterize the crack-tip stress and strain fields. As defined by Rice [164], the J-Integral in two dimensions is given by: 


$$
J=\int_{\tau}\left(W n_{1}-T_{i} \frac{\partial u_{i}}{\partial x_{1}}\right) d s
$$

$W$ is the strain energy density given by,

$$
W=\int_{0}^{\varepsilon_{i j}} \sigma_{i j} d \varepsilon_{i j}
$$

$T_{i}$ is $\sigma_{i j} n_{j}$, the traction normal to the contour $\Gamma$ and $u_{i}$ is the displacement vector. The $\mathrm{J}$-integral is evaluated along a closed contour surrounding the crack-tip as shown schematically for the 2-D case in Figure 3.7. As shown by Rice [164], the J-integral represents the change in potential energy associated with crack advance in nonlinear elastic and linear elastic materials. In addition, he has also shown that the J-integral is path-independent, meaning that the J-integral will have the same value on any admissible path that encloses the crack-tip. The path independence of the J-integral is based on the assumption that the strain energy density represents a unique function from which the stresses can be uniquely derived $[165,166]$. This assumption enforces the condition that proportional loading occurs in the plastically deformed region at the crack-tip, meaning that the ratio of principal stresses do not change with time. Rice [164] also proved for linear-elastic problems that the J-integral was equivalent to the energy release rate. From this it can be established that the J-integral is related to the stress-intensity factor as follows:

$$
J=G=\frac{K^{2}}{E^{\prime}}
$$


where $E^{\prime}=E(1-v)$ for plane strain and $E^{\prime}=E$ for plane stress problems.

For anisotropic materials, $\mathrm{Wu}$ et al. [145] have shown, based on the continuously distributed dislocation theory (CDDT), that the J-integral under small-scale mode-I dominance conditions can be expressed as:

$$
J=\frac{1}{2} K_{i} F_{i j}^{-1} K_{j}
$$

where $F_{\mathrm{ij}}$ is the characteristic elastic matrix for the material, and it reduces to a diagonal matrix with $F_{11}=F_{22}=G /(1-v)$ and $F_{33}=G$, for isotropic material, where $G$ is the shear modulus and $v$ is the Poisson's ratio.

For complicated engineering materials, solutions of the J-integral need to be sought out using numerical methods of incremental plasticity theory in conjunction with the appropriate material constitutive laws. For example, Zhao and co-workers [56] have used the finite element method to estimate the J-integral for notch root thumbnail cracks in a single-edge notch specimen. The analyses were conducted using an incremental elasticplastic constitutive law based on either the von Mises or the Hill yield criterions, for isotropic and anisotropic elastic-plastic cases respectively, and without strain-hardening, i.e. elastic-perfectly plastic material behaviour. They considered single-edge notch specimens with [001] as the loading axis, two notch orientations, [100] and [110], and three crack sizes with an aspect ratio $a / c=0.5$ under loading conditions for small-scale yielding and large-scale plasticity. Among their findings it was reported that the Jintegral variation around the crack front was different for each notch orientation. It was 
also reported that the J-integral was generally higher for the $[100]$ notch orientation than the $[110]$ notch orientation.

Brocks et al. [165] and Brocks and Scheider [166] have conducted detailed investigations on the use of incremental plasticity constitutive theories in finite-element simulations as applied to estimating J-integrals in fracture problems. Among their findings, it was reported that the J-integral is path-dependent when incremental plasticity theories are used to describe the plastic material behaviour. This path dependence has been attributed to the re-arrangement of stresses in the plastically deformed material ahead of the crack that causes a change in loading direction in stress-space, resulting from the incremental plasticity yield condition, where the loading is no longer proportional in the plastically deformed region. The proportionality of loading is a necessary condition to preserve the path independent properties of the J-integral. Therefore, these results may challenge the validity of J-integral to be considered as a crack-tip parameter. However, Brocks and Scheider [166] have also shown that for small-scale and contained yielding that path independent J-integral estimates could be obtained as long as the contours were chosen outside of the plastic deformed region. Contained yield conditions would be mostly found in practical engineering fracture problems.

\subsection{Numerical Methods for Fracture Mechanics Parameter Estimation}

For many practical fracture mechanics problems analytical solutions for the stressintensity factor and J-integral are unavailable and therefore numerical methods must be used to obtain estimates of these parameters. Among the numerical methods available, the finite-element method has emerged as one of the most advanced and capable numerical methods for estimation of LEFM and EPFM crack-tip parameters. Therefore, 
this review will focus on FEM techniques for estimating the stress-intensity factor and Jintegral.

Gallagher [167], Liebowitz and Moyer [168] and Ingraffea and Wawrzynek [169], among others, have reviewed the application of finite element methods for stress-intensity factor calculations. The techniques available for extraction of stress-intensity factors from FEM solutions include:

- displacement field approximation method,

- stress field approximation method,

- virtual crack extension (energy) method,

- virtual crack-closure technique, and

- application of the J-integral (energy) method.

Among these techniques, the J-integral approach, as implemented within the finite element method, is the most attractive for a number of reasons. For example, special quarter-point crack-tip elements and orthogonal finite element meshes that exactly represent the crack-tip stress and displacement fields are unnecessary if the exact solutions for the crack-tip stress and displacement fields are not required [170]. In addition, many commercial FEM codes include capabilities for estimating the J-integral as an additional post-processing procedure in elastic and elastic-plastic problems. Because of the path-independent properties of the J-integral, it can be calculated on paths remote from the crack-tip so that accurate results can be obtained using relatively coarse FEM meshes [170]. Lastly, the J-integral is widely accepted as a fracture mechanics parameter for both elastic and elastic-plastic materials. 
The commercial FEM program ABAQUS [171] includes a procedure to calculate the Jintegral based on the virtual crack extension (VCE) method described by Parks [172, 173]. The J-integral is not evaluated by the line integral described by equation 3.12 , but instead is discretized into an area integral in two dimensions or a volume integral for 3D problems (see Reference [166]). The discretization of equation 3.12 in the finite element model is formulated as a set of linear algebraic equations, which relate the total potential energy changes caused by small amount of crack advance, $\Delta a$, at each of the crack front nodal positions to the J-integral values at the same crack front nodal positions. Further details on this procedure can be found in the ABAQUS Theory Manual [174] and Reference [166].

The stress-intensity factors in linear elastic materials under mixed-mode loading conditions can be obtained from $[174,175]$ :

$$
J=\frac{1}{8 \pi}[K]^{T}[B]^{-1}[K]
$$

where $[K]=\left[K_{I}, K_{I I}, K_{I I I}\right]$ and $[B]$ is the pre-logarithmic energy factor matrix [174]. For isotropic linear elastic materials $[B]$ is diagonal, and therefore the mixed mode stress intensity factors are related to the J-integral by [174]:

$$
J=\frac{1}{E^{\prime}}\left(K_{I}^{2}+K_{I I}^{2}\right)+\frac{1}{2 G} K_{I I I}^{2}
$$

where $G$ is the shear modulus and $E^{\prime}$ was defined earlier. For anisotropic elastic materials the pre-logarithmic energy factor matrix is no longer diagonal and other procedures are required to evaluate the stress-intensity factors from the J-integral. 
In the commercial FEM program ABAQUS [171], the mixed-mode stress-intensity factors for anisotropic and isotropic elastic materials are calculated using the interaction integral method (IIM) proposed by Shih and Asaro [176]. The IIM was developed based on the superimposing an auxiliary pure Mode I, II or III crack-tip fields onto the actual crack-tip fields for the problem under consideration. The total J-integral, $J_{\text {tot }}^{\alpha}$, then consists of contributions from the actual crack-tip fields, $J$, and those associated with the auxiliary crack-tip fields, $J_{\text {aux }}^{\alpha}$, where the superscript $\alpha$ denotes the mode of the auxiliary crack-tip field. The interaction integral, $J_{\text {int }}^{\alpha}$, is defined by:

$$
J_{i n t}^{\alpha}=J_{t o t}^{\alpha}-J-J_{a u x}^{\alpha}
$$

When the interaction integrals for all three Modes of fracture are calculated a system of equations is obtained from which $K_{I}, K_{I I}$ and $K_{I I I}$ can be determined. Additional details on the finite element implementation and calculation of the interaction integrals are described in the ABAQUS Theory Manual [174].

\subsection{Fatigue Crack Growth in Polycrystalline Materials}

\subsubsection{Characterization of Fatigue Crack Growth}

Early fatigue crack growth (FCG) studies mainly concentrated on polycrystalline materials and specimens containing long pre-cracks. This warrants the application of the LEFM for isotropic small-scale yielding cases. The FCG properties of materials are generally characterized under constant load amplitude loading (see ASTM E647 [177]). The results obtained from such tests when plotted as crack length versus number of cycles resemble those shown schematically Figure 3.8 . The fatigue crack propagation rate 
is obtained from the slope of the $a$ versus $N$ curve, but the raw data cannot be applied directly to life prediction of structures because the crack growth behaviour is unique to the specimen geometry and applied loading conditions. Often, a fracture mechanics crack-tip parameter, such as the stress-intensity factor, is used to correlate with the experimental FCG rates. The well-correlated relationship, i.e., $d a / d N$ as a function of $\Delta K$, is considered to be an intrinsic property of the material [135].

\subsubsection{Application of Linear Elastic Fracture Mechanics}

Under constant amplitude cyclic loading conditions, and assuming that small-scale yielding conditions are satisfied and the crack is large relative to characteristic microstructural dimensions, the driving force for crack extension is controlled by the stress-intensity factor range, $\Delta K$, defined as

$$
\Delta K=K_{m a x}-K_{m i n}
$$

where $K_{\max }$ and $K_{\min }$ are the stress intensity factors at the maximum and minimum stress amplitudes ( $S_{\max }$ and $S_{\min }$ ), respectively. It is implicitly assumed when fatigue crack growth rate (FCGR) data are characterized by stress-intensity factor range that fatigue cracks in specimens and components with the same $\Delta K$ should exhibit the same FCG properties if the materials are identical.

When fatigue crack propagation data from laboratory test specimens are plotted as crack growth rate, $d a / d N$, versus $\Delta K$ on a $\log -\log$ scale, the relationship for most materials exhibit a sigmoidal shape curve as shown schematically in Figure 3.9. Referring to this Figure, three distinct regions of FCG behaviour can be identified, Region I, Region II and Region III. In Region I the FCGR increases abruptly with small changes in $\Delta K$ and the 
$\Delta K$ at which the FCGR tends to zero is termed the threshold stress intensity factor range, $\Delta K_{t h}$. The threshold stress-intensity range is often reached with a load-shedding experimental procedure in which $\Delta K$ is reduced at a constant rate as described in ASTM E647 [177]. Presumably FCG from defects will not occur or they will grow imperceptibly slow when the design loads are small enough that $\Delta K$ is less than the threshold stressintensity factor range for the defect under consideration. Strictly speaking this behaviour is not true for small fatigue cracks, which will be discussed in a later section. It is found in practice that because of the crack growth rates are slow in Region $I$, that $\Delta K_{t h}$ obtained from FCGR tests is particularly sensitive to environment, microstructure, mean stress, crack closure and other factors $[50,135]$. The effect of mean stress on fatigue crack propagation is expressed in terms of the stress ratio defined as:

$$
R=\frac{\sigma_{\min }}{\sigma_{\max }}=\frac{K_{\min }}{K_{\max }}
$$

The apparent mean stress $(R)$ dependence of the fatigue threshold has been attributed to fatigue crack closure [135].

As first postulated and experimentally verified by Elber [178], crack closure occurs as a result of the crack faces coming into contact during a portion of a fatigue loading cycle so that there is a minimum opening stress level, $\sigma_{o p}$, necessary to open the crack surfaces and provide the driving force for crack extension. The stress range that causes crack extension to occur is referred to as effective stress range, which is given by:

$$
\sigma_{e f f}=\sigma_{m a x}-\sigma_{o p}
$$


where $\sigma_{\text {eff }}$ is the effective stress range, $\sigma_{\max }$ is the maximum applied stress amplitude during a fatigue cycle and $\sigma_{o p}$ is the applied stress necessary to open the crack faces. The effective stress-intensity factor range $\left(\Delta K_{e f f}\right)$ for crack extension is then given by:

$$
\Delta K_{e f f}=K_{m a x}-K_{o p}
$$

where $K_{\max }$ and $K_{o p}$ represent the stress-intensity factor associated with the maximum stress amplitude and opening stress amplitudes, respectively.

Crack closure effects in fatigue crack growth are believed to arise from one or more of the following three mechanisms;

- plasticity induced crack closure,

- oxide induced crack closure,

- roughness induced crack closure.

Each of these crack closure mechanisms is shown schematically in Figure 3.10.

Plasticity induced crack closure results from residual plastic deformation and ensuing compressive residual stresses that develop in the wake of a fatigue crack as shown schematically in Figure 3.10a. In the absence of other crack closure mechanisms, plasticity induced crack closure is dependent on the crack length. When fatigue cracks are short, there is insufficient plastic wake behind the crack tip to develop significant closure levels, but as the crack length increases a gradual transition in the crack closure level occurs until steady-state closure conditions are attained, which merge with long-crack 
closure levels $[179,180]$. This has been confirmed both from numerical simulations $[181$, $182]$ and analytical models $[146,180]$ of fatigue crack closure, as well as from experimental measurements [183]. Therefore, the absence of crack closure in the short crack regime is believed to be one of the main reasons why short cracks propagate faster than long cracks in the same material $[8,184]$. For long fatigue cracks, the threshold development has been associated with a rise in crack opening loads as the applied load is reduced using load-shedding procedures [180]. Since small cracks must grow to some specific length to attain comparable closure levels to long fatigue cracks, $\Delta K_{\text {eff }}$ is larger for small cracks until crack closure similitude is attained, and therefore small crack growth can occur below $\Delta K_{t h}$ associated with long fatigue cracks.

Oxide induced crack closure is caused by the build-up of oxidation products on the fatigue crack surfaces. This build-up of oxidation products on the fracture surface acts like a wedge between the crack surfaces, resulting in contact of the two crack surfaces sooner than if the crack surfaces are un-oxidized [185].

Roughness induced crack closure results from the crack faces being displaced in the direction parallel to the crack growth [186] causing a mismatch of the crack surfaces. This mismatch in the crack surfaces results in premature crack closure because small asperities generated during crack advancement, come into contact during load reversal. In the absence of other crack closure mechanisms, the minimum crack opening stress, $\sigma_{o p}$, is dependent on the topology of the fracture surface, with rougher surfaces enhancing the crack closure effect. 
The crack closure effects, as defined by equation 3.22 would cause crack growth to arrest when the applied $\Delta K$ approaches the crack closure level, resulting in Region I crack growth. In Region II the FCGR exhibits a linear dependence on $\Delta K$. This region is usually referred to as the Paris regime because the relationship between $d a / d N$ versus $\Delta K$ is characterized by Paris' equation [187]:

$$
\frac{d a}{d N}=C \Delta K^{m}
$$

where $C$ and $m$ represent the intercept and slope, respectively, of the linear curve fit to the Region II FCGR data. In practice the intercept $C$ is found to depend on the mean stress, and therefore is usually stated as a function of $R[50]$. Because FCG rates in Region II are higher, the FCG behaviour is relatively insensitive to microstructure, and load ratio and environmental effects are less pronounced than crack growth in Region I $[50,135]$.

Region III is characterized by FCG rates that are significantly higher than in the Paris region and is usually associated with unstable crack growth. Fatigue crack growth in this region is most notably affected by factors such as stress ratio and the state of stress (plane stress or plane strain) at the crack-tip [50, 135]. In Region III, the FCGR rate asymptotically approaches the fracture toughness of the material, $K_{c}$, or the mode I plane strain fracture toughness, $K_{l c}$, under plane strain conditions.

\subsubsection{Application of Elastic-Plastic Fracture Mechanics}

The J-integral was derived based on the fundamental assumption of monotonic deformation based on the deformation theory of plasticity. Strictly speaking it is no 
longer valid as a crack-tip parameter for characterizing fatigue crack propagation under cyclic loading because the premise of J-integral is monotonic loading conditions [188]. In spite of this apparent limitation, there has been some success reported using the cyclic Jintegral, $\Delta J$, to characterize fatigue crack growth under both small-scale yielding and elastic-plastic conditions [189-192]. The cyclic J-integral for 2-D stationary cracks as defined by Tanaka [193] for a remote stress change $\left(\sigma_{\infty}\right)_{j}-\left(\sigma_{\infty}\right)_{i}$ is given by:

$$
\Delta J_{j / i}=\int_{\tau}\left(\Delta W d x_{2}-\Delta T_{m} \frac{\partial \Delta u_{m}}{\partial x_{1}} d s\right)
$$

where $\Delta W, \Delta T_{m}$ and $\Delta u_{m}$ represent the relative changes in between the strain energy density, traction and displacement, respectively given by:

$$
\begin{gathered}
\Delta W=\int_{\left(\varepsilon_{m n}\right)_{i}}^{\left(\varepsilon_{m n}\right)_{j}}\left[\sigma_{k l}-\left(\sigma_{m n}\right)_{i}\right] d \varepsilon_{k l} \\
\Delta T_{m}=\left(T_{m}\right)_{j}-\left(T_{m}\right)_{i} \\
\Delta u_{m}=\left(u_{m}\right)_{j}-\left(u_{m}\right)_{i}
\end{gathered}
$$

In the case of small-scale yielding, where the linear-elastic approximation holds it can be shown $[188,193]$ that under plane stress conditions for an isotropic elastic material that:

$$
\Delta J_{j / i}=\frac{\Delta K_{j / i}^{2}}{E}
$$


This result is significant in that it implies under small-scale yielding conditions the linear elastic and elastic-plastic solutions should collapse onto approximately the same fatigue crack propagation curves.

It has been shown that FCGR data when plotted as a function of the cyclic J-integral on a log-log scale collapse into the characteristic three region fatigue crack growth rate curve similar to that observed in the case of linear-elastic materials, as shown in Figure 3.11 [191, 192]. Dowling and Begley [194] proposed that the linear portion of the curve can be fitted by power-law expression similar to Paris' law, which is given by:

$$
\frac{d a}{d N}=C(\Delta J)^{m}
$$

where $C$ and $m$ represent the intercept and slope, respectively, of the $\log -\log$ linear fit.

Lambda [195] has noted that evaluation of the cyclic J-integral in elastic-plastic materials is not as straightforward as taking the difference between the monotonic J-integral values associated with the maximum and minimum loads, i.e.

$$
\Delta J \neq J_{\max }-J_{\min }
$$

Therefore, to evaluate the cyclic J-integral from numerical solutions, such as FEM, one must instead directly evaluate the line-integral in equation 3.24, which can only be used in case of 2-D bodies. 
Findley et al. [196] and Kim and co-workers [197] have developed approximate solutions for estimating the cyclic J-integral for thumbnail cracks in round bars and flat plates, respectively. Their empirical models were derived from finite element calculations in which the Ramberg-Osgood (R-O) constitutive law was used to describe the non-linear material behaviour. Since these empirical equations were developed using the R-O constitutive law, they are only suitable for isotropic materials.

Staroselsky and co-workers [198] have suggested an alternative approach for characterizing elastic-plastic fatigue crack growth in materials based solely on the monotonic J-integral value, which can be estimated using numerical methods. This approach does not consider crack closure effects and it is assumed that for small positive stress ratios $(0.0-0.2)$ that the contribution of the J-integral associated with the minimum load is negligible. Based on these conditions the cyclic J-integral can be approximated by its monotonic value associated with the maximum load, i.e. $\Delta J \approx J_{M A X}$ and the crack growth law specified in equation 3.29 is then given by:

$$
\frac{d a}{d N}=C(J)^{m}
$$

This approach was considered further in this investigation.

\subsubsection{Small Fatigue Crack Growth}

When crack growth rates were observed from naturally-generated (as opposed to artificially introduced) small fatigue cracks, it was often found that an anomalous

behavior is manifested in Region I, particularly near and below the long-crack fatigue threshold, $\Delta K_{\mathrm{th}}$, as shown schematically in Figure 3.12, where the long-crack $\Delta K$ - 
similitude breaks down. This anomalous behaviour and lack of similitude based on $\Delta K$ has been attributed to $[135,184]$ :

- small fatigue cracks being more notably affected by microstructure, such as grain boundaries (in polycrystalline materials) and strengthening precipitates, environment and crack closure mechanisms than physically long fatigue cracks,

- violation of the small-scale yielding conditions on which $\Delta K$ is based when the cyclic crack-tip plastic zone size approaches that of the crack length.

Notably, small fatigue cracks can exhibit remarkably higher growth rates below and near $\Delta K_{t h}$. As crack propagation continues, it is often observed that small-fatigue crack growth rates will merge with long FCG rates when tested under the same conditions, as shown in Figure 3.12.

\subsubsection{Definitions for Small Fatigue Cracks}

For understanding the small-fatigue crack growth phenomena, small fatigue cracks are generally classified based on the Kitagawa-Takahashi diagram, shown in Figure 3.13. From this diagram the small crack regime consists of two sub-regimes designated the microstructurally short and physically small crack regimes, respectively. In the microstructurally small crack regime the crack length is comparable to microstructural dimensions, such as the grain size in polycrystalline materials or inter-particle spacing in precipitation strengthened materials. Hence, breakdown of the similitude between small and long crack growth behaviour for the microstructurally small crack regime can be attributed to microstructural heterogeneity [199]. Conceptually, the fracture mechanics parameters such as stress-intensity factor and the J-integral for isotropic materials cannot 
be used to unambiguously characterize fatigue crack growth in this regime because microstructural heterogeneity violates the assumptions used in deriving these parameters.

The physically small crack regime considers cracks that can be long relative to characteristic microstructural dimensions but are merely small (less than 1000-2000 $\mu \mathrm{m}$ ) [135], or cracks that are small compared to cyclic plastic zone size ahead of the propagating crack (mechanically small cracks) $[135,200]$. This later case can be encountered in smooth fatigue specimens under high stresses, which result in fatigue cracks with a large plastic zone size, or for fatigue cracks that nucleate and propagate through the plastically deformed region at the root of a macroscopic notch, as shown schematically Figure 3.3. In this case the small-scale yielding assumption is violated and $\Delta K$ is no longer valid as a crack-tip parameter. Elastic-plastic fracture mechanics parameters such as the J-integral have been considered to offer a possible means accounting for the differences between small and long fatigue crack behaviour when plasticity is considered significant $[49,50,135]$.

In addition to the two small crack regimes shown in Figure 3.13, fatigue cracks can also be considered small when anomalous crack growth occurs as a consequence of the crack being affected by environmental processes, such as oxidation and stress-corrosion, which are referred to as chemically short fatigue cracks [135]. For the purpose of discussing small crack growth in single-crystal superalloys fatigue cracks will be considered "small" if they are physically small surface (thumbnail) cracks less that $2000 \mu \mathrm{m}$ in length, and "short" in the case of physically small (less than $2000 \mu \mathrm{m}$ ) through-thickness cracks. 


\subsubsection{Mechanisms of Small Fatigue Crack Growth}

The growth of small cracks in polycrystalline materials usually occurs in two successive stages; Stage I (also called crystallographic or Mode II) crack growth and Stage II (noncrystallographic, Mode I or normal mode). During Stage I crack growth the crack is initially confined within a single grain and propagation occurs along specific crystallographic planes. After the crack propagates beyond a single grain there is a gradual transition to Stage II crack growth, which becomes exclusively Stage II after the crack length exceeds several times the grain size, thus merging into the long-FCG behavior.

The transition from small to long fatigue crack behavior, on one hand, has been thought to be due to the rising of crack closure from zero in the small-crack regime to a steady level in the long-crack regime $[49,135]$. But, on the other hand, it can also be attributed to grain boundary impedance (blocking of slip bands) [201-203]. Both mechanisms can be operating at the same time, but measurements of crack closure for naturally-generated small cracks are difficult and therefore rarely reported.

\subsubsection{Effect of Macroscopic Notches}

As noted earlier in this Chapter, fatigue cracks can nucleate from stress-raising features such as macroscopic notches. Small fatigue cracks emanating from the root of a notch can exhibit anomalous FCG behaviour because they are influenced by the elastic-plastic strain fields associated with the notch, as shown schematically in Figure 3.3b. In some cases this anomalous FCG initially involves accelerated fatigue crack propagation, followed by a deceleration at increasing crack lengths, and finally acceleration such that the small fatigue crack growth rate curve merges with long fatigue crack propagation data 
resulting in the characteristic "fish hook" FCGR curve as illustrated in Figure 3.12 [49, $50,135,199,204]$.

The apparent small crack effect at notches has been attributed to several factors including notch root plasticity [205] and the effects of crack closure [49]. When the applied stress in a notched specimen is sufficiently high that plasticity occurs at the notch root, this plasticity contributes to an initial acceleration in crack growth rate [51]. As the crack length increases the small crack develops a plastic wake that is confined within the plastically deformed region in the notch root resulting in crack closure. Finite element simulations performed by McClung and Sehitoglu $[181,182]$ on notched specimens show that crack opening levels initially start out very low (approximately $K_{\min }$ for $R=0$ ) but increase quickly with increasing crack length. Fatigue experiments conducted by Tanaka and Nakai [51] have shown that crack closure levels can become sufficiently high in notched specimens to cause crack deceleration and in some cases complete arrest of small fatigue cracks. As small fatigue cracks continue to propagate after this deceleration phase, they accelerate to merge with long FCG behaviour because the crack closure levels stabilize and the crack grows beyond the notch plastic field. McClung and Sehitoglu $[181,182]$ have shown from FEM calculations simulating crack closure, that crack opening loads did not stabilize until the crack was sufficiently long as to no longer be influenced by the notch root fields, that is the crack resides in the region in which the maximum stresses associated with an uncracked notched body decay approximately to nominal far-field values. 


\subsection{Fatigue Crack Formation and Propagation in Single-Crystal Superalloys}

In a holistic lifing approach the total fatigue life for a component is considered to consist of four phases, as shown schematically in Figure 3.14. The first three phases, crack nucleation, small crack growth and steady crack growth are the most important to consider when designing components. The first two phases, crack nucleation and small crack growth, will be discussed further in regard to crack growth in single-crystal superalloys.

\subsubsection{Crack Nucleation in Single-Crystal Superalloys}

Fatigue crack nucleation and growth can occur in ductile metallic materials even when cyclic loads are significantly lower than the materials elastic limit. Although the mechanisms associated with fatigue crack nucleation are generally understood, at least in a mechanistic sense, there are no commonly accepted quantitative theories for describing the crack nucleation process [206]. However, it is known that crack nucleation involves irreversible slip on one or more slip planes that is accompanied by progressive cyclic softening or hardening resulting in the localization of strain. There are number of microstructural and geometrical factors that facilitate the localization of strain during cyclic loading including:

- pre-existing stress-raising features such as macroscopic notches or surface roughness,

- localized regions of the material that are microstructurally "soft" compared to the bulk material,

- general surface roughening produced during fatigue by slip offsets,

- shearable precipitates, 
- coherent or incoherent secondary phases that are brittle

- materials defects such as shrinkage or porosity and non-metallic inclusions.

When no pre-existing material defects are present, fatigue crack nucleation generally originates from the material surface. In single-crystal superalloys surface crack nucleation can occur by several different mechanisms depending on temperature, cyclic frequency, applied stress and environment. At low temperatures (below $760^{\circ} \mathrm{C}$ ) and high cyclic frequencies, slip localization in the form persistent slip bands (PSB's) can occur on octahedral slip systems with the maximum resolved shear stress in single-crystal superalloys $[12,100,103,117,119,206]$. Persistent slip bands are in essence highly localized region of slip in which the plastic strain amplitude can be 100 times higher than the surrounding matrix [206]. The formation of PSB's in single-crystal superalloys can occur in a localized or extended manner. Local PSB formation occurs at lower stress amplitudes and involves octahedral slip that is confined to the $\gamma$-matrix channels. Higher stress amplitudes favor the formation of extended PSB's that shear through the $\gamma$-matrix and $\gamma^{\prime}$ precipitates on octahedral planes as shown in Figure 3.15.

In addition to stress amplitude, the transition from localized to extended PSB formation appears to be closely related to the chemistry of the $\gamma^{\prime}$ phase. It has been reported that compositional modifications to the single-crystal alloy PWA1480, principally made to alter the $\gamma^{\prime}$ phase composition, can cause precipitate shearing under conditions that the conventional alloy does not [5]. At the free surfaces, PSB's manifest as intrusions and extrusions of material that contribute to a general increase in surface roughness, as depicted in Figure 3.16, that ultimately lead to the formation of a fatigue crack. In singlecrystals oriented for multiple-slip, PSB's can form on multiple slip systems that intersect at free surfaces contributing to fatigue crack formation [5]. 
At lower temperatures non-metallic carbide and boride phases have an important role in formation of fatigue cracks in single-crystal superalloys $[31,36,48,69,207]$. Because of the brittle nature of these phases they can become cracked at some point prior to the component entering service. Gell and Leverant [69] have reportedly observed carbide phases in platelet form that contained pre-existing cracks in Mar-M200 single-crystals. These pre-cracked carbides were observed to function as crack nucleation sites [69]. Initially sound brittle phases can also become cracked under monotonic and cyclic loading. Under conditions conducive to planar slip these brittle phases can become cracked by a slip impingement mechanism or they may also function as stress raisers thereby initiating planar slip within the material. The size of these brittle phases also appears to have a strong effect on the crack nucleation process in single-crystal superalloys. Gell and Leverant [69] have reported that Mar-M200 material containing large carbides $(0.30 \mathrm{~mm})$ cracked upon initial loading to the proportional limit and exhibited short fatigue lives, while material containing smaller carbides had markedly improved fatigue lives under strain-controlled fatigue testing.

In addition to brittle phases other intrinsic material quality defects, such as shrinkage porosity and interdendritic $\gamma-\gamma$ 'eutectic phase have an important role in nucleating fatigue cracks in single-crystal superalloys, as shown in Figure 3.17. Among these defects, shrinkage porosity is the most important. At low and intermediate temperatures (427$760^{\circ} \mathrm{C}$ ), interdendritic shrinkage porosity is the primary cause reported for lowtemperature crack nucleation in carbide-free single crystal superalloys $[5,31,33,34,37$, $38,44,45,163,208,209]$. However, it has been observed that crack nucleation in smooth fatigue samples tested in air involves a competitive process between surface crack nucleation and subsurface nucleation from pores. This effect has been attributed to the slip homogenizing effect of surface oxidation which results in more uniformly distributed 
and smaller surface shear displacements [163]. Subsurface pores, on the other hand not only act as stress raisers they also function as a free surface from which localized slip band formation can occur. Because subsurface shrinkage pores are under vacuum the slip dispersal effect of oxidation is precluded and persistent slip band formation can more readily occur. In single-crystal fatigue specimens containing macroscopic notches it has been reported that crack nucleation is strongly influenced by pore proximity to the notch surface and the pore size [48]. In general, shrinkage pores that are closer to the notch surface and/or larger in size tend to greatly reduce the number of cycles required to nucleate and form a macroscopic fatigue crack. It has also been reported that fatigue crack nucleation from pores in notched single-crystals subjected to uniaxial loading frequently occurs on planes that are inclined to the plane normal to the applied loading [208, 209].

At elevated temperature fatigue crack nucleation is a competitive process between oxidation damage mechanisms (in air) and subsurface defects. When single-crystal alloys are tested in air environments and high temperatures $\left(>800^{\circ} \mathrm{C}\right)$, oxidation can change the material characteristics both at the physical and chemical levels. Physically, surface oxidation in single-crystal superalloys can occur with different kinetics because of localized regions of compositional heterogeneity. Such sites include surface connected carbide and eutectic $\gamma-\gamma^{\prime}$ phases as well as compositional heterogeneity related to the retained dendritic cast structure after solution heat treatment. During cyclic loading, the integrity of the oxide layer can be compromised as a result of thermal expansion [186, $210]$ and/or mechanical property [186] incompatibilities between the oxide layer and substrate material. The end result being that the oxide layer grows heterogeneously into the substrate forming oxide spikes as illustrated in Figure 3.18. Numerous investigators have reported that oxide spikes function as preferential sites for crack nucleation in 
directional solidified and single-crystal superalloys $[32,118,211,212]$. The formation of oxide spikes is not the only mechanism by which oxidation can cause fatigue crack nucleation. Ohtani and co-workers [213] have reported that the fatigue cracks can nucleate from the boundaries of individual oxide grains on the surface of smooth fatigue specimens and then propagate into the substrate material.

Chemically, oxidation can alter the material causing local material embrittlement and formation of a region depleted of oxide-forming elements. In single-crystal superalloys surface oxidation depletes the subsurface region of oxide forming elements, such as aluminum, resulting in the formation of a $\gamma^{\prime}$ precipitate free zone as shown in Figure 3.19. Because single-crystal superalloys rely predominantly on $\gamma^{\prime}$ precipitates for high temperature strength, the precipitate free zone is expected to have inferior mechanical properties to the bulk material. It has been reported [69] in a wrought nickel-base superalloy that plastic deformation concentrates in the precipitate free zone resulting in the nucleation of fatigue cracks.

\subsubsection{Small Fatigue Crack Growth in Nickel-base Single-Crystal Superalloys}

Study of small fatigue crack growth in nickel-base single-crystal superalloys is of more practical importance than considering long cracks, because they are used to fabricate gas turbine vanes and blades that are not designed to tolerate long cracks. This argument may be supported by several factual reasons. Firstly, it has been observed from fatigue experiments that growth of naturally-generated fatigue cracks less than $300 \mu \mathrm{m}$ in length can constitute $60-80 \%$ of total life in single-crystal superalloys [31, 32]. Secondly, it has been suggested that because fatigue cracks predominantly nucleate and propagate from intrinsic material quality defects that are several to tens of microns in size (pores and secondary phases) in single-crystal superalloys, these defects should be considered as 
small fatigue cracks when adopting a fracture mechanics approach for component lifeprediction [214]. Lastly, fatigue in highly stressed components in gas turbine engines is predominantly a LCF problem in which the critical crack lengths may be close to or within the physically small crack regime [215].

Despite its engineering relevance, fatigue crack growth behavior from naturallygenerated small cracks has not been widely studied and reported in the literature, even though the nickel-base single-crystal superalloys have gone through 4 to 5 generations of development. Some observations on both long and small cracks in nickel-base single crystal superalloys are reviewed in the following sections.

\subsubsection{Mechanisms of Fatigue Crack Propagation in Single-Crystal Superalloys}

As opposed to polycrystalline materials, the growth of fatigue cracks in single-crystal superalloys do not usually follow the sequence from Stage I to Stage II growth. The presence or absence of Stage I and Stage II crack propagation mechanisms in singlecrystal superalloys depend on testing frequency, environment, temperature, and stress state at the crack-tip, which influence slip planarity and $\gamma^{\prime}$ shearing and bypass mechanisms discussed in Chapter 2.

In single-crystal materials, Stage I crack growth predominantly occurs under conditions that are conducive to planar slip, i.e. high cyclic loading frequency and low testing temperatures. In contrast, Stage II crack growth predominantly occurs under conditions that are conducive to slip dispersal, i.e. high temperatures and lower testing frequencies, particularly with environmental effects, e.g. oxidation. Two recent studies have examined the influence of temperature and cycling frequency on the process of crack initiation and propagation in cube oriented PWA1484 [29] and CMSX-2 [45] single-crystals. The FCG 
behavior of CMSX-2 is shown in Figure 3.20. Based on the results reported in theses two studies, the crack propagation mechanisms can be summarized into three specific temperature regimes:

- Low Temperatures $\left(\mathrm{T}<427^{\circ} \mathrm{C}\right.$ [29]): Crack growth in samples tested at $427^{\circ} \mathrm{C}$ and $595^{\circ} \mathrm{C}$ occurred primarily along octahedral planes independent of testing frequency. In some cases, crack growth was confined to only one octahedral slip system resulting in fatigue crack inclined at $45^{\circ}$ to the applied load axis, similar to Stage I cracks in polycrystalline materials. In other samples, fatigue cracks propagated on several different octahedral planes resulting in large crystallographic octahedral facets on the fracture surface, similar in appearance to the fracture surface shown in Figure 3.21. The crack growth mode was found to be independent of testing frequency.

- Intermediate Temperatures $\left(600<\mathrm{T}<871^{\circ} \mathrm{C}\right.$ [29]): Crack growth in samples tested at 760 and $871^{\circ} \mathrm{C}$ were more complex than at lower temperatures. In some samples, cracks propagated along several octahedral planes up to $200 \mu \mathrm{m}$, then changed to Stage II propagation mode. Stage II cracks were observed to propagate in the $\gamma$-matrix channels along $\gamma-\gamma^{\prime}$ interfaces and in a trans-precipitate noncrystallographic (TPNC) mode. Higher testing frequencies promoted Stage I crystallographic crack growth features. In contrast, lower testing frequencies caused predominantly Stage II fatigue crack propagation.

- High Temperatures $\left(\mathrm{T}>871^{\circ} \mathrm{C}[45]\right)$ : Crack growth in samples tested at $950^{\circ} \mathrm{C}$ are dominated by stage II crack growth with cracks propagating in the $\gamma$-matrix channels along $\gamma-\gamma^{\prime}$ interfaces as illustrated in Figure 3.22. 
In addition to temperature and loading frequency affecting the crack propagation mechanisms, transitions between fatigue Stage I and II crack growth have been observed to depend on $\Delta K$. Telesman and Ghosn $[27,28,30]$ have reported for long crack growth in single-crystal superalloy PWA1480 tested at room temperature, that crack growth can change from crystallographic growth on a single octahedral plane to the TPNC mode. This transition was observed at $\Delta K$ in the range of 2.5 to $8 \mathrm{MPa}^{1 / 2}$ during load shedding, used to experimentally measure the fatigue threshold $\Delta K_{t h}$, and under constant amplitude ( $K$-increasing) testing procedures. They reported that after the transition from crystallographic to TPNC crack propagation mechanisms, the FCGR appeared to be independent of $\Delta K$ and thus deviated from the normal sigmoidal $d a / d N-\Delta K$ behaviour associated with long crack growth. Since their fatigue experiments tests were performed at $R=0.5$, crack closure was not a factor in the transition in crack propagation mechanism nor the apparent acceleration in the fatigue crack growth rates at low $\Delta K$. Crack-tip constraint conditions (plane stress or plane strain) were also observed to influence the fatigue crack growth mechanisms for thru-thickness cracks in $\mathrm{C}(\mathrm{T})$ specimens $[28,30]$. Stage I crystallographic crack growth was observed regardless of applied $\Delta K$ where the crack intersected the free surface of the $\mathrm{C}(\mathrm{T})$ specimen and plane stress conditions existed. Stage II crack propagation was observed at low $\Delta K$ at the midthickness plane of the fatigue specimen where material constraint imposed plane strain conditions at the crack-tip. At higher $\Delta K$ a transition between Stage II and Stage I crack growth was observed at the specimen mid-plane with faceting becoming more pronounced as $\Delta K$ was increased.

Chan and coworkers $[15,16]$ have also observed TPNC and crystallographic crack propagation and transitions between these two crack propagation mechanisms in the single-crystal superalloy PWA1484 tested at $593^{\circ} \mathrm{C}$. Among their findings they reported 
TPNC crack growth rates were higher at low $\Delta K$ than crystallographic crack growth and the $\Delta K_{t h}$ was lower for TPNC fatigue cracks. Above a certain $\Delta K$, depending on temperature and cyclic frequency, the crack growth rate for Stage I crystallographic cracks was higher than cracks propagating by the TPNC growth mechanism. They also reported that transition between TPNC and crystallographic crack propagation was promoted under mixed-mode (Mode I and Mode II) loading conditions. During threshold testing using a load shedding procedure and under Mode I loading, they observed that crystallographic fatigue cracks would deflect from the crystallographic plane and propagate on a non-crystallographic crack plane to the lower TPNC fatigue threshold.

Joyce et al. [216] have also reported changes in fatigue crack propagation mechanisms in CMSX-4 tested at $650^{\circ} \mathrm{C}$ in air and vacuum environments and two crack propagation directions $\langle 100\rangle$ and $\langle 110\rangle$ with loading along the $<001\rangle$ crystal direction. They reported macroscopically planar crack growth along the $\{100\}$ plane, which transitioned to cooperative slip along alternating $\{111\}$ slip planes at intermediate $\Delta K$ levels producing a facetted (Stage I) fracture surface features, and finally transitions to extended $\{11\}$ slip plane cracks above a critical $\Delta K$ level. Crystallographic facets were also observed on the side faces of fatigue specimens with a $<100>$ crack growth direction at low to intermediate $\Delta K$ levels. These observations and those of Chan and coworkers [15, $16]$ and Telesman and Ghosn $[27,28,30]$ for crack growth in single-crystal superalloys may also be relevant even for small crack growth. It has been suggested that changes in crack propagation mechanisms can result in significantly different fatigue crack propagation kinetics. Additional factors that can promote changes in crack propagation mechanisms in single-crystal superalloys and their effect on crack propagation rates are discussed further in the following section. 


\subsubsection{Factors Influencing Small Crack Growth}

\subsubsection{Crystallographic Orientation}

The majority of small and long fatigue crack propagation studies in single-crystal superalloys consider only unaxial loading and $\langle 100\rangle$ primary orientations. This may in part be due to the exclusive use of cube oriented single-crystals for turbine airfoils because this is the natural solidification direction in single-crystal cast structures and the favorable anisotropic properties of the $\langle 100\rangle$ orientation. There do not appear to be reports of the effect of primary crystallographic orientation on the behaviour for small fatigue cracks in the literature, but a number of researchers [20, 21, 42], among others, have investigated the influence of primary orientation on long fatigue crack propagation behavior. Henderson and Martin [20] have reported that long FCG rates in SRR99 single

crystals having $\langle 011\rangle$ primary orientation are higher than crack propagation rates in $\langle 111\rangle$ and $\langle 100\rangle$ orientations tested at 650 and $850^{\circ} \mathrm{C}$, while the $\langle 111\rangle$ orientation exhibited the lowest crack propagation rates. They attributed the primary orientation dependence on long crack propagation rates to the degree of slip reversibility at the crack-tip. Primary orientations that minimized the number of octahedral slip systems to accommodate crack-tip shear, e.g. $\langle 011\rangle$, exhibited substantial cross-slip at the crack front, which minimized crack-tip slip reversibility and resulted in higher FCG rates [20].

In notched fatigue specimens, a second crystallographic direction $\langle h k l\rangle$ can be used to define the direction of crack propagation in single crystal nickel-base superalloys in relation to the primary single-crystal orientation. This is referred to as the secondary orientation, as described in an earlier Chapter. For clarity, a schematic diagram defining the secondary orientation in notched single-crystal fatigue specimen has been provided in 
Figure 3.23. The influence of secondary orientation on small crack fatigue crack propagation is somewhat controversial, with some authors reporting no secondary orientation effects [36, 47], and others reporting a strong secondary orientation dependence on the fatigue crack propagation properties for small fatigue cracks $[33,34$, 40]. In studies that have reported secondary orientation effects on the fatigue crack propagation rate of small fatigue cracks, FCG rates were higher in specimens in which the secondary orientation was aligned the $\langle 001\rangle$ crystal direction than the $\langle 011\rangle$ crystal direction. In the study conducted by Defresne and Remy [33, 34], the crack opening loads were measured for mechanically short through-thickness cracks in single-edge notch fatigue samples (machined to be short from a long crack configuration generated by load shedding procedures). They determined that the secondary orientation dependence of the FCGR at $650^{\circ} \mathrm{C}$ in test specimens with $\langle 011\rangle$ and $\langle 001\rangle$ crack propagation directions could be rationalized by taking crack closure effects into consideration.

On the other hand, Wu et al. [145] have shown, based on an elastic-perfectly plastic strip yielding model, that the energy release rate and the crack tip opening displacement (CTOD) are lower in the $\langle 011\rangle$ orientation than the $\langle 001\rangle$ orientation, even under smallscale yielding conditions. The physically intrinsic properties were considered to reconcile the orientation dependence of the fatigue crack growth behavior in single crystal Udimet 720 at room temperature.

\subsubsection{Crack Closure Effects}

In single-crystal superalloys tested in laboratory air and elevated temperatures, significant oxidation of the crack faces has been reported to occur [44, 45], an example of which is shown in Figure 3.24. A number of researchers [20, 21, 25, 39, 40, 42, 44-47, 216, 217] have attributed differences in FCG behaviour in single-crystal superalloys tested in air 
and vacuum environments at elevated temperatures to oxide induced crack closure effects. Okazaki et al. [217] have experimentally measured closure levels for naturallygenerated small fatigue cracks in CMSX-2 single-crystals tested at $600,750,850$ and $950^{\circ} \mathrm{C}$ in air. The fatigue specimens were tested under controlled loading conditions to ensure that the ratio of applied stress to the temperature dependent yield strength of the material was constant in each of the fatigue experiments. They reported the crack opening ratio, characterized by the ratio $\Delta K_{\text {eff }} / \Delta K$, decreases with increasing test temperature indicating that the effective driving force for crack extension was reduced by an oxidation induced crack closure mechanism. The effect of environment on fatigue crack growth behaviour in single-crystal superalloys will be considered in more detail in the following section.

Roughness induced crack closure behaviour has been reportedly observed in singlecrystal superalloys in which fatigue cracks propagate in a crystallographic manner. Chan and workers $[17,18]$ have observed macroscopic evidence of burnishing damage and emission of debris from crystallographic fatigue cracks in Mar-M200 single-crystals fatigue tested at room temperature. Gell and Leverant [162] have reported the presence of rub marks on slip steps that extend above the main fracture surface of crystallographic fatigue cracks in a directionally solidified nickel-base superalloy fatigue tested at room temperature. Howland [218] has also reported the presence of fretting damage on the fracture surface of corner crack specimens of the single-crystal superalloy SRR-99 fatigue tested at room temperature and $R=0.1$. He did not observe evidence of fretting damage on specimens tested at higher stress ratios $(R=0.5$ and $R=0.8)$ indicating the full range of the stress-intensity factor contributed to crack growth under higher $R$ conditions. 


\subsubsection{Environment}

Environmental effects on fatigue crack growth in Ni-base single crystal superalloys often occur at high temperatures, mainly through two mechanisms: i) oxidation induced change in the crack growth kinetics, and ii) oxidation induced crack closure. The degree to which the environment will affect short crack growth behavior is dependent on the crack-tip exposure time to the operating environment, which is influenced by testing frequency, loading conditions (stress, strain ratio and temperature). It also depends on the $\Delta K$ level, the relative contribution from pure mechanical fatigue, i.e., slip, and the kinetics of oxidation. In addition to these factors, the FCGR can also influence the degree to which environmental effects will contribute to fatigue crack propagation. When the fatigue crack propagates by slip faster than the environment can change the material ahead of the crack tip, the environmental effects will be diminished [69].

Gell and coworkers [69] have investigated the effects of testing environment on the propagation of fatigue cracks in cube oriented Mar-M200 single crystals tested in vacuum and air during fully reversed strain-controlled fatigue conditions $(R=-1)$. They have reported that $\mathrm{FCG}$ rates were higher at $927^{\circ} \mathrm{C}\left(1700^{\circ} \mathrm{F}\right)$ in vacuum than in air. The higher FCG rates in vacuum were attributed to the degree of crack re-sharpening of Stage II cracks during the compressive half of the loading cycle. Stage II cracks formed in the vacuum environment were observed to be sharper than Stage II cracks in samples tested in air. The process of crack tip re-sharpening during Stage II crack growth for air and vacuum environments is shown schematically in Figure 3.25. It is clear from their investigation that oxidation not only influences crack closure behavior, but also can contribute to crack tip blunting. In addition to oxidation, crack tip blunting can also occur as a result of creep strain increasing the crack tip root radius, which leads to lower fatigue crack propagation rates $[219,220]$. 
Schubert and colleagues [47] found a lower fatigue threshold for short fatigue cracks in vacuum than air in the single-crystal superalloy CMSX-4 tested at $5 \mathrm{~Hz}$ and $750^{\circ} \mathrm{C}$. This change in threshold was attributed to increased crack closure associated with oxidation of the fracture surfaces for tests conducted in laboratory air. On the other hand, Defresne and Remy [33] have reported a complete absence of a fatigue threshold for long fatigue cracks in CMSX-2 single-crystals tested at $650^{\circ} \mathrm{C}$ under vacuum. Joyce et al. [216] reported higher FCG rates were observed in vacuum than in air in CMSX-4 long crack growth rate tests conducted at $650^{\circ} \mathrm{C}$ using a 4 second trapezoidal cycle incorporating a 1 second hold at the maximum and minimum loads. They attributed this behaviour to oxidation induced closure effects and increased slip homogenization from oxidation of the fracture surfaces suppressing the transition to faster Stage I crystallographic crack growth.

In a study by Okazaki and coworkers [44,217], the influence of temperature on the short and long crack propagation behavior in DS CM247LC and cube oriented CMSX-2 single crystals was investigated, at test temperatures of $600,750,850$ and $950^{\circ} \mathrm{C}$. Among their findings, it was reported that the short FCG rates in both alloys increased considerably (two to three orders of magnitude) when the test temperature was increased from 600 to $950^{\circ} \mathrm{C}$. They speculated that the strong temperature dependence of the small FCG rates was due to increased environmental attack at higher temperatures. This viewpoint was supported by post-test microstructural analysis of fatigue specimens, which showed that oxidation of the crack-tip was accompanied by a formation of a $\gamma^{\prime}$ depleted zone that was more extensive at higher test temperatures. 


\subsubsection{Microstructure}

Besides influencing deformation mechanisms and fatigue crack propagation mechanisms, cyclic loading at high temperatures can cause microstructure changes in nickel-base superalloys. During fatigue cycling at high temperatures the regular array of cuboidal $\gamma^{\prime}$ precipitates in single-crystal nickel-base superalloys can directionally coarsen to form a plate or continuous morphology, referred to as $\gamma^{\prime}$ rafting. Mughrabi and coworkers [221] have reported on the effects of TMF cycle on the raft development in cube oriented, $\langle 100\rangle$, CMSX-6 single crystals. Out-of-phase (OP) TMF cycling causes rafts to form parallel to the stress axis (p-type), whereas in-phase (IP) TMF cycling causes rafts to form normal to the stress axis (n-type). Both n-type and p-type types of rafting are shown in Figure 3.26. Okazaki and co-workers [45] have reported that $\gamma^{\prime}$ rafting (n-type) at the crack tip in CMSX-2 during creep-fatigue loading at $950^{\circ} \mathrm{C}$. They did not report any affect of the rafted precipitate structure on the small fatigue crack propagation behavior. In a related study Okazaki et al. $[44,217]$ reported $\gamma^{\prime}$ rafting and $\gamma^{\prime}$ depletion near the crack faces was more pronounced for small fatigue cracks than long fatigue cracks in CMSX-2 tested at 850 and $950^{\circ} \mathrm{C}$.

The effect of $\gamma^{\prime}$ rafting on long FCG, has been studied by Ai and co-workers [11, 12]. They reported an increase in long crack FCG rates and decrease in threshold in CMSX-2, that was thermal mechanically treated (TMT) prior to fatigue testing at $950^{\circ} \mathrm{C}$ to produce a rafted $\gamma^{\prime}$ structure, with the rafts aligned parallel to the crack growth direction (n-type). The difference in long crack growth rates between the TMT and standard microstructure was attributed to differences between the crack propagation paths observed in the TMT and "virgin" material. Fatigue cracks in the TMT specimens were observed to be exclusively confined to the $\gamma$-matrix channels adjacent to the $\gamma^{\prime}$ rafts. Stage II fatigue 
cracks in the standard microstructure were observed to branch between the $\gamma^{\prime}$ precipitates, resulting in a more tortuous crack propagation path and enhanced roughness induced crack closure. 


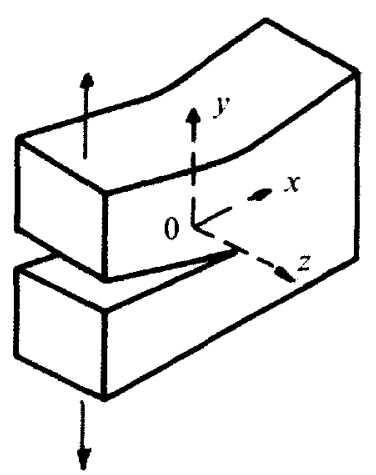

(a)

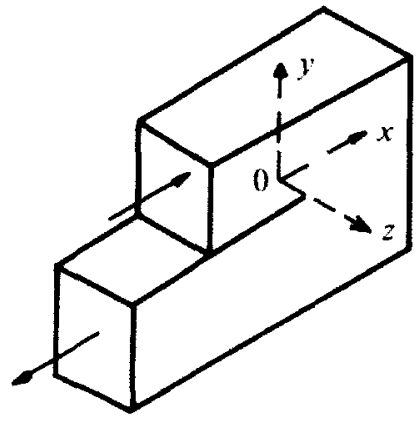

(b)

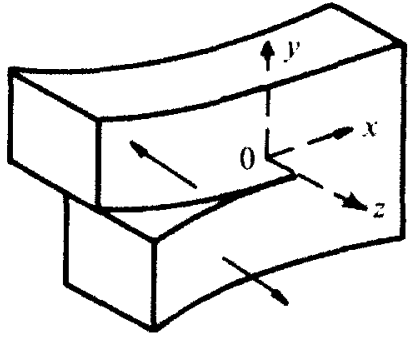

(c)

Figure 3.1 - The three independent modes for crack opening [135]. (a) Mode I or tensile opening mode, (b) Mode II or in-plane shearing mode and (c) Mode III or anti-plane shearing mode.

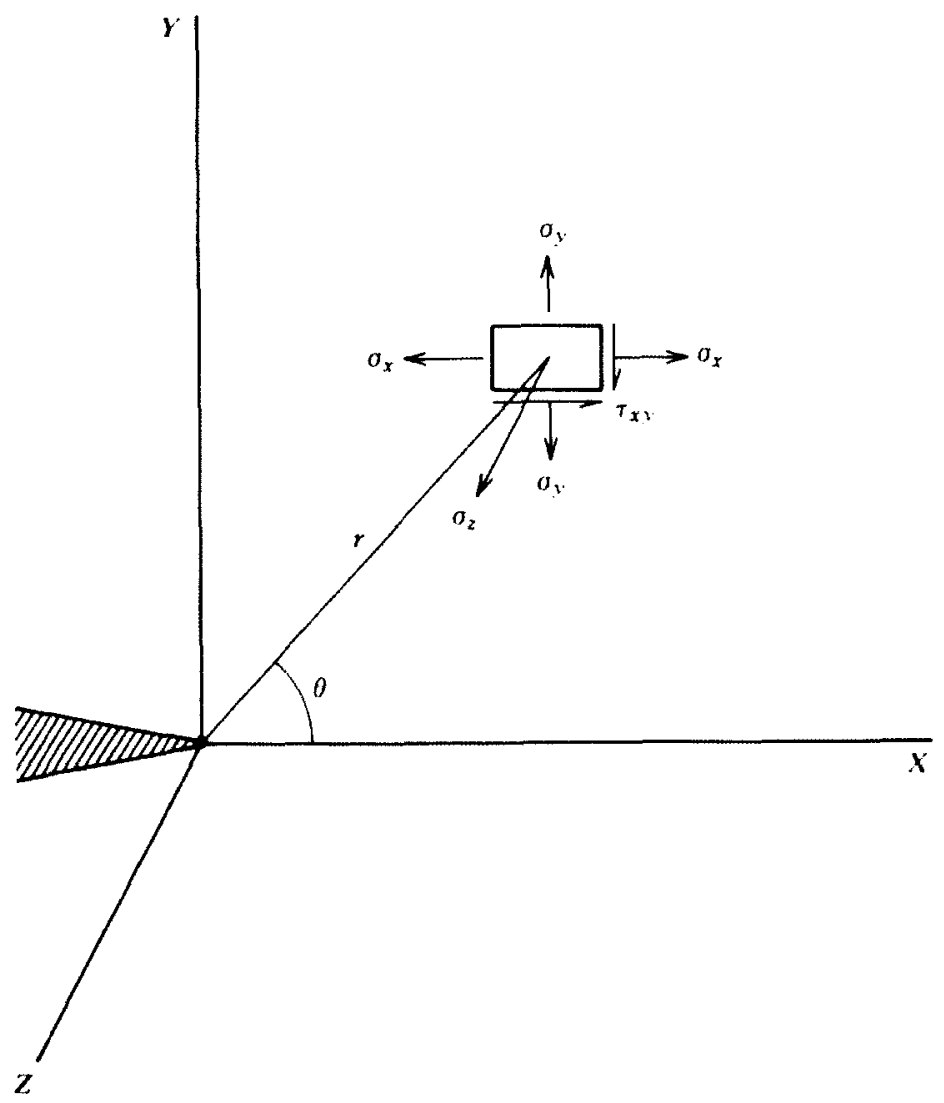

Figure 3.2 - Schematic showing polar coordinate system at the crack-tip [133]. 


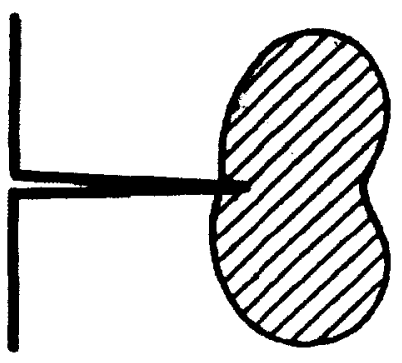

(a)

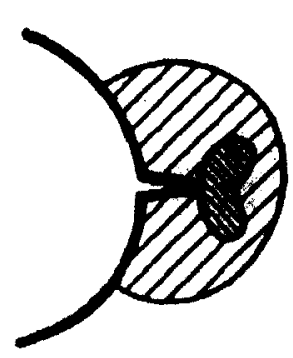

(b)

Figure 3.3 - Schematic showing mechanically (or physically) small fatigue cracks and associated plastic zone sizes in (a) smooth and (b) notched fatigue specimens [49].

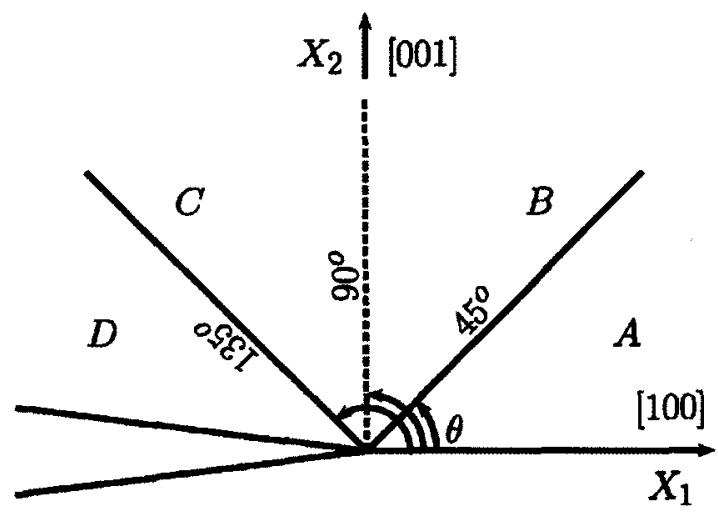

a)

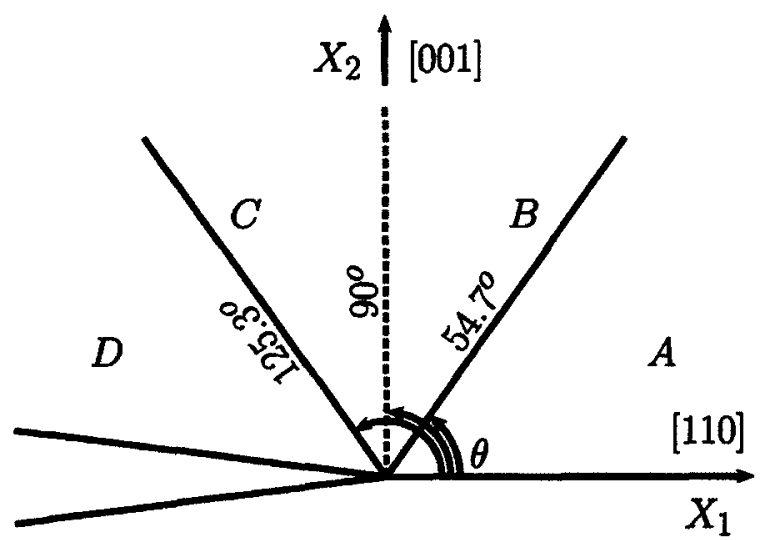

b)

Figure 3.4 - Crystallographic slip sector boundaries for 2-D cracks; a) [001] loading axis with [100] crack growth direction and b) [001] loading axis with [110] crack growth direction. The Cartesian components of the stress tensor is constant in sectors $A, B, C$ and $D$. The sector boundaries are denoted by rays with a given angle of theta where those associated with octahedral slip are denoted by solid rays and dashed rays for cubic slip [143]. 


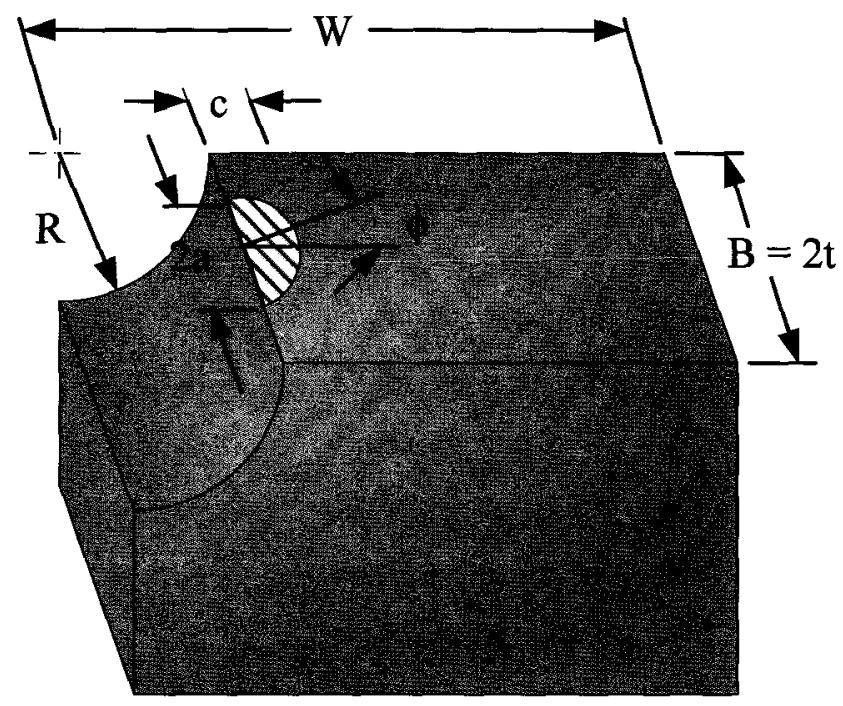

Figure 3.5 - Nomenclature for a semi-elliptical surface crack in a 3-D body.

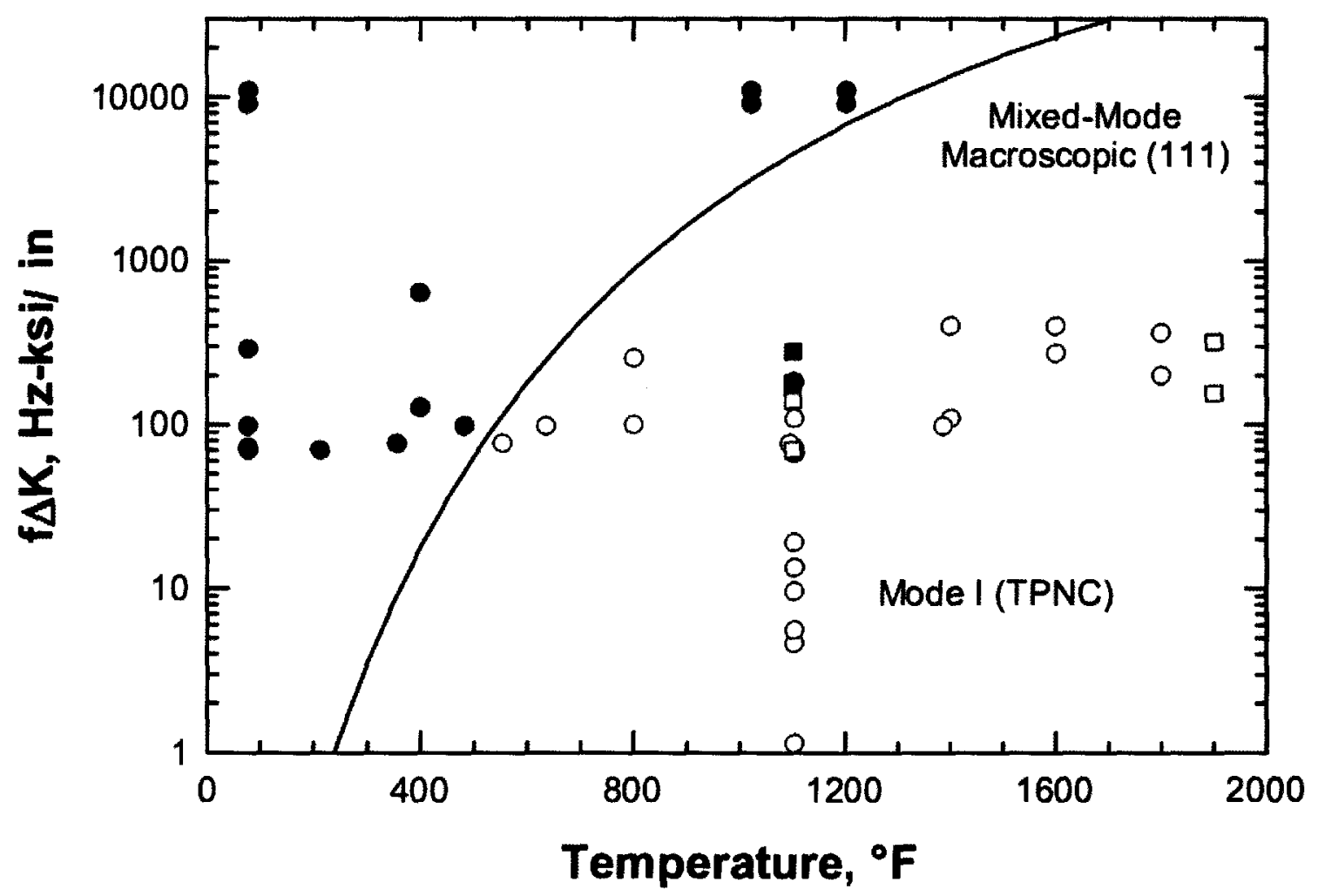

Figure 3.6 - Fatigue crack growth mechanisms map for PWA1484 single-crystals [157]. Note: line denotes transition boundary between mixed-mode octahedral (crystallographic) crack growth and Mode I transprecipitate non-crystallographic growth (TPNC). 


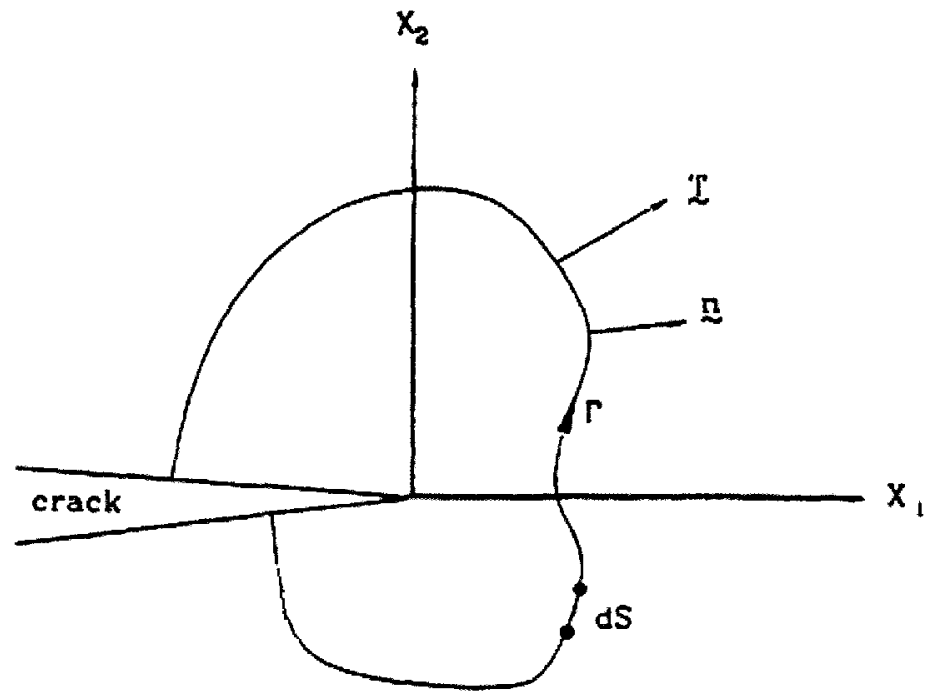

Figure 3.7 - Definition of the J-integral for a 2-D crack consisting of a closed contour $\Gamma$ originating from the lower crack surface which goes clockwise around the crack-tip to the upper crack surface[138].

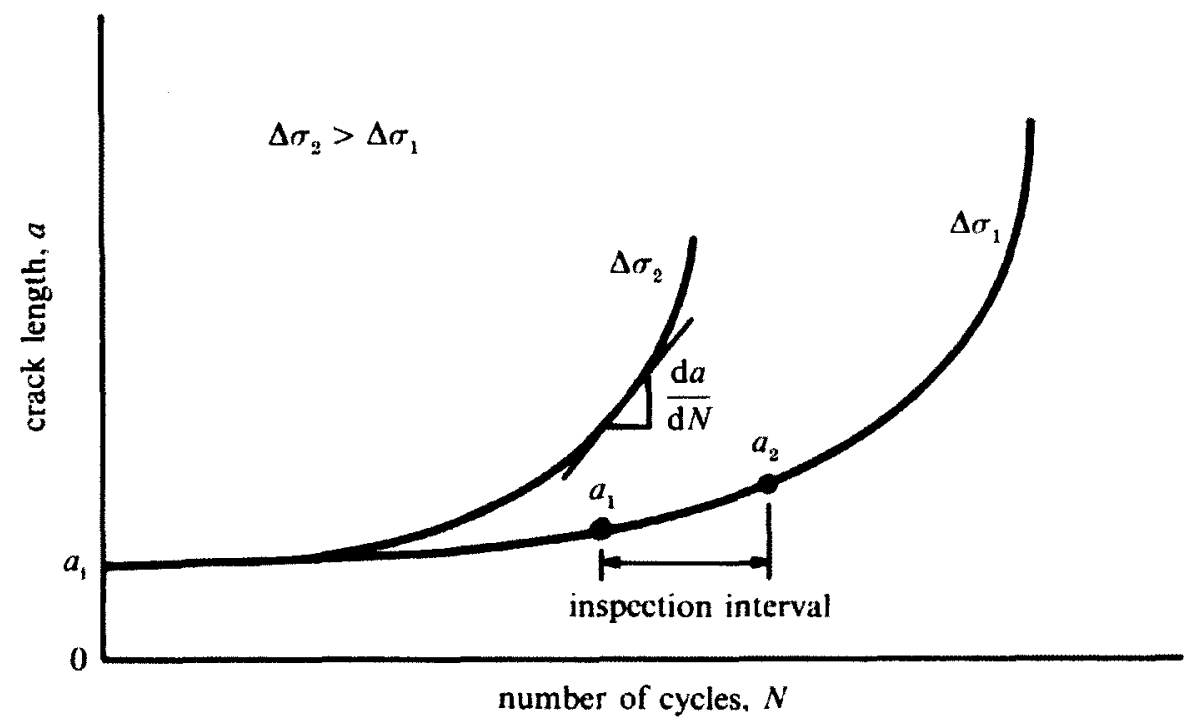

Figure 3.8 - Typical crack growth behaviour obtained from laboratory specimens tested under constant stress amplitude loading [135]. 


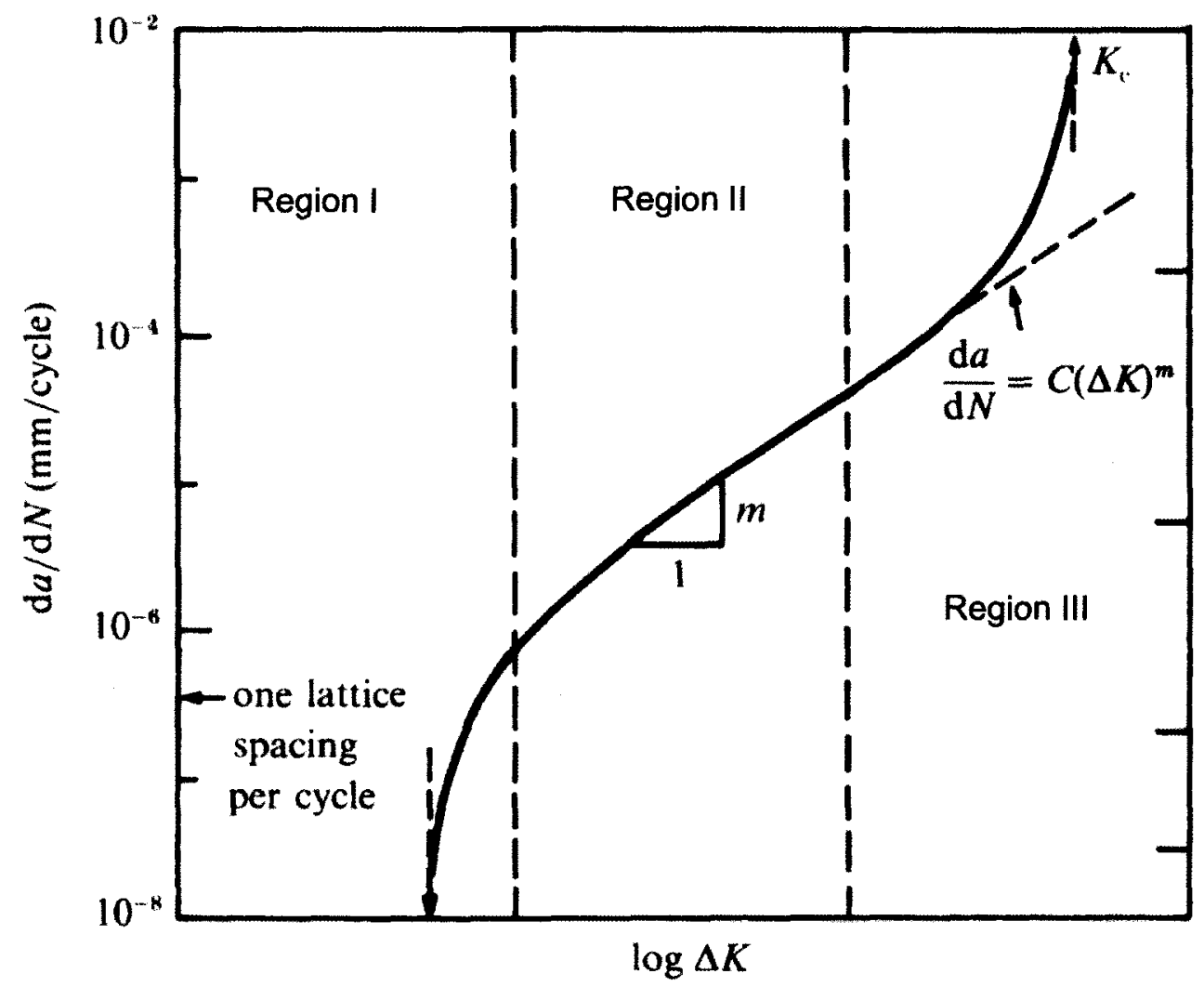

Figure 3.9 - Schematic illustrating the three different regions of stable crack propagation for long-fatigue cracks (adapted from [135]).

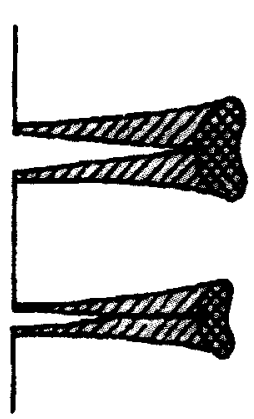

(a)

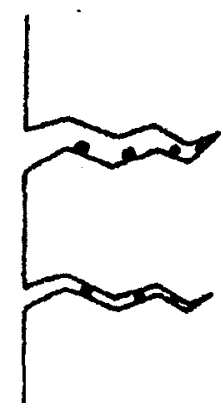

(b)

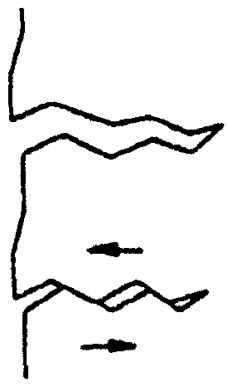

(c)

Figure 3.10 - Three mechanisms of fatigue crack closure: a) plasticity induced crack closure, b) oxide induced crack closure, and c) roughness induced crack closure. Adapted from Reference [185]. 


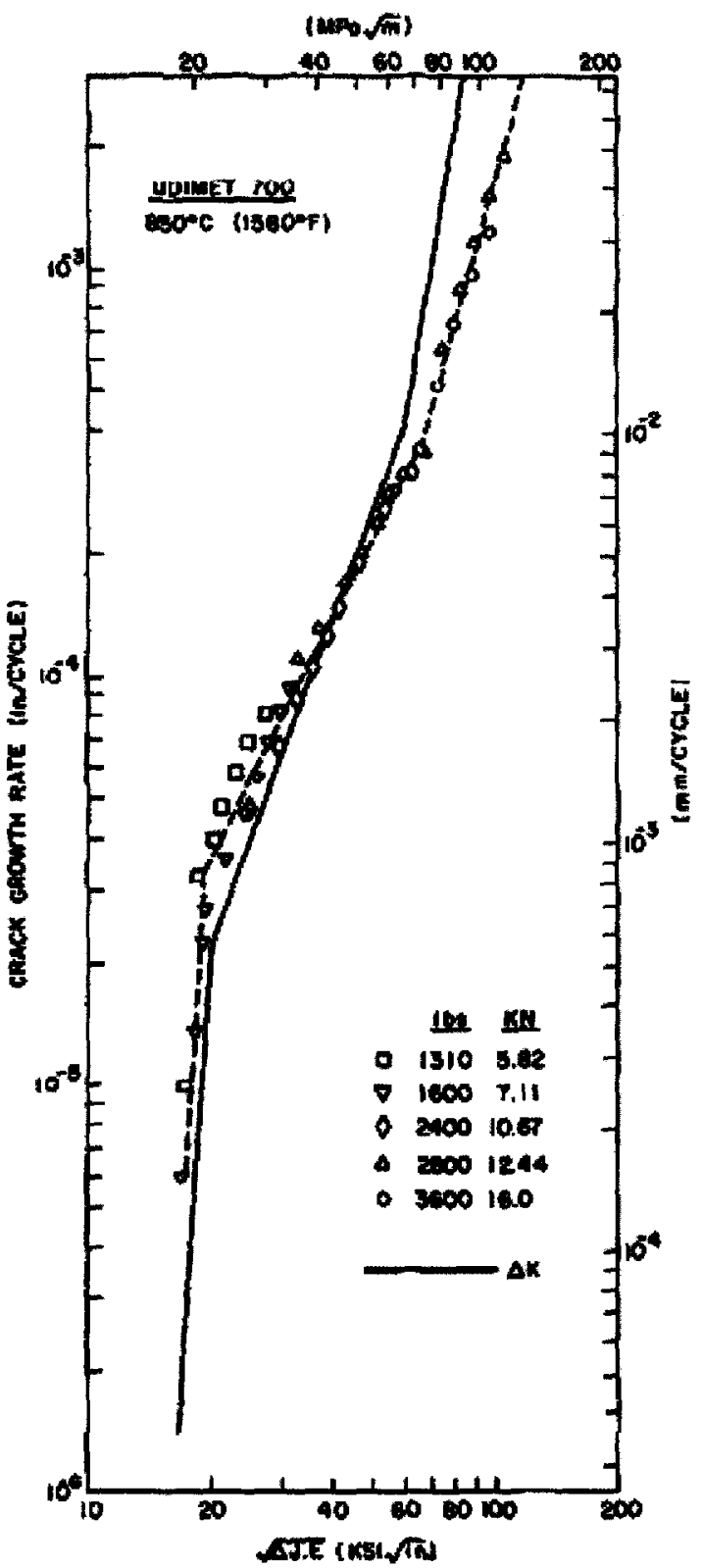

Figure 3.11 - Fatigue crack growth behaviour for Udimet 700 tested at $850 \mathrm{C}$ showing three region crack growth behaviour [192]. Note: solid line represents linear-elastic and dashed line represents elastic-plastic characterization of the fatigue crack growth behaviour. 


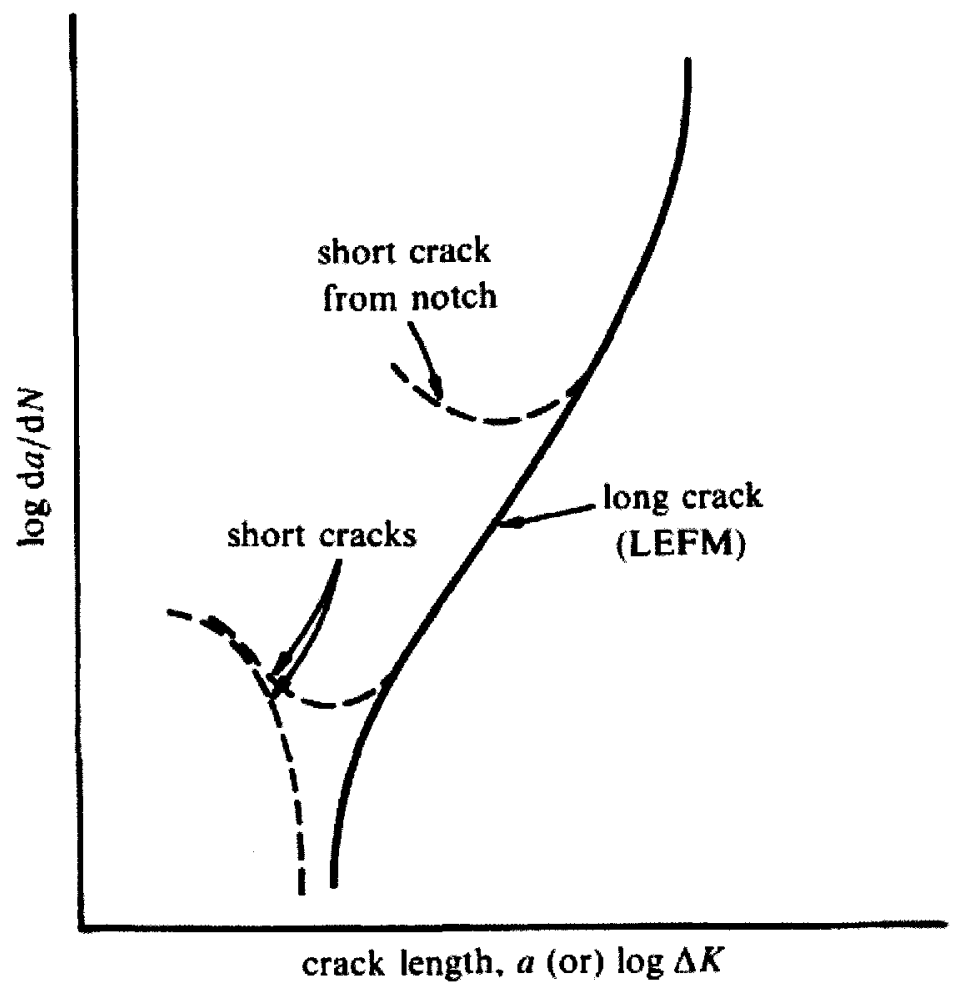

Figure 3.12 - Schematic showing typical characteristics of small (or short) and long fatigue cracks [135].

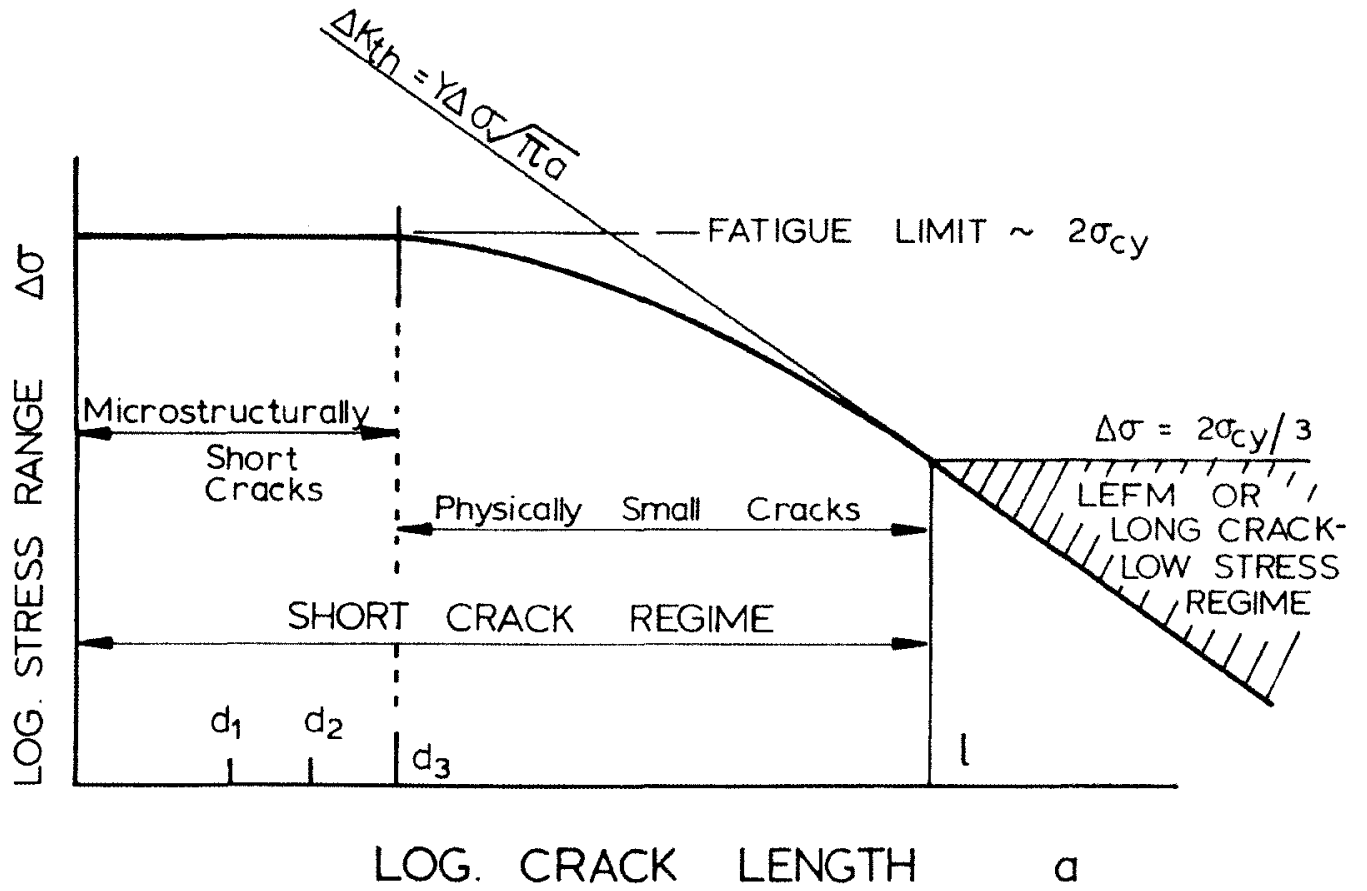

Figure 3.13 - Schematic of the Kitagawa-Takahashi curve showing the small (or short) and long crack growth regimes (after Miller and de los Rios [222]). 


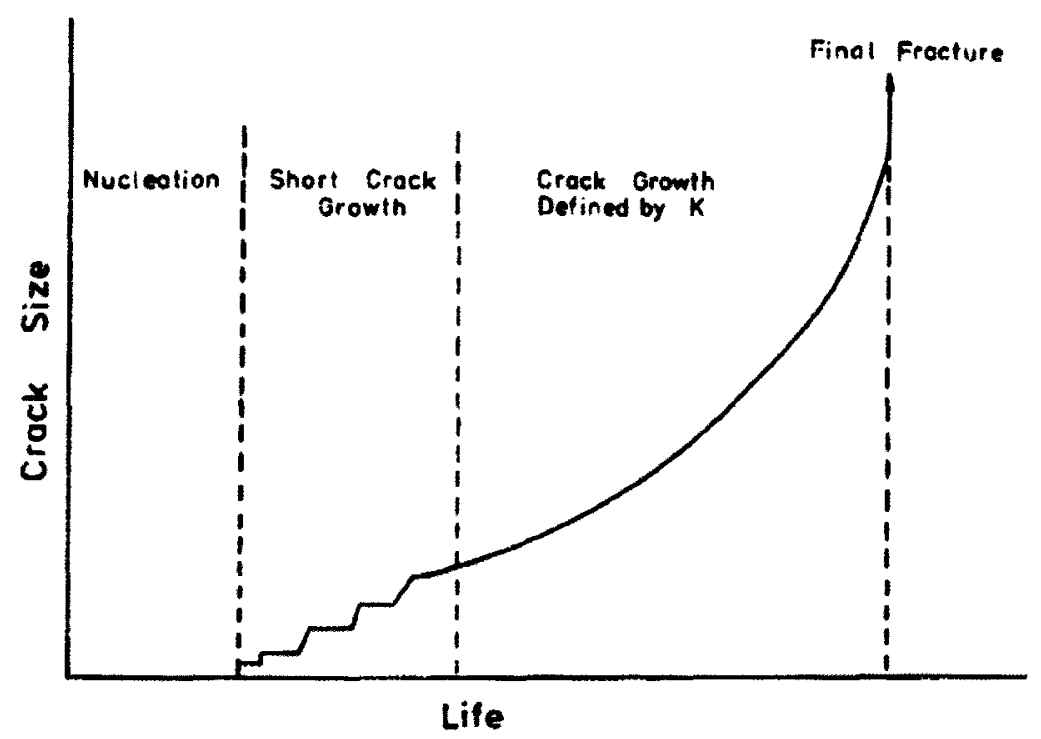

Figure 3.14 - Schematic of crack size versus life curve showing the four phase of fatigue crack formation and growth $[8]$.

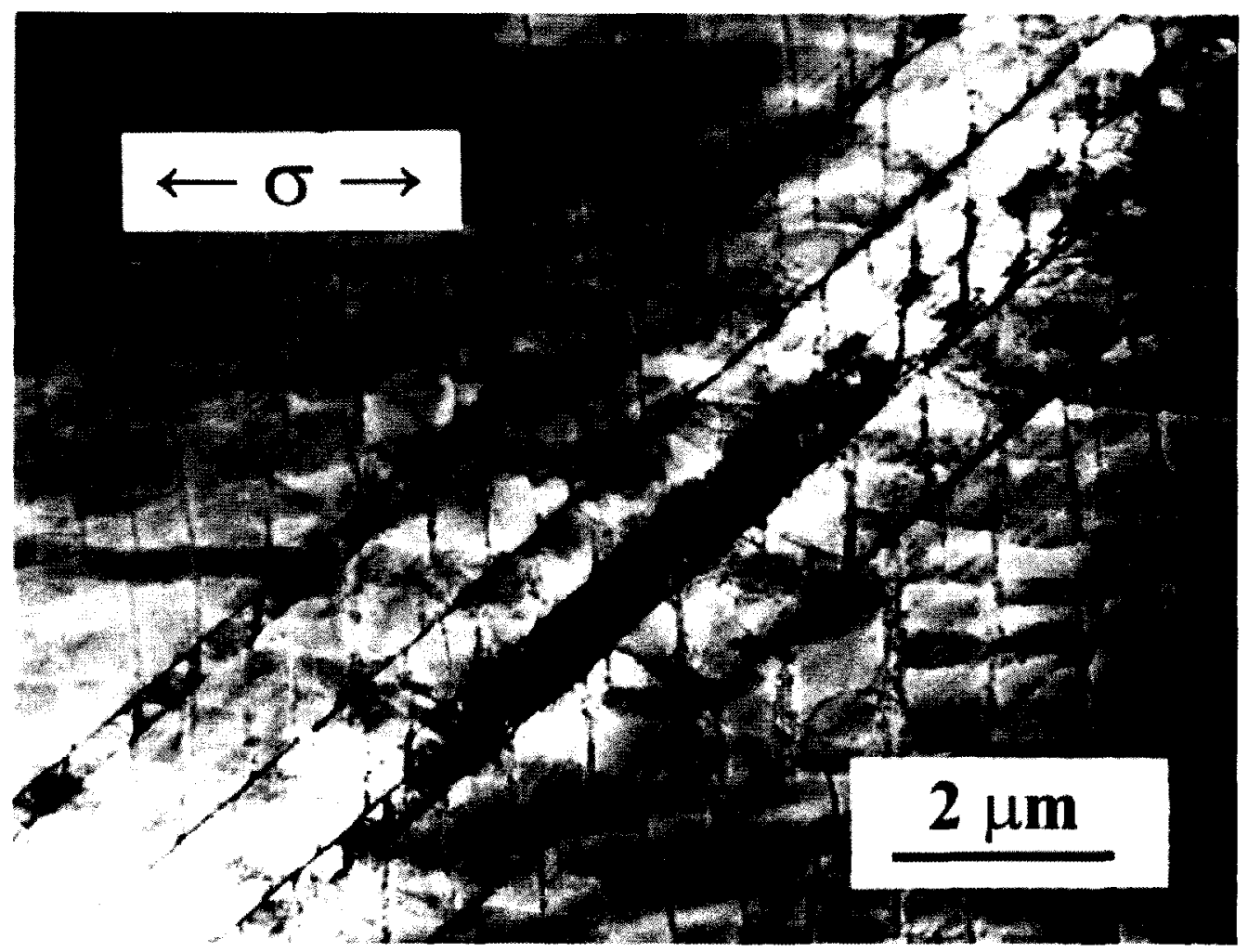

Figure 3.15 - TEM micrograph showing persistent slip bands in $<100>$-oriented singlecrystal superalloy tested at $700^{\circ} \mathrm{C}[206]$. 


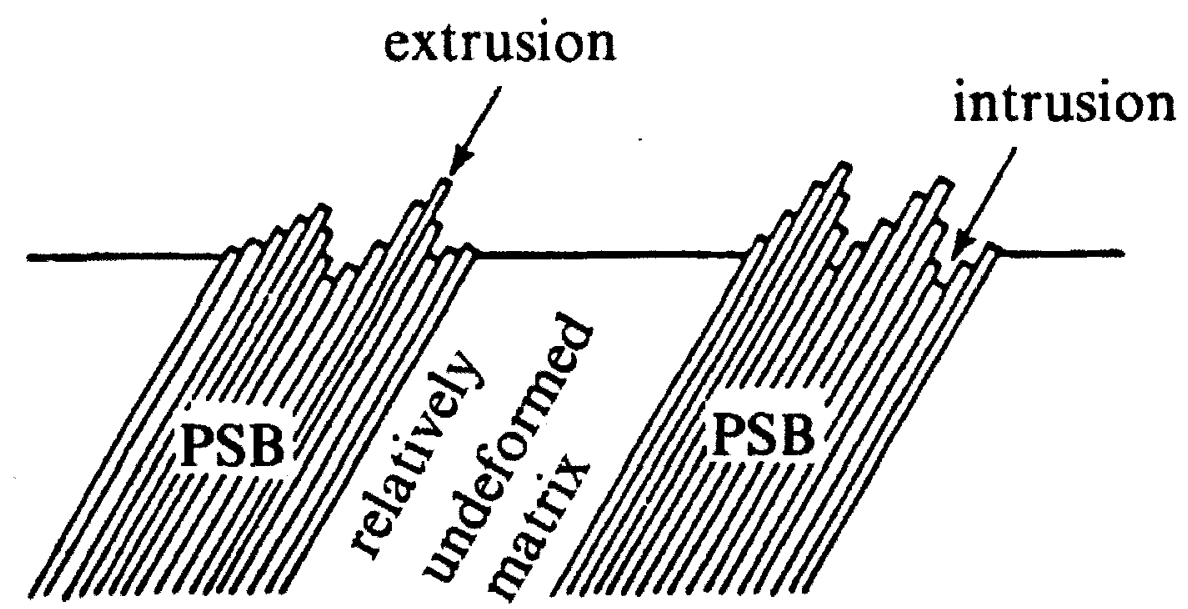

Figure 3.16 - Schematic showing rough extrusion-intrusion surface topography caused by persistent slip bands [135].

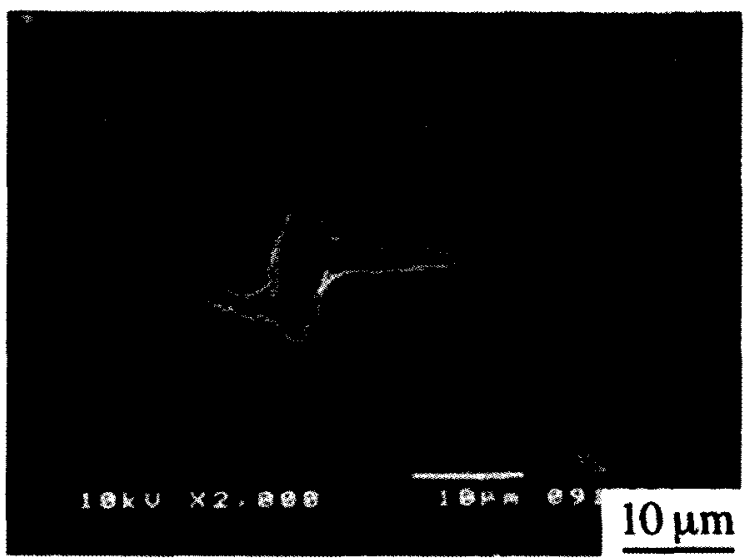

(a)

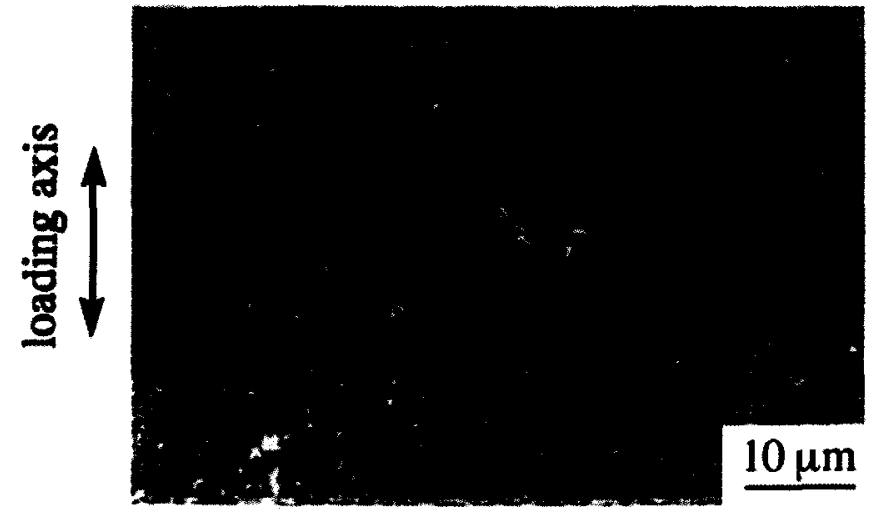

(b)

Figure 3.17 - Naturally nucleated surface fatigue crack sites in single-crystal superalloy CMSX-2, (a) surface connected shrinkage porosity and (b) surface connected eutectic $\gamma-\gamma$ ' nodule [45]. 


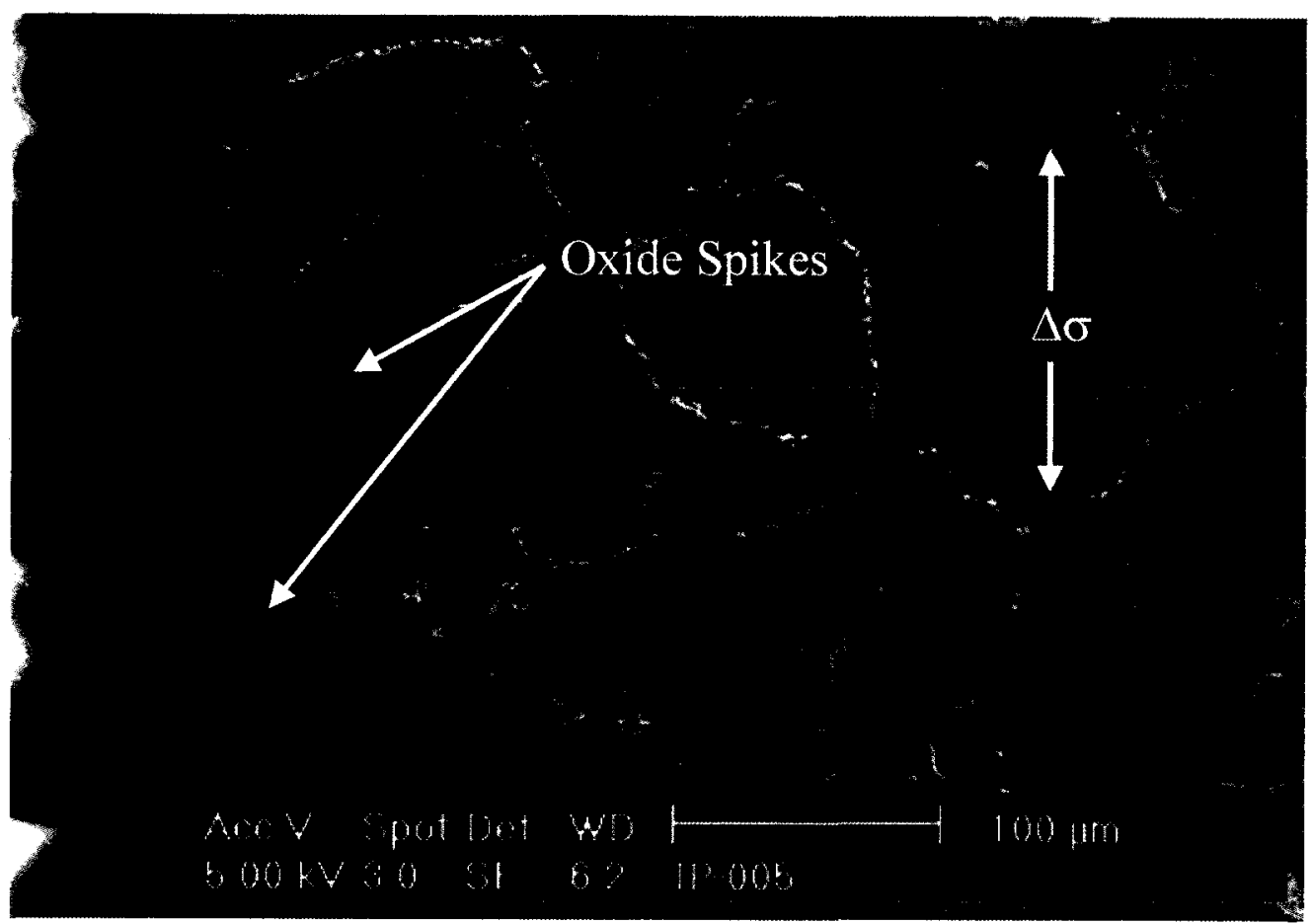

Figure 3.18 - Oxide spikes formed during high temperature fatigue in a nickel-base superalloy [223].

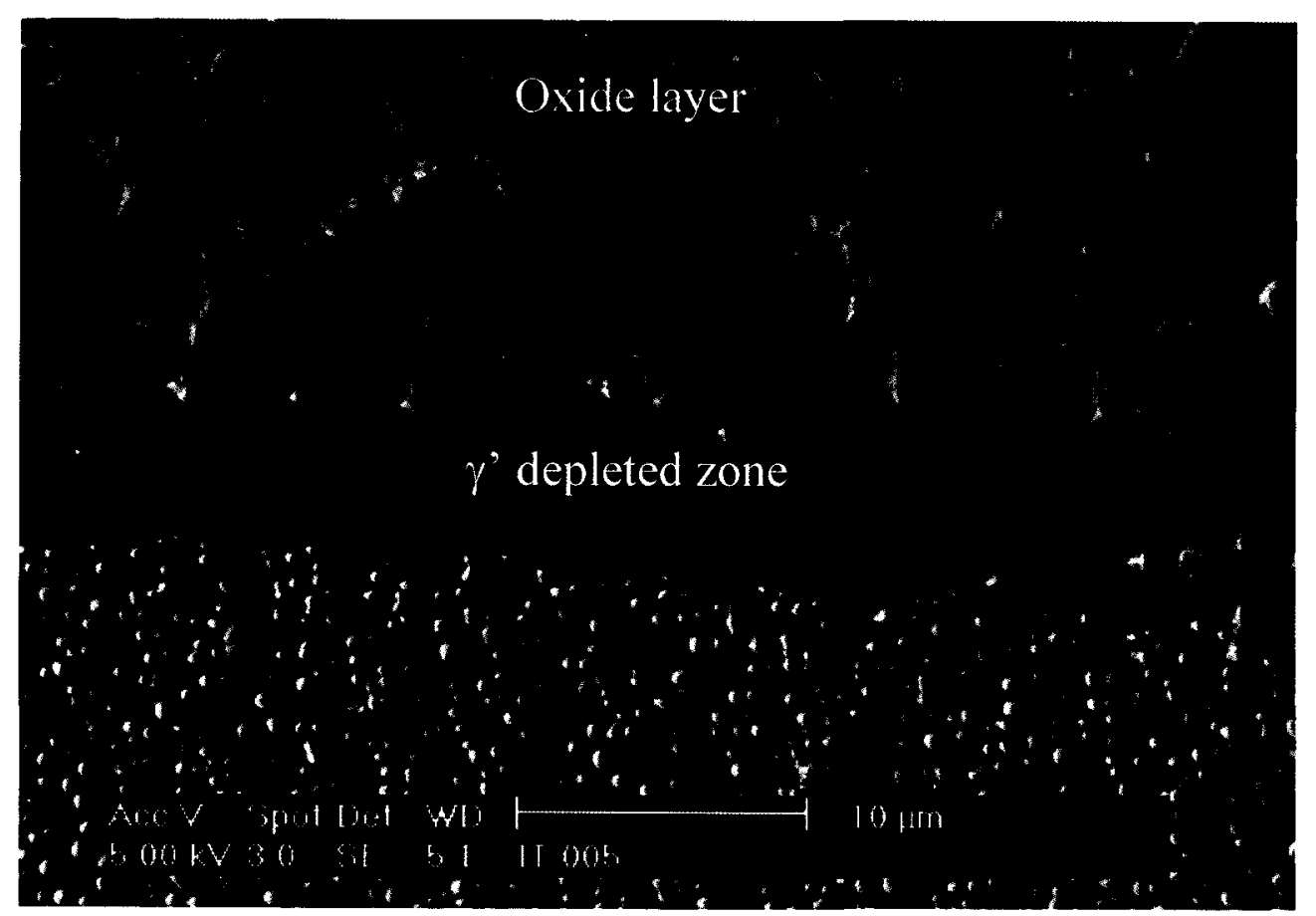

Figure $3.19-\gamma^{\prime}$ depleted zone formed during high temperature fatigue in a nickel-base superalloy [223]. 


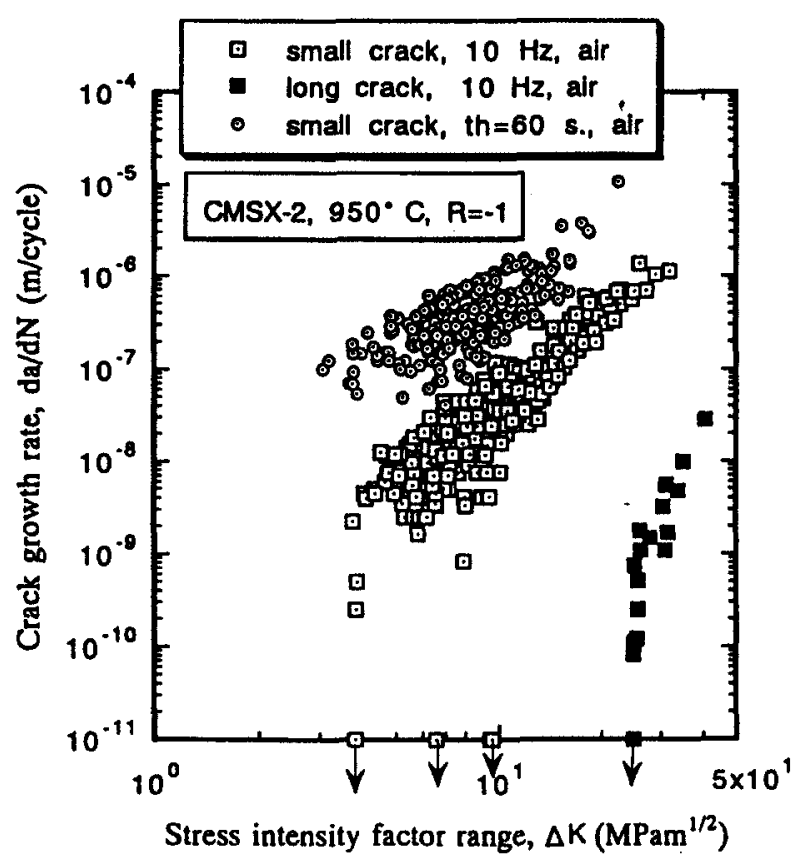

Figure 3.20 - Small and long fatigue crack growth rates correlated with $\Delta \mathrm{K}$ for singlecrystal CMSX-2 tested at $950^{\circ} \mathrm{C}$ under creep-fatigue and continuous cycling conditions [45].
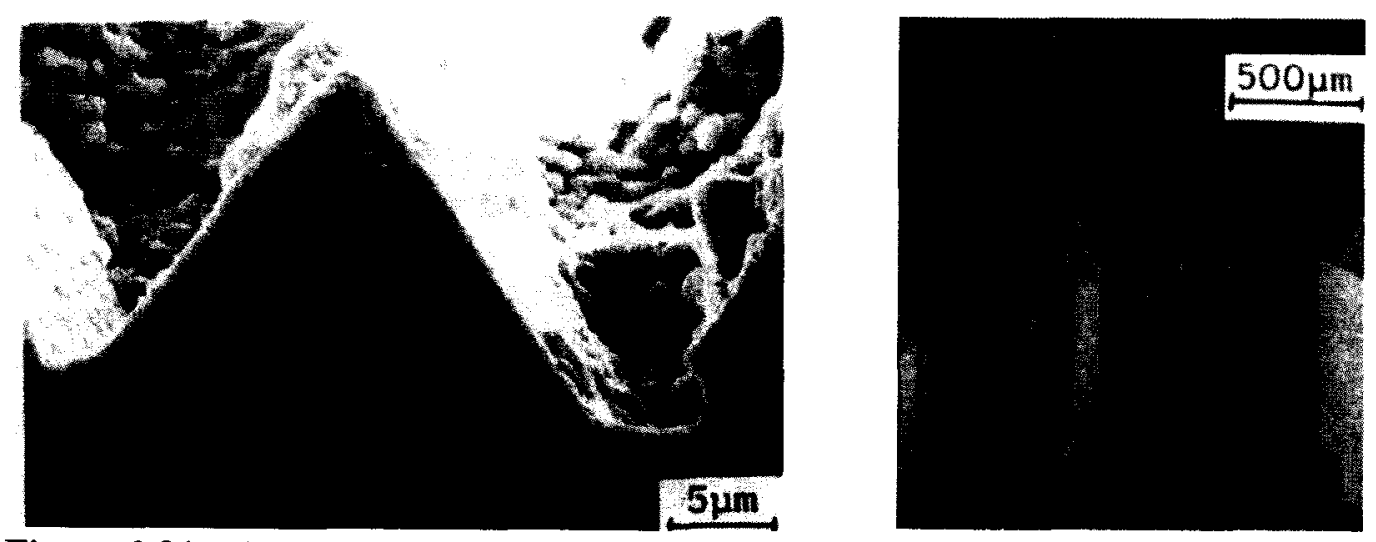

Figure 3.21 - Faceted crack growth on octahedral slip planes in single-crystal superalloy CMSX-2 tested at room temperature; a) profile view, and b) top view. Micrographs adapted from Reference [40]. 


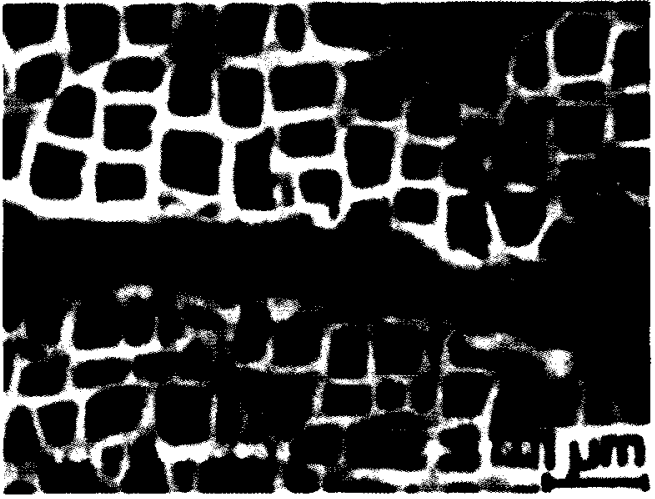

a)

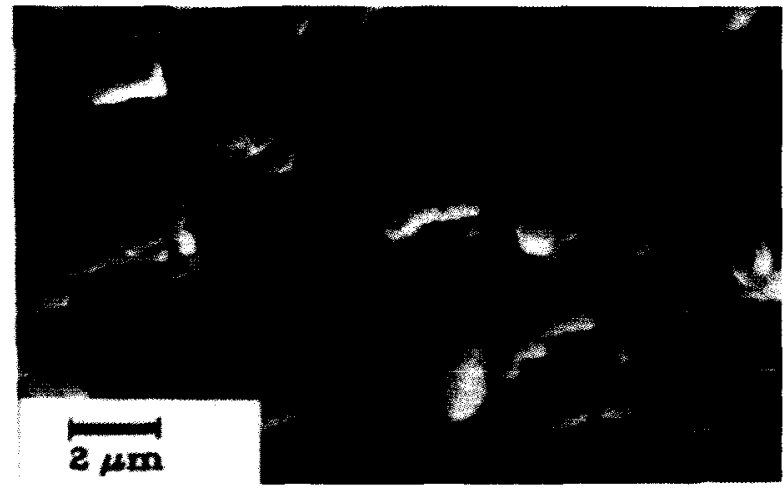

b)

Figure 3.22 - High temperature fatigue crack growth along $\gamma-\gamma$ ' interface in the singlecrystal superalloy CMSX-2; a) profile view showing crack propagating along the $\gamma$-matrix channels, and b) fracture surface showing a propagating fatigue crack that is avoiding the $\gamma$ ' precipitates. a) adapted from Reference [45], and b) adapted from Reference [13].

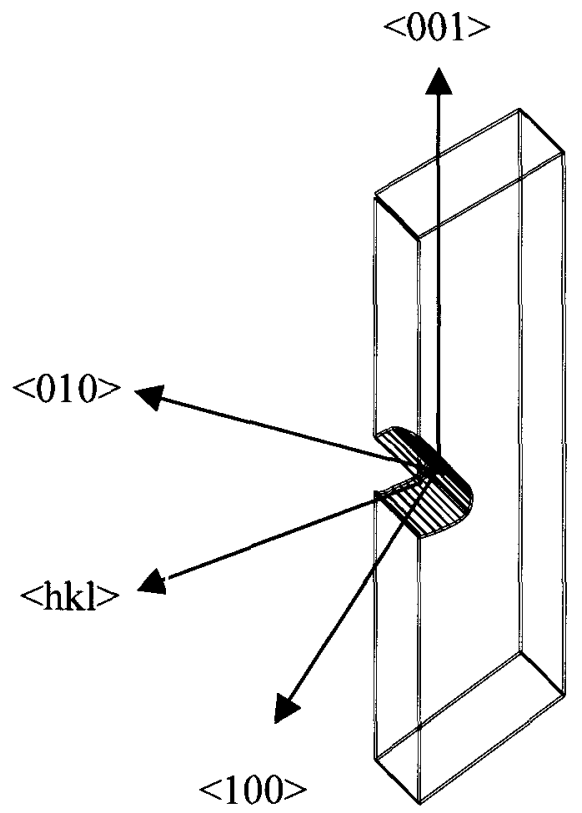

Figure 3.23 - Schematic defining the secondary orientation for a notched single-crystal fatigue specimen. Note: the direction denoted by the indices $<$ hkl $>$ represents the crystallographic direction perpendicular to the notch face. 


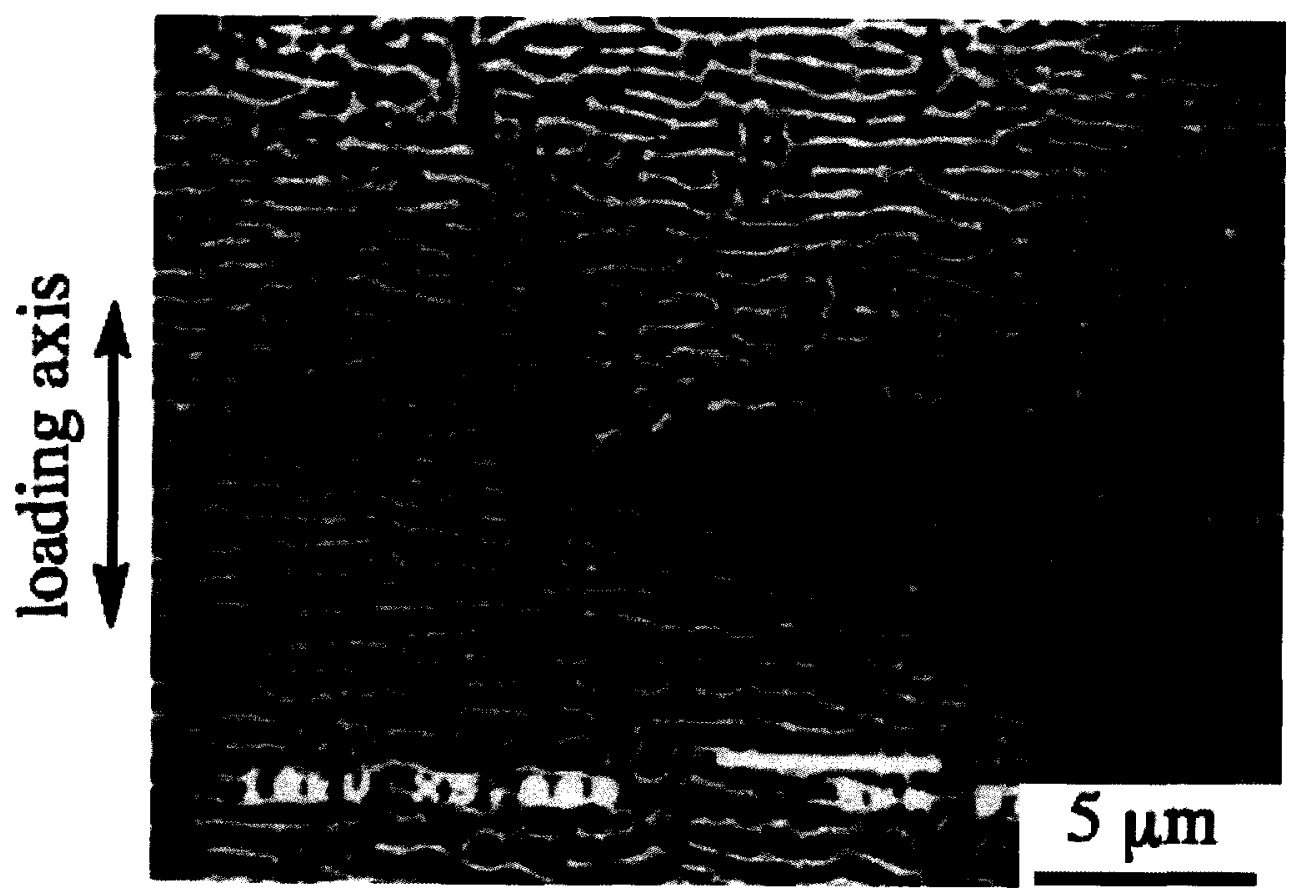

Figure 3.24 - Scanning electron micrograph showing an oxidized fatigue crack in singlecrystal superalloy CMSX-2 [45].

a)

)

b)

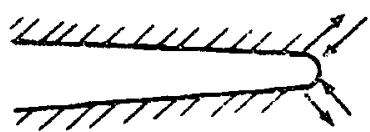

c)

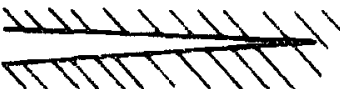

d)

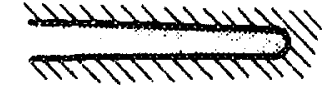

e)

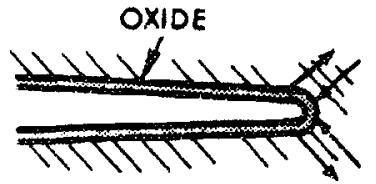

f)

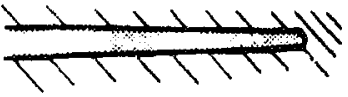

Figure 3.25 - Schematic of Stage II crack growth in vacuum [a, b, c] and in air [e, f, g] [69]. 

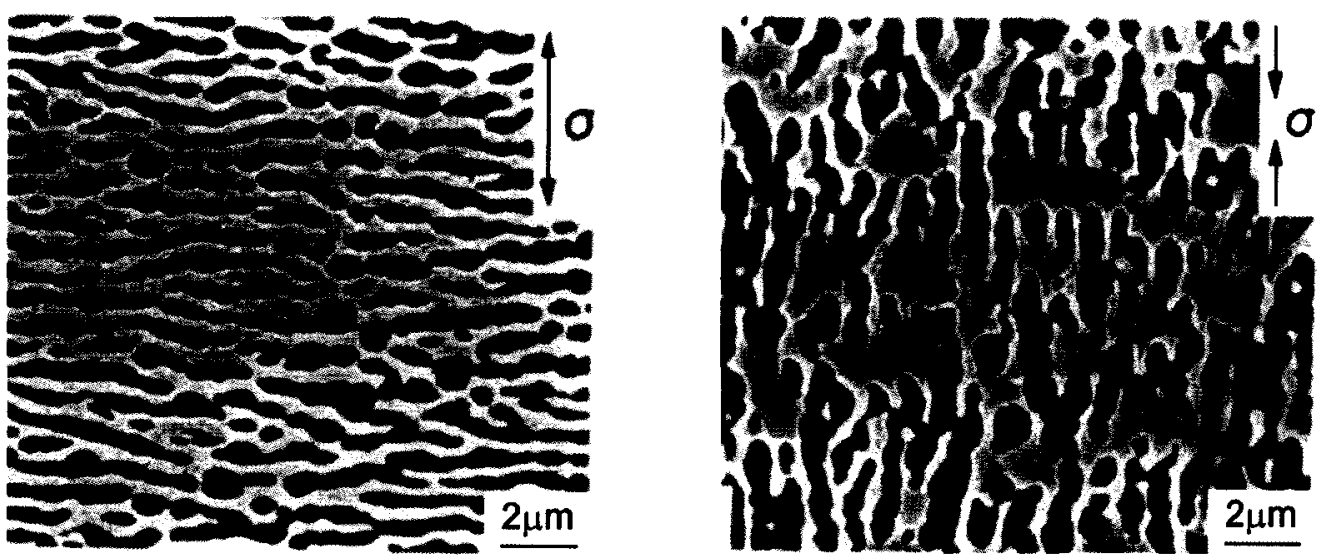

Figure 3.26 - Directionally coarsened $\gamma^{\prime}$ precipitates after IP and OP TMF testing: a) IP ntype (normal to applied stress) rafting, and b) OP p-type rafting (parallel to applied stress). Note the $\gamma^{\prime}$ phase appears dark in the micrographs. Adapted from Reference [221]. 


\section{Literature Review: Single-Crystal Constitutive Models}

\subsection{Introduction}

Nickel-base single-crystal superalloys have attracted considerable interest for use in gas turbine blade and vane applications because of their exceptional high temperature mechanical properties. However, translating the improved structural margins these alloys offer into functional components requires design and analysis tools that account for not only elastic deformation, but the possibility that appreciable inelastic strains can occur. Constitutive equations specify the functional relationships between the stresses and strains, with consideration of elastic and inelastic deformations, and therefore are indispensible for structural analysis calculations. As discussed in previous Chapters, single-crystal superalloys exhibit complex elastic-plastic anisotropy, which must be taken into consideration when formulating constitutive equations to describe the elastic and inelastic material response. The constitutive material models that have been developed for single-crystal superalloys are generally formulated based one of the following approaches:

1. A macroscopic phenomenological approach that considers deformation that occurs at macroscopic (continuum) length scales [224, 225].

2. A micro-phenomenological approach in which the constitutive equations are introduced at the crystallographic slip-system length scale [114, 226-229].

The macroscopic phenomenological models are usually developed from classical continuum plasticity theories originally proposed for isotropic materials. Where modern constitutive theories deviate from classical plasticity is that all inelastic strain is assumed 
to be governed by a single flow equation, i.e. inelastic strains from creep and plasticity are treated by a unified framework. These models generally consist of a yield criterion, various state variables with evolution equations used to model the effects of monotonic and cyclic strain hardening behaviours, and a flow rule that describes the evolution of the inelastic strain in terms of the state variables and stress. Various modifications are made to the constitutive equations to account for single-crystal anisotropy. Rate dependent (viscoplastic) material response is accounted for in the formulation of the flow rule. Nouailhas and Chaboche [225] have proposed a macroscopic phenomenological model based on this framework to model the cyclic viscoplastic response of single-crystal superalloys. The details of this model will be discussed further in Section 4.2.

Micro-phenomenological models were derived from classical rate-independent crystalline slip theory in which the shear stresses and strains are calculated on slip planes and slip directions that are considered active, i.e. the set of slip systems on which the resolved shear stress exceeds the critical resolved shear stress to cause plastic flow. The constitutive equations that describe the inelastic shear strains and hardening behaviour of the individual slip systems are generally formulated from rate-independent or viscoplastic constitutive equations used for modeling inelastic deformation at the macroscale. This is why these constitutive theories are described as being micro-phenomenological. One example that will be discussed further in this review is the single-crystal microphenomenological model proposed by Han and coworkers [226].

In the following sections specific forms of macroscopic phenomenological and the microphenomenological constitutive models will be reviewed. In particular, the Nouailhas and Chaboche macroscopic phenomenological and the micro-phenomenological model developed by Han and co-workers will be reviewed. 


\subsection{Nouailhas and Chaboche Phenomenological Constitutive Model for Single-Crystal Superalloys [225]}

The Nouailhas and Chaboche phenomenological constitutive model was developed to reduce the complexity associated with crystal plasticity theories and more importantly to reduce the number of material parameters to be identified from mechanical test data. It is based on a generalization of a unified constitutive model proposed by Chaboche [230232] for isotropic materials. Cubic single-crystal anisotropy is taken into account by introducing additional material parameters that describe the elastic-plastic anisotropy. Below is a summary of the functional form of this model, expressed in the principal material axes, for the isothermal case.

The total strain rate, $\dot{\boldsymbol{\varepsilon}}$, is based on the additive decomposition of strains into elastic, $\dot{\boldsymbol{\varepsilon}}^{e l}$ and inelastic $\dot{\varepsilon}^{\text {in }}$ strain rate components:

$$
\dot{\varepsilon}=\dot{\varepsilon}^{e l}+\dot{\varepsilon}^{i n}
$$

The elastic strain is related to the stress by the generalized form of Hooke's law:

$$
\sigma=C: \varepsilon^{e l}
$$

where $C$ represents fourth-order anisotropic elastic stiffness tensor and the second-order tensor $\sigma$ is the stress.

The yield criterion and function is based on a generalization of the von Mises yield criterion by introducing a fourth-order tensor $\boldsymbol{M}$ to describe the initial yield anisotropy and plastic strain induced anisotropy: 


$$
f=\sqrt{\frac{3}{2}(s-\chi): M:(s-\chi)}-k \leq 0
$$

where $f$ represents the yield surface, $k$ is the initial size of the yield surface, $s$ and $\chi$ are the deviatoric components of the stress and back stress tensors, respectively. By definition the back stress is deviatoric; the deviatoric stress is calculated using the following expression (in indicial notation):

$$
s_{i j}=\sigma_{i j}-\frac{1}{3} \delta_{i j} \sigma_{k k}
$$

where $\delta_{i j}$ is the Kronecker delta. The main purpose of the back stress state variable is to model the Bauschinger effect observed under cyclic loading, i.e. the apparent reduction in yield stress upon load reversal after some plastic deformation has occurred during a reversed and symmetrical loading cycle. Physically, the back stress represents the internal stress that develops when dislocations pile up against obstacles, such as grain boundaries, or precipitates. These internal stresses impose additional resistance to further dislocation motion, the effects of which are observable in the macroscopic scale in uniaxial tensile (or compressive) curves as post-yield hardening or the Bauschinger effect under reversed cyclic loading. Geometrically, the back stress tensor shifts the yield surface in principal stress space, as shown schematically in Figure 4.1. In this model, kinematic hardening is assumed have a short range effect on the constitutive material behaviour, such that it saturates quickly resulting in no additional shift in the yield surface. In this model two non-linear kinematic hardening state variables were specified, one to model the large non-linearity associated with initial plastic flow and the second to correctly model the 
kinematic hardening response at higher inelastic strains. The evolution law for each of the kinematic hardening variables is specified by:

$$
\dot{\chi}^{q}=\frac{2}{3} N^{q}: \dot{\varepsilon}^{i n}-Q^{q}: \chi^{q} \dot{p}
$$

where the two additional fourth-order tensors, $\boldsymbol{N}^{q}$ and $\boldsymbol{Q}^{q}$, were introduced to describe the anisotropic kinematic hardening response of the material. The first term on the righthand-side of equation 4.5 predicts linear strain hardening and the second term represents dynamic recovery. The non-linearity is controlled by the values specified for $\boldsymbol{N}^{q}$ and $\boldsymbol{Q}^{q}$. Higher values for $N^{q}$ result in more non-linear strain hardening behaviour and the value specified for $\boldsymbol{Q}^{q}$ controls the rate of saturation of the back stress.

The total back stress is obtained by summing the contribution from each kinematic hardening law:

$$
\chi=\sum_{q} \chi^{q}
$$

To account for rate dependence of the materials constitutive response a viscoplastic potential based on the same formulation as proposed by Chaboche [230-232] was used:

$$
\Omega=\frac{K}{n+1}\left\langle\frac{f}{K}\right\rangle^{n+1}
$$


where $n$ is the viscosity exponent and $K$ represents a scalar viscous drag stress. The drag stress physically represents the initial resistance of the crystal lattice to dislocation motion. It is possible to specify an evolution equation for the drag stress (see Reference [231]), but it is assumed to be constant in this model. The viscosity exponent controls the rate dependence of the material's constitutive behaviour, with higher values translating into reduced rate dependence. Both $n$ and $K$ are strongly temperature dependent which is confirmed by the temperature-dependent mechanical properties of single-crystal superalloys discussed in Chapter 2. The Macaulay brackets denote the following:

$$
\langle u\rangle= \begin{cases}u & u>0 \\ 0 & u \leq 0\end{cases}
$$

The viscoplastic strain rate is obtained from the normality hypothesis:

$$
\dot{\varepsilon}^{i n}=\frac{\partial \Omega}{\partial \sigma}=\dot{p} \frac{\partial f}{\partial \sigma}=\frac{3}{2}\left\langle\frac{f}{K}\right\rangle^{n} \frac{M:(s-\chi)}{\sqrt{\frac{3}{2}(s-\chi): M:(s-\chi)}}
$$

with

$$
\dot{p}=\sqrt{\frac{3}{2} \dot{\varepsilon}^{i n}: M^{-1}: \dot{\varepsilon}^{i n}}=\left\langle\frac{f}{K}\right\rangle^{n}
$$

and $\dot{p}$ denoting the accumulated inelastic strain rate, or viscoplastic multiplier. The second term in equation 4.9 that is obtained by the partial differentiation of the current yield surface with respect to the stress tensor, i.e.: 


$$
n=\frac{M:(s-\chi)}{\sqrt{\frac{3}{2}(s-\chi): M:(s-\chi)}}
$$

specifies the direction of plastic flow, $\boldsymbol{n}$.

For cubic single-crystal materials Nouailhas and Chaboche [225] have shown that when the fourth-order anisotropy tensors $\boldsymbol{M}, \boldsymbol{Q}^{q}$, and $\boldsymbol{N}^{q}$ are converted to concise tensor notation and crystal symmetry and inelastic strain incompressibility are taken into consideration, these anisotropy tensors have only two independent material constants. In Voigt notation these tensors have the same form $[225,226]$ :

$$
]_{i j}=\left[\begin{array}{cccccc}
]_{11}-[]_{12} & 0 & 0 & 0 & 0 & 0 \\
& {[]_{11}-U_{12}} & 0 & 0 & 0 & 0 \\
& & \left.[]_{11}-\right]_{12} & 0 & 0 & 0 \\
& & & ]_{44} & 0 & 0 \\
& \text { symm. } & & & {[]_{44}} & 0 \\
& & & & & {[]_{44}}
\end{array}\right]
$$

It is usually assumed that the couple []$_{11}-[]_{12}$ has a unitary value so that isotropic deformation response is obtained when the mechanical loading takes place along the principal material directions, i.e. one of the $\langle 100\rangle$ crystallographic directions. One interesting feature of this model is the decoupling of single-crystal anisotropy as indicated by the two independent anisotropy material parameters, one for the direct stress and on for the shear stress terms. Because of this decoupling, the anisotropic material parameters can easily be identified from uniaxial test data obtained from specimens with 
$\langle 100\rangle$ and $\langle 111\rangle$ crystallographic orientations aligned with the mechanical loading direction [225].

\subsubsection{Critique}

This constitutive model introduces material anisotropy through material parameters that are specified as fourth-order tensors. As established by Nouailhas and Chaboche for cubic single-crystal materials, these fourth-order anisotropy tensors each contain two independent material parameters. Because these anisotropy parameters are defined from constitutive material tests for the $\langle 100\rangle$ and $\langle 111\rangle$ crystallographic orientations predicted constitutive material response near these crystallographic orientations should be excellent. On the other hand, the constitutive material response for other crystallographic orientations is defined in essence by a "smearing" of the parameters identified for $\langle 100\rangle$ and $\langle 111\rangle$ crystallographic orientations and consequently a close match to the actual constitutive material response is not expected. This is indeed the case.

Swanson and coworkers [233] have demonstrated the Hill's quadratic anisotropic yield criterion, which is similar to the yield criterion employed by this model, predicts a monotonic variation in yield stress with crystallographic orientation, as shown in Figure 4.2. Although the example they presented was hypothetical, i.e. the $\langle 111\rangle$ yield strength was assumed to be double that of $\langle 100\rangle$, this monotonic variation in yield stress does not match the yield characteristics of single-crystal superalloys. More sophisticated yield functions have been proposed by Nouailhas et al. [234] that predict yielding characteristics that more closely match observed material behaviour. The last and perhaps most serious limitation of the yield criterion employed in this model is that it does not

differentiate between tensile and compressive stresses, and therefore does not properly 
account for the tension-compression yield anisotropy observed in single-crystal superalloys. As discussed in Chapter 2 tension-compression yield anisotropy is significant (at low temperatures) for crystallographic orientations that deform by octahedral slip.

In Chapter 2 it was discussed that single-crystal superalloys exhibit little strain rate dependence at low temperatures under uniaxial monotonic and cyclic loading. To replicate this nearly strain rate independent behaviour requires a large viscosity exponent to be specified in the flow equation. The specification of a large viscosity exponent results in a numerically "stiff" set of equations for predicting the constitutive material response. In addition, because this constitutive model utilizes a single flow rule it is implicitly assumed that the strain rate dependence is the same for all crystallographic orientations. This is not physically correct because different crystallographic orientations can deform by different deformation mechanisms, i.e. octahedral or cubic slip or combinations of both slip mechanisms. Furthermore, it can be inferred that strain rate dependence would be more prominent in crystallographic orientations that promote multiple slip because of the possibility of dislocation interactions on different slip systems. The use of a single flow rule cannot account for these potential differences in flow mechanisms.

In the formulation presented by Nouailhas and Chaboche the effects of cyclic softening and hardening were not taken into consideration. However, it is straightforward to account for cyclic hardening effects by specifying an evolution equation for the yield surface size $k$. In spite of the limitations of this constitutive model, it has been demonstrated that the relatively good predictions of the cyclic constitutive material response can be obtained [225]. Among the advantages of this model is its commonality 
with constitutive equations implemented in FEM and its relatively small number or material parameters to identify. This results in a model that is simple to implement numerically and straightforward to use in the analysis of single-crystal components. In the next section a more sophisticated constitutive model that considers the crystallographic slip processes that govern the deformation behaviour of single-crystal superalloys is presented.

\subsection{Han et al. Micro-phenomenological Constitutive Model for Single- Crystal Superalloys [226]}

The single-crystal micro-phenomenological constitutive model proposed by Han and coworkers was developed from classical rate-independent crystal plasticity theories. Similar to other models proposed based on this crystal plasticity framework, the constitutive equations are specified at the macroscopic and microscopic length scales. Below is a summary of the functional form of this model, expressed in the principal material axes, for the isothermal case.

At the macroscopic level, the total strain rate is decomposed into elastic and inelastic strain rate components:

$$
\dot{\varepsilon}=\dot{\varepsilon}^{e l}+\dot{\varepsilon}^{i n}
$$

Similar to the Nouailhas and Chaboche [225] phenomenological model the, the elastic strain at the macroscopic scale is defined by the generalized Hooke's law:

$$
\sigma=C: \varepsilon^{e l}
$$


Based on crystalline slip theory the inelastic strain rate at the macroscale is obtained by summing the inelastic shear strain rates on the active slip systems, i.e. those slip systems in which the resolved shear stress exceeds the critical resolved shear stress to initiate plastic flow. For nickel-base single-crystal superalloys, 18 slip systems are generally considered in the crystallographic model. The slip systems that are considered include the 12 octahedral $[110]\{111\}$ and 6 cubic $[110]\{100\}$ slip systems, as summarized in Tables 4.1 and 4.2, respectively. The inelastic shear strain rates on each of the active slip systems is obtained by first calculating the resolved shear stress, $\tau^{s}$ onto each of the crystallographic slip planes using the generalized form of Schmid's law:

$$
\tau^{s}=m^{s}: \sigma
$$

where the second-order tensor, $\boldsymbol{m}^{\boldsymbol{s}}$ is defined by:

$$
m_{i j}^{s}=\frac{1}{2}\left(n_{i}^{s} l_{j}^{s}+n_{j}^{s} l_{i}^{s}\right)
$$

the superscript, $s,(s=1-18)$ denotes the slip system under consideration and $n_{i}^{s}$ and $l_{i}^{s}$, represent the slip plane normal vector and slip direction, respectively. The stress tensor in this case must be specified in the crystallographic coordinate system. The macroscopic inelastic strain rate is obtained by summing the shear strain rates, $\dot{\gamma}^{s}$, on each of the active slip systems:

$$
\dot{\varepsilon}^{i n}=\sum_{s \in s^{a c t}} m^{s} \dot{\gamma}^{s}
$$


where $s \in s^{a c t}$ implies that the summation is only performed on slip systems that are active.

The constitutive equations used to describe shear strain rate on each of the active slip systems are formulated from a macroscopic viscoplastic framework proposed by Chaboche $[231,232,235]$ written at the microscale. The yield criterion for the octahedral and cubic slip systems are defined by:

$$
\begin{aligned}
& f_{o}^{s}=\left|\tau_{o}^{s}-x_{o}^{s}\right|-k_{o}-r_{o}^{s} \leq 0 \\
& f_{c}^{s}=\left|\tau_{c}^{s}-x_{c}^{s}\right|-k_{c}-r_{c}^{s} \leq 0
\end{aligned}
$$

where $x^{s}$ and $r^{s}$ are the back stress and the isotropic hardening, respectively, for each slip plane, and $k_{o}^{s}$ and $k_{c}^{s}$ represent the critical resolved shear stress for the octahedral and cubic slip planes. The back stress and isotropic hardening state variables are defined separately for the octahedral and cubic slip systems. The evolution of the back stress is defined by the following evolution equations:

$$
\begin{aligned}
& \dot{x}_{o}^{s}=c_{o} \dot{\gamma}_{o}^{s}-d_{o} x_{o}^{s}\left|\dot{\gamma}_{o}^{s}\right| \\
& \dot{x}_{c}^{s}=c_{c} \dot{\gamma}_{c}^{s}-d_{c} x_{o}^{s}\left|\dot{\gamma}_{c}^{s}\right|
\end{aligned}
$$

where $c_{o}$ and $c_{c}$ represent the linear kinematic hardening modulus, $d_{o}$ and $d_{c}$ represent the modulus for the dynamic recovery terms, and the subscripts $o$ and $c$ denote the 
octahedral or cubic slip systems. The behaviour of the non-linear kinematic hardening rules is similar to that of the Nouailhas and Chaboche [225] phenomenological constitutive model discussed in the previous section.

Isotropic hardening is incorporated into the crystalline slip constitutive equations to model the cyclic hardening or softening that is observed in symmetrical fully reversed strain controlled loading or the plastic shakedown observed in stress controlled fatigue tests. Physically, isotropic hardening represents the gradual development of a cyclic dislocation structure in the material, such as softening caused by shearing of strengthening precipitates or the hardening associated with dislocations forming Orowan loops around strengthening precipitates. The evolution equations for the isotropic hardening state variable are specified by:

$$
\begin{aligned}
& \dot{r}_{o}^{s}=\left(q_{o}-b_{o} r_{o}^{s}\right)\left|\dot{\gamma}_{o}^{s}\right| \\
& \dot{r}_{c}^{s}=\left(q_{c}-b_{c} r_{c}^{s}\right)\left|\dot{\gamma}_{c}^{s}\right|
\end{aligned}
$$

where $q$ and $b$ represent two material parameters that describe the deformation induced isotropic hardening. Geometrically, the isotropic hardening evolution equations cause an expansion or contraction of the yield surface associated with each slip system. At the macroscopic scale isotropic hardening causes the yield characteristics to change as shown schematically in Figure 4.3. The form of the evolution equations have been selected to be representative of observed cyclic deformation behaviour, such that the isotropic hardening equation saturates at a value of $r_{0}^{s}+q$ at large accumulated inelastic shear strain rates, $\left|\dot{\gamma}^{s}\right|$, at a rate that is controlled by value specified for $b$. 
The viscoplastic shear strain rate is derived from a potential function that is similar in form to that defined for the Nouailhas and Chaboche [225] model and using the normality hypothesis. The inelastic shear strain rate for each slip system is specified by:

$$
\begin{aligned}
& \dot{\gamma}_{o}^{s}=\left\langle\frac{\left|\tau_{o}^{s}-x_{o}^{s}\right|-k_{o}-r_{o}^{s}}{K_{o}}\right\rangle^{n_{o}} \operatorname{sign}\left(\tau_{o}^{s}-x_{o}^{s}\right) \\
& \dot{\gamma}_{c}^{s}=\left\langle\frac{\left|\tau_{c}^{s}-x_{c}^{s}\right|-k_{c}-r_{c}^{s}}{K_{c}}\right\rangle^{n_{c}} \operatorname{sign}\left(\tau_{c}^{s}-x_{c}^{s}\right)
\end{aligned}
$$

where $K_{o}$ and $K_{c}$ are state variables that represent the viscoplastic drag stress for the octahedral and cubic slip systems, respectively, and $n_{o}$ and $n_{c}$ are the viscoplastic exponents. The viscosity terms are strongly temperature dependent. In this model the drag stress is assumed to have a constant value, i.e. no evolution equation is specified. The first term of the flow equations represent the viscoplastic shear strain rate multiplier:

$$
\left|\dot{\gamma}^{s}\right|=\left\langle\frac{f}{K}\right\rangle^{n}
$$

The term $\operatorname{sign}\left(\tau^{s}-x^{s}\right)$ specifies the direction of the plastic flow on each of the active slip systems.

\subsubsection{Critique}

The primary advantage of constitutive models formulated based on crystallographic slip is that they more physically correctly link the microstructural deformation mechanisms 
and the macroscopic constitutive material behaviour of single-crystal superalloys. However, there are limitations associated with this particular model and other constitutive equations based on crystallographic slip theory that should be noted.

For crystallographic orientations that deform isotropically under uniaxial loading, such as $\langle 100\rangle$ and $\langle 111\rangle$, crystallographic slip constitutive models predict that slip occurs homogeneously because the resolved shear stress on the octahedral (for $\langle 100\rangle$ oriented specimens) and cube slip systems (for $\langle 111\rangle$ oriented specimens) that are active have the same magnitude. As noted in Chapter 2 single-crystal superalloys at low temperatures deform by slip on discrete slip systems, and therefore do not deform homogeneously. This heterogeneity of slip is not accounted for in the formulation of constitutive models based on crystallographic slip. In the Han and coworkers model it is possible to specify different values for the critical resolved shear stress, $k$, on each slip system that would mimic discrete slip phenomena; however, it is unclear if such an approach is physically valid.

In addition to slip homogenization, crystallographic formulations developed for singlecrystal superalloys also treat the material at the $\gamma-\gamma^{\prime}$ microstructural scale as being homogeneous. That is, the effect of $\gamma^{\prime}$ morphology and volume fraction are not explicitly taken into consideration in the formulation, nor are changes in microstructure that occur at elevated temperature. Presumably the effects of microstructure and microstructural changes on the constitutive material response are embodied in the formulation of the kinematic and isotropic hardening laws, and the flow rule. Because the constitutive material parameters have little or no physical connection with the microstructure, the constitutive model parameters are specific to an alloy and microstructural condition. 
Micro-mechanical constitutive models, such as the model proposed by Tinga and coworkers [236], can account for microstructural factors $\left(\gamma^{\prime}\right.$ volume fraction and morphology) and microstructural evolution without having to discretize the model at the $\gamma-\gamma^{\prime}$ microstructural scale. However, in this model it was assumed that the $\gamma^{\prime}$ precipitates deform elastically, even though it has been established that inelastic deformations do indeed occur, such as $\gamma^{\prime}$ shearing discussed in Chapter 2. It is clear that additional research is needed to develop constitutive models that more rigoursly establish a connection between microstructure and the formulation of the constitutive equations.

The constitutive model proposed by Han and coworkers specifies the same critical resolved shear stress regardless if loading is tensile or compressive. It is therefore, incapable of predicting the tension and compression anisotropy associated with octahedral slip in single-crystal superalloys. Other crystallographic slip constitutive models, such as those proposed by Jordan and Walker [227] and Dame and Stouffer [114] have formulations that consider the cube cross-slip process that is believed to be responsible for the tension-compression yield anisotropy. It is possible to modify the yield function for octahedral slip to include cube cross-slip model, such as that proposed by Pope and Ezz [237], but this would require additional material parameters (and constitutive tests) to be identified. In spite of these limitations, constitutive models that are formulated based crystallographic slip such as that proposed by Han and coworkers do provide reasonable predictions of single-crystal constitutive material response at the macroscale. However, to utilize the capabilities of this constitutive model or the Nouailhas and Chaboche model reviewed earlier in Section 4.2 for structural analysis calculations, they must first be implemented into structural analysis codes. This requires the development of robust and efficient numerical integration schemes to integrate the 
rate equations used to describe the constitutive material response. This issue is discussed further in the following section.

\subsection{Numerical Integration Methods}

Given the important role that constitutive equations play in inelastic structural calculations using the finite element method, considerable research has been devoted to developing numerical integration algorithms for the rate equations used to describe the constitutive material behaviour. Several good reviews on this subject are presented by Simo and Hughes [238], Saleebe and Li [239], and Li and Saleebe [240]. Numerous researchers have developed numerical integration schemes for specific constitutive equations [241-244] or have presented generalized frameworks [245-249] for rate independent and rate dependent plasticity. In general, these integration algorithms are based on the generalized mid-point rule in which the initial conditions are established by assuming the deformation is entirely elastic. If plastic flow occurs the equations are solved iteratively, mainly via a return mapping algorithms $[239,240]$, to obtain updated values for the stress and state variables while ensuring the plastic flow conditions are satisfied. An overview of the general numerical approach based on the generalized midpoint integration rule is discussed in the following section.

\subsubsection{Numerical Integration Techniques}

The constitutive equations presented earlier are represented by a series of coupled and non-linear first-order differential equations having the form: 


$$
\begin{gathered}
\dot{y}_{1}(t)=f_{1}\left(y_{1}(t), y_{2}(t), \ldots, y_{n}(t)\right) \\
\dot{y}_{2}(t)=f_{2}\left(y_{1}(t), y_{2}(t), \ldots, y_{n}(t)\right) \\
\vdots \\
\dot{y}_{n}(t)=f_{n}\left(y_{1}(t), y_{2}(t), \ldots, y_{n}(t)\right)
\end{gathered}
$$

with the initial state,

$$
\dot{y}_{i}(0)=\dot{y}_{i}\left(t_{k}\right)
$$

where $\dot{y}$ represents an arbitrary first-order differential equation and $t$ denotes time. The objective is to integrate equation 4.27 between the initial state at $t=t_{k}$ and the final state at $t_{k+1}=t_{k}+\Delta t$, where the subscripts $k$ and $k+1$ denote the initial and final state and $\Delta t$ represents the increment of time. Differential equations, such as those in equation 4.27 can be solved numerically using algorithms developed from the generalized mid-point integration rule. Using the generalized mid-point rule equation 4.27 can be restated in incremental form as follows:

$$
\begin{gathered}
y_{i}\left(t_{k+1}\right)=y_{i}\left(t_{k}\right)+\Delta t f_{i}\left(y_{1}\left(t_{k+\theta}\right), y_{2}\left(t_{k+\theta}\right), \ldots, y_{n}\left(t_{k+\theta}\right)\right) \\
y_{i}\left(t_{k+\theta}\right)=\theta y_{i}\left(t_{k+1}\right)+(1-\theta) y_{i}\left(t_{k}\right)
\end{gathered}
$$

where $y_{i}\left(t_{k+1}\right)$ represents the current (best) estimate for $y_{i}$ at $t_{k+1}$ and $\theta$ is an integration parameter. Several possible integration schemes are possible, depending on the value selected for $\theta$, such as: 


$$
\begin{array}{ll}
\theta=0 & \text { Forward Euler (Explicit) } \\
\theta=\frac{1}{2} & \text { Midpoint Rule } \\
\theta=1 & \text { Backward Euler (Implicit) }
\end{array}
$$

Numerical algorithms developed based on the forward Euler rule are simple to implement, but impose severe restrictions on the maximum increment size and is inefficient for convergence [239]. Consequently, implicit integration methods are preferred because of better convergence properties and ease of calculating the consistent tangent stiffness matrix required to improve the global convergence properties of implicit FEM codes.

After restating the constitutive equations in incremental form one obtains a coupled series of non-linear algebraic equations that can be solved iteratively by linearizing these equations about a trial solution state for the stress and state variables. In general, the Newton-Raphson (N-R) method is used to conduct the linearization about the trial

solution state (in vector form), $\left[y_{i}\right]=\left[y_{i}\right]^{k}$, where the superscript $\mathrm{k}$ represents the solution iteration index. Applying the N-R method about the trial solution state and gives:

$$
\left[y_{i}\right]^{k+1}=\left[y_{i}\right]^{k}-\left[J_{i j}\right]^{-1}\left[f_{j}\right]^{k}
$$

with,

$$
\left[J_{i j}\right]=\left[\frac{\partial f_{i}}{\partial y_{j}}\right]
$$


where $\left[y_{i}\right]^{k}$ represents the current best guess for the solution, $\left[y_{i}\right]^{k+1}$ represents an improved estimate of the solution which brings the residual functions, $\left[f_{j}\right]^{k}$, closer to zero and $\left[J_{i j}\right\rfloor$ represents the gradients of the residual functions or so-called Jacobian matrix. Since equation 4.31 linearizes the constitutive equations, an improved estimate of the stress and state variables can be obtained using an iterative scheme consisting entirely of basic linear algebra operations. The iterative solution procedure is continued until the difference between two successive iterations is smaller than a user specified tolerance. The application of the forgoing numerical approach to the constitutive model used in this investigation will be discussed in Chapter 6 . 
Table 4.1 - Slip plane normal and slip directions for the 12 FCC octahedral slip systems.

\begin{tabular}{|c|c|c|}
\hline Octahedral Slip System & Slip Direction $\left[l_{1}, l_{2}, l_{3}\right]$ & Slip Plane Normal $\left\{n_{1}, n_{2}, n_{3}\right\}$ \\
\hline 1 & {$[1 / \sqrt{2}, 0,-1 / \sqrt{2}]$} & $\{1 / \sqrt{3}, 1 / \sqrt{3}, 1 / \sqrt{3}\}$ \\
\hline 2 & {$[-1 / \sqrt{2}, 1 / \sqrt{2}, 0]$} & $\{1 / \sqrt{3}, 1 / \sqrt{3}, 1 / \sqrt{3}\}$ \\
\hline 3 & {$[0,-1 / \sqrt{2}, 0,1 / \sqrt{2}]$} & $\{1 / \sqrt{3}, 1 / \sqrt{3}, 1 / \sqrt{3}\}$ \\
\hline 4 & {$[0,1 / \sqrt{2},-1 / \sqrt{2}]$} & $\{-1 / \sqrt{3}, 1 / \sqrt{3}, 1 / \sqrt{3}\}$ \\
\hline 5 & {$[-1 / \sqrt{2},-1 / \sqrt{2}, 0]$} & $\{-1 / \sqrt{3}, 1 / \sqrt{3}, 1 / \sqrt{3}\}$ \\
\hline 6 & {$[1 / \sqrt{2}, 0,1 / \sqrt{2}]$} & $\{-1 / \sqrt{3}, 1 / \sqrt{3}, 1 / \sqrt{3}\}$ \\
\hline 7 & {$[-1 / \sqrt{2}, 0,-1 / \sqrt{2}]$} & $\{-1 / \sqrt{3},-1 / \sqrt{3}, 1 / \sqrt{3}\}$ \\
\hline 8 & {$[1 / \sqrt{2},-1 / \sqrt{2}, 0]$} & $\{-1 / \sqrt{3},-1 / \sqrt{3}, 1 / \sqrt{3}\}$ \\
\hline 9 & {$[0,1 / \sqrt{2}, 0,1 / \sqrt{2}]$} & $\{-1 / \sqrt{3},-1 / \sqrt{3}, 1 / \sqrt{3}\}$ \\
\hline 10 & {$[0,-1 / \sqrt{2}, 0,-1 / \sqrt{2}]$} & $\{1 / \sqrt{3},-1 / \sqrt{3}, 1 / \sqrt{3}\}$ \\
\hline 11 & {$[1 / \sqrt{2}, 1 / \sqrt{2}, 0]$} & $\{1 / \sqrt{3},-1 / \sqrt{3}, 1 / \sqrt{3}\}$ \\
\hline 12 & {$[-1 / \sqrt{2}, 0,1 / \sqrt{2}]$} & $\{1 / \sqrt{3},-1 / \sqrt{3}, 1 / \sqrt{3}\}$ \\
\hline
\end{tabular}

Table 4.2 - Slip plane normal and slip directions for 6 FCC cubic slip systems.

\begin{tabular}{|c|c|c|}
\hline Cubic Slip System & Slip Direction $\left[l_{1}, l_{2}, l_{3}\right]$ & Slip Plane Normal $\left\{n_{1}, n_{2}, n_{3}\right\}$ \\
\hline 13 & {$[1 / \sqrt{2}, 1 / \sqrt{2}, 0]$} & $\{0,0,1\}$ \\
\hline 14 & {$[-1 / \sqrt{2}, 1 / \sqrt{2}, 0]$} & $\{0,0,1\}$ \\
\hline 15 & {$[1 / \sqrt{2}, 0,1 / \sqrt{2}]$} & $\{0,1,0\}$ \\
\hline 16 & {$[-1 / \sqrt{2}, 0,1 / \sqrt{2}]$} & $\{0,1,0\}$ \\
\hline 17 & {$[0,1 / \sqrt{2}, 1 / \sqrt{2}]$} & $\{1,0,0\}$ \\
\hline 18 & {$[0,-1 / \sqrt{2}, 1 / \sqrt{2}]$} & $\{1,0,0\}$ \\
\hline
\end{tabular}




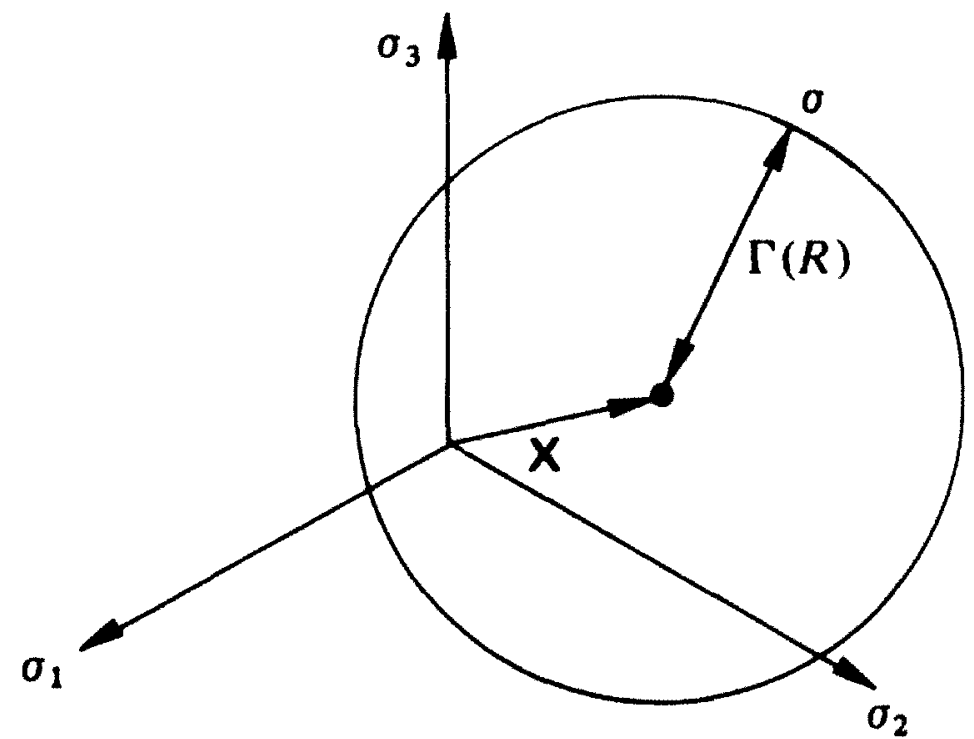

Figure 4.1 - Schematic definition of the back stress. The back stress, $X$, causes a kinematic shift to the yield surface, $\Gamma(R)$, in deviatoric stress space [250].

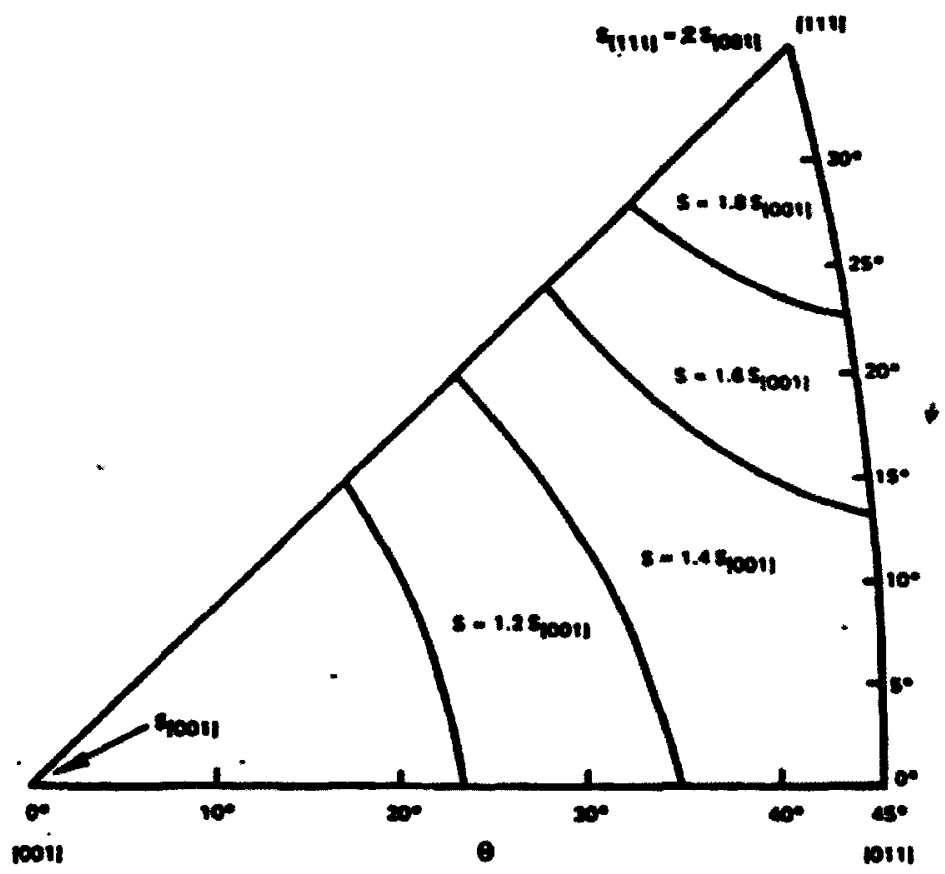

Figure 4.2 - Monotonic variation in yield strength of cubic single crystal materials predicted by the Hill yield function with $Y S_{[001]}=0.5 * Y S_{[111]}[233]$. 


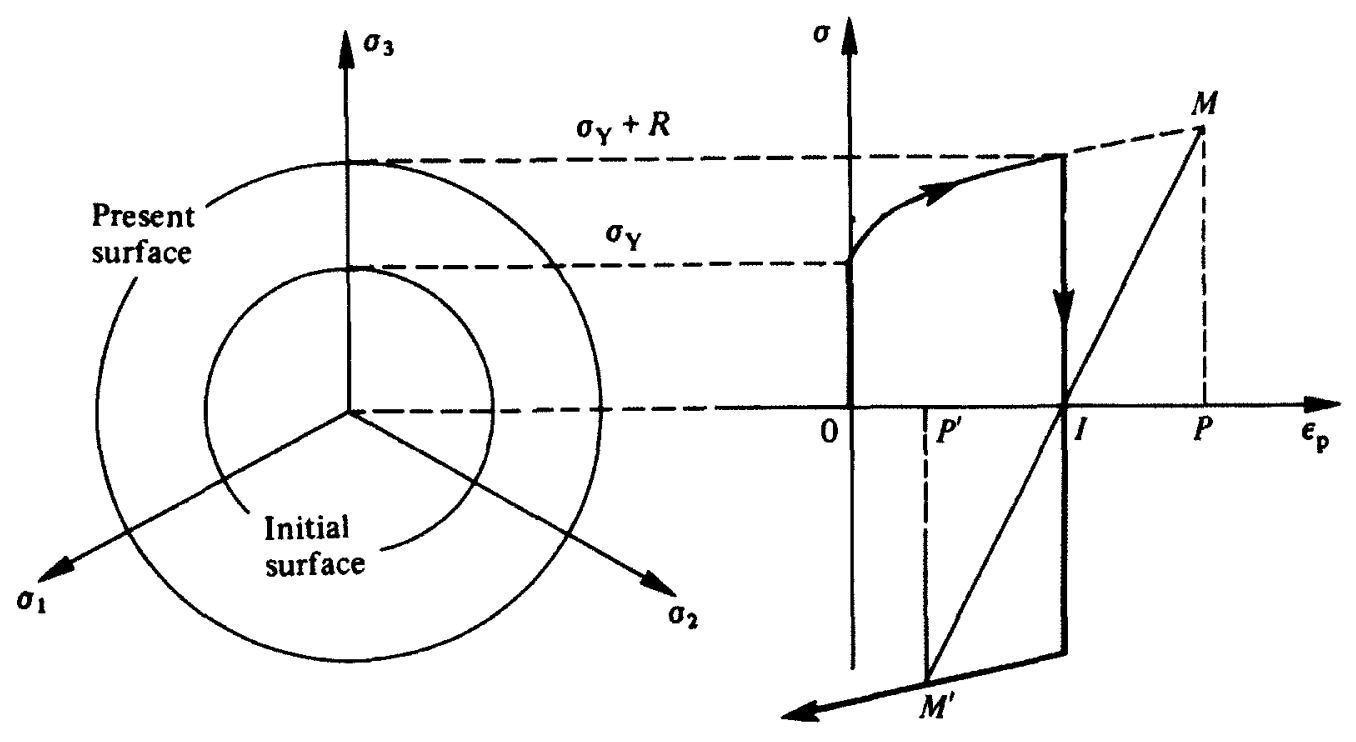

Figure 4.3 - Schematic illustrating the macroscopic definition of isotropic hardening. Isotropic hardening causes an expansion, $R$, to the initial yield surface of radius $\sigma_{\mathrm{Y}}$. Note: The deformation response depicted in right half of the Figure shows the effect of isotropic hardening on reversed plastic loading [250]. 


\section{Materials and Experimental Methods}

\subsection{Experimental Materials}

At the start of this research program second generation single-crystal superalloys were the state-of-the-art alloys used for fabrication of gas turbine airfoils. Although third generation single-crystal superalloys had been developed in the late 1990's, these alloys have never achieved same level of commercial acceptance as the second generation alloys. Therefore, second generation alloys were considered as the subject of this study. Based on successful casting experiments to produce single-crystal test bars in the Carleton University Bridgman casting furnace [73], it was decided to purchase polycrystalline master alloy and recast the material into single-crystal test bars.

\subsubsection{Alloy Selection and Procurement}

The second generation nickel-base single-crystal superalloy PWA1484 was selected as the material to be evaluated in this investigation. This alloy was developed by Pratt \& Whitney to offer significantly improved creep-rupture strength, thermal fatigue and oxidation resistance when compared to the first production single-crystal nickel-base superalloy PWA1480 [84]. It was also the first production single-crystal superalloy to incorporate rhenium in its alloy chemistry [84] to improve high temperature creep properties. This alloy was selected for the following reasons:

1. Compatibility with pre-existing PWA1484 single-crystal test bars that could be used for seed crystals;

2. The casting parameters, such as superheat and withdrawal rate, were established for casting this alloy in the Carleton University Bridgman furnace [73]; and, 
3. No short fatigue crack propagation results have been reported for this particular single-crystal superalloy.

Having selected the material to be utilized in this research, a 25-lb heat of PWA1484 master alloy was purchased from Sophisticated Alloys of Butler PA. The material was vacuum induction melted and cast into 8 5/8-in $(\sim 16 \mathrm{~mm})$ diameter bars approximately 36-in $(\sim 915 \mathrm{~mm})$ in length. In addition to supplying the material, Sophisticated Alloys also provided report detailing the chemistry of the master alloy heat. The contents of this report are listed in Table 5.1. Trace element analysis results were not supplied for the master alloy material. For comparison purposes the accepted composition range for each of chemical constituents in PWA1484 is also included Table 5.1. It may be observed that all alloy additions except for aluminum were within PWA1484 alloy chemistry specifications. In spite of the aluminum content being slightly lower $(0.4 \mathrm{Wt} . \%)$ than the target alloy chemistry, the material was accepted because of the significant lead-time associated with taking delivery of a second heat of material.

\subsubsection{Ceramic Molds}

High purity single-bore alumina tubes were purchased from Vesuvius McDanel of Beaver Falls PA to function as single-crystal casting molds. The ceramic tubes were of a double open-ended design having nominal dimensions of 5/8-in $(\sim 16 \mathrm{~mm})$ I.D., 7/8-in $(\sim 22 \mathrm{~mm})$ O.D. $x$ 9-in $(\sim 230 \mathrm{~mm})$ long with smooth internal surface finish (Vesuvius McDanel Part \#98A310830-06-09). After several casting trials with these molds, it was observed that large porosity was consistently present at the top of the test bars, as shown in Figure 5.1, which reduced the number of test specimens that could be obtained from each test bar to one specimen. In later castings, ceramic tubes (Vesuvius McDanel Part \#98A310830-06-12) with an overall length of 12-in ( 300 mm) were used for single- 
crystal castings which nominally yielded sufficient material for fabrication of two test specimens.

\subsection{SX Casting Procedure}

\subsubsection{Carleton University DS Casting Facility}

The Carleton University DS casting facility consists of a vertical Bridgman casting furnace manufactured by Centorr Vacuum Industries. The details of the furnace and ancillary equipment are shown in Figure 5.2. The furnace consists of a vacuum/pressure shell of stainless steel construction that is divided into two separate chambers and includes an integrated water-jacket for cooling purposes as shown schematically in Figure 5.3. The upper chamber, or furnace hot zone, contains two-pairs of tungsten-mesh heating elements, divided into an upper and lower zone, which are rated at $25 \mathrm{kVA}$ and $20 \mathrm{kVA}$, respectively, providing a maximum temperature capability of $2000^{\circ} \mathrm{C}$. The power supply for the heating elements consisted of two step-down transformers with silicon controlled rectifiers (SCR) to control power switching to the heating elements. A Honeywell DCP 700 dual channel programmable temperature controller was used for closed-loop temperature control of the upper and lower hearting elements. The temperatures within the furnace hot-zone were measured using Type-C thermocouples. A Honeywell DPR 1000 multi-channel temperature data logger was used for continuous monitoring of the furnace temperatures. Heat shields consisting of multiple layers of molybdenum sheet material are used to separate the heating elements from the vacuum containment vessel.

The lower chamber, or furnace cold zone, is separated from the furnace hot zone by retractable baffles to reduce heat loss and to create and maintain a high thermal gradient 
necessary to achieve directional solidification. The lower chamber features a door to enable loading and unloading of the furnace. A water-cooled copper chill plate is used to support the casting mold and to extract heat from the casting during withdrawal from the furnace hot zone. The chill plate is integrated into a motorized pedestal system that allows the casting mold to be raised into the furnace hot zone and withdrawn at a controlled rate. A detailed view of the chill plate and pedestal system can be observed in Figure 5.4. The pedestal assembly consists of a direct current (DC) stepper motor, speed reduction gear box and ball-screw drive. The vacuum system comprises a mechanical roughing pump (Leybold D30A dual-stage rotary vane mechanical pump) and diffusion pump. The diffusion pump was added to the facility to enable high vacuum (approximately $10^{-5}$ torr or $1.3 \times 10^{-2} \mathrm{~Pa}$ ) casting capability [73]. In addition to vacuum casting capabilities, the furnace can also be backfilled to a small positive pressure (approximately 2 psi or $13.8 \mathrm{kPa}$ ) with inert gas, such as argon, to reduce evaporative losses of alloy constituents that have low vapor pressures.

\subsubsection{Seed Crystal Casting Process}

In Chapter 2 it was discussed that it is common industrial practice to use casting molds incorporating crystal selectors for producing single-crystal components. However, production of crystal selector casting molds requires specialized equipment to carry out the lost wax mold manufacturing process and substantial time and experience to produce casting molds with sufficient structural strength to ensure mold integrity during casting. Instead of utilizing the crystal selection technique in this work, single-crystal seeds were used to initiate single-crystal solidification. This seed crystal casting technique was selected for the following reasons: 
1. A small supply of PWA1484 (produced by PCC Airfoils Minerva OH, USA) and CMSX-10 (produced by Howmet Whitehall MI, USA) single-crystal test bars were available from which seed crystals could be obtained; and

2. The seed crystal materials were accompanied by reports that indicated the longitudinal orientation of each test bar was within $10^{\circ}$ of the $[100]$ crystal direction.

The details of the seed crystal casting process are shown schematically in Figure 5.5. It may be noted that unlike conventional investment casting practices in which the molten alloy charge is held in a separate crucible and subsequently poured into the casting mold, the master alloy charge was instead melted in the casting mold and the seed crystal was used to initiate single-crystal solidification.

The seed crystal casting process utilized in this work consisted of the following steps:

1. Preparation of the seed crystal and master alloy ingot material;

2. Preparation of the casting mold;

3. Insertion of the casting mold, master alloy and seed crystal into the casting furnace;

4. Raising the casting mold into the furnace hot zone and heating the material to the appropriate superheat temperature; and

5. Withdrawing the casting mold and material from the furnace hot zone at a constant withdrawal rate to obtain a single-crystal test bar.

Additional details on each of these steps are described in the following paragraphs. 
The first step in the seed crystal casting process involved preparation of the master alloy material. Preparation of the master alloy material consisted of two steps. First, the master alloy ingots were ground to remove the surface oxide layer and residual casting mold debris, and to provide clearance between the mold and master alloy ingots to accommodate the different thermal expansion characteristics of the mold and master alloy materials. After grinding the master alloy ingots were cut into segments approximately 2 -in in length $(\sim 50 \mathrm{~mm})$ using an abrasive cut-off saw, cleaned ultrasonically in a bath of acetone to remove surface contaminants and carefully hot-air dried to ensure a stain-free surface finish.

Preparation of the seed crystals involved three steps. First, the PWA1484 test bar used for preparing the seed crystals was machined to a diameter approximately 0.005 to 0.010 -in ( 0.127 to $0.254 \mathrm{~mm}$ ) smaller than the internal diameter of the casting mold. This small clearance was necessary to accommodate thermal expansion mismatch between the casting mold and seed crystal materials, and to minimize the nucleation of stray grains caused by molten metal flowing into the gap between the seed crystal and casting mold. After machining, the secondary orientation of the seed crystal material was determined using metallographic techniques and permanently marked by scribing two reference lines along the entire length of the test bar as shown schematically in Figure 5.6. Specific details on the process used to determine the secondary orientation of seed material are described in a later section. The last step for seed crystal preparation involved machining 1-in $(25.4 \mathrm{~mm})$ long seeds crystals from the single-crystal test bar and scribing a line onto one end to indicate the secondary crystallographic orientation, as shown schematically in Figure 5.7. The seed crystals were cleaned in a manner similar to the master alloy ingots prior to casting. 
Approximately one-half of the single-crystal test bars used in this investigation were cast using seed crystals machined from CMSX-10 test bars. This material was used after the supply of PWA1484 seed crystals was exhausted. The CMSX-10 seed crystals were prepared in a manner similar to the PWA1484 seed crystals. However, unlike the PWA1484 seed crystal material, the [101] secondary crystallographic orientation was identified on the CMSX-10 test bars by the foundry that supplied the material. The same process as described for marking the secondary orientation on the PWA1484 seed crystals was followed for preparation of the CMSX-10 seed crystals.

The casting molds were prepared by first passing a clean cloth through the inside of the mold to remove dust and debris that could contaminate the casting. After cleaning, the casting molds were tightly wrapped with a 6-in by 12 -in $(15 \mathrm{~cm}$ by $30 \mathrm{~cm})$ sheet of 0.020 -in $(0.5 \mathrm{~mm})$ molybdenum foil secured in place by molybdenum wire. The purpose of the molybdenum foil was to provide containment of the molten alloy in the event of a casting mold failure. A spacer disk was fabricated from 0.5 -in $(12.7 \mathrm{~mm})$ thick zirconia fiber board (Zircar Zirconia Inc. ZYFB-3) and placed in the bottom of the casting mold so that it was situated between the seed crystal and the copper chill plate, as shown schematically in Figure 5.5. The function of the spacer disk was to ensure that the seed crystal was partially inside the furnace hot zone when the pedestal and chill plate was in the fully extended position for casting and to control heat transfer to the chill plate.

After preparation of the casting mold the seed crystal was inserted into the top of the mold so that it was positioned on top of the zirconia spacer disk. Several master alloy ingots were then inserted until the alloy charge was flush with the top of the mold. The casting mold with alloy charge was then placed onto the chill-plate mold support stand as shown in the schematic in Figure 5.5. The casting mold was then raised into the furnace 
hot zone and the retractable baffles were closed around the casting mold while leaving sufficient clearance to allow for unimpeded withdrawal of the casting. The loading door of the furnace was then closed to permit vacuum system operation.

Prior to furnace operation, the furnace chamber was evacuated using the roughing pump and back-filled with ultra-high purity argon gas several times in succession to ensure a clean casting environment. After completing the final argon back-fill, the furnace chamber was evacuated using the roughing and high-vacuum diffusion pump to attain a vacuum of approximately $4 \times 10^{-4}$ torr $\left(5.3 \times 10^{-2} \mathrm{~Pa}\right)$. After attaining sufficient vacuum to safely activate the heating system, the upper and lower heating element power was enabled and a progressive heating routine was followed to prevent damage to the heating elements. The progressive heating process consisted of a sequence of heating ramps to specific temperature end-levels. The following heating sequence was followed:

1. Ramp to $100^{\circ} \mathrm{C}$ at $5^{\circ} \mathrm{C} / \mathrm{min}$;

2. Ramp to $500^{\circ} \mathrm{C}$ at $10^{\circ} \mathrm{C} / \mathrm{min}$;

3. Ramp to $1400^{\circ} \mathrm{C}$ at $20^{\circ} \mathrm{C} / \mathrm{min}$;

4. Ramp to $1500^{\circ} \mathrm{C}$ at $10^{\circ} \mathrm{C} / \mathrm{min}$; and

5. Ramp to $1580^{\circ} \mathrm{C}$ at $5^{\circ} \mathrm{C} / \mathrm{min}$.

After reaching the casting superheat temperature $\left(1580^{\circ} \mathrm{C}\right)$ the casting was soaked for 15 minutes to ensure complete melting of the charge and to ensure that some portion of the single-crystal seed was back-melted prior to withdrawal. The casting was then withdrawn from the furnace hot-zone at a constant rate of $8-\mathrm{in} / \mathrm{hr}(\sim 20 \mathrm{~cm} / \mathrm{hr})$. The final casting superheat temperature and withdrawal rate was determined using previous work by Sarri [73] as a guideline and by performing a number of trial castings. 


\subsubsection{Determination of the Seed-Crystal Secondary Crystallographic Orientation}

Typically, the orientation of single-crystal materials is assessed using the back-reflection or transmission Laue x-ray diffraction techniques. The equipment necessary to conduct a Laue analysis was unavailable for this work, and therefore an alternative method was devised using the single-crystal macrostructure to determine the secondary crystallographic orientation. It was noted in Chapter 2 that the dendritic structure of cubic single-crystal materials naturally aligns with the cube $\langle 100\rangle$ crystal directions. Using this property of cubic materials, the secondary orientation of the PWA1484 seeds was established by polishing the ends of the seed-crystal using the procedures described in a later section (see Section 5.3.1) followed by macroetching the seed to reveal the cast dendritic structure. An example of the macrostructure is shown in Figure 5.8. After macroetching a Vickers microhardness indenter was used to indent a series of points aligned with the secondary dendrite arms. The cube secondary orientation was indicated by scribing a line to connect the series of indentations. The seed-crystal was then examined using optical microscopy to verify that the scribed line was aligned with the indentations and the cube secondary orientation.

\subsection{Microstructural Evaluation}

\subsubsection{Sample Preparation}

Metallographic samples were prepared by sectioning the material using a high-speed abrasive saw and mounting the sections in a thermal setting resin. All metallographic samples were ground using progressively finer grades of silicon carbide (SiC) paper (180 to 600 grit), followed by polishing with $9 \mu \mathrm{m}$ and $3 \mu \mathrm{m}$ diamond paste and $0.01 \mu \mathrm{m}$ colloidal silica suspension. 


\subsubsection{Macrostructure Samples}

To reveal the cast structure of the single-crystal material for optical microscopy analysis, metallographic samples were etched in a solution containing $10 \mathrm{ml} \mathrm{HCl,} 10 \mathrm{ml} \mathrm{HNO}, 0.3$ $\mathrm{g} \mathrm{MoO}_{3}$ (molybdic acid), and $15 \mathrm{ml} \mathrm{H}_{2} \mathrm{O}$. The metallographic samples were submerged in the etchant and gently agitated for $20-25$ seconds. It was observed that the best macrostructure contrast was obtained when the etchant had discolored slightly after etching several samples. This etchant dissolves the $\gamma^{\prime}$-phase leaving the $\gamma$-matrix intact. At low magnifications this etching procedure was found to be effective for revealing the dendritic structure, spurious grain defects and the presence of eutectic $\gamma-\gamma$.

\subsubsection{Microstructure Sample Preparation}

To reveal the size and morphology of the $\gamma^{\prime}$ precipitate structure in the as-cast and heattreated condition, metallographic samples were electolytically etched in a solution containing $48 \mathrm{ml} \mathrm{H}_{2} \mathrm{SO}_{4}, 12 \mathrm{ml} \mathrm{H}_{3} \mathrm{PO}_{4}$, and $48 \mathrm{ml} \mathrm{HNO}_{3}$, for $20-30$ seconds at 6.5 volts. This etching procedure was effective at removing the $\gamma$-matrix while leaving the $\gamma$ ' precipitates and other phases intact for microscopic examination using optical or scanning electron microscopy. Samples prepared using this etchant were used to estimate the $\gamma^{\prime}$ precipitate size, morphology, and volume fraction, as well as revealing the presence of residual eutectic $\gamma-\gamma^{\prime}$ in the fully heat-treated condition.

\subsubsection{Optical and Scanning Electron Microscopy Equipment}

Detailed microstructural analysis of metallographic samples and fractographic analysis of fatigue test samples was undertaken using a Philips XL-30 S-FEG high resolution scanning electron microscope (SEM). This SEM was equipped with secondary electron (SE) and backscatter electron (BSE) detectors, and energy-dispersive x-ray spectroscopy hardware for compositional analysis. Selected metallographic samples and fatigue 
specimen fracture surfaces were examined optically at low magnification using a Nikon SMZ 1000 stereo microscope and at higher magnification using an Olympus PMG 3 optical microscope.

\subsection{Test Bar Heat Treatment}

The mechanical properties of single-crystal superalloys critically depend on the ability to homogenize the segregated cast structure, fully solution the as-cast $\gamma^{\prime}$-phase and reprecipitate the $\gamma^{\prime}$-phase with a controlled size and distribution. Consequently, the development of microstructural characteristics to optimize high temperature mechanical properties and microstructural stability in single-crystal superalloys depends on proper design and application of heat treatments. In the following sections the macro and microstructural details of the as-cast single-crystal test bars produced by Bridgman casting in this investigation are discussed. This is followed by a discussion on the development and application of a heat treatment process for the single-crystal test bars.

\subsubsection{As-Cast Macro and Microstructure of Single-Crystal Test Bars}

After casting, metallographic samples were prepared from the as-cast test bars and etched using the macrostructure etchant described earlier. These metallographic samples were examined using optical microscopy to determine if the material was free of casting defects, such as large casting pores and spurious grains, which could compromise the mechanical properties of the fatigue and constitutive behaviour test specimens. The metallographic samples were prepared from transverse sections cut from the middle and top sections of each cast test bar to ensure that single-crystal solidification occurred along the entire of length of the casting. Longitudinal metallographic samples were prepared from selected single-crystal bars to examine the as-cast longitudinal dendritic structures. In addition to investigating the macroscopic microstructural features of the single-crystal 
test bars, selected metallographic samples were etched using the electrolytic etchant described earlier and examined by SEM to investigate the as-cast microstructural features.

\subsubsection{Longitudinal and Transverse Section Solidification Structures}

To examine the solidification structures in the longitudinal direction, one single-crystal test bar was sectioned along its entire length by wire electrical discharge machining (EDM), polished and macroetched to reveal the dendritic solidification structure. The longitudinal section from the bottom third of the test bar is shown in Figure 5.9. The seed crystal and start of new single-crystal solidification is readily apparent in this figure. A more detailed optical examination of this longitudinal section was undertaken to examine the solidification structures at the interface between the seed crystal and new singlecrystal growth, the results of which are shown in Figure 5.10. The solidification structure consists of the un-melted seed crystal, a mushy zone caused by partial melting of the seed crystal and new single-crystal growth. Although it is not apparent in Figure 5.10, the primary dendrite arms extend along the entire length of the casting and are aligned with the $<100>$ crystallographic direction. More importantly, the primary dendrites in the new single-crystal growth are aligned with the crystallographic orientation of the seed crystal. The interdendritic regions are decorated with eutectic $\gamma-\gamma^{\prime}$ phase which appears as the white phase in the optical micrograph. It is apparent that the primary dendrite arm spacing is finer in the new single-crystal growth than the seed crystal. This was investigated further by examining metallographic samples prepared from transverse sections removed from the test bars.

As noted earlier, transverse cross-sections were cut from the middle and top sections of the single-crystal test bars to ensure that single-crystal solidification occurred along the 
entire length of the casting. Optical examination of transverse sections cut from the middle and top of the test bars did not reveal any significant differences in the as-cast solidification structures. A representative transverse section that was cut from the middle section of a single-crystal test bar and macroetched to reveal the as-cast dendritic structure is shown in Figure 5.11. The solidification structure consists of primary dendrites with a PDAS of approximately $281 \pm 75 \mu \mathrm{m}$ and secondary dendrites that grow along cube directions normal to the primary dendrites. Also apparent in this Figure are islands of eutectic $\gamma-\gamma^{\prime}$ phase (appears as the white phase in the optical micrograph) that decorate the interdendritic regions. Due to the limited resolution of optical microscopy a more detailed investigation of the as-cast microstructural features was undertaken using scanning electron microscopy. The results of this investigation are discussed in the following section.

\subsubsection{As-cast Microstructural Features}

A more detailed analysis of the as-cast solidification structures was undertaken using transverse sections cut from the single-crystal test bars. These metallographic samples were etched using the electrolytic process described earlier to remove the $\gamma$-matrix material and reveal $\gamma^{\prime}$-phase and interdendritic constituents. The SEM micrograph depicted in Figure 5.12 shows an individual dendrite and the interdendritic regions. It is apparent from this micrograph that there are two distinctly different size distributions for the $\gamma^{\prime}$-phase. The $\gamma^{\prime}$-phase within the primary dendrite core and secondary dendrite arms appears to be finer than at the periphery of the dendritic structure, i.e. near the interdendritic regions. The size variation of the $\gamma^{\prime}$-phase is more apparent in the micrograph shown in Figure 5.13. As shown in Figure 5.14, the $\gamma^{\prime}$-phase within the dendrite core mainly have a cubic morphology (approximate size $0.5 \mu \mathrm{m}$ ) with some

precipitates appearing to be in the process of evolving into an ogdoadically diced 
morphology. The larger $\gamma^{\prime}$ precipitates (approximately $1-1.2 \mu \mathrm{m}$ ) found at the periphery of the dendrite appear to have distinctive ogdoadically diced morphology, as indicated in Figure 5.15.

In addition to examining the $\gamma^{\prime}$ precipitate size, distribution and morphology, a more detailed analysis of the interdendritic constituents was undertaken. As shown in Figure 5.16 , the main constitutes of the interdendritic regions were eutectic $\gamma-\gamma^{\prime}$ phase, and massive primary $\gamma^{\prime}$ precipitates. The phase that appears to be bright white in the BSE micrograph was identified by SEM-EDS to be a tantalum-rich carbide. This phase was only sparingly observed in the as-cast material but indicates that the PWA1484 master alloy ingots contained trace amounts of carbon deviating from the nominal alloy chemistry for PWA1484. In addition to this apparent alloy chemistry anomaly, it was noted earlier, the composition of the Sophisticated Alloys master alloy used in this investigation deviated from the nominal alloy chemistry range for PWA1484. Before conducting solution and aging heat treatments on this alloy, a detailed investigation was undertaken to determine what effects these compositional anomalies could have on the solution heat treatment process. This is discussed further in the following section.

\subsubsection{Differential Scanning Calorimetry (DSC) Analysis}

As indicated earlier the master alloy material procured for this research program had the nominal composition of PWA1484, but chemical analysis results indicated that the aluminum content was below the accepted limits. It was unclear what affect this may have on the ability to apply the homogenization heat treatment parameters established for PWA1484, or if new parameters would need to be developed. Therefore, an investigation was undertaken using the differential scanning calorimetry (DSC) method to measure the alloy phase transition temperatures. The focus of this investigation was to determine the 
alloy solidus temperature, which influences the capacity to carry out the homogenization heat treatment. In addition to testing the single-crystal test bars, the thermal properties for the PWA1484 seed-crystal material were also measured for comparison purposes.

The DSC samples were prepared from $2 \mathrm{~mm}$ thick transverse sections cut from a singlecrystal test bar and from the PWA1484 seed crystal material. DSC samples with a mass of approximately $90 \mathrm{mg}-100 \mathrm{mg}$ were obtained by cutting the transverse section into a number of cubes with an edge length of approximately $2 \mathrm{~mm}$ using a low-speed diamond wafering saw. Samples having a mass greater than $100 \mathrm{mg}$ were ground using 180 grit $\mathrm{SiC}$ paper until the final sample mass was within the allowable sample mass range for the DSC equipment. The samples were then cleaned ultrasonically in reagent grade acetone and hot air dried to a stain-free finish before conducting the DSC tests.

The DSC thermal analysis tests were conducted using a Netzsch 404-C Pegasus DSC equipped with a high temperature furnace to allow measurements to be carried out between room temperature and $1500^{\circ} \mathrm{C}$. A high temperature DSC measurement head equipped with type-S thermocouples was used for the measurements. A full temperature range calibration was performed prior to the DSC measurements by melting various standard commercial purity metals. An alumina crucible was used to contain the test sample and an empty alumina crucible was used as the DSC reference. The DSC tests were performed using linear heating and cooling profiles at a rate of $20 \mathrm{~K} / \mathrm{min}$ and under a $50 \mathrm{ml} / \mathrm{min}$ flowing argon gas atmosphere. The endothermic peaks associated with solidsolid and solid-liquid phase transitions (during heating) were identified from the heatflow versus temperature traces recorded during the DSC tests and analyzed using Netzsch Proteus software package. The alloy solidus temperature was identified from the extrapolated onset temperature associated with the liquidus endothermic peak. The 
liquidus onset temperature was determined from the intercept of a tangent line drawn through the leading slope of the liquidus endothermic peak and the baseline.

The DSC curves obtained from PWA1484 cast by PCC Airfoils and the Sophisticated Alloys version of PWA1484 cast into single-crystal form in the Carleton University Bridgman facility are shown in Figure 5.17. The DSC results indicate that the liquidus temperatures, as determined from temperature at the maximum endothermic peak for each alloy, were different (Sophisticated Alloys PWA1484 $-1380^{\circ} \mathrm{C}$ versus PCC PWA1484 $\left.-1395^{\circ} \mathrm{C}\right)$. However, the extrapolated tangent-onset for the solidus to liquidus phase transformation indicates that the incipient melting temperatures for the Sophisticated Alloys version of PWA1484, and PWA1484 produced by PCC Airfoils, were remarkably similar (Sophisticated Alloys $-1345^{\circ} \mathrm{C}$ versus PCC PWA1484 $1350^{\circ} \mathrm{C}$ ). It has been established that higher heating rates in DSC analyses, such as the 20 $\mathrm{K} / \mathrm{min}$ heating rate used in this investigation, can promote errors in the interpretation of DSC results because the sample temperature can lag behind the heating furnace temperature [251]. However, based on the excellent agreement between incipient melting temperatures established from the DSC results for Sophisticated Alloys PWA1484 and PCC PWA1484, it was decided that the standard heat treatment process specified by Cetel and Dhul [84] for PWA1484 could be used with Sophisticated Alloys PWA1484. The heat treatment procedures are discussed in the following section.

\subsubsection{Test Bar Heat Treatment}

The standard three-step heat treatment developed for PWA1484 consists of the following steps [84]: 
- Homogenizing heat treatment at $2400^{\circ} \mathrm{F}\left(\sim 1315^{\circ} \mathrm{C}\right)$ for 4 hours, followed by gas fan quench cooling,

- Diffusion heat treatment at $1975^{\circ} \mathrm{F}\left(\sim 1079^{\circ} \mathrm{C}\right)$ for 4 hours, followed by gas fan quench cooling,

- Secondary aging heat treatment at $1300^{\circ} \mathrm{F}\left(704^{\circ} \mathrm{C}\right)$ for 24 hours.

The homogenizing heat treatment is used to solution the coarse as-cast $\gamma^{\prime}$ and eutectic $\gamma$ $\gamma^{\prime}$, and more importantly to reduce the segregation present in the as-cast structure. Fine primary $\gamma^{\prime}$ precipitates form during cooling from the homogenizing temperature. The diffusion heat treatment step is normally utilized to facilitate the diffusion bonding of oxidation resistant coatings (used as part of TBC system) with the single-crystal substrate but also is used to optimize the primary $\gamma^{\prime}$ size and distribution. The secondary aging heat treatment results in the precipitation of ultra fine $\gamma^{\prime}$, which improves the intermediate temperature strength of single-crystal superalloys. As indicated by the gas fan quench processes used to cool the material between heat treatment steps, the standard industry practice is to conduct the heat treatment of finished single-crystal components in vacuum to prevent oxidation of the component prior to application of the thermal barrier coating.

In this investigation the single-crystal test bars were heat treated inside a radiation furnace (Linberg Blue Model BF51333C) in laboratory air. Because the test bars were significantly larger than the fatigue and constitutive material test specimens used in this investigation, oxidation of the test bars was considered not to be detrimental because the oxidized layer would be removed during specimen manufacturing As noted earlier, during metallographic sample preparation the single-crystal test bars were sectioned in the middle resulting in two bars approximately $80 \mathrm{~mm}$ in length from which test specimens would be machined. To ensure that the secondary crystallographic information 
for the cast test bar was retained after heat treatment, the secondary orientation of the test bars was permanently marked onto the ends of the test bars by machining a $0.1 \mathrm{~mm}$ groove to a depth of $1 \mathrm{~mm}$ using wire electrical discharge machining (EDM). To reduce the time required to apply heat treatments, three specimen sized test bars were heat treated simultaneously. To monitor the test bar temperature during heat treatment a type$\mathrm{S}$ thermocouple sheathed in an alumina tube was situated in close proximity to the test bars. Before carrying out the heat treatments, the appropriate furnace temperature set points were established by conducting trial homogenizing heat treatments on scrap test bars.

The standard heat treatment schedule for PWA1484 was nominally followed but several deviations from this procedure should be noted. To reduce the risk of incipient melting during the homogenizing heat treatment step, the temperature of the single-crystal test bars was gradually increased to the homogenization temperature according to the following steps:

- Homogenize at $1290^{\circ} \mathrm{C}$ for 2 hours,

- Ramp the furnace temperature from $1290^{\circ} \mathrm{C}$ to $1305^{\circ} \mathrm{C}$ over 2 hours, followed by a 2 hour hold at $1305^{\circ} \mathrm{C}$,

- Ramp the furnace temperature from $1305^{\circ} \mathrm{C}$ to $1315^{\circ} \mathrm{C}$ over 2 hours, followed by a 4 hour hold at $1315^{\circ} \mathrm{C}$,

- Remove the bars from the furnace and air-cool.

The total time required to conduct the homogenizing heat treatment was 12 hours. The standard diffusion and secondary aging heat treatment steps were followed except the bars were air-cooled after application of these heat treatment steps. After finishing the 
heat treatments, metallographic samples were prepared from selected test bars to characterize the heat treated microstructure. The results from this investigation are discussed in the following sections.

\subsubsection{Macrostructural Features after Heat Treatment}

Metallographic samples were prepared by sectioning heat treated test bars and etching the samples using the electrolytic etching procedure described earlier to reveal the $\gamma^{\prime}$ size and morphology. Before conducting detailed microstructural analysis, the macrostructure of the heat treated material was investigated using scanning electron microscopy. A composite image consisting of eight backscatter electron SEM images was assembled to show the residual cast dendritic structure and to characterize the interdentritic shrinkage porosity. This composite image is shown in Figure 5.18. It can be observed that the residual cast dendritic structure is significantly less apparent than observed in the as-cast material, suggesting that microsegregation was significantly reduced during the homogenizing heat treatment. This last point is speculative because a quantitative analysis to estimate the microsegregation in the as-cast and heat treated conditions was not performed. It is also apparent in Figure 5.18 that shrinkage porosity is present in some interdendritic regions. A quantitative analysis was undertaken to estimate the area fraction and pore size distribution using the image analysis capabilities of UTHSCSA* Image Tool version 3. To obtain a more representative estimate of the pore area fraction and pore size the image analyses were performed on several different composite images obtained from metallographic samples cut from the middle and top sections of the test bars. The minimum, average and maximum pore sizes measured by image analysis were approximately 4, 9 and $23 \mu \mathrm{m}$, respectively. Most pores were spherical in shape and no

\footnotetext{
${ }^{*}$ University of Texas Health Sciences Center
} 
pores had an aspect ratio (the ratio of the maximum and minimum dimensions) exceeding 4.0. The average pore area fraction was approximately $0.15 \%$.

\subsubsection{Microstructural Features after Heat Treatment}

In addition to investigating the macrostructural features after heat treatment, a detailed microstructural investigation was conducted to quantify size, distribution and volume fraction of the $\gamma^{\prime}$ phase, as well as to characterize secondary phases present after heat treatment. As shown in Figure 5.19, some residual eutectic $\gamma-\gamma$ ' and shrinkage porosity was observed in the interdendritic regions. A quantitative analysis of the eutectic $\gamma-\gamma^{\prime}$ was not undertaken, but the occurrence of this phase appeared to be significantly reduced in comparison with the as-cast material. This result was expected, because the purpose of the homogenizing heat treatment is not only to reduce microsegregation, but to solution the eutectic $\gamma-\gamma^{\prime}$ as well reducing its occurrence after heat treatment. The $\gamma^{\prime}$ size and morphology after heat treatment is shown in Figure 5.20. Two distinctly different $\gamma$ ' precipitates sizes were observed; large primary $\gamma^{\prime}$ (Figure 5.20a), which formed during cooling from the homogenizing temperature and later coarsened during the diffusion heat treatment, and hyperfine secondary $\gamma^{\prime}$ (Figure 5.20b), which forms during the secondary aging heat treatment. The size and volume fraction of the primary $\gamma^{\prime}$ was estimated using image analysis techniques. It was determined that the average size and volume fraction of the primary $\gamma^{\prime}$ precipitates was approximately $0.3 \mu \mathrm{m}$ and $62 \%$, respectively.

\subsection{Constitutive Material Behaviour Testing}

Tensile tests were performed to establish the constitutive behaviour of the single-crystal material used in this investigation. The tensile tests were performed using an MTS computer controlled closed-loop servo-hydraulic test machine in accordance with the test 
practices described in ASTM-E8 [252]. The test machine was equipped with a three-zone furnace (MTS Model 653.04) and an air-cooled high-temperature axial extensometer with a gage length of $12 \mathrm{~mm}$ (MTS Model 632.54F-14). Additional details of the experimental setup are shown in Figure 5.21. The tensile test specimens were machined according to the drawing shown in Figure 5.22. As discussed in Chapter 2, single-crystal superalloys in general, exhibit little viscoplastic behaviour at the test temperature $\left(650^{\circ} \mathrm{C}\right)$ considered in this investigation. However, for completeness and to quantify the strain rate dependence of the material employed in this investigation, total strain rate controlled tensile tests were contucted at strain rates of $10^{-2}, 10^{-3}, 10^{-4}$ and $10^{-5} \mathrm{sec}^{-1}$ at $650^{\circ} \mathrm{C}$ for tensile specimens with a nominal $\langle 100\rangle$ orientation. Two tensile tests were performed at each strain rate. The tensile test results will be discussed in the following section.

\subsubsection{Tensile Test Results}

The constant total strain-rate tensile curves for $\langle 100\rangle$ Sophisticated Alloys PWA1484 alloy at $650^{\circ} \mathrm{C}$ are shown in Figure 5.23. In the linear-elastic portion of the tensile curves it appears that the apparent elastic modulus is rate dependent. It is believed that this apparent rate dependence is due to the relatively lower resolution and sensitivity of the extensometer in the elastic region [253] and crystallographic orientation differences between of the individual tensile specimens. One thing that is apparent from the tensile curves is that little rate dependence is exhibited by the material at $650^{\circ} \mathrm{C}$. This can be more closely scrutinized by comparing the saturated flow stress values for tensile tests conducted at total strain rates of $10^{-2}$ and $10^{-5} \mathrm{sec}^{-1}$. In spite of the three order of magnitude change in the total strain rate, a difference of approximately $40 \mathrm{MPa}$ (approximately 4\%) was observed. The predominantly rate independent constitutive material response agrees with the reported low-temperature single-crystal superalloy 
constitutive behaviour discussed in Chapter 2. Therefore, the material was considered rate independent in this investigation.

\subsection{Small Fatigue Crack Growth Rate Testing}

As discussed in Chapter 1, one of the objectives of this investigation was to investigate the effect of secondary crystallographic orientation on crack formation and small FCG for naturally-generated small fatigue cracks in a single-crystal superalloy. A single-edge notched (SEN) specimen design (see Figure 5.24) that approximately represents the stress concentration $\left(\mathrm{K}_{\mathrm{t}} \sim 2.7\right)$ present in a turbine blade fir tree attachment region was specified for the FCG experiments. A total of 24 small FCGR tests were performed; 12 for calibration purposes and 12 to characterize the effect of secondary crystallographic orientation on small FCG properties.

All FCGR tests were performed on single-crystal specimens with a $\langle 010\rangle$ tensile axis and with two different nominal crack growth directions $\langle 101\rangle$ and $\langle 100\rangle$, as shown schematically in Figure 5.25. In Figure 5.25 the nomenclature specified by Reed and coworkers [52] was adopted for the fatigue crack growth specimens with [010]-[101] and [010]-[100] crystallographic orientations (first set of Miller indices specifies the tensile axis and the second set specifies the crack growth direction) which will hereinafter be referred to as orientation $A$ and orientation $B$, respectively. The [100] and [101] crack growth directions were considered in this investigation because these two secondary orientations have the largest variation in transverse elastic properties and symmetry of the material anisotropy precludes out-of-plane distortions that complicate stress analysis of the specimen. It is anticipated that if secondary orientation affects the propagation behaviour of small fatigue cracks that these two notch orientations will define the upper 
and lower bounds for other notch orientations along the [100] and [101] boundary of the standard stereographic triangle.

The SEN specimens were machined by Metcut Research Inc. of Cincinnati OH using conventional practices and finished by low-stress grinding followed by longitudinal polishing to the specified surface finish. To ensure that the secondary orientation of the SEN specimens met specifications, Metcut preserved the secondary orientation markings from the single-crystal test bars sent for machining. Special consideration was given to machining the notch. The notch was machined slightly undersized by wire EDM and finished to specified tolerances by low-stress grinding using a rotary grinding tool. The use of a rotary grinding tool to finish the notch machining process ensured that the grinding marks were circumferential to the notch. To reduce the risk that the sharp edges present at the corners of the notch from machining operations did not facilitate the nucleation of corner cracks, the sharp edges near the notch were broken using low stress grinding operations.

Small FCGR tests were conducted at a temperature of $650^{\circ} \mathrm{C}$ to approximately represent the metal temperature in the blade root of a first-stage turbine blade. The FCGR tests were performed according to the testing practices described in ASTM-E647 [177]. All FCGR tests were conducted under constant load amplitude of $29 \mathrm{kN}$ using a $1 \mathrm{~Hz}$ triangular waveform and a stress ratio $(\mathrm{R})$ of 0.1 . The FCGR tests were performed in an MTS computer controlled servo-hydraulic test machine. A custom Visual Basic program was written using the MTS 793 software application program interface (API) to program and control the test machine and to acquire and process crack growth rate data during testing. A single-zone split box furnace designed for fracture mechanics testing (ATS Series 3600) was used for specimen heating. Additional details of the FCGR test setup 
are shown in Figure 5.26. The fatigue crack formation and small crack growth processes were monitored using a Matelec CGM-7 ACPD measurement system. Additional details on the ACPD hardware, crack probe setup, and equipment and calibration tests are discussed in the following section.

\subsubsection{ACPD Crack Growth Monitoring System}

The Matelec CGM-7 ACPD measurement system was used to monitor the growth of small fatigue cracks in the present investigation. This system consists of a current source that generates a constant amplitude alternating current, PD signal preamplifier and filtering hardware and amplifier with phase sensitive detection circuitry. The current supply is capable of generating a highly stable alternating current with a frequency ranging from $500 \mathrm{~Hz}$ to $230 \mathrm{kHz}$ and has a maximum current capability of $5 \mathrm{~A}$. Potential signals measured at the ACPD voltage probes are amplified by the preamplifier and then pass through the phase detection circuitry which separates the ACPD signal into a resistive (in-phase with the current) and inductive ( $90^{\circ}$ out-of-phase with the current) components. The total ACPD signal consisting of the resistive and inductive components is affected by PD probe placement and other factors and therefore is unsuitable for measuring crack growth [254].

The resistive (in-phase) component is relatively immune to small PD probe placement errors that cause inductive pickup and thus changes in this signal can be related to the crack growth in fatigue specimens. The CGM-7 unit features analog voltage outputs that output the in-phase PD signal, which was used to detect and monitor small crack growth in this investigation. The CGM-7 unit also has built-in signal filtering capabilities to block stray alternating currents that could generate noise in the ACPD signals. The filter settings can be adjusted to specific cutoff frequencies of $0.1 \mathrm{~Hz}, 80 \mathrm{~Hz}$, and $1 \mathrm{kHz}$; a 
filter setting of $1 \mathrm{kHz}$ was used in this investigation. In the following section details of the ACPD PD and current probe design and placement will be briefly discussed.

\subsubsection{ACPD Current and PD Probe Setup}

The ACPD current and PD probe setup developed by Kearsey [255] was employed in this investigation. The PD and current probe setup is shown in Figure 5.27. It was demonstrated [255] that this current and PD probe arrangement facilitated high resolution small crack growth rate measurements and provided a minimum detectable crack depth of approximately $80 \mu \mathrm{m}$. The current probe was fabricated from two lengths of 0.032 -in $(\sim 0.81 \mathrm{~mm})$ diameter Chromel wire. One length of wire was bent to conform to the notch shape to focus the current in the notch root. As shown in Figure 5.28, the current probe wires were twisted together up to the attachment points on the SEN specimen to reduce the current loop area. The current probe wires were attached to the specimen using resistance spot welding and further secured using Cotronics Resbond 919 ceramic adhesive at the wire attachment points. The PD probes were assembled from two lengths of 0.020 -in $(\sim 0.50 \mathrm{~mm})$ diameter Chromel wire. The crack length monitoring probe (working probe) was attached diagonally across the notch using resistance spot welding, as shown in Figure 5.28. A reference probe was also attached $5 \mathrm{~mm}$ below the notch by resistance spot welding, as indicated in Figure 5.28. The PD probes were twisted up to their attachment points to minimize inductive pickup from the current supply wires.

A series of tests were performed to optimize the current source settings (frequency and applied current) and PD amplifier gain settings. From these tests it was established that current settings of $120 \mathrm{kHz}$ and $4.5 \mathrm{~A}$, and a PD amplifier gain setting of 1000 represented a good compromise between crack detection resolution and PD signal noise, 
and more importantly, prevented saturation of the PD signal amplifiers within the small crack size range (crack depths between $0-\sim 1000 \mu \mathrm{m}$ ) of interest in this investigation.

\subsubsection{ACPD Data Acquisition and Signal Analysis}

The ACPD signals for the working and reference probes were acquired from the analog PD signal outputs on the Matelect CGM-7 unit using the analog to digital conversion hardware built into the MTS 793 digital control system. To ensure that the crack was open during data acquisition and for cycle-to-cycle consistency, the PD signals were acquired at the maximum load during each loading cycle. It was observed during preliminary tests of the ACPD system that significant noise (approximately $\pm 1 \mathrm{mV}$ ) existed in the PD signals acquired at the maximum load. Since the detection and monitoring of small fatigue crack growth using potential difference techniques require the ability to detect small changes in PD voltage with relatively low signal to noise ratios, additional measures were taken to reduce this noise both during testing and during the analysis of the fatigue crack propagation data.

In addition to using the analog filtering capabilities of the CGM-7 ACPD system, as noted earlier, digital filtering was applied during the fatigue crack growth rate tests by averaging a series of $\mathrm{PD}$ voltage readings acquired during 10 loading cycles. In practice, more sophisticated PD averaging schemes have been developed, such as an adaptive fatigue crack growth rate measurement scheme in which the number of PD cycles averaged depends on the fatigue crack growth rate, i.e. more PD readings are averaged for slower fatigue crack growth rates [256]. However, the data averaging scheme used in this investigation was observed to be effective in reducing the PD signal noise amplitude (after amplification) to approximately $\pm 0.25 \mathrm{mV}$. This level of noise was sufficiently

small to enable changes in PD signal of approximately $2 \mathrm{mV}$ to be unambiguously 
detected during the ACPD calibration tests but was insufficient for conducting analysis of the fatigue crack growth rate data. Therefore, additional data processing procedures were implemented after the fatigue experiments were completed to further reduce the effect of ACPD signal noise before transforming the PD voltage to a change in PD.

To further reduce the effect of ACPD signal noise a local regression smoothing procedure was applied to the PD versus cycles data obtained during the fatigue experiments. The local regression smoothing procedure introduced by Cleveland [257], referred to as LOESS (locally weighted scatter smoothing), was used to smooth the data. The LOESS technique is a data smoothing procedure in which a smoothed estimate for $y_{\mathrm{i}}$ corresponding to a particular $x_{\mathrm{i}}$ is obtained by performing a weighted regression analysis on a subset (or band) of data points which contains the observation $\left(x_{\mathrm{i}}, y_{\mathrm{i}}\right)$ being evaluated. The weights are assigned to each data point in the band using a weighting function $\left(w_{\mathrm{i}}\right)$ such that the weights decline from 1 at $x_{\mathrm{i}}$ to 0 based on their distance from $x_{\mathrm{i}}$. In the present work a tri-cube weighting function was used. A smoothed estimate for $y_{\mathrm{i}}$ is obtained by performing a weighted least-squares regression analysis using either a linear or quadratic polynomial function. A linear function was used in present case. The bandwidth, that is the number of points to which the polynomial is fitted, is specified by a smoothing parameter $\left(\alpha_{p}\right)$ that ranges from 0 to 1 . A small value for $\alpha_{p}\left(\alpha_{p}=0.04\right)$ was used in the present case to avoid obscuring rapid changes in $y_{\mathrm{i}}$ (the PD voltage reading) that were observed at higher fatigue crack propagation rates. The LOESS smoothing was performed using a Microsoft Excel function developed by Peltier Technical Services, Inc. [258]. Using the LOESS data smoothing technique the scatter in the working probe PD readings was reduced to $\pm 0.05 \mathrm{mV}$, which enabled straightforward identification of the onset of fatigue crack growth from the recorded PD values. 
Changes in specimen temperature during testing, current and PD probe placement, or instrumentation characteristics can cause changes in measured PD voltages. To compensate for these effects the measured working probe voltage can be normalized by a reference voltage measured at a location on the specimen that is unaffected by the growing fatigue crack. Specifically, the working probe PD measurement is divided by the ratio $V_{r e f} / V_{r e f 0}$ where $V_{r e f}$ is the instantaneous reference voltage measurement and $V_{\text {ref } 0}$ represents the initial reference voltage measurement [177]. As discussed previously, the ACPD probe setup used for the SEN specimen considered in the present work included a reference voltage probe. It was observed during fatigue tests that the reference voltage measurement was affected by the propagating fatigue crack and therefore was not used in the analysis of the crack growth data. Instead the reference probe was monitored to verify that the ACPD equipment was stable during the crack growth rate tests before a fatigue crack formed and propagated.

\subsubsection{ACPD Crack Length Calibration Tests}

To determine the crack length-potential drop relationship and establish the crack shape development as a function of crack size, 12 calibration (6 orientation A and 6 orientation B) tests were performed to cover a crack size range of $200-1200 \mu \mathrm{m}$. In order to maximize the number of calibration points obtained from the calibration tests, a marker banding procedure was implemented. The marker banding procedure consisted of interrupting the fatigue crack growth rate test after a specific change in PD signal was detected and then resuming fatigue cycling at a stress ratio of $R=0.7$ with the same maximum load amplitude $(29 \mathrm{kN})$. Fatigue cycling was continued until a small change in the PD signal was detected $(\sim 1 \mathrm{mV})$ after which, the original fatigue cycling parameters were restored $(\mathrm{R}=0.1)$. A marker band (or beach mark) is introduced by this process 
because the change in stress ratio alters the crack growth kinetics. Although $K_{\max }$ is nominally the same for each loading condition the driving force for crack extension ( $\Delta K$ ) is smaller for $\mathrm{R}=0.7$ loading resulting in a substantial decrease in fatigue crack propagation rate. Macroscopically, this change in crack growth rate is apparent on the fatigue specimen fracture surface, as shown in Figure 5.29. The marker banding process was repeated at different PD values to obtain several crack length-potential drop calibration points per calibration test.

After completing the calibration test, a static load of $23 \mathrm{kN}$ was applied for one hour while the specimen was at the test temperature to heat tint the fracture surface. The test specimen was then cooled to room temperature and statically loaded until separation of the specimen into two halves for fractographic analysis. The fracture surfaces were examined using SEM. It was discovered that SEM-BSE imaging provided good contrast for delineating the marker bands from the nominal fatigue crack growth regions on the specimen fracture surfaces (see Figure 5.29). Multiple SEM-BSE images of the fracture surface were taken to generate a detailed composite image of the fracture surface for crack geometry measurements using image analysis techniques. All calibration test specimens were observed to contain a single dominant thumbnail crack after fatigue testing. As shown in Figure 5.29, the thumbnail cracks were macroscopically planar and oriented normal to the applied load consistent with non-crystallographic Mode I (Stage II) crack growth at the macroscale. The PD voltage data associated with each marker band were extracted from the PD versus cycles data acquired during the calibration test (see example shown in Figure 5.30). It is evident from Figure 5.30 that PD voltage increases as the fatigue crack propagates. The discontinuities in the PD versus cycles curve indicate when the load ratio was changed to introduce a marker band. 
The crack depth, surface length and area were determined for each calibration test specimen using the measurement capabilities in UTHSCSA Image Tool version 3. These measurements were then related to the change in PD voltage to generate a calibration curve for each SEN specimen orientation (orientation A and B). The crack depth, half surface crack length and crack area versus PD change calibration curves for orientation A crack growth specimens are shown in Figures 5.31 to 5.33, respectively. Similar calibration curves for orientation B fatigue specimens are presented in Figures 5.34 to 5.36. It was determined that a power law most closely fitted the experimental calibration data, specifically:

$$
c, a, A=C_{1}(\triangle P D)^{n_{1}}
$$

where $c, a$ and $A$ represent the crack depth, the half surface crack length and crack area, respectively, $\triangle P D$ is the change in ACPD signal (in $\mathrm{mV}$ ), and $C_{1}$ and $n_{1}$ are the curve fitting parameters. The curve fitting parameters for each specimen orientation are summarized in Table 5.2. Closer examination of these calibration curves shows that the minimum detectable crack size was approximately $235 \mu \mathrm{m}$ deep based on a PD voltage change of $1.0 \mathrm{mV}$. Analysis of the fatigue crack propagation data considered crack depths in the range of 200 to $1000 \mu \mathrm{m}$ to respect a minimum PD voltage change of approximately $1.0 \mathrm{mV}$. A comparison of the calibration curves derived for both specimen orientations was performed using the fitted calibration curves. The crack depth versus PD change calibration curves for orientation A and B are compared in Figure 5.37. It is apparent that the calibration curves for each orientation are different. It was speculated that this apparent orientation dependence of the ACPD calibration curves could be rationalized by considering the crack shape and its effect on the crack area. 
To investigate the orientation dependence of the crack shape, the measured crack aspect ratios, $a / c$, were plotted as function of the normalized crack depth, $c / W$, for both specimen orientations. The results for orientation A and B are shown independently in Figure 5.38 and Figure 5.39, respectively. A linear curve fit was determined to provide the best fit to this data, i.e.,

$$
\frac{a}{c}=L_{1}\left(\frac{c}{W}\right)+L_{2}
$$

where $L_{1}$ and $L_{2}$ are the curve fitting parameters. The curve fit parameters for both orientations are summarized in Table 5.3. The crack aspect ratio versus normalized crack depth curves were compared for orientation $\mathrm{A}$ and $\mathrm{B}$, as shown in Figure 5.40. It is apparent that the crack aspect ratios were different for both orientations. The effect of crack area on the PD change was investigated to determine if similitude between the two orientations could be achieved. The calibration curves for the crack area versus PD change were compared for orientation $\mathrm{A}$ and $\mathrm{B}$, as shown in Figure 5.41. It can be observed that the agreement between the calibration curves for orientation A and B is excellent. This implies that the orientation dependence of the ACPD crack calibration curves was primarily due to the crack aspect ratio and its evolution as function of crack depth being different for each specimen orientation. Furthermore, because the crack aspect ratio is dependent on specimen orientation it must be taken into consideration when calculating the fracture mechanics parameters to characterize the fatigue crack growth behaviour.

Although the ACPD calibration curves based on crack area resolve the orientation apparent orientation dependence of the ACPD calibration curves, the crack size ( $c$ and $a$ ) 
versus $P D$ change calibration curves were used to analyze the ACPD data. These relationships were used because they could be applied directly to the crack growth data without having to perform supplementary calculations to convert the crack area into fundamental crack dimensions.

\subsubsection{Fatigue Data Analysis}

A representative plot of the ACPD working probe voltage (absolute) versus cycles for a small fatigue crack growth rate test is shown in Figure 5.42. Similar to the ACPD calibration tests discussed earlier it can be observed that the working probe voltage increases with number of cycles once a small fatigue crack has formed and begins to propagate. In order to determine the fatigue crack growth rate the ACPD curves must be transformed into a PD change $(\triangle P D)$ versus cycles $(\mathrm{N})$ curve. This transformation was performed by first identifying the working probe PD voltage at the onset of FCG, that is the point at which the PD readings deviate from a tangent line fitted through the steadystate PD voltage, as shown schematically in Figure 5.43. After the onset PD voltage $\left(P D_{0}\right)$ was identified the change in working probe PD voltage was calculated using,

$$
\triangle P D=P D_{N}-P D_{0}
$$

where $P D_{N}$ is the PD voltage associated with a particular cycle $\mathrm{N}$.

Fatigue analysis software developed by Fracture Technology Associates (FTA), Automated Fatigue Crack Growth Analysis version 3.03.02 [259], was used to perform the calculations to transform the measured PD change versus cycles data into FCGR curves. The software uses the data reduction techniques described in ASTM E647 [177] to perform the FCGR calculations. User defined empirical equations (of a specific form) 
can be specified for the PD change versus crack size and crack-tip parameter solutions to enable fatigue crack growth rate calculations to be performed on any laboratory fatigue specimen geometry. In addition, the software includes capabilities to correct the PD crack length measurements using visual crack length measurements obtained during testing, or after testing is completed. The visual crack geometry correction features were not used in the present investigation. Other capabilities of the crack analysis software are described in Reference [259].

The FTA analysis software transforms the PD voltage versus cycles data to crack lengths using built-in expressions for common laboratory specimens, user defined polynomial expressions (up to $5^{\text {th }}$ order), or a look-up table consisting of voltage change and normalized crack length (such as $c / W$ ) data pairs. A look-up table was used for this transformation since the power law equations defined for PD voltage change versus crack length relationship could not be accurately represented by a polynomial expression. The fatigue crack growth rate was calculated using the incremental polynomial method described in ASTM E647 [177]. In this procedure, a second-order polynomial is fitted to seven successive $a, N$ (or $c, N$ ) data points using the least squares method to minimize the deviation between the observed and fitted crack lengths over the range $a_{i-3}<a<a_{i+3}$ (or $c$ ). The crack length at the midpoint of the fitted polynomial, designated by $\hat{a}$ (or $\hat{c}$ ), is calculated at that mid-point cycle count, $N_{i}$, which is precisely known. The fatigue crack growth rate is calculated from the derivative of the fitted polynomial evaluated at $N_{i}$. The value of the crack-tip parameter, such as $\Delta K$, associated with the FCGR is calculated using the fitted crack length, $\hat{a}$ (or $\hat{c}$ ). One problem with this procedure is that the first and last three points in the $a$ or $c$ versus $N$ data set are discarded during the FCGR calculations. To overcome this issue, the FCGR was calculated for the first and 
last three data points in the $a$ (or $c$ ) versus $N$ data set using the modified-secant method described in Reference [259].

The incremental polynomial procedure provides some smoothing of the FCGR data. However, substantial scatter in crack growth rates may be observed when crack growth data are recorded too frequently. To reduce the scatter associated with the fatigue crack growth rate calculations, the FTA analysis software includes two types of data filters. These filters are used to specify a minimum crack length change, $\Delta a$ (or $\Delta c$ ), and the maximum change in cycles, $\Delta N$, before a crack growth rate calculation is performed. In this investigation these filters were configured as follows:

- Minimum $\Delta a$ (or $\Delta c)=20 \mu \mathrm{m}$, and

- Maximum $\Delta N=100$ cycles.

The FTA analysis software uses polynomial expressions for calculation of the crack-tip parameter ( $\Delta K$ or $J$ in the present case) associated with a specific crack growth rate and crack length. These polynomial fitting parameters (up to $5^{\text {th }}$ order) can be specified by the user, but must conform to the stress-intensity factor solution for the compact tension specimen geometry specified in ASTM E647 [177]. For a fourth-order polynomial, the stress-intensity factor range solution takes on the following form:

$$
\Delta K=\frac{\Delta P}{B \sqrt{W}} \frac{\left(2+\alpha_{g}\right)}{\left(1-\alpha_{g}\right)^{3 / 2}}\left(C_{1}+C_{2} \alpha_{g}+C_{3} \alpha_{g}^{2}+C_{4} \alpha_{g}^{3}+C_{5} \alpha_{g}^{4}\right)
$$

where $\Delta P$ is the load range, $B$ is the specimen thickness, $W$ is the specimen width, $C_{n}$ are the polynomial coefficients, and $\alpha_{g}=a / W$, the crack size to ligament ratio. The 
geometrical parameters in equation 5.4 are specific to the compact tension specimen and therefore are not applicable for surface cracks in a SEN specimen However, with judicious selection of the geometrical parameters this equation can be adapted to calculate the appropriate stress intensity factors in the FTA post processing software. These calculations, however, must be performed independently for the crack growth in the $a$ and $c$ directions. Further details on the procedures to adapt this equation to the SEN specimen and identification of the polynomial coefficients are discussed further in Chapter 8.

One of the primary objectives of this investigation was to quantitatively establish the effect of secondary crystallographic orientation, if any, on the propagation of naturallygenerated, notch root small fatigue cracks. Indeed, this is a complex problem because of variability that is inherent in FCGR data, even when the experiments are performed under identical testing conditions. Therefore, to ensure that any differences in FCGR between the two secondary crystallographic orientations considered in the present study are statistically significant, the variability in crack growth rate must be quantified. This requires both a population of FCGR data obtained using the same test parameters and statistical processing of the fatigue crack growth rate curves obtained for both secondary crystallographic orientations.

In the present study, a population of FCGR data was established by concentrating on one testing condition with 6 replicate tests for each secondary crystallographic orientation. The variability in the crack growth rate is established by statistical analysis of the FCGR data to determine the mean crack FCGR and it standard deviation as a function of $\Delta K$ or the J-integral. The statistical analysis procedures described by Clark and Hudak [260] and 
McKeighan et al. [261] were employed in this study. These procedures are described later in this section.

Statistical processing of the fatigue data requires comparison of multiple FCGR curves consisting of the crack growth rate $(d a / d N)$ as a function of $\Delta K$ or the J-integral. This task is facilitated by comparing the FCG rates at the same $\Delta K$ or the J-integral level. However, FCGR data are usually processed on the basis of crack length change or cycle intervals, and therefore the $\Delta K$ or the J-integral level for each crack growth curve will be different. To overcome this issue, a crack growth rate data interpolation procedure devised by McKeighan et al. [261] was applied to the FCGR data so that crack growth rate comparisons could be conducted at the same $\Delta K$ or the J-integral level. This interpolation procedure is described in this section.

\subsubsection{Crack Growth Rate Data Interpolation Method}

The crack growth rate data interpolation procedure consists of dividing the range (the highest minus the lowest $\Delta K$ or the J-integral for the data set) of $\Delta K$ or the J-integral over which the crack growth data were obtained into fixed intervals. In the case of $\Delta K$ the crack growth rates were interpolated using an interval of $1 \mathrm{MPa}-\mathrm{m}^{1 / 2}$. A smaller interval size of $0.2 \mathrm{MPa}-\mathrm{mm}$ was used for the J-integral since the crack growth data set spans a smaller J-integral range. The interpolation was conducted in $\log \mathrm{da} / \mathrm{dN}-\log \Delta K$ (or Jintegral) space between two points that bridge the $\Delta K$ or the J-integral interval using an interpolation function. In the present case, the Paris relationship (equation 3.23) was used for $\Delta K$ data interpolation and the Dowling-Begley (equation 3.29) relationship was used for the J-integral. Additional details on the interpolation procedure can obtained from Reference [261]. 


\subsubsection{Statistical Analysis of Crack Growth Rate Data}

Once all fatigue crack growth rate data sets were interpolated to the same $\Delta K$ or the Jintegral intervals the mean and standard deviation of the crack growth rates $(d a / d N)$ were calculated at a given $\Delta K$ or the J-integral level. These statistical parameters were calculated in $\log d a / d N$ space. The statistical calculations were performed individually on the crack growth rate data sets obtained from orientation $\mathrm{A}$ and $\mathrm{B}$ specimens. The variability in the FCG rates was then calculated at each $\Delta K$ or the J-integral level based on the FCGR ratio, $10^{4 \mathrm{~b}}$, given by:

$$
10^{4 b}=\frac{10^{(M+2 b)}}{10^{(M-2 b)}}
$$

where $M$ is the mean crack growth rate and $2 b$ represents 2 standard deviations from the mean. To assess the effect of secondary crystallographic orientation on the FCGR, the mean crack growth rate curves obtained for orientation A and B specimens were compared. Additional details on the comparison procedure are described in Chapter 10.

\subsection{Concluding Remarks}

Master alloy ingots having the nominal composition of the second generation singlecrystal superalloy PWA1484 were procured for recasting into single-crystal test bars. A Bridgeman casting process that made use of a single-crystal seed to initiation singlecrystal solidification was successfully utilized to produce the single-crystal test bars used in this investigation. 
The as-cast and heat treated macro and microstructures of the single-crystal test bars was investigated using optical and scanning electron microscopy techniques. In the as-cast state, the dendritic structure was apparent and small interdendritic shrinkage pores were observed at that macroscale. The as-cast microstructure consisted of coarse primary $\gamma$ ' precipitates in a $\gamma$ matrix, eutectic $\gamma-\gamma^{\prime}$ and massive $\gamma^{\prime}$ precipitates in the interdendritic regions, and tantalum-rich carbides which were rarely observed. After application of the standard heat treatment procedures for PWA1484 the microstructure consisted of $0.3 \mu \mathrm{m}$ primary $\gamma^{\prime}$ precipitates with a volume fraction of approximately $62 \%$ and hyperfine secondary $\gamma^{\prime}$ in a $\gamma$ matrix, eutectic $\gamma-\gamma^{\prime}$ and tantalum-rich carbides. The as-cast dendritic structure was much less apparent after solution heat treatment and the occurrence of the eutectic $\gamma-\gamma^{\prime}$ phase was significantly reduced from the as-cast condition.

Tensile tests were performed to establish the $\langle 100\rangle$ constitutive material behaviour at $650^{\circ} \mathrm{C}$ for the single-crystal alloy considered in this investigation. The tensile tests were performed under constant total strain rate conditions with strain rates between $10^{-2}$ and $10^{-5} \mathrm{sec}^{-1}$. In spite of testing at total strain rates that varied by 3 orders of magnitude, the constitutive material behaviour exhibited little rate dependence, and therefore was considered to be rate independent.

The current focusing ACPD technique was successfully implemented to facilitate the detection of surface cracks and the measurement of small fatigue crack growth properties. It was shown that this technique was capable of reliably detecting surface-flaws with crack depths as small as $200 \mu \mathrm{m}$, which is approximately $2 \times$ larger than reported in reference [255]. The detection of smaller cracks was primarily inhibited by the subsurface nucleation of fatigue cracks at shrinkage porosity (see Chapter 9) and the 
higher test temperatures utilized in this investigation, which reduce the sensitivity of the current focusing ACPD technique.

Fatigue crack growth rate experiments were performed on single-edge notch fatigue specimens with a [010] crystallographic orientation aligned with the loading axis and with [101] (orientation A) and [100] (orientation B) crack growth orientations. A marker banding technique was utilized to investigate the variation in crack shape with crack size and to develop calibration curves that relate the ACPD voltage change to crack size for a crack depth range of $200-1000 \mu \mathrm{m}$. Separate calibration curves were identified for orientation A and B fatigue specimens. Additional small fatigue crack growth rate tests were performed without the marker banding technique to investigate the effect of secondary crystallographic orientation on the FCG behaviour of small fatigue cracks. The results of these FCGR experiments are discussed in Chapters 9 and 10. 
Table 5.1 - Target composition range for PWA1484 and the actual composition of the master alloy material (heat $\# 01091032 \mathrm{~A}$ ) received from Sophisticated Alloys.

\begin{tabular}{|l|c|c|c|c|c|c|c|c|c|}
\hline \multirow{2}{*}{ Material } & \multicolumn{10}{|c|}{ Element (Wt. \%) } \\
\cline { 2 - 10 } & Cr & Co & Mo & W & Ta & Al & Hf & Re & Ni \\
\hline PWA1484 & $4.75-$ & $9.50-$ & $1.70-$ & $5.60-$ & $8.40-$ & $5.50-$ & $0.05-$ & $2.80-$ & Bal. \\
& 5.25 & 10.50 & 2.10 & 6.20 & 9.00 & 5.80 & 0.15 & 3.20 & \\
\hline Heat \#01091032A & 5.04 & 9.97 & 2.02 & 5.85 & 8.43 & 5.12 & 0.09 & 2.92 & Bal. \\
\hline
\end{tabular}

Table 5.2 - ACPD power law calibration curve fitting parameters.

\begin{tabular}{|c|c|c|c|c|c|c|}
\hline \multirow{2}{*}{ Orientation } & \multicolumn{2}{|c|}{ Crack Depth, c } & \multicolumn{2}{c|}{ 1/2 Surface Length, a } & \multicolumn{2}{c|}{ Crack Area } \\
\cline { 2 - 7 } & $\begin{array}{c}\mathbf{C}_{\mathbf{1}} \\
(\mu \mathbf{m} / \mathbf{m V})\end{array}$ & $\mathbf{n}_{\mathbf{1}}$ & $\begin{array}{c}\mathbf{C}_{\mathbf{1}} \\
(\mu \mathbf{m} / \mathbf{m V})\end{array}$ & $\mathbf{n}_{\mathbf{1}}$ & $\begin{array}{c}\mathbf{C}_{\mathbf{1}} \\
\left(\mathbf{m m}^{\mathbf{2}} / \mathbf{m V}\right)\end{array}$ & $\mathbf{n}_{\mathbf{1}}$ \\
\hline $\mathrm{A}$ & 222.042 & 0.297 & 169.984 & 0.410 & 0.065 & 0.697 \\
\hline $\mathrm{B}$ & 252.805 & 0.289 & 172.123 & 0.419 & 0.067 & 0.689 \\
\hline
\end{tabular}

Table 5.3 - ACPD Crack aspect ratio calibration curve fitting parameters.

\begin{tabular}{|c|c|c|}
\hline \multirow{2}{*}{ Orientation } & \multicolumn{2}{|c|}{ Crack Aspect Ratio, a/c } \\
\cline { 2 - 3 } & $\mathrm{L}_{1}$ & $\mathrm{~L}_{2}$ \\
\hline $\mathrm{A}$ & 6.503 & 0.738 \\
\hline $\mathrm{B}$ & 6.238 & 0.698 \\
\hline
\end{tabular}



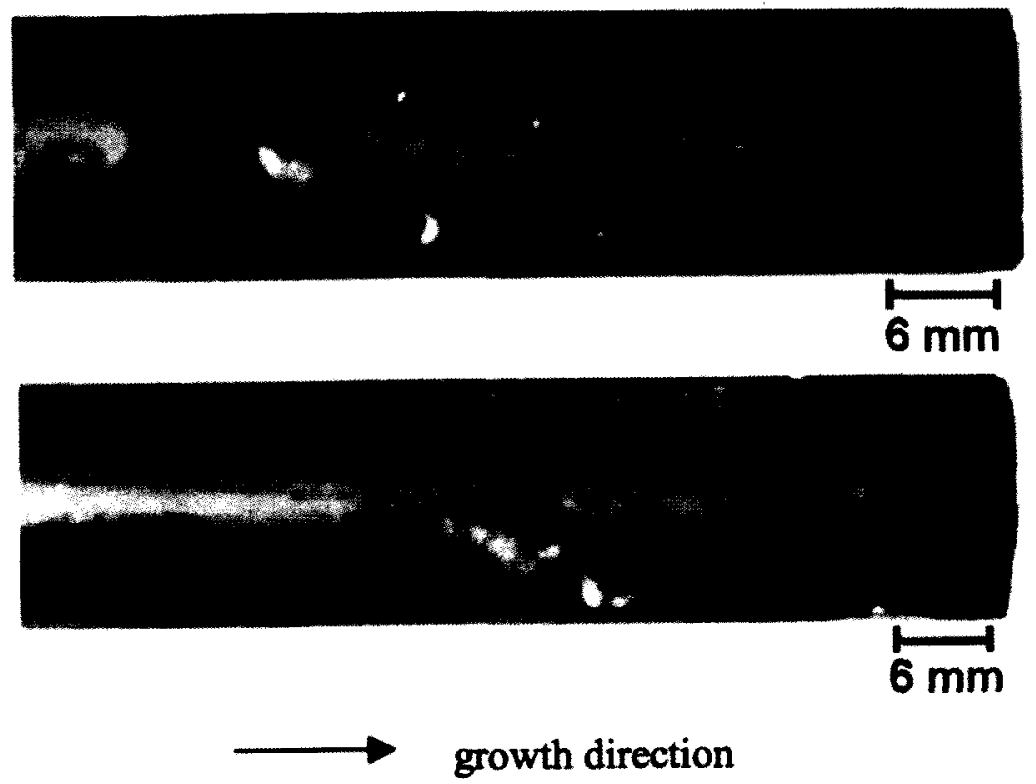

Figure 5.1 - PWA1484 test bars with large pores located near the top of the casting.

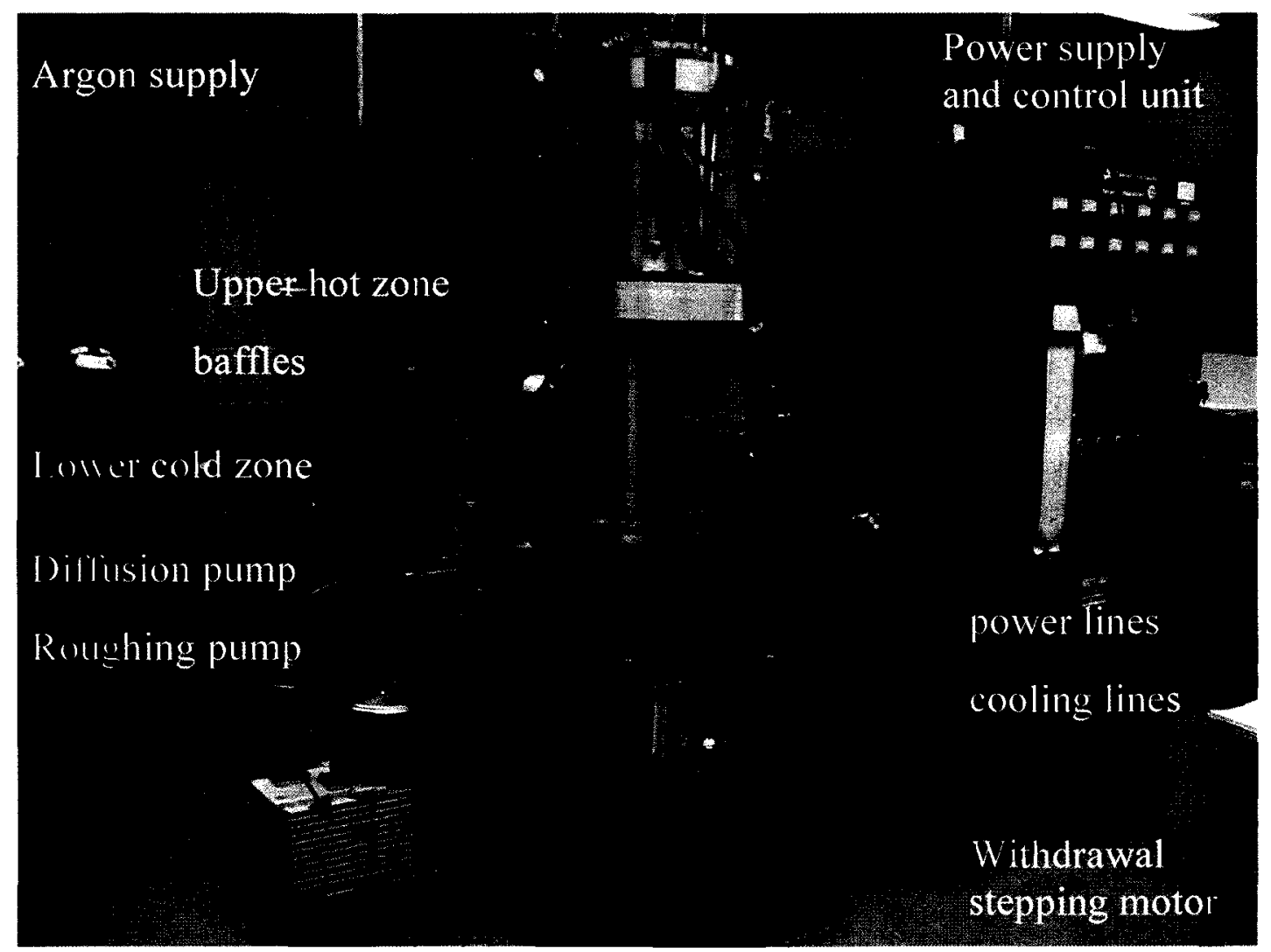

Figure 5.2 - Carleton University pilot scale Bridgman casting furnace. 


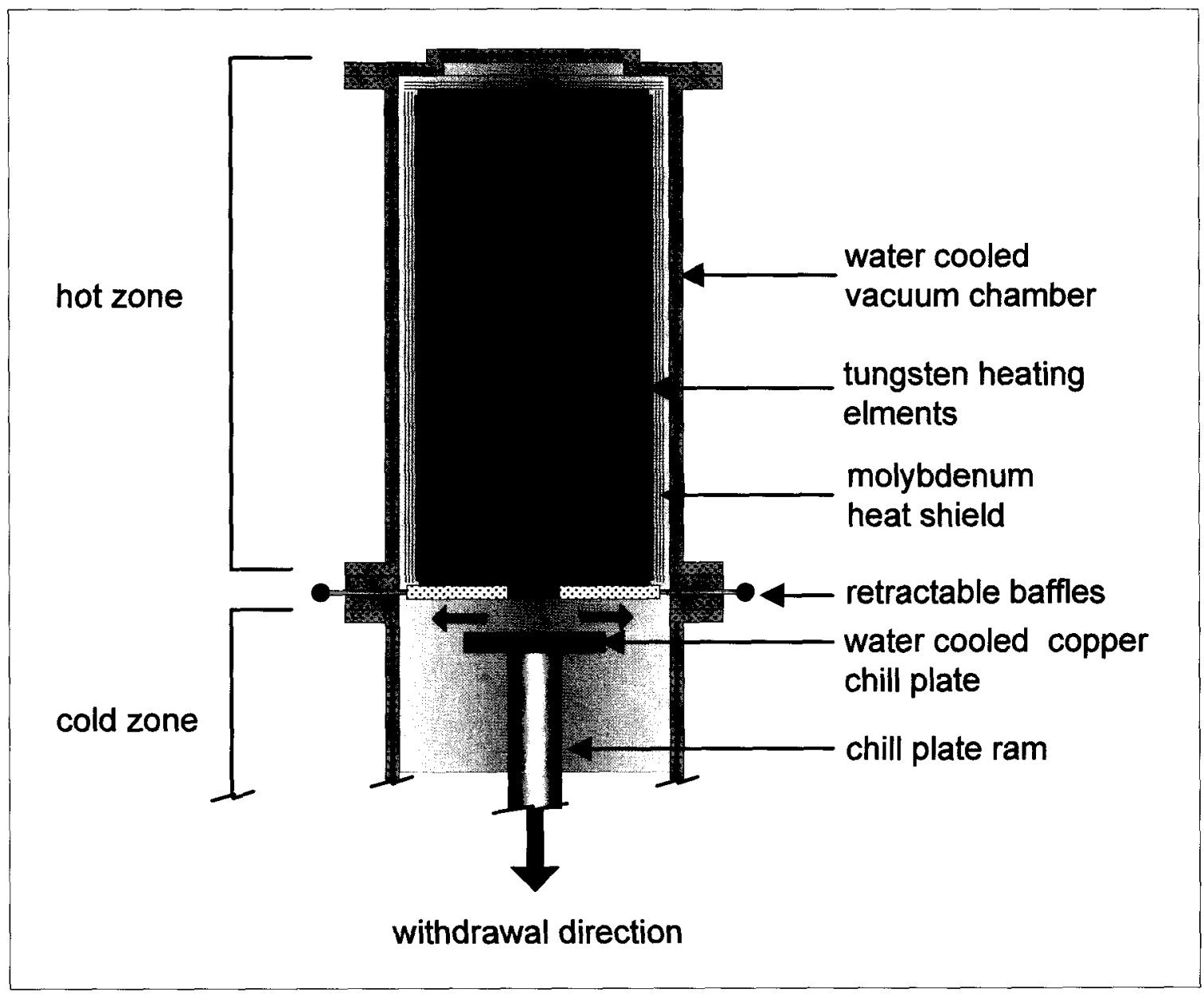

Figure 5.3 - Schematic diagram showing major components of the Bridgeman casting furnace. 


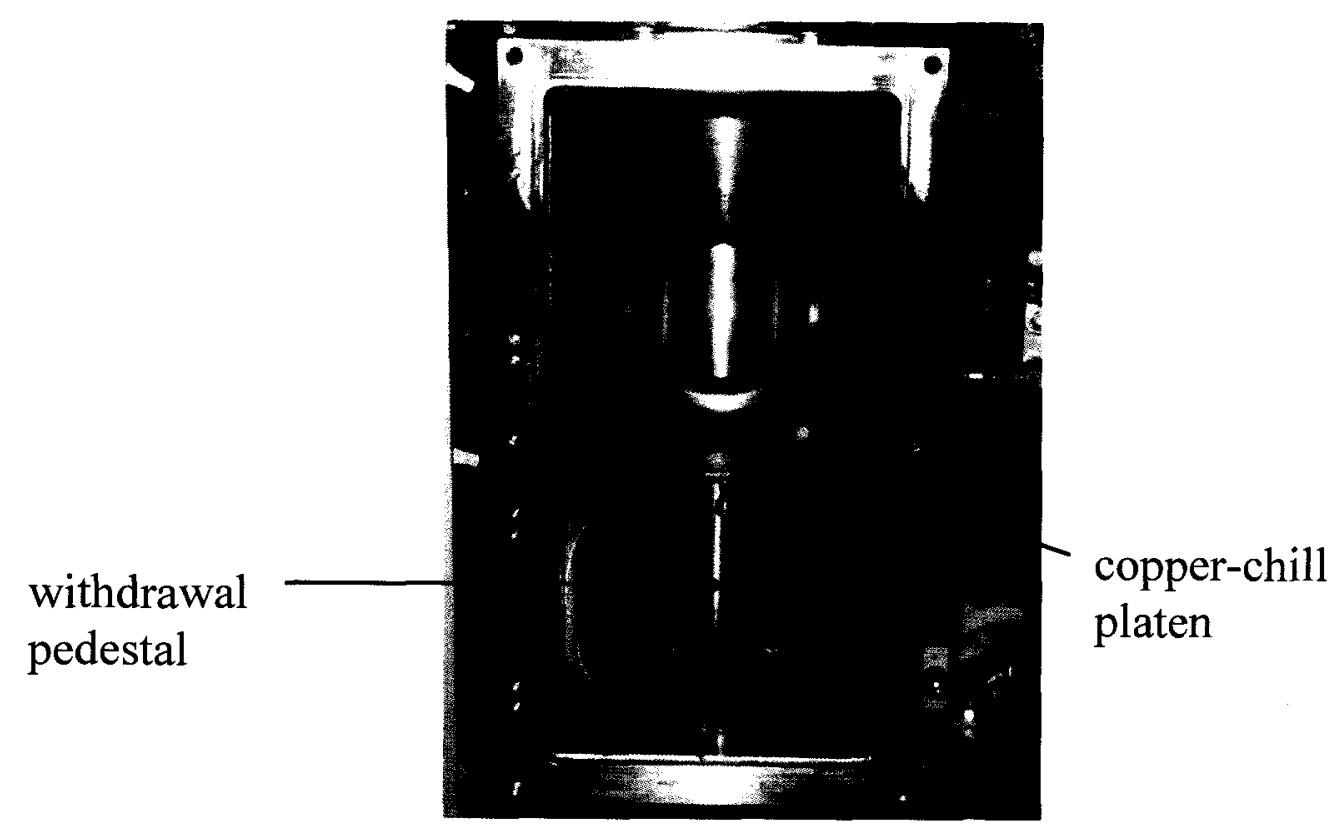

Figure 5.4 - Detailed view of Bridgman furnace cold zone.

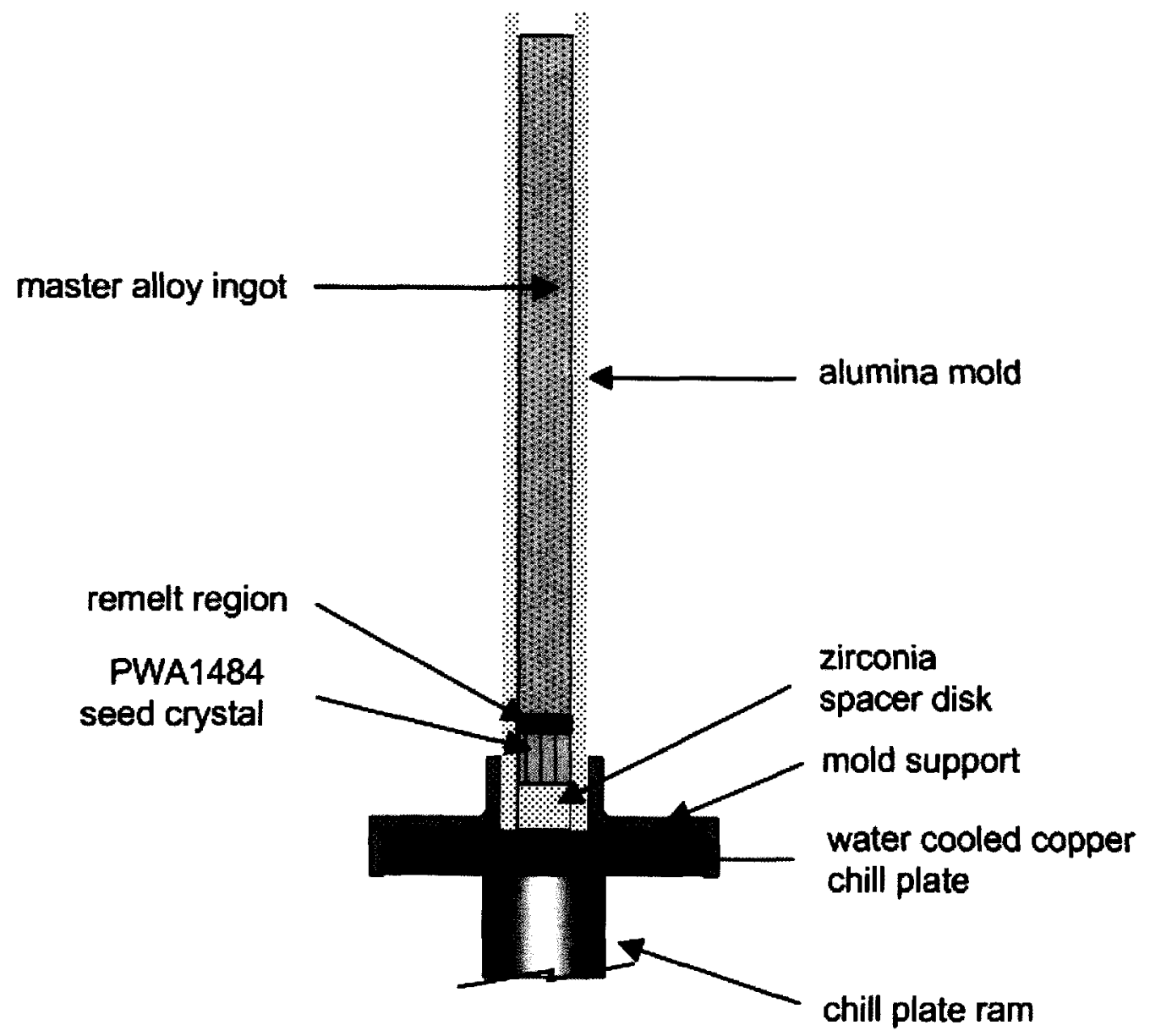

Figure 5.5 - Detailed schematic of the single-crystal seed casting setup. 


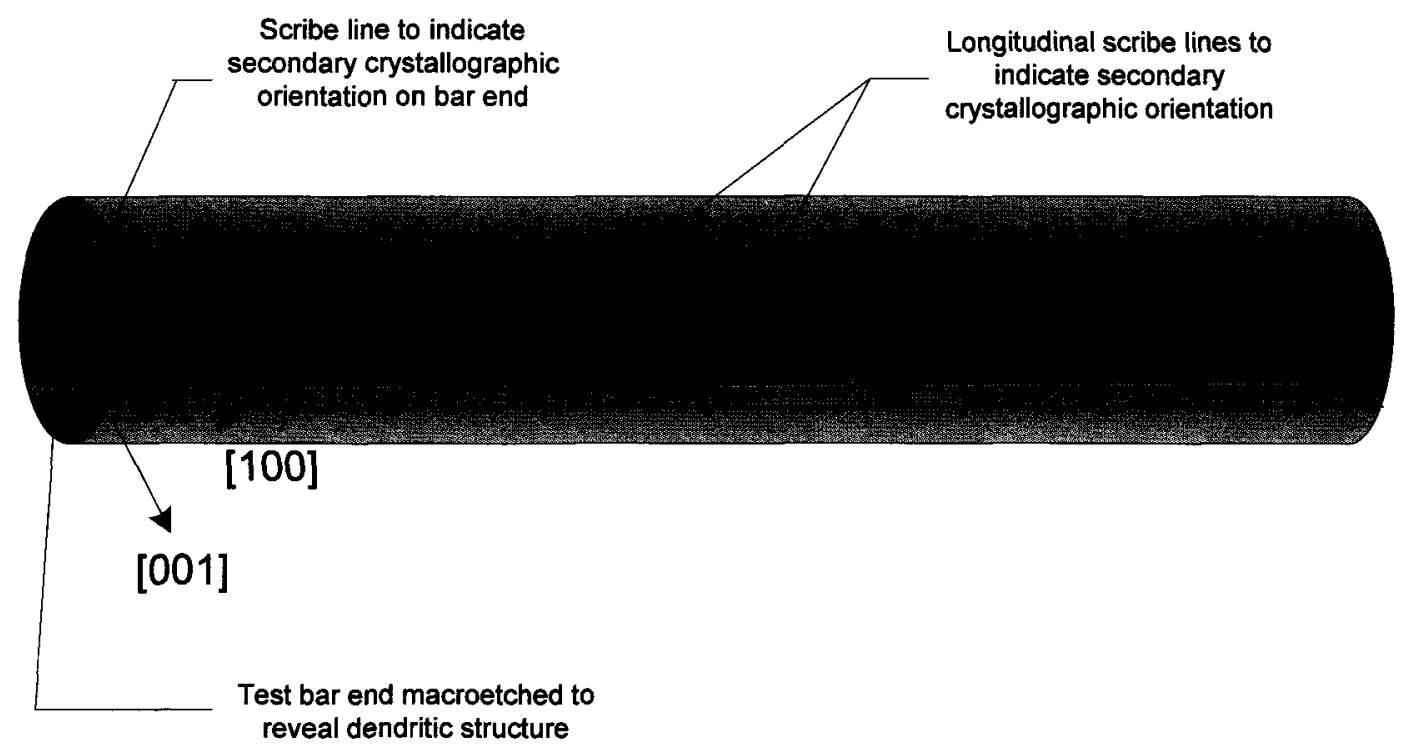

Figure 5.6 - Schematic showing the method used to mark the secondary orientation of the seed crystal test bar.

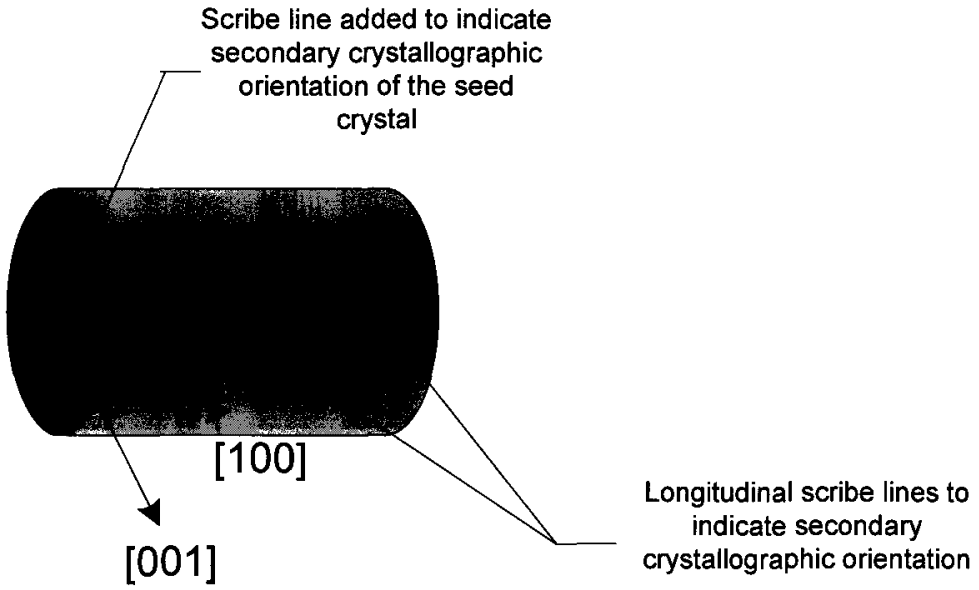

Figure 5.7 - Schematic showing the identification of the secondary orientation for the seed crystal from the longitudinal scribe lines added to the seed crystal test bar. 


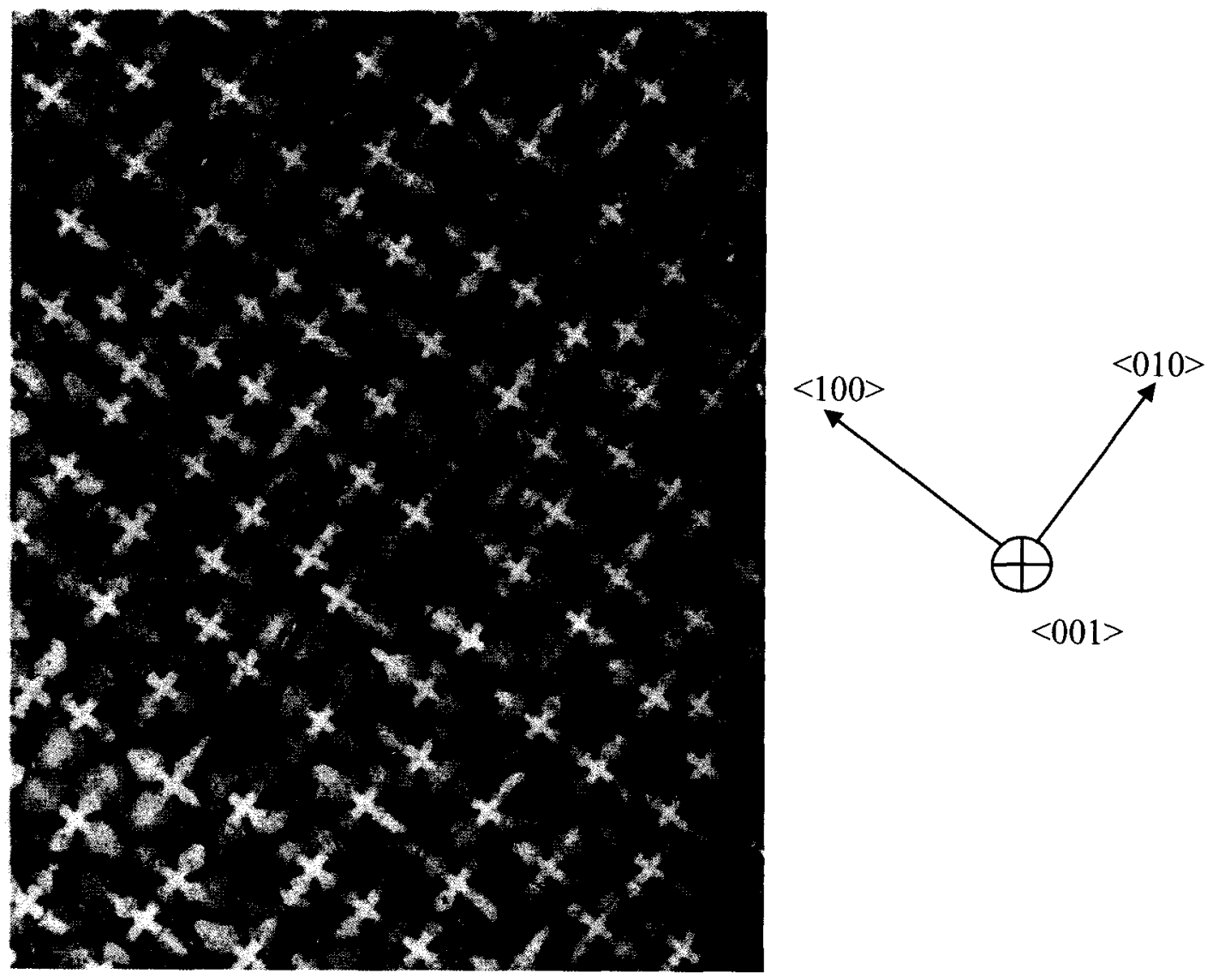

Figure 5.8 - Transverse section showing the dendrite structure in cube oriented SX PWA 1484 in the as-cast condition [262]. The arrows at the right indicate the crystal orientation with respect to the dendritic structure. 


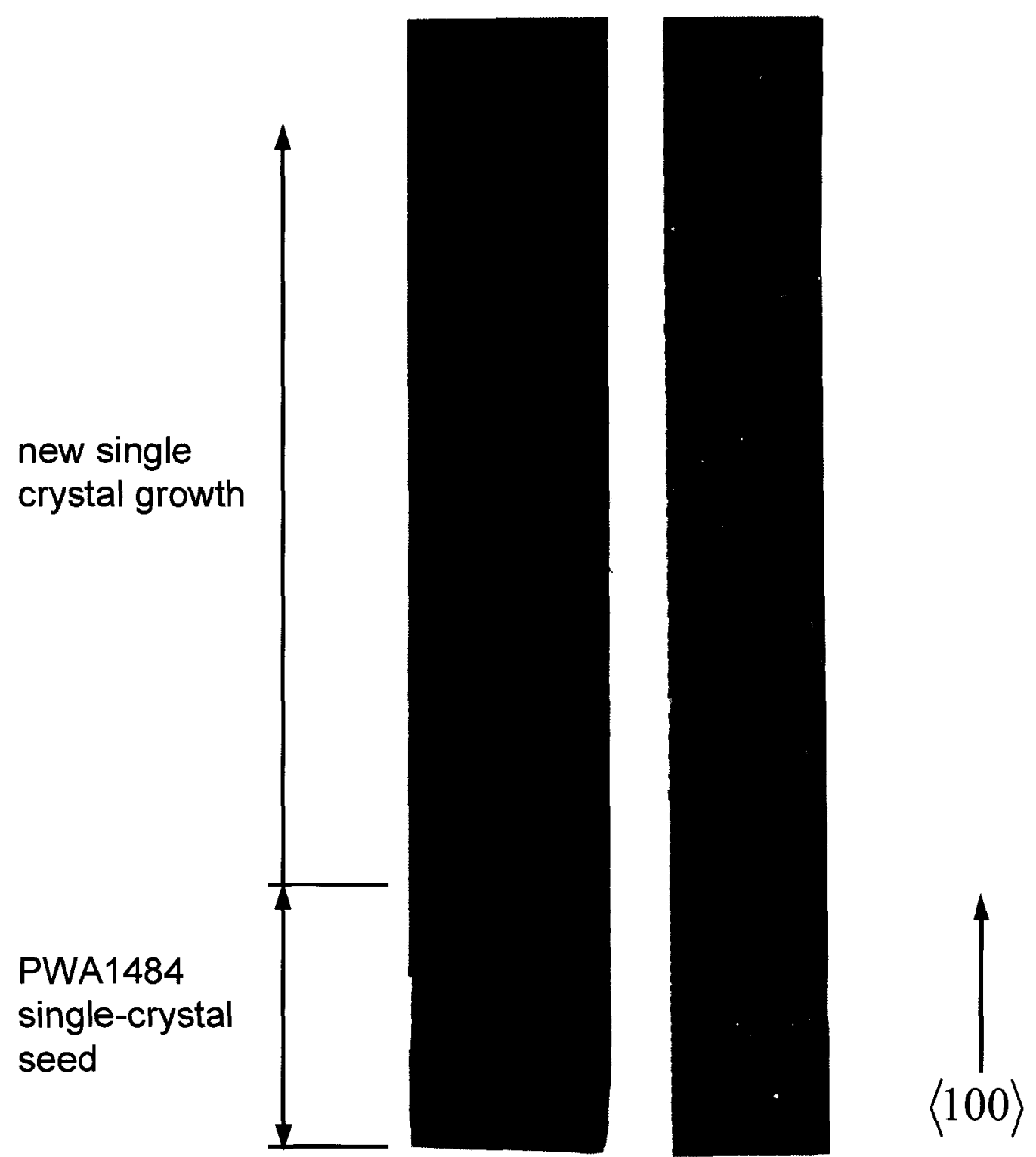

Figure 5.9 - Partial longitudinal cross section showing the seed crystal and new singlecrystal growth in a Bridgman cast single-crystal test bar. 

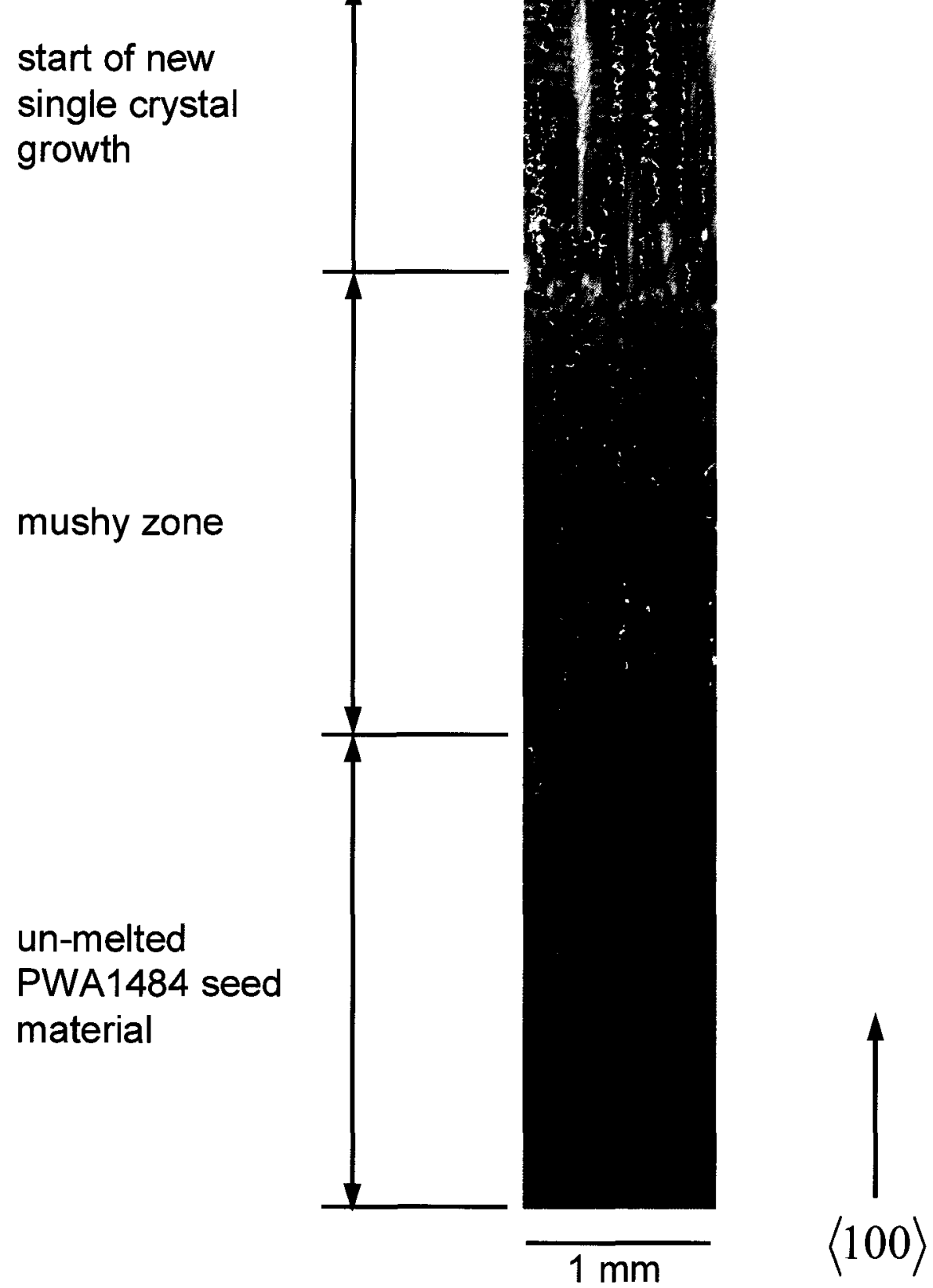

Figure 5.10 - Composite optical micrograph showing a close up view of the longitudinal single-crystal dendritic macrostructure near the seed crystal. 


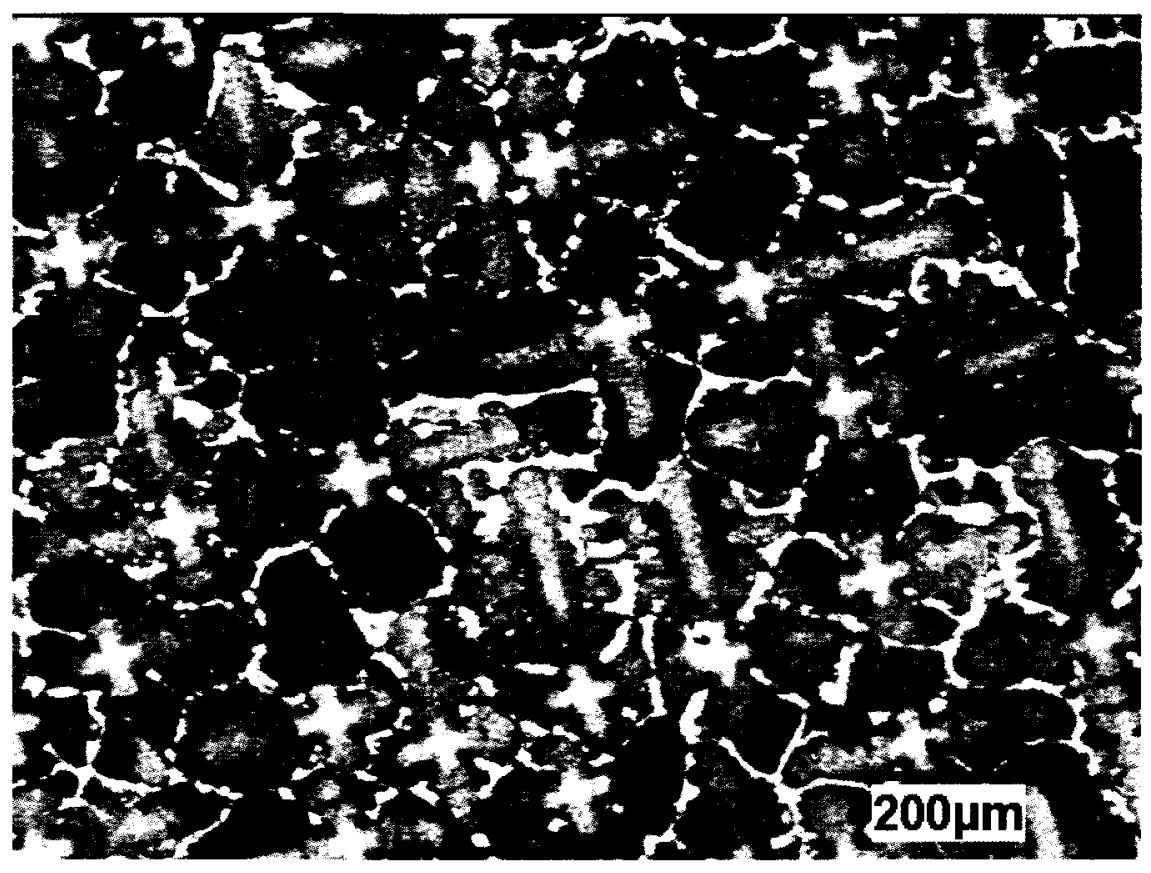

Figure 5.11 - Optical micrograph showing the as-cast solidification structure in a transverse section cut from the middle of a single-crystal test bar.

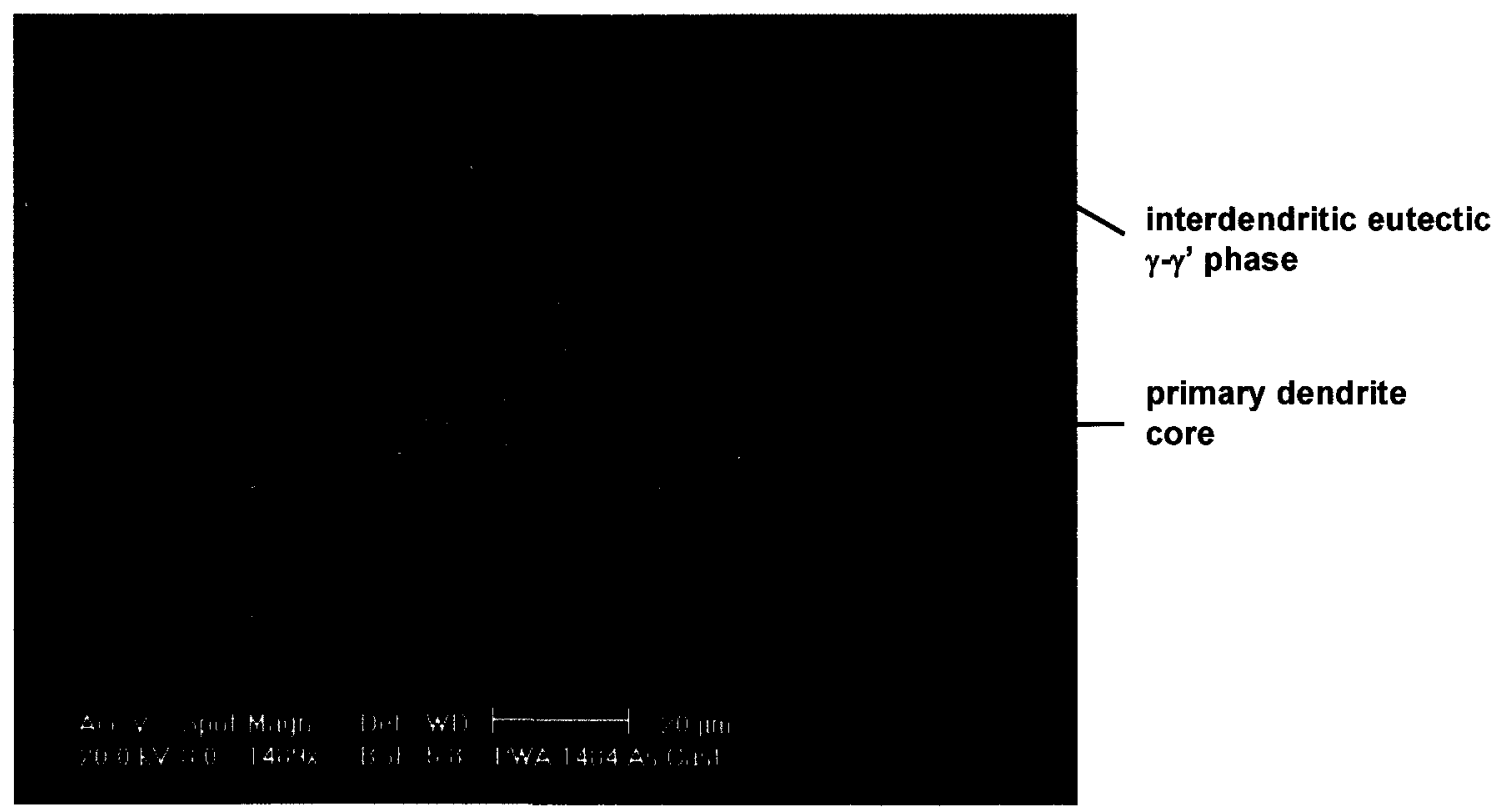

Figure 5.12 - Backscatter electron micrograph showing the variation in $\gamma^{\prime}$ size within an individual primary dendrite core, the secondary dendrite arms and the interdendritic regions. 
coarse $\gamma^{2}$ phase near interdendritic region

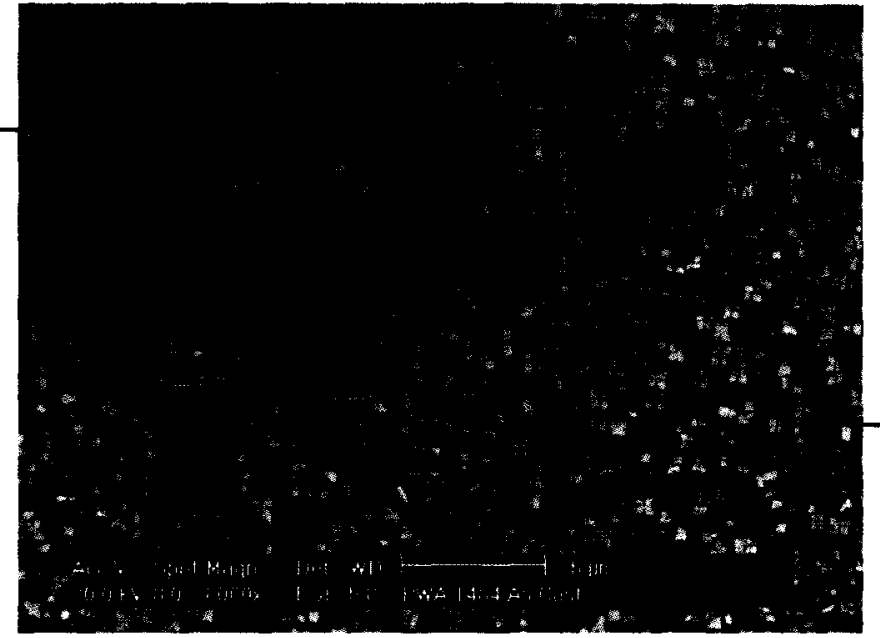

finer $\gamma^{\prime}$ phase near the primary dendrite core and within secondary dendrite arms

Figure 5.13 - Backscatter electron micrograph showing higher magnification view of the region indicated in Figure 5.12. The variation in $\gamma^{\prime}$ size between the primary dendrite core, secondary dendrite arms and the periphery of the dendritic structure is more apparent.

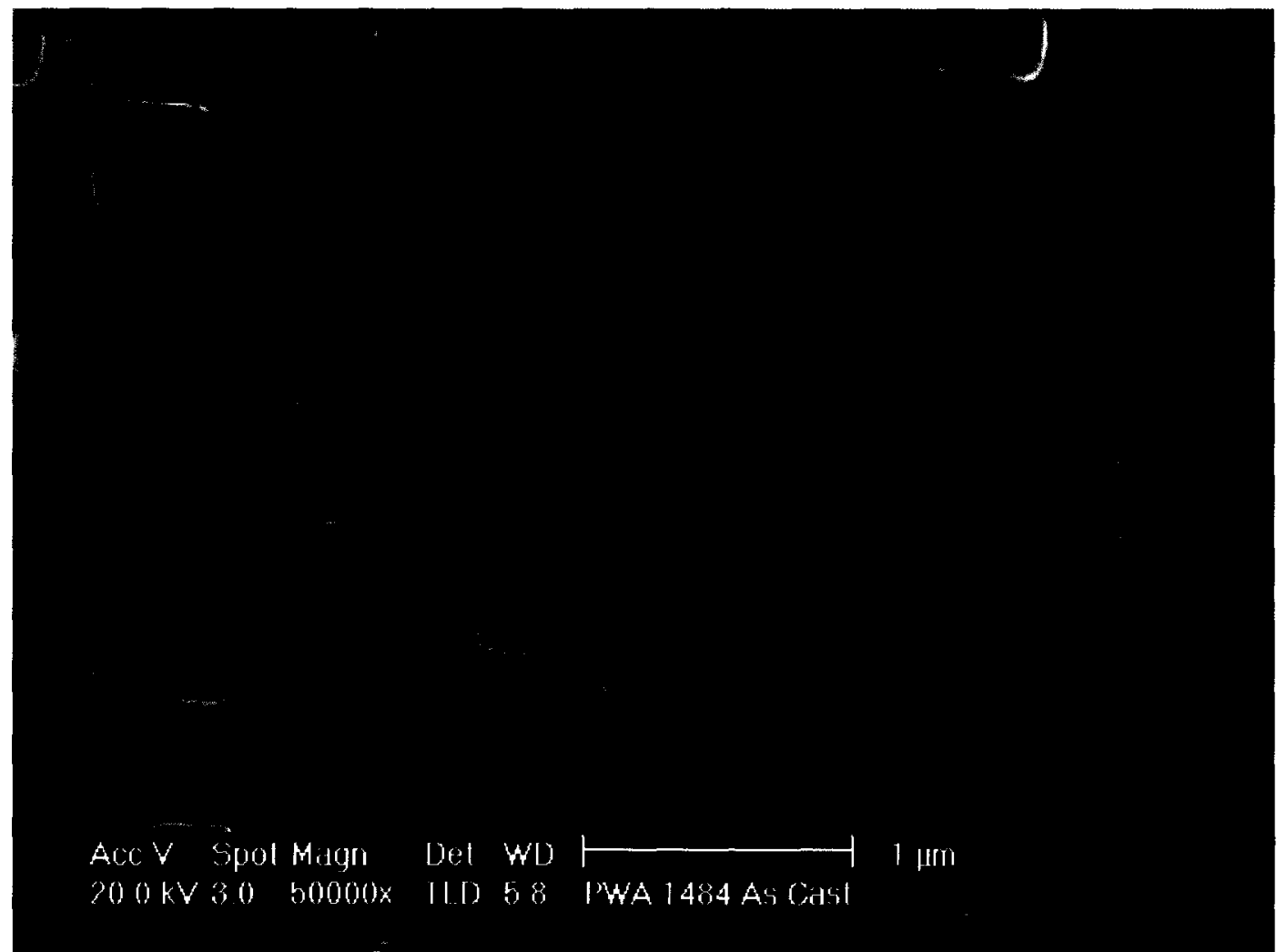

Figure 5.14 - SEM micrograph showing the finer $\gamma$ '-phase located within the primary dendrite core. 


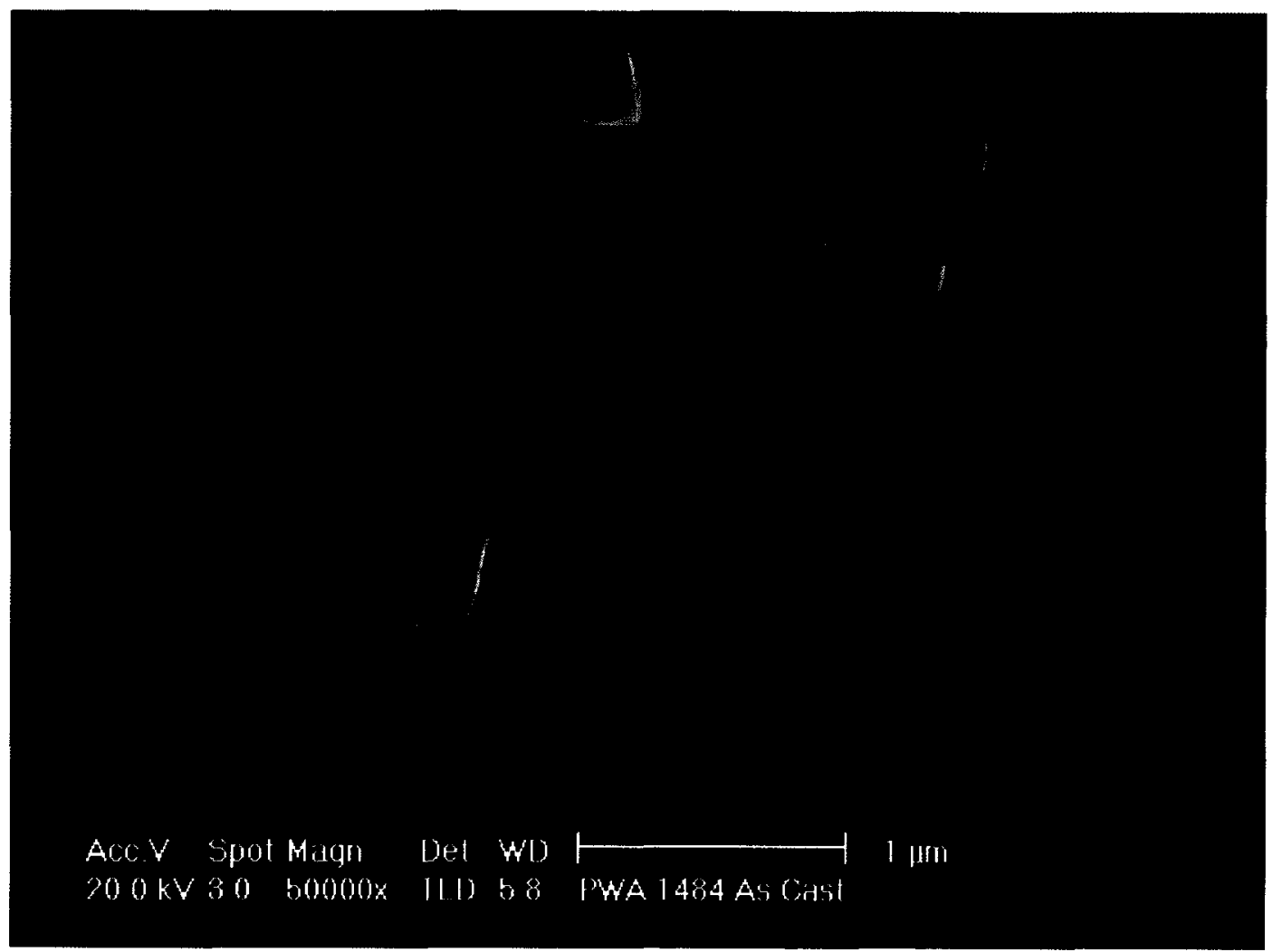

Figure 5.15 - SEM micrograph showing the larger $\gamma$ '-phase located between the dendrite core and interdendritic regions.

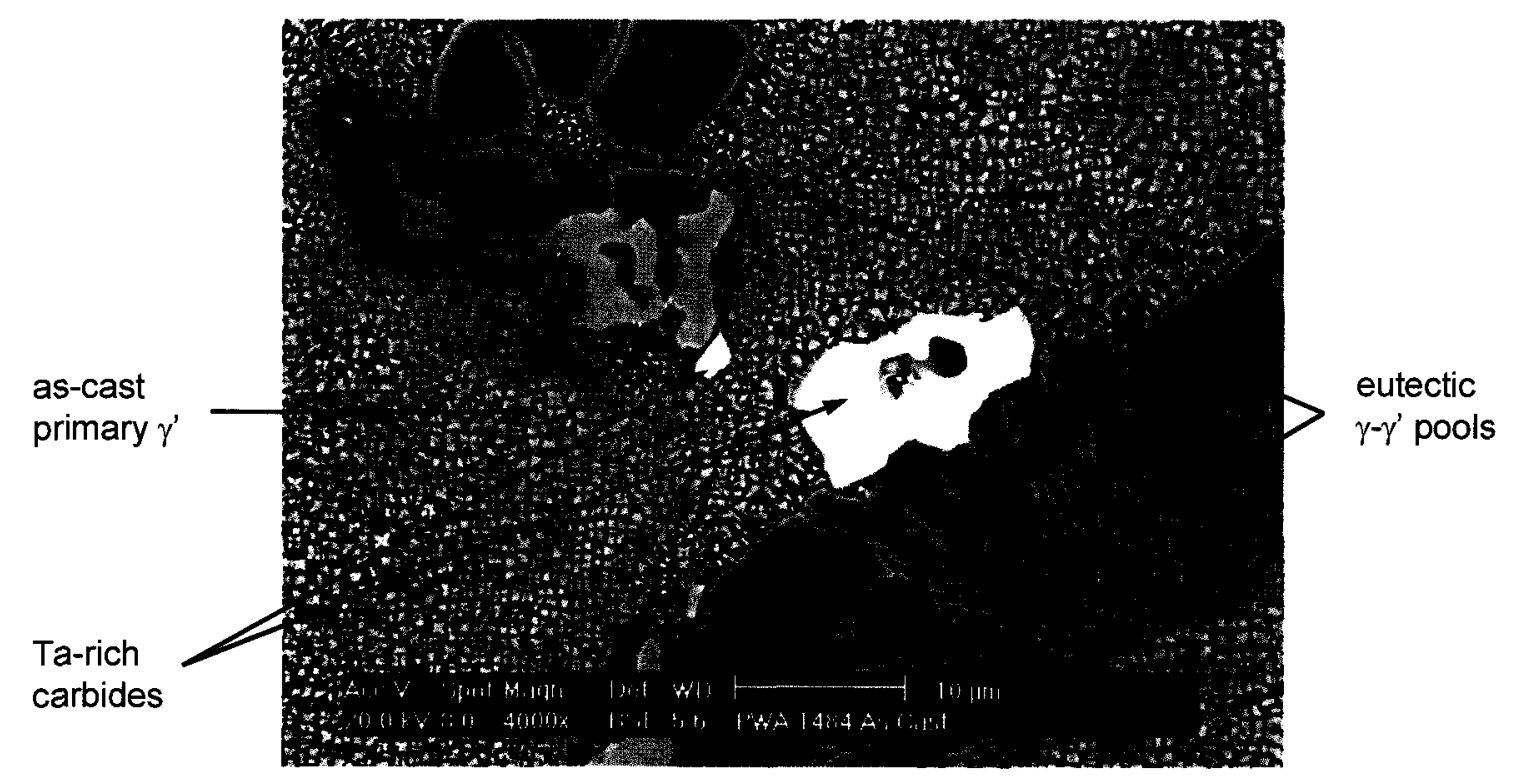

Figure 5.16 - SEM backscatter electron image showing the interdendritic constituents in ascast PWA1484. 


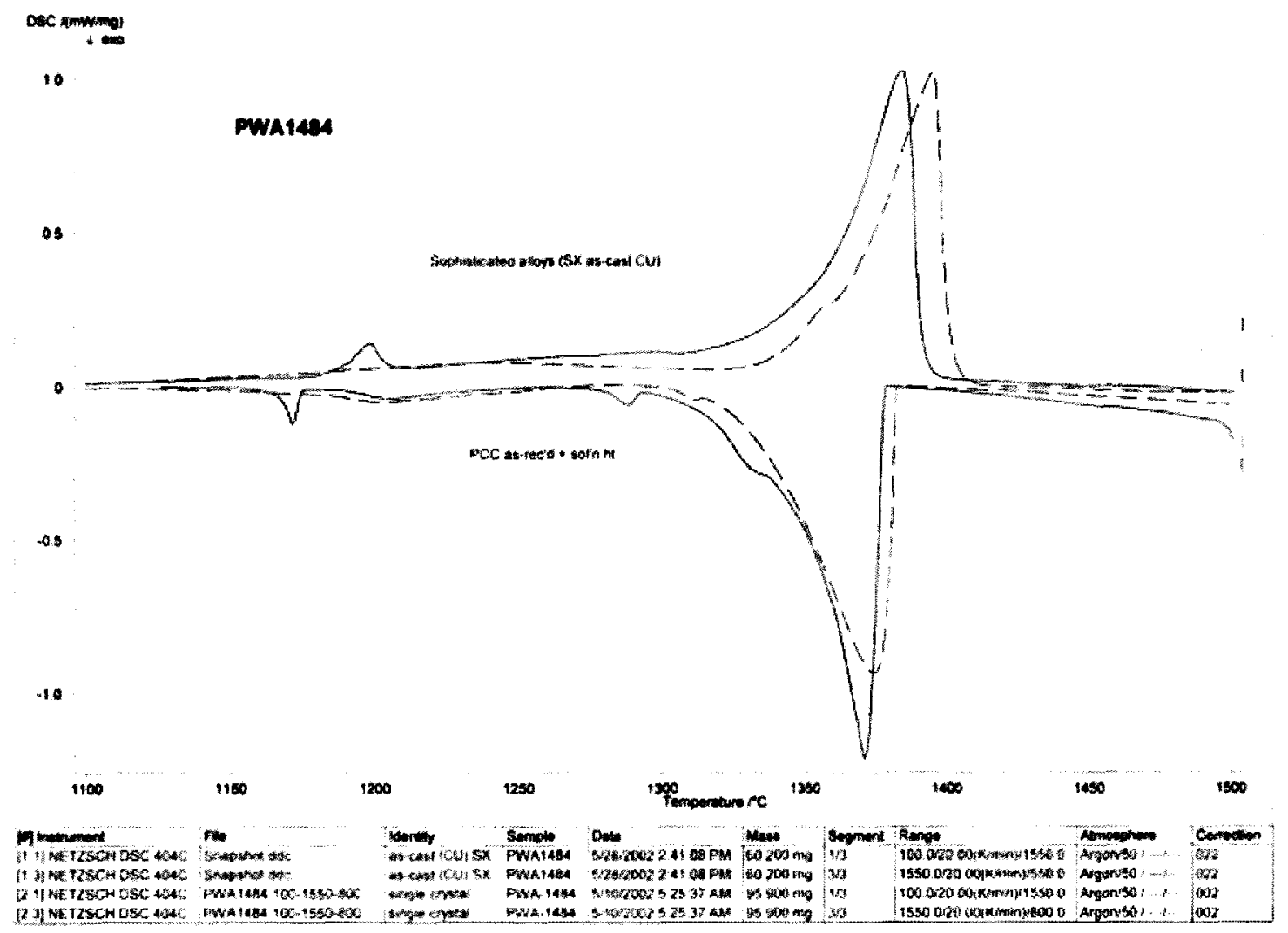

Figure 5.17 - DSC curves comparing PWA1484 material cast by PCC Airfoils to the Sophisticated Alloys version of PWA1484 after casting into single-crystal form in the Carleton University Bridgman casting facility. 


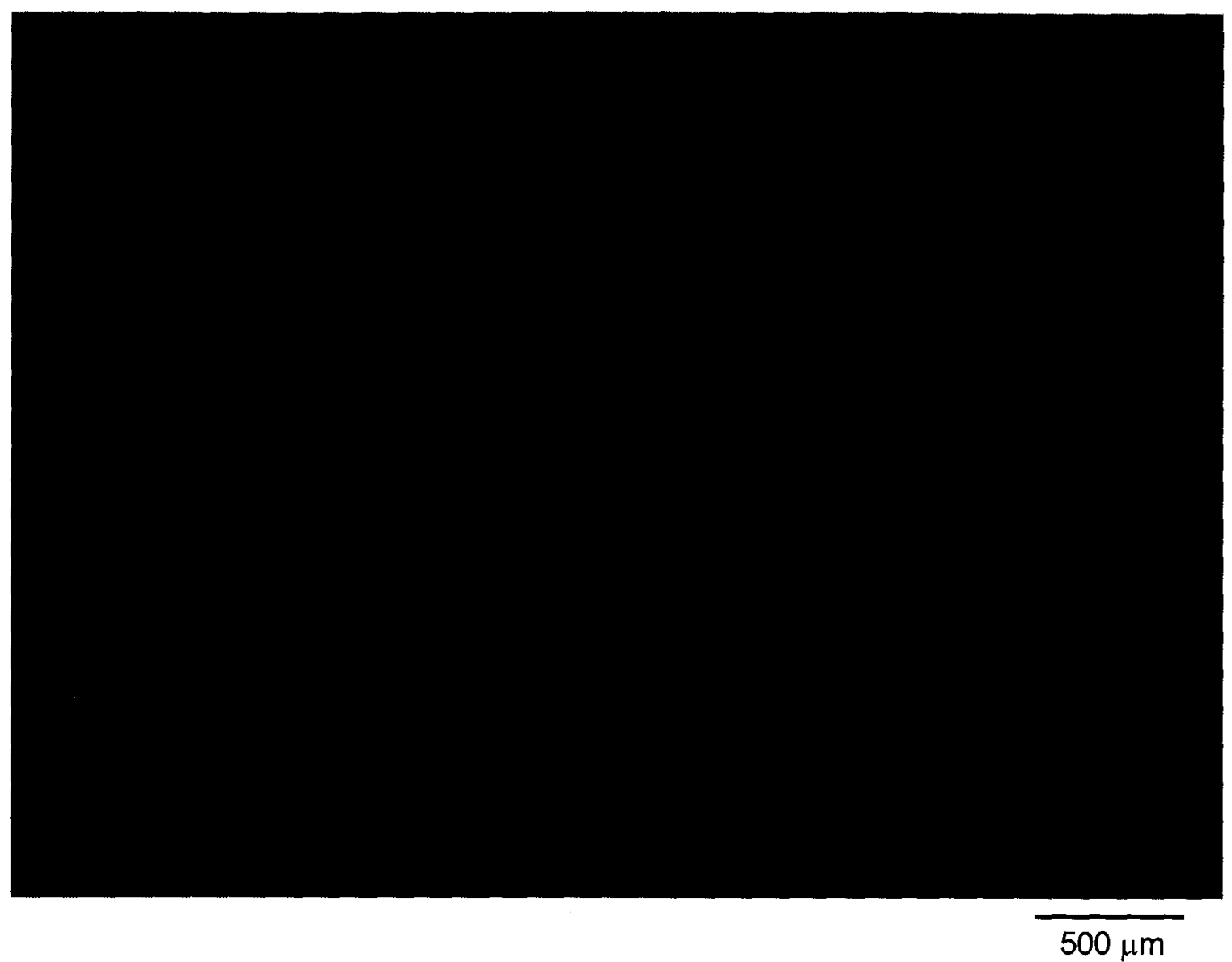

Figure 5.18 - Composite BSE-SEM image showing the single-crystal macrostructure after heat treatment. 

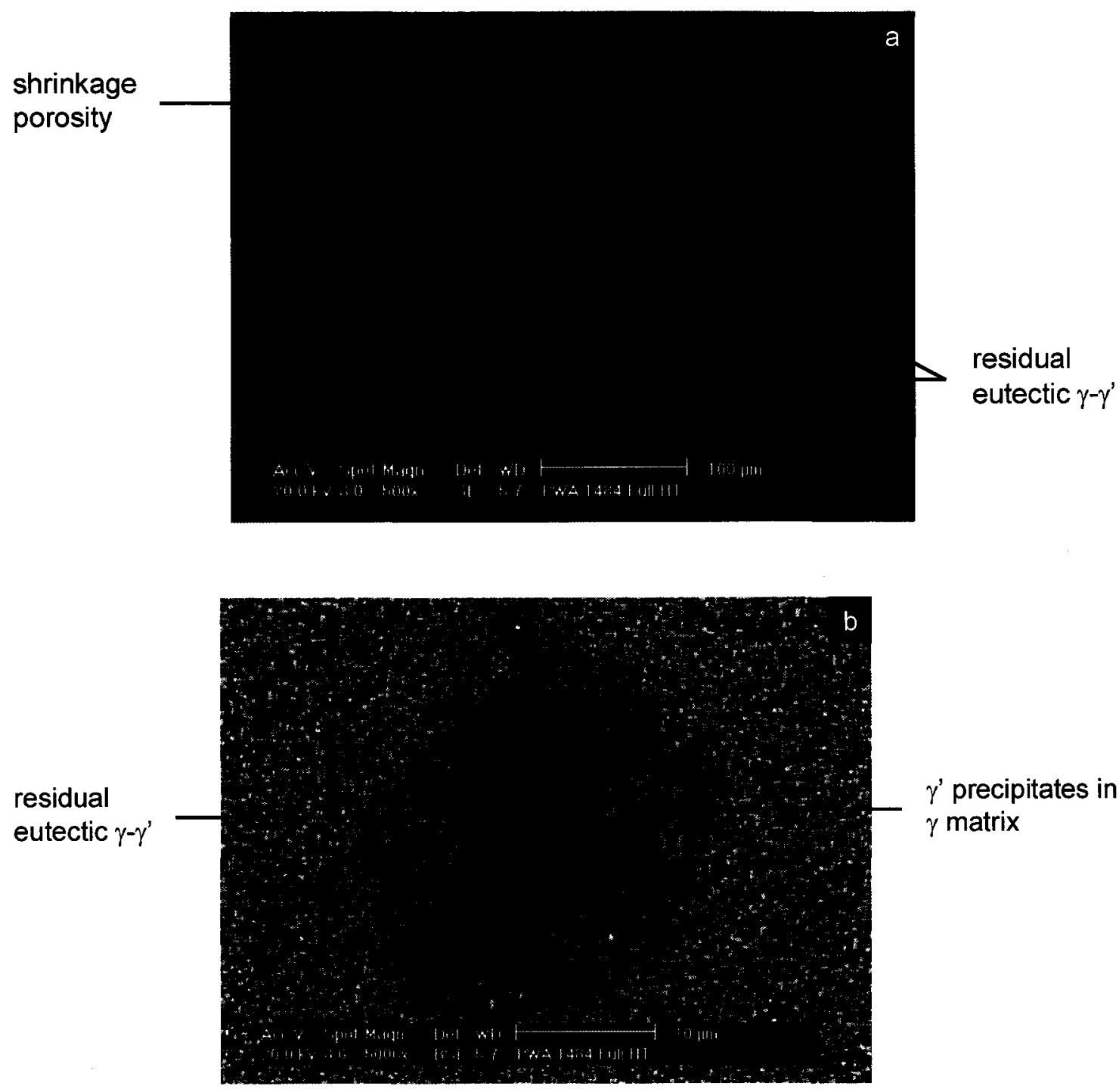

Figure 5.19 - SEM micrographs showing selected microstructural features after heat treatment; a) low magnification image showing residual $\gamma-\gamma$ ' eutectic phase and an shrinkage pore in the interdendritic regions, and b) higher magnification view showing $\gamma-\gamma$, eutectic phase. 

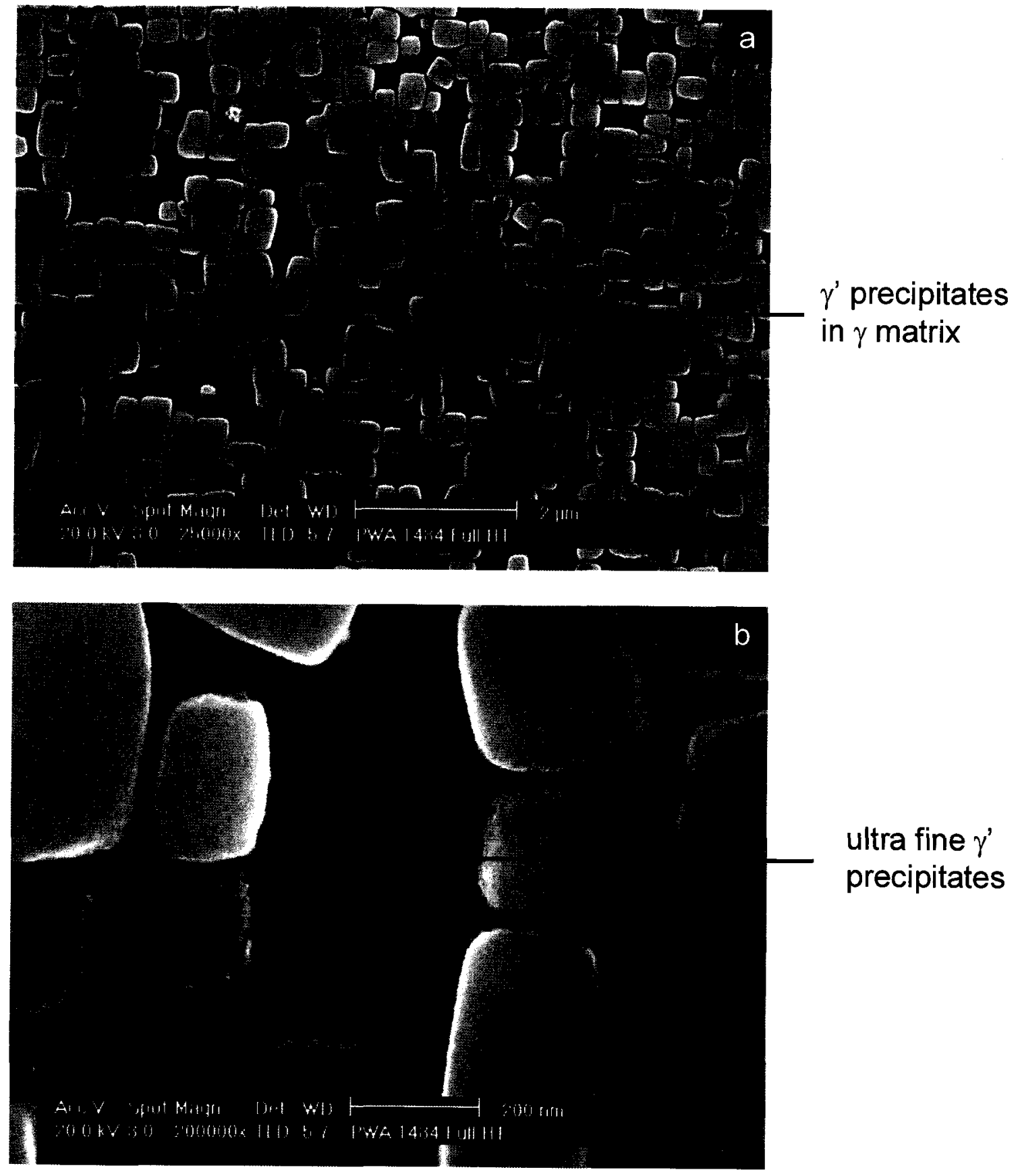

Figure 5.20 - SEM micrographs showing $\gamma^{\prime}$-phase morphology after heat treatment; a) primary $\gamma^{\prime}$-phase, and b) secondary $\gamma^{\prime}$-phase. 


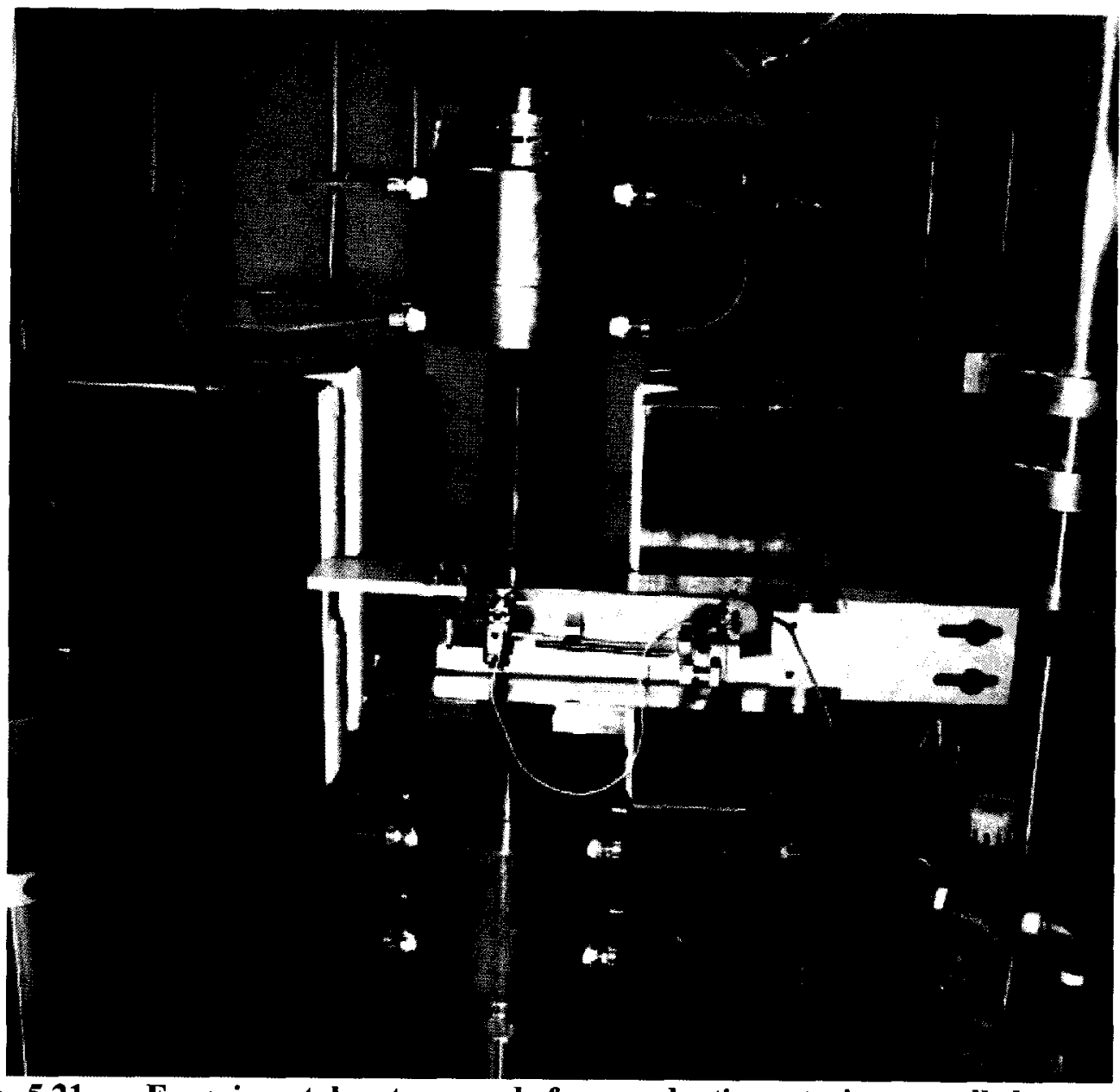

Figure 5.21 - Experimental setup used for conducting strain-controlled constitutive material tests.

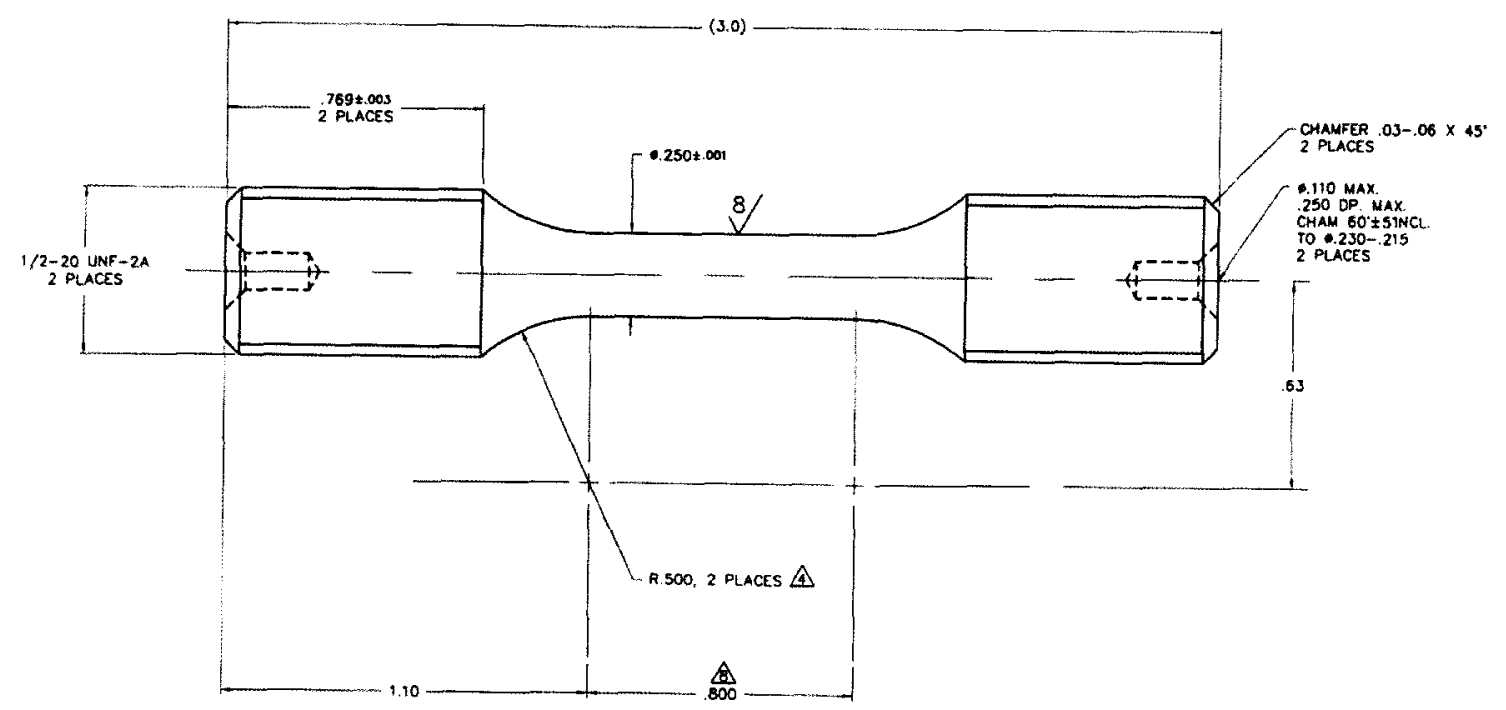

Figure 5.22 - Specimen geometry used for the constitutive material tests. Note: specimen dimensions are in inches. 


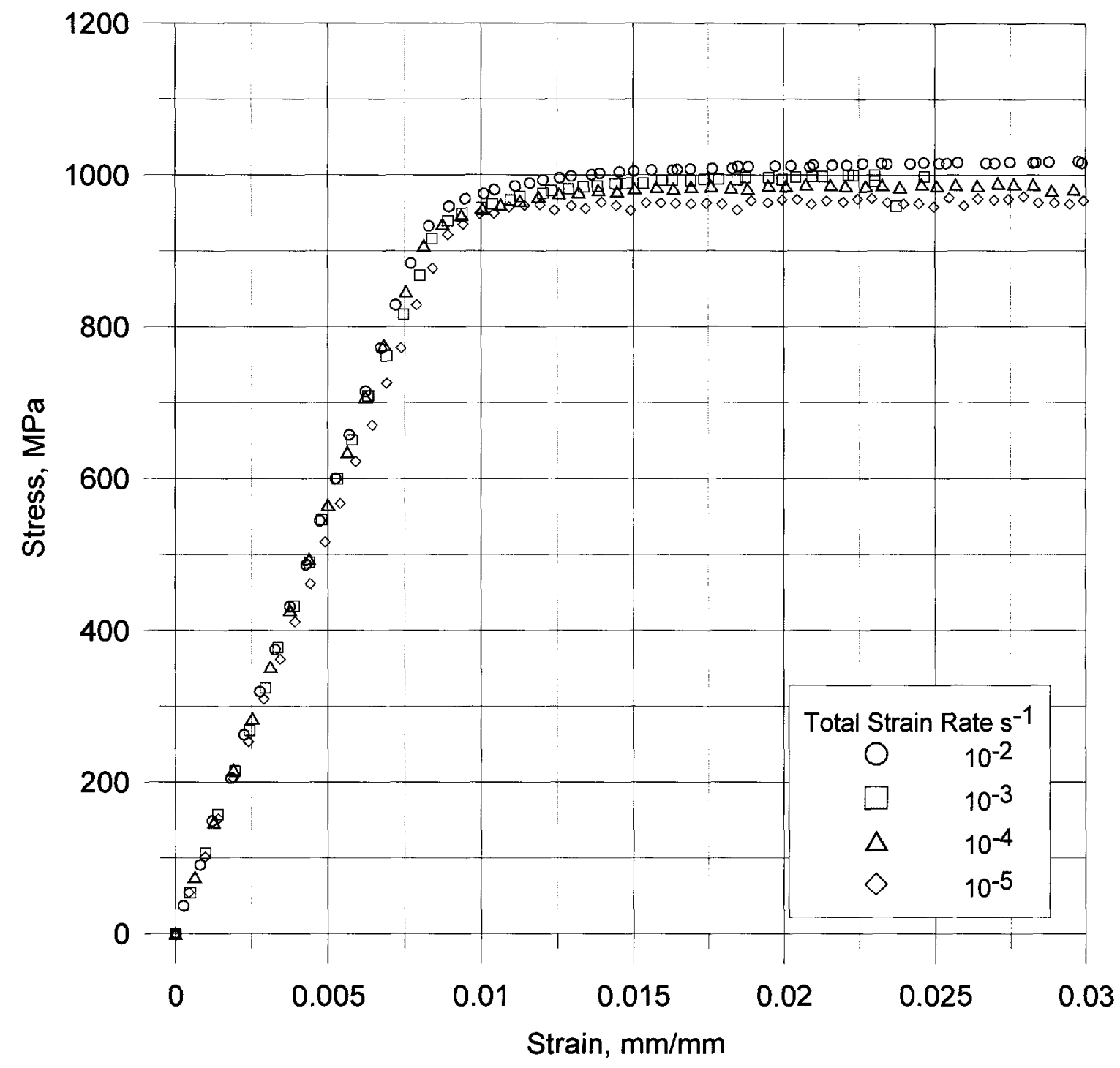

Figure 5.23 - Constant total strain-rate engineering stress-strain tensile test curves for $<100>$ tensile axis Sophisticated Alloys PWA1484 alloy tested at $650^{\circ} \mathrm{C}$. 

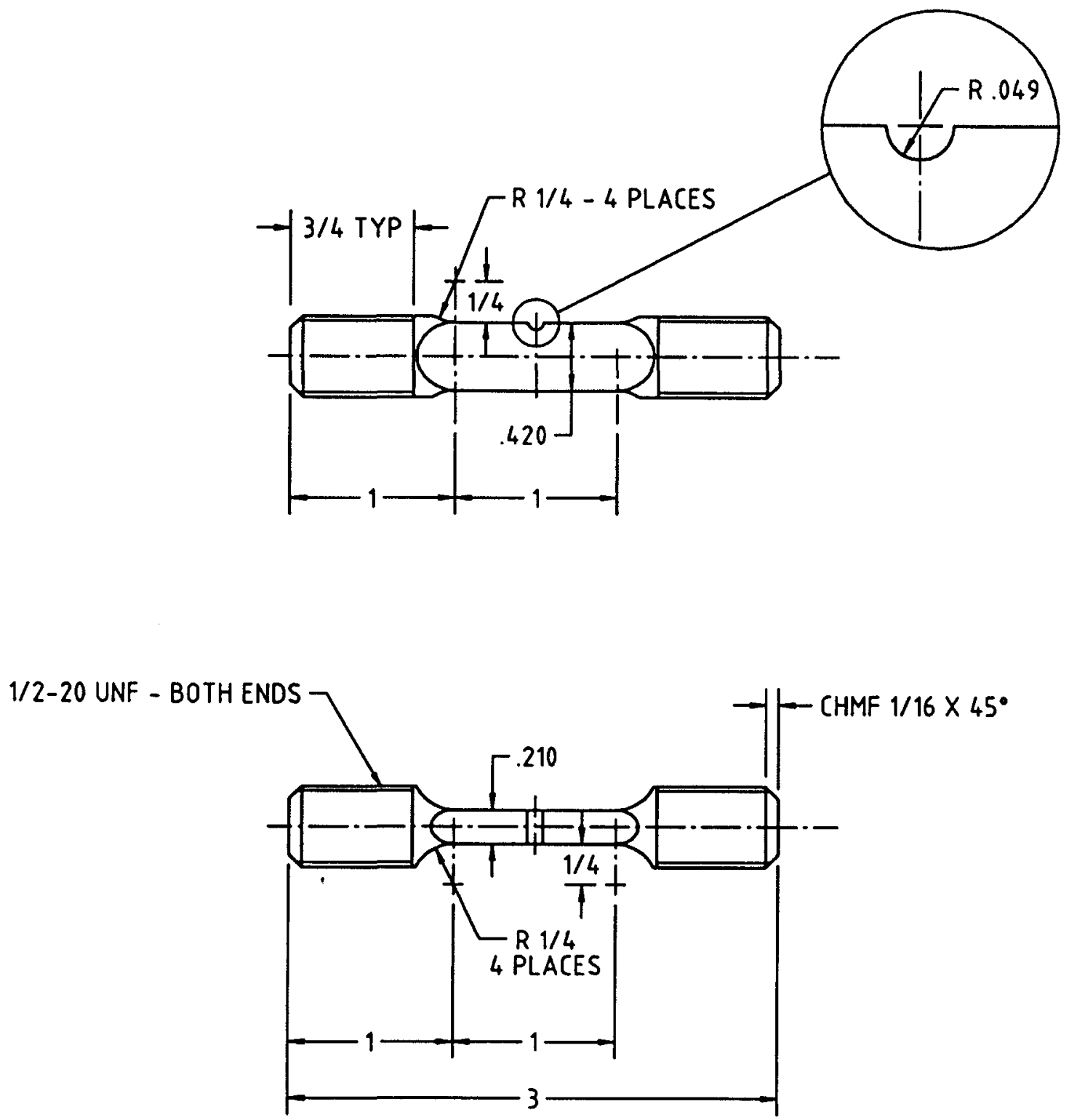

Figure 5.24 - Single-edge notch specimen geometry used for small fatigue crack growth characterization. Note: specimen dimensions are in inches. 


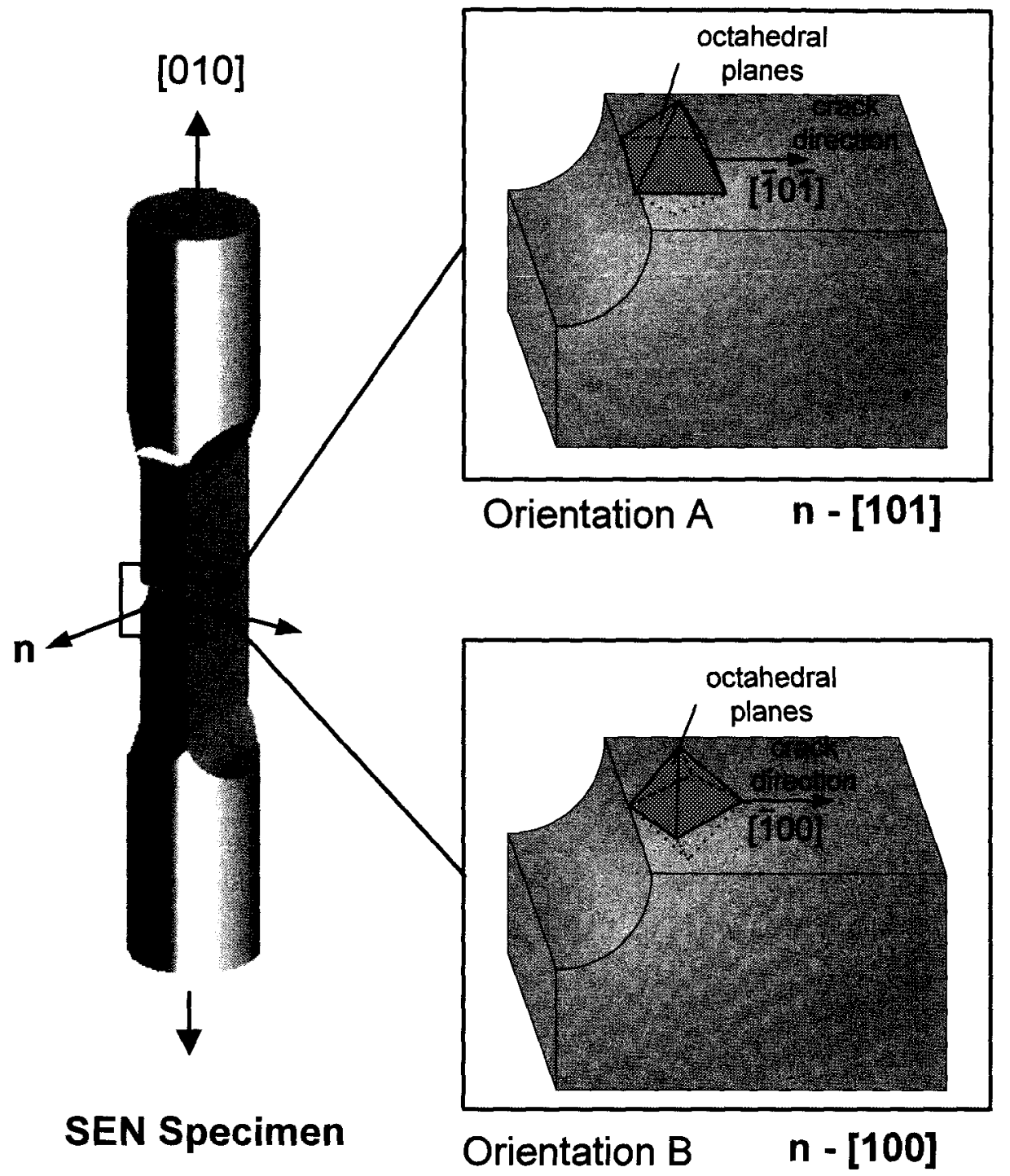

Figure 5.25 - Schematic showing SEN specimen and details in the notch region for orientations $A[010]-[101]$ and $B[010]-[100]$. 


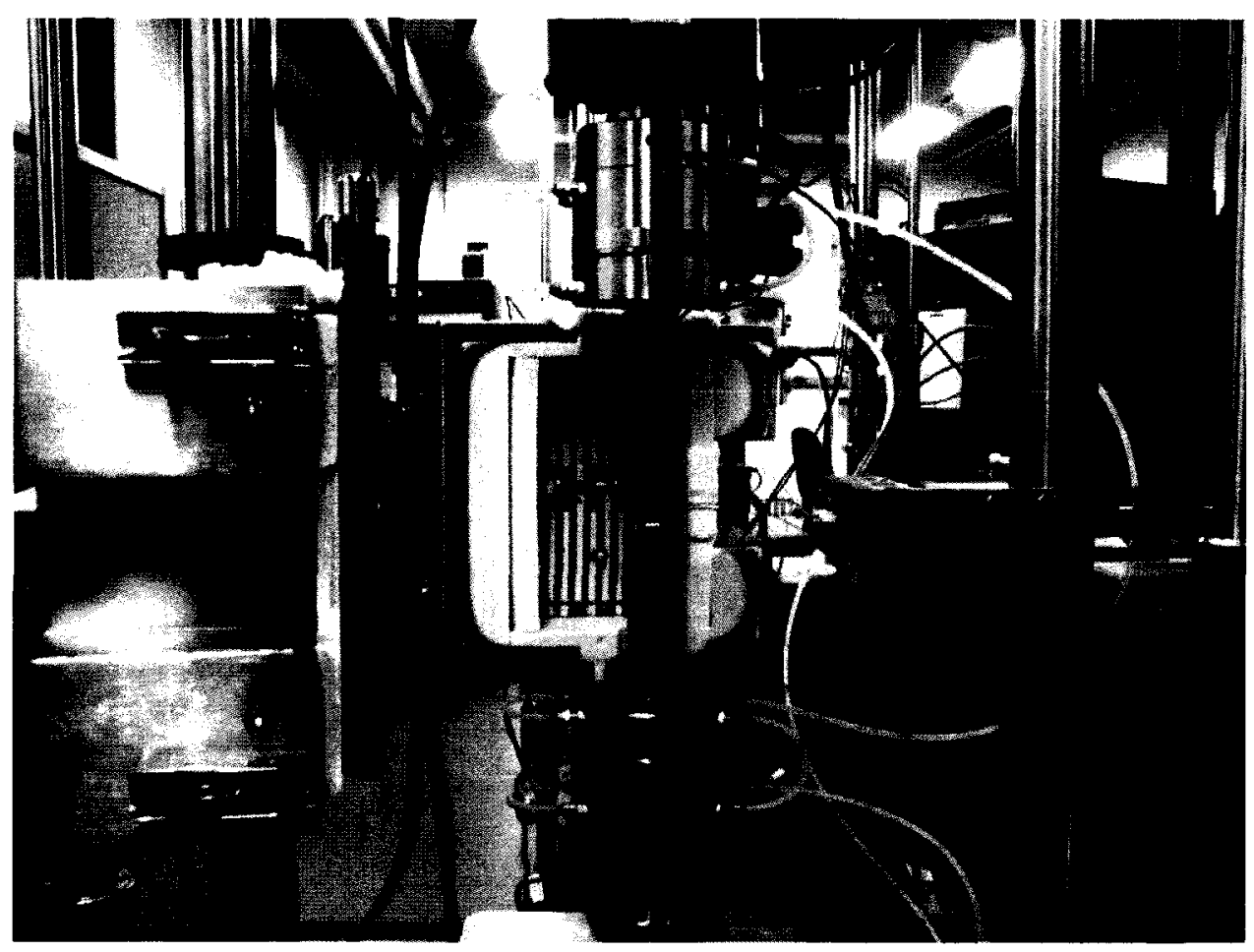

Figure 5.26 - General overview of mechanical test setup used for FCGR testing.

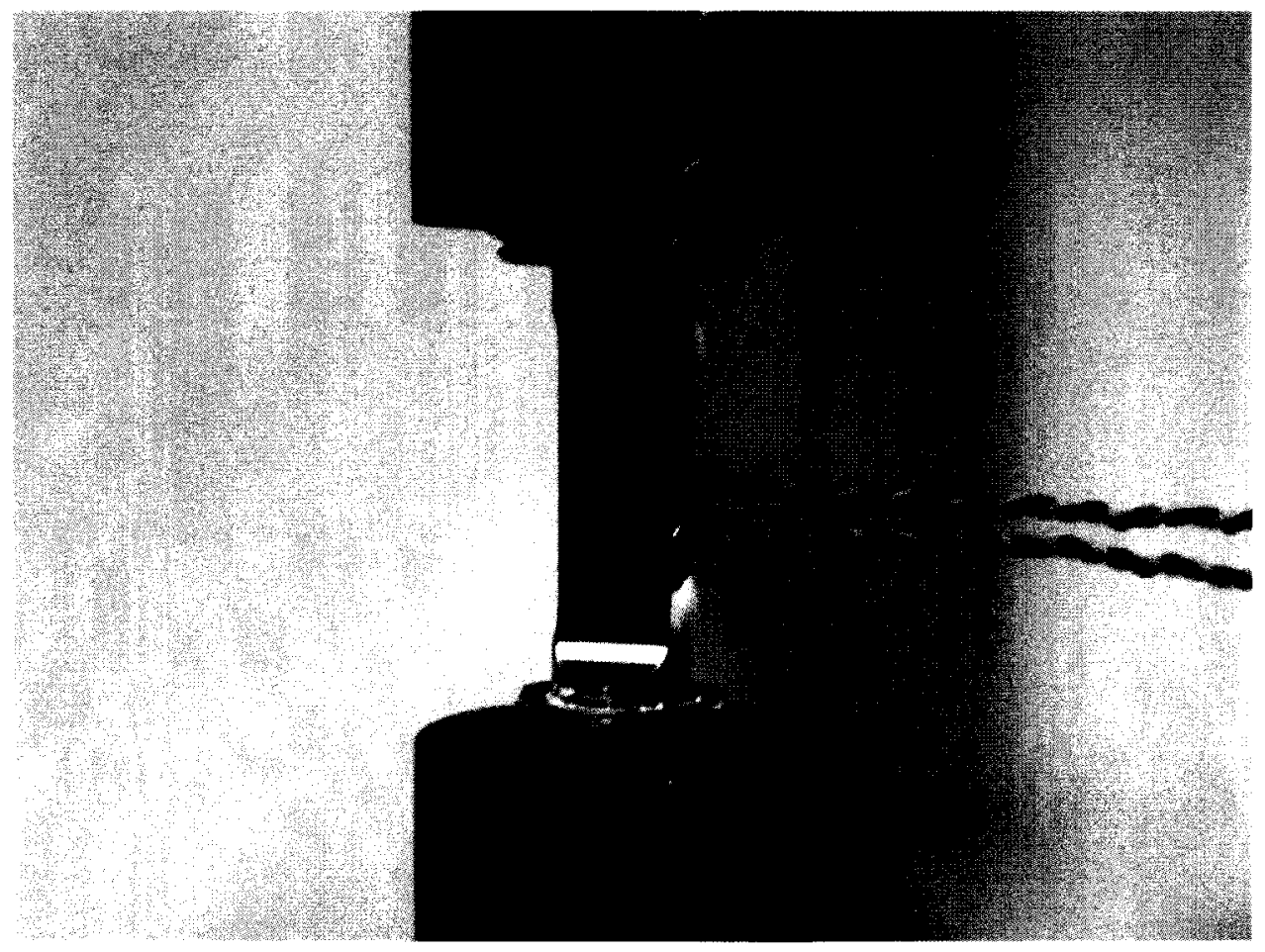

Figure 5.27 - ACPD current and voltage probe arrangement. 


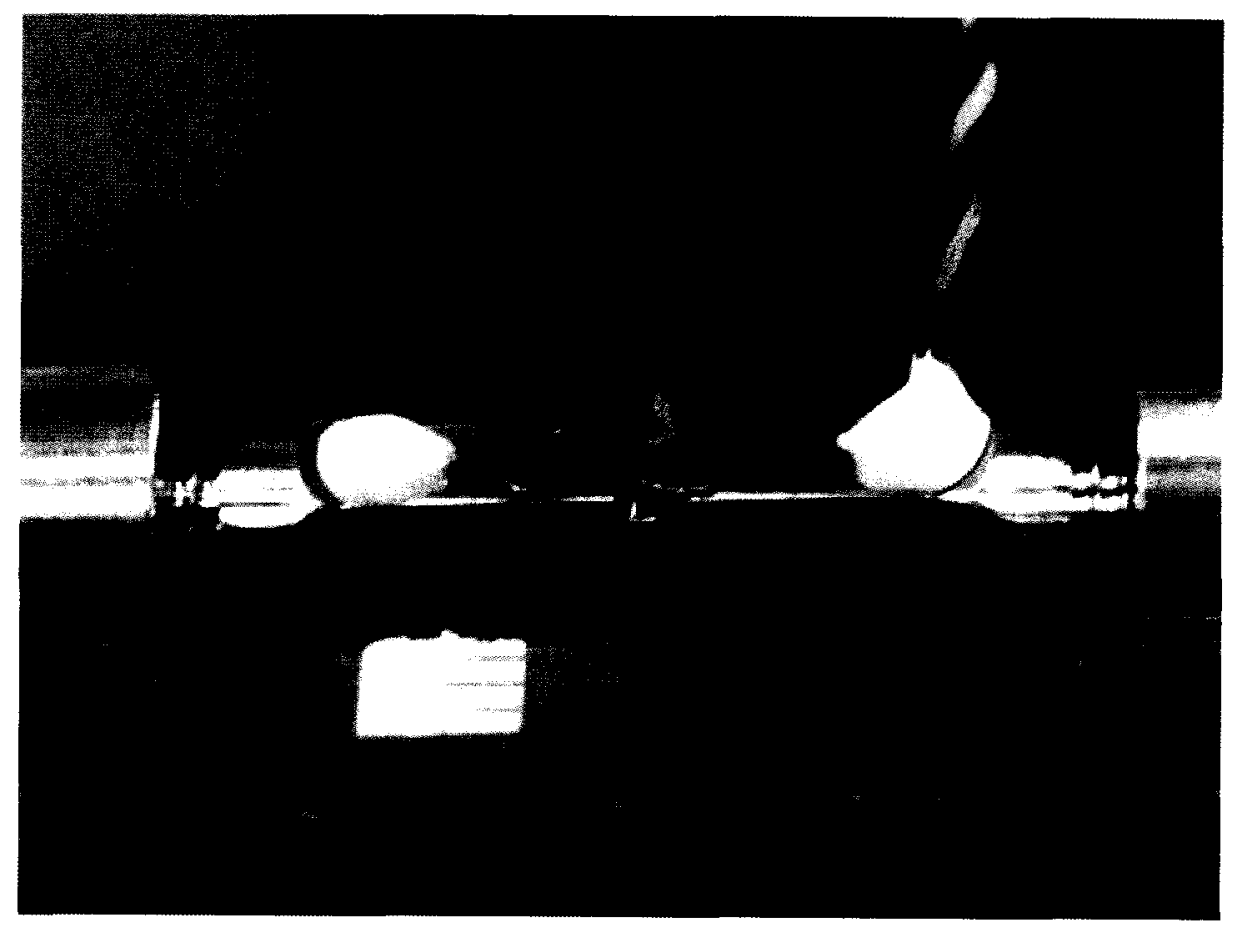

Figure 5.28 - Close-up view of the ACPD current and voltage probe arrangement.

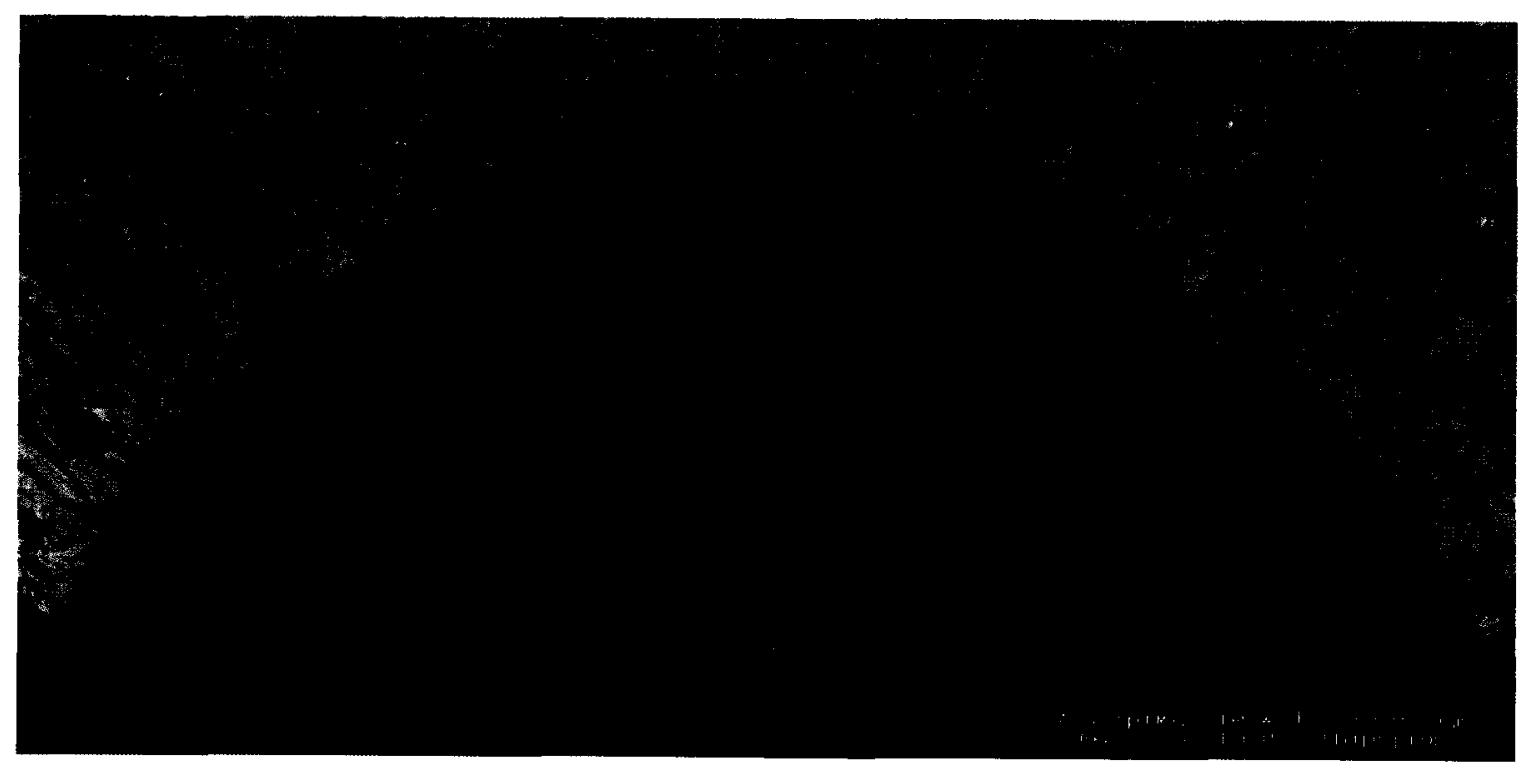

Figure 5.29 - ACPD crack calibration specimen fracture surface. 


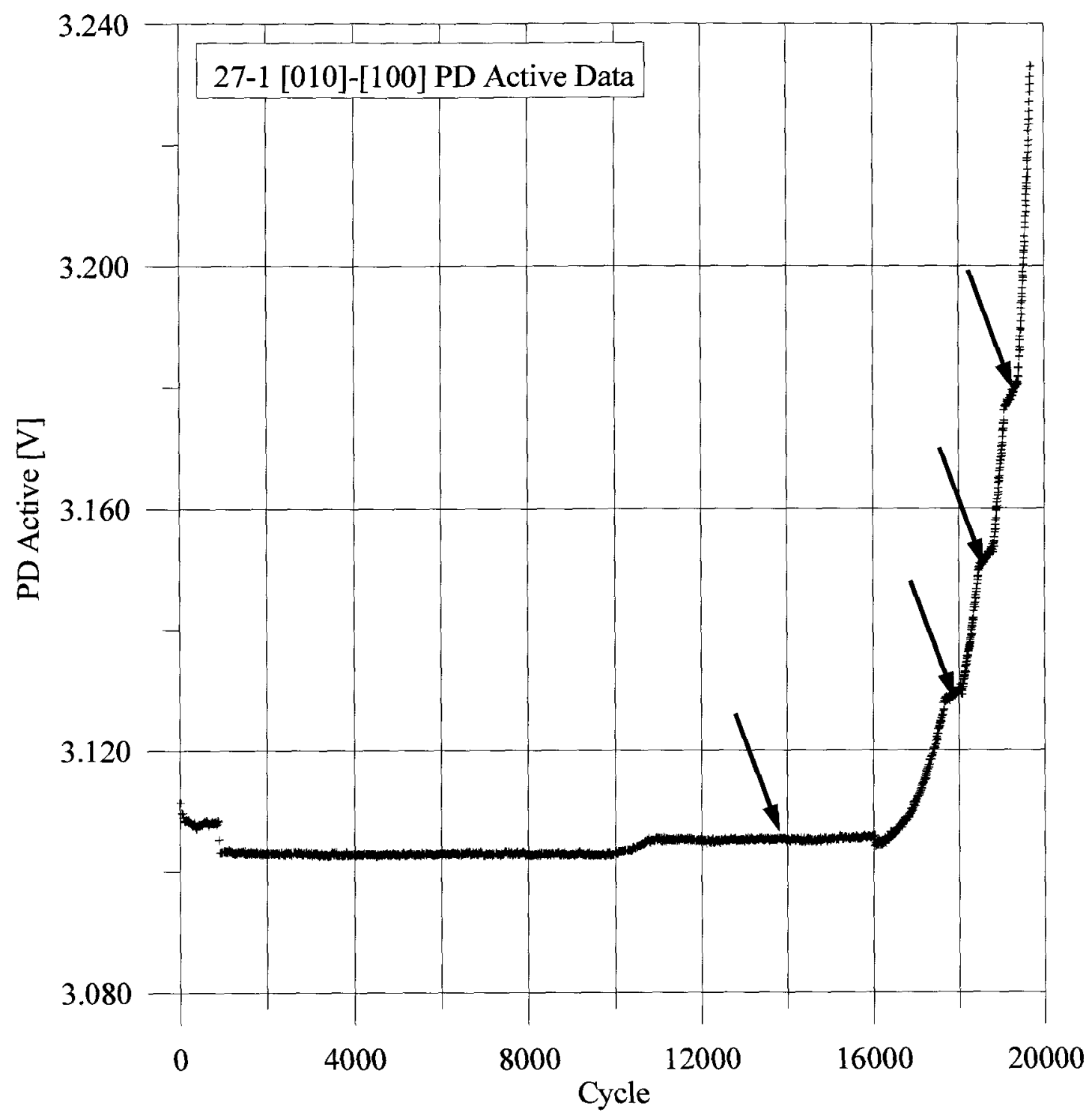

Figure 5.30 - Recorded PD versus cycle data for an ACPD calibration test. Note: arrows indicate change from $R=0.1$ to $R=0.7$ loading to introduce a marker band. 


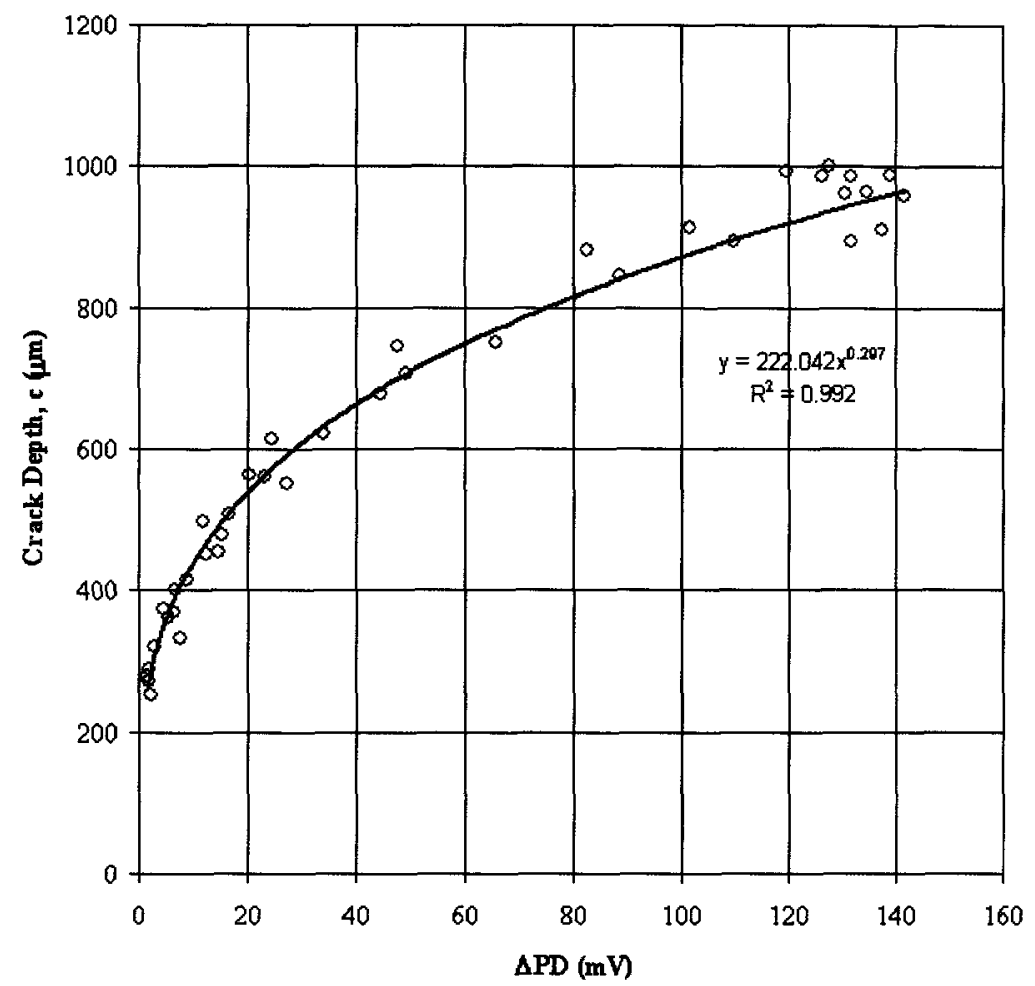

Figure 5.31 - Orientation A crack depth, $c$, versus PD change calibration curve.

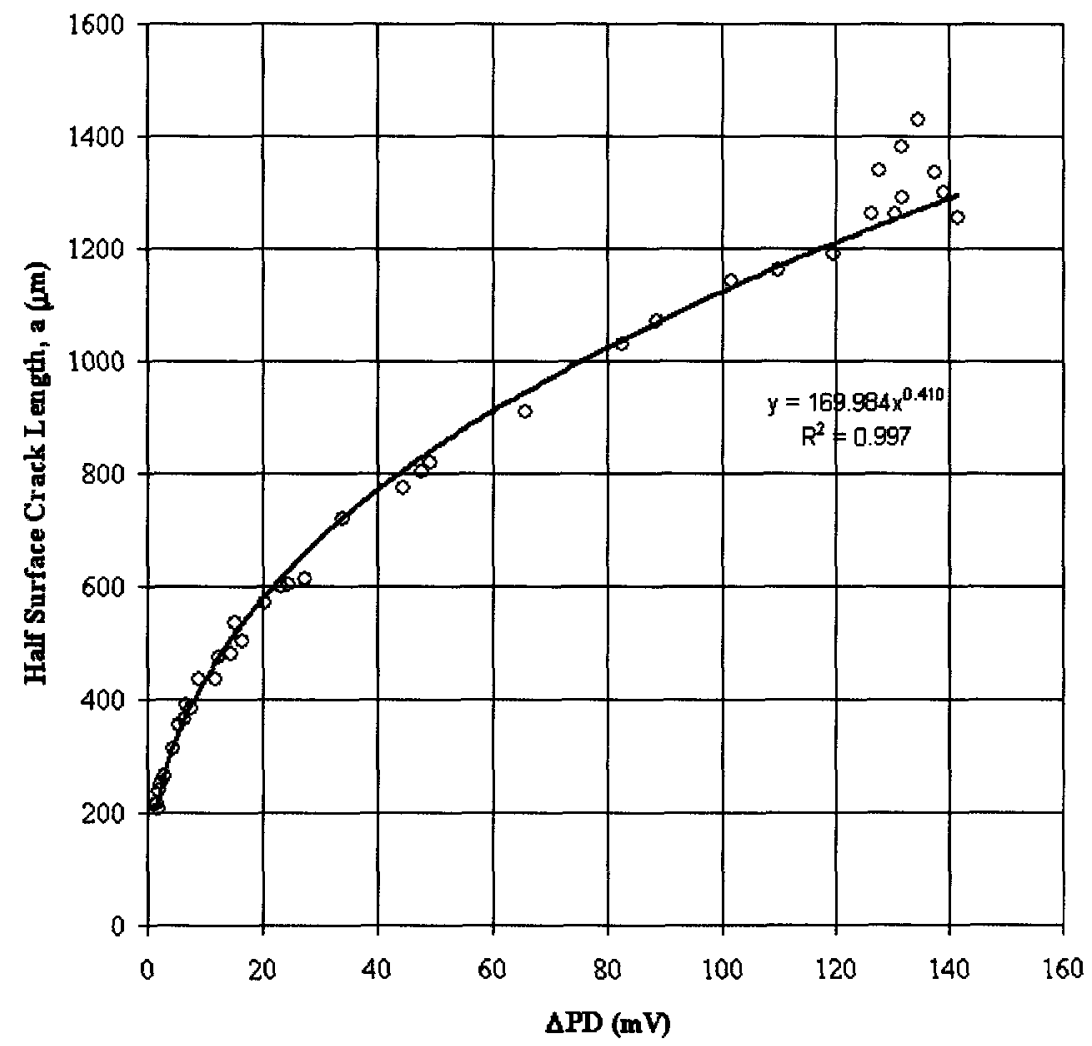

Figure 5.32 - Orientation A half surface crack length, $a$, versus PD change calibration curve. 


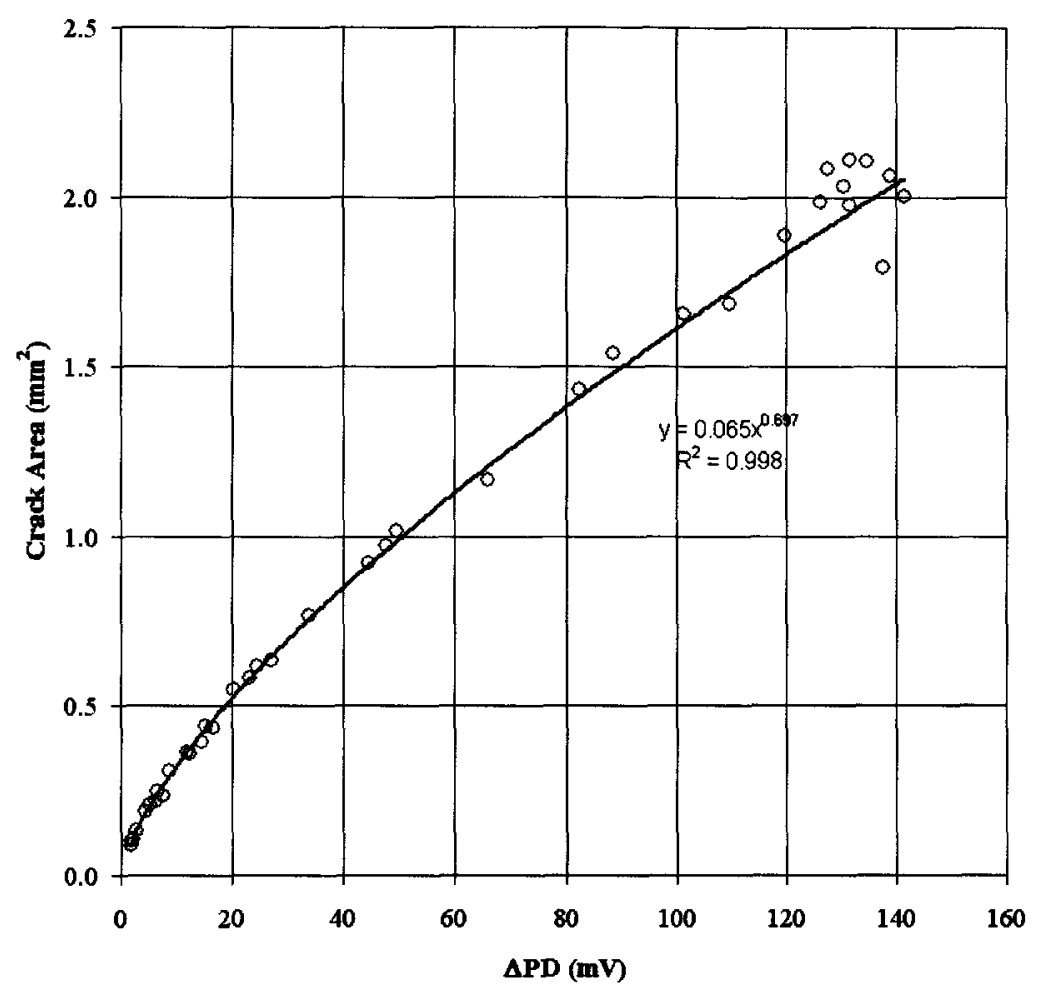

Figure 5.33 - Orientation A crack area versus PD change calibration curve.

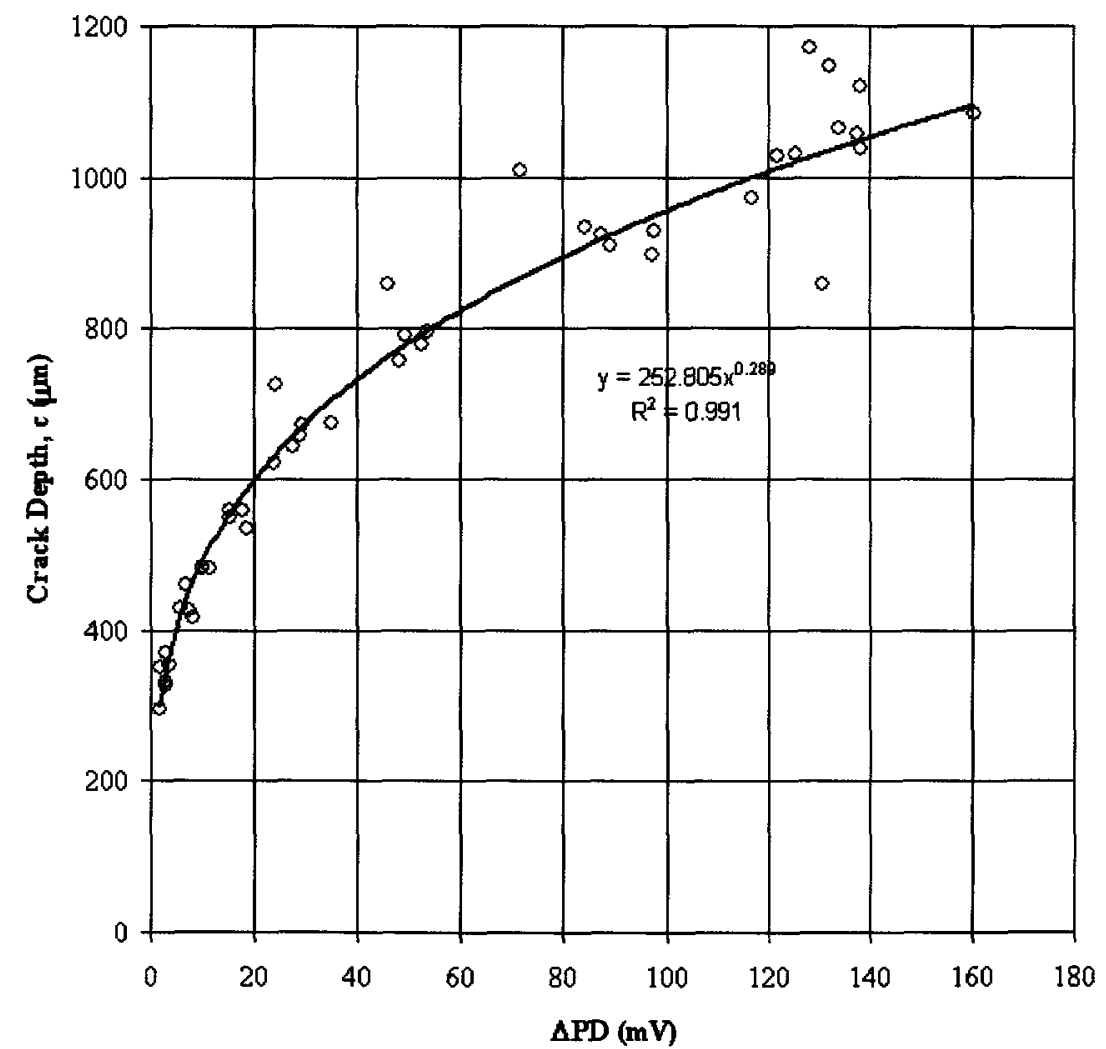

Figure 5.34 - Orientation B crack depth, $c$, versus PD change calibration curve. 


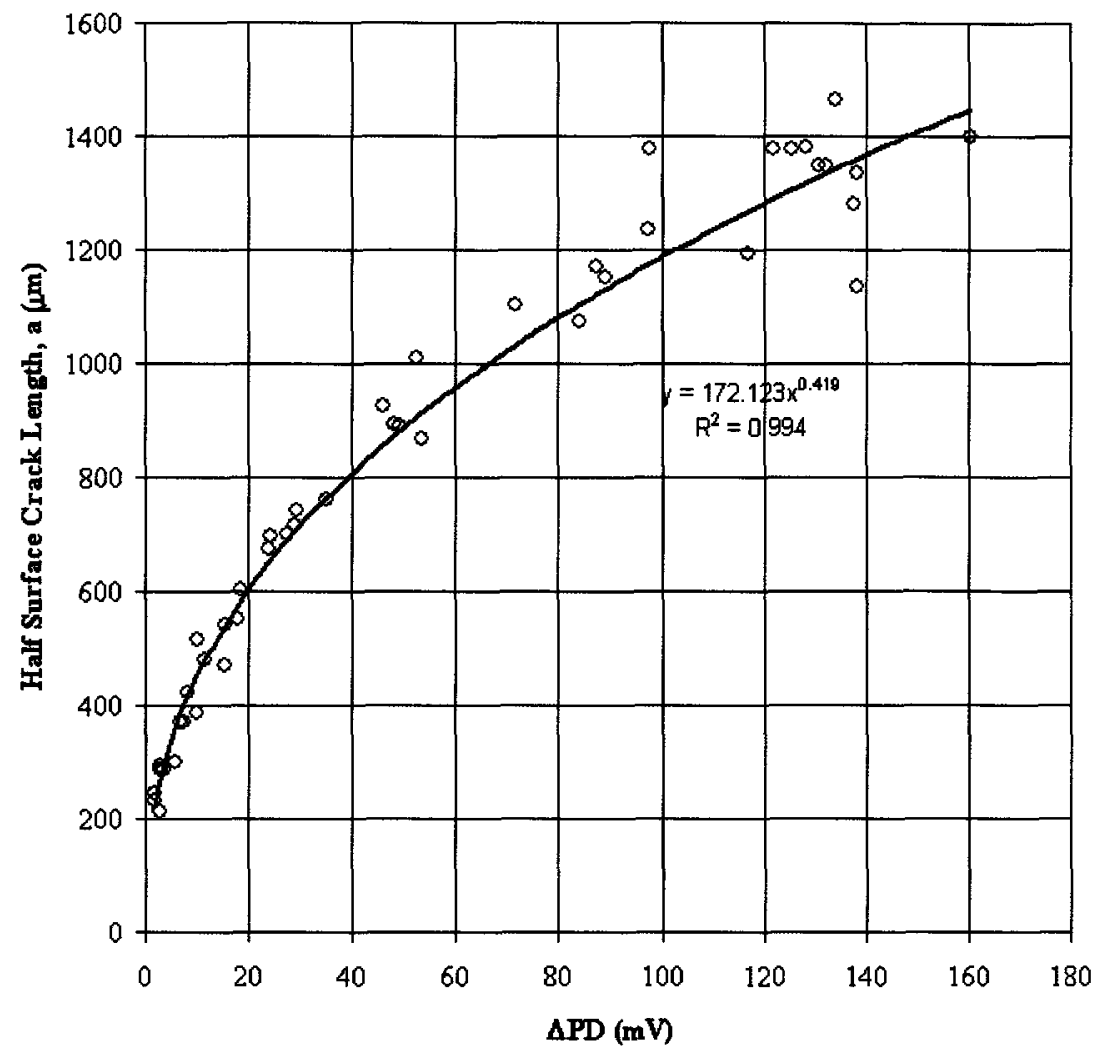

Figure 5.35 - Orientation B half surface crack length, $a$, versus PD change calibration curve.

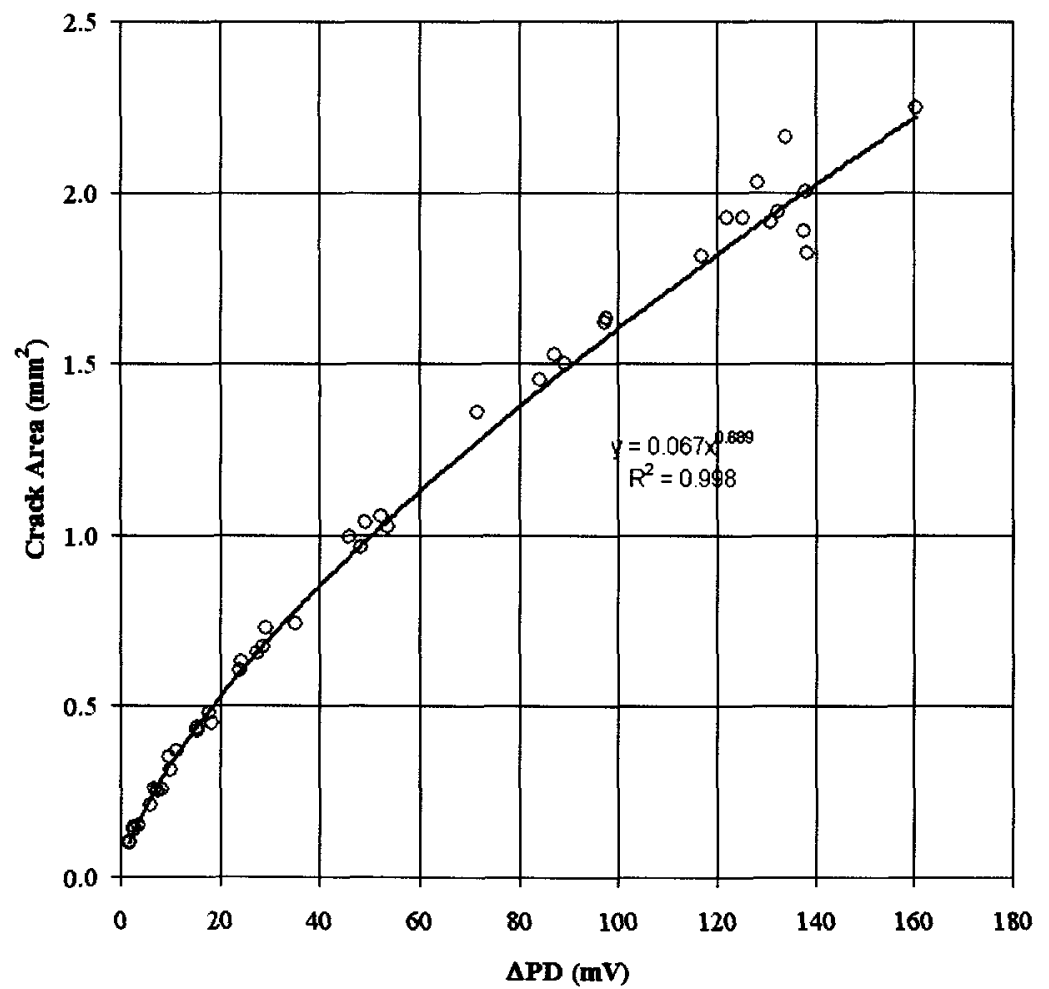

Figure 5.36 - Orientation B crack area versus PD change calibration curve. 


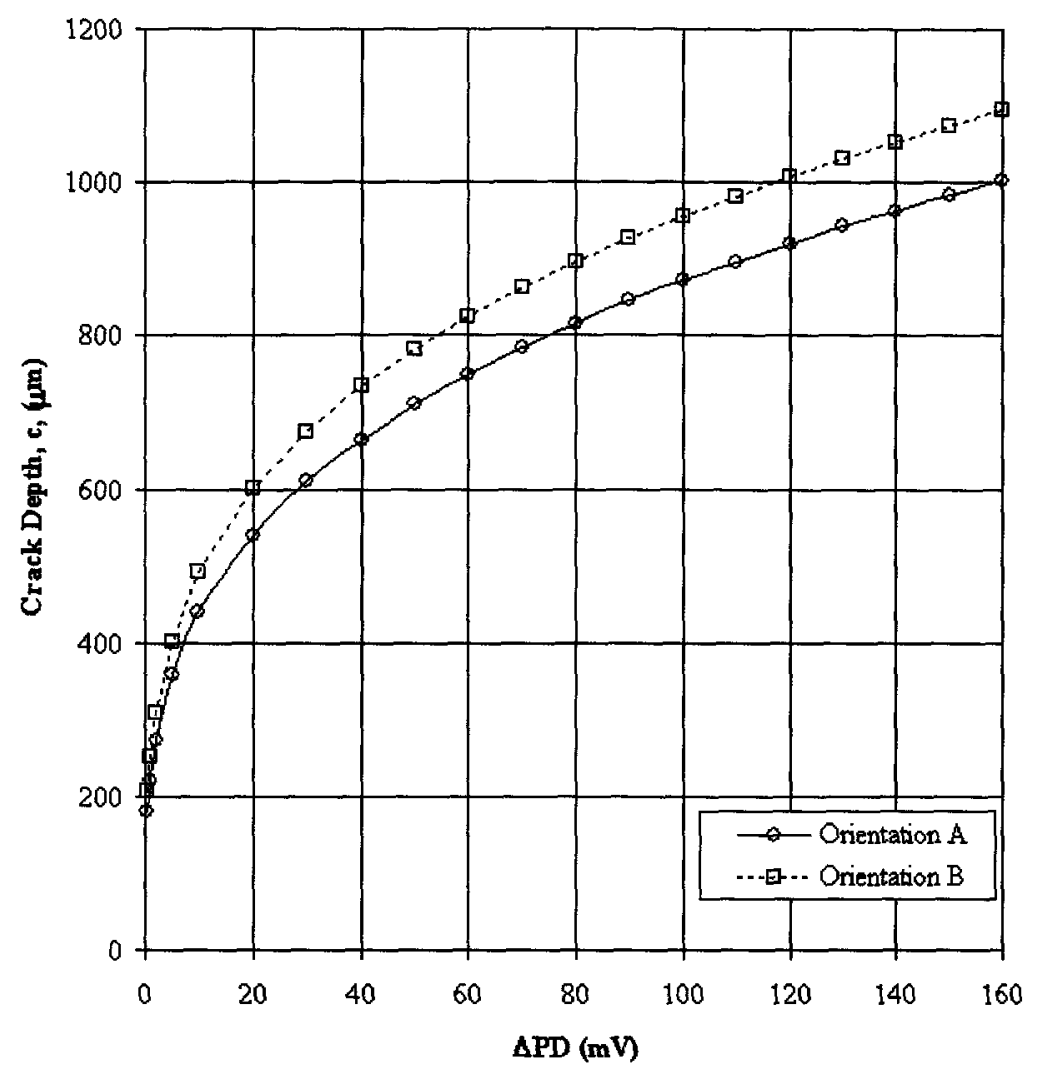

Figure 5.37 - Comparison of crack depth, $c$, versus PD change calibration curves for orientation $A$ and $B$.

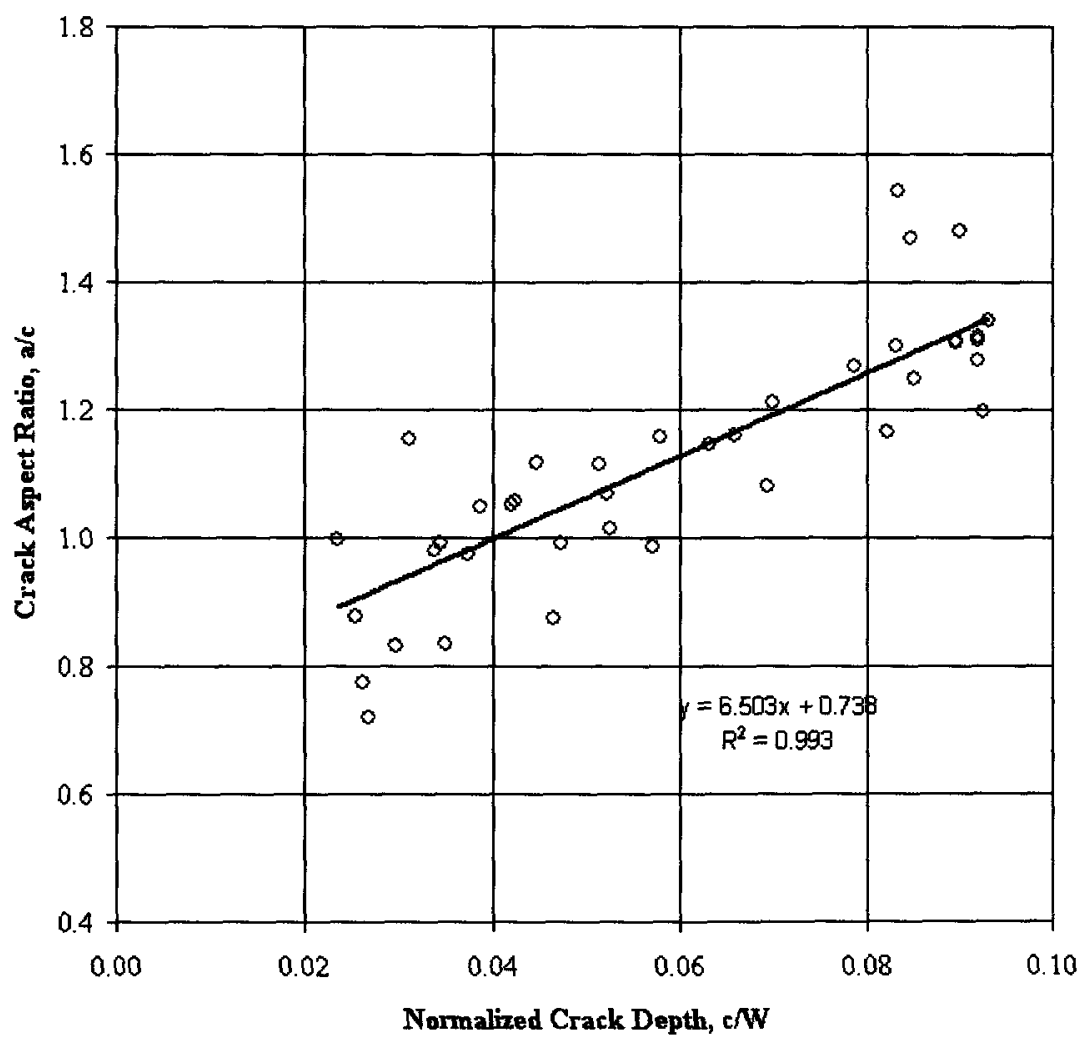

Figure 5.38 - Orientation A crack aspect ratio, $a / c$, versus normalized crack depth, $c / W$. 


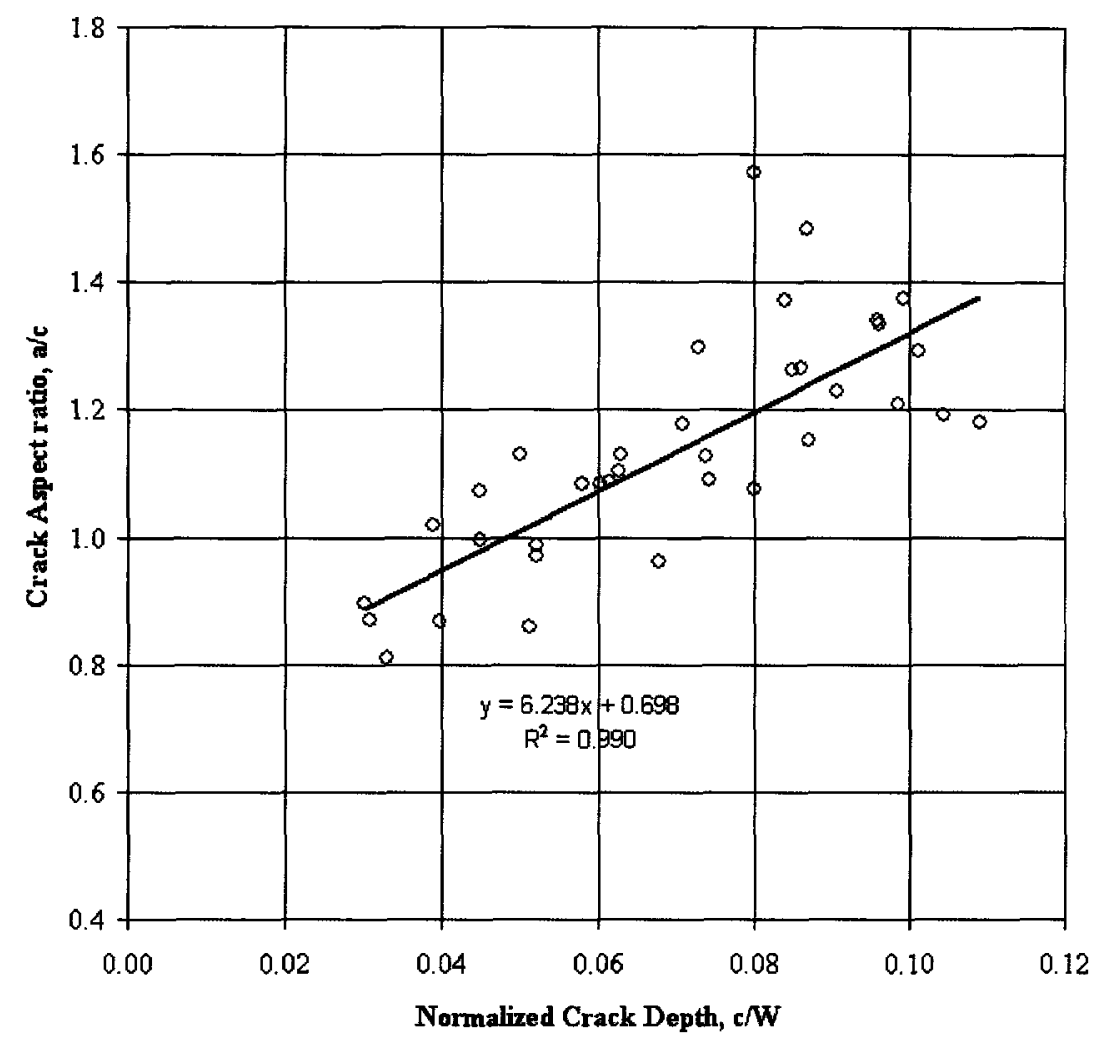

Figure 5.39 - Orientation B crack aspect ratio, $a / c$, versus normalized crack depth, $c / W$.

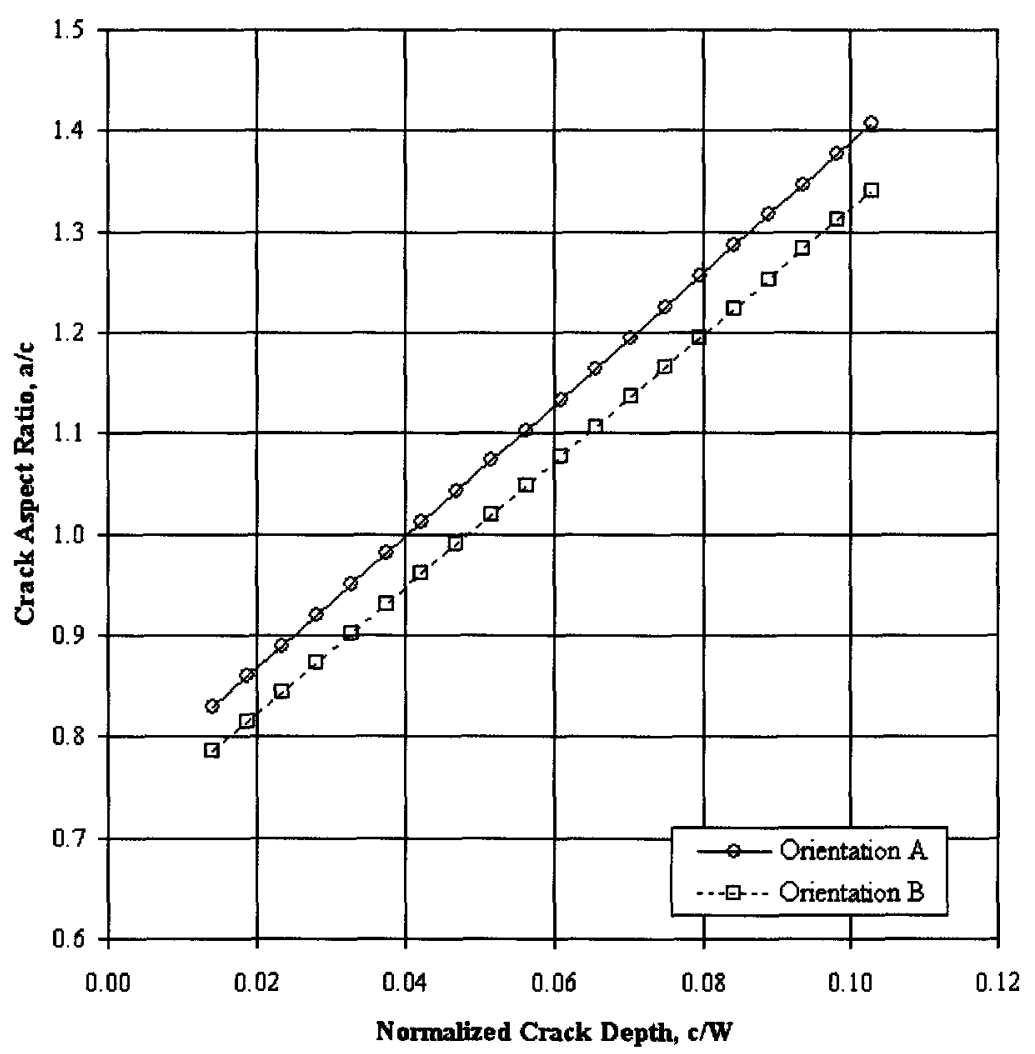

Figure 5.40 - Comparison of crack aspect ratio calibration curves for orientations A and B. 


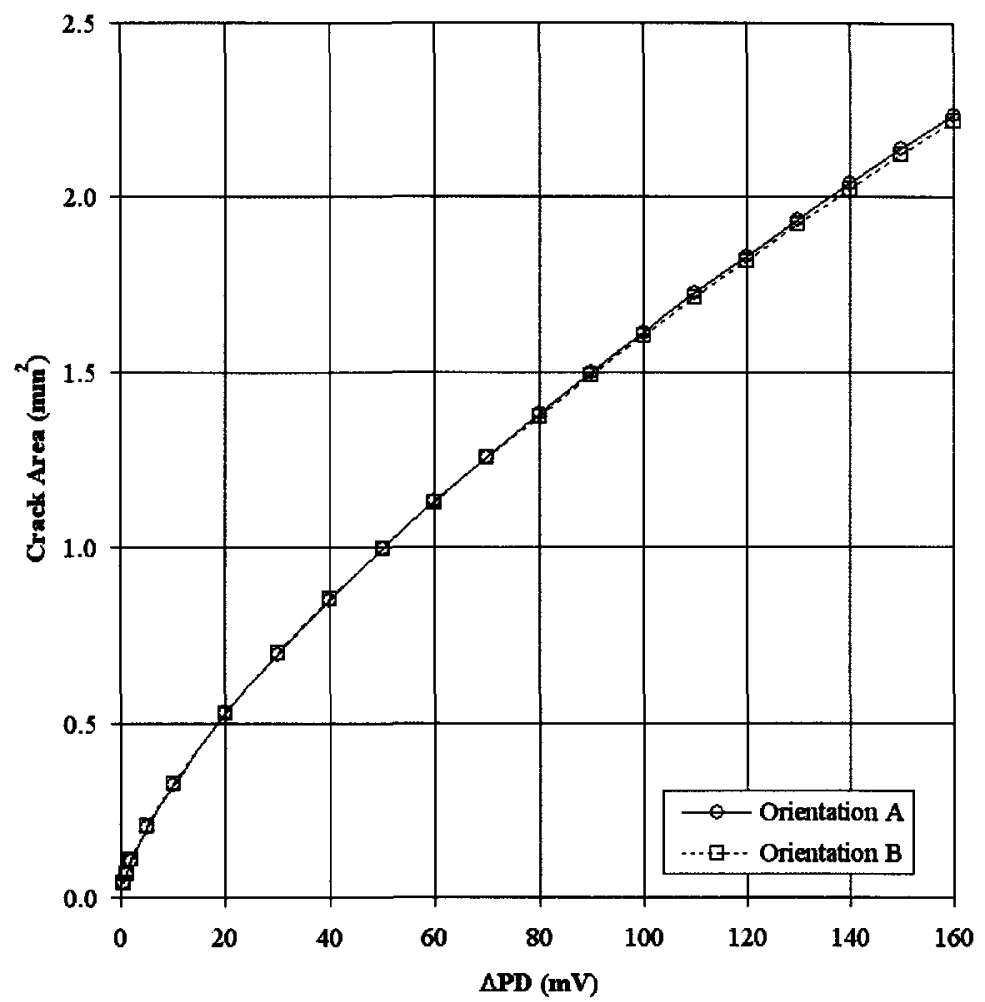

Figure 5.41 - Comparison of crack area calibration curves for orientations $A$ and $B$.

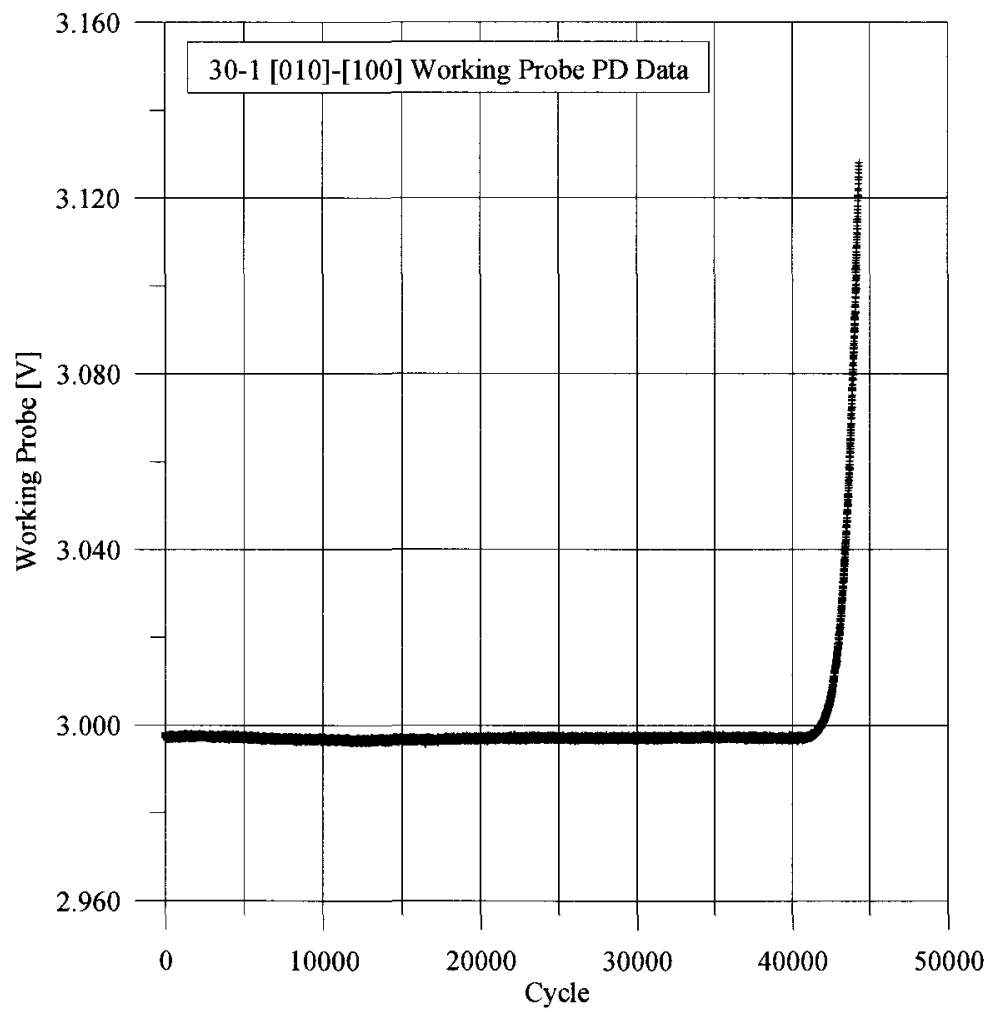

Figure 5.42 - Typical variation in working probe voltage versus cycles for a small fatigue crack growth rate test. 


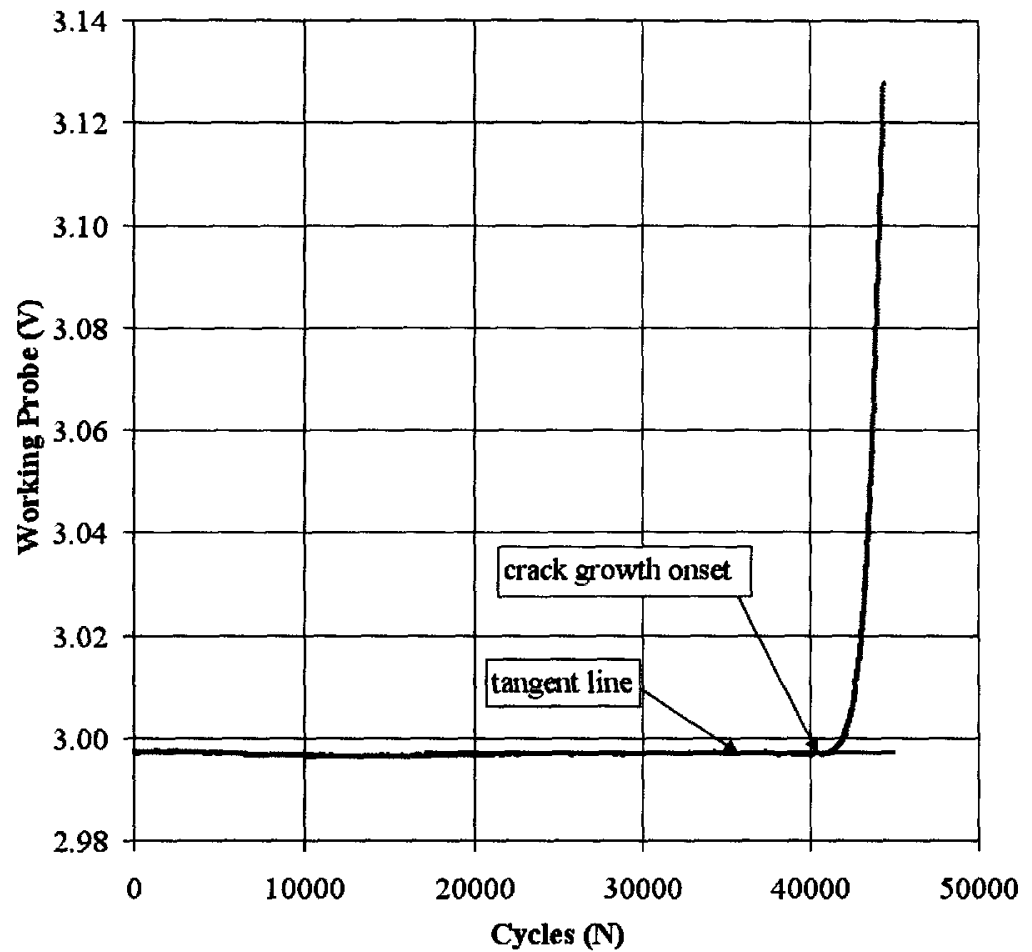

Figure 5.43 - Schematic showing method used to determine the onset PD voltage associated with fatigue crack growth. 


\section{Finite Element Implementation of a Single-Crystal}

\section{Constitutive Model}

\subsection{Introduction}

Finite element analysis has become an indispensible tool used in the design and development process for a wide range of engineering components. For, gas turbine engines, it is commonly used to establish the thermal and mechanical loads that must be sustained by engine components, identify life-limiting locations, to extract information needed for component life prediction and to establish structural design margins. These activities require both heat transfer and mechanical structural analyses to be undertaken. While both analyses are crucial elements to the design process, the attention of this chapter focuses on the requirements for conducting mechanical structural analyses to establish stress-strain distributions at the component scale.

The finite element method, as applied to structural mechanics problems, involves the iterative solution of the discretized form of the momentum balance (equilibrium) equation while satisfying the deformation compatibility in the continuum. The solution process is divided into a number of time increments and involves global and local calculations [263]. At the global scale, the equilibrium equation is solved using FEM procedures incorporated into FEM numerical codes. At the local or material point scale, the constitutive equations describing the material deformation response are integrated numerically using the incremental strains and set of material state variables as inputs to the integration procedure. Although commercial FEM codes provide some basic constitutive material behaviors that can be utilized for structural analysis, in general, such

models are inadequate in the sense of rate dependency but are adequate for rate- 
independent nonlinear problems. Constitutive material behaviours for modeling the anisotropic yield and hardening characteristics of single-crystals are limited or nonexistant in many commercial finite element codes. To overcome this limitation finite element solvers such as ABAQUS allow the user to define constitutive theories in a very general way via user software subroutines, which perform the local material point calculations.

In this chapter the finite element implementation of a micro-phenomenological constitutive model for cubic single-crystals is presented. The constitutive equations were implemented as a user subroutine denoted UMAT in the commercial finite element code ABAQUS/Standard. The single-crystal constitutive model considered in this investigation is similar to micro-phenomenological constitutive model presented in Chapter 4; however, a rate independent formulation was adopted because of the predominantly rate independent constitutive behaviour observed during the constitutive material tests (see Chapter 5). The FEM implementation is formulated based on the implicit, backward Euler time-integration procedure and solved iteratively via an elastic predictor and plastic-corrector return mapping algorithm. In addition to discussing the general numerical approach, a procedure for identifing the constitutive material model parameters is presented. Finally, the application of the constitutive model and predicted material response are demonstrated.

\subsection{Single-Crystal Micro-Phenomenological Constitutive Model}

The micro-phenomenological constitutive model employed in this investigation is formulated similar to the single-crystal model proposed by Han and coworkers [226] that was discussed in Chapter 4; however, these equations have been reformulated for rate independent material behaviour and isotropic hardening was not considered. Equations 
4.13 to 4.17 are retained in the present formulation and for conciseness will not be repeated here. Unlike the micro-phenomenological model presented in Chapter 4, two back stress state variables are employed in the present formulation to model the postyield constitutive material response. The first back stress is defined by a non-linear evolution equation having the Armstrong-Frederic form:

$$
\dot{x}^{s[1]}=\left[c^{s[1]} \operatorname{sign}\left(\tau^{s}-\bar{x}^{s}\right)-d^{s[1]} x^{s[1]}\right] \dot{\gamma}^{s}
$$

A linear evolution equation was specified for the second back stress:

$$
\dot{x}^{s[2]}=c^{s[2]} \dot{\gamma}^{s} \operatorname{sign}\left(\tau^{s}-\bar{x}^{s}\right)
$$

Separate back stress evolution equations are specified for each of the octahedral and cubic slip systems. The total back stress for each slip system is obtained by summing both back stress state variables for each slip system,

$$
\bar{x}^{s}=\sum_{i=1}^{2} x^{s[i]}
$$

The yield functions associated with the octahedral slip and cubic systems are defined by:

$$
\begin{aligned}
& f_{o}^{s}(\sigma, \bar{x})=\left|\tau_{o}^{s}-\bar{x}_{o}^{s}\right|-k_{o}^{s} \leq 0 \\
& f_{c}^{s}(\sigma, \bar{x})=\left|\tau_{c}^{s}-\bar{x}_{c}^{s}\right|-k_{c}^{s} \leq 0
\end{aligned}
$$


The evolution of the macroscopic inelastic strain for rate independent plasticity using a multiple yield surface criterion is described by Koiter's rule (after Simo and Hughes [238]):

$$
\dot{\boldsymbol{\varepsilon}}^{i n}=\sum_{s=1}^{m} \dot{\gamma}^{s} \partial_{\sigma} f^{s}
$$

which naturally leads to the result presented earlier in equation 4.17 . Excluding the three independent elastic constants, the micro-phenomenological constitutive model utilizes 8 material parameters, 4 for the cubic slip systems and 4 for the octahedral slip systems. A procedure for obtaining the material parameters from uniaxial tensile data is described in section 6.6.3.

\subsection{Numerical Implementation of the Constitutive Model}

When the subroutine UMAT is called, it is provided with the total strain increment, $\Delta \varepsilon_{n+1}$, and time increment, $\Delta t$, for step $n+1$ of the global FEM solution and the values

for the stress and state variables from the last converged solution step, $n$. The function of the subroutine UMAT is to update the variables for step $n+1$, which include the macroscopic stress tensor, $\sigma_{n+1}$, and inelastic strain tensor, $\varepsilon_{n+1}^{i n}$, and the inelastic shear strain, $\gamma_{n+1}^{s}$, and back stress, $x_{n+1}^{[i] s}$, for each crystallographic slip system. The subroutine UMAT must also calculate the tangent stiffness matrix for the constitutive model at step $n+1$ as required for an iterative N-R solution of the global finite element problem. Using the backward Euler method (equation 4.29 with $\theta=1$ ), the variables at the end of the step for micro-phenomenological model are expressed as: 


$$
\begin{gathered}
\varepsilon_{n+1}^{i n}=\varepsilon_{n}^{i n}+\dot{\varepsilon}_{n+1}^{i n} \Delta t \\
x_{n+1}^{s[1]}=x_{n}^{s[1]}+\dot{x}_{n+1}^{s[1]} \Delta t \\
x_{n+1}^{s[2]}=x_{n}^{s[2]}+\dot{x}_{n+1}^{s[2]} \Delta t \\
\gamma_{n+1}^{s}=\gamma_{n}^{s}+\dot{\gamma}_{n+1}^{s} \Delta t \\
\sigma_{n+1}=C:\left(\varepsilon_{n+1}-\varepsilon_{n+1}^{i n}\right)
\end{gathered}
$$

From equations 4.13 and 6.6 the stress at the end of the increment (equation 6.10) may also be expressed by:

$$
\sigma_{n+1}=C:\left(\varepsilon_{n}^{e l}+\Delta \varepsilon_{n+1}-\Delta \varepsilon_{n+1}^{i n}\right)
$$

The quantity given by:

$$
\sigma^{\text {trial }}=C:\left(\varepsilon_{n}^{\epsilon l}+\Delta \varepsilon_{n+1}\right)
$$

represents the elastic trial stress and $C: \Delta \varepsilon_{n+1}^{i n}$ represents the plastic corrector. Combining equations $4.17,6.6$ and 6.9 , and substituting into equation 6.11 one obtains:

$$
\sigma_{n+1}=\sigma^{t r i a l}-C: \sum_{s \in s^{a c t}} m^{s} \Delta \gamma_{n+1}^{s} \operatorname{sign}\left(\tau_{n+1}^{s}-\bar{x}_{n+1}^{s}\right)
$$


where $s \in s^{a c t}$ represents the set of active slip systems. The method for determining the active set of slip systems is discussed in Appendix A. The resolved shear stress on each slip system is obtained by substituting equation 6.13 into equation 4.15 :

$$
\tau_{n+1}^{s}=m^{s}:\left[\sigma^{\text {trial }}-\sum_{s \in s^{s c t}} m^{s} \Delta \gamma_{n+1}^{s} \operatorname{sign}\left(\tau_{n+1}^{s}-\bar{x}_{n+1}^{s}\right)\right]
$$

After substituting equations 6.1 and 6.9 into equation 6.7 the first back stress, $x^{s[1]}$, becomes:

$$
x_{n+1}^{s[1]}=x_{n}^{s[1]}+\left[c^{s[1]} \operatorname{sign}\left(\tau_{n+1}^{s}-\bar{x}_{n+1}^{s}\right)-d^{s[1]} x_{n+1}^{s[1]}\right] \Delta \gamma_{n+1}^{s}
$$

Equation 6.15 can be manipulated to obtain a closed-form expression for the first back stress at the end of the increment in terms of the $x_{n}^{s[1]}$ and $\Delta y_{n+1}^{s}$ :

$$
x_{n+1}^{s[1]}=\frac{x_{n}^{s[1]}+c^{s[1]} \Delta \gamma_{n+1}^{s} \operatorname{sign}\left(\tau_{n+1}^{s}-\bar{x}_{n+1}^{s}\right)}{1+d^{s[1] \Delta \gamma_{n+1}^{s}}}
$$

Combining equations $6.2,6.8$ and 6.9 the second back stress, $x^{s[2]}$, can be expressed as:

$$
x_{n+1}^{s[2]}=x_{n}^{s[2]}+c^{s[2]} \Delta \gamma_{n+1}^{s} \operatorname{sign}\left(\tau_{n+1}^{s}-\bar{x}_{n+1}^{s}\right)
$$

After substitution of $6.14,6.16$ and 6.17 into the yield function (equation 6.4) one obtains the final result: 


$$
\begin{aligned}
& f_{n+1}^{s}\left(\sigma_{n+1}, \bar{x}_{n+1}^{s}\right)=\mid m^{s}: \sigma^{t r i a l}-m^{s}: C: \sum_{t \in t^{a c t}} m^{t} \Delta \gamma_{n+1}^{t} \operatorname{sign}\left(\tau_{n+1}^{t}-\bar{x}_{n+1}^{t}\right) \\
& -\frac{x_{n}^{s[1]}+c^{s[1]} \Delta \gamma_{n+1}^{s} \operatorname{sign}\left(\tau_{n+1}^{s}-\bar{x}_{n+1}^{s}\right)}{1+d^{s[1]} \Delta \gamma_{n+1}^{s}}-x_{n}^{s[2]} \\
& -c^{s[2]} \Delta \gamma_{n+1}^{s} \operatorname{sign}\left(\tau_{n+1}^{s}-\bar{x}_{n+1}^{s}\right) \mid-k^{s}
\end{aligned}
$$

in which the discretized equations for each slip system have been reduced to a series of $s \in s^{a c t}$ scalar equations defining the algorithmic consistency condition, in which there are $s \in s^{a c t}$ unknown $\Delta \gamma_{n+1}^{s}$ to determine. Equation 6.18 can be solved iteratively using the N-R method discussed earlier in Chapter 4. Development of a complete stress update algorithm based on the N-R method is presented in Appendix A.

\subsection{Consistent Tangent Stiffness Matrix}

In addition to the constitutive integration routine returning the updated values for the stress and state variables its purpose is to also return the tangent stiffness matrix for each of the element integration points. It is an essential quantity in implicit FEM codes because corrections to the incremental displacement field are calculated from the tangent stiffness, which assists the global FEM solution in achieving force equilibrium via a N-R iterative procedure. Numerous researchers $[241,244,247,249]$ have stated the importance of properly formulating the tangent stiffness matrix in terms of the linearized constitutive equations so as to obtain a tangent stiffness matrix that is consistent with the integration algorithm. While it should be emphasized that the tangent stiffness does not affect the accuracy of the FEM solution it has been shown [241, 244] to affect the number of equilibrium iterations necessary to achieve global convergence. At the material point scale, the solution to the constitutive equations is obtained when the 
residuals converge within some specified tolerance. Physically this means that based on the incremental displacement field, the solution at the integration point is at state of equilibrium. The tangent stiffness represents the change in stress when the displacement field - the total strain — is perturbed from this equilibrium state, which can be expressed mathematically as follows:

$$
C_{n+1}^{e p}=\frac{d \sigma_{n+1}}{d \varepsilon_{n+1}}
$$

The tangent stiffness for the micro-phenomenological model is developed from the incremental elastic-plastic stress-strain relationship, that is:

$$
\sigma_{n+1}=C:\left(\epsilon_{n+1}-\epsilon_{n+1}^{i n(k+1)}\right)
$$

Differentiating equation 6.20 with-respect-to the total strain one obtains:

$$
\frac{\partial \sigma_{n+1}}{\partial \varepsilon_{n+1}}=C-C: \frac{\partial \varepsilon_{n+1}^{i n}}{\partial \varepsilon_{n+1}}
$$

All that remains is to define the last partial derivative in equation 6.21 . Recalling that the inelastic strain is a function of $\Delta y^{s}$, one may use the chain rule to define this partial derivative in the following form:

$$
\frac{\partial \varepsilon_{n+1}^{i n}}{\partial \varepsilon_{n+1}}=\frac{\partial \varepsilon_{n+1}^{i n}}{\partial \Delta \gamma_{+1}^{s}} \otimes \frac{\partial \Delta \gamma_{n+1}^{s}}{\partial \varepsilon_{n+1}}
$$


The first partial derivative in equation 6.22 is obtained by differentiating 4.17 :

$$
\frac{\partial \varepsilon_{n+1}^{i n}}{\partial \Delta \gamma_{n+1}^{s}}=\sum_{s \in s^{a c t}} m^{s} \operatorname{sign}\left(\tau_{n+1}^{s}-\bar{x}_{n+1}^{s}\right)
$$

and after applying the chain rule again the second partial derivative is defined by:

$$
\frac{\partial \Delta \gamma_{n+1}^{s}}{\partial \varepsilon_{n+1}}=\left(\frac{\partial \Delta \gamma_{n+1}^{s}}{\partial R_{n+1}^{t}}\right)^{-1} \frac{\partial R_{n+1}^{t}}{\partial \varepsilon_{n+1}}
$$

The first partial derivative in equation 6.24 represents the inverse of the solution Jacobian matrix defined by equation A.5. The second partial derivative is obtained by differentiating the residual equation A.1:

$$
\frac{\partial R_{n+1}^{t}}{\partial \varepsilon_{n+1}}=m^{t}: C \operatorname{sign}\left(\tau_{n+1}^{t}-\bar{x}_{n+1}^{t}\right)
$$

Combining equations 6.21 to 6.25 and noting that summation over the slip system index $t$ is necessary, one obtains the final result for the tangent stiffness matrix:

$$
C_{n+1}^{e p}=C-\sum_{s \in s^{a c t}} \sum_{t \in t^{a c t}} C: m^{s} \operatorname{sign}\left(\tau_{n+1}^{s}-\bar{x}_{n+1}^{s}\right)\left(\frac{\partial \Delta \gamma_{n+1}^{s}}{\partial R_{n+1}^{t}}\right)^{-1} \otimes m^{t}: C \operatorname{sign}\left(\tau_{n+1}^{t}-\bar{x}_{n+1}^{t}\right)
$$

Equation 6.26 represents the consistent tangent stiffness since it is derived from the linearized form of the constitutive equations. 


\subsection{Constitutive Material Data}

As discussed in Chapter 5, uniaxial constitutive tests were performed on the material used in this study to assess the monotonic tensile behaviour. The purpose of undertaking these tests was to develop a data set from which an appropriate constitutive law could be selected and to identify the constitutive material parameters. The tensile tests were conducted at $650^{\circ} \mathrm{C}$ on specimens oriented in the $[010]$ material direction. Unfortunately, tensile data for [010] material direction alone is inadequate for determining the orientation dependence of the mechanical properties. Therefore, tensile test data for specimens oriented in the [111] material direction was obtained from the literature. The tensile test results published by Alden [132] for René N4 was used to supplement the PWA1484 [010] tensile test data. Although the published data is for a different alloy than the one considered in the present work and was conducted at a higher test temperature $\left(760^{\circ} \mathrm{C}\right)$, this was the only published data that could be obtained that included a complete disclosure of the tensile testing parameters and sufficient data for extraction of the constitutive material parameters.

Tensile data for the [111] material orientation was obtained from test results reported by Alden [132] for René N4. The tensile tests were conducted under constant total strain rate conditions with total strain rates of $1.0 \times 10^{-4}$ and $6.0 \times 10^{-4} \mathrm{sec}^{-1}$. These strain rates were within the range of strain rates considered in the PWA1484 [010] constitutive tests described in Chapter 5. The tensile response for René N4 with a [111] material orientation is shown in Figure 6.1. It may be noted that two sets of tensile test data are presented on this figure. Two tensile curves are shown on the same plot because one specimen was used to obtain tensile data at two different strain rates using a so-called double tensile test. In this type of test, tensile loading is commenced at slow strain rate 
$\left(6.0 \times 10^{-4} \mathrm{sec}^{-1}\right)$ to a predetermined strain level, then the load is removed, and finally the specimen is reloaded at a higher strain rate $\left(1.0 \times 10^{-4} \mathrm{sec}^{-1}\right)$ until the tensile test is discontinued or specimen failure occurs. Referring to Figure 6.1 it may be observed that this material exhibits little rate dependence at $760^{\circ} \mathrm{C}$, and therefore it was assumed that both tensile curves could be combined into a single tensile curve. The stress-strain data were extracted from Alden's [132] tensile data based on this assumption, and used to estimate the constitutive material parameters for the [111] crystallographic orientation.

\subsection{Identification of the Constitutive Material Parameters}

\subsubsection{Transformation of Stress and Strain Tensors}

In structural analysis problems tensor quantities, such as stress and strain, are usually referenced to the geometric or global coordinate system, which is convenient when conducting analysis of components. It is often necessary to express tensor quantities in terms of coordinate systems that are oriented differently. One such instance is when the analysis involves anisotropic materials. Since the constitutive equations stated earlier were formulated in the material coordinate system, all tensor quantities (e.g. stress and strain) must be referred to the material coordinate system.

The transformation law for Cartesian tensors can be developed by first deriving the transformation needed to relate the global and material coordinate systems. Consider two Cartesian coordinate systems that share common origin with $X, Y, Z$ representing the global coordinate system and $X^{\prime}, Y^{\prime}, Z^{\prime}$ representing the material coordinate system. The relationship between both coordinate systems is shown schematically in Figure 6.2. For cubic materials, the relationship between the global and material coordinate systems can be expressed in terms of two rotations; the first rotation about the material $Z$ '-axis by the 
angle $\theta$ and a second rotation, about the new $X^{\prime}$-axis, by the angle $\psi$, as illustrated Figure 6.3. Since it is sometimes convenient to use the standard stereographic triangle to show the material orientation, the relationship between the angles $\theta$ and $\psi$ and the geometrical features of the standard stereographic triangle are illustrated in Figure 6.4. Each of the basis vectors $X_{i}$ that define the global coordinate system can be expressed as functions of the material coordinate system basis vectors $X_{j}^{\prime}$ through the transformation tensor $Q_{i j}$ :

$$
X_{i}=Q_{i j} X_{j}^{\prime}
$$

The second-rank transformation tensor, $Q_{i j}$, contains the direction cosines that transform $X_{j}^{\prime}$ to the $X_{i}$ coordinate system which are derived such that they satisfy orthogonality conditions [264].

Using the orthogonality conditions one may derive the remaining direction cosines that transform the global to the material coordinate system. The final result for the present case is:

$$
[Q]=\left[\begin{array}{ccc}
\cos \theta & \sin \theta \cos \psi & \sin \theta \sin \psi \\
-\sin \theta & \cos \theta \cos \psi & \cos \theta \sin \psi \\
0 & -\sin \psi & \cos \psi
\end{array}\right]
$$

The transformation between the material and global coordinate systems is given by the matrix transpose of equation 6.28 : 


$$
[Q]^{T}=\left[\begin{array}{ccc}
\cos \theta & -\sin \theta & 0 \\
\sin \theta \cos \psi & \cos \theta \cos \psi & -\sin \psi \\
\sin \theta \sin \psi & \cos \theta \sin \psi & \cos \psi
\end{array}\right]
$$

The symmetry of the stress and strain tensor can be exploited to express these tensors in Voigt vector notation, therby simplifying the resulting mathematical form for transforming the stress and strain tensors. Adopting the ABAQUS stress and strain vector notation, i.e. $\{\boldsymbol{\sigma}\}=\left\{\sigma_{11}, \sigma_{22}, \sigma_{33}, \sigma_{12}, \sigma_{13}, \sigma_{23}\right\}$ and $\{\varepsilon\}=\left\{\varepsilon_{11}, \varepsilon_{22}, \varepsilon_{33}, \gamma_{12}, \gamma_{13}, \gamma_{23}\right\}$ where $\gamma_{\mathrm{xy}}$ is the engineering shear strain $\left(\gamma_{x y}=2 \varepsilon_{x y}\right)$, the transformation of the stress and strain tensors can be conducted by means of a transformation matrix:

$$
[A]=\left[\begin{array}{cccccc}
Q_{11}^{2} & Q_{12}^{2} & Q_{13}^{2} & 2 Q_{11} Q_{12} & 2 Q_{11} Q_{13} & 2 Q_{12} Q_{13} \\
Q_{21}^{2} & Q_{22}^{2} & Q_{23}^{2} & 2 Q_{21} Q_{22} & 2 Q_{21} Q_{23} & 2 Q_{22} Q_{23} \\
Q_{31}^{2} & Q_{32}^{2} & Q_{33}^{2} & 2 Q_{31} Q_{32} & 2 Q_{31} Q_{33} & 2 Q_{32} Q_{33} \\
Q_{11} Q_{21} & Q_{12} Q_{22} & Q_{13} Q_{23} & Q_{12} Q_{21}+Q_{11} Q_{22} & Q_{13} Q_{21}+Q_{11} Q_{23} & Q_{11} Q_{23}+Q_{13} Q_{22} \\
Q_{11} Q_{31} Q_{12} Q_{23} & Q_{13} Q_{33} & Q_{11} Q_{32}+Q_{12} Q_{31} & Q_{11} Q_{33}+Q_{13} Q_{31} & Q_{11} Q_{33}+Q_{13} Q_{32} \\
Q_{21} Q_{31} & Q_{22} Q_{32} & Q_{32} Q_{33} & Q_{21} Q_{32}+Q_{22} Q_{31} & Q_{21} Q_{33}+Q_{23} Q_{31} & Q_{22} Q_{33}+Q_{23} Q_{32}
\end{array}\right]
$$

In vector-matrix form the transformations for stress and strain are given by:

$$
\begin{gathered}
\left\{\sigma^{\prime}\right\}=[A]\{\sigma\} \\
\left\{\varepsilon^{\prime}\right\}=[R][A][R]^{-1}\{\varepsilon\}
\end{gathered}
$$

where $[R]$ is the Reuter matrix defined by [115]: 


$$
[R]=\left[\begin{array}{llllll}
1 & 0 & 0 & 0 & 0 & 0 \\
0 & 1 & 0 & 0 & 0 & 0 \\
0 & 0 & 1 & 0 & 0 & 0 \\
0 & 0 & 0 & 2 & 0 & 0 \\
0 & 0 & 0 & 0 & 2 & 0 \\
0 & 0 & 0 & 0 & 0 & 2
\end{array}\right]
$$

\subsubsection{Elastic Constants}

As discussed in Chapter 2 single-crystal nickel-base superalloys have cubic crystal symmetry, and therefore the elastic properties of these materials are described using three independent elastic constants, $E_{[010]}, Y_{0010]}$ and $G_{[010]}$, which represent the elastic modulus, Poisson ratio and shear modulus referenced to the principal material axes. These elastic constants can obtained from measurements of the uniaxial elastic properties from the principal material orientation and a second arbitrary orientation, and performing calculations using anisotropic elasticity theory.

The strain response of a tensile specimen loaded by a uniaxial stress, $\sigma$, applied along a principal material direction is given by:

$$
\varepsilon=S: \sigma
$$

where the forth-order tensor $\boldsymbol{S}$ represents the compliance coefficients. For cubic symmetry materials there are three independent compliance coefficients which can be specified as functions of $E_{[010]}, v_{[010]}$ and $G_{[010]}$. Using concise tensor notation the compliance coefficients are: 


$$
\begin{gathered}
S_{11}=\frac{1}{E_{[010]}} \\
S_{12}=-\frac{\nu_{[010]}}{E_{[010]}} \\
S_{44}=\frac{1}{2 G_{[010]}}
\end{gathered}
$$

For uniaxial loading along any other crystal direction [h $\mathrm{k} \mathrm{l}$ ], the stress and strain measured during the tensile test must be transformed to the principal material axes. The stress and strain transformations can be performed using the methods described in the previous section. From anisotropic linear elasticity theory it has been established that the uniaxial stress and strain for any crystallographic orientation, represented by, $\sigma_{[\mathrm{hk}]}$ and $\varepsilon_{[\mathrm{hkl}}$, respectively, can be represented in terms of the three compliance coefficients using the following relationship [265]:

$$
\varepsilon_{[h k l]}=\sigma_{[h k l]}\left[S_{11}-2\left(S_{11}-S_{12}-\frac{S_{44}}{2}\right)\left(t_{1}^{2} t_{2}^{2}+t_{1}^{2} t_{3}^{2}+t_{2}^{2} t_{3}^{2}\right)\right]
$$

where $t_{i}$ represent the direction cosines, which in the present case are given by:

$$
\begin{aligned}
& t_{1}=\sin \theta \cos \psi \\
& t_{2}=\cos \theta \cos \psi \\
& t_{3}=-\sin \psi
\end{aligned}
$$

Where The quantity, $a_{[\mathrm{hkl}]} / \sigma_{[\mathrm{hkl}]}$, is immediately recognizable as the inverse of the apparent elastic modulus, $1 / E_{[\mathrm{hkl}]}$. The apparent elastic modulus can be obtained from the linear-elastic portion of the uniaxial stress-strain curve. 
Stouffer and Dame [115] have noted that tensile compliance measurements from the principal orientation and two other crystallographic orientations are insufficient to determine the elastic parameters because of coupling between $S_{11}, S_{12}$ and $S_{44}$ in equation 6.36. This dilemma is resolved by measuring $E_{[010]}$ and $v_{[010]}$ from a uniaxial tensile specimen loaded in one of the principal material directions. The shear modulus, $G_{[010]}$, is then obtained from uniaxial tensile test results from any other crystallographic orientation after solving equation 6.36 for $S_{44}$.

Measurements of the Poisson ratio were not obtained from the [010] tensile tests performed in this investigation. Nissley and coworkers [266] have presented elastic compliance and apparent elastic modulus measurement results for the single-crystal superalloy PWA1480 over a wide range of test temperatures. From their published results $S_{12}=-3.9 \times 10^{-6} \mathrm{MPa}^{-1}$ and the apparent elastic modulus $E_{[010]}=103,421 \mathrm{MPa}$ for PWA1480 at $650^{\circ} \mathrm{C}$. Substituting these values into equation 6.35 (b) one obtains $v_{[010]} \approx$ 0.4 .

The elastic coefficients used in the present investigation were obtained by extracting the apparent elastic modulus from the $10^{-2} \sec ^{-1}[010]$ tensile curve shown in Figure 5.23, and the [111] tensile curves presented by Alden [132] for René N4. The compliance coefficients were then determined from the apparent elastic modulii and using 6.36 with $v_{[010]}=0.4$. The three stiffness coefficients were then calculated by assembling the compliance coefficients into Voigt matrix form and calculating the matrix inverse. The elastic stiffness constants and compliance coefficients are summarized in Table 6.1. 


\subsubsection{Inelastic Constitutive Material Parameters}

Han and co-workers [226] describe various procedures used to identify the material constants using monotonic and cyclic data for constitutive equations similar to those described in Chapter 4. Their procedure involves deriving analytical equations from the constitutive material law which describe the macroscopic stress and strain response obtained from simple constitutive material tests, e.g. uniaxial tensile and cyclic loading tests. However, due to inelastic anisotropy single-crystal materials can exhibit complex multiaxial deformation, even under uniaxial loading, that cannot be described using closed-form analytical expressions. Therefore, the procedure to identify the constitutive material parameters must consider specific crystallographic orientations in which the apparent inelastic deformation behaviour is macroscopically "isotropic", i.e. initially

round uniaxial specimens remain round after inelastic deformation. The [010] and [111] crystallographic orientations fulfill this requirement under uniaxial loading [226]. The procedures described by Han and co-workers [226] for identifying the inelastic constitutive material parameters have been adapted to the constitutive model considered in this investigation. The procedure for identifying the material constants will be discussed in the following sections.

\subsubsection{Analytical Equations for Uniaxial Loading}

The analytical equations used to identify the constitutive material parameters are derived from the constitutive equations. For uniaxial loading applied in the [010] crystallographic direction the applied macroscopic stress, $\sigma_{[010]}$, and macroscopic inelastic strain, $\varepsilon_{[010]}^{i n}$, are aligned with the principal material directions. The resolved shear stress acting on the 12 octahedral and 6 cubic slip systems can be calculated using equations 4.15 and 4.16 to determine which slip systems contribute to inelastic deformation. The final result is: 


$$
\begin{aligned}
& \tau^{1}=\tau^{6}=\tau^{7}=\tau^{12}=\tau^{13}=\tau^{14}=\tau^{15}=\tau^{16}=\tau^{17}=\tau^{18}=0 \\
& \tau^{2}=\tau^{4}=\tau^{8}=\tau^{10}=\frac{1}{\sqrt{6}} \sigma_{[010]} \\
& \tau^{3}=\tau^{5}=\tau^{9}=\tau^{11}=-\frac{1}{\sqrt{6}} \sigma_{[010]}
\end{aligned}
$$

It is apparent that 8 octahedral slip systems have non-zero resolved shear stresses which are equal in magnitude. The resolved shear stress on the remaining 4 octahedral and 6 cubic slip systems are all zero. Since the resolved shear stresses on each of the eight octahedral slip planes (with non-zero resolve shear stresses) have the same magnitude, the inelastic shear strain rate slip on each slip system is also equal. The macroscopic inelastic strain rates in the material coordinate system are obtained from equation 4.17:

$$
\begin{aligned}
& \dot{\varepsilon}_{11}^{i n}=\dot{\varepsilon}_{33}^{i n}=-\frac{1}{2} \dot{\varepsilon}_{22}^{i n} \\
& \dot{\varepsilon}_{12}^{i n}=\dot{\varepsilon}_{13}^{i n}=\dot{\varepsilon}_{23}^{i n}=0 \\
& \text { with, } \\
& \dot{\varepsilon}_{22}^{i n}=\dot{\varepsilon}_{[010]}^{i n}=\frac{8}{\sqrt{6}} \dot{\gamma}_{o}^{s}
\end{aligned}
$$

Equation 6.39 (c) can be inverted to obtain the inelastic shear strain rate as a function of the macroscopic inelastic strain rate in the global coordinate system:

$$
\dot{\gamma}_{o}^{s}=\frac{\sqrt{6}}{8} \dot{\varepsilon}_{[010]}^{i n}
$$

The result presented in equation 6.40 can be substituted into each of the back stress evolution equations (equations 6.1 and 6.2) to obtain the back stress rates on each of the 
active octahedral slip systems as a function of the global inelastic strain rate. Carrying out this substitution and integrating the back stress evolution equations explicitly from the initial conditions $\varepsilon_{[010]}^{i n}=0, x_{o}^{[1]}=0$ and $x_{o}^{[2]}=0$ and dropping the superscript $s$ since the octahedral material parameters are identical one obtains:

$$
\begin{aligned}
& x_{o}^{[1]}=\frac{c_{o}^{[1]}}{d_{o}^{[1]}}\left[1-\exp \left(-\frac{\sqrt{6}}{8} d_{o}^{[1]} \varepsilon_{[010]}^{i n}\right)\right] \\
& x_{o}^{[2]}=\frac{\sqrt{6}}{8} c_{o}^{[2]} \varepsilon_{[010]}^{i n}
\end{aligned}
$$

After substituting equations 6.38 into the octahedral yield function, equation 6.4(a) one obtains the analytical equation for unaxial loading in the [010] direction:

$$
\sigma_{[010]}=\sqrt{6} x_{o}^{[1]}+\sqrt{6} x_{o}^{[2]}+\sqrt{6} k_{o}
$$

with $x_{o}^{[1]}$ and $x_{o}^{[2]}$ defined by equation 6.41 .

A similar analytical equation can be developed for uniaxial loading along the [111] crystallographic direction. For uniaxial loading along the global $\mathrm{Y}$ axis (see Figure 6.3) the macroscopic stress and inelastic strain in the global coordinate system are defined by $\sigma_{[11]}$ and $\varepsilon_{[11]}^{i n}$, respectively. Since the global stress and inelastic strain are not aligned with the principal material directions, they must be transformed to the material coordinate system using the procedures described earlier. The stresses in the material coordinate system are: 


$$
\sigma_{11}=\sigma_{22}=\sigma_{33}=\sigma_{12}=-\sigma_{13}=-\sigma_{23}=\frac{1}{3} \sigma_{[111]}
$$

To determine which of the 18 slip systems are active during monotonic tensile loading along the [111] crystal direction, the resolved shear stress is calculated using Schmid's law (equation 4.15):

$$
\begin{aligned}
& \tau^{1}=\tau^{5}=\tau^{12}=\tau_{o}, \tau^{3}=\tau^{4}=\tau^{11}=-\tau_{o} \\
& \tau^{2}=\tau^{6}=\tau^{7}=\tau^{8}=\tau^{9}=\tau^{10}=0 \\
& \tau^{13}=\tau^{16}=\tau^{18}=-\tau_{c} \\
& \tau^{14}=\tau^{15}=\tau^{17}=0
\end{aligned}
$$

with,

$$
\begin{aligned}
\tau_{o} & =\frac{\sqrt{2}}{3 \sqrt{3}} \sigma_{[111]} \\
\tau_{c} & =\frac{\sqrt{2}}{3} \sigma_{[111]}
\end{aligned}
$$

Since the non-zero resolved shear stresses exist on both the cubic and octahedral slip systems, both slip systems potentially can contribute to inelastic deformation. However, the resolved shear stress for cubic slip is higher than that for octahedral slip by a factor of $\sqrt{3}$, and therefore at small inelastic strains, those which are primarily of interest in infinitesimal strain problems, octahedral slip systems will not be active for [111] uniaxial loading. Furthermore, Han and coworkers [226] have shown that the octahedral contribution to the inelastic deformation for the [111] crystallographic orientation is negligible. Therefore, the octahedral slip contribution will not be considered further in the 
development of the analytical uniaxial equation. It may also be noted that the resolved shear stresses on each of the three cubic slip systems (with non-zero resolve shear stresses) have the same magnitude, and therefore the inelastic shear strain rate slip on each slip system is also equal. The macroscopic inelastic strain rates in the material coordinate system are obtained from the flow rule (equation 4.17) and then are transformed back to the global coordinate system with the final result being:

$$
\begin{aligned}
& \dot{\varepsilon}_{11}^{i n}=\dot{\varepsilon}_{33}^{i n}=-\frac{1}{2} \dot{\varepsilon}_{22}^{i n} \\
& \dot{\varepsilon}_{12}^{i n}=\dot{\varepsilon}_{13}^{i n}=\dot{\varepsilon}_{23}^{i n}=0 \\
& \text { with, } \\
& \dot{\varepsilon}_{22}^{i n}=\dot{\varepsilon}_{[111]}^{i n}=\sqrt{2} \dot{\gamma}_{c}^{s}
\end{aligned}
$$

Equation 6.46 (c) can be inverted and then substituted into the back stress evolution equations (equations 6.1 and 6.2) to obtain the back stress rates on each of the cubic slip planes (with non-zero resolved shear stresses) as a function of the global inelastic strain rate. Similar to the analytical expressions developed for uniaxial loading along the [010] crystallographic direction, the back stress evolution equations can be integrated explicitly from the initial conditions $\varepsilon_{[111]}^{i n}=0, x_{c}^{[1]}=0$ and $x_{c}^{[2]}=0$ and dropping the superscript $s$, since it is assumed the cubic material parameters are identical for each of the cubic slip systems, one obtains:

$$
\begin{aligned}
& x_{c}^{[1]}=\frac{c_{c}^{[1]}}{d_{c}^{[1]}}\left[1-\exp \left(-\frac{1}{\sqrt{2}} d_{c}^{[1]} \varepsilon_{[111]}^{i n}\right)\right] \\
& x_{c}^{[2]}=\frac{1}{\sqrt{2}} c_{c}^{[2]} \varepsilon_{[111]}^{i n}
\end{aligned}
$$


After substituting equations 6.45(b) into the octahedral yield function, equation 6.4(b), one obtains the analytical equation for uniaxial loading in the [111] direction:

$$
\sigma_{[111]}=\frac{3}{\sqrt{2}} x_{c}^{[1]}+\frac{3}{\sqrt{2}} x_{c}^{[2]}+\frac{3}{\sqrt{2}} k_{c}
$$

with $x_{c}^{[1]}$ and $x_{c}^{[2]}$ defined by equation 6.47 . It may observed that the analytical equations for uniaxial loading along the [111] crystallographic direction (equations 6.47 and 6.48) are identical in form to those presented for [010] uniaxial loading (equations 6.41 and 6.42). These equations are function of the cubic and octahedral inelastic material parameters, the applied stress and the inelastic strain. Therefore, the analytical equations can be presented in a more generic form for conducting regression analyses to determine the inelastic material parameters. The generic analytical equation for uniaxial loading is:

$$
\sigma=\frac{c^{[1]^{\prime}}}{d^{[1]^{\prime}}}\left[1-\exp \left(-d^{[1]^{\prime}} \varepsilon^{i n}\right)\right]+c^{[2]^{\prime}} \varepsilon^{i n}+k^{\prime}
$$

with,

$$
\begin{aligned}
& k^{\prime}=\sqrt{6} k_{o} \\
& c^{[1]^{\prime}}=\frac{3}{4} c_{o}^{[1]} \quad d^{[1]^{\prime}}=\frac{3}{4} c_{o}^{[1]} \quad c^{[2]^{\prime}}=\frac{3}{4} c_{o}^{[2]}
\end{aligned}
$$

for the octahedral inelastic material parameters and,

$$
\begin{aligned}
& k^{\prime}=\frac{3}{\sqrt{2}} k_{c} \quad d^{[1]^{\prime}}=\frac{1}{\sqrt{2}} d_{c}^{[1]} \quad c^{[2]^{\prime}}=\frac{3}{2} c_{c}^{[2]} \\
& c^{[1]^{\prime}}=3 c_{c}^{[1]}
\end{aligned}
$$


for the cubic slip inelastic material parameters. In the following section a regression analysis procedure for identifying the inelastic material parameters is derived from the generic analytical equation for uniaxial loading.

\subsubsection{Regression Analysis}

From the generic analytical equation for uniaxial loading it may be observed that two variables, $\sigma$ and $\varepsilon^{\text {in }}$ are required to identify the inelastic material parameters. During monotonic uniaxial tensile testing, the recorded test variables include the stress and total strain. The inelastic strain can be determined by subtracting the elastic strain from the total strain response recorded during the uniaxial tensile test. The elastic strain component depends on the apparent elastic modulus which can easily be evaluated from

the linear-elastic region of the monotonic tensile curve for $[010]$ and $[111]$ oriented single-crystals. Once the apparent elastic modulus is known, the inelastic strain can be calculated from the following:

$$
\varepsilon^{i n}=\varepsilon-\frac{\sigma}{E}
$$

where $\sigma$ is the applied stress, $\varepsilon$ is the total axial strain and $E$ is the apparent elastic modulus. Now, all that is required is to identify a numerical procedure to identify the constitutive material parameters from the generic uniaxial equations.

The back stress material constants and the critical resolved shear stress for the octahedral and cubic constitutive equations can be identified by developing a least squares regression procedure from the generic uniaxial equation. The regression equations are described by a set of non-linear equations given by: 


$$
R^{2}=\sum_{i=1}^{n}\left(Y_{i}-\bar{Y}_{i}\right)^{2}
$$

with

$$
\begin{aligned}
Y_{i} & =\sigma_{i} \\
\bar{Y}_{i} & =k^{\prime}+\frac{c^{[1]^{\prime}}}{d[1]^{\prime}}\left[1-\exp \left(-d^{[1]^{\prime}} \varepsilon_{i}^{i n}\right)\right]+c^{[2]^{\prime}} \varepsilon_{i}^{i n}
\end{aligned}
$$

where $R$ represents the residual. Separate equations are formulated for [010] and $[111]$ uniaxial tensile test data to obtain the octahedral and cubic inelastic material parameters. Equation $6.53(\mathrm{c})$ is non-linear in $d^{[1]}$, but it can be linearized by assuming an initial value for $d^{[1]}$, [267]. For fixed $d^{[1]}$, the optimum values for $k, c^{[1]}$ and $c^{[2]}$ are determined when,

$$
\begin{aligned}
& \frac{\partial R^{2}}{\partial k^{\prime}}=\sum_{i=1}^{n}\left\{Y_{i}-k^{\prime}-\frac{c^{[1]^{\prime}}}{d^{[1]^{\prime}}}\left[1-\exp \left(-d^{[1]^{\prime}} \varepsilon_{i}^{i n}\right)\right]-c^{[2]^{\prime}} \varepsilon_{i}^{i n}\right\}=0 \\
& \frac{\partial R^{2}}{\partial c^{[1]^{\prime}}}=\sum_{i=1}^{n}\left\{Y_{i}-k^{\prime}-\frac{c^{[1]^{\prime}}}{d^{[1]^{\prime}}}\left[1-\exp \left(-d^{[1]^{\prime}} \varepsilon_{i}^{i n}\right)\right]-c^{[2]^{\prime}} \varepsilon_{i}^{i n}\right\} \\
& \frac{1}{d[1]^{\prime}}\left[1-\exp \left(-d^{[1]^{\prime}} \varepsilon_{i}^{i n}\right)\right]=0 \\
& \frac{\partial R^{2}}{\partial c^{[2]^{\prime}}}=\sum_{i=1}^{n}\left\{Y_{i}-k^{\prime}-\frac{c^{[1]^{\prime}}}{d^{[1]^{\prime}}}\left[1-\exp \left(-d^{[1]^{\prime}} \varepsilon_{i}^{i n}\right)\right]-c^{[2]^{\prime}} \varepsilon_{i}^{i n}\right\} \varepsilon_{i}^{i n}=0
\end{aligned}
$$

This system of linear equations can be solved using any number of solution techniques for linear equations. In the present investigation these equations were solved using Gaussian elimination. It should be noted that the values obtained for $k^{\prime}, c^{[1]}$ and $c^{[2]}$ are determined for a fixed $d^{[1]}$, represent only "locally" optimized values. The global optimum for all inelastic parameters is obtained by repeating the regression analysis process with different starting values for $d^{[1]}$, until the sum of the residuals squared is a 
globally minimum value. Using this regression analysis procedure, the constitutive material parameters were identified for octahedral slip and cubic slip from the [010] and [111] tensile curves described earlier. The inelastic material parameters for octahedral and cubic slip determined using the regression analysis procedure are presented in Table 6.2 .

\subsection{Comparison of Calculations to Experimental Data}

After completing the finite element implementation of the rate independent microphenomenological constitutive model and determining the constitutive material parameters, a number of simulations were conducted to validate the UMAT subroutine. All numerical tests were performed using ABAQUS/Standard and only uniaxial loading cases were considered. Since the calculations were performed only for uniaxial loading conditions, it was unnecessary to model the actual geometry of the test specimens. Instead, these tests were conducted on a single 20-node continuum brick element (ABAQUS C3D20 element type) loaded under simulated uniaxial and strain controlled loading. The calculations were performed to compare with the experimental uniaxial tensile curves and to demonstrate the predicted constitutive response of the UMAT subroutine under conditions for which no experimental data were available.

\subsubsection{Monotonic Deformation Behaviour}

The monotonic loading simulations were performed to compare the predicted constitutive material response to the [010] uniaxial tensile curve obtained in this investigation and the [111] uniaxial tensile curve presented by Alden [132]. The simulations considered strain controlled loading up to a total strain of $0.03 \mathrm{~mm} / \mathrm{mm}$. A comparison between the predicted tensile curve for $[010]$ and the experimental data obtained in this investigation 
is shown in Figure 6.5. It may be observed that the predicted stress response is within $2 \%$ of the experimental values, and therefore it may be concluded the agreement is excellent. The predicted tensile curve for [111] uniaxial loading is compared with the [111] tensile results presented by Alden [132] in Figure 6.6. The predicted stress response is generally within $3.5 \%$ of the experimental tensile curve.

\subsubsection{Cyclic Deformation Behaviour}

Additional simulations were performed to investigate the predicted response of the UMAT subroutine under more demanding uniaxial cyclic loading conditions. The simulations were performed for [010] and [111] crystallographic orientations under fully reversed uniaxial strain-controlled loading. Since no relevant experimental data was available for comparison, only the predicted constitutive material response is presented. The predicted first-cycle stress-strain hysteresis loop for [010] uniaxial loading and 0.02 $\mathrm{mm} / \mathrm{mm}$ strain range is presented in Figure 6.7. A similar stress-strain hysteresis loop for [111] uniaxial loading and $0.01 \mathrm{~mm} / \mathrm{mm}$ strain range is shown in Figure 6.8. Kinematic hardening effects are apparent in both the [010] and [111] stress-strain hysteresis loops, that is the apparent onset of yielding during the compressive portion of the loading cycle is lower than the apparent onset of yielding during the first quarter cycle in tension.

\subsection{Concluding Remarks}

A strain rate-independent micro-phenomenological constitutive model formulated based on a crystal plasticity framework for infinitesimal strain was implemented for 3-D finite element simulations using the ABAQUSIStandard UMAT subroutine interface. The stress update algorithm was formulated using an elastic-predictor plastic-corrector return mapping based on implicit (backward Euler) integration. The consistent tangent stiffness 
matrix was derived from the stress update algorithm. A method for deriving the anisotropic elastic and inelastic material constants from uniaxial tensile curves for the [010] and [111] crystallographic orientations was presented. The UMAT subroutine was demonstrated to provide adequate predictions of the [010] and [111] uniaxial tensile curves from which the material parameters were derived. It was also demonstrated that the UMAT subroutine could be used to predict the constitutive material response for fully reversed uniaxial cyclic loading conditions, but without cyclic fatigue data it is not possible to validate the predicted constitutive material behaviour. Additional experimental data from tests under multiaxial loading conditions would also be necessary to validate the UMAT subroutine for more demanding loading conditions. 
Table 6.1 - Elastic stiffness constants and compliance coefficients (in Voigt notation) determined from the $[010]$ and $[111]$ tensile curves.

\begin{tabular}{|c|c|c|c|c|c|}
\hline $\mathrm{C}_{11}(\mathrm{MPa})$ & $\mathrm{C}_{12}(\mathrm{MPa})$ & $\mathrm{C}_{44}(\mathrm{MPa})$ & $\mathrm{S}_{11}\left(\mathrm{MPa}^{-1}\right)$ & $\mathrm{S}_{12}\left(\mathrm{MPa}^{-1}\right)$ & $\mathrm{S}_{44}\left(\mathrm{MPa}^{-1}\right)$ \\
\hline 257,525 & 171,718 & 126,167 & $8.324 \times 10^{-6}$ & $-3.330 \times 10^{-6}$ & $7.926 \times 10^{-6}$ \\
\hline
\end{tabular}

Table 6.2 - Inelastic material parameters for octahedral and cubic slip.

\begin{tabular}{|l|c|c|c|c|}
\hline Parameters & $\mathrm{k}_{\mathrm{o}}(\mathrm{MPa})$ & $c_{\boldsymbol{o}}^{[1]}(\mathrm{MPa})$ & $d_{\boldsymbol{o}}^{[1]}$ & $c_{\boldsymbol{o}}^{[2]}(\mathrm{MPa})$ \\
\hline Octahedral Slip & 326.6 & 430,267 & 5,043 & 2,100 \\
\hline Parameters & $\mathrm{k}_{\mathrm{c}}(\mathrm{MPa})$ & $c_{c}^{[1]}(\mathrm{MPa})$ & $d_{c}^{[1]}$ & $c_{c}^{[2]}(\mathrm{MPa})$ \\
\hline Cubic Slip & 230.9 & 913,748 & 5,207 & 2,651 \\
\hline
\end{tabular}




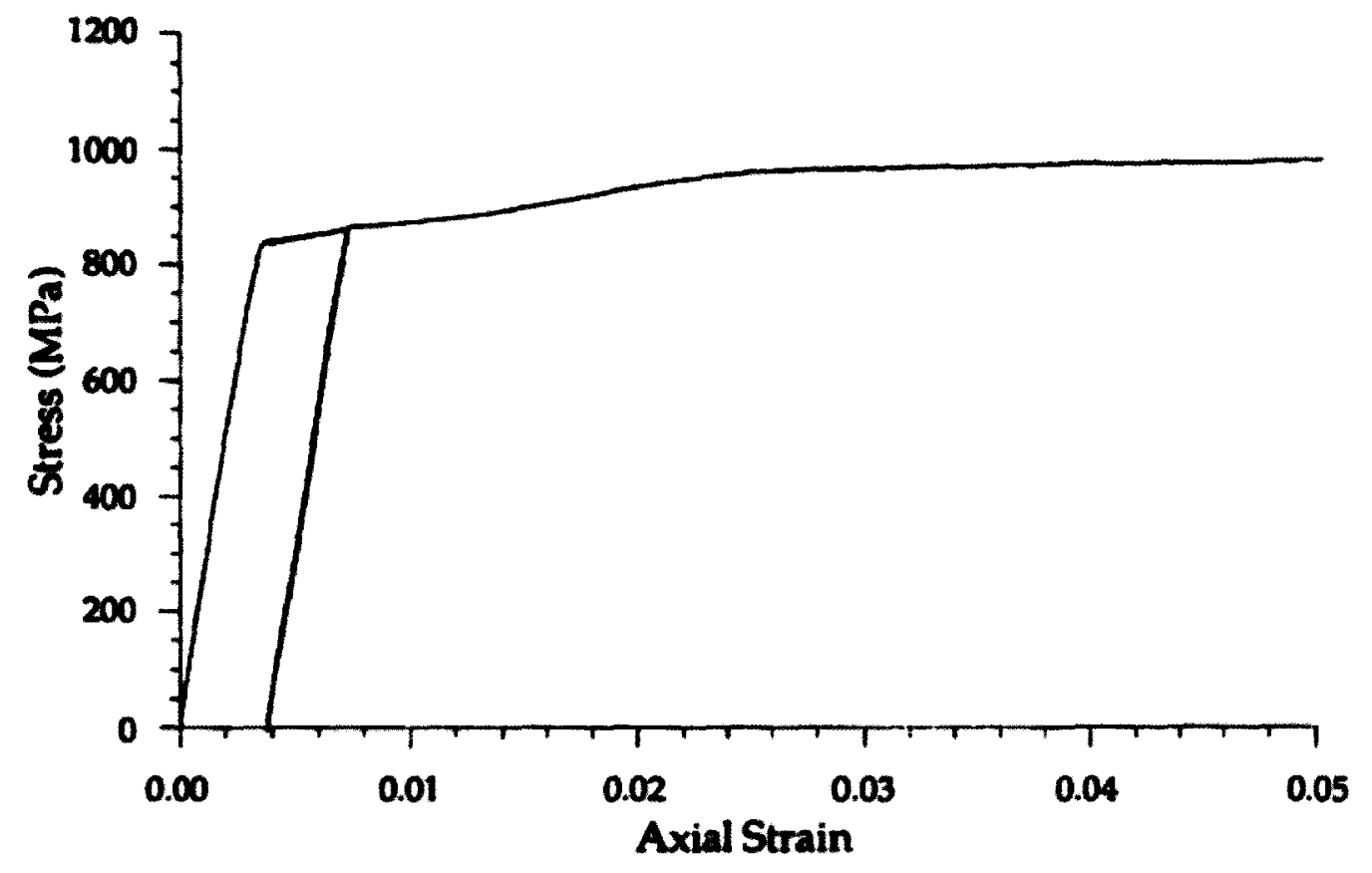

Figure 6.1 - Engineering stress-strain curve for René N4 with [111] material orientation tested at $760^{\circ} \mathrm{C}$ using a double tensile test (total strain rates were $6.0 \times 10^{-4} \sec ^{-1}$ and $6.0 \times 10^{-4}$ $\left.\sec ^{-1}\right)[132]$.

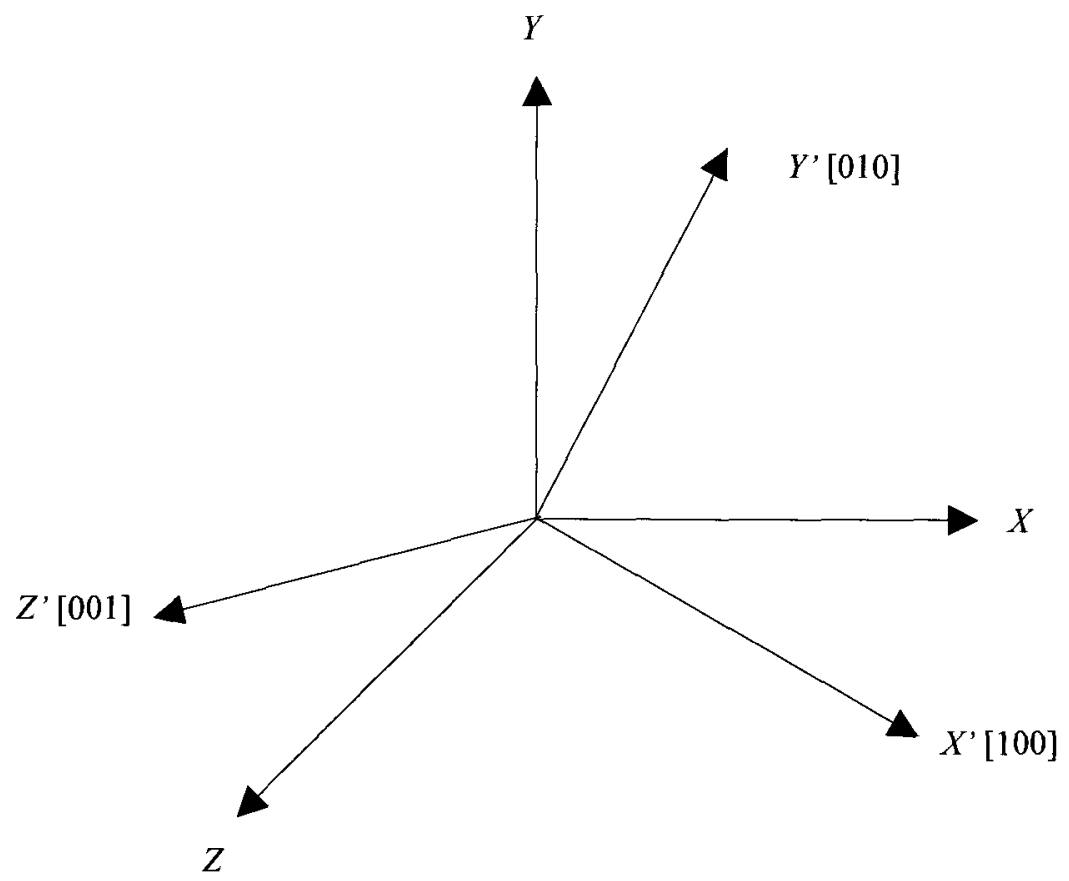

Figure 6.2 - Schematic showing the relationship between the material $\left(X^{\prime}, Y^{\prime}, Z^{\prime}\right)$ and global $(X, Y, Z)$ coordinate systems. 


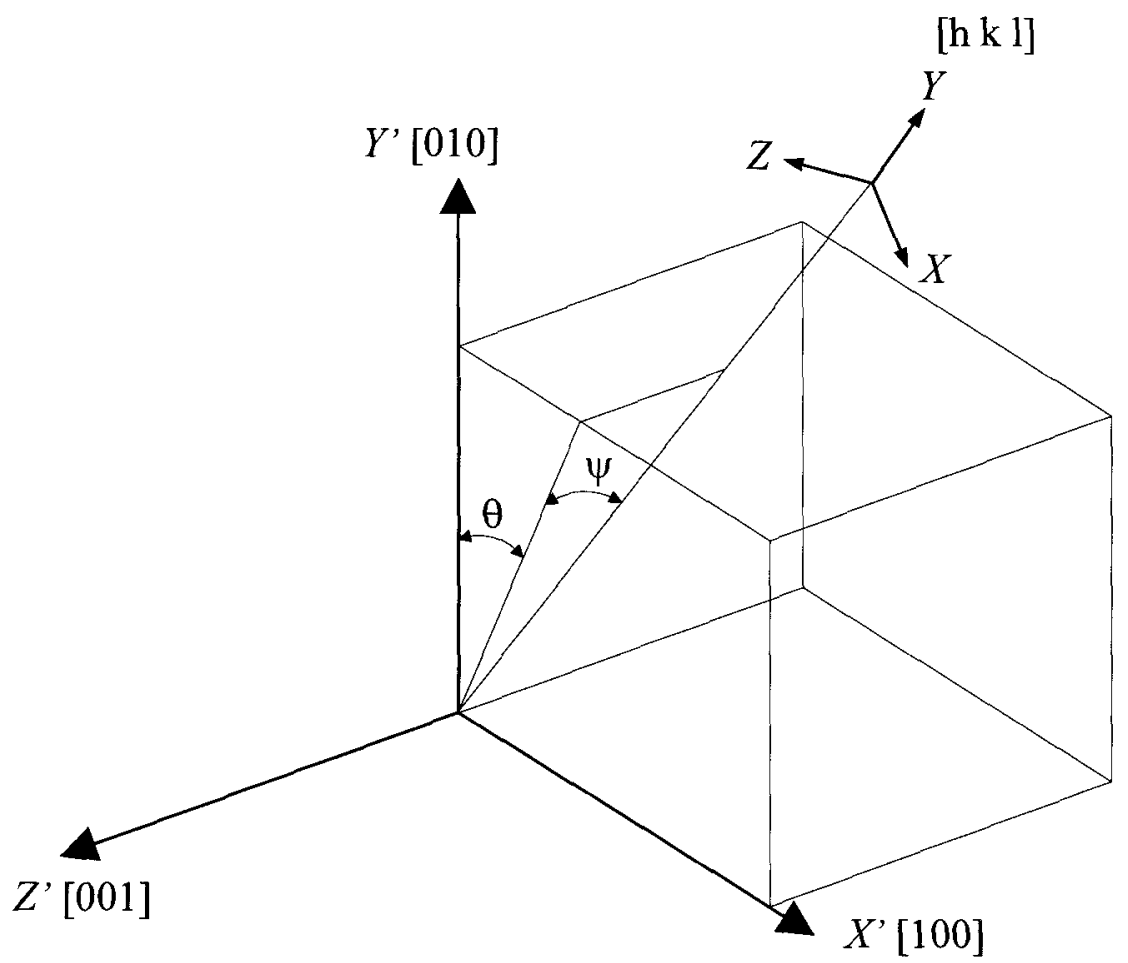

Figure 6.3 - Transformation from material cooridinate system $\left(X^{\prime}, Y^{\prime}, Z^{\prime}\right)$ and global cooridinate system $(X, Y, Z)$ with arbitrary miller indices [h $\mathrm{k} l]$ along $Y$ axis using two rotations.

[111]

$$
\left(\theta=45^{\circ}, \psi=35.264^{\circ}\right)
$$
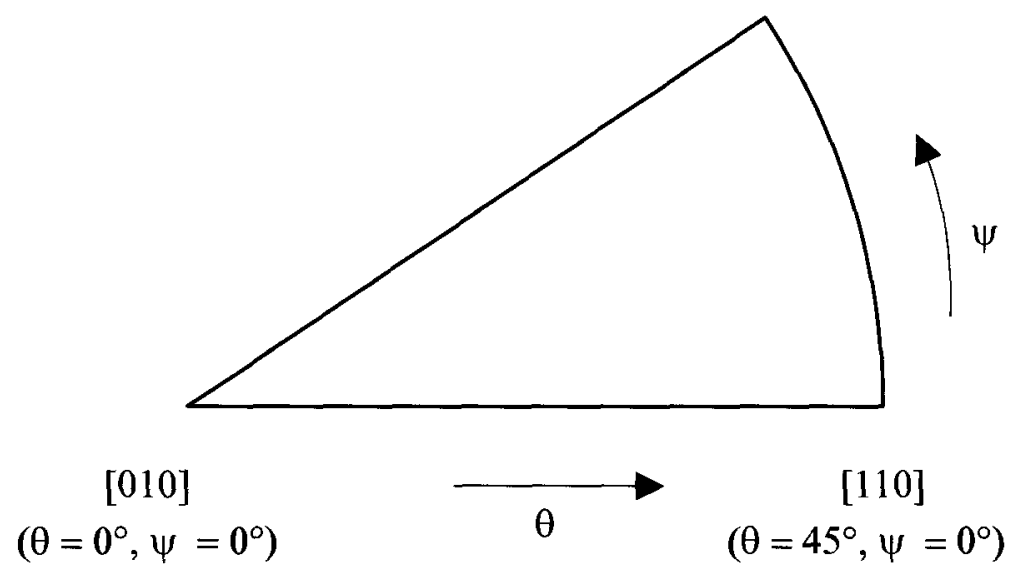

Figure 6.4 - Schematic showing relationship between the cooridinate system transformation angles and the standard stereographic triangle. 


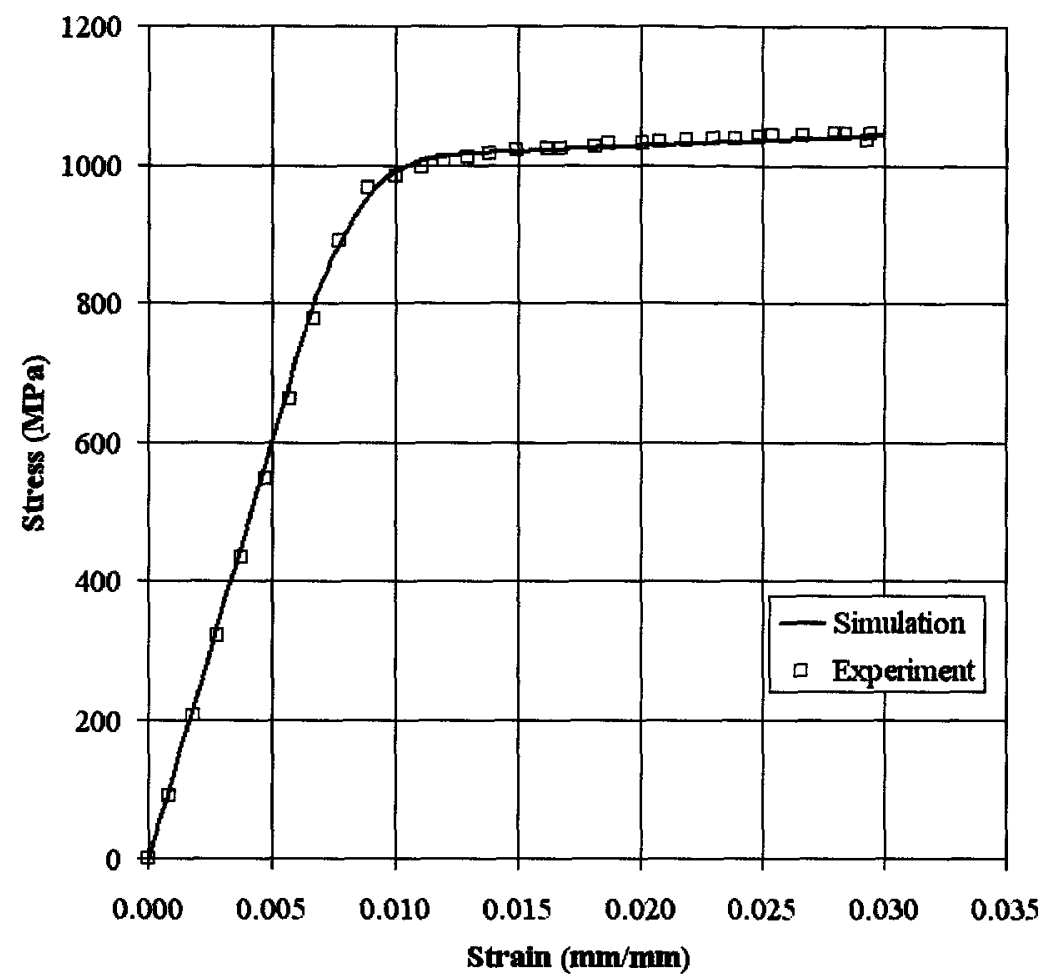

Figure 6.5 - Comparison of predicted and experimental uniaxial tensile curves for loading along the $[010]$ crystallographic direction.

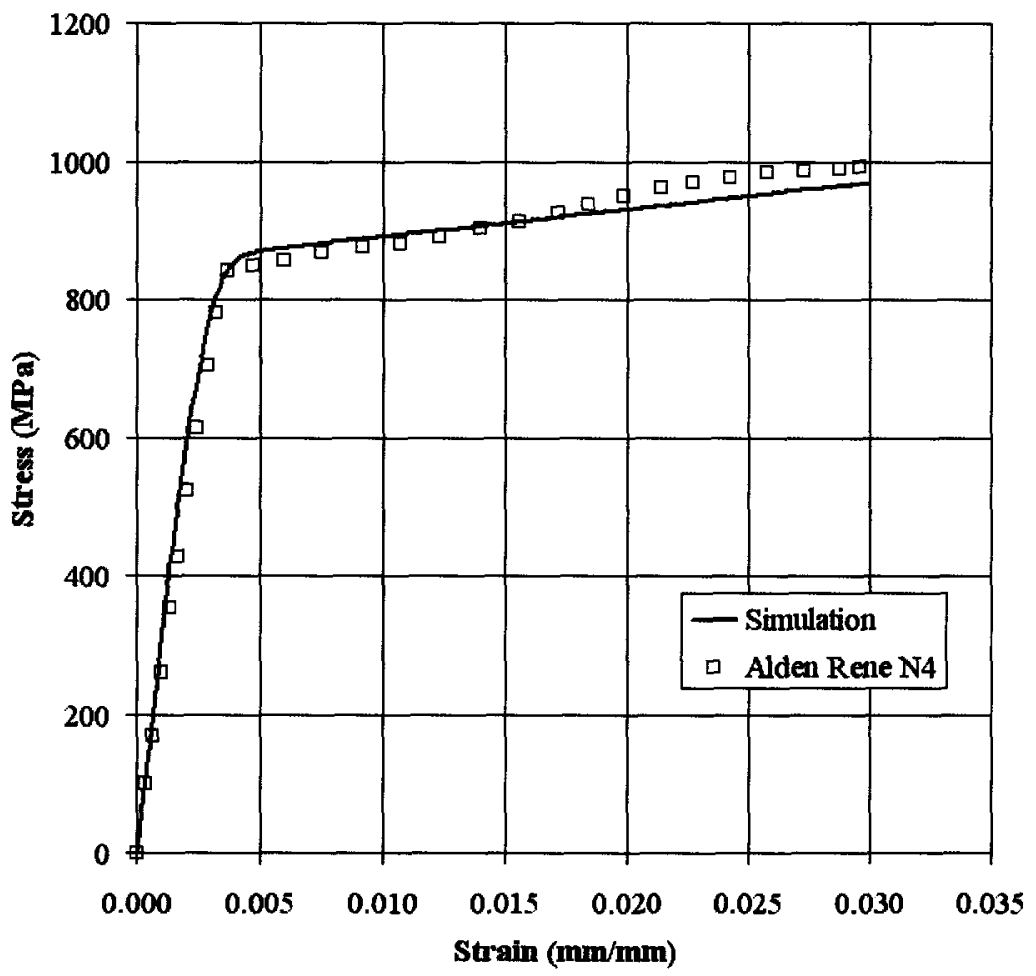

Figure 6.6 - Comparison of predicted and experimental uniaxial tensile curves for loading along the [111] crystallographic direction. Note: experimental data after Alden [132]. 


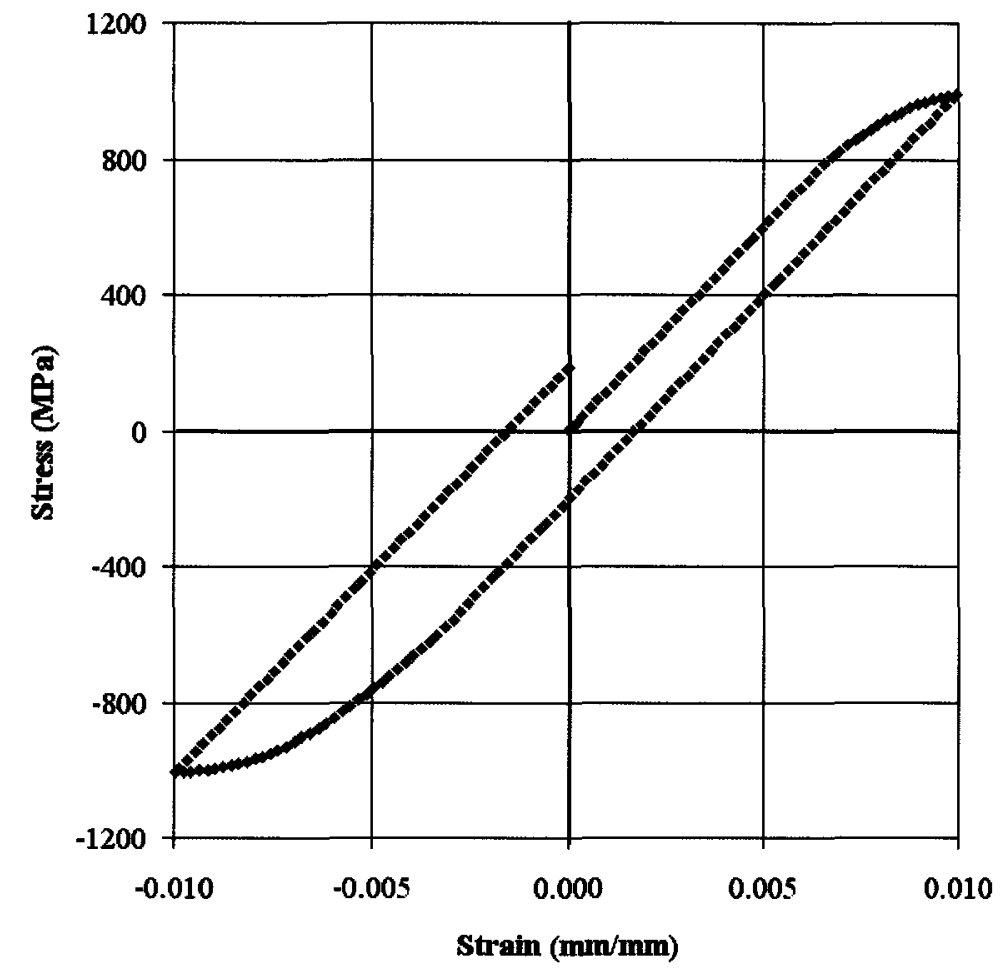

Figure 6.7 - Predicted first cycle stress-strain hysteresis loop for uniaxial loading along [010] crystallographic direction.

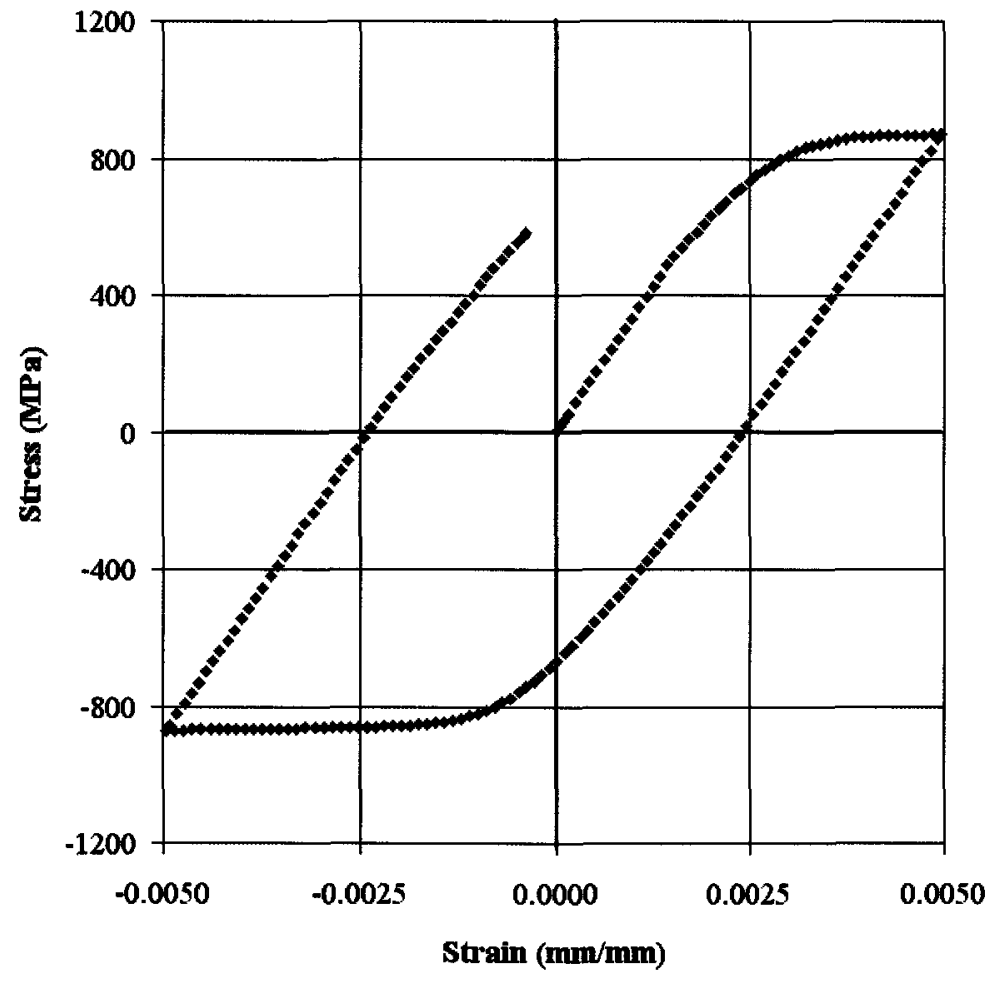

Figure 6.8 - Predicted first cycle stress-strain hysteresis loop for uniaxial loading along [111] crystallographic direction. 


\section{Finite Element Modeling}

\subsection{Introduction}

Both linear elastic and elastic-plastic fracture mechanics have been demonstrated to be indispensible for characterizing crack growth behaviour in ductile materials. For practical problems, closed-form solutions for the stress-intensity factor and J-integral are rarely available. Numerical techniques such as FEM can be used to perform calculations to estimate these crack-tip parameters. As discussed in Chapter 3, some empirical equations for the stress-intensity factor and J-integral have been developed from analytical approaches. However, these empirical solutions were developed for specific laboratory specimen geometries and for isotropic materials, and therefore they can not be universally applied to other specimen geometries and their application to anisotropic materials is unclear.

Consequently, FEM simulations were performed to calculate the anisotropic stressintensity factors and J-integrals for notch root small fatigue cracks in the SEN specimen considered in this investigation. Elastic and elastic-plastic anisotropy and crack geometry $(a / c, c, a)$ were considered in the calculations to estimate the fracture mechanics

parameters. In addition to conducting finite element simulations to analyze notch root cracks, elastic and elastic-plastic analyses of the SEN specimen were performed to estimate the notch root stress and strain fields. In this chapter only the analytical approach and FEM models are described. The results of the FEM simulations are presented and discussed in Chapter 8. 


\subsection{Software}

Three commercial software programs were used to develop the FE models and to carry out the calculations. MSC.Patran version 2003r1 was used to define the FEM model of the SEN specimen. ABAQUS/Standard version 6.5 was used to perform the FE calculations and ABAQUS/Viewer version 6.5 was used for post-processing the calculation results. In addition to commercial software, the ABAQUS user material subroutine discussed in the Chapter 6 was used in the elastic-plastic simulations.

\subsection{Geometry of the Specimen Modeled}

The geometry of the SEN specimen is presented in Figure 5.24. The notch, located on one edge of the specimen, is semi-circular and the specimen thickness in the gage length is constant. The specimen dimensions used to create the FE model are summarized in Table 7.1. In other investigations [268, 269], it was assumed for specimens such as the SEN specimen used in this investigation, that the geometry of the specimen can be simplified by considering it to be a notched bar of a constant thickness. Mechanical loads are then applied assuming either constant stress or uniform displacement far-field boundary conditions, as depicted in Figure 7.1. An alternative approach is considered for the specimen used in this investigation. If it is assumed that a condition of zero-bending exists where the SEN specimen is threaded into the test machine grips, i.e. the test machine grips are much stiffer than the specimen, then the FEM model should also include the portions of the specimen gage section up to the first thread, as shown in Figure 7.2. Based on this assumption, a uniform displacement condition exists at the first

thread of the SEN specimen and specifying the loading boundary conditions for the models is simplified. 


\subsection{Finite Element Models for Notch root Stress and Strain Analysis}

\subsubsection{Finite Element Models}

A three-dimensional FEM procedure, based on infinitesimal strain assumptions, was used to calculate the elastic and elastic-plastic stress and strain fields in the notch root under tensile loading conditions. As discussed in Chapter 5, two single-crystal specimen orientations considered in this investigation possess crystallographic symmetries that do not cause out-of-plane distortions. In addition to crystallographic symmetry, the geometrical and loading symmetry of the specimen can be exploited to reduce to the size of the FE model and thus decrease the calculation time. Taking advantage of the crystallographic and geometrical symmetries about the planes A-A and B-B shown in Figure 7.2, only one quarter of the SEN specimen was modeled.

Several FEM models were prepared with different degrees of mesh refinement in the notch area to investigate the effect of the FE mesh size on the calculated results. The FE model shown in Figure 7.3 represents the most refined FE mesh used to calculate the stress and strain fields within the notch. All finite elements were three-dimensional, second-order, hexahedron (brick) isoparametric elements with three degrees of freedom (DOF) per node (ABAQUS C3D20 element type). The model shown in Figure 7.3, consists of 10,040 elements and 44,905 nodes, which generated 134,715 DOF before the boundary conditions were applied.

\subsubsection{Boundary Conditions}

The symmetric boundary conditions were enforced by fixing the displacement of the nodes on plane A-A (Figure 7.2) in the z-direction and the nodes on plane plane B-B (Figure 7.2) in the y-direction, as shown schematically in Figure 7.4. Mechanical loading was applied by specifying a constant displacement in the y-direction to the end of the 
specimen FE model, as shown schematically in Figure 7.5. To replicate the mechanical loading used in the fatigue crack growth rate tests, the displacement was specified such that the total $y$-direction reaction force for the nodes on the $y=0$ plane was $14.5 \mathrm{kN}$.

\subsection{Finite Element Models for Crack Analysis}

\subsubsection{Finite Element Models}

A three-dimensional FEM procedure, based on the infinitesimal strain assumption, was used to estimate the stress-intensity factor (linear elastic) and the J-integral (elasticplastic) as a function of crack size and secondary crystallographic orientation. The calculation of these crack-tip parameters were performed only for monotonic loading conditions, assuming a stationary crack without prior loading history. Assuming that a semi-elliptical crack exists at the notch root, the crack is symmetrical about the mid-plane of the SEN specimen (Figure 3.5) and exploiting crystallographic and geometrical symmetries, only one quarter of the SEN specimen was modeled. The assumption was made based on the fractographic observations from the ACPD calibration tests (Chapter 5), in which each specimen was observed to contain a single Mode I thumbnail crack.

A total of $18 \mathrm{FE}$ models were prepared to cover a crack depth range of $200-1000 \mu \mathrm{m}$ in $100 \mu \mathrm{m}$ increments. The crack aspect ratios were specified independently for orientations A and B based on the crack aspect ratio relationships (see Figure 5.40 and parameters in Table 5.3) determined from the ACPD calibration tests. The crack size and geometrical properties for these FE models are summarized in Table 7.2. All finite elements were three-dimensional, second-order, reduced integration, hexahedron (brick) isoparametric

elements with three DOF per node (ABAQUS C3D20R element type). Reduced 
integration elements were used in the FE models to reduce the computational time and as suggested by Kim et al. [197] to avoid problems associated with incompressibility.

The stress-intensity factor and elastic-plastic J-integral calculations were performed using the domain integral method (J-integral) capabilities in ABAQUS. Since the J-integral is theoretically path-independent, the values were calculated remote from the crack-tip. As discussed in Chapter 3, when estimating crack-tip parameters remote from the crack-tip using the J-integral, special elements required to simulate the local stress and strain fields are unnecessary. Therefore, brick elements were used to define the crack-tip, as illustrated in Figure 7.6. For the linear-elastic simulations acceptable estimates of the stress-intensity factor were obtained using coarser and non-orthogonal FE meshes. However, to achieve more accurate solutions and convergence of the elastic-plastic constitutive models, a relatively finer mesh was necessary in the notch-crack area than that used to estimate the notch root fields. For the elastic-plastic analyses, the FE mesh size used in the analyses was dependent on the crack size, two extremes of which are illustrated in Figure 7.7. The FE mesh shown in Figure 7.7a was for a model with a smaller crack size $(c=200 \mu \mathrm{m})$ contained 19,208 elements and 84,291 nodes with 252,873 DOF. The FE mesh for a model with a larger crack $(c=1000 \mu \mathrm{m})$ shown in Figure $7.7 \mathrm{~b}$ contained 38,408 elements and 163,685 nodes with 491,055 DOF. The FE models for crack sizes between $c=200 \mu \mathrm{m}$ and $c=1000 \mu \mathrm{m}$ contained numbers of elements, nodes and DOF that were between these two examples.

As discussed in Chapter 3, the elastic-plastic J-integral is inherently path dependent when using constitutive laws based on incremental plasticity. To investigate this matter further, the J-integral was evaluated on a large number of contours (up to 14) surrounding the crack-tip. The conventional method for defining the contours is to specify a node set that 
containing a single node at each crack front position, as shown schematically in Figure 7.8. When requesting J-integral calculations on multiple contours, ABAQUS automatically identifies additional concentric rings of nodes enclosing the crack-tip as shown schematically in Figure 7.9. The estimated J-integral values were extracted from the crack front positions as a function of the crack-front parametric angle as defined in Figure 7.10 .

In addition to conducting elastic-plastic simulations to estimate the J-integral, calculations were performed on the same FE models using isotropic and cubic anisotropic elastic constitutive material behaviour. These additional finite element calculations were performed to extract both the isotropic and anisotropic stress-intensity factors as a function of the crack-front parametric angle (Figure 7.10). The mixed-mode stress intensity factors were calculated using the interaction integral capabilities included in ABAQUS/Standard. Because non-singular crack-tip elements were used in the FE models, the stress-intensity factors were extracted from contours remote from the cracktip.

\subsubsection{Boundary Conditions}

The symmetry boundary condition on plane A-A (Figure 7.2) was enforced as described previously for the model used to conduct notch root fields analysis. For plane B-B (Figure 7.2) the symmetry boundary condition was enforced by applying a $\mathrm{y}=0$ displacement constraint only to the nodes that define the crack-free ligament, as shown in Figure 7.11. Similar to the models used to calculate the notch root fields, the load was applied by specifying a constant y-displacement boundary condition on the end of the specimen (Figure 7.5). The displacement was specified to ensure that the total y-direction reaction force at plane $\mathrm{B}-\mathrm{B}$ was equivalent to $14.5 \mathrm{kN}$, and therefore its magnitude 
depended on the crack size embedded in the FE model. Only monotonic loading was considered in the FEM calculations, i.e. loading increases from 0 and $14.5 \mathrm{kN}$. Usually, two or three trial simulations were necessary to determine the correct displacement boundary condition to apply to the model.

\subsection{Constitutive Material Properties}

The FEM calculations were performed using linear elastic and elastic-plastic material properties. For the linear elastic analyses, calculations were performed using the isotropic elastic properties summarized in Table 7.3 and the cubic anisotropic elastic properties summarized in Table 6.1. The isotropic elastic properties were selected to approximately simulate the elastic constitutive response of a uniaxially loaded single-crystal superalloy with a $[100]$ crystallographic orientation. The elastic-plastic analyses were performed using micro-phenomenological constitutive model described in Chapter 6. The elastic properties summarized in Table 6.1 and the inelastic constitutive law material parameters listed in Table 6.2 were used with this model.

The FEM model geometry was defined in a 3-D Cartesian global coordinate system and by default ABAQUS specifies the local material orientation to be aligned with this coordinate system. However, when conducting FEM simulations with anisotropic constitutive material behaviours an alternate local material orientation must be specified to obtain the correct constitutive material response when the global and local material coordinates systems do not coincide. For orientation B, the global coordinate system for the FEM model and the local (material) coordinate systems are aligned. This was not the case for orientation A. For this orientation, the correct material orientation for the finite 
elements was specified by defining a local Cartesian coordinate system rotated $45^{\circ}$ about the $y$-axis of the global coordinate system, as shown in Figure 7.12. 
Table 7.1 - Summary of SEN specimen dimensions from Figure 5.24.

\begin{tabular}{|c|c|c|}
\hline Dimension & in & $\mathrm{mm}$ \\
\hline $\mathrm{R}$ & 0.049 & 1.245 \\
\hline $\mathrm{t}$ & 0.105 & 2.667 \\
\hline $\mathrm{W}$ & 0.420 & 10.668 \\
\hline $\begin{array}{c}\text { Distance between } \\
\text { specimen threads }\end{array}$ & 1.5 & 38.1 \\
\hline Gage Length & 1.0 & 25.4 \\
\hline Maximum Diameter & 0.5 & 12.7 \\
\hline
\end{tabular}

Table 7.2 - Crack sizes for the finite element models used to estimate the elastic and elasticplastic crack-tip parameters.

\begin{tabular}{|c|c|c|}
\hline Orientation & $\begin{array}{c}\text { Crack Depth, } c, \\
(\mu \mathrm{m})\end{array}$ & $a / c$ \\
\hline \multirow{9}{*}{$\mathrm{A},[010]-[101]$} & 200 & 0.86 \\
\hline & 300 & 0.92 \\
\hline & 400 & 0.98 \\
\hline & 500 & 1.04 \\
\hline & 600 & 1.10 \\
\hline & 700 & 1.16 \\
\hline & 800 & 1.23 \\
\hline & 900 & 1.29 \\
\hline & 1000 & 1.35 \\
\hline \multirow{9}{*}{$\mathrm{B},[010]-[100]$} & 200 & 0.82 \\
\hline & 300 & 0.87 \\
\hline & 400 & 0.93 \\
\hline & 500 & 0.99 \\
\hline & 600 & 1.05 \\
\hline & 700 & 1.11 \\
\hline & 800 & 1.17 \\
\hline & 900 & 1.22 \\
\hline & 1000 & 1.28 \\
\hline
\end{tabular}


Table 7.3 - Elastic material properties used in the isotropic linear elastic FEM calculations.

\begin{tabular}{|c|c|c|}
\hline Analysis Type & $\mathrm{E}(\mathrm{MPa})$ & $v(\mathrm{MPa})$ \\
\hline Isotropic & 120,134 & 0.4 \\
\hline
\end{tabular}




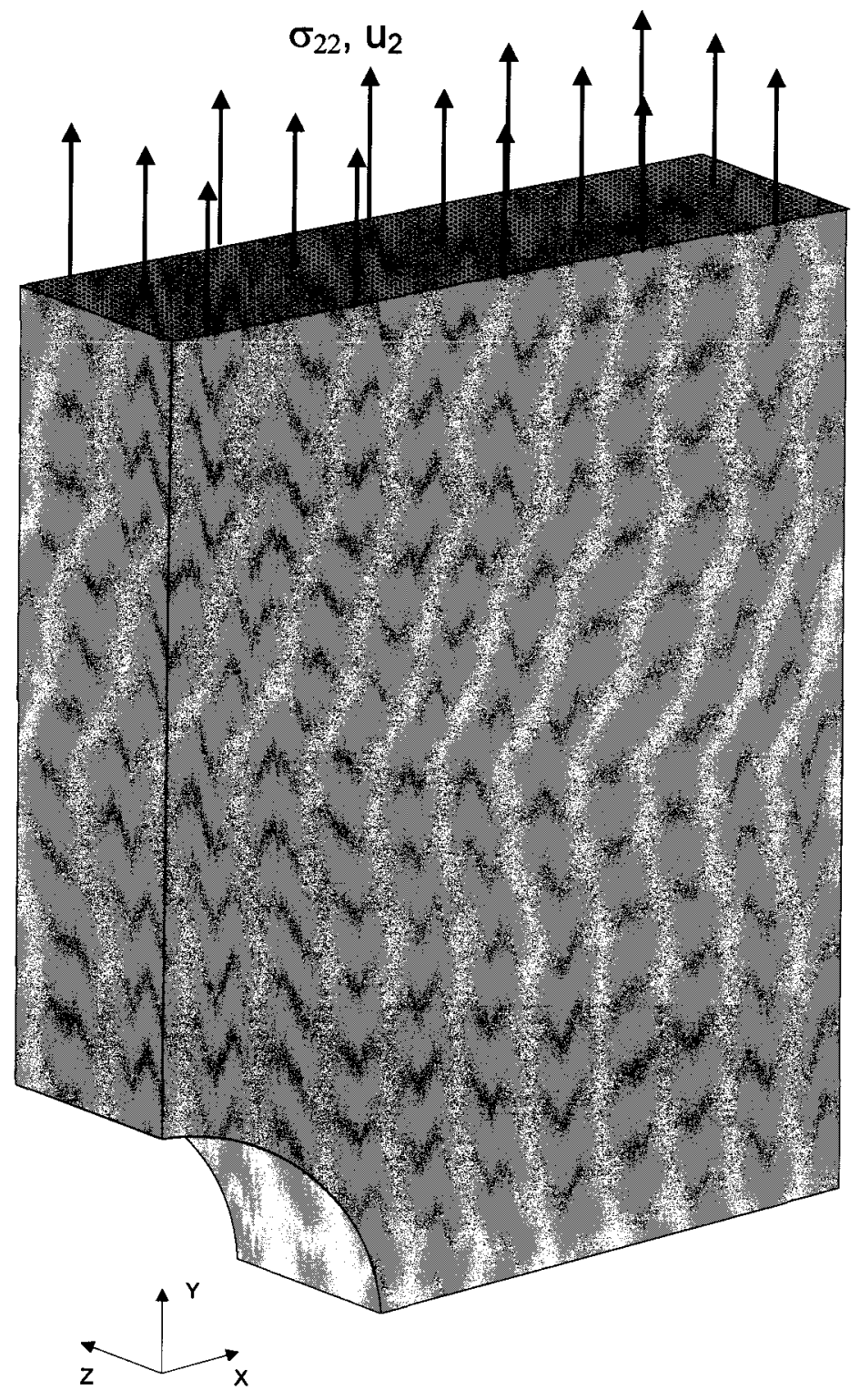

Figure 7.1 - Schematic of a notched rectangular bar with constant stress $\left(\sigma_{22}=\right.$ const.) or constant displacement $\left(u_{2}=\right.$ const. $)$ loading boundary conditions. 

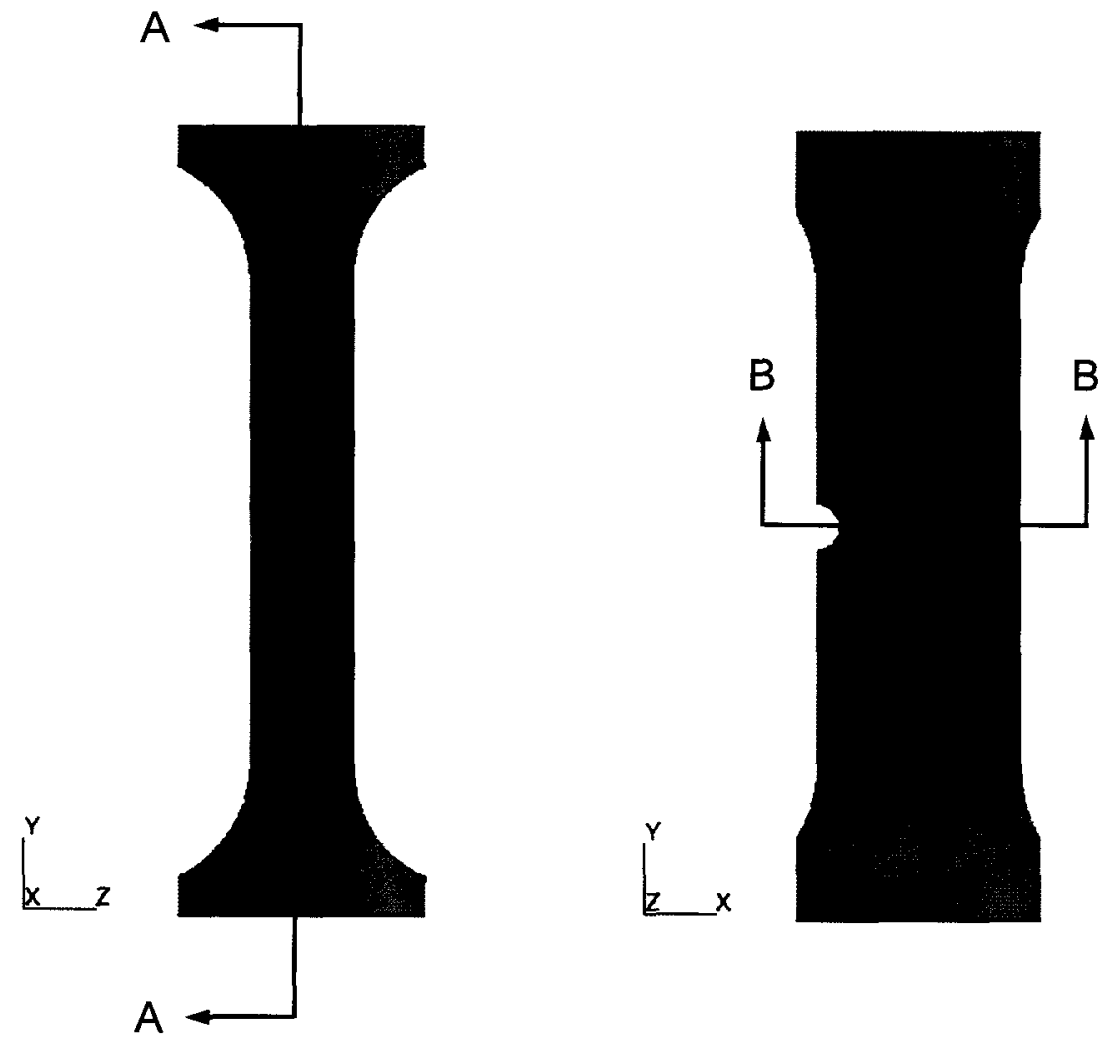

Figure 7.2 - Schematic of a SEN specimen with threaded regions removed.
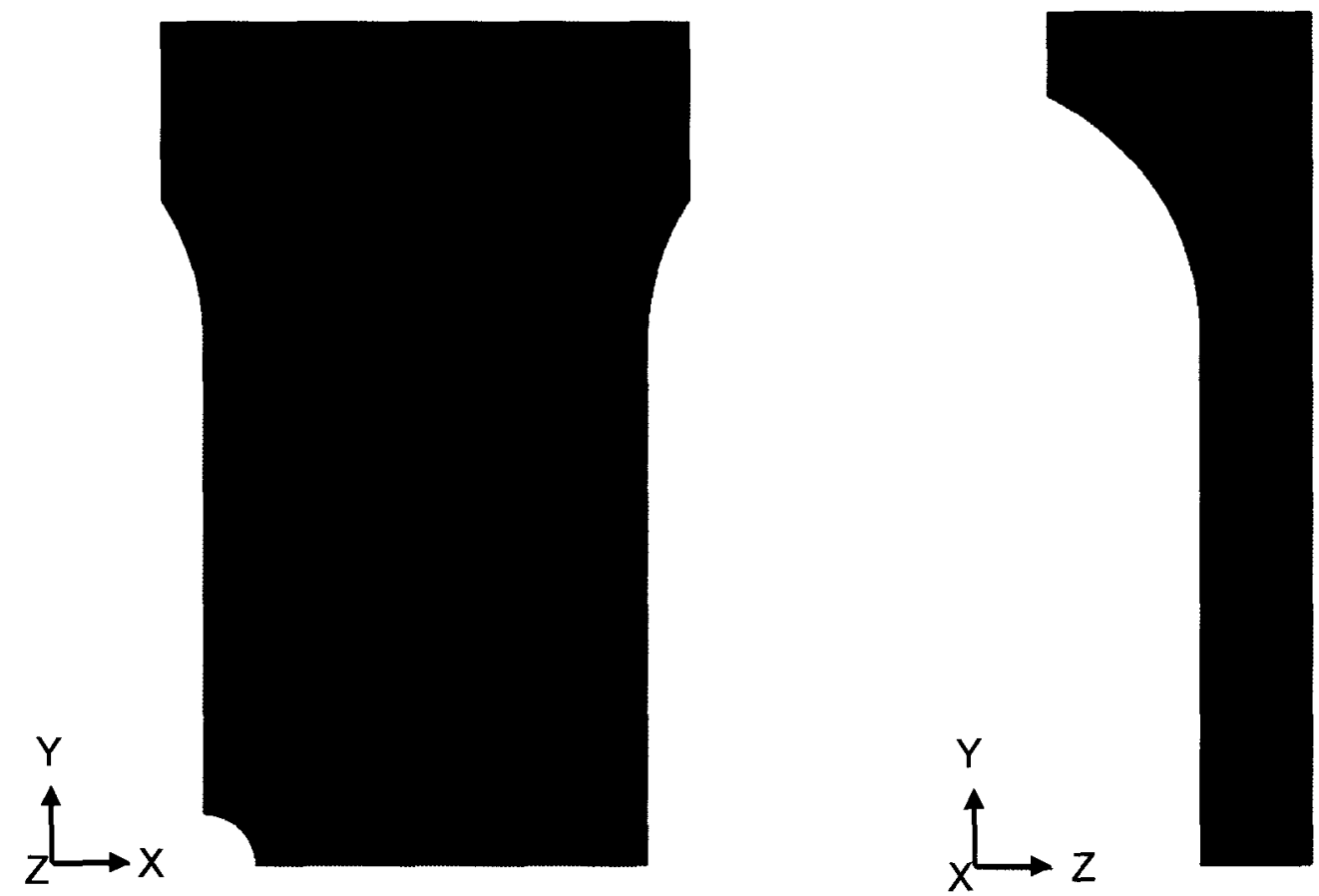

Figure 7.3 - Finite element model for notch stress and strain field analysis. 


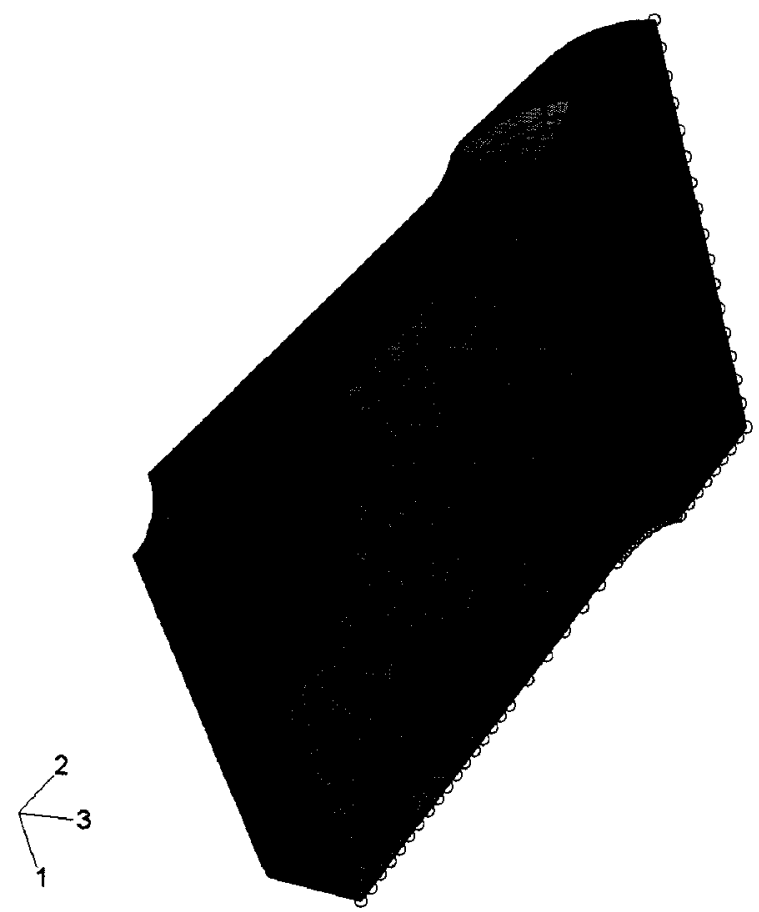

a)

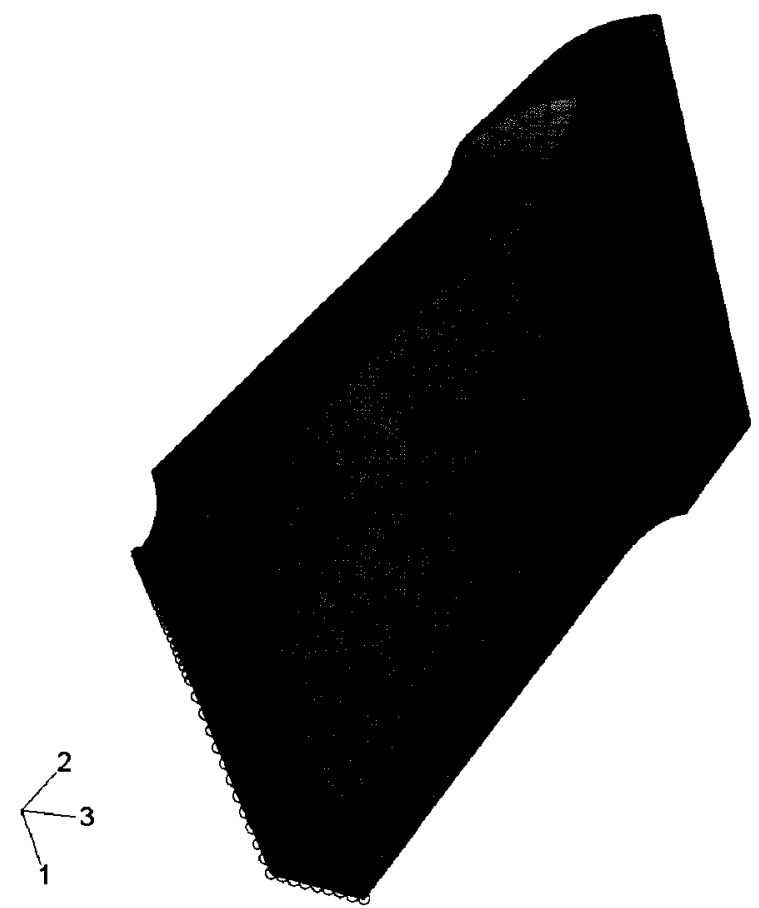

b)

Figure 7.4 - Finite element mesh of the SEN specimen showing symmetry enforcing boundary conditions: a) $u_{3}=0$ on plane $A-A$ (see Figure 7.2), and b) $u_{2}=0$ on plane B-B (see Figure 7.2). Note: highlighted nodes (in red) denote the nodes on which the constraints were applied. 


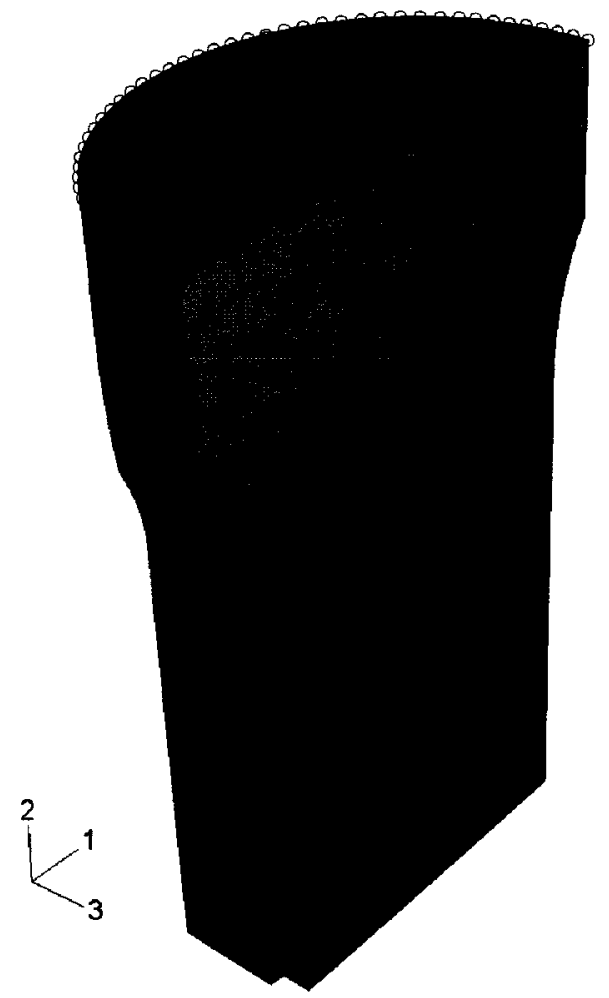

Figure 7.5 - Finite element mesh of the SEN specimen showing the loading boundary conditions. A fixed $y$-displacement ( $u_{2}=$ const.) was applied to the nodes highlighted in red.

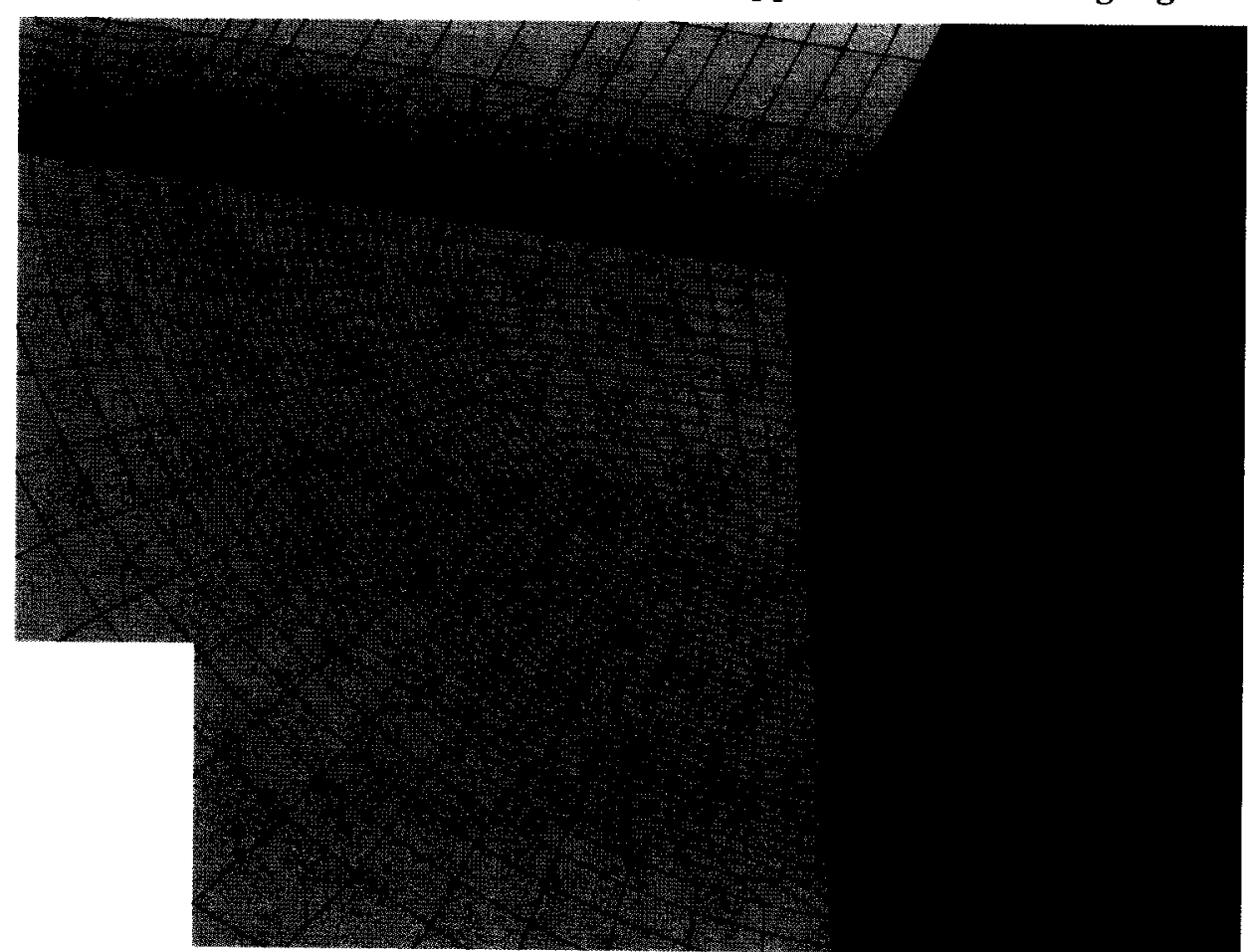

Figure 7.6 - Finite element mesh showing the crack front defined by continuum brick elements. Note: the nodes defining the crack front are highlighted in red. 

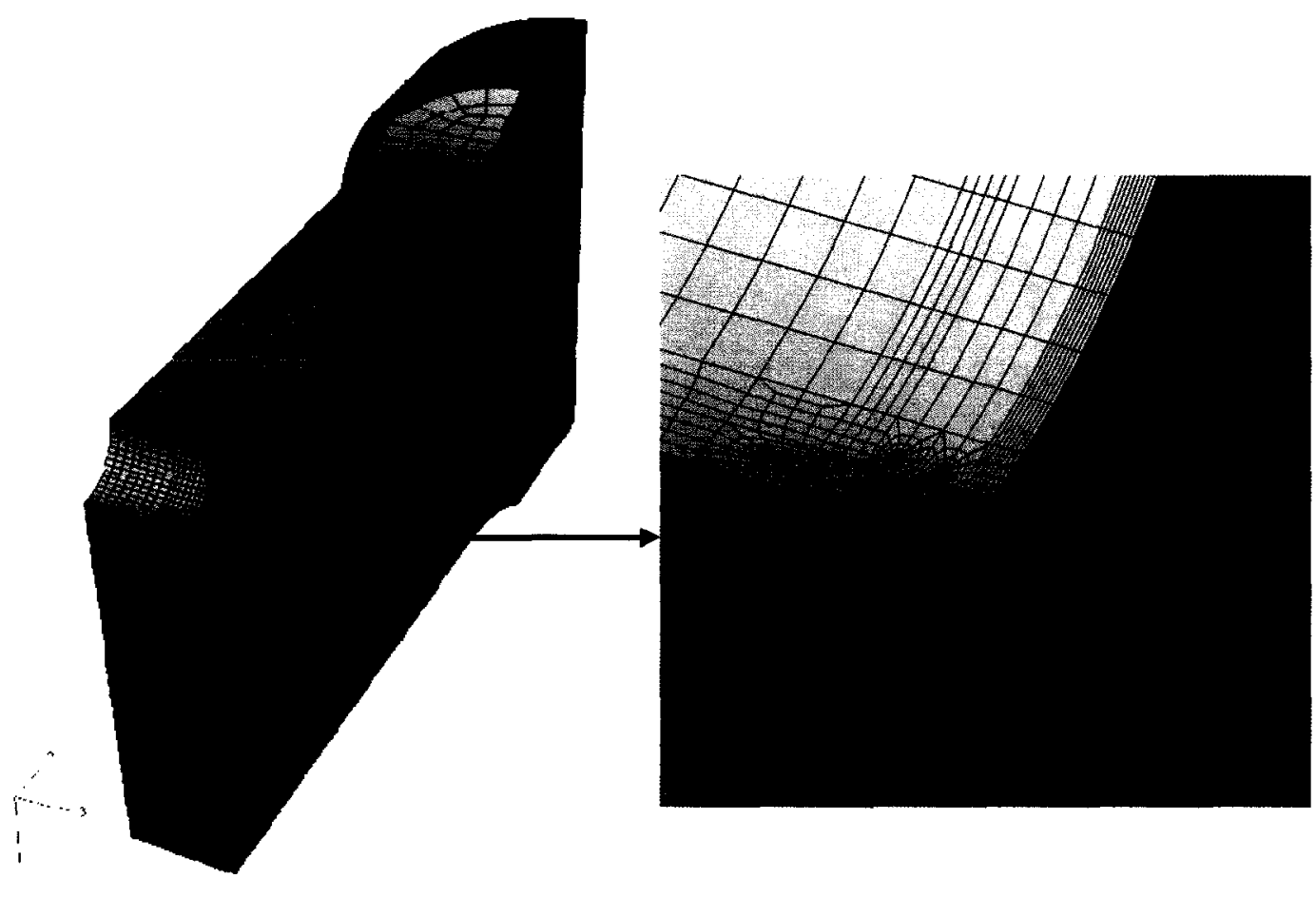

a)
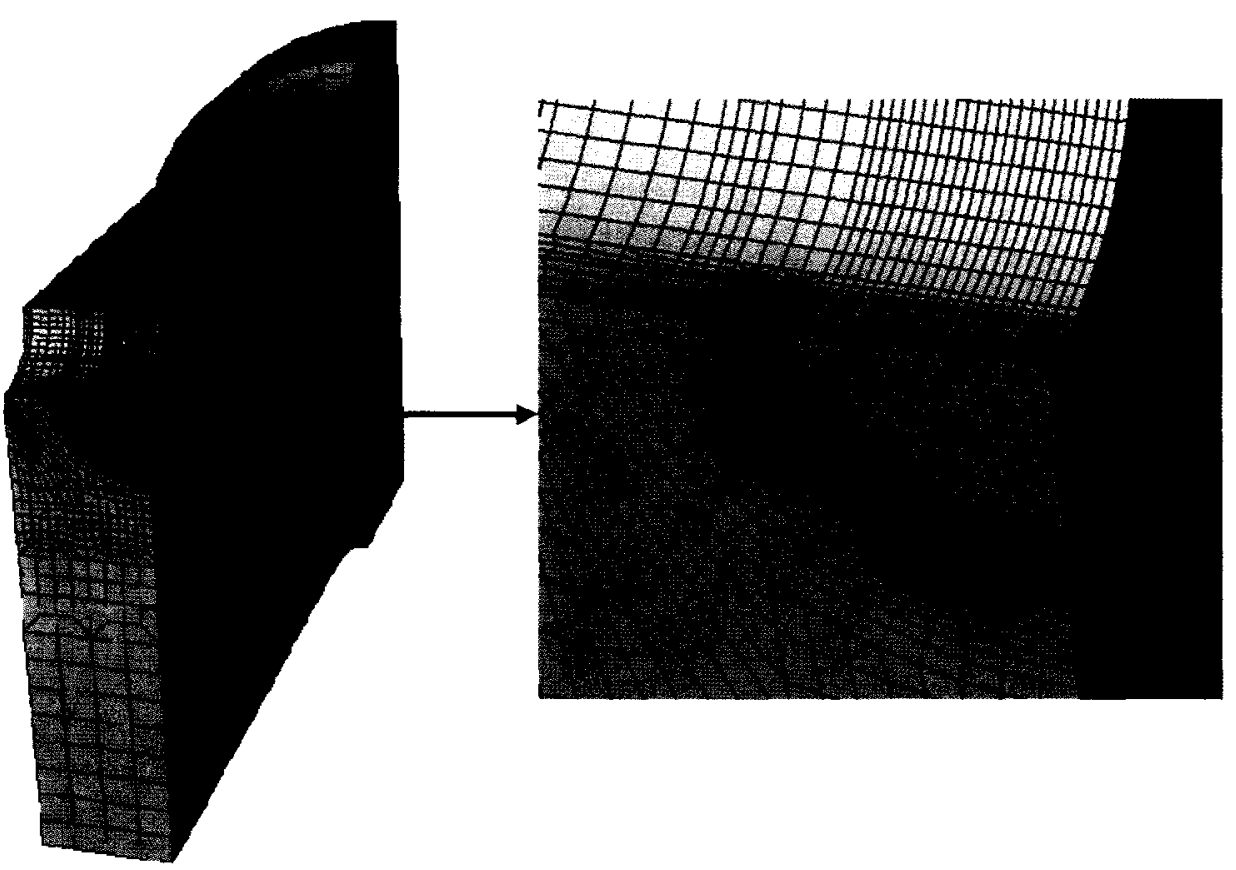

b)

Figure 7.7 - Finite element mesh details for SEN specimens containing notch root semielliptical cracks: a) $c=200 \mu \mathrm{m}$ deep, $a / c=0.86$ and b) $c=1000 \mu \mathrm{m}, a / c=1.35$ cracks. Note nodes defining the crack front are highlighted in red. 


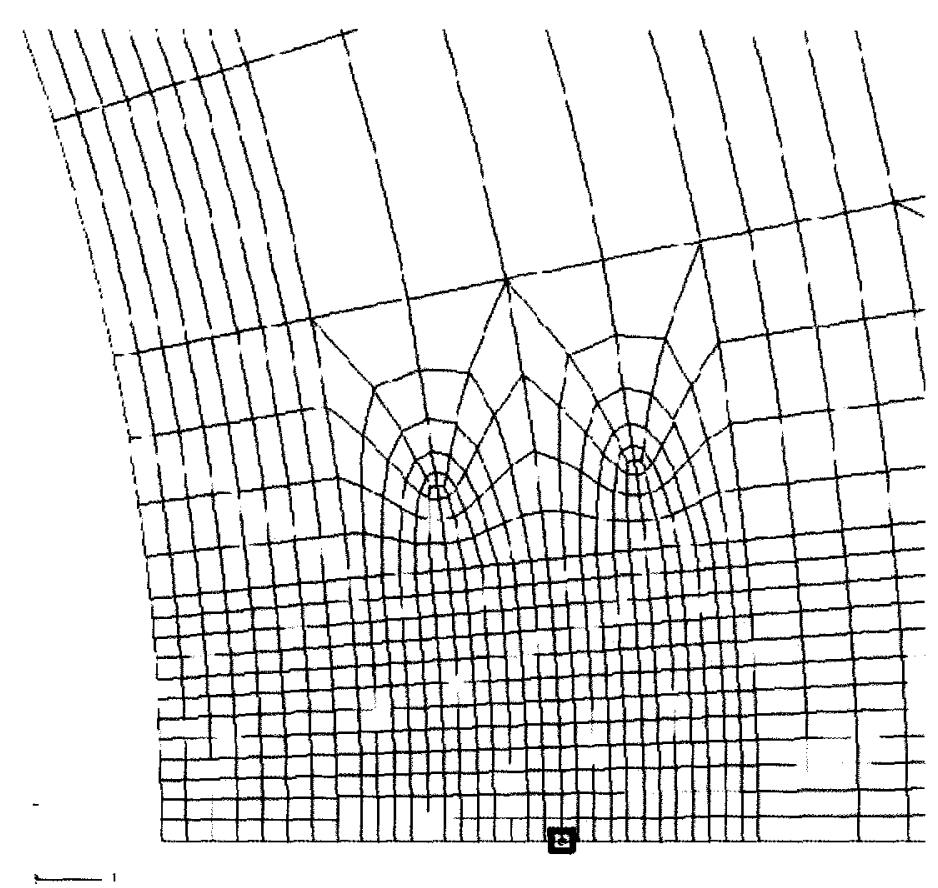

Figure 7.8 - Schematic showing the conventional method for defining a contour by means of specifying a node set containing a single node (in the present case) for J-integral calculations. Note: single crack front node is highlighted in red.

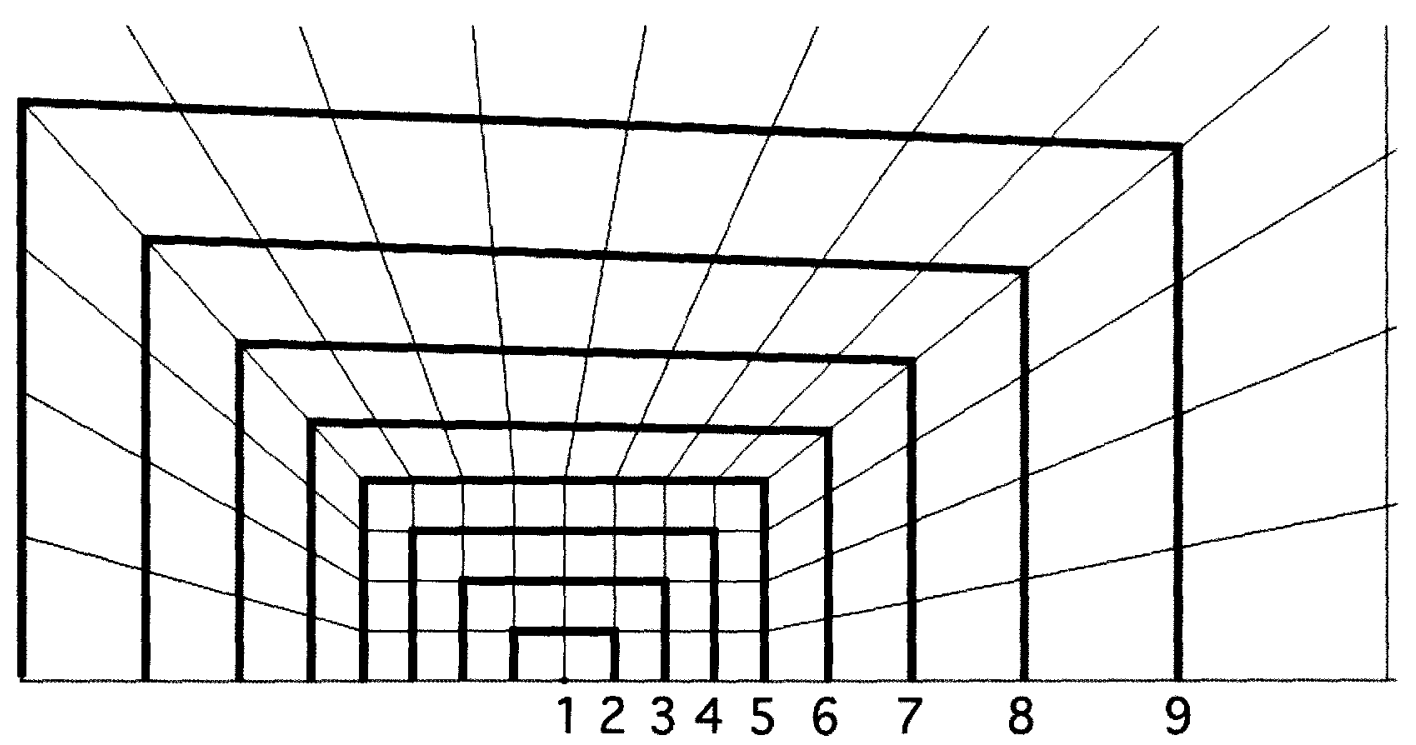

Figure 7.9 - Schematic showing contours automatically defined by ABAQUS for J-integral calculations when multiple paths are requested (in this case 9 paths) [166]. Note: contour 1 is defined by the single node defining the crack front. 


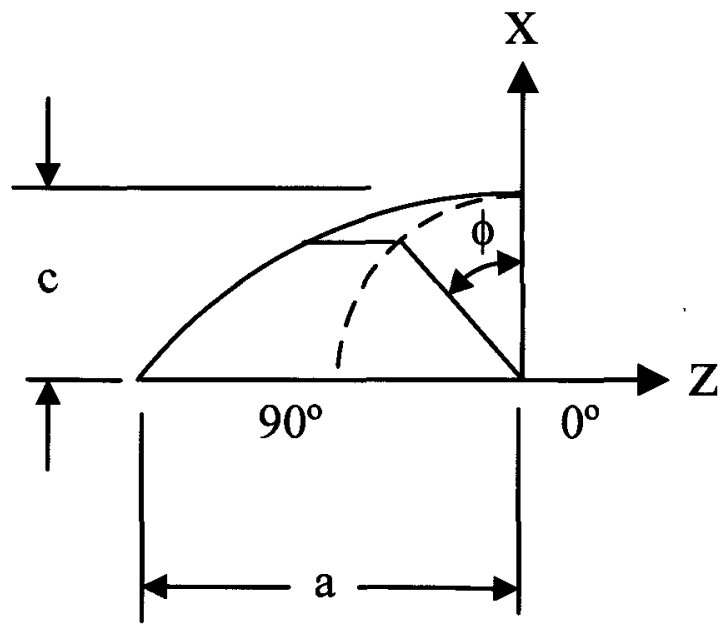

Figure 7.10 - Definition of the crack-front parametric angle, $\phi$, for a semi-elliptical crack.

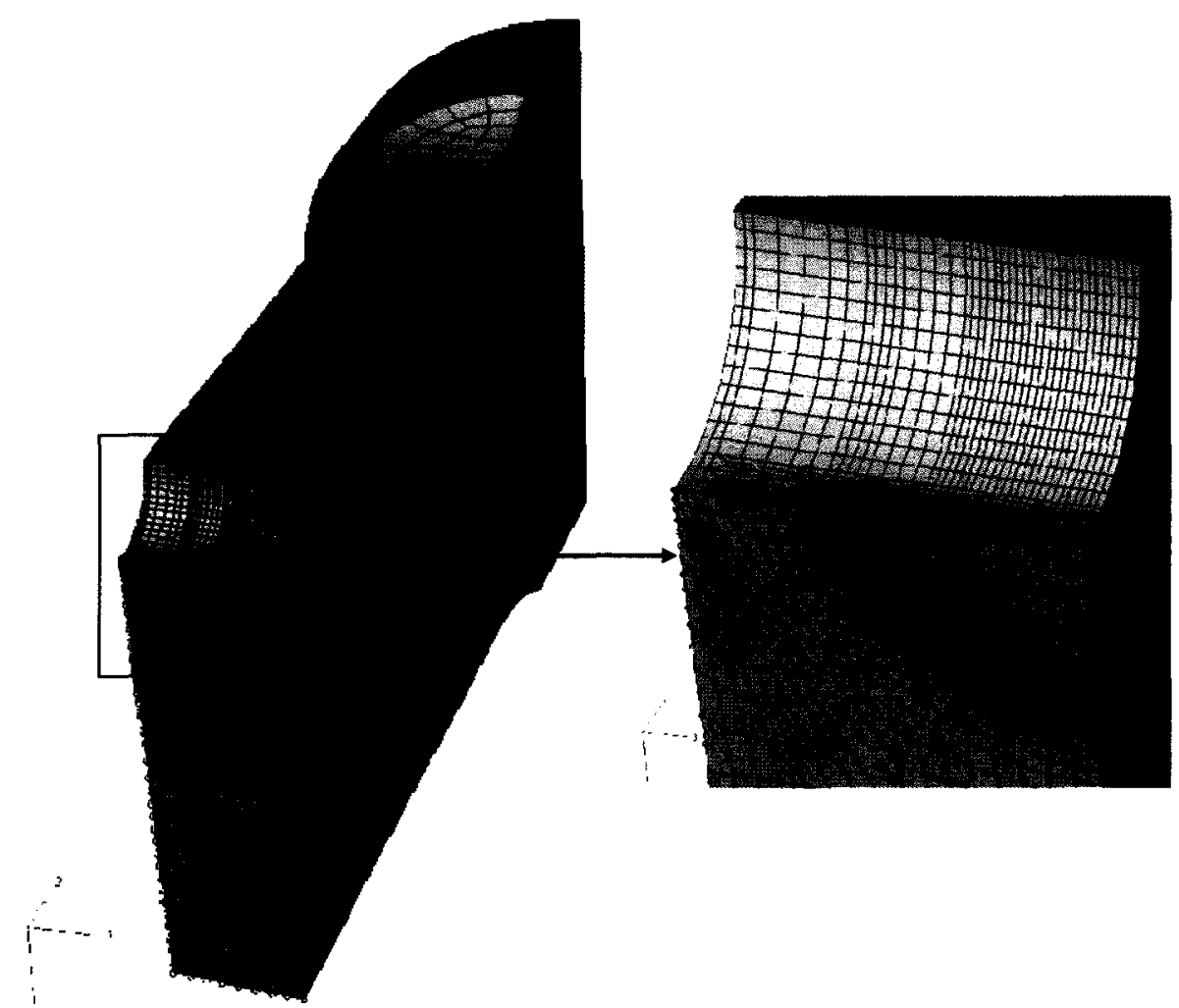

Figure 7.11 - Finite element mesh of the SEN specimen showing $y$-direction $\left(u_{2}=0\right)$ constraint applied to enforce the symmetry boundary condition. Note: nodes highlighted in red indicate the nodes that were constrained. 


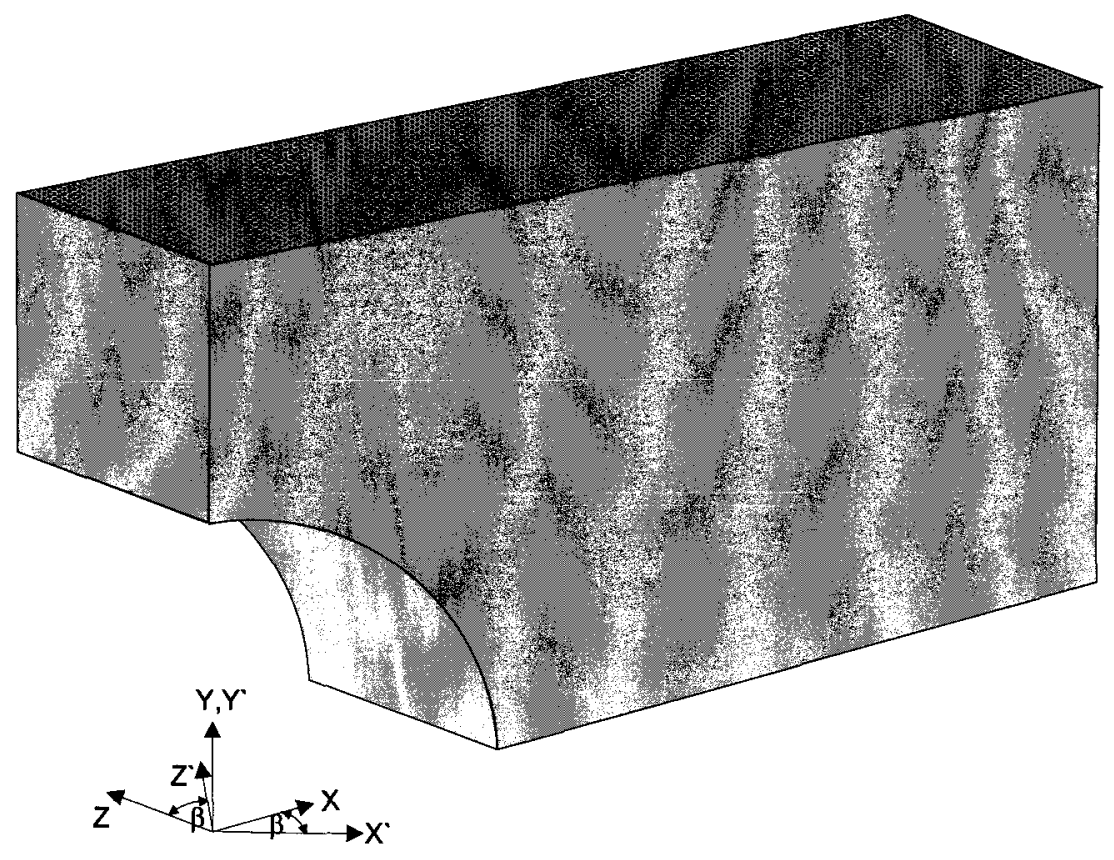

Figure 7.12 - Schematic definition of a local material orientation for orientation A SEN specimens. The local (material) coordinate system $X^{\prime}, Y^{\prime}, Z^{\prime}$ is defined by a rotation of the global coordinate system $X, Y, Z$ by an angle $\beta$ of $45^{\circ}$ about the global $Y$-axis. 


\section{Finite Element Modeling Results and Discussion}

\subsection{Introduction}

Finite element analyses were performed to evaluate the anisotropic notch root stress and strain fields and to estimate the stress-intensity factor (linear elastic) and J-integral (elastic-plastic) crack-tip parameters. In Chapter 7 a general description of the modeling approach, material constitutive behaviours, and loading and boundary conditions were presented. In this chapter the results of the FEM simulations performed to evaluate the notch root fields and crack-tip parameters are presented and discussed.

\subsection{SEN Specimen Notch Fields Analysis}

\subsubsection{Linear Elastic Notch Root Analyses}

Linear elastic finite element simulations were performed, assuming isotropic and cubic anisotropic elastic constitutive material behaviour, to calculate the notch root stress fields for the SEN specimen described in Figure 5.24. The main purpose of these calculations was to identify the effect of elastic anisotropy and the notch orientation on the resulting stress fields and the elastic stress-concentration factors.

Contour plots of the von Mises stresses in the vicinity of the notch obtained from the FEM results for isotropic and for cubic anisotropy (orientations $A$ and $B$ ) are shown in Figures 8.1 - 8.3. For elastic isotropy (Figure 8.1), it can be observed that the maximum stress occurs at the notch root. However, when elastic anisotropy was considered the maximum stress does not occur at the specimen minimum cross-section as predicted by the isotropic elastic analysis. In order to identify the location where the stress was a maximum in the anisotropic models, the angular variation in the von Mises stress 
distribution was extracted from the FEM results along a path of nodes defining the semicircular notch shape at the symmetry plane (inset diagram in Figure 8.4). These results are shown in Figure 8.4 for the isotropic and anisotropic elastic models. It can be observed that the von Mises stress was highest for the isotropic model (approximately 7.5\% higher than orientation B) and higher for orientation $\mathrm{B}$ than orientation $\mathrm{A}$ by approximately $8.2 \%$. It is also apparent for the anisotropic models that the maximum von Mises stress occurs at an angle of $30^{\circ}$ from the notch root.

The von Mises stress was extracted along the nodal paths shown in Figures 8.5a and 8.5b for the isotropic and anisotropic models, respectively, to characterize the stress distribution in the specimen thickness direction. The results are presented in Figure 8.6. The maximum von Mises stress for orientation B was located at symmetry plane A-A in Figure 7.2. For the isotropic and orientation A anisotropic models, the maximum von Mises stress was located approximately $1.3 \mathrm{~mm}$ and $1.8 \mathrm{~mm}$, respectively, from symmetry plane A-A. Since the differences between the stress at the local maxima and symmetry plane A-A were less than $1 \%$, it was assumed that the maximum von Mises stress coincided with the plane A-A when calculating the stress concentration factors.

Stress concentration factors were calculated from the FEM results to quantify the effect of elastic anisotropy on the notch root stress fields. The stress concentration factor, $K_{f}$, is defined as:

$$
K_{f}=\frac{\sigma_{\max }}{\sigma_{n o m}}
$$


where $\sigma_{\max }$ is the maximum stress within the notch and $\sigma_{n o m}$ is the nominal stress. For ductile materials, Garrell and co-workers [270] have suggested that the maximum von Mises stress should be used when estimating the stress concentration factor. As suggested by Pilkey [271], the nominal stress was calculated based on the minimum cross sectional area of the specimen. For the SEN specimen shown in Figure 5.24, the nominal stress was calculated from the maximum applied load, $P_{\max }$, and using the nominal specimen dimensions:

$$
\sigma_{n o m}=\frac{P_{\max }}{2 t(W-R)}
$$

The geometrical parameters $t, W$ and $R$ in equation 8.2, which define the specimen dimensions, are summarized in Table 7.1. After substituting the appropriate values into equation 8.2 , the nominal stress was determined to be approximately $577 \mathrm{MPa}$. The elastic stress concentration factors obtained from the linear-elastic FEM simulations are listed in Table 8.1. As indicated in Figure 8.4, the maximum von Mises stress in the anisotropic models does not coincide with notch root from which the nominal stress was calculated. Therefore, it is unclear if the stress concentration factors for the anisotropic models have any physical meaning.

\subsubsection{Elastic-Plastic Analysis}

The elastic-plastic stress and strain fields in the notch were investigated using the microphenomenological constitutive model described in Chapter 6. To facilitate a comparison between the predicted notch stress fields and results reported by Nissley and coworkers [272] and Meyer et al. [209], contour plots of the maximum principal stress were plotted instead of the von Mises stress. These contour plots are shown in Figures 8.7 and 8.8 for 
orientations $\mathrm{A}$ and $\mathrm{B}$, respectively. It can be observed that the maximum principal stress does not occur at the notch root as would be predicted for an isotropic material.

To determine the location of the maximum principal stress in the notch, this variable was extracted along a circumferential path at the notch root coinciding with symmetry plane A-A. The results are shown in Figure 8.9. It can be observed that the maximum principal stress for orientation A and B SEN specimens is located at an angle of approximately $24^{\circ}$ from the minimum specimen cross-section. The maximum principal stress for orientation B was approximately $7.4 \%$ higher than that predicted for orientation A. Meyer and coworkers [209] performed similar FEM calculations for a double-edge notch specimen with a $\langle 100\rangle$ primary orientation using the Walker single-crystal plasticity model [272]. They reported that the maximum principal stress was located at an angle of $23^{\circ}$ from the minimum specimen cross-section, thus confirming the results obtained in this investigation.

In addition to comparing the maximum principal stress to Meyer and coworker's [209] FEM results, the location of the maximum principal strain in the notch was also examined. Contour plots showing the maximum principal strain distribution in the SEN specimen notch are shown in Figures 8.10 and 8.11 for orientations $A$ and $B$, respectively. It can be observed that the maximum principal strain is located at the minimum specimen cross-section, which is similar to the findings reported by Meyer and colleagues [209].

A more detailed investigation was undertaken to characterize the effect of secondary crystallographic orientation on the inelastic notch strain fields. Contour plots showing the distribution of the equivalent plastic strain in the notch were plotted as shown in Figures 
8.12 and 8.13 for orientations $\mathrm{A}$ and $\mathrm{B}$, respectively. For the micro-phenomenological model the equivalent plastic strain was defined by:

$$
\begin{aligned}
& \varepsilon^{e q}=\int_{0}^{t} \dot{\varepsilon}^{e q} d t \\
& \text { with, } \\
& \dot{\varepsilon}^{e q}=\sqrt{\frac{2}{3} \varepsilon^{i n}: \dot{\varepsilon}^{i n}}
\end{aligned}
$$

It is apparent in Figures 8.12 and 8.13 that the notch plasticity was localized and confined within the specimen notch although the distribution appears to be different for orientations $\mathrm{A}$ and $\mathrm{B}$. The effect of secondary orientation on the equivalent plastic strain is more apparent when its distribution is plotted around the circumference of the notch at symmetry plane A-A, as shown in Figure 8.14. Examining this Figure, it is apparent the magnitude of the equivalent plastic strain is significantly higher (up to 1.7 times higher) for orientation $\mathrm{A}$ than $\mathrm{B}$. For orientation $\mathrm{A}$, there also appears to be a very prominent peak in the equivalent plastic strain distribution approximately $28^{\circ}$ from the notch root, which closely coincides with the angular position where the highest maximum principal stress was located (Figure 8.9). It should be noted that closer examination of the equivalent plastic strain contour plot for orientation B (Figure 8.13) indicates the maximum equivalent plastic strain does not coincide with symmetry plane A-A nor with the angular position where the maximum principal stress was located (Figure 8.9). For orientation $\mathrm{B}$, the maximum equivalent plastic strain is located approximately $2.1 \mathrm{~mm}$ from symmetry plane A-A along the z-direction (direction 3 in Figure 8.13) and at an angular position between 17 and $19^{\circ}$ from the minimum specimen cross-section. 
In equation 8.3 the equivalent plastic strain is defined from the macroscopic inelastic strain rate, which is a function of the individual shear strain rates on the active slip systems. To investigate the role of cubic and octahedral shear strains on the macroscopic plastic strain distribution, the cumulative shear strain rates for the octahedral and cubic slip systems were calculated during the FEM calculations. The cumulative (equivalent) octahedral and cubic shear strains were defined by [144]:

$$
\begin{aligned}
& \gamma_{c u m}^{o}=\int_{0}^{t} \dot{\gamma}_{c u m}^{o} d t \\
& \text { with, } \\
& \dot{\gamma}_{c u m}^{o}=\sum_{s=1}^{12}\left|\dot{\gamma}_{o}^{s}\right| \\
& \text { and, } \\
& \gamma_{c u m}^{c}=\int_{0}^{t} \dot{\gamma}_{c u m}^{c} d t \\
& \text { with, } \\
& \dot{\gamma}_{c u m}^{c}=\sum_{s=1}^{6}\left|\dot{\gamma}_{c}^{s}\right|
\end{aligned}
$$

Contour plots of the cumulative octahedral shear strain are presented in Figures 8.15 and 8.16 for orientations $\mathrm{A}$ and $\mathrm{B}$, respectively. The secondary orientation dependence of the octahedral shear strain distribution at plane A-A is more apparent when plotted function of the notch circumferential angle, as shown in Figure 8.17. Referring to this Figure, it is apparent that the cumulative octahedral strain was highest at the notch root for orientation $\mathrm{A}$ and at a location approximately $12^{\circ}$ from the notch root for orientation $\mathrm{B}$. The maximum cumulative octahedral strain for orientation A was approximately $15 \%$ higher than the maximum value for orientation B. Similar contour plots showing the cumulative cubic shear strain for orientations $\mathrm{A}$ and $\mathrm{B}$ are shown in Figures 8.18 and 8.19, 
respectively. The circumferential distribution of the cumulative cubic shear strain at plane A-A is also shown in Figure 8.20. A significant peak in the cumulative cubic shear strain is observed at $28^{\circ}$ from the notch root for orientation $\mathrm{A}$ and $37^{\circ}$ from the notch root for orientation B. The maximum cumulative cubic shear strain for orientation A was 1.9 times larger than the maximum value calculated for orientation B.

Several researchers [273-275] have reported on the stress fields in notched single-crystal superalloy laboratory specimens based on anisotropic linear elastic finite element analyses. The work published by Siddiqui and coworkers [275] is one of the most comprehensive because the authors have considered both octahedral and cubic slip in their analyses. In their investigation the primary orientation of the specimens was $\langle 001\rangle$ and two secondary orientations were considered, $\langle 110\rangle$ and $\langle 100\rangle$, equivalent to orientations A and B used in this investigation. They conducted anisotropic linear elastic finite element analyses on these two specimen orientations to predict the slip system activity within the specimen notch. The authors assumed that slip systems were active when the resolved shear stress on a particular slip system exceeded the critical resolved shear stress. They reported that both octahedral and cubic slip activity occurred within the specimen notch. Their results seem to corroborate the results in this investigation. However, their results cannot be compared directly to those obtained in this work because they considered a different notch geometry (saw-cut notch), and they predicted slip activity based on anisotropic elastic finite element calculations. The later point can be confirmed by comparing the elastic and elastic-plastic circumferential stress distributions at the notch, i.e. Figures 8.4 and 8.9 , from which it is apparent that the stress distributions in the notch depends on the constitutive material behaviour used in the analysis. 


\subsection{Analysis of SIF's for Semi-Elliptical Notch Root Cracks}

The mixed-mode stress-intensity factors for notch root semi-elliptical cracks were calculated using the interaction integral capabilities in ABAQUS/Standard. It may be noted that because the model cracks were located at the notch root and were normal to the applied load the only non-zero stress intensity factors were calculated for Mode I. The stress-intensity factors were extracted as a function of the crack-front parametric angle as defined in Figure 7.10. Before presenting the isotropic and anisotropic stress-intensity factor results, the results of a validation study used to verify the FE modeling approach discussed in Chapter 7 is presented.

\subsubsection{Validation of SIF Calculations}

As discussed in Chapter 3 the main advantage of the using the domain integral approach, i.e. the J-integral, for the calculation of stress-intensity factors in the finite element method is that special quarter point crack-tip elements are unnecessary when the exact crack-tip stress and displacement fields are not needed. However, to verify that special crack-tip elements were unnecessary, an isotropic linear elastic FE analysis was conducted for $a / c=1.0$ (semi-circular), $c=0.4 \mathrm{~mm}$ notch root crack for comparison with results reported in the literature. The elastic properties used in the analysis are summarized in Table 8.2. The results of this validation test were compared with the FEM results reported by Beres and Koul [269] for a DEN specimen with $R / W=1 / 8$, which most closely matched the geometry of the SEN specimen used in this investigation. For comparison with their finite element results, the stress-intensity factors were normalized to obtain the boundary correction factors, $F_{s n}$, defined by: 


$$
F_{s n}=\frac{K_{I}}{S \sqrt{\pi c / Q}}
$$

where $S$ is the far-field applied stress, $c$ is the crack depth and $Q$ is the elliptical shape factor approximated by equation 3.8.

The calculated SIF's were converted to boundary correction factors for comparison with Beres and Koul's [269] FEM results. The boundary correction factors were extracted from contours that were remote from the crack-tip, to ensure that the saturated far-field values were obtained. As shown in Figure 8.21, the stress-intensity factors obtained from the fourth contour surrounding the crack-tip converged to the far-field saturated value. The boundary correction factors obtained in this investigation were compared with those reported by Beres and Koul [269], as shown in Figure 8.22. A detailed comparison of the differences between the boundary correction factors is shown in Figure 8.23. It can be observed that the boundary correction factors obtained in this investigation are generally higher than the reported values by up to $6.5 \%$. This difference can be attributed to three factors. First, the SEN specimen introduces additional bending that would cause the calculated stress-intensity factors to be higher. In addition, the ratio of the geometrical parameter $(R / W)$ for the SEN specimen does not exactly match the DEN specimen considered in Beres and Koul's [269] analysis. Lastly, the discretization of the crackfront was much finer than used in Beres and Koul's [269] analysis. Raju and Newman [149] have published stress-intensity factor calculation results for surface cracks in plates that demonstrate that calculated stress-intensity factors are lower when too coarse of FE mesh is used in the analysis. Since the differences between reported and the present stress-intensity factors calculations can be rationalized, it is concluded that using non- 
singular elements at the crack-tip provided reasonable estimates of the stress-intensity factors and thus the modeling approach is deemed to be acceptable.

\subsubsection{SIF's for Notch Root Semi-Elliptical Cracks}

As discussed in Chapter 3, the stress-intensity factor depends on crack and specimen geometry, applied loading and the elastic material properties. The specimen geometry and loading conditions in the fatigue crack growth rate experiments conducted in this investigation were constant, but elastic anisotropy and the specimen orientation relative to the material orientation and crack geometry are variables in the analysis. In order to evaluate the effect of elastic anisotropy and secondary crystallographic orientation on the stress-intensity factor in an efficient manner, two representative crack geometries $(c=0.2$ $\mathrm{mm}$ with $a / c=0.86$ and $c=1.0 \mathrm{~mm}$ with $a / c=1.35)$ were assumed in the FEM calculations.

The variation in the stress-intensity factors for a semi-elliptical notch root cracks with $c=$ $0.2 \mathrm{~mm}$ with $a / c=0.86$ and $c=1.0 \mathrm{~mm}$ with $a / c=1.35$ are shown in Figures 8.24 and 8.25 , respectively. The variation in the stress-intensity factors along the crack front for the isotropic models appear to be similar to that reported by Beres and Koul [268]. The maximum stress-intensity factor for the isotropic and anisotropic cases is observed near the notch surface (near $\phi=90^{\circ}$ ). Findley and coworkers [196] have suggested this indicates that a propagating fatigue crack will grow faster along the $a$-axis, and therefore $a / c$ will increase as the crack grows larger. This agrees with the variation in the crack shape determined from the ACPD calibration tests, i.e. a/c increases as the crack depth increases as discussed in Chapter 5. It is also apparent in these figures that elastic anisotropy has a marked effect on the variation in stress-intensity factors along the crackfront. Most notably, a local minimum in the stress-intensity factor is observed for 
orientation A between crack-front angles of approximately 20 and $50^{\circ}$, depending on the crack size. The variation in the stress-intensity factors for orientation B is significantly different than observed for orientation $\mathrm{A}$ and the isotropic models. Comparing the stressintensity factors obtained for two different crack sizes (Figures 8.24 and 8.25), it is clear that the stress-intensity factors were higher for the larger crack size, which is in agreement with reported [268] behaviour.

The differences between the isotropic and anisotropic stress-intensity factors as a function of the crack front parametric angle are shown in Figures 8.26 and 8.27 for the $c$ $=0.2 \mathrm{~mm}$ and $c=1.0 \mathrm{~mm}$ cracks respectively. For the $c=0.2 \mathrm{~mm} a / c=0.86$ crack geometry, the isotropic analysis appears to over predict the stress-intensity factors along most of the crack front. The largest difference is observed at $\phi=45^{\circ}(10 \%)$ for orientation A and $\phi=90^{\circ}(18 \%)$ for orientation B. A crack size effect is also apparent when comparing Figures 8.26 and 8.27 . For the $c=1.0 \mathrm{~mm} a / c=1.35$ crack geometry, the isotropic analysis in general under-predicts the anisotropic stress-intensity factors along most of the crack front. The largest differences are observed at $\phi=0^{\circ}(10 \%)$ for orientation $\mathrm{A}$ and $\phi=57.5^{\circ}(18 \%)$ for orientation $\mathrm{B}$. It is apparent that stress-intensity factors obtained from an isotropic elasticity analysis do not correctly predict the anisotropic stress-intensity factor variation and magnitude along the crack front for notch root semi-elliptical cracks.

The secondary orientation dependence of the calculated stress-intensity factors is compared for both crack geometries $(c=0.2 \mathrm{~mm} \mathrm{a/c}=0.86$ and $c=1.0 \mathrm{~mm} a / c=1.35)$ by plotting the differences between orientation A and B stress-intensity factors, as shown in Figure 8.28. In general, the stress-intensity factors at the $\phi=0^{\circ}$ and $\phi=90^{\circ}$ are higher for orientation $\mathrm{A}$ than $\mathrm{B}$ by $5-10 \%$ and $10-12 \%$ at these crack front positions, 
respectively. At other crack front positions, however, the anisotropic stress-intensity factors are lower for orientation A than B, particularly for crack front positions in the range of $\phi=20^{\circ}-80^{\circ}$. These results suggest that the elastic anisotropy effects on the stress-intensity factors for notch root semi-elliptical cracks are significant and should be taken into consideration in the analysis of small fatigue cracks. Interestingly, when other researchers have reported a significant secondary orientation dependence on the growth short through-thickness fatigue cracks, orientation $\mathrm{B}$ tends to exhibit higher fatigue crack propagation rates than orientation $\mathrm{A}[33,34,47,52,216]$. This would suggest the driving force for crack extension, i.e. the anisotropic stress-intensity factors, should be higher for orientation $B$ than $A$. The results obtained in this investigation indicate the stressintensity factors for embedded notch root cracks were generally higher for orientation $\mathrm{A}$ than B. This finding suggests the stress-intensity factor may be unsuitable for characterizing the effects of elastic anisotropy on small fatigue crack growth in singlecrystal superalloys. The suitability of the stress-intensity factor for characterizing the small fatigue crack growth data obtained in this investigation will be considered further in Chapter 10.

Tinga $[54,55]$ has suggested that there are two ways in which elastic anisotropy can affect the calculated stress-intensity factors. The first involves the addition of elastic anisotropy to the stress-intensity factor calculation method. Snyder and Cruse [276] have shown that this first effect is quite small for cracks in isotropic and anisotropic elasticity when cracks were exposed to the same nominal stress field. Presumably, this effect is small in the present work because the same stress-intensity factor extraction techniques were used in finite element calculations. The second factor involves the effect of elastic anisotropy on the stress fields at the crack-tip. Tinga $[54,55]$ concluded that this second 
factor had a larger effect on calculated stress-intensity factors and noted that this factor is taken into consideration during the finite element calculations.

It has been established that elastic anisotropy has little effect on Mode I stress-intensity factors for cracks in semi-infinite bodies [136]. Therefore, the effects of elastic anisotropy on stress-intensity factors in finite bodies, i.e. laboratory fatigue specimens, is mainly due to finite geometrical effects on the anisotropic elastic stress and strain fields. Chan and Cruse [53] have shown for through-thickness cracks in C(T) specimens that the effect of elastic anisotropy on the stress-intensity factors was insignificant and that established stress-intensity factor solutions could be used in the analysis of fatigue crack growth data obtained from single-crystal $\mathrm{C}(\mathrm{T})$ specimens. On the other hand Tinga [54, 55] has demonstrated that elastic anisotropy has a significant effect on calculated stressintensity factors for thumbnail cracks in single-crystal corner crack fatigue specimens. He reported that Mode I anisotropic stress-intensity factors were over a factor of $2 \times$ higher than the isotropic stress-intensity factors for thumbnail corner cracks. It is not apparent from his published results what assumptions could have contributed to the substantial differences between the isotropic and anisotropic stress-intensity factors; however, his reported effects of elastic anisotropy are inconsistent with the results obtained in the present investigation and the results published by other researchers $[53,157]$.

From an analytical perspective, it is generally assumed when modeling fatigue cracks, to estimate crack-tip parameters such as the stress-intensity factor or the J-integral, that the fatigue crack is located at the notch root, i.e. the minimum specimen cross-section. This is assumed in the empirical models developed to estimate the stress-intensity factors for through thickness and surface cracks in notched specimens for isotropic elastic materials that were discussed in Chapter 3. Similarly, the anisotropic elastic-plastic FEM results 
reported by Zhao and co-workers [56] were based on semi-elliptical cracks embedded at the notch root. In this investigation it was also assumed that fatigue cracks were embedded at the notch root; however, it should be recognized that if the range of the elastic stresses are assumed to control the nucleation and growth of fatigue cracks that the analysis should consider the possibility that fatigue cracks can form in the more highly stressed region away from the notch root. Finite element calculations performed to estimate the crack-tip parameters should take this possibility into consideration. Presumably, if fatigue cracks were to form in the high stress region of the notch the stress-intensity factors for the anisotropic cases would be higher and the variation along the crack front could be different than the results obtained from the present analysis. To verify this, further finite element simulations would need to be performed for Mode I cracks that are assumed to be located in this high stress region. This exercise is left for follow-up work to this investigation.

\subsection{Analysis of The J-Integral for Semi-Elliptical Notch Root Cracks}

Crack-tip parameters such as the J-integral are known to depend on several factors including the applied loading, constitutive material behaviour, and the crack and specimen geometry. In this investigation the loading and specimen geometry and constitutive material behaviour are constant, but the crack geometry and material orientation are the variables in the analyses. To systematically investigate the effect of elastic-plastic anisotropy and secondary crystallographic orientation, two representative crack geometries were assumed in the analyses $(c=0.2 \mathrm{~mm}$ with $a / c=0.86$ and $c=1.0$ $\mathrm{mm}$ with $a / c=1.35$ ). In this section, the effects of these two factors on the elastic-plastic J-integral are presented and discussed. 
As discussed in Chapter 3, elastic-plastic J-integral values calculated using incremental constitutive theories are known to exhibit some path dependence, which depends on the constitutive theory and the extent of plastic deformation. The path independence of the Jintegral was investigated for the elastic-plastic constitutive model used in present investigation. The results of this investigation are presented and discussed in following section.

\subsubsection{Path Independence of the J-integral}

The path independence of the J-integral was investigated using the constitutive model described in Chapter 6. From several trial analyses it was established that the J-integral path dependence was more prominent for larger crack sizes. Therefore, the largest crack size considered in the finite element simulations, i.e. $c=1.0 \mathrm{~mm}$ with $a / c=1.35$ was used to investigate the path dependence of the J-integral. The investigation focused on the three crack-front positions; at the maximum crack depth $\left(\phi=0^{\circ}\right)$, at the notch surface $(\phi$ $=90^{\circ}$ ), and the crack front location where the maximum J-integral was calculated. The Jintegral was calculated on up to 14 contours that were specified as discussed in Chapter 7.

The variation of the J-integral calculated from different contours is shown in Figure 8.29. In this figure, higher contour numbers are further from the crack-tip. It can be observed that J-integral asymptotically converges to constant values on contours evaluated far from the crack-tip, which presumably represents the far-field J-integral. Brocks and Scheider [166] examined the path dependence of the J-integral for through thickness cracks in compact tension specimens under small and contained yielding, and have reported similar asymptotic convergence behaviour. Based on the results obtained from this investigation, 
it was assumed that J-integral values calculated on contours taken as far as possible from the crack-tip represented the path independent far-field J-integral.

\subsubsection{Elastic-Plastic J-Integral for Notch Root Semi-Elliptical Cracks}

The J-integral variation along the crack front was investigated using identical crack geometries to those used in the stress-intensity factor calculations. However, before proceeding with detailed calculations of the J-integral, the mesh size dependence of calculated J-integral was investigated for the largest embedded crack model $(c=1.0 \mathrm{~mm}$ $a / c=1.35)$.

The calculated J-integral variation along the crack-front for two levels of mesh refinement with 32 and 40 elements defined along the crack-front is shown in Figure 8.30. Comparing the results for both levels of mesh refinement it is apparent the calculated J-integral values are generally the same along the entire crack-front, except where the crack-front intersects the notch surface $\left(\phi=90^{\circ}\right)$. The J-integral at the notch surface $\left(\phi=90^{\circ}\right)$ was approximately $40 \%$ lower for the FE model containing the finer mesh (40 elements along the crack-front). A similar, but smaller mesh size dependence was observed in simulations that considered smaller crack sizes $(c<1 \mathrm{~mm})$. Since the calculated J-integral values were generally the same up to the crack front position nearest the notch surface, that is the $N-1$ crack front position (where $N$ represents the number nodes defining the crack front and $N$ coincides with the notch surface), the J-integral at the notch surface was assumed to be the J-integral calculated at the $\mathrm{N}-1$ crack front position.

The variation in the J-integral for semi-elliptical $c=0.2 \mathrm{~mm} a / c=0.86$ and $c=1.0 \mathrm{~mm}$ $a / c=1.35$ notch root cracks are shown in Figures 8.31 and 8.32, respectively. It can be 
observed that the $\mathrm{J}$-integral variation along the crack front is distinctly different for the two anisotropic notch orientations and crack sizes under elastic-plastic loading conditions. For the smaller crack size $(c=0.2 \mathrm{~mm} a / c=0.86)$ the $\mathrm{J}$-integral variation for orientation $\mathrm{A}$ increases from the deepest part of the $\operatorname{crack}\left(\phi=0^{\circ}\right)$ to a maximum value at $\phi=45^{\circ}$ and then decreases to a minimum value at the notch surface $\left(\phi=90^{\circ}\right)$. On the other hand, for orientation B and the same crack size, the J-integral variation exhibits two local maxima; one at deepest part of the crack $\left(\phi=0^{\circ}\right)$ and the second at the $\phi=65^{\circ}$ crack front position. After $\phi=65^{\circ}$ the J-integral decreases to a minimum value at the notch surface $\left(\phi=90^{\circ}\right)$. The J-integral at the two local maxima were higher than the maximum J-integral calculated for orientation $\mathrm{A}$, with the maximum difference being observed at the deepest part of the crack $\left(\phi=0^{\circ}\right)$ where the calculated J-integral was approximately $2 \times$ higher for orientation $B$ than orientation A. A more detailed comparison of the differences between the J-integral along the crack front for orientations $\mathrm{A}$ and $\mathrm{B}$ and both crack geometries is presented in Figure 8.33. In this Figure values above or below $0 \%$ difference indicate the $\mathrm{J}$-integral for orientation $\mathrm{B}$ is higher or lower respectively, than the J-integral for orientation $\mathrm{A}$.

The crack size dependence on the J-integral variation along the crack front is apparent when comparing Figures 8.31 and 8.32. It can be observed that the J-integral was generally higher along the entire crack-front for the larger crack size. It is also apparent the J-integral variation for the larger crack size $(c=1.0 \mathrm{~mm} a / c=1.35)$ is significantly different than calculated for the smaller crack size $(c=0.2 \mathrm{~mm} a / c=0.86)$. For orientation $\mathrm{A}$, the $\mathrm{J}$-integral increases monotonically from the $\phi=0^{\circ}$ crack-front position to a maximum value at $\phi=75^{\circ}$ then decreases to a minimum value at the notch surface $(\phi$ $=90^{\circ}$ ). For orientation $\mathrm{B}$ the $\mathrm{J}$-integral variation along the crack-front still exhibits two local maxima but the maximum at $\phi=0^{\circ}$ is much less apparent than observed for the 
smaller crack size. The J-integral variation along the crack-front for orientation B decreases monotonically from the $\phi=0^{\circ}$ crack-front position to a local minimum value at $\phi=45^{\circ}$, then increases to a maximum value at $\phi=80^{\circ}$ and then decreases to a minimum value at the notch surface $\left(\phi=90^{\circ}\right)$. Similar to the smaller crack size the J-integral was generally higher for orientation $\mathrm{B}$ than $\mathrm{A}$ along the majority of the crack-front as indicated in Figure 8.33. The maximum difference in the calculated J-integral along the crack-front was observed at $\phi=80^{\circ}$ where the J-integral for orientation B was higher than orientation A by approximately $36 \%$.

The anisotropic stress-intensity factor results presented earlier indicated that the stressintensity factors for notch root semi-elliptical cracks were higher at the $\phi=0^{\circ}$ and $\phi=$ $90^{\circ}$ crack front positions for orientation A than B. In contrast, the elastic-plastic J-integral results suggest the opposite is the case, i.e. the J-integral is higher for orientation $\mathrm{B}$ than A at $\phi=0^{\circ}$ and $\phi=90^{\circ}$ crack front positions. Also, when comparing Figure 8.28 and Figure 8.33 the effects of elastic-plastic anisotropy and secondary crystallographic orientation on the J-integral appear to be much larger than the elastic anisotropy effects on the stress-intensity factor. The finite element results presented by Zhao and coworkers [56] also have indicated the J-integral was higher for orientation $B$ than $A$ in their anisotropic elastic perfectly plastic finite element results for semi-elliptical notch root cracks, although the reported differences between these two orientations were much smaller than results obtained in the present investigation.

As discussed earlier, other researchers have observed significant differences in the growth of short through-thickness orientation B exhibits higher crack propagation rates than orientation A. Defresne and Remy [33] have rationalized the apparent secondary orientation dependence short fatigue crack growth on the basis of crack closure, which 
they reported was higher for orientation A than B. They did not indicate why shielding was more prominent for cracks in orientation A fatigue specimens; however, their fatigue tests were conducted in air at $650^{\circ} \mathrm{C}$ where environmental effects on fatigue crack growth, such as oxide induced crack closure or crack-tip blunting could be significant.

On the other hand, Reed and coworkers [52] have investigated the propagation of short through-thickness fatigue cracks in single-crystal Udimet 720 at room temperature in air and vacuum, in which environmental effects on fatigue crack growth, such as oxide induced crack closure, would be negligible. They reported fatigue crack growth was faster in orientation $B$ than orientation A fatigue specimens. One factor that could contribute to a secondary orientation dependence on fatigue crack growth rates is the transition from Stage II non-crystallographic crack growth to the intrinsically faster Stage I extended crystallographic mechanism of fatigue crack propagation. As discussed in Chapter 3, the transition between Stage II and Stage I crack growth (or vice-versa) depends on temperature, stress state and cyclic loading frequency, among other factors. Reed et al. [52] reported the crack propagation mechanisms were identical (predominantly Stage II non-crystallographic crack growth) for both secondary crystallographic orientations. Since environmental effects were precluded under the test conditions employed in their investigation, this suggests the driving force crack extension was indeed higher for orientation B than A. This apparent secondary orientation dependence on crack propagation rate could potentially be rationalized using the elasticplastic $\mathrm{J}$-integral results obtained in this investigation.

In Chapter 3 it was discussed that several numerical and experimental studies have been conducted by various researchers to investigate the localization of plastic deformation in single-crystal materials. Flouriot and coworkers [143] have shown from FEM 
calculations that strain localization into specific slip sectors and the magnitude of plastic shear strain for remote tensile (Mode I) loading mainly depend on orientation of the loading axis and the crack relative to the principal material (crystallographic) directions. A comparison of the cube and octahedral slip activity at the $\phi=0^{\circ}$ and $\phi=90^{\circ}$ crack front positions was conducted for the $c=0.2 \mathrm{~mm} \mathrm{a/c}=0.86$ crack geometry and both secondary crystallographic orientations to determine if differences in slip activity at the crack-tip could rationalize the effect of elastic-plastic anisotropy on the J-integral.

Contour plots of cumulative octahedral shear strain for orientation A and B are presented in Figures 8.34 and 8.35 for $\phi=0^{\circ}$ and $\phi=90^{\circ}$ crack front positions, respectively. Strain localization is apparent and both the relative magnitude of the cumulative octahedral shear strain and the angle between the concentrated slip band and the crack plane are different for both secondary orientations. Qualitatively, both the magnitude of the cumulative octahedral shear strain and its extent from the crack-tip appear to be higher for orientation B than orientation A. As demonstrated by Flouriot and coworkers [143], a much finer FE mesh than used in the present investigation would be necessary to accurately resolve any quantitative differences in apparent slip activity. The concentrated slip band angles in relation to the crack plane shown in Figures 8.34 and 8.35 agree relatively well with theoretical angles of $54.7^{\circ}$ for orientation A and $45^{\circ}$ for orientation $B$ predicted by the asymptotic solutions presented by Rice [141].

Contour plots of cumulative cube shear strain for orientation A and B are presented in Figures 8.36 and 8.37 for $\phi=0^{\circ}$ and $\phi=90^{\circ}$ crack front positions, respectively. It is apparent that cube shear strain localization occurs at the crack-tip and the relative magnitude of the cumulative cube shear strain was different for orientation A and orientation B. Finite element simulations performed by Flouriot and coworkers [143] 
have also demonstrated cube slip localization for through-thickness cracks in a singlecrystal superalloy. Comparing the contour plots shown in Figures 8.36 and 8.37 suggests both the magnitude of the cumulative cubic shear strain and its extent from the crack-tip is higher for orientation $\mathrm{B}$ than orientation $\mathrm{A}$. Combining this observation with the octahedral slip activity shown in Figures 8.34 and 8.35, indicates the J-integral at the $\phi=$ $0^{\circ}$ and $\phi=90^{\circ}$ crack-front positions are higher for orientation B primarily due to more intense slip activity at the crack-tip than orientation A.

\subsection{Relationships Between Crack-tip Parameters and Crack Geometry}

In addition to conducting the FEM calculations discussed earlier in this Chapter, additional simulations were performed to estimate stress-intensity factors and J-integrals for post-processing of the small FCG data obtained from the fatigue experiments. As discussed in Chapter 7, the variation in crack geometry with notch orientation and crack depth were considered in these analyses (Table 7.2). However, as indicated in Figures 5.38 and 5.39 and discussed earlier, some variability in crack geometry was observed during the ACPD calibration tests, particularly at larger crack sizes, that deviates from the nominal crack geometry assumed in the analyses. This variability was not taken into consideration in the FEM calculations. Accounting for these variations in crack geometry would require the development of sophisticated empirical models such as those proposed by Newman [146] for stress-intensity factors, or by Findley et al. [196] and Kim et al. [197] for the J-integral. This would require more extensive FEM simulation work than considered in the present investigation, and should be considered as an exercise for future work. 
The fatigue crack growth properties for surface flaws are generally assessed from the crack growth rate at the surface and deepest portions of the crack-front, i.e. the notch surface $\left(\phi=90^{\circ}\right)$ and maximum crack depth $\left(\phi=0^{\circ}\right)$ in the present case. The crack-tip parameters associated with these two crack-front positions were extracted from the FEM simulation results to develop polynomial expressions for post-processing of the fatigue crack growth data obtained from the small fatigue crack growth experiments. In the following sections the results from the FEM calculation will be presented. The methodology used for transforming the crack-tip parameters into the specific form of polynomial expressions required by FTA fatigue crack growth rate post-processing software will also be presented.

\subsubsection{Anisotropic Stress-Intensity Factors}

The variation in the anisotropic stress-intensity factors as a function of crack depth are summarized in Table 8.3 and presented in Figure 8.38 for orientations A and B. It can be observed for the range of crack sizes considered in the FEM simulations that the stressintensity factors for orientation A were generally higher by approximately $4-15 \%$ than orientation B. This agrees with the FEM simulation results presented earlier (section 8.3.2) when the stress-intensity factors for orientations A and B were compared with equal crack geometry parameters.

\subsubsection{J-Integral}

The variation in $\mathrm{J}$-integral as a function of crack depth for orientations $\mathrm{A}$ and $\mathrm{B}$ are summarized Table 8.4 and presented graphically in Figure 8.39. It is apparent that elasticplastic anisotropy has a significant effect on the J-integral at the $\phi=90^{\circ}$ and $\phi=0^{\circ}$ crackfront positions since this parameter was significantly higher for orientation B than A over the entire range of crack sizes $(200 \mu \mathrm{m}<c<1000 \mu \mathrm{m})$ considered in the calculations. The effect of anisotropy is more evident at the deepest part of the crack-front $\left(\phi=0^{\circ}\right)$, 
particularly for smaller crack sizes. The difference between the J-integral at the deepest part of the crack $\left(\phi=0^{\circ}\right)$ for orientations A and B was approximately $85 \%$ for the smallest crack depth $(c=200 \mu \mathrm{m})$ and was approximately $15 \%$ for the largest crack depth $(c=1000 \mu \mathrm{m})$. Comparing these differences to the J-integral results presented earlier (section 8.4.2), indicates that the small differences in crack geometry determined from the ACPD calibration tests for orientations $\mathrm{A}$ and $\mathrm{B}$ has negligible effect on the Jintegral.

\subsubsection{Polynomial Expressions for Crack Growth Rate Data Analysis}

As discussed in Chapter 5, the FTA crack growth analysis software uses polynomial functions of a specific form to relate the crack-tip parameters to loading condition and to the crack and the specimen geometry. In the present investigation the stress-intensity factor and J-integral for the notch surface $\left(\phi=90^{\circ}\right)$ and maximum crack depth $\left(\phi=0^{\circ}\right)$ were expressed in terms of fourth-order polynomials having the same form as equation 5.4. Adopting the crack nomenclature shown in Figure 3.5, the polynomial coefficients, $C_{n}$, were obtained by rearranging equation 5.4 as follows for the $\phi=0^{\circ}$ crack-front position,

$$
\begin{aligned}
& C_{1}+C_{2} \alpha_{g}+C_{3} \alpha_{g}^{2}+C_{4} \alpha_{g}^{3}+C_{5} \alpha_{g}^{4}=\frac{(\Delta K, J) 2 t \sqrt{W}\left(1-\alpha_{g}\right)^{3 / 2}}{\Delta P\left(2+\alpha_{g}\right)} \\
& \text { with, } \\
& \alpha_{g}=c / W
\end{aligned}
$$

and, 


$$
C_{1}+C_{2} \alpha_{g}+C_{3} \alpha_{g}^{2}+C_{4} \alpha_{g}^{3}+C_{5} \alpha_{g}^{4}=\frac{(\Delta K, J) W \sqrt{2 t}\left(1-\alpha_{g}\right)^{3 / 2}}{\Delta P\left(2+\alpha_{g}\right)}
$$

with,

$$
\alpha_{g}=a / t
$$

for the $\phi=90^{\circ}$ crack-front position. It should be noted that the units of equations 8.6 and 8.7 do not match those of the J-integral. However, the use of these equations enables one to "deceive" the FTA post processing software to output the correct values for the Jintegral when analyzing the fatigue crack growth rate data. The polynomial coefficients were obtained by fitting a fourth-order polynomial to the dependent and independent variables, i.e. the right-hand-side of equations 8.6 and 8.7 and $\alpha_{g}$, respectively, using least squares regression. For the anisotropic stress-intensity factor and J-integral, separate polynomial coefficients were obtained for orientations A and B. The polynomial fitting coefficients for the isotropic and anisotropic stress-intensity factors and the anisotropic elastic-plastic J-integral are summarized in Table 8.5. These polynomial coefficients are only valid for the crack and specimen geometry parameters and loading conditions assumed in the FEM calculations.

\subsection{Concluding Remarks}

\subsubsection{Notch root Stress and Strain Fields Analysis}

Finite element calculations were performed to estimate the notch root stress and strain fields for the single-crystal SEN specimens employed in this investigation. The effect of single-crystal anisotropy was investigated using two notch orientations [101] (orientation A) and $[100]$ (orientation B) with the loading axis of the specimen aligned with the [010] crystallographic direction. The notch root fields were obtained for different constitutive 
material behaviours including isotropic and cubic anisotropic elasticity, and an elasticplastic micro-phenomenological model formulated based on crystalline slip theory. From these calculations the following have been established:

For the isotropic elastic model, the maximum notch root stresses and strains coincide with the minimum specimen cross section, i.e. the notch root. On the other hand, the maximum stresses in the notch root were predicted to be located at an angle of approximately $30^{\circ}$ from the minimum specimen cross-section for the cubic anisotropic elastic models. The stresses in the notch root were lower for orientation A than orientation B under the same far-field loading conditions. The notch root stresses were also predicted to be lower for the cubic anisotropic elastic models than the isotropic elastic model.

For the micro-phenomenological elastic-plastic constitutive model, the maximum notch root stresses for orientations A and B were located at an angle of approximately $24^{\circ}$ from the minimum specimen cross-section. The notch root stresses were higher for orientation B than orientation A. For orientation A, the highest plastic strain in the notch root occurs at an angle of approximately $28^{\circ}$ from the minimum specimen cross-section and coincides with the mid-thickness of the specimen. The maximum equivalent plastic strain in the notch root for orientation B was located approximately $2.1 \mathrm{~mm}$ from symmetry plane A-A (see Figure 7.2 for definition) of the specimen and at an angular position between 17 and $19^{\circ}$ from the minimum specimen cross-section.

\subsubsection{Fracture Mechanics Parameters for Notch Root Semi-Elliptical Cracks}

Finite element calculations were performed to estimate the stress-intensity factors and Jintegral for notch root semi-elliptical cracks $(200 \mu \mathrm{m}<c<1000 \mu \mathrm{m})$ for the SEN 
specimen used in this investigation. Similar to the notch fields analysis models the effect of elastic and elastic-plastic anisotropy was investigated for two notch orientations [101] (orientation A) and [100] (orientation B) with the loading axis of the specimen aligned with the $[010]$ crystallographic direction. The crack geometry parameters were specified independently for orientations $\mathrm{A}$ and $\mathrm{B}$ based on the crack aspect ratio relationships determined from the ACPD calibration tests described in Chapter 5. The stress-intensity factors were calculated assuming both isotropic and cubic anisotropic elastic properties. The micro-phenomenological elastic-plastic constitutive model described in Chapter 6 was utilized for the elastic-plastic J-integral calculations. From the FEM calculations the following have been established:

For the linear elastic finite element solutions it was demonstrated that accurate estimates of the stress-intensity factors could be obtained using the interaction integral technique without resorting to specialized quarter-point crack-tip elements. The variation of the stress-intensity factors along the crack-front was different for isotropic and cubic anisotropic elastic models along the entire crack-front under the same far-field loading conditions and with the same crack geometry parameters. For smaller notch root cracks, the isotropic stress-intensity factors generally over predict the anisotropic stress-intensity factors along the crack front. The isotropic stress-intensity factors under predict the anisotropic stress-intensity factors for larger notch root crack sizes. In general, the anisotropic stress-intensity factors for orientation A were within $\pm 20 \%$ of the stressintensity factor values for orientation B. At the notch surface $\left(\phi=90^{\circ}\right)$ and deepest part of the crack front $\left(\phi=0^{\circ}\right)$, the anisotropic stress intensity factors were higher for orientation A than B. Generally, it can be concluded that the effects of elastic anisotropy 
on the stress-intensity factors for small notch root semi-elliptical cracks must be taken into consideration.

The path independence of the J-integral was investigated using the microphenomenological constitutive model described in Chapter 6. It was demonstrated for small-scale and confined yielding conditions that the J-integral was path independent on contours evaluated far from the crack-tip.

For the micro-phenomenological elastic-plastic constitutive model, the J-integral variation along the crack-front exhibited marked secondary orientation dependence. In particular, the J-integral at the deepest part of the crack was significantly higher (up to a factor of $2 \times$ ) for orientation B than orientation $A$. The effect of elastic-plastic anisotropy on the J-integral was less apparent for larger crack sizes but the J-integral was generally higher at the notch surface $\left(\phi=90^{\circ}\right)$ and the deepest part of the crack $\left(\phi=0^{\circ}\right)$ for orientation B. Localization of plastic shear strain into specific slip sectors at the crack-tip was observed in the FEM calculation results. In general, the slip sectors corresponded to theoretically predicted values reported in the literature. 
Table 8.1 - Linear elastic stress concentration factors and location of maximum von Mises equivalent stress.

\begin{tabular}{|l|c|c|c|}
\hline \multicolumn{1}{|c|}{ Model } & $\begin{array}{c}\text { Maximum von } \\
\text { Mises Equivalent } \\
\text { Stress (MPa) }\end{array}$ & $\begin{array}{c}\text { Angular Position } \\
(\mathrm{deg})\end{array}$ & $\begin{array}{c}\text { Stress Concentration } \\
\text { Factor, } \mathrm{K}_{\mathbf{t}}\end{array}$ \\
\hline Isotropic & 1589 & 0.0 & 2.75 \\
\hline $\begin{array}{l}\text { Orientation A (cubic } \\
\text { anisotropic) }\end{array}$ & 1366 & 30.0 & 2.37 \\
\hline $\begin{array}{l}\text { Orientation B (cubic } \\
\text { anisotropic) }\end{array}$ & 1479 & 30.0 & 2.56 \\
\hline
\end{tabular}

Table 8.2 - Finite element model parameters for validation of extracted SIF's from ABAQUS/Standard using non-singular crack-tip elements.

\begin{tabular}{|c|c|c|}
\hline $\begin{array}{c}\text { Far-field Stress, } \\
(\mathrm{MPa})\end{array}$ & $\begin{array}{c}\text { Elastic Modulus, E, } \\
(\mathrm{GPa})\end{array}$ & Poisson Ratio, $v$ \\
\hline 500 & 100,000 & 0.3 \\
\hline
\end{tabular}

Table 8.3 - Calculated anisotropic stress-intensity factors for a crack depth range of 0.2 to $1.0 \mathrm{~mm}$ for orientations $A$ and $B$.

\begin{tabular}{|c|c|c|c|c|c|c|}
\hline Model & $\begin{array}{c}\text { Crack } \\
\text { Depth, } \\
(\boldsymbol{\mu m})\end{array}$ & $\mathbf{c} / \mathbf{W}$ & $\mathbf{a} / \mathbf{t}$ & $\mathbf{a} / \mathbf{c}$ & $\begin{array}{c}\mathbf{K}, \boldsymbol{\phi}=\mathbf{0}^{\circ} \\
\left(\mathbf{M P a}-\mathbf{m}^{\mathbf{0 . 5}}\right)\end{array}$ & $\begin{array}{c}\mathbf{K}, \boldsymbol{\phi}=\mathbf{9 0} \\
\left(\mathbf{M P a}-\mathbf{m}^{\mathbf{0} .5}\right)\end{array}$ \\
\hline & 200 & 0.0187 & 0.0644 & 0.86 & 20.93 & 26.09 \\
& 300 & 0.0281 & 0.1035 & 0.92 & 25.01 & 31.84 \\
& 400 & 0.0375 & 0.1472 & 0.98 & 28.07 & 36.05 \\
& 500 & 0.0469 & 0.1954 & 1.04 & 30.49 & 38.71 \\
& 600 & 0.0562 & 0.2482 & 1.10 & 32.60 & 42.18 \\
& 700 & 0.0656 & 0.3056 & 1.16 & 34.63 & 44.68 \\
& 800 & 0.0750 & 0.3675 & 1.23 & 36.49 & 50.53 \\
& 900 & 0.0844 & 0.4340 & 1.29 & 38.42 & 51.93 \\
& 1000 & 0.0937 & 0.5051 & 1.35 & 40.45 & 53.73 \\
\hline \hline \multirow{5}{*}{ Orienation A } & 200 & 0.0187 & 0.0611 & 0.81 & 18.83 & 22.41 \\
& 300 & 0.0281 & 0.0982 & 0.87 & 22.97 & 26.90 \\
& 400 & 0.0375 & 0.1397 & 0.93 & 26.15 & 30.96 \\
& 500 & 0.0469 & 0.1856 & 0.99 & 28.65 & 33.80 \\
& 600 & 0.0562 & 0.2359 & 1.05 & 30.60 & 36.81 \\
& 700 & 0.0656 & 0.2905 & 1.11 & 33.29 & 39.37 \\
& 800 & 0.0750 & 0.3496 & 1.17 & 33.90 & 42.88 \\
& 900 & 0.0844 & 0.4130 & 1.22 & 35.54 & 44.59 \\
& 1000 & 0.0937 & 0.4808 & 1.28 & 37.31 & 47.09 \\
\hline
\end{tabular}


Table 8.4 - J-integral for a crack depth range of 0.2 to $1.0 \mathrm{~mm}$ for orientations A and B based on the micro-phenomenological constitutive model.

\begin{tabular}{|c|c|c|c|c|c|c|}
\hline Model & $\begin{array}{c}\text { Crack } \\
\text { Depth, c, } \\
(\mathbf{m})\end{array}$ & $\mathbf{c} / \mathbf{W}$ & $\mathbf{a} / \mathbf{t}$ & $\mathbf{a} / \mathbf{c}$ & $\begin{array}{c}\mathbf{J}, \mathbf{\Xi}=\mathbf{0} \\
(\mathbf{M P a}-\mathbf{m m})\end{array}$ & $\begin{array}{c}\mathbf{J}, \mathbf{\Xi}=\mathbf{9 0} \\
(\mathbf{M P a}-\mathbf{m m})\end{array}$ \\
\hline & 200 & 0.0187 & 0.0644 & 0.86 & 2.12 & 2.21 \\
& 300 & 0.0281 & 0.1035 & 0.92 & 3.28 & 2.89 \\
& 400 & 0.0375 & 0.1472 & 0.98 & 4.13 & 3.65 \\
& 500 & 0.0469 & 0.1954 & 1.04 & 4.89 & 4.23 \\
Orientation A & 600 & 0.0562 & 0.2482 & 1.10 & 5.51 & 5.00 \\
& 700 & 0.0656 & 0.3056 & 1.16 & 6.05 & 5.88 \\
& 800 & 0.0750 & 0.3675 & 1.23 & 6.76 & 6.71 \\
& 900 & 0.0844 & 0.4340 & 1.29 & 7.51 & 7.61 \\
& 1000 & 0.0937 & 0.5051 & 1.35 & 8.40 & 9.04 \\
\hline \hline \multirow{5}{*}{ Orientation B } & 200 & 0.0187 & 0.0611 & 0.81 & 3.93 & 2.51 \\
& 300 & 0.0281 & 0.0982 & 0.87 & 5.19 & 3.31 \\
& 400 & 0.0375 & 0.1397 & 0.93 & 6.11 & 4.17 \\
& 500 & 0.0469 & 0.1856 & 0.99 & 6.79 & 4.84 \\
& 600 & 0.0562 & 0.2359 & 1.05 & 7.26 & 5.66 \\
& 700 & 0.0656 & 0.2905 & 1.11 & 7.60 & 6.68 \\
& 800 & 0.0750 & 0.3496 & 1.17 & 8.16 & 7.43 \\
& 900 & 0.0844 & 0.4130 & 1.22 & 8.82 & 8.29 \\
& 1000 & 0.0937 & 0.4808 & 1.28 & 9.63 & 9.36 \\
\hline
\end{tabular}

Table 8.5 - Fourth order polynomial coefficients for computation of the stress-intensity factors and J-integral at the deepest part of the crack $\left(\phi=0^{\circ}\right)$ and the notch surface $(\phi=$ $90^{\circ}$ ). Note: In equations 8.6 and 8.7 the force must be specified in $\mathrm{N}$ and geometrical parameters must be in $\mathrm{mm}$ to return the correct values for $\Delta \mathrm{K}$ and the J-integral.

\begin{tabular}{|c|c|c|c|c|c|}
\hline \multirow{2}{*}{ Model } & \multicolumn{5}{|c|}{ Polynomial Fitting Parameter } \\
\hline & $\mathrm{C}_{1}$ & $\mathrm{C}_{2}$ & $\mathrm{C}_{3}$ & $\mathrm{C}_{4}$ & $\mathrm{C}_{5}$ \\
\hline Anisotropic $\Delta \mathrm{K} \phi=0^{\circ}$ Orientation $\mathrm{A}$ & 0.08 & 8.10 & -136.13 & 1176.58 & -3838.64 \\
\hline Anisotropic $\Delta \mathbf{K} \phi=90^{\circ}$ Orientation $\mathrm{A}$ & 0.13 & 1.99 & -11.10 & 23.68 & -18.65 \\
\hline Anisotropic $\Delta \mathrm{K} \phi=0^{\circ}$ Orientation B & 0.08 & 5.77 & -53.68 & 90.18 & 850.87 \\
\hline Anisotropic $\Delta \mathrm{K} \phi=90^{\circ}$ Orientation B & 0.12 & 1.50 & -7.74 & 15.12 & -11.24 \\
\hline Anisotropic J Micro-Phenomenological $\phi=0^{\circ}$ Orientation A & $-4.69 \mathrm{E}-04$ & $8.21 \mathrm{E}-02$ & -1.29 & 10.28 & -26.63 \\
\hline Anisotropic J Micro-Phenomenological $\phi=90^{\circ}$ Orientation A & 3.33E-04 & $6.03 \mathrm{E}-03$ & $-1.91 \mathrm{E}-02$ & $2.38 \mathrm{E}-02$ & $-1.15 \mathrm{E}-02$ \\
\hline Anisotropic J Micro-Phenomenological $\phi=0^{\circ}$ Orientation B & $-1.03 \mathrm{E}-04$ & $9.93 \mathrm{E}-02$ & -1.63 & 11.54 & -22.39 \\
\hline Anisotropic J Micro-Phenomenological $\phi=90^{\circ}$ Orientation B & $3.63 \mathrm{E}-04$ & $7.68 \mathrm{E}-03$ & $-2.58 \mathrm{E}-02$ & $3.58 \mathrm{E}-02$ & $-2.10 \mathrm{E}-02$ \\
\hline
\end{tabular}




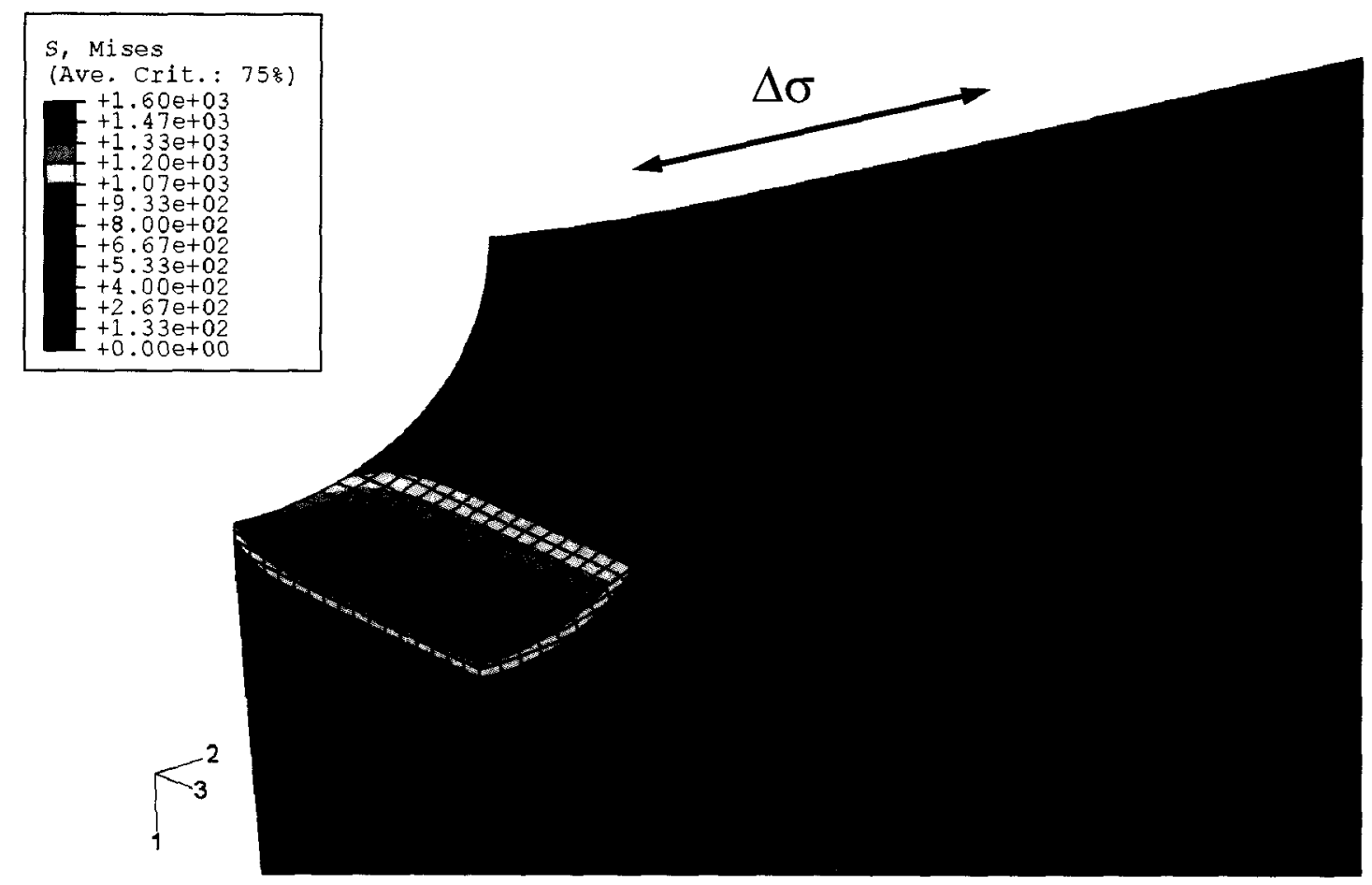

Figure 8.1 - Contour plot showing the von Mises equivalent stress distribution at the notch root for isotropic linear elasticity.

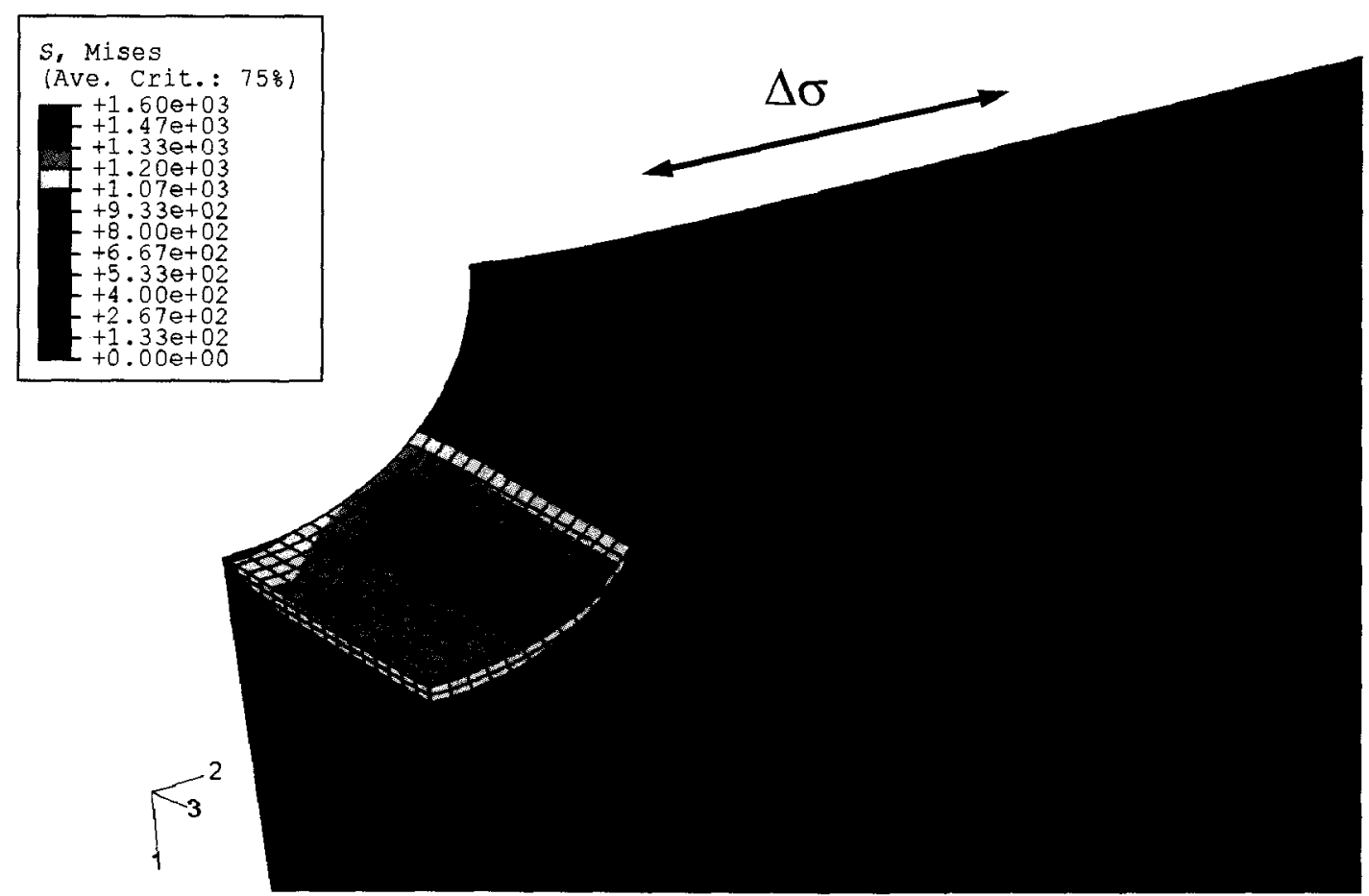

Figure 8.2 - Contour plot showing the von Mises equivalent stress distribution at the notch root for orientation A with cubic anisotropic elastic properties. 


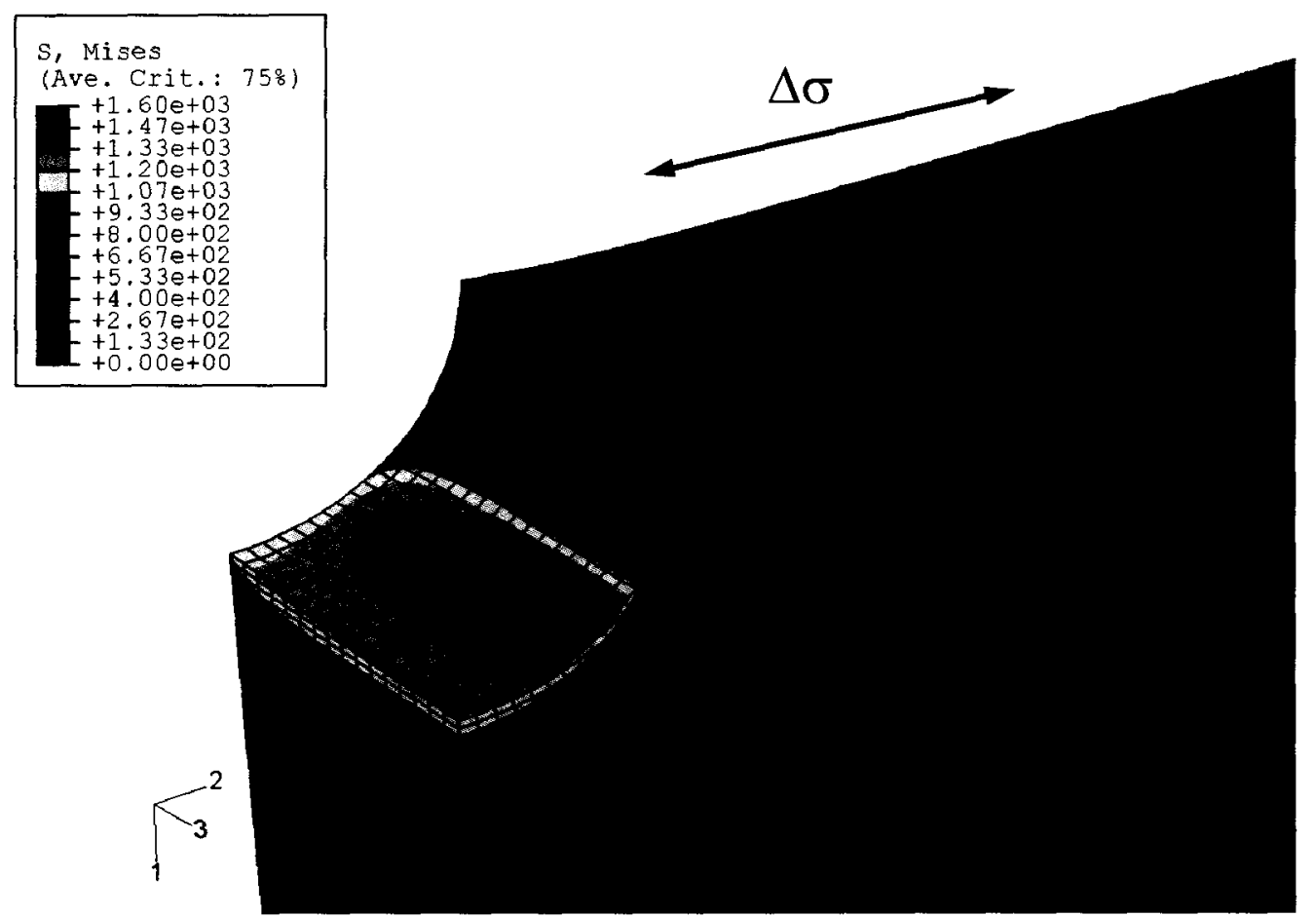

Figure 8.3 - Contour plot showing the von Mises equivalent stress distribution at the notch root for orientation $B$ with cubic anisotropic elastic properties.

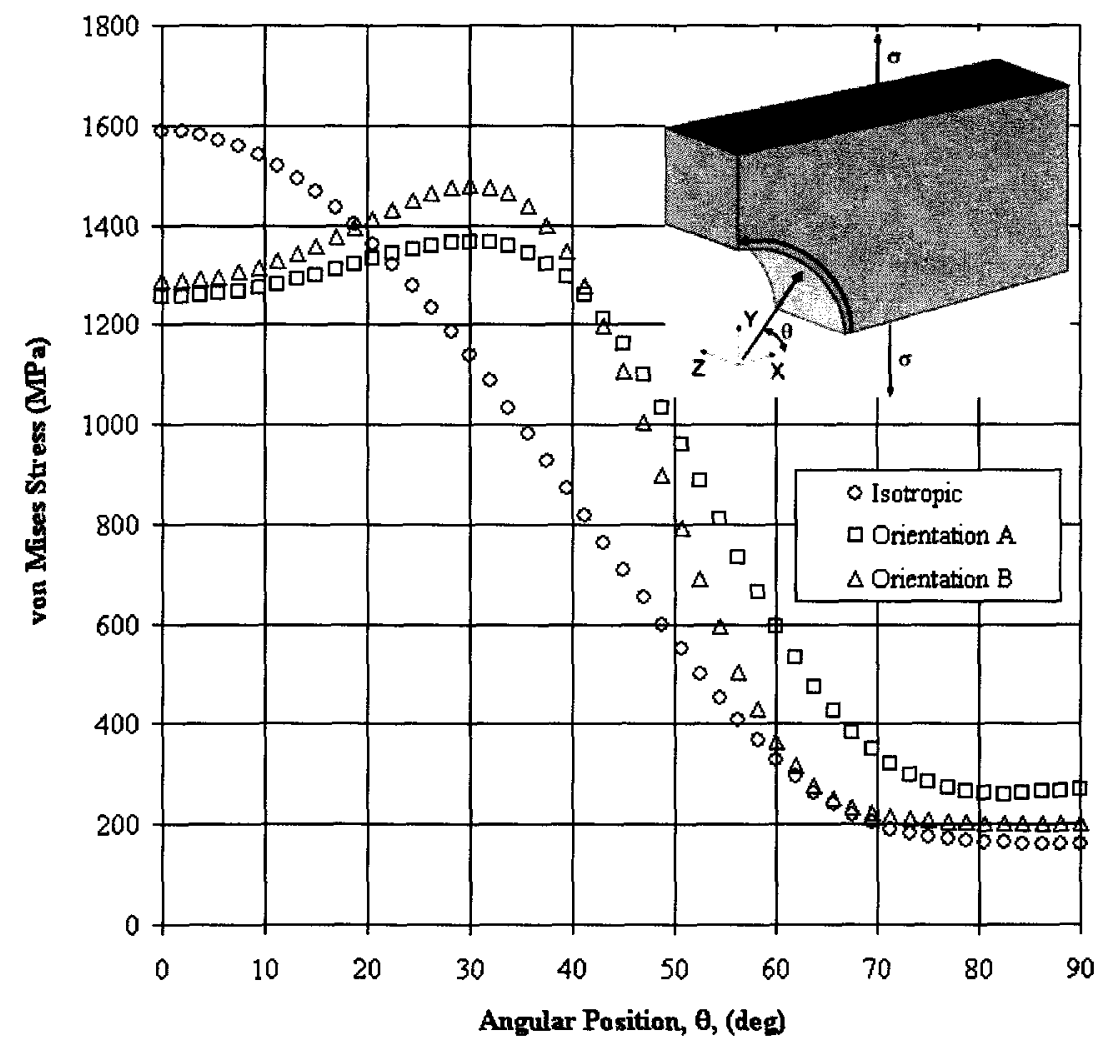

Figure 8.4 - Calculated circumferential variation of von Mises equivalent stress distribution within the SEN specimen notch using linear elastic constitutive material behaviour. 

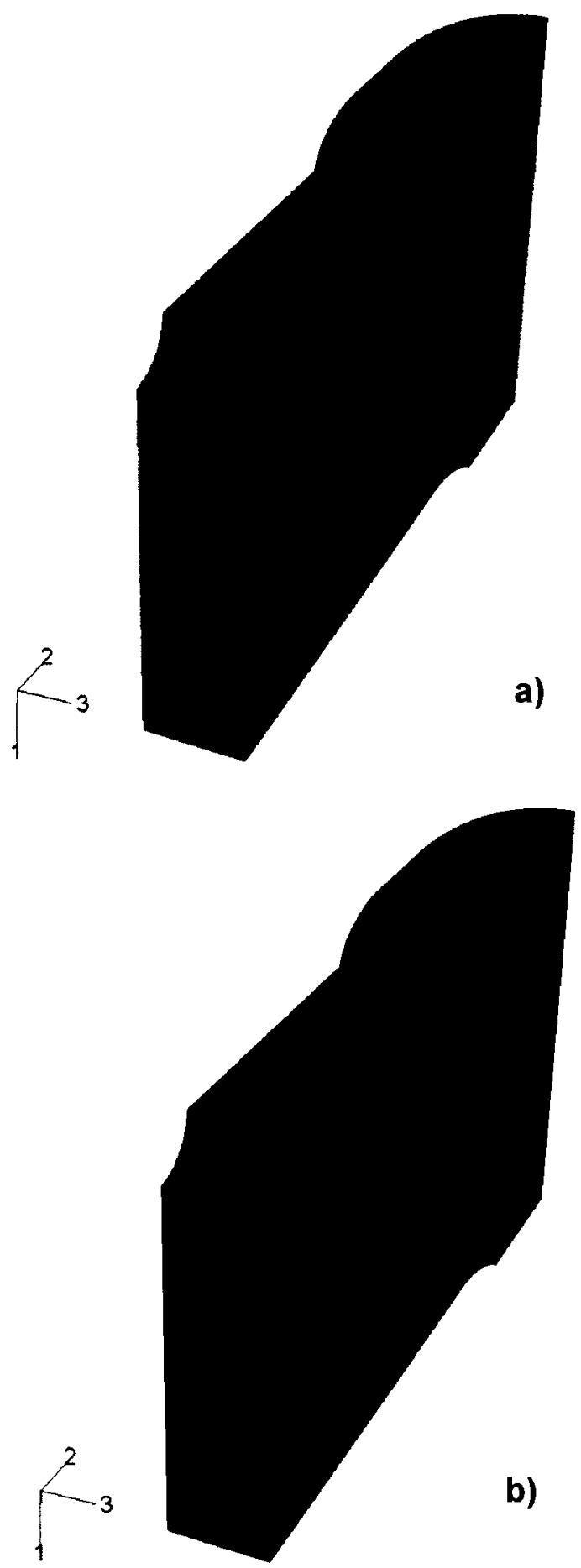

Figure 8.5 - Finite element model of SEN specimen showing the paths used for extracting the stress distribution along the specimen thickness (z-direction) in the notch: a) isotropic and $b$ ) anisotropic cases. Note: red lines indicate the paths. 


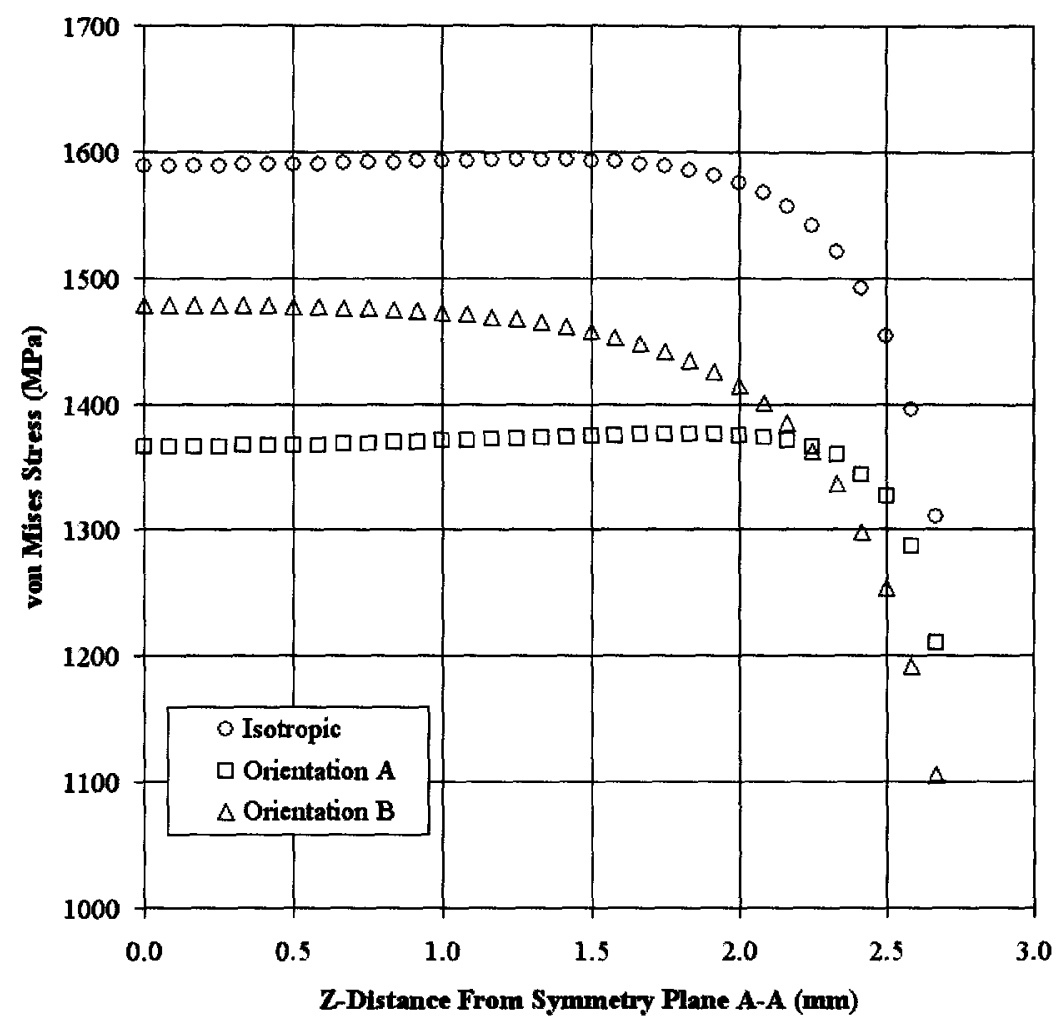

Figure 8.6 - Calculated variation of the von Mises stress in the SEN specimen thickness direction using linear elastic constitutive material behaviour.

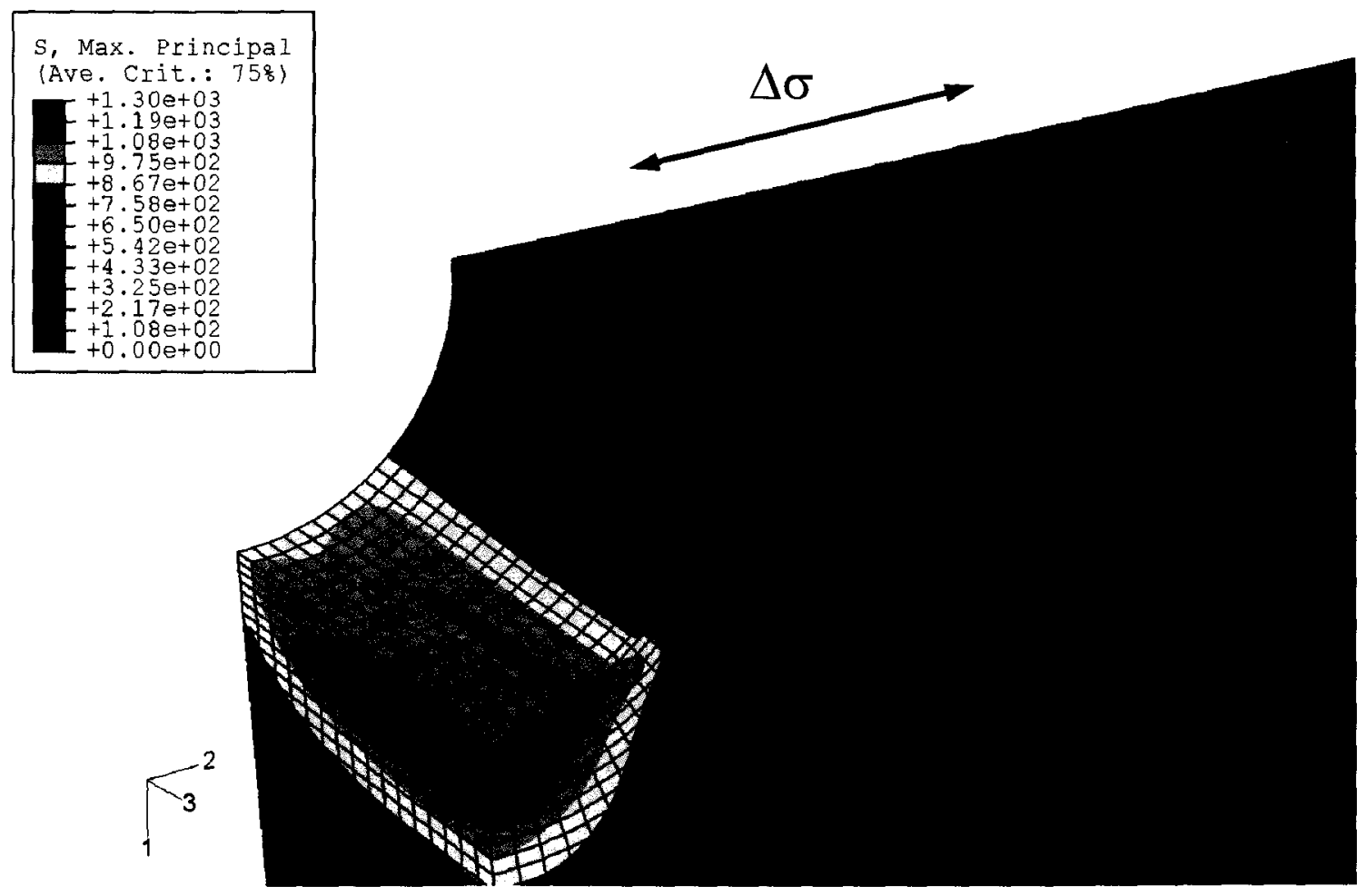

Figure 8.7 - Maximum principal stress contour plot for orientation A. 


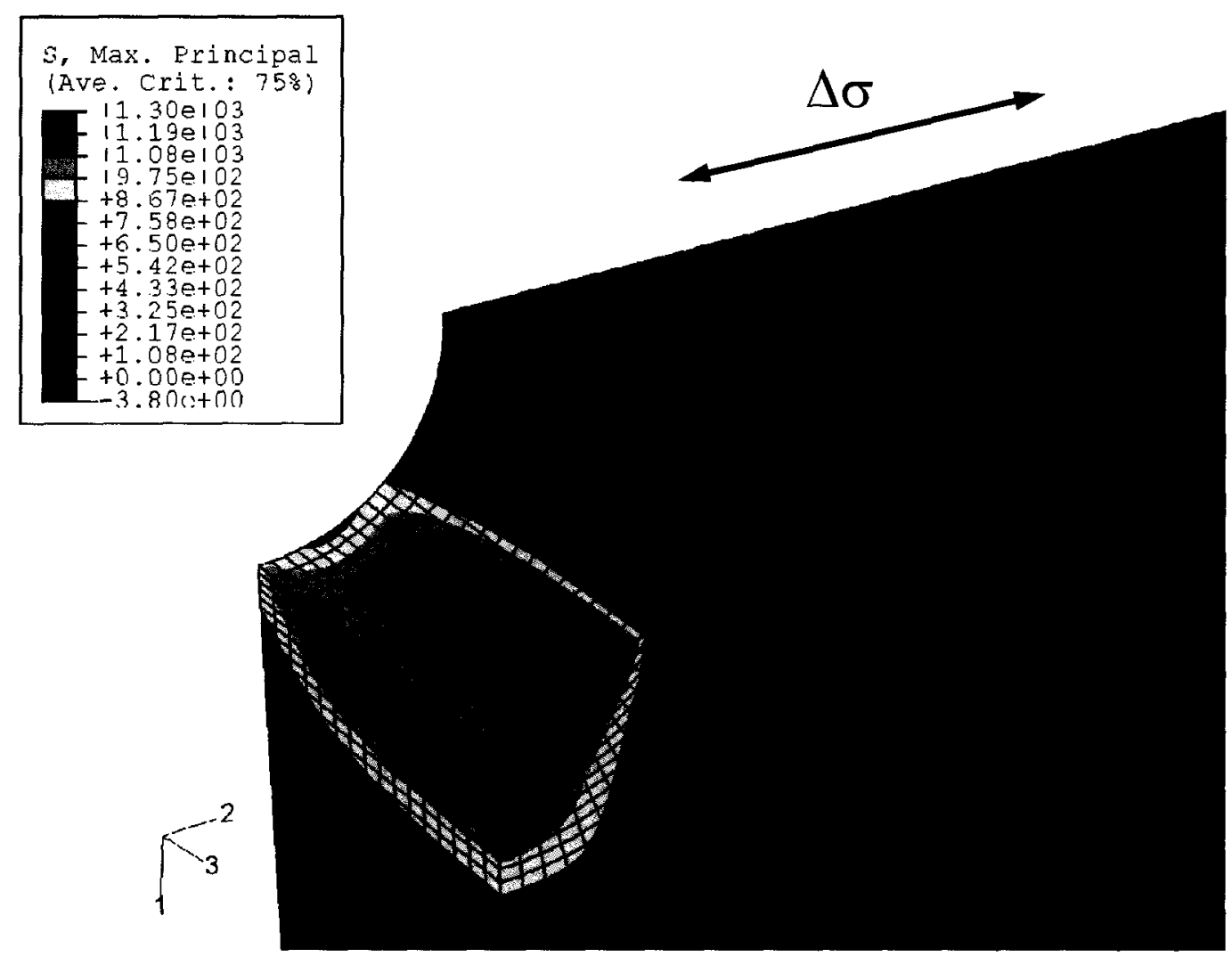

Figure 8.8 - Maximum principal stress contour plot for orientation B.

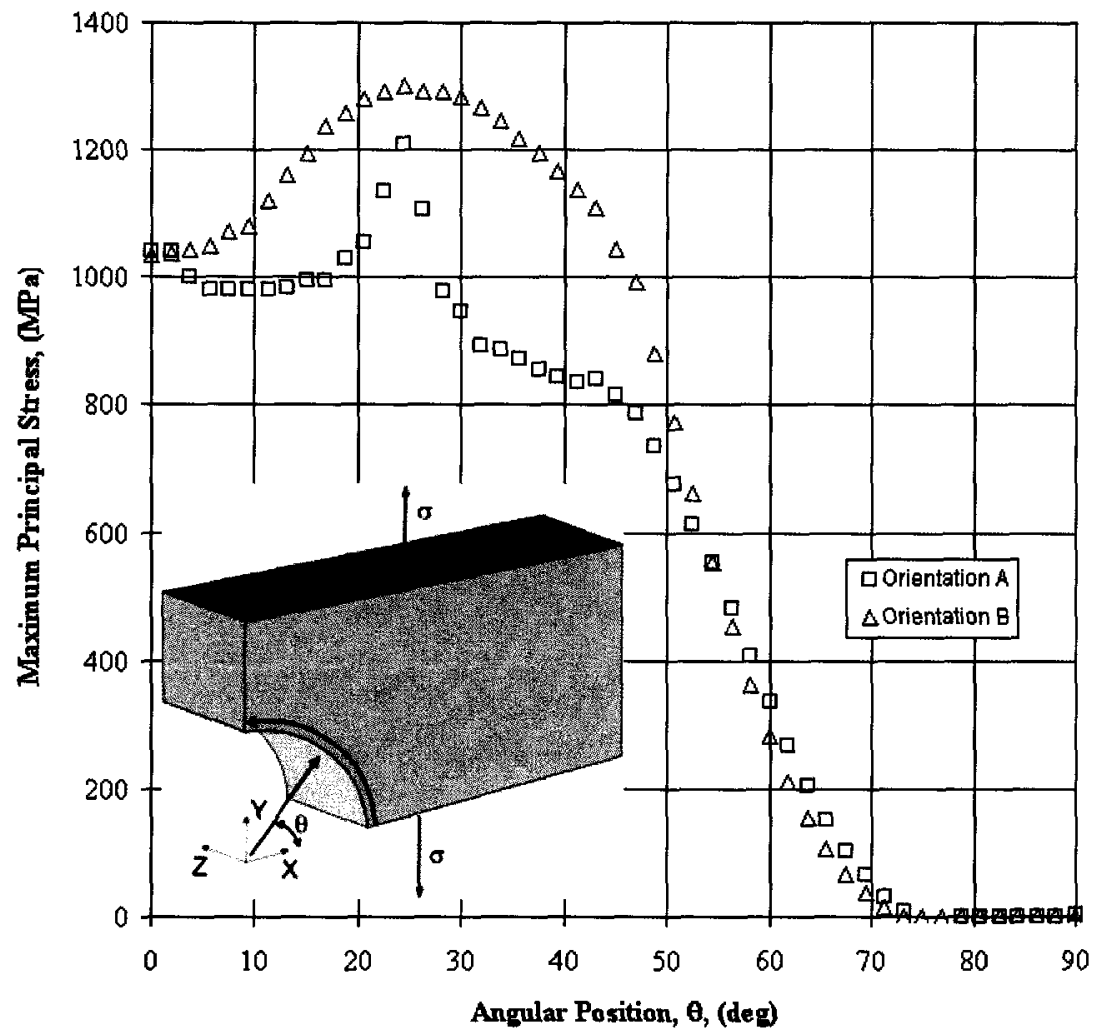

Figure 8.9 - Calculated circumferential variation in the maximum principal stress. 


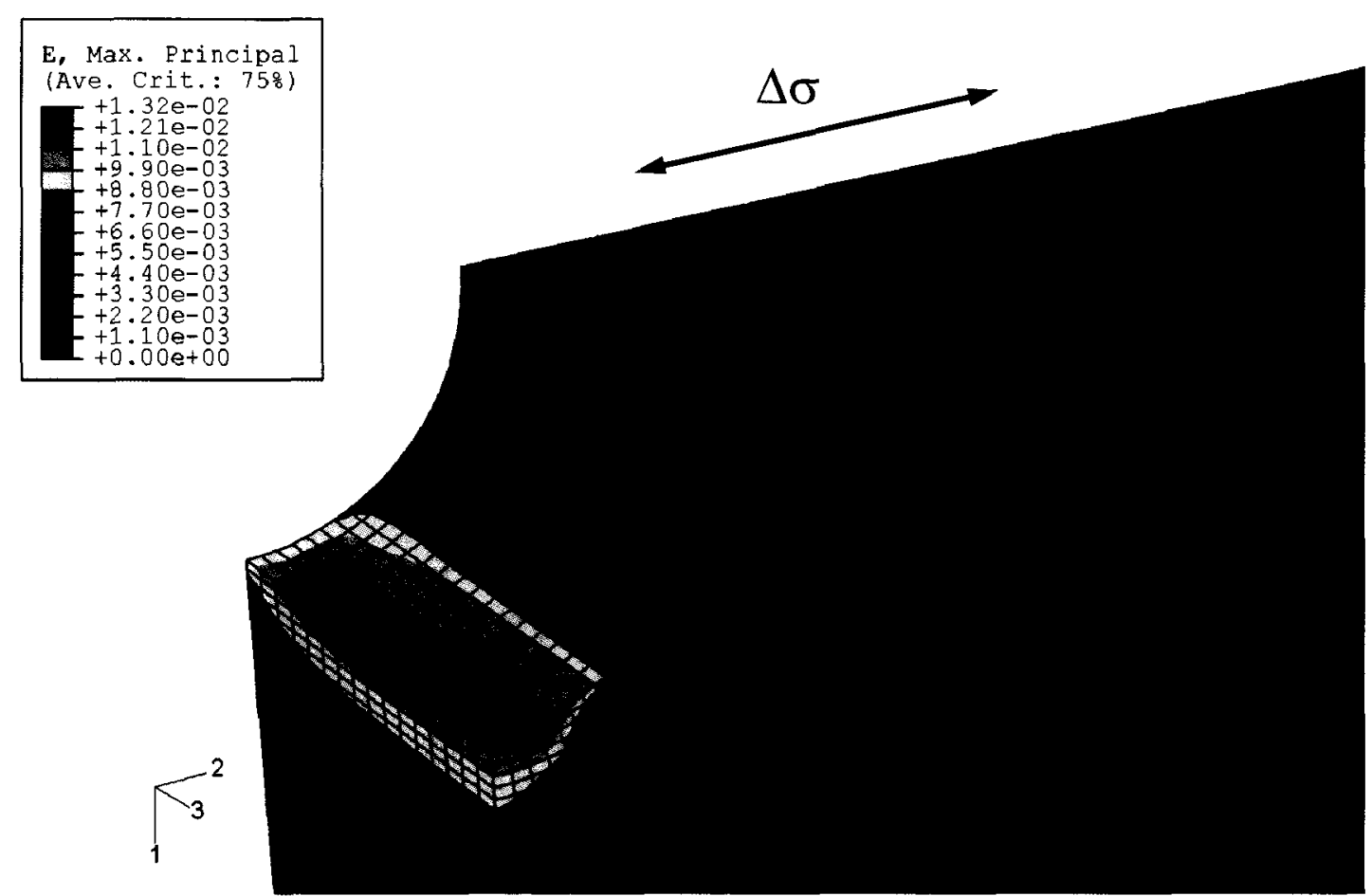

Figure 8.10 - Maximum principal strain contour plot for orientation A.

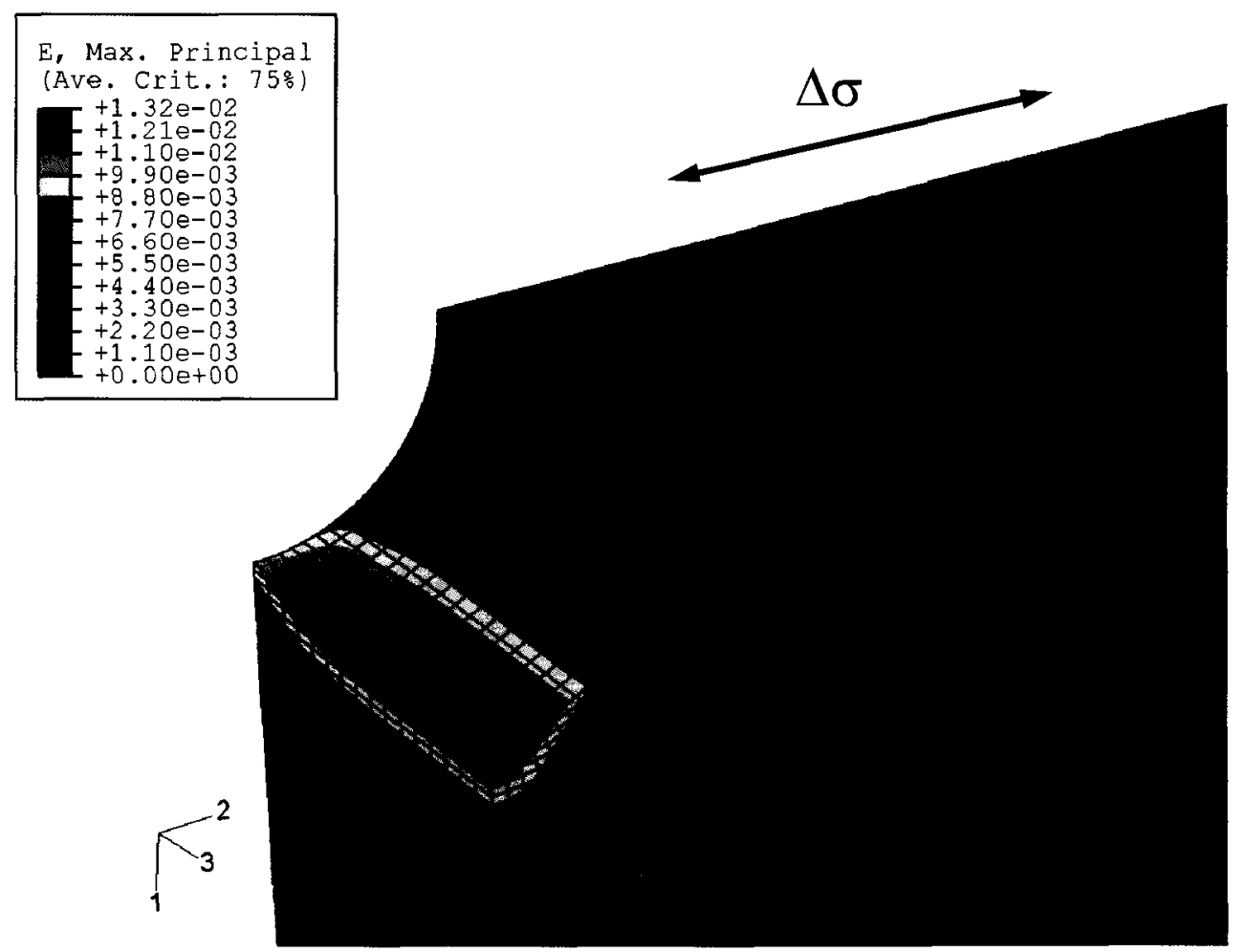

Figure 8.11 - Maximum principal strain contour plot for orientation B. 


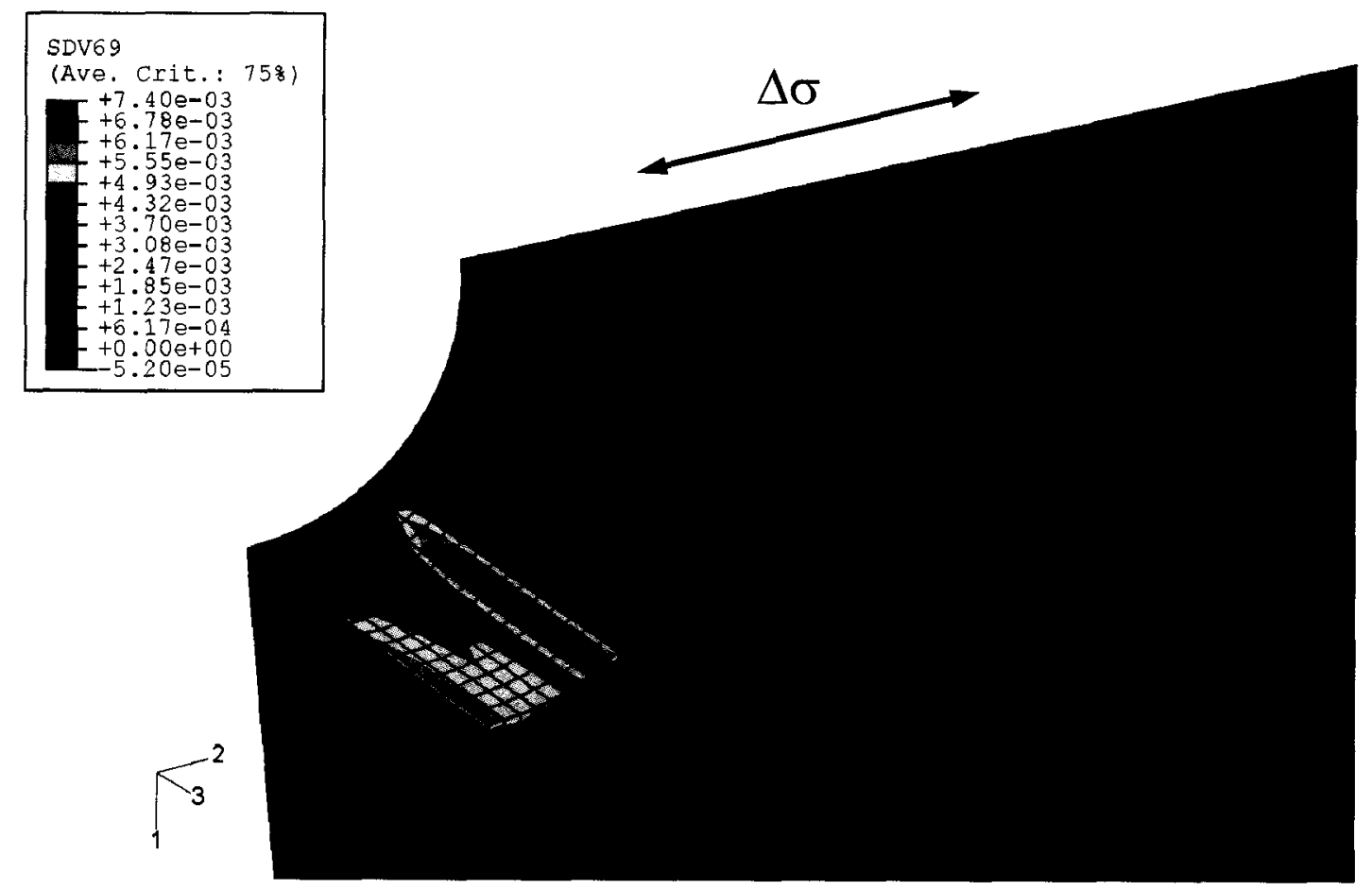

Figure 8.12 - Equivalent plastic strain contour plot for orientation A.

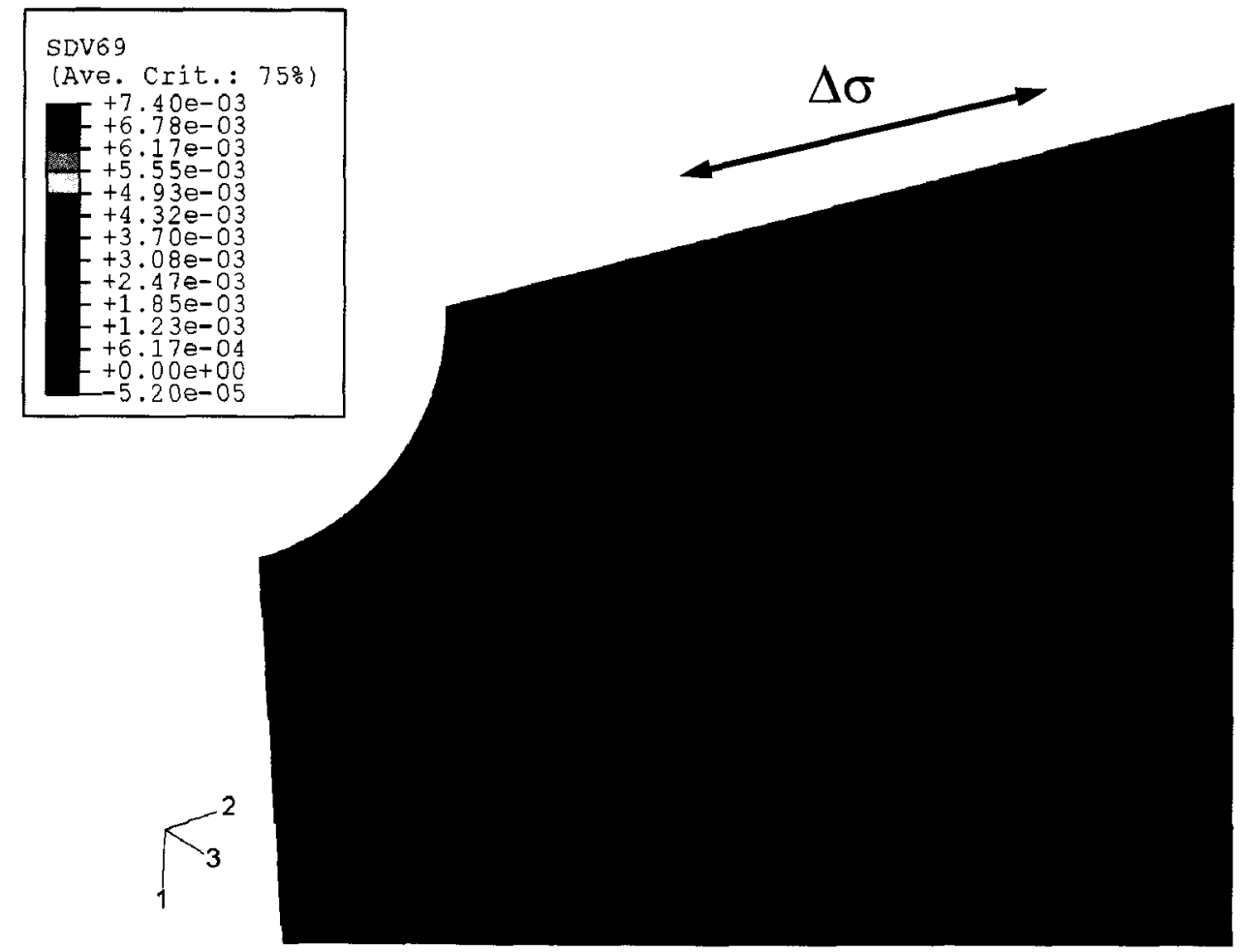

Figure 8.13 - Equivalent plastic strain contour plot for orientation B. 


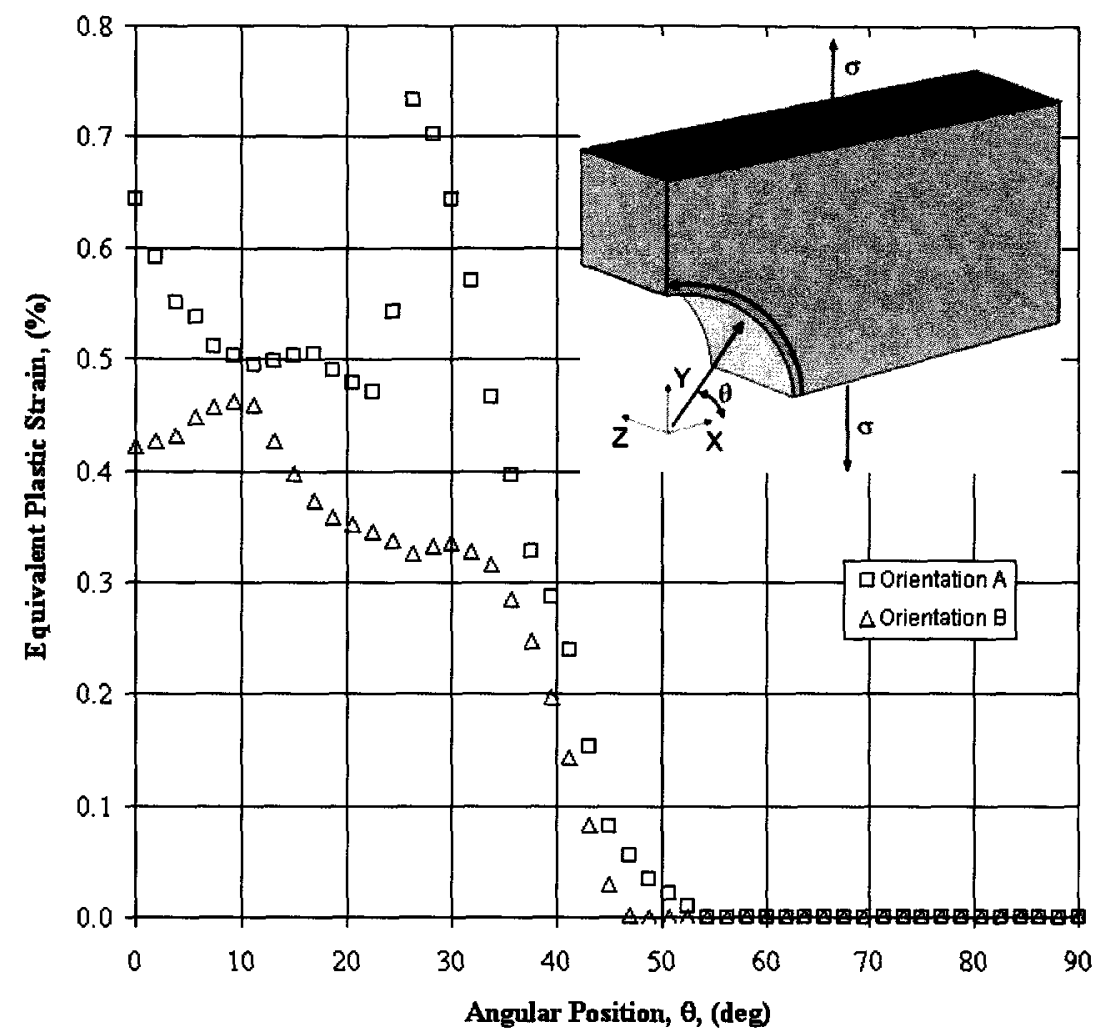

Figure 8.14 - Equivalent plastic strain distribution within the SEN specimen notch.

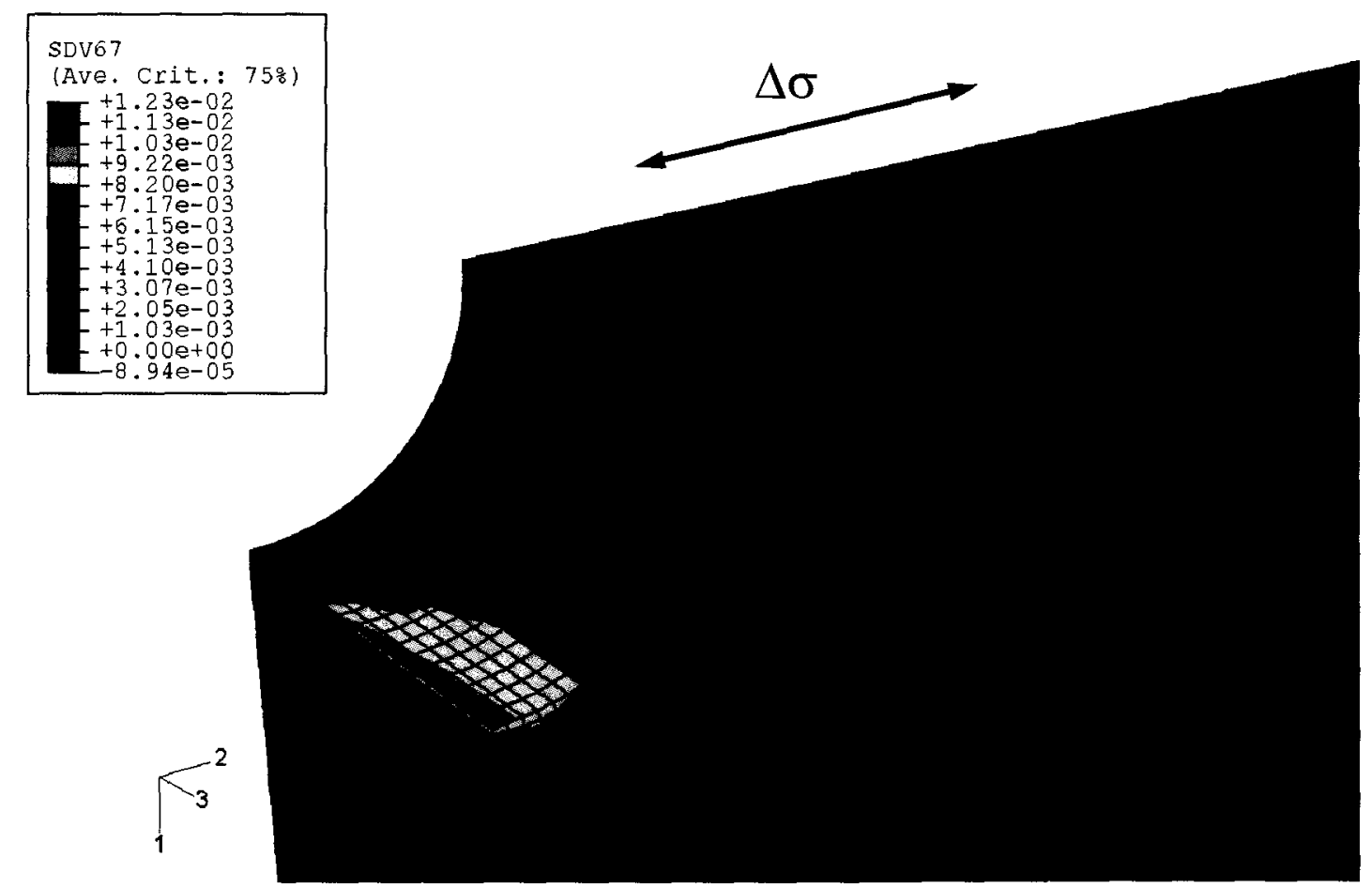

Figure 8.15 - Cumulative octahedral shear strain contour plot for orientation A. 


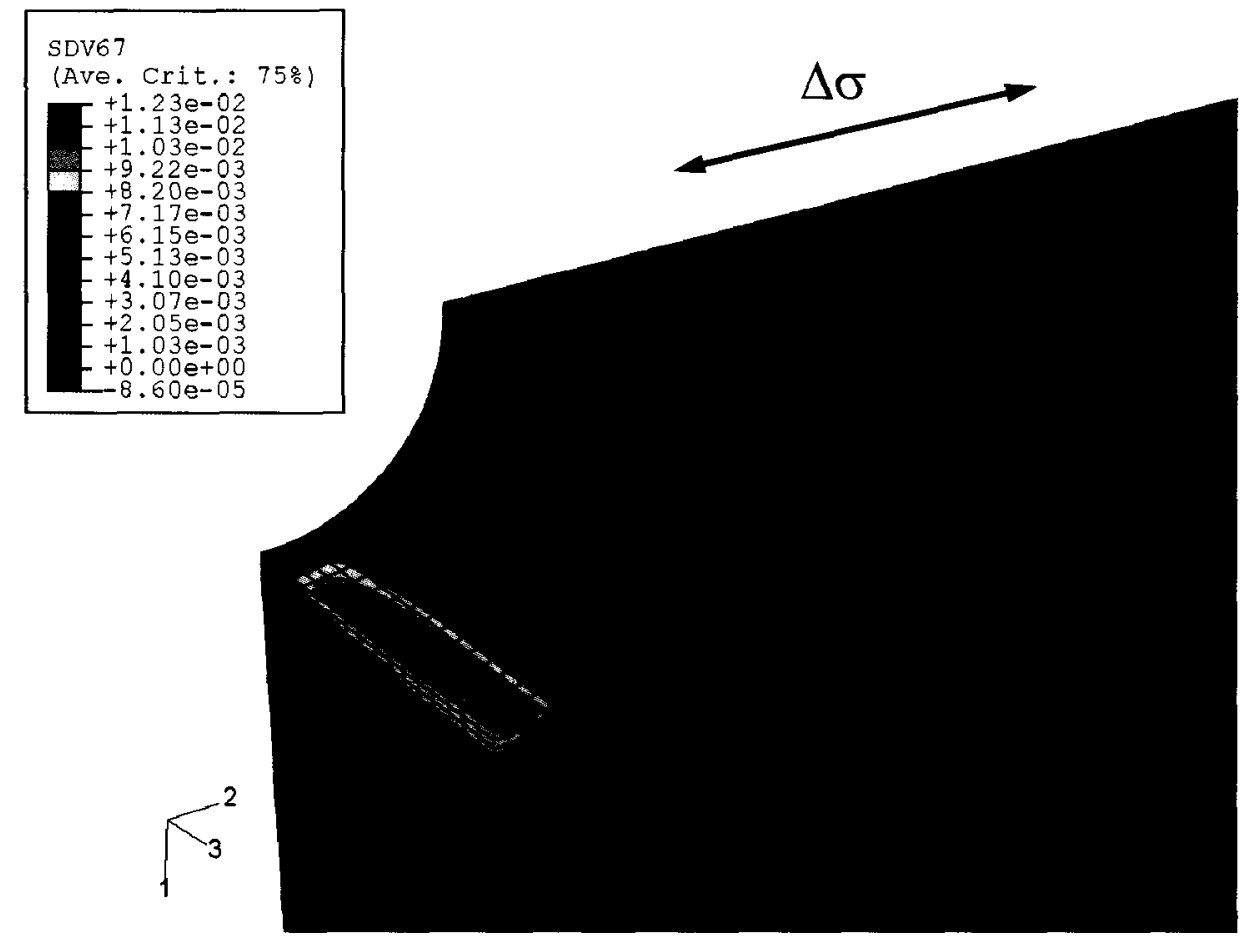

Figure 8.16 - Cumulative octahedral shear strain contour plot for orientation B.

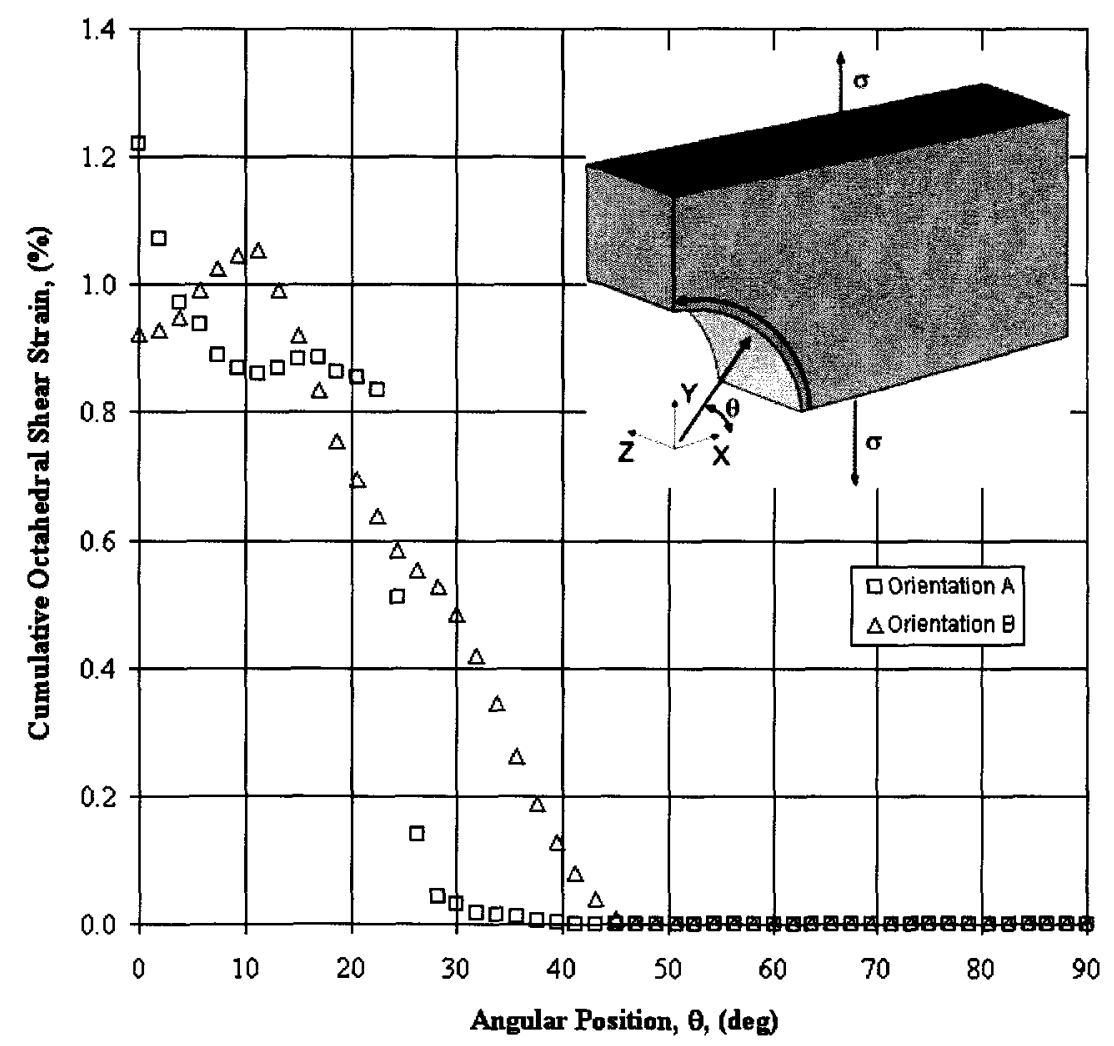

Figure 8.17 - Calculated circumferential variation in the cumulative octahedral shear strain. 


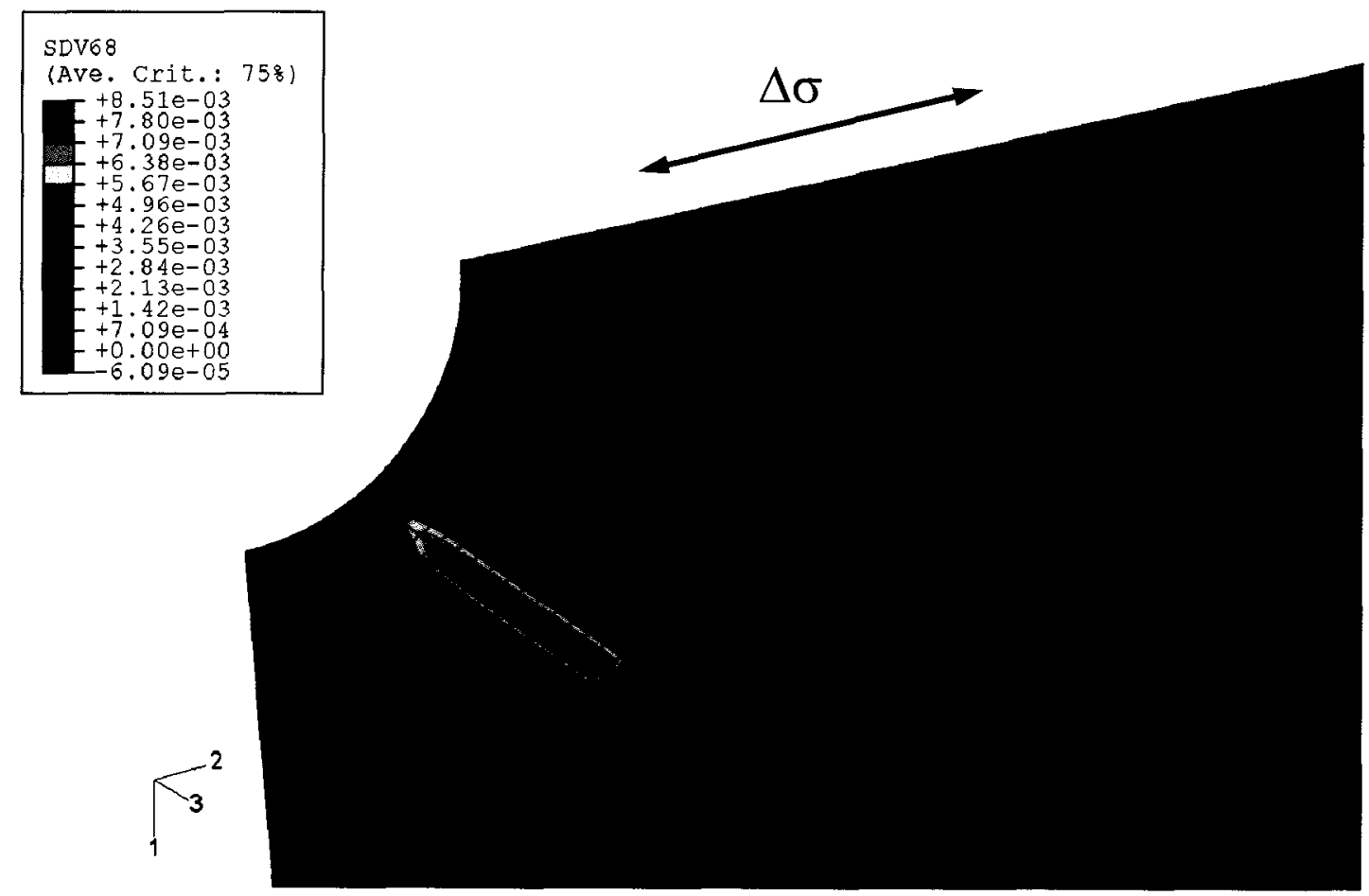

Figure 8.18 - Cumulative cubic shear strain contour plot for orientation A.

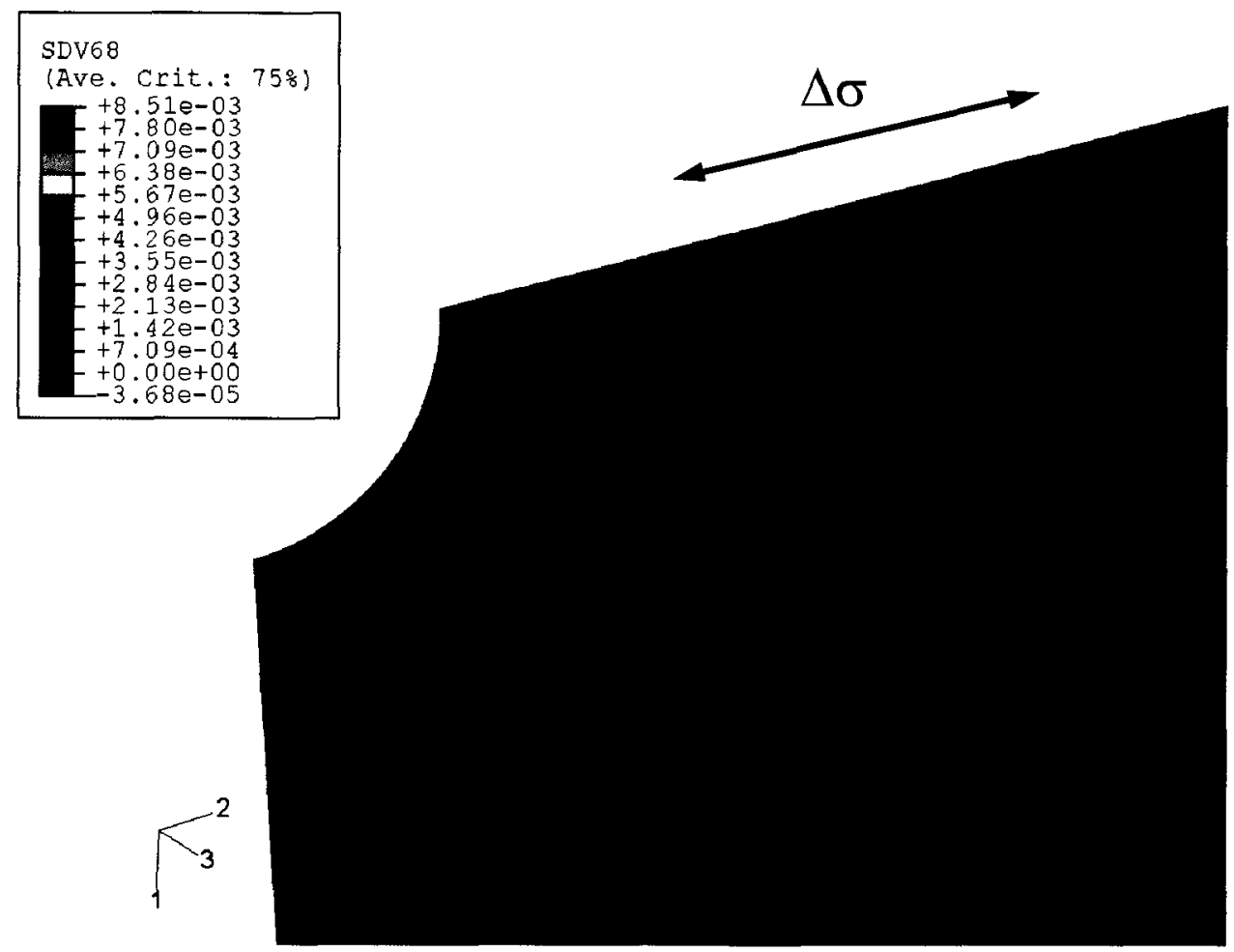

Figure 8.19 - Cumulative cubic shear strain contour plot for orientation B. 


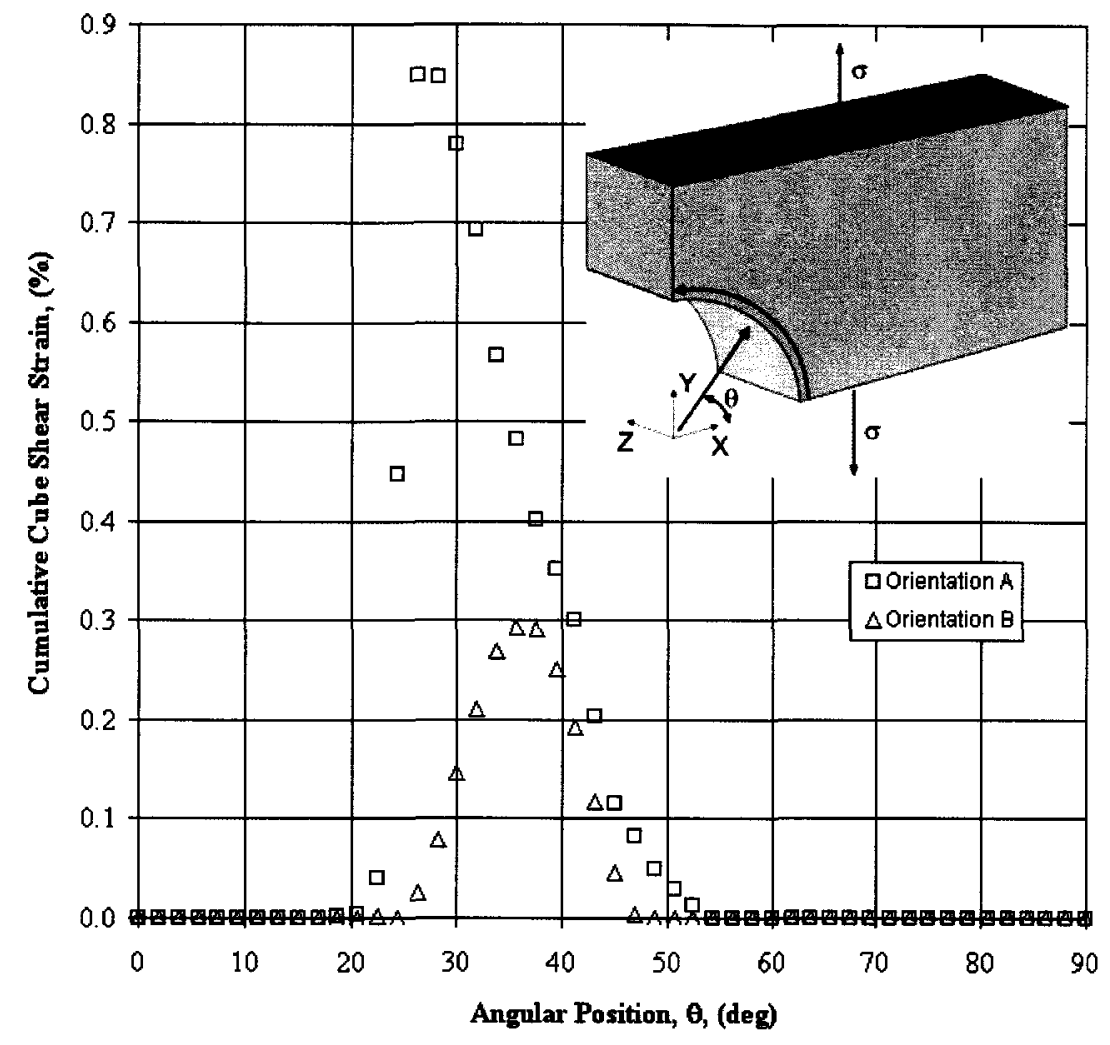

Figure 8.20 - Calculated circumferential variation in the cumulative cubic shear strain.

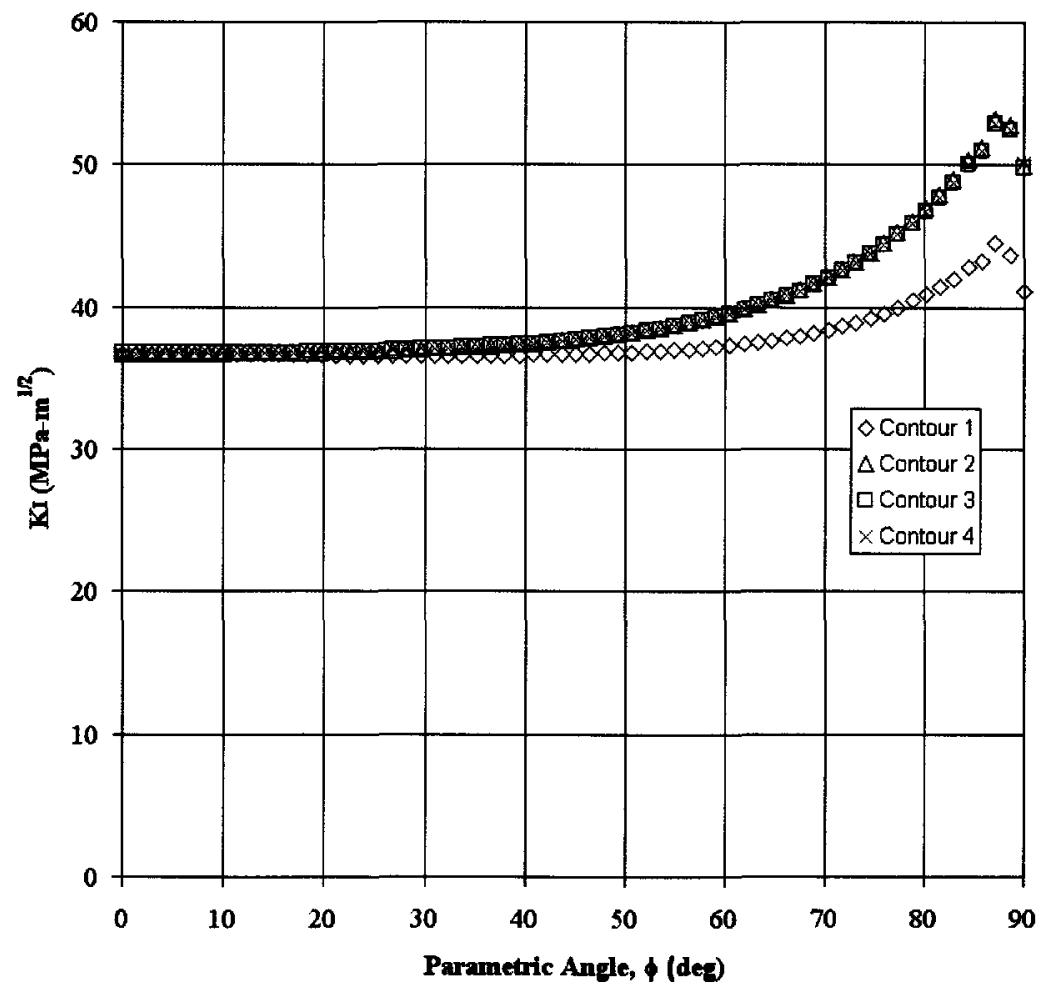

Figure 8.21 - Convergence of the stress-intensity factors calculated along different crack front contours for $c=1.0 \mathrm{~mm} a / c=1.35$ notch root crack. 


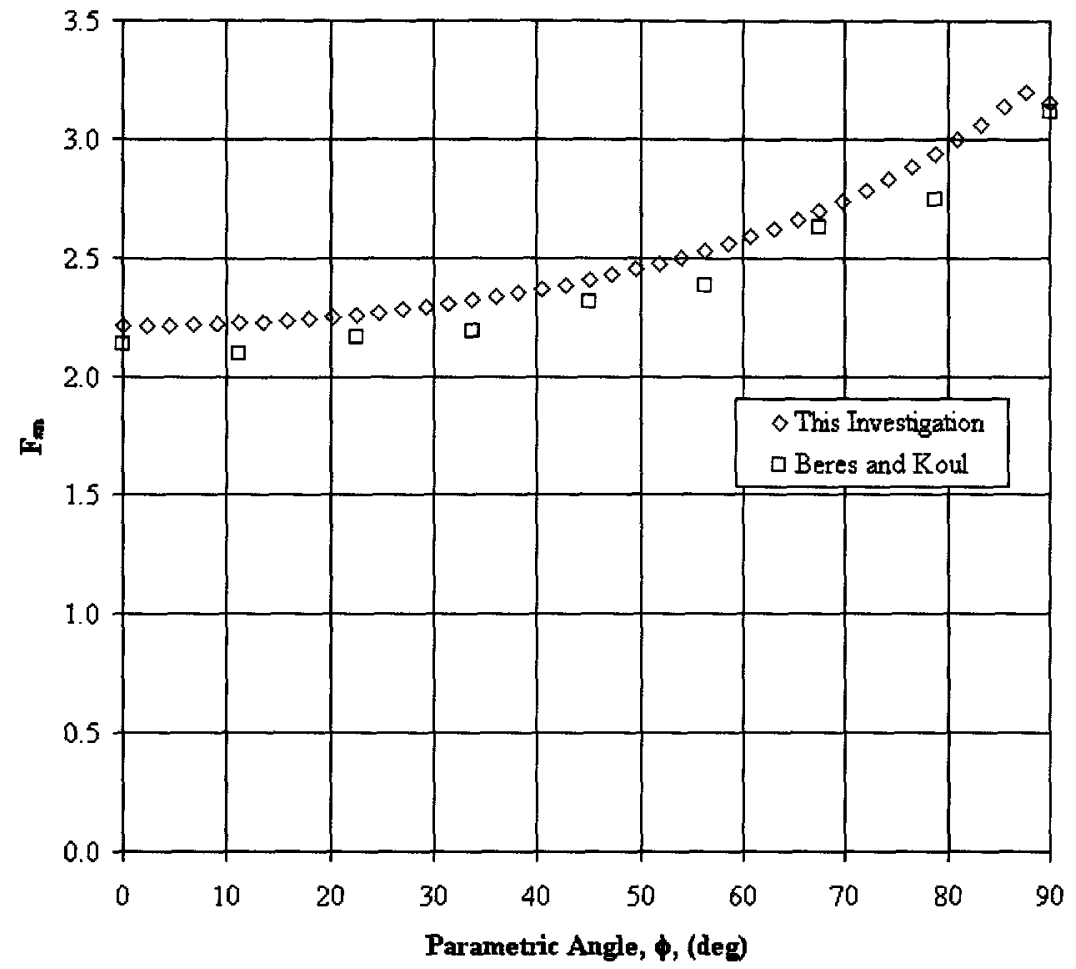

Figure 8.22 - Comparison of boundary correction factors for an $a / c=1.0, c=0.4 \mathrm{~mm}$ crack in the SEN specimen used in this investigation (ABAQUS), and results reported by Beres and Koul for a DEN specimen [269].

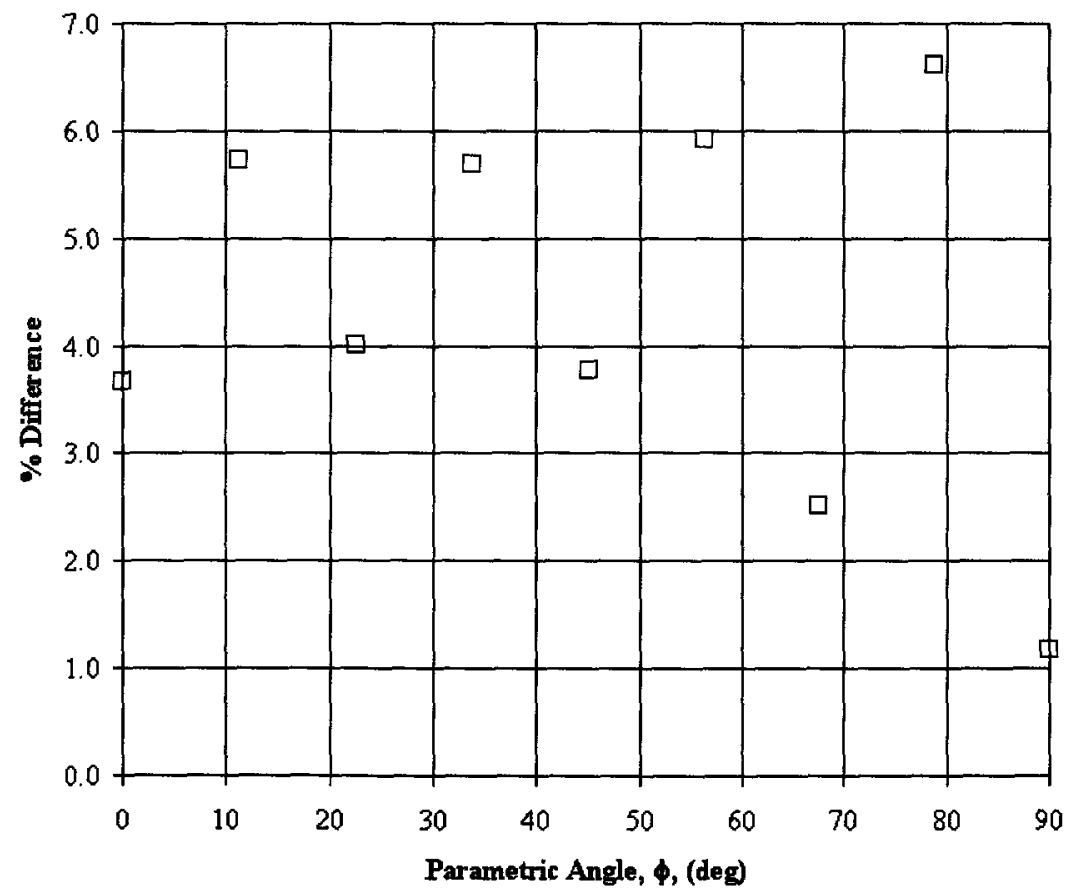

Figure 8.23 - Comparison of differences in boundary correction factors for an $a / c=1.0, c=$ $0.4 \mathrm{~mm}$ crack in the SEN specimen used in this investigation (ABAQUS), and results reported by Beres and Koul for a DEN specimen [269] 


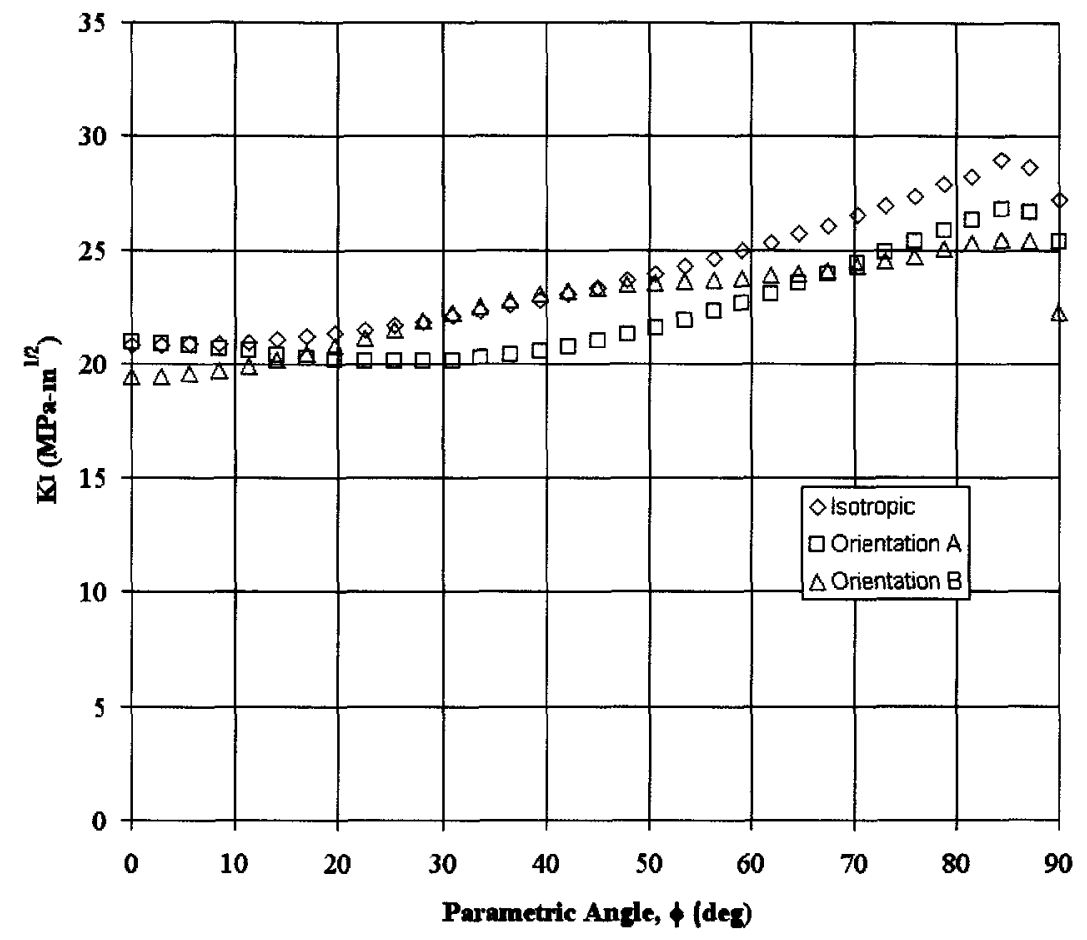

Figure 8.24 - Absolute values of the stress-intensity factor for the SEN specimen for $c=0.2$ $\mathrm{mm} a / c=0.86$ crack.

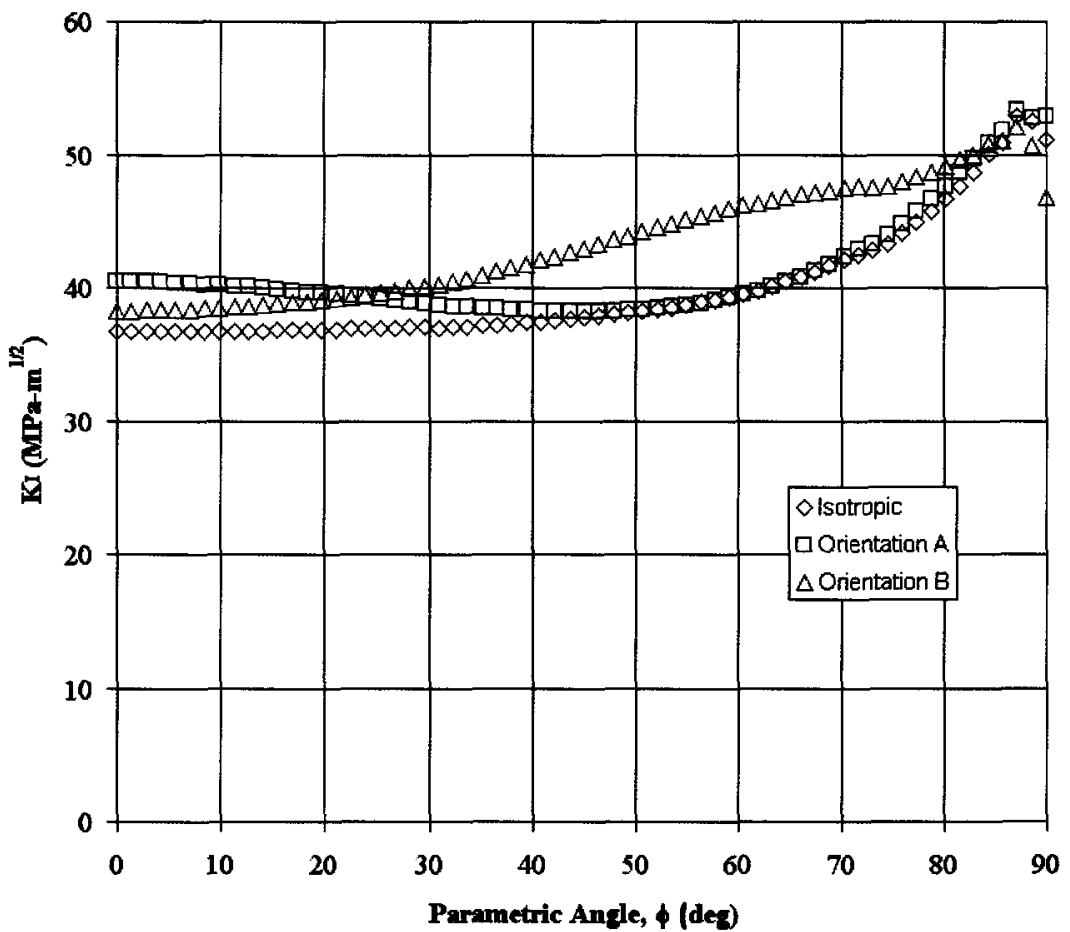

Figure 8.25 - Absolute values of the stress-intensity factor for the SEN specimen for $c=1.0$ mm $a / c=1.35$ crack. 


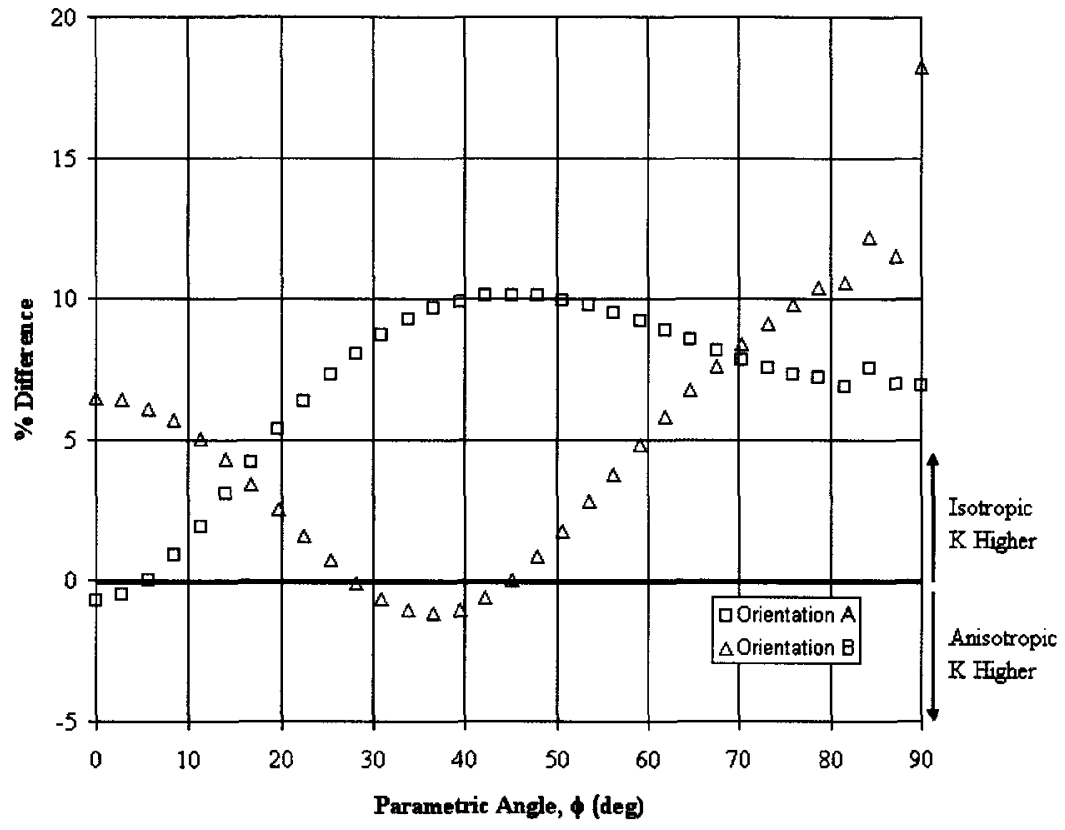

Figure 8.26 - Comparison of differences between the isotropic and anisotropic stressintensity factors for orientations $A$ and $B$ and $c=0.2 \mathrm{~mm} a / c=0.86$ crack.

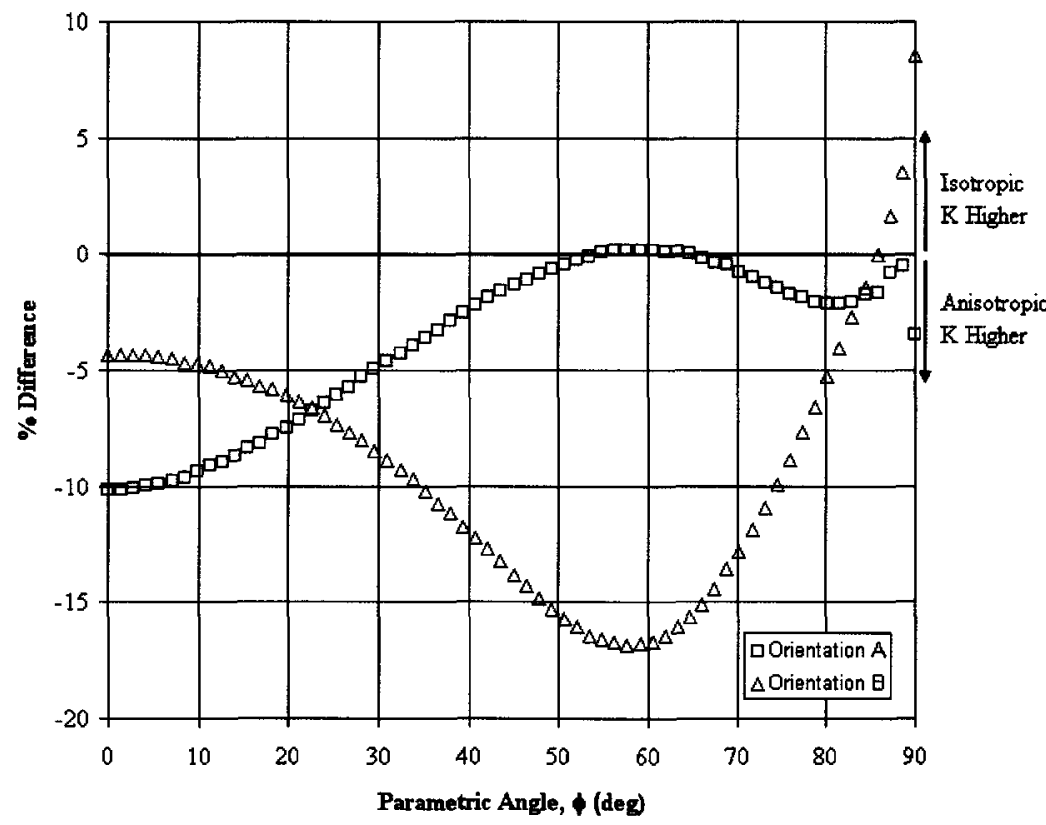

Figure 8.27 - Comparison of differences between the isotropic and anisotropic stressintensity factors for orientations $A$ and $B$ for $c=1.0 \mathrm{~mm} a / c=1.35$ crack. 


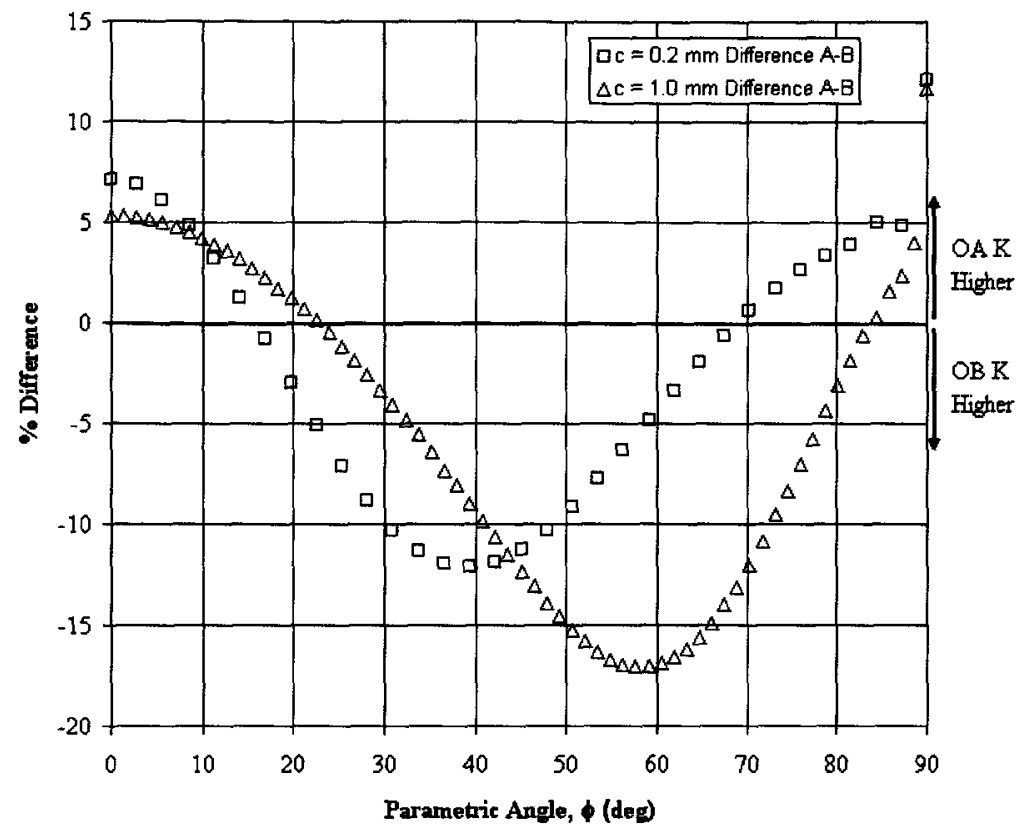

Figure 8.28 - Comparison of differences between orientations $A$ and $B$ anisotropic stressintensity factors for $c=0.2 \mathrm{~mm} a / c=0.86$ and $c=1.0 \mathrm{~mm} a / c=1.35$ crack geometries.

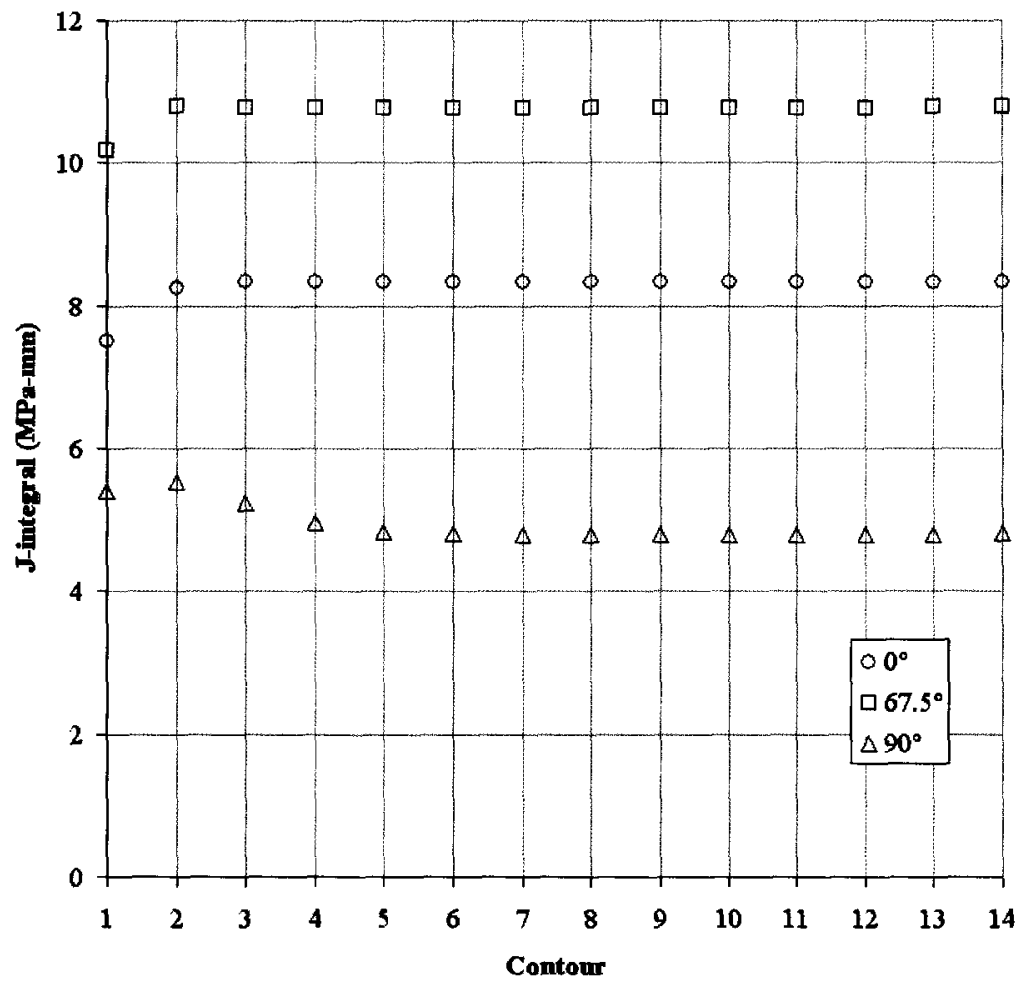

Figure 8.29 - Path dependence of the J-integral evaluated from different crack front contours at the deepest part of the crack $\left(\phi=0^{\circ}\right)$, the notch surface $\left(\phi=90^{\circ}\right)$ and the crack front position where the calculated J-integral was highest $\left(\phi=67.75^{\circ}\right)$. 


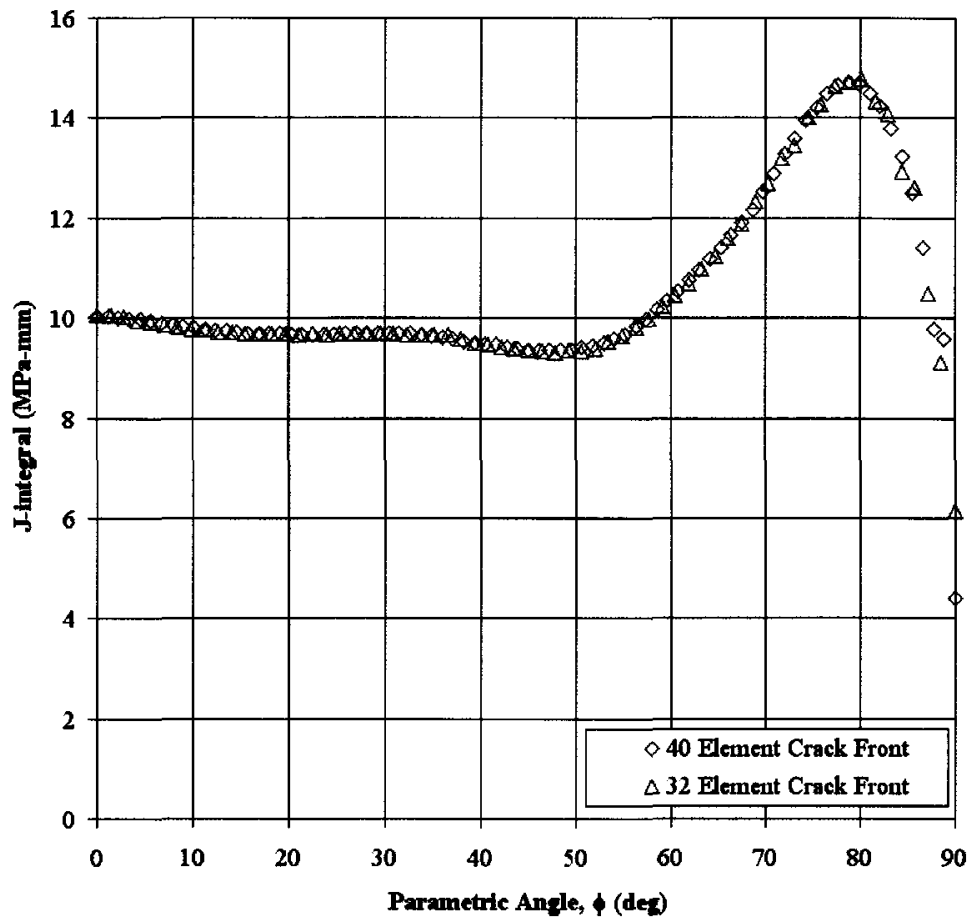

Figure 8.30 - Effect of crack-front mesh refinement on the J-integral for $c=1.0 \mathrm{~mm} a / c=$ 1.35 crack.

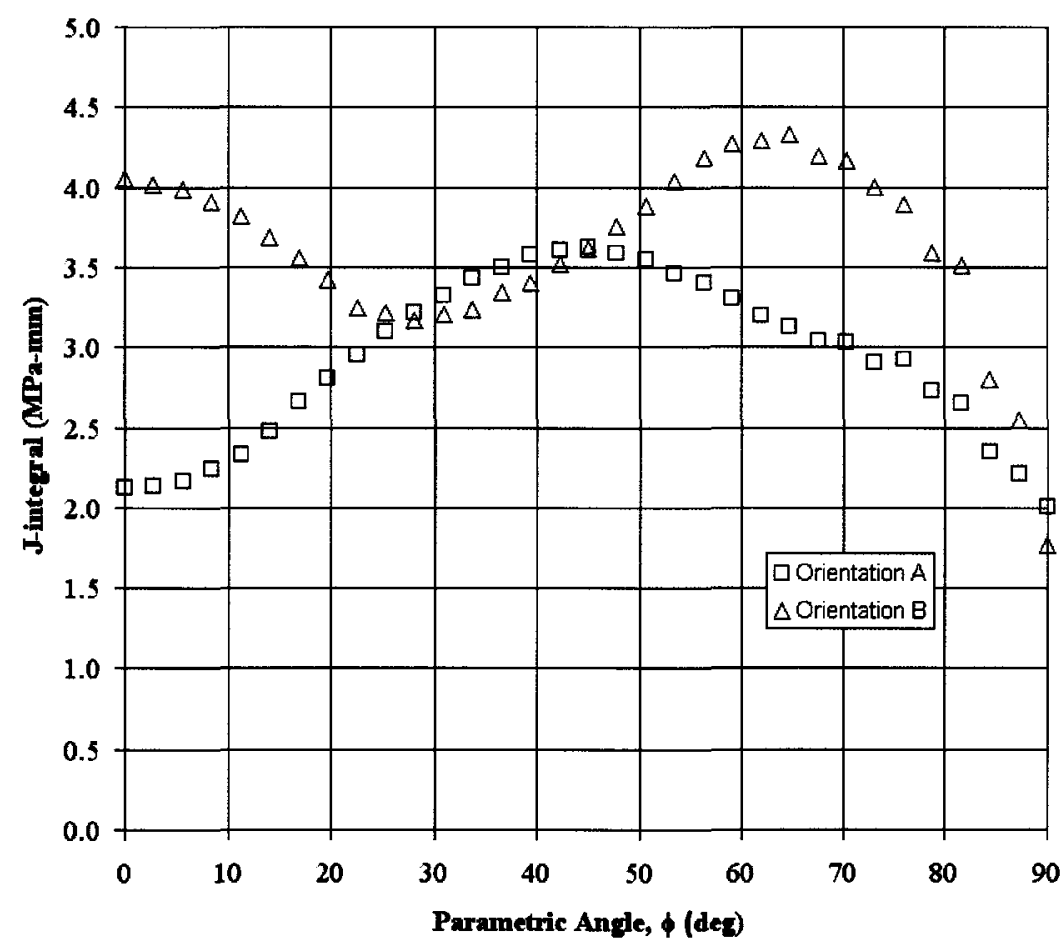

Figure 8.31 - J-integral variation along the crack front for $c=0.2 \mathrm{~mm} a / c=0.86$ crack. 


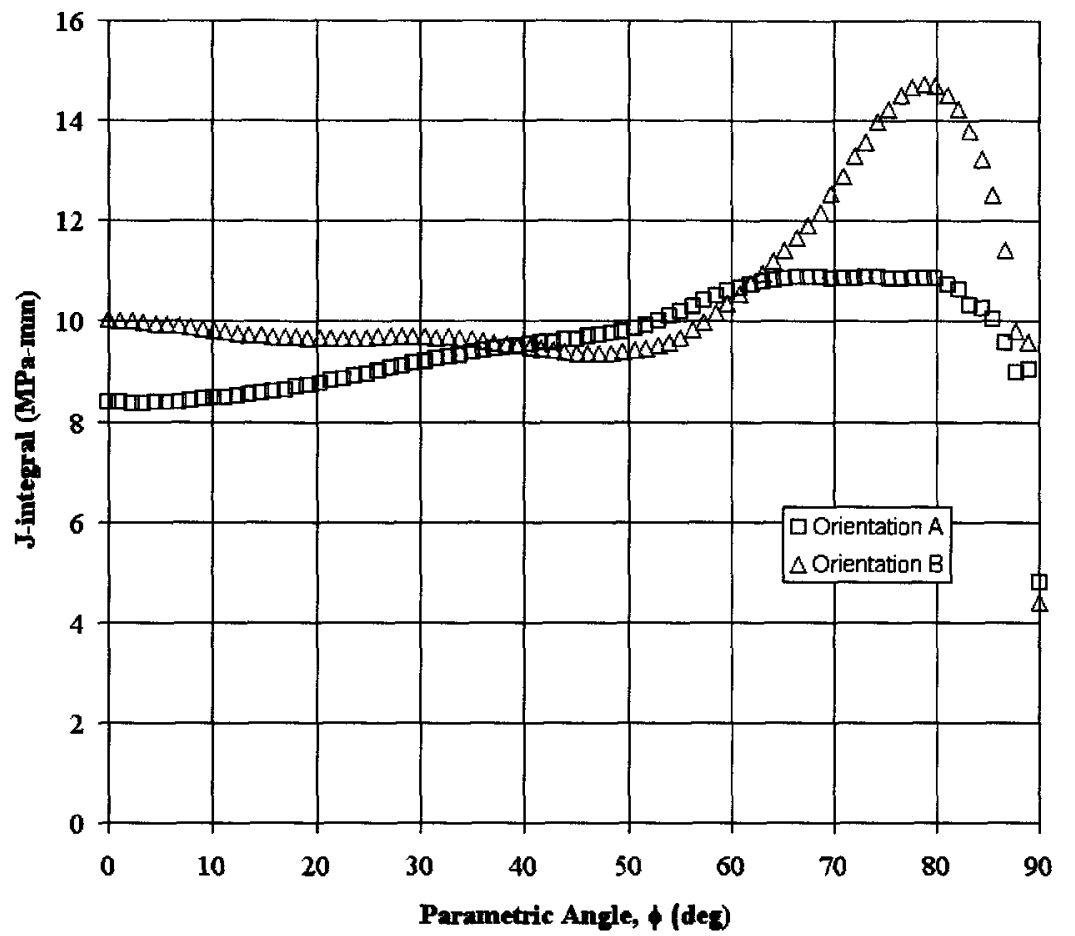

Figure 8.32 - J-integral variation along the crack front for $c=1.0 \mathrm{~mm} a / c=1.35 \mathrm{crack}$.

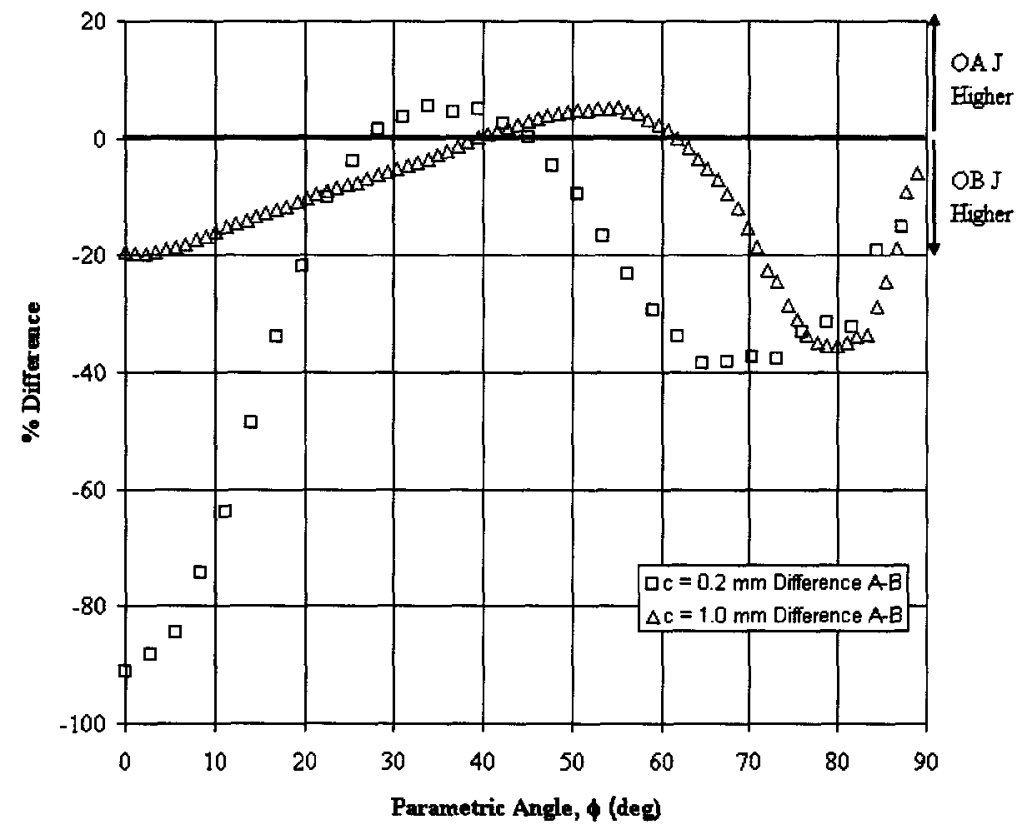

Figure 8.33 - Comparison of differences between orientation $A$ and $B$ anisotropic elasticplastic J-integral values for $c=0.2 \mathrm{~mm} a / c=0.86$ and $c=1.0 \mathrm{~mm} a / c=1.35$ crack geometries. 

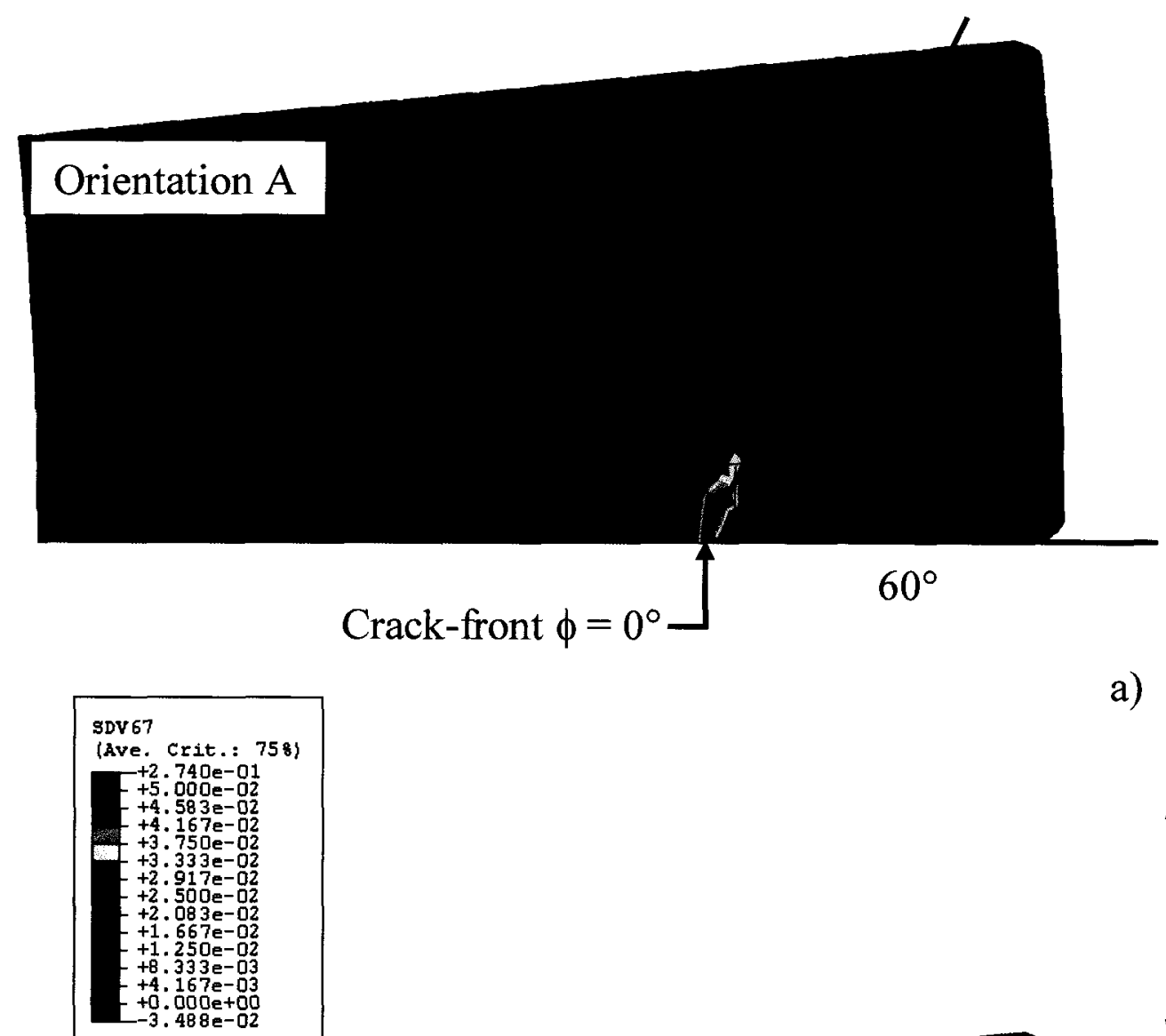

a)

Orientation B

$$
\text { Crack-front } \phi=0^{\circ} \int^{\circ}
$$

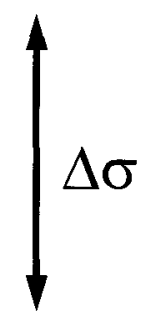

b)

Figure 8.34 - Contour plot showing equivalent octahedral shear strain slip fields at the crack-tip ( $\phi=0^{\circ}$ crack-front position) for $c=0.2 \mathrm{~mm} a / c=0.86$ crack; a) orientation $A$ and b) orientation $B$. Note: the approximate slip-sector angles are indicated on the contour plots. 

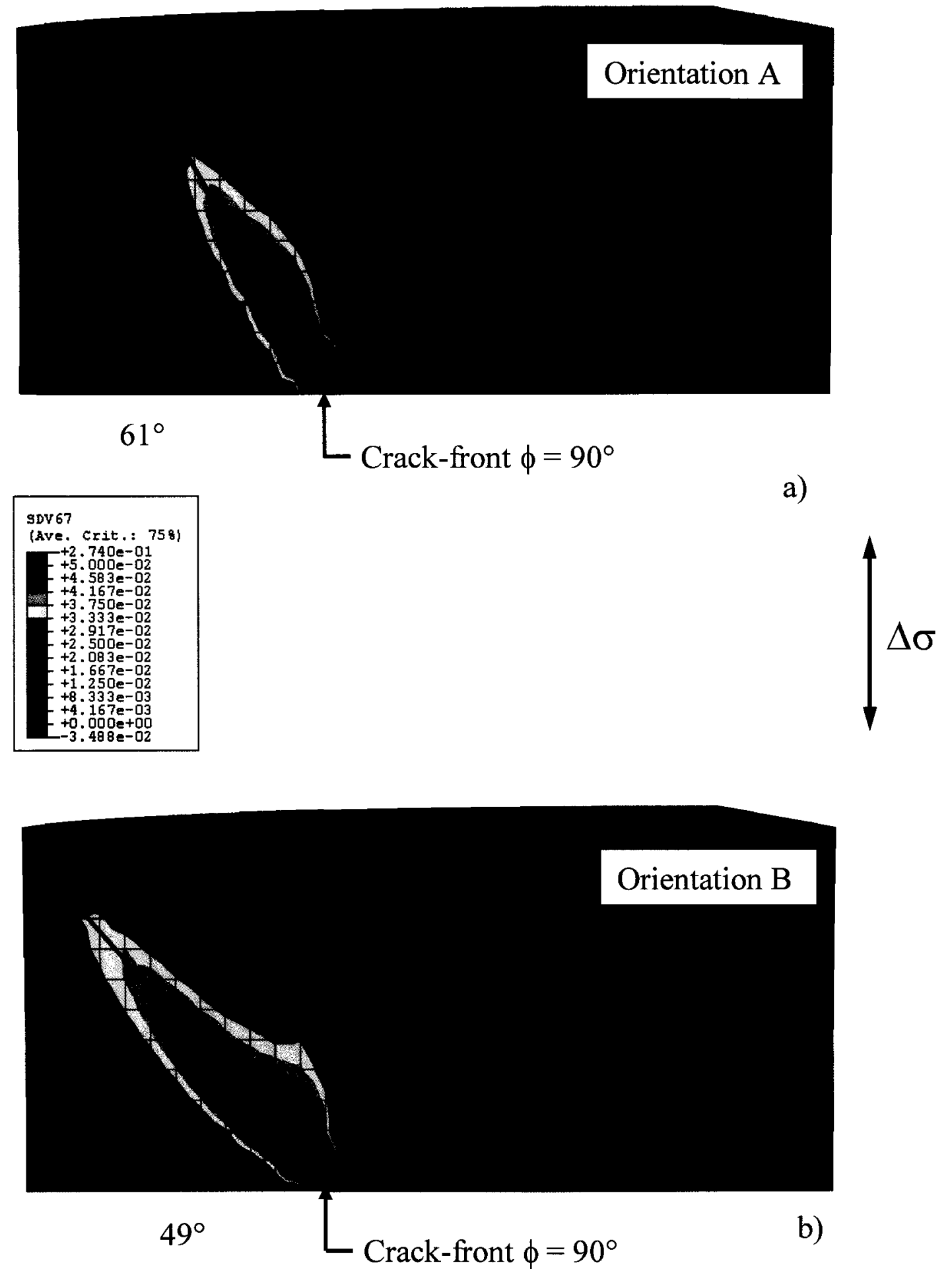

Figure 8.35 - Contour plot showing equivalent octahedral shear strain slip fields at the crack-tip ( $\phi=90^{\circ}$ crack-front position) for $c=0.2 \mathrm{~mm} a / c=0.86 \mathrm{crack}$; a) orientation $A$ and b) orientation $B$. Note: the approximate slip-sector angles are indicated on the contour plots. 


\section{Orientation A}

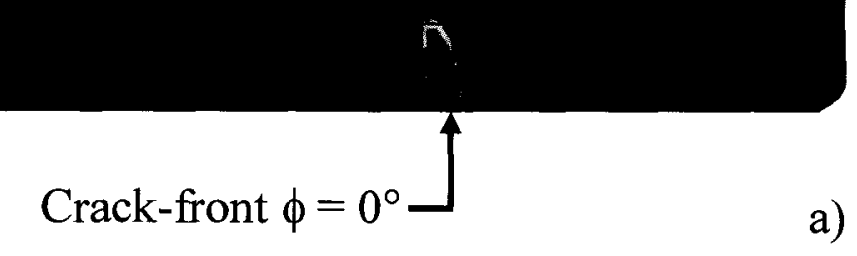

\begin{tabular}{|c|}
\hline sDv68 \\
(Ave. crit.: 758 ) \\
$+3.295 \mathrm{e}-01$ \\
$+5.000 \mathrm{e}-02$ \\
$+4.593 \mathrm{e}-02$ \\
$+4.167 \mathrm{e}-02$ \\
$+3.750 \mathrm{e}-02$ \\
$+3.333 \mathrm{e}-02$ \\
$+2.917 \mathrm{e}-02$ \\
$+2.500 \mathrm{e}-02$ \\
$+2.083 \mathrm{e}-02$ \\
$+1.667 \mathrm{e}-02$ \\
$+1.250 \mathrm{e}-02$ \\
$+8.333 \mathrm{e}-03$ \\
$+4.167 \mathrm{e}-03$ \\
$+0.000 \mathrm{e}+00$ \\
$-5.167 \mathrm{e}-02$ \\
\hline
\end{tabular}

\section{Orientation B}

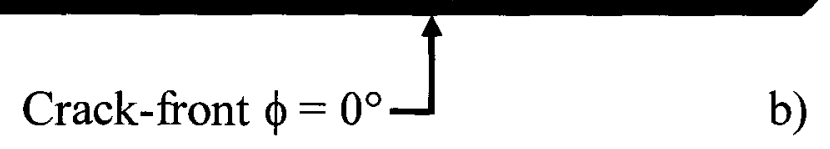

Figure 8.36 - Contour plot showing equivalent cubic shear strain slip fields at the crack-tip ( $\phi=0^{\circ}$ crack-front position) for $c=0.2 \mathrm{~mm} a / c=0.86 \mathrm{crack}$; a) orientation $A$ and b) orientation $B$. 


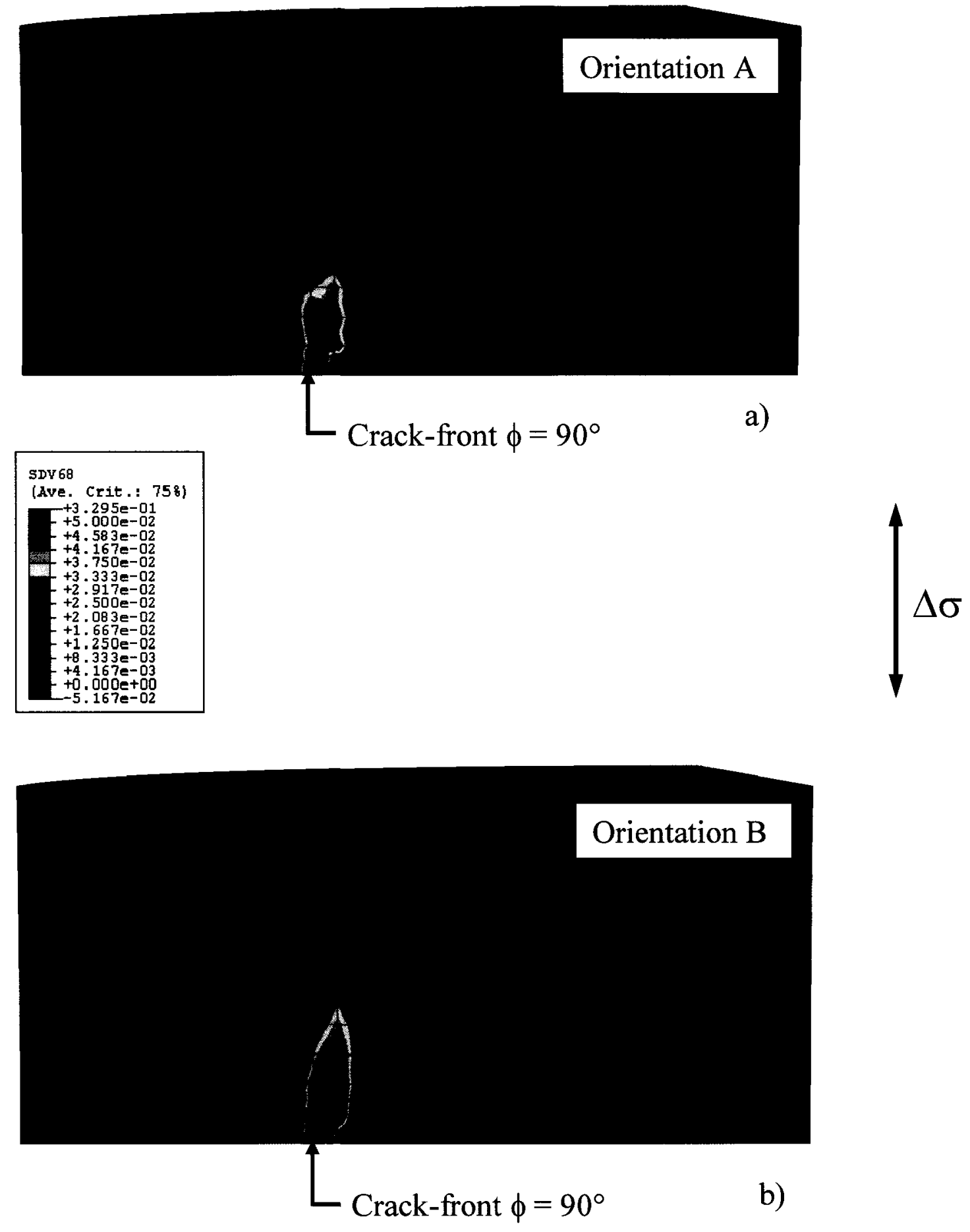

Figure 8.37 - Contour plot showing equivalent cubic shear strain slip fields at the crack-tip $\left(\phi=90^{\circ}\right.$ crack-front position) for $c=0.2 \mathrm{~mm} a / c=0.86$ crack; a) orientation $A$ and b) orientation $B$. 


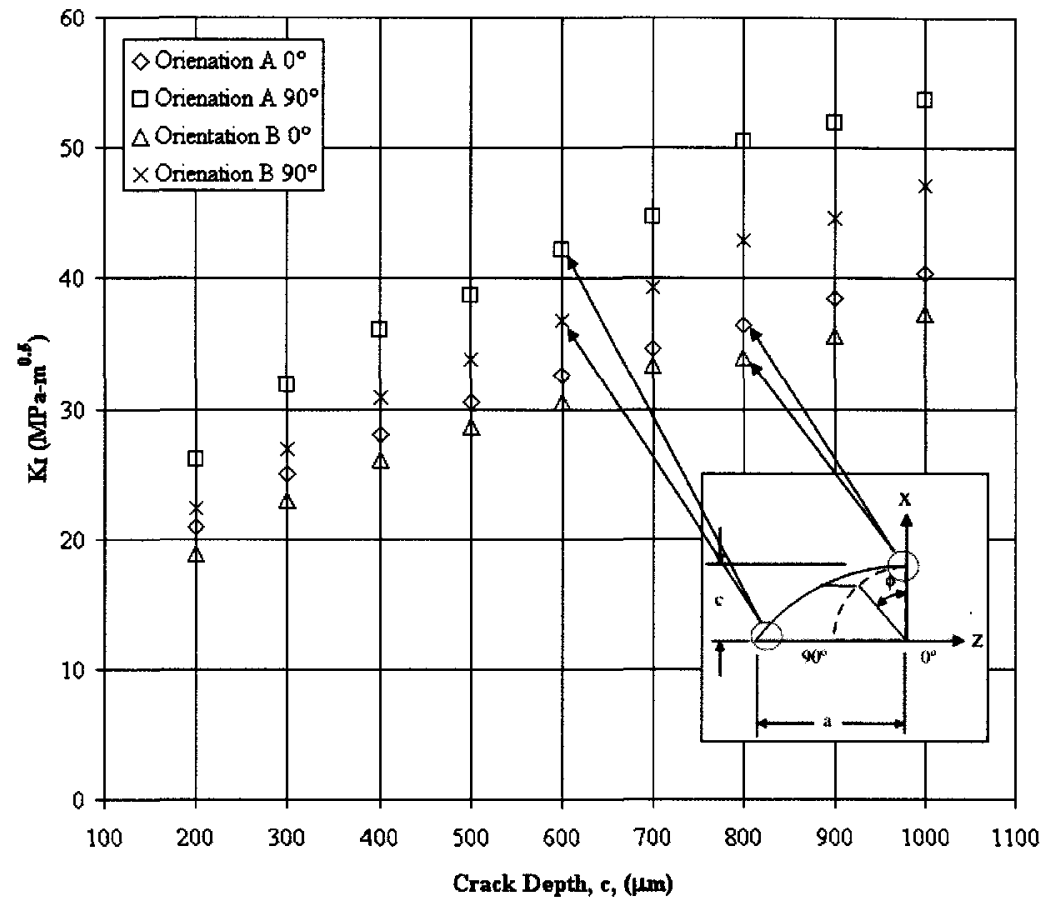

Figure 8.38 - Variation in the absolute anisotropic stress-intensity factor at the deepest part of the crack $\left(\phi=0^{\circ}\right)$ and the notch surface $\left(\phi=90^{\circ}\right)$ as function of crack depth for orientation $A$ and $B$.

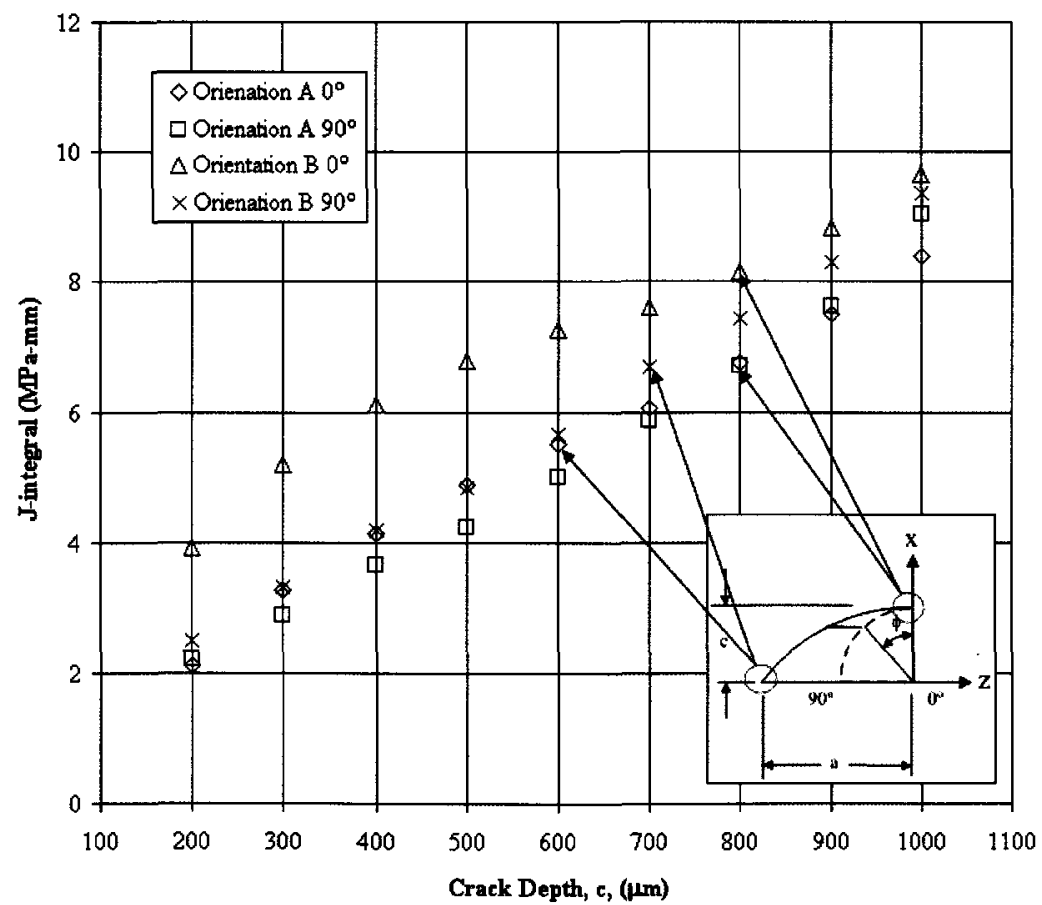

Figure 8.39 - Variation in the J-integral at the deepest part of the crack $\left(\phi=0^{\circ}\right)$ and the notch surface $\left(\phi=90^{\circ}\right)$ as function of crack depth for orientation $A$ and $B$. 


\section{Fractographic Analysis of Fatigue Crack Growth Rate Specimens - Results and Discussion}

\subsection{Introduction}

Detailed fractographic analysis of the FCGR specimens was undertaken using optical and scanning electron microscopy techniques to identify fatigue crack nucleation sites and to study the microscopic aspects of FCG. The optical microscopy study mainly focused on characterizing the angular position and location of the dominant fatigue cracks in the notch root. The SEM analyses were used to identify the crack nucleation sites, their proximity to the notch root, and to characterize the microscopic features on the fracture surfaces at different stages of FCG. In the following sections, the typical results of these analyses are presented and discussed.

\subsection{Fractographic Observations and Discussion}

This investigation concentrated on the effect of secondary crystallographic orientation on the formation of naturally generated fatigue cracks and their subsequent propagation as small fatigue cracks in notched fatigue specimens. The fatigue experiments were performed under identical loading and environmental conditions and were terminated after a fatigue crack approximately $1000 \mu \mathrm{m}$ deep $(c \sim 1000 \mu \mathrm{m})$ formed in the notch root. After interrupting the fatigue tests, the specimens were removed from the test machine for examination using optical and scanning electron microscopy to determine the location and crack path of the dominant fatigue crack within the specimen notch and to observe the fracture mode.

Representative crack paths observed at the notch surface for orientation A and B SEN specimens are shown in Figure 9.1. Macroscopically, the crack paths were approximately 
perpendicular to the applied loading direction throughout the entire crack size range considered in this investigation. This finding validates the treatment of small fatigue cracks as Mode I cracks as was assumed in the FEM calculations conducted in this investigation. Some crystallographic crack growth features are apparent at the notch surface for both orientations, but in general, the notch surface crack paths for orientation A specimens appeared to be smoother on a macroscopic scale than those observed in orientation B specimens. The notch surface crack paths for orientation B fatigue specimens were observed to have more extensive crystallographic facet features, particularly near the crack tips, which indicate a transition from Stage II to Stage I crack growth occurs at higher stress-intensities.

Defresne and Remy [33] have reported that crack paths for through-thickness short fatigue cracks in CMSX-2 tested under similar experimental conditions to those considered in this investigation were macroscopically smoother for orientation A than orientation B fatigue specimens. This agrees with the present findings. As discussed in Chapter 3, Stage II crack growth predominantly occurs under conditions that are conducive to slip dispersal, i.e. high temperatures and lower testing frequencies, environmental effects such as oxidation and state of stress. Since the temperature, testing frequency and environment were identical for each of the fatigue experiments in this investigation, the stress state is one factor that could promote the Stage II to Stage I crack propagation mechanism transition observed in the orientation B SEN specimens. Various researchers $[24,28,29,52,161]$ have proposed the resolved normal and shear stress intensity factors or the ratio of these two factors [52] to define the conditions under which transitions from Stage II to Stage I crack propagation mechanism occur. The resolved normal and shear stress intensity factors for octahedral slip have different values in orientation A and B specimens since the octahedral planes, as shown in Figure 5.25, are 
oriented differently. Analyses conducted by Reed and coworkers [52] have shown that the resolved shear stress and normal stress parameters are higher in orientation B than orientation A specimens. This could promote the Stage II to Stage I crack growth transition observed in the orientation B SEN specimens utilized in this investigation.

One additional factor that could contribute to the Stage II to Stage I crack propagation mechanism transition observed in orientation B fatigue specimens is differences in the transverse stiffness. The apparent elastic modulus for orientation A can be determined using equation 6.36 and 6.37 (with $\theta=45^{\circ}$ and $\psi=0^{\circ}$ ) and the compliance coefficients summarized in Table 6.1. The apparent elastic modulus in the crack growth direction for orientation A is $223 \mathrm{GPa}$ and for orientation B is $120 \mathrm{GPa}$. The relative differences in transverse stiffness between orientation A and B specimens could be important for the shear band decohesion mechanism which promotes the transition from Stage II to Stage I crack growth.

The anisotropic elastic-plastic FEM calculations performed in this investigation utilized a constitutive model formulation based on crystallographic slip. Since the elastic-plastic constitutive model was formulated based on a crystallographic slip framework, it does conceptually embody some aspects of physical deformation processes in single-crystal superalloys. One interesting observation from the FEM simulation results was that slip occurred in concentrated slip sectors, which are consistent with that of both octahedral and cubic slip. Therefore, in the presence of a stress-raising feature, the crystallographic slip constitutive model predicts inhomogeneous slip phenomena. Interestingly, some physical evidence of this localized deformation was observed when examining the crack path in the notch root. As shown in Figure 9.2, $\{111\}$ slip band traces were also apparent on the notch surface. The slip band traces were aligned with the crystallographic facets in 
orientation B specimens (Figure 9.2 b) but were perpendicular to the applied load in orientation A specimens (Figure 9.2 a). These slip band traces were generally observed near the crack-tips indicating that higher stress-intensities are required for the slip bands to be apparent at the macroscale. Moreover, the FEM results indicated the J-integral and the extent and magnitude of the equivalent octahedral slip at the crack tip was higher for orientation B than orientation A specimens. This finding at least qualitatively suggests the Stage II to Stage I crack growth mechanism transition could occur more readily in orientation B than orientation A fatigue specimens. It does not, however, quantitatively establish the conditions under which this transition occurs.

During the process to examine the dominant notch root crack and to characterize its crack path, several small secondary fatigue cracks were also observed within the specimen notch root. Representative examples of these observed cracks are shown in Figure 9.3. The secondary fatigue crack shown in Figure 9.3a nucleated from a surface connected micropore while Figure $9.3 \mathrm{~b}$ appears to subsurface in origin. The small size of the secondary fatigue cracks indicates that they either formed early in life and arrested, or they formed much later than the dominant fatigue crack and grew to a limited size prior to test interruption. It addition to observing secondary fatigue cracks it was also observed that undissolved eutectic $\gamma-\gamma^{\prime}$ was more heavily oxidized than the surrounding $\gamma-\gamma^{\prime}$ matrix material.

Defresne and Remy [48] used replication techniques to study the formation of notch root fatigue cracks in CMSX-2 tested under similar experimental conditions to those considered in this investigation, but they tested their fatigue specimens at different stress amplitudes. For fatigue tests performed at higher stress amplitudes, they reported that fatigue cracks were detectable in surface replicas after $1 \%$ of fatigue life from surface 
connected pores and that a small number of cycles were necessary to propagate cracks originating from subsurface defects until they were apparent on the notch surface. Their finding suggests the incubation phase for notch root fatigue cracks represents an insignificant portion of the fatigue life and the majority of fatigue life is spent propagating small fatigue cracks from these material discontinuities. Based on their findings it is speculated that the secondary fatigue cracks observed in the present study formed early in life and arrested. However, additional fatigue tests employing an interrupted testing procedure to periodically obtain surface replicas would be necessary to establish when the secondary fatigue cracks formed in the present study. Since the secondary cracks were observed to be physically small, limited in number, and below the crack detection resolution capabilities of the ACPD equipment, they could not be unambiguoulsy detected from the recorded ACPD signal associated with the dominant fatigue crack.

In addition to examining the macroscopic features of the fatigue cracks in each SEN specimen, optical microscopy and image analysis techniques were used to quantitatively determine the position of the fatigue crack in the specimen notch root. The position of the fatigue crack in the notch root was characterized by two parameters, $\theta_{c}$ and $l_{c}$, which represent the angle from the minimum specimen cross section and the distance from the centerline of the specimen to the mid-point of the surface crack as depicted in Figure 9.4. The results of this investigation are summarized in Table 9.1. In general, the majority of fatigue cracks were located within $15-25^{\circ}$ of the notch root, which appears to agree quite well with the maximum principal stress location identified from the elastic-plastic finite element notch stress results presented in the Chapter 8 (Figures $8.7-8.9$ ). This finding also agrees with Meyer and colleagues' [209] investigation of fatigue crack growth in PWA1480 DEN specimens with $\langle 001\rangle$ primary orientation and $\langle 100\rangle$ and $\langle 210\rangle$ notch 
orientations. They reported that fatigue cracks predominantly formed on a plane that was approximately 22 to $30^{\circ}$ from the minimum specimen cross-section.

The elastic-plastic finite element simulation results for notch root stress fields indicated that the maximum principal stress coincides with the centerline of the SEN specimen, i.e. symmetry plane A-A in Figure 7.2. Naturally, it is intuitive to assume that fatigue cracks will predominantly nucleate at the centerline of the specimen, i.e. $l_{c}=0 \mathrm{~mm}$; however, close examination of the measurements summarized in Table 9.1 indicates that this is not the case. The position of the cracks from the center line of the specimen cannot be rationalized from principal stress distribution predicted by the elastic-plastic FEM simulations. This finding suggests that location where the fatigue crack nucleates depends on factors in addition to the notch root stresses. One potential explanation for this finding is that fatigue crack formation can occur from surface connected and subsurface material discontinuities, the size and distribution of which is stochastic in nature, and therefore fatigue crack formation depends not only the notch-root stress and strain fields but also the characteristics of the material discontinuities. A more detailed investigation of the specimen fracture surfaces was undertaken to identify both the crack nucleation sites associated with the dominant fatigue crack and their characteristics.

After examining the notch surface to characterize the dominant fatigue crack $(c \sim 1000$ $\mu \mathrm{m})$, the fatigue specimens were cycled to fracture to examine and characterize the fracture surface using scanning electron microscopy. The fracture surface shown in Figure 9.5a is representative of the majority of fatigue specimens, that is a single semielliptical crack originating from a single crack nucleation site. Defresne and Remy [33] have reported similar findings for naturally generated fatigue cracks in CMSX-2. Two fatigue specimens were observed to contain a single dominant fatigue crack formed by 
coalescence of two fatigue cracks on different planes and with separate crack nucleation sites (Figure 9.5b). In general, the fracture surfaces were macroscopically planar but some crack bifurcation was clearly evident on the fracture surfaces of both orientation A and B specimens. The occurrence of crack bifurcation becomes more apparent at higher stress-intensity factor ranges as shown in Figure 9.6.

Representative crack nucleation sites are shown in Figures 9.7a and 9.7b for orientation A and B specimens, respectively. In general, fatigue cracks nucleated from one or two subsurface pores and the relative size and depth of the pores from the notch surface was different in each fatigue specimen. The subsurface crack nucleation sites are surrounded by a circular region that intersects with the surface of the notch, which indicates the extent of subsurface crack growth that occurs in a vacuum environment. Once the subsurface crack breaks through the notch surfaces, it grows into a semi-elliptical shape and propagates in a laboratory air environment. Similar observations have been reported for subsurface fatigue crack formation in single-crystal superalloys [33, 34, 48, 117, 277]. Unlike the anisotropic subsurface fatigue crack growth Anton [117] observed in an experimental single-crystal superalloy, the circular subsurface crack growth region indicates that crack growth occurs equally at all positions along the crack front, i.e. there is no secondary orientation dependence of fatigue crack growth for subsurface fatigue cracks that grow macroscopically on $\{100\}$ crystallographic planes. This is in contrast to the marked secondary orientation dependence of fatigue crack growth under vacuum reported by Joyce and coworkers [216] for through-thickness fatigue cracks.

Close examination of Figure 9.7 indicates that the circular region delineating the extent of subsurface crack growth does not appear to be symmetrical around the subsurface pore. Since the extent of the subsurface crack growth region was small and confined within the 
plastically deformed region in the notch root, stress gradient effects would have only a small influence on the crack shape development and thus crack growth via expansion of these defects should be symmetrical. It may be noted, however, that undissolved interdendritic eutectic $\gamma-\gamma^{\prime}$ was observed adjacent to the subsurface pores, as shown in Figure 9.7, and the eutectic phase was approximately at the center of the circular subsurface crack growth region. Therefore, it is apparent that $\gamma-\gamma^{\prime}$ eutectic to some extent affects small fatigue crack growth.

Unlike the $\gamma-\gamma$ ' eutectic-matrix decohesion mechanism reported by Deluca and Annis [7] for PWA1480 single crystals tested at low temperatures, it is apparent that small fatigue cracks propagated through the $\gamma-\gamma^{\prime}$ eutectic phase in the specimens of this study. It is speculated that fatigue cracks propagate faster through $\gamma-\gamma$ ' eutectic because the microstructural characteristics of the $\gamma-\gamma$ ' eutectic phase are different than the surrounding $\gamma-\gamma^{\prime}$ matrix material. Although this suggests that an asymmetric crack shape should develop rather than the observed circular shape of the subsurface crack growth region, it is possible that the crack growth kinetics through $\gamma-\gamma^{\prime}$ eutectic are substantially faster than the nominal $\gamma-\gamma^{\prime}$ matrix material. This would in effect promote uniform crack growth in the surrounding $\gamma-\gamma^{\prime}$ matrix material. If this was the case it is unclear if the $\gamma-\gamma^{\prime}$ eutectic should be treated as a discontinuity with the same crack formation potency as subsurface pores. Because of the small size of the $\gamma-\gamma^{\prime}$ eutectic and its heterogeneous distribution it would indeed be a difficult task to measure the crack growth kinetics in $\gamma-\gamma^{\prime}$ eutectic to resolve this issue.

In addition to conducting a general characterization of the crack nucleation sites, quantitative measurements of the size and distance of these discontinuities from the notch 
root were obtained. The size of the subsurface pores was characterized by two dimensions, $2 c$ and $2 a$, which represent the pore diameter parallel to the notch and the crack growth direction, respectively. The distance from the center of the subsurface pore to the notch surface was characterized by dimension, $d_{\mathrm{p}}$. The results obtained from this investigation are summarized in Table 9.2. It may be noted that two fatigue specimens were observed to have multiple (two) subsurface nucleation sites; the relevant dimensions for both subsurface pores are reported in Table 9.2.

A more detailed examination of the specimen fracture surfaces was undertaken using scanning electron microscopy to elucidate the micromechanisms of fatigue growth and to establish if the fatigue crack propagation mechanisms were dependent on secondary crystallographic orientation. Representative micrographs are shown in Figures 9.8 and 9.9 for orientations $\mathrm{A}$ and $\mathrm{B}$, respectively. In the subsurface crack growth region the fracture surface is microscopically flat and no crystallographic facets are apparent. Although some of the details in this region are obscured by heavy oxidation, it is speculated that the crack path deviates around the primary $\gamma^{\prime}$ precipitates resulting in $\{100\}$ planar crack growth within the $\gamma$ matrix. The fracture surface becomes progressively rougher at the microscopic scale at higher stress-intensity factor ranges but the crack path is identical to that observed in the subsurface crack growth region. It is also more apparent that the crack path deviates around the $\gamma^{\prime}$ precipitates as indicated by the series of cube faces on the fracture surface which are approximately the same size as the $\gamma^{\prime}$ precipitates (see Figure 9.9). At the microscopic scale the fatigue crack propagation mechanisms appear to be similar for orientation A and B fatigue specimens.

The predominantly non-faceted crack growth observed in the present work is consistent with the non-crystallographic crack growth reported by Defresne and Remy [33] for 
naturally-generated small fatigue cracks in CMSX-2 single crystals. This does not preclude the possibility that the non faceted fracture surface was faceted at a very fine scale. Crompton and Martin [219] have shown that macroscopic (001) crack growth observed in Mar-M002 single-crystal specimens consisted of microscopic (111) facets approximately $3 \mu \mathrm{m}$ in size. However, microscopic facets of this size would have been apparent on the specimen fracture surface during the detailed SEM examinations. The fracture surfaces mainly consisted of cubic features, which were generally consistent with the size of the $\gamma^{\prime}$ precipitates. This finding suggests that crack growth occurs by another mechanism than microscopic Stage I crack growth.

Antolovich and colleagues [13] have proposed a mechanism to explain the noncrystallographic crack growth observed in single-crystal superalloys at elevated temperatures. They proposed that fatigue cracks which exhibit a nominal $\{100\}$ crack plane occurs by crack growth on a combination of $\{100\}$ and $\{111\}$ type crystallographic planes at the microscopic scale. Dislocation motion resulting from cyclic loading causes damage to accumulate ahead of the advancing fatigue crack on several adjacent $\{111\}$ crystallographic planes between the $\gamma^{\prime}$ precipitates, leading to the formation of microcracks in the $\gamma$ channels. These microcracks arrest when they encounter $\gamma^{\prime}$ precipitates, which have much higher strength than the $\gamma$ matrix at intermediate temperatures. Additional cyclic loading results in a build-up of dislocation density at the $\gamma^{\prime}$ precipitate causing damage to the $\gamma-\gamma^{\prime}$ interface. Once a critical amount of damage is accumulated at the $\gamma-\gamma^{\prime}$ interface, the crack then propagates around the $\gamma^{\prime}$ precipitate and continues propagating along $\{111\}$ crystallographic planes in the $\gamma$ matrix until the next $\gamma$ ' precipitate is encountered. The formation of secondary cracks, which were observed in the present investigation, are explained by this same mechanism. 
The non-crystallographic crack growth mechanism proposed by Antolovich and coworkers [13] could also be environmentally assisted due to differences in the oxidation characteristics of the $\gamma$ matrix and $\gamma^{\prime}$ precipitates. Pieraggi and Dabosi [278] have reported the $\gamma$-matrix oxidizes faster than $\gamma^{\prime}$ in the single-crystal superalloy CMSX-2. Defresne and Remy [33] have suggested that the increased oxidation kinetics of the $\gamma$ matrix could cause oxidation embrittlement, which would assist fatigue crack propagation along the $\{100\} \gamma$ matrix channels in air. The increased dislocation density in the $\gamma$ matrix and at the $\gamma-\gamma$ ' interfaces would promote short-circuit diffusion enhancing this oxidation embrittlement effect. In addition, oxidation at the crack-tip reduces slip reversibility promoting the activation of additional slip systems at the crack tip which suppress the formation of extended planar slip bands that cause the transition from Stage II to Stage I crack growth. Additional constraint imposed by the small notch-root cracks considered in the present investigation, which are more severe than for through-thickness fatigue cracks, could also contribute to suppression of the Stage II to Stage I crack growth mode transition and promote the $\gamma$ phase decohesion crack propagation mechanism.

\subsection{Concluding Remarks}

Fractographic examinations were conducted on the SEN fatigue specimens tested in this investigation to characterize the dominant fatigue crack position within the notch root and crack path on the notch surface, identify the crack nucleation site(s) and to identify the micromechanisms of fatigue crack propagation. In general, most fatigue cracks were observed to coincide with $\{100\}$ planes that were located at an angular position ranging from $15-25^{\circ}$ from the minimum specimen cross section. This finding provides strong confirmation of the critical (high stress) region identified by the elastic-plastic finite 
element simulation results presented in Chapter 9 . Some variability was observed in the crack position in the notch root that cannot be accounted for based on the elastic-plastic FEM results. The fatigue crack paths along the notch root were macroscopically planar but orientation B exhibited more crystallographic facetted features than orientation A. The dominant fatigue crack was usually accompanied by several smaller secondary fatigue cracks that appeared to nucleate from subsurface and surface-connected interdendritic porosity.

Small fatigue cracks predominantly nucleated from subsurface interdendritic pores and the relative size and depth of the pores from the notch surface was different in each fatigue specimen. Initial crack growth occurred by expansion of these material discontinuities into a circular shape until the subsurface crack reached the notch surface after which the fatigue cracks developed into semi-elliptical surface cracks. The semielliptical cracks were macroscopically planar and propagated as Stage II noncrystallographic cracks on a plane normal to the applied loading. Fatigue crack propagation at the microscale involved crack propagation along the $\{100\}$ crystallographic $\gamma$ matrix channels as indicated by the presence of cube faces on the fracture surface which are approximately the same size as the $\gamma^{\prime}$ precipitates. There were no apparent differences in the fatigue crack propagation mechanisms at the microscale for orientation A and B SEN specimens. 
Table 9.1 - Crack positions in the specimen notch. Note: $\theta_{\mathrm{c}}$ represents the angular position from the minimum specimen cross section (the notch root) and $l_{c}$ represents the distance from the center line of the specimen to the mid-point of the surface crack.

\begin{tabular}{|c|c|c|c|}
\hline Orientation & Specimen ID & $\theta_{\mathrm{c}}(\mathrm{deg})$ & $\mathrm{l}_{\mathrm{c}}(\mathrm{mm})$ \\
\hline \multirow{4}{*}{$\mathrm{A}$} & $39-2$ & 3 & 0.15 \\
\cline { 2 - 4 } & $40-1$ & 17 & 0.38 \\
\cline { 2 - 4 } & $41-1$ & 21 & 1.50 \\
\cline { 2 - 4 } & $41-2$ & 23 & 1.41 \\
\cline { 2 - 4 } & $42-2$ & 17 & 0.32 \\
\hline & $44-2$ & 11 & 0.55 \\
\hline \multirow{4}{*}{ B } & $22-1$ & 25 & 0.84 \\
\cline { 2 - 4 } & $23-1$ & 4 & 1.62 \\
\cline { 2 - 4 } & $26-1$ & 15 & 1.35 \\
\cline { 2 - 4 } & $29-1$ & 17 & 1.07 \\
\cline { 2 - 4 } & $30-1$ & 24 & 1.16 \\
\cline { 2 - 4 } & $35-2$ & 23 & 0.85 \\
\hline
\end{tabular}

Table 9.2 - Size of subsurface pores that nucleated fatigue cracks and their proximity to the SEN specimen notch surface. Note: the subscripts 1 and 2 denote the primary and secondary crack nucleation sites.

\begin{tabular}{|c|c|c|c|c|c|c|c|}
\hline Orientation & Specimen ID & $\mathrm{d}_{\mathrm{p} 1}(\mu \mathrm{m})$ & $2 \mathrm{c}_{1}(\mu \mathrm{m})$ & $2 \mathrm{a}_{1}(\mu \mathrm{m})$ & $\mathrm{d}_{\mathrm{p} 2}(\mu \mathrm{m})$ & $2 \mathrm{c}_{2}(\mu \mathrm{m})$ & $2 \mathrm{a}_{2}(\mu \mathrm{m})$ \\
\hline \multirow{5}{*}{$\mathrm{A}$} & $39-2$ & 141 & 27 & 16 & & & \\
\cline { 2 - 10 } & $40-1$ & 37 & 20 & 27 & & & \\
\cline { 2 - 10 } & $41-1$ & 65 & 44 & 35 & & & \\
\cline { 2 - 10 } & $41-2$ & 61 & 22 & 19 & & & \\
\cline { 2 - 10 } & $42-2$ & 133 & 31 & 34 & & & \\
\hline \multirow{5}{*}{ B } & $44-2$ & 28 & 21 & 25 & & & \\
\cline { 2 - 10 } & $22-1$ & 70 & 39 & 25 & 86 & 13 & 21 \\
\cline { 2 - 10 } & $23-1$ & 93 & 48 & 34 & & & \\
\cline { 2 - 9 } & $26-1$ & 132 & 52 & 58 & & & \\
\cline { 2 - 9 } & $29-1$ & 52 & 34 & 32 & & & \\
\cline { 2 - 9 } & $30-1$ & 130 & 12 & 20 & & & \\
\cline { 2 - 9 } & $35-2$ & 43 & 40 & 40 & 25 & 25 & 19 \\
\hline
\end{tabular}




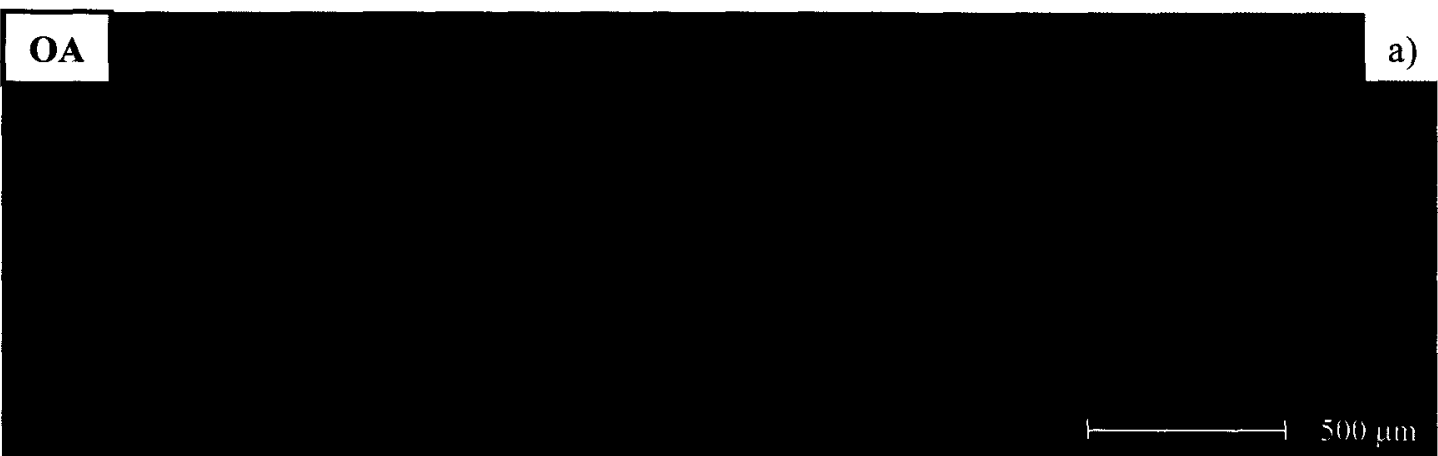

\section{OB}

Figure 9.1 - Back scatter electron micrographs showing representative crack paths on the notch surface; a) orientation $A, b)$ orientation $B$. 


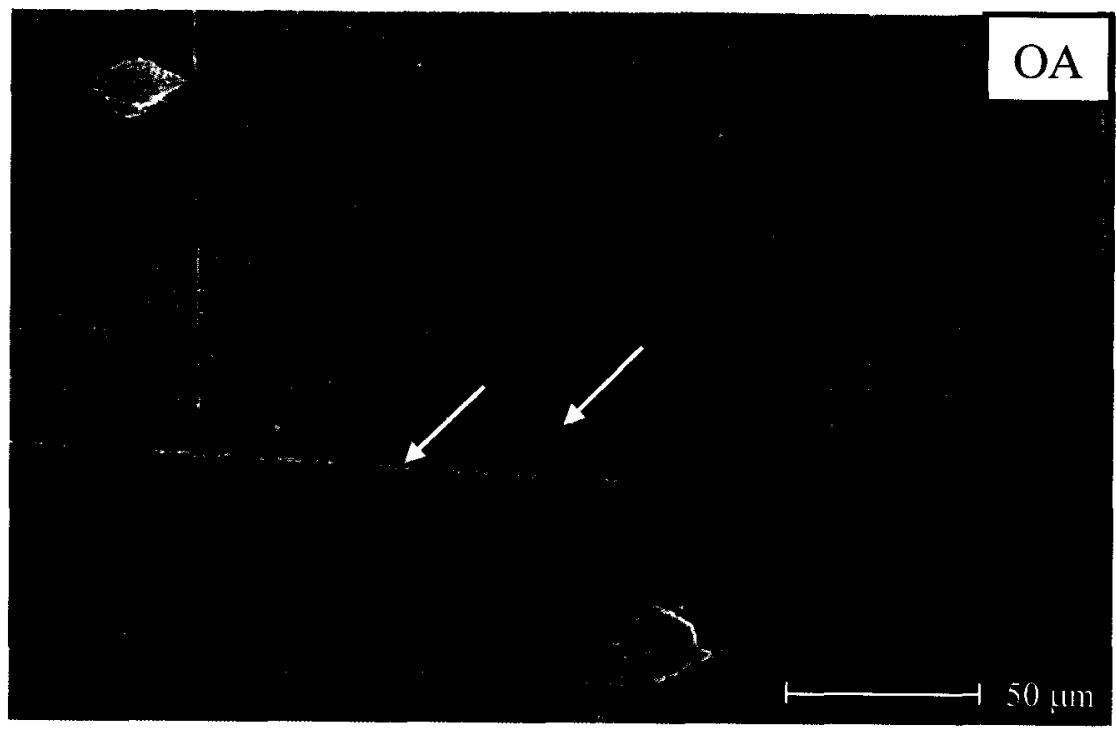

a)
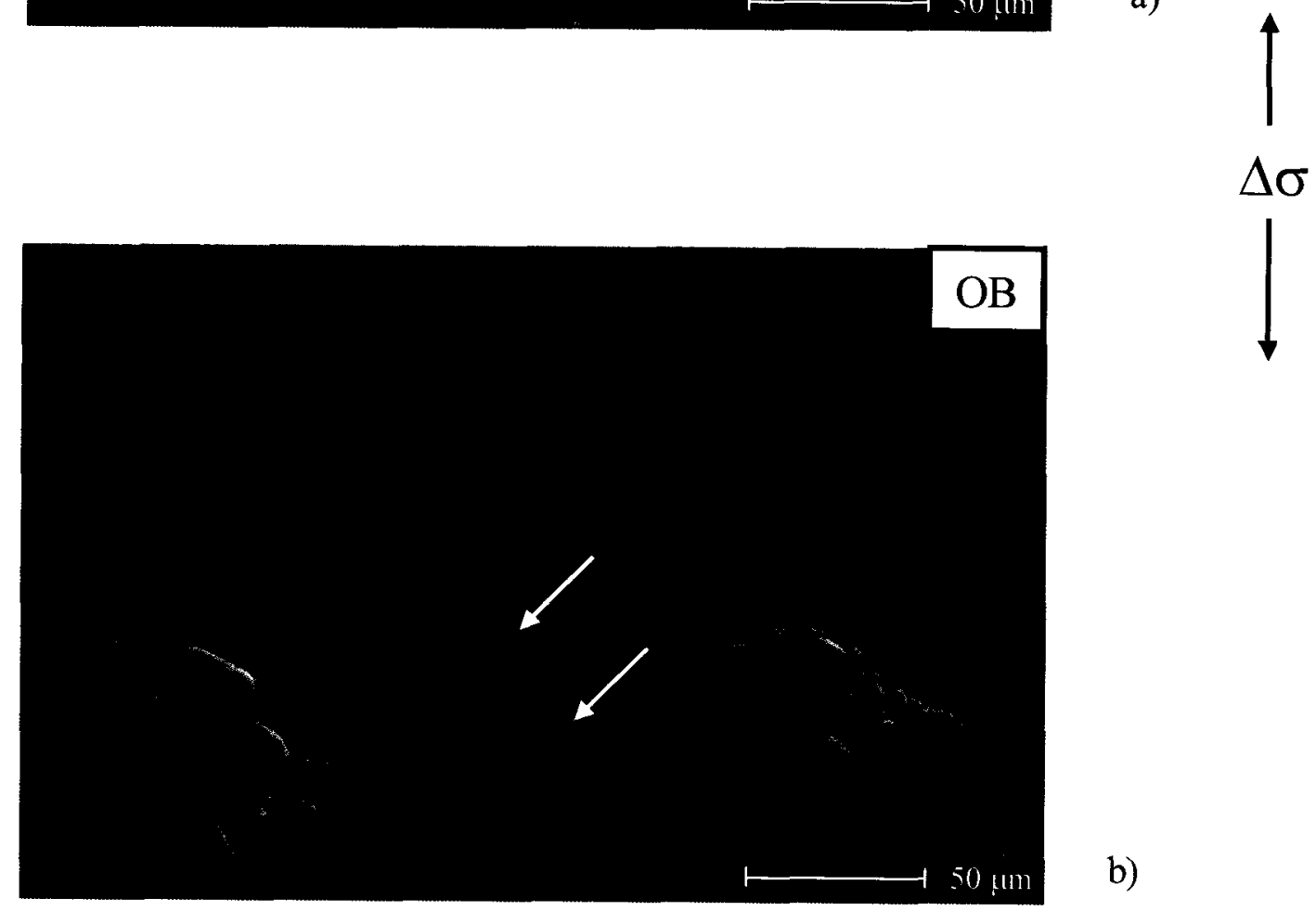

Figure 9.2 - Scanning electron micrographs showing octahedral slip traces on the notch surface: a) orientation $A$ and b) orientation $B$. 


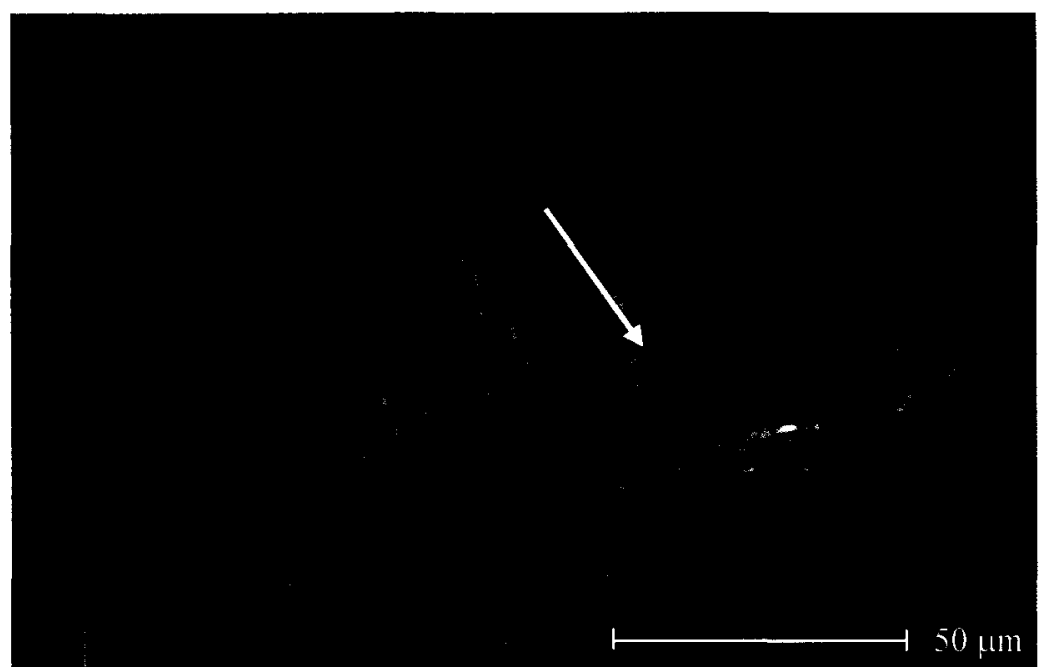

a)

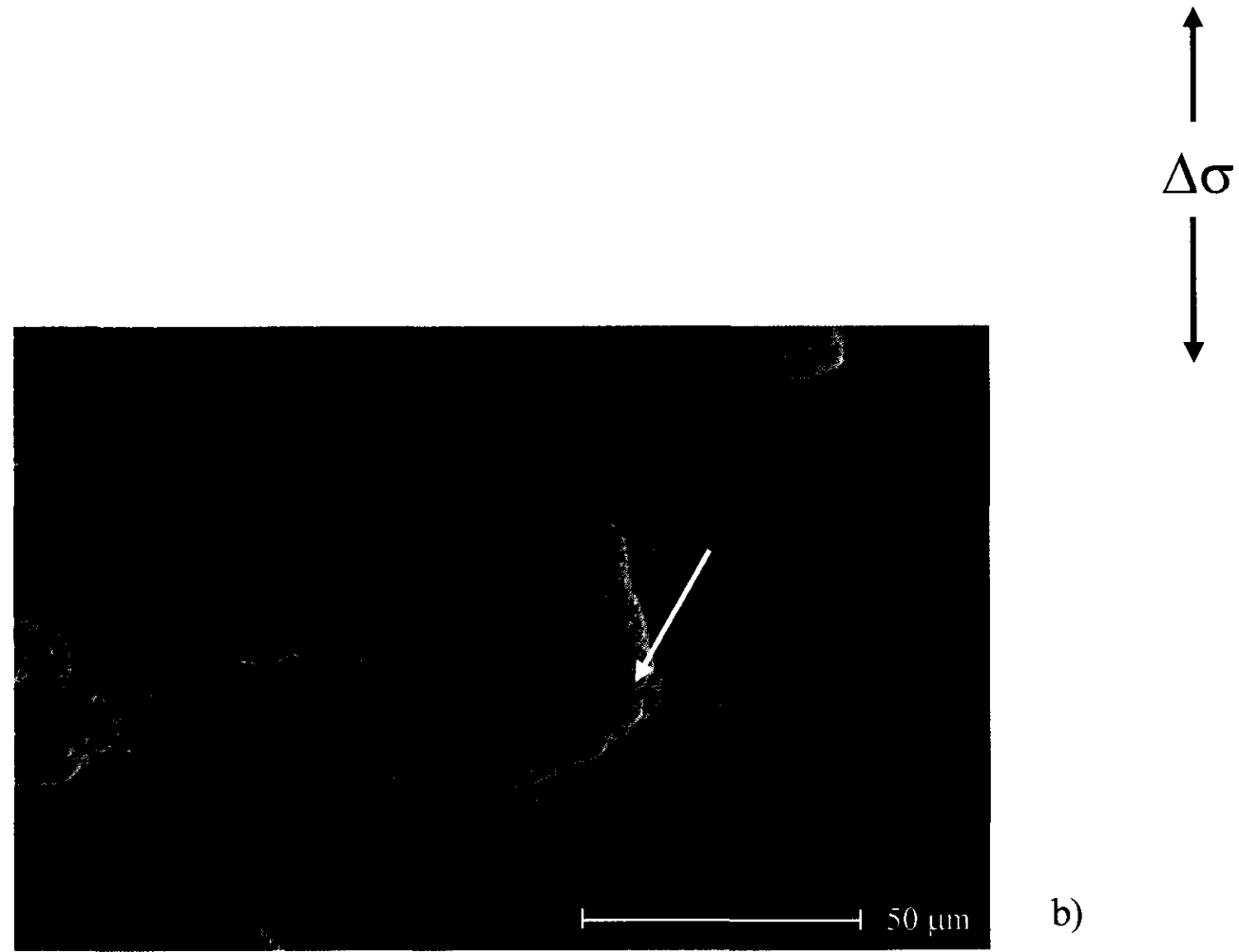

Figure 9.3 - Scanning electron micrographs showing secondary crack nucleation sites observed in the specimen notch: a) surface connected shrinkage porosity and b) subsurface nucleated crack breaking through the notch surface. 


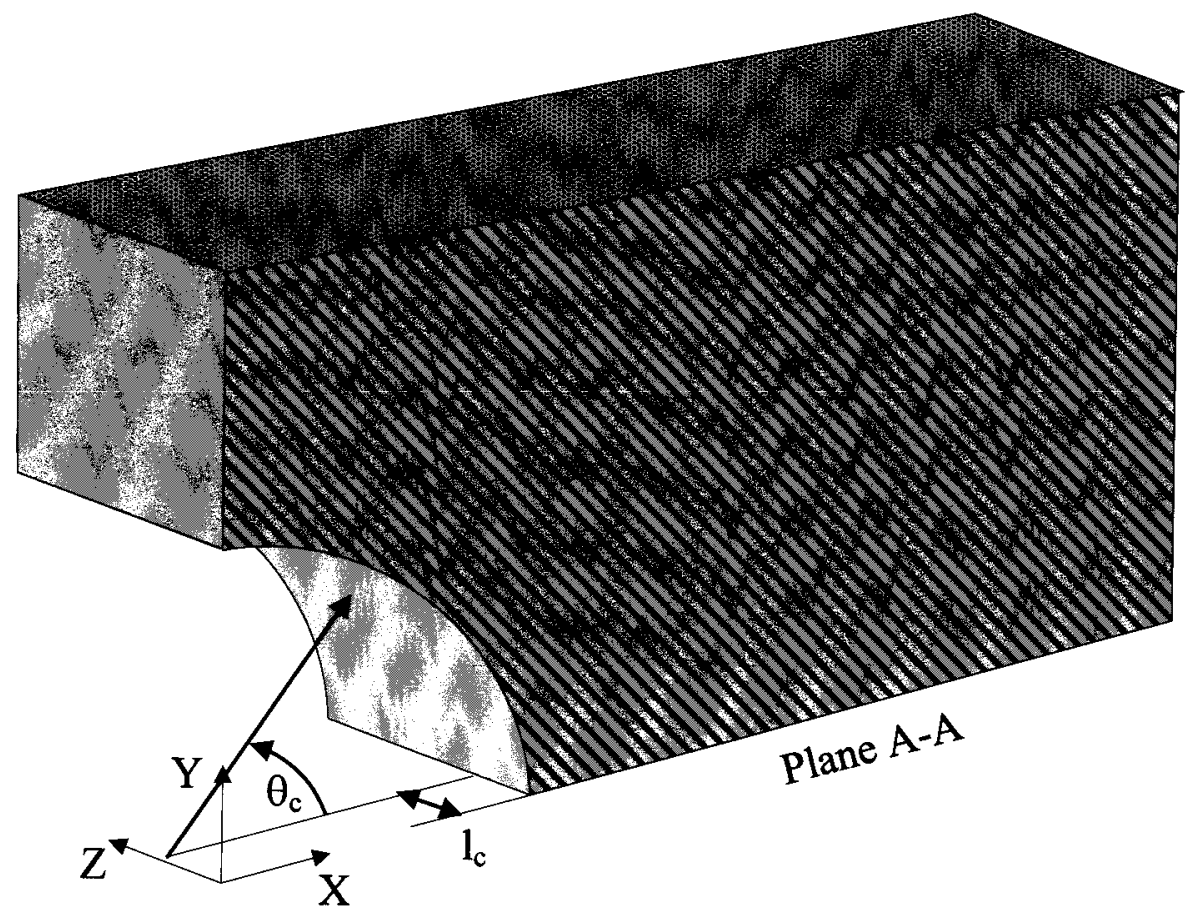

Figure 9.4 - Schematic showing definition of parameters $\theta_{c}$ and $l_{c}$ used to characterize dominant fatigue crack position in the notch. 

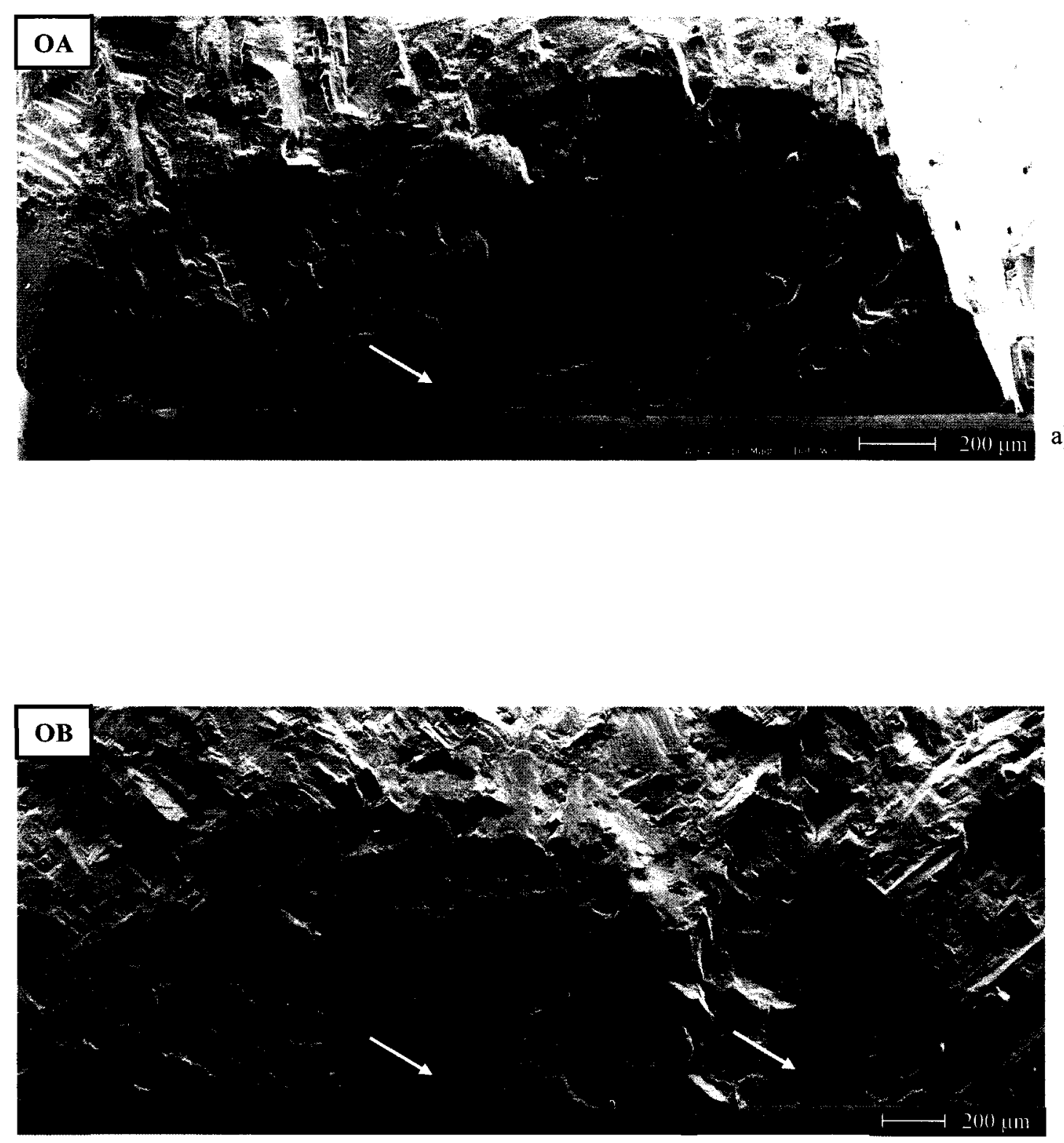

b)

Figure 9.5 - Scanning electron micrographs showing representative fracture surfaces: a) single dominant fatigue crack in orientation $A$ fatigue specimen and b) specimen containing multiple (two) fatigue cracks (orientation B). Note: black arrow indicates the crack propagation direction and yellow arrows indicate crack nucleation sites. 


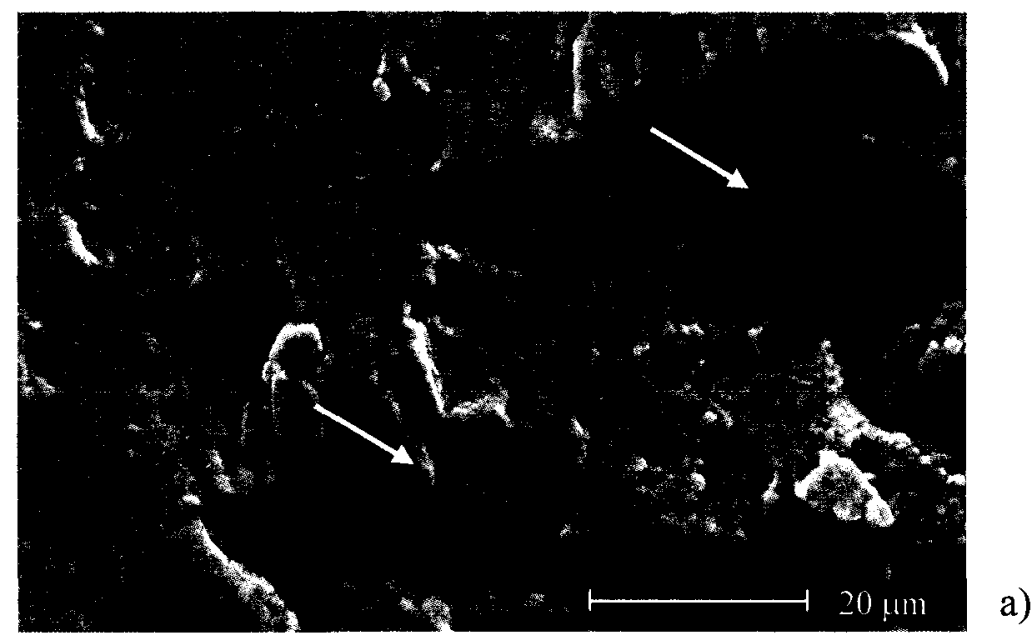

a)

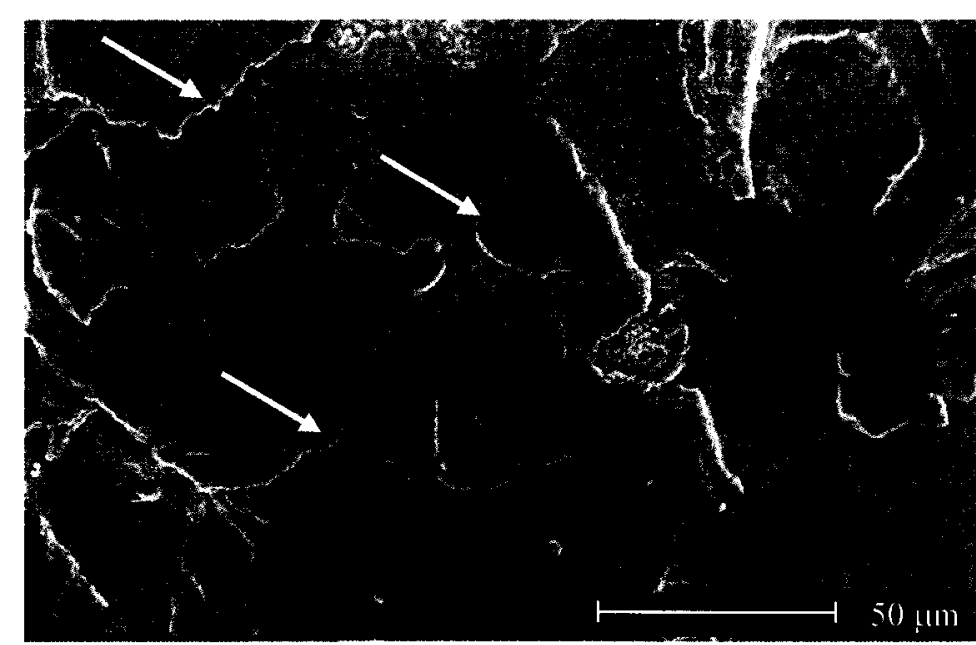

b)

Figure 9.6 - Secondary crack formation observed on fracture surfaces: a) $c \sim 200 \mu \mathrm{m}, \Delta \mathrm{K} \sim$

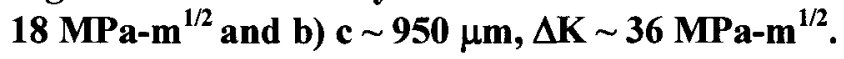




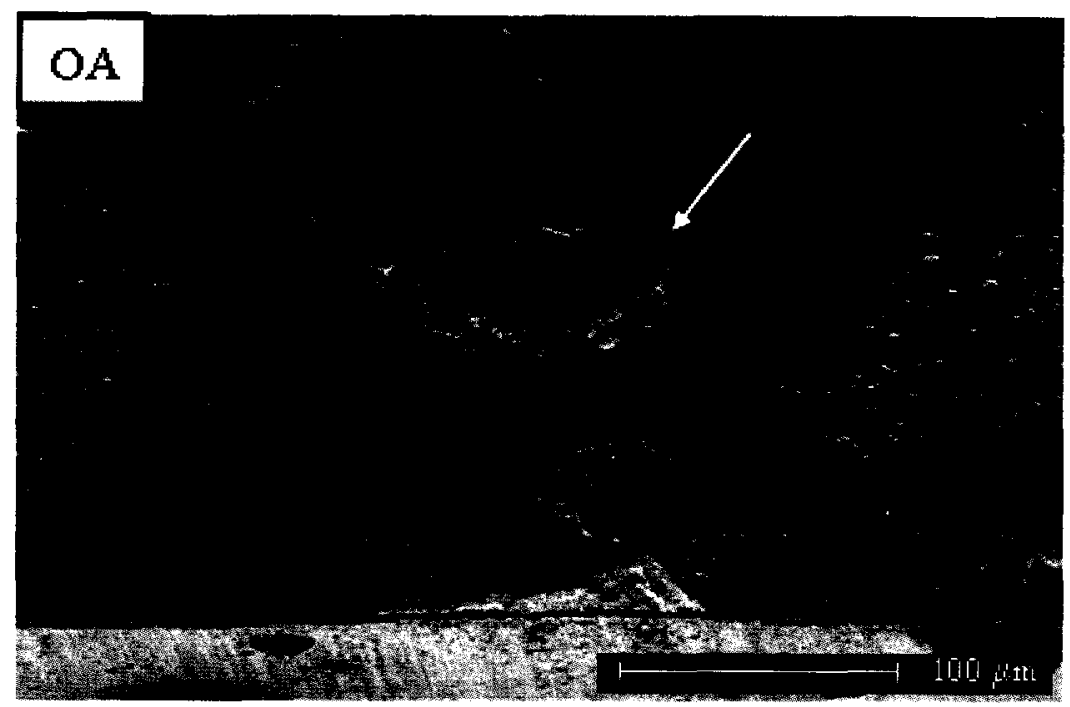

a)

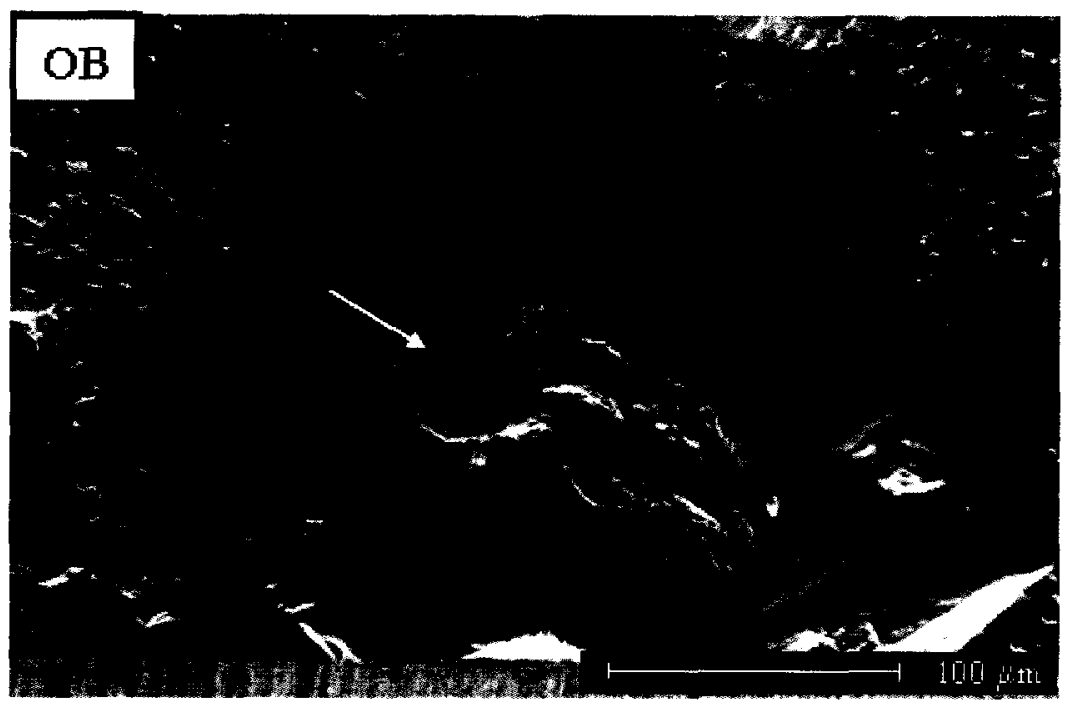

b)

Figure 9.7 - Scanning electron micrographs showing typical crack nucleation sites: a) orientation $A$ and $b$ ) orientation $B$. Note: black arrow indicates the crack propagation direction. 


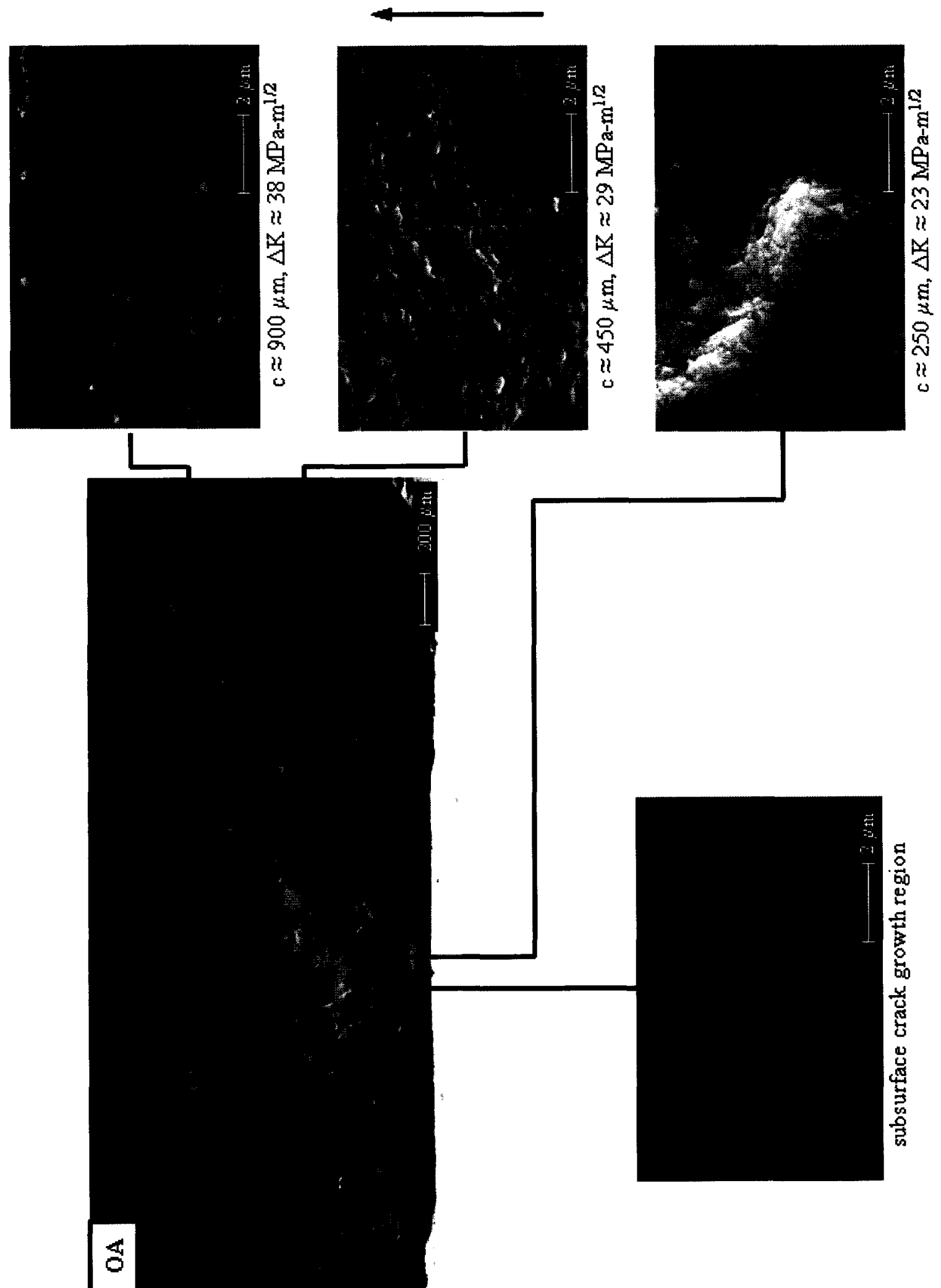

Figure 9.8 - Scanning electron micrographs showing representative details of orientation $A$ SEN specimen fracture surface at different crack lengths. Note: arrow indicates the crack propagation direction. 


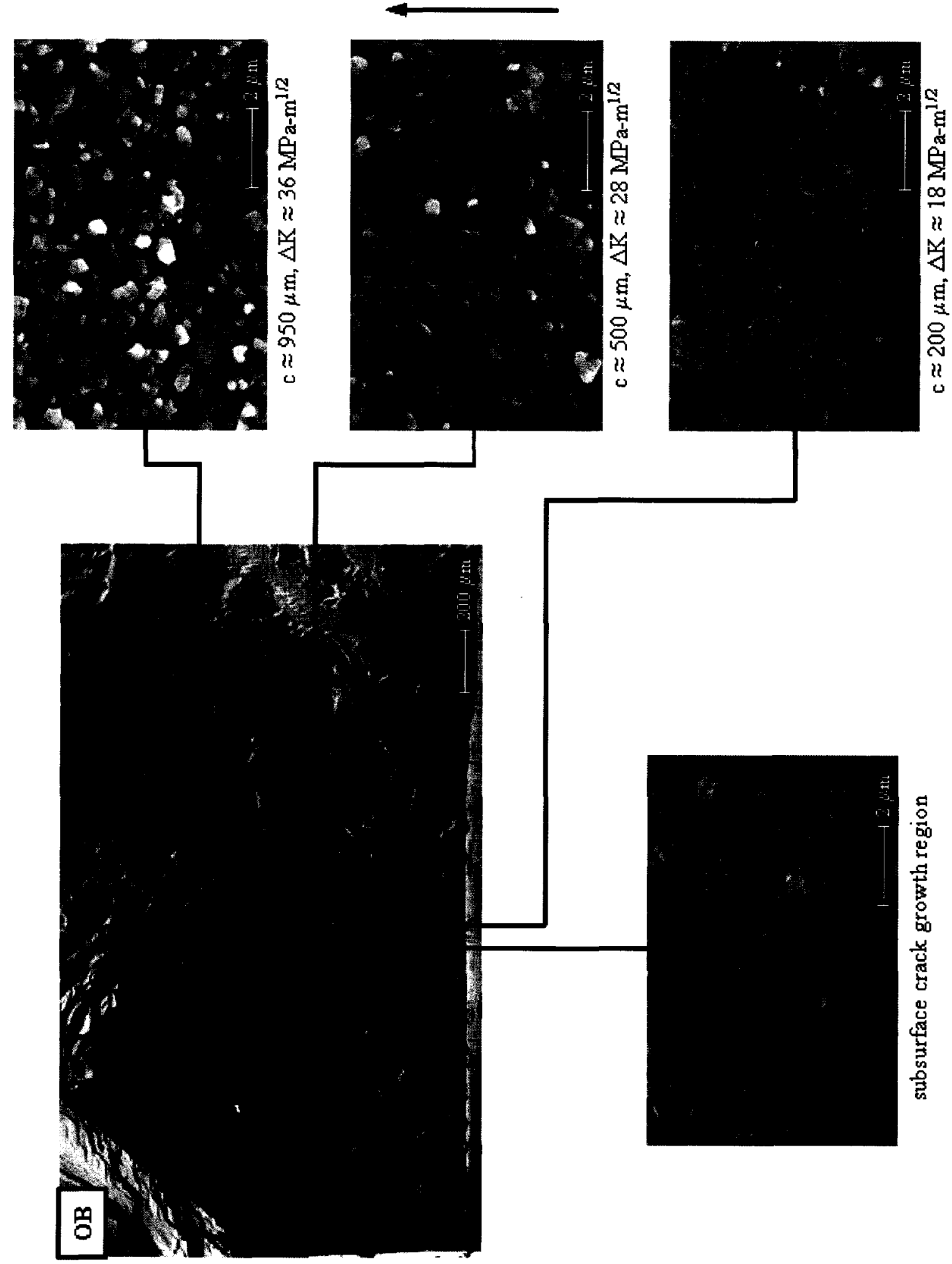

Figure 9.9 Scanning electron micrographs showing representative details of orientation $B$ SEN specimen fracture surface at different crack lengths. Note: arrow indicates the crack propagation direction. 


\section{Fatigue Crack Growth Rate Test Results and Discussion}

\subsection{Introduction}

In this investigation FCGR data were obtained for naturally generated small fatigue cracks in SEN fatigue specimens with two orientations [010]-[101] (orientation A) and [010]-[100] (orientation B). The current focusing ACPD technique was successfully used to monitor fatigue crack growth during the fatigue experiments. Empirical equations describing the variation in the anisotropic elastic $\Delta K$ and the elastic-plastic J-integral as a function of crack size over the range of $200<c<1000 \mu \mathrm{m}$ were presented in Chapter 8 . These empirical equations were used in conjunction with the FCGR data analysis procedures described in Chapter 5 to generate FCGR curves for naturally-generated small cracks emanating from SEN specimen notch. In addition to obtaining the small FCGR curves, crack initiation life data were extracted from the post-processed FCGR data.

This chapter will discuss the observed variability in crack initation life and investigate the factors that contribute to this variability. The next section considers the application of the anisotropic stress-intensity factor and the elastic-plastic J-integral, obtained from FEM calculations, to the analysis of the small FCGR test results. The small FCGR curves will then be presented and the effects of secondary crystallographic orientation on the small FCG properties will be discussed. Finally, a fracture mechanics methodology, utilizing the small fatigue crack growth data obtained in this investigation, is used to predict the crack initation life behaviour for small fatigue cracks originating from subsurface pores. 


\subsection{Crack Initiation Life}

As discussed in earlier chapters, the safe-life approach to component life-cycle management defines a component failure as the number of cycles to initiate an engineering crack with a probability of occurrence of 1 in 1000. The definition of crack initiation varies among researchers, but usually it is considered to conform with the appearance of a surface crack with a length of $760 \mu \mathrm{m}$ (1/32 in.). This definition for crack initiation was adopted in the present investigation. The crack initiation life is therefore defined by the number of cycles to form a thumbnail crack with a surface length, $2 a$, of $760 \mu \mathrm{m}$. The crack initiation life was determined from the crack length and cycle count data generated by the FTA crack growth analysis software when post-processing the small FCG test data. This section will primarily focus on presenting and discussing the sources of crack initiation life variability observed in the fatigue experiments.

The crack initiation life for smooth and notched fatigue specimens tested under load (or stress) controlled conditions is traditionally established from the applied stress range versus crack initiation life curves. However, in the present investigation the fatigue crack growth rate experiments concentrated on identical loading conditions (stress range, stress ratio, etc.) to establish the variability in small FCG properties. Since the fatigue experiments concentrated on one stress range, the stress-life curve only reflects the variability in the crack initiation life at one stress range rather than representing the traditional stress-life design curve that contains data from experiments conducted under different loading conditions.

The crack initiation life results from the 12 FCGR experiments are summarized in Table 10.1 and presented on a stress-life curve, as shown in Figure 10.1. Two things are 
apparent when examining this figure. First, there is considerable variability in the crack initiation life data with crack initiation lives spanning a factor of $2-4 \times$. This level of scatter in fatigue life is not unusual, as other researchers have reported fatigue lives in single-crystal superalloys spanning between $10-100 \times$ under the same applied stress range [48, 279]. Second, the crack initiation life exhibits a marked secondary crystallographic orientation dependence in which orientation B specimens generally exhibit shorter crack initiation lives than orientation A specimens. It is unclear, if this orientation dependence would be apparent at different applied stress ranges than those considered in this study. Defresne and Remy [48] have reported that at high stress ranges a small secondary orientation dependence of fatigue life in notched CMSX-2 specimens was apparent for specimens with $[100]$ notches exhibiting longer lives than specimens with other secondary crystallographic orientations. However, at a lower stress ranges they reported that scatter in the fatigue lives masked any apparent secondary orientation dependence. In the present study, a more detailed examination of the fatigue specimens was undertaken to determine what additional factors could contribute to both the variability and the secondary orientation dependence in the crack initiation lives.

In the previous chapter, fractographic observations indicated that subsurface interdendritic pores were the primary crack nucleation sites and that initial crack growth mainly occurred by expansion of these material discontinuities. One potential source for variability in the fatigue lives could be related to the relative size and distribution of these material discontinuities. Therefore, a more detailed examination of the crack initiation lives was undertaken using the subsurface pore dimensions and notch proximity data presented earlier in Table 9.2. 
The crack initiation lives as a function of total subsurface pore area is shown in Figure 10.2. The total subsurface pore area includes the area of primary and secondary subsurface pores for fatigue specimens containing two subsurface crack nucleation sites. It is apparent that larger subsurface pores, as indicated by the total pore area, are more detrimental and result in shorter crack initiation lives. The crack initiation lives for orientation B specimens appear to be shorter than orientation A specimens containing similar sized pores. This suggests that some other factor than the pore size causes the apparent secondary crystallographic orientation dependence on the crack initiation lives. Interestingly, the results presented in Figure 10.2 also suggest the size of the subsurface pores is larger in orientation B specimens. It should be noted, however, that two orientation B fatigue specimens contained multiple (two) subsurface crack nucleation sites and the total pore area reflects the additive contribution of both crack nucleation sites. If the primary subsurface crack nucleation sites for these specimens are taken into consideration, i.e. the secondary pore area is excluded, this apparent trend vanishes.

Defresne and Remy [48] have reported that fatigue lives notched CMSX-2 single-crystal specimens were generally shorter for naturally-generated fatigue cracks originating from larger subsurface pores. They also reported that when fatigue cracks originate from surface connected porosity, that fatigue lives were generally a factor of $10 \times$ shorter than cracks formed from subsurface defects. Such differences in fatigue life could be due to differences in crack propagation behaviour in air and vacuum environments. However, some researchers $[40,216]$ have reported that crack growth in single-crystal superalloys are generally faster in vacuum than in air environments when fatigue cracks propagate by the same mechanisms (Stage II or Stage I crack growth). One additional factor that could contribute to the differences in fatigue life between surface connected and subsurface crack nucleation sites is the stress-intensity factors associated with surface and subsurface 
cracks. For subsurface defects, additional constraint is imposed by surrounding material, and therefore the constraint conditions can be considered plane strain. On the other hand, for surface connected small cracks, the constraint conditions where the crack-tip intersects the free surface of the notch are different. The constraint conditions at the notch surface can be considered plane stress, and therefore the stress intensity factors are higher leading to higher crack propagation rates for surface connected small cracks.

In addition to considering the subsurface pore size the proximity of these defects to the notch root was also considered a factor in the crack initiation lives. The crack initiation life is plotted as a function of the distance between the subsurface pore and the notch surface, as shown in Figure 10.3. In the case of multiple subsurface crack nucleation sites, only the distance associated with the dominant crack nucleation site is plotted. The only discernable trend is that orientation B fatigue specimens generally exhibit shorter crack initiation lives than orientation A specimens. Again this suggests that some other factor is responsible for the shorter crack initiation lives exhibited by orientation B specimens.

The lack of correlation between crack initiation lives and the distance of subsurface pores from the notch surface is intriguing. Subsurface discontinuities that are further from the notch surface spend a greater portion of the crack initiation life propagating in a vacuum environment. As discussed earlier, subsurface defects in general have lower stressintensity factor ranges than surface connected cracks under the same loading conditions due to additional constraint from surrounding material. If it is assumed that crack propagation behaviour is similar in air and vacuum and the initial defect sizes are similar, then crack initiation lives should be shorter for subsurface pores that are located closer to the notch surface. Therefore, some trend should be apparent in Figure 10.3. However, as 
indicated in Table 9.2 the initial size of subsurface pores that resulted in the formation of fatigue cracks vary from specimen-to-specimen as does the distance between these defects and the notch surface. This is perhaps why there is no apparent connection between the crack initiation life and the distance between the subsurface crack origin and the notch root.

The analysis of both the size of subsurface pores that resulted in formation of fatigue cracks and the distance between these defects and the notch root revealed that differences in crack initiation lives for orientation A and B SEN specimens could not be rationalized based on these parameters. This suggests that crack initiation life is dependent on some additional factors, such as the local stresses acting upon the subsurface defect. Since fatigue cracks were observed to predominantly form where the maximum principal stress was predicted in the elastic-plastic FEM calculations, it was assumed that the maximum principal stress controls the nucleation of fatigue cracks from subsurface pores. The local stress was extracted from the elastic-plastic notch root finite element model based on the $\theta_{\mathrm{c}}$ and $l_{\mathrm{c}}$ parameters used to characterize the location of the dominant notch root fatigue crack in each specimen (see Table 9.1).

The crack initiation life as a function of the local maximum principal stress acting at the crack nucleation site is shown in Figure 10.4. It can be observed that a reasonable correlation was achieved between the crack initiation life and the local stress acting at the crack nucleation site. Furthermore, the secondary orientation dependence of the crack initiation lives appears to be due to the stress being larger in orientation B specimens. However, substantial scatter (approximately $2-4 \times$ ) is still apparent in the crack initiation lives, which indicates that local stress does not completely resolve the variability. This suggests that pore size, the distance between the pore and the notch surface and the local 
stress acting at the pore must all be taken into consideration when predicting crack initiation life. One life prediction method that allows these factors to be taken into consideration simultaneously is fracture mechanics. A fracture mechanics approach to predicting fatigue crack initiation life is discussed later in this Chapter.

\subsection{Small Fatigue Crack Growth Test Results and Discussion}

\subsubsection{Application of Fracture Mechanics Parameters to FCGR Data}

In this investigation the crack growth directions at the notch surface $\left(\phi=90^{\circ}\right)$ and the maximum crack depth $\left(\phi=0^{\circ}\right)$ conform to orthogonal $\langle 100\rangle$ or $\langle 101\rangle$ crystal directions. Theoretically, the crack growth rates should be the same along a family of crystallographic directions in cubic single-crystals provided that the driving force for crack extension is equivalent. This viewpoint is supported by the circular crack growth region surrounding subsurface crack nucleation sites as discussed in Chapter 9. Therefore, it is expected that with an appropriate selection for the crack-tip parameter, the crack growth rate curves at the surface of the notch $(d a / d N)$ and at the maximum crack depth $(d c / d N)$ should collapse onto a single crack growth rate curve. This was investigated with the fatigue crack propagation data obtained in this study.

The FCGR ( $d a / d N$ and $d c / d N$ ) is plotted as a function of the anisotropic stress-intensity factor range for orientation $\mathrm{A}$ and $\mathrm{B}$ SEN specimens in Figures 10.5 and 10.6, respectively. In general, it is apparent the anisotropic stress-intensity factor range collapses the FCG rates, $d a / d N$ and $d c / d N$, onto a single crack propagation curve within a factor of $1-1.5 \times$. This finding suggests the crack growth rate at the notch surface and the deepest part of the crack front can be characterized by a single crack propagation curve.

Furthermore, it suggests the anisotropic stress-intensity factor correctly accounts for 
material anisotropy and variations in the stress distribution at both crack front positions. The apparent success of $\Delta K$ in correlating the small crack $d a / d N$ and $d c / d N$ curves could be the result of "isotropy" associated with crack growth along orthogonal but crystallographically equivalent directions and the predominantly small-scale yielding conditions within the notch root. Zhao and coworkers [56] have shown that the anisotropic elastic-plastic J-integral for small notch root fatigue cracks can be expressed in terms of the stress-intensity factor under small-scale yielding conditions.

The FCGR ( $d a / d N$ and $d c / d N$ ) is plotted as a function of the elastic-plastic J-integral for orientation A and B SEN specimens in Figures 10.7 and 10.8, respectively. Unlike the anisotropic stress-intensity factor range, it is apparent the J-integral fails to collapse $d a / d N$ and $d c / d N$ onto a single crack growth rate curve. The difference between the two crack growth rate curves varies but was generally within $1-3 \times$. As discussed in Chapter 8, the FEM calculations showed a mesh size dependence for the J-integral calculated at the notch surface. To resolve this ambiguity, it was assumed that the J-integral at the notch surface was a value calculated from an adjacent contour. The J-integral from the adjacent contour was higher than the value calculated at the notch surface. This would shift the $d a / d N$ crack growth rate curve to the right and into closer agreement with the $d c / d N$ crack growth rate curve. However, if the actual notch surface J-integral was used as the crack-tip parameter for the $d a / d N$ crack growth rate curve, the correlation would be much poorer than the results presented because the $d a / d N$ crack growth rate curve would be shifted further to the left.

One possible explanation for this discrepancy is that the elastic-plastic FEM simulations only considered notch root cracks, i.e. the fatigue crack was assumed to be located at the minimum specimen cross-section. The fractographic analysis of the fatigue specimens 
after testing showed that the fatigue cracks do not form at the notch root but on a plane located $15-25^{\circ}$ from the minimum specimen cross-section. It is likely that the elasticplastic J-integral would be more notably affected by localization of plastic strain and the anisotropic stress distribution within the notch where the fatigue cracks were predominantly observed to form. Investigating such effects would require that the exact location where the crack forms in the notch root to be taken into consideration in the FEM calculations. Due to variability in the crack formation locations it would not be practical to conduct numerous and computationally expensive non-linear calculations that consider the exact position of each fatigue crack in the notch fields and how crack position affects the elastic-plastic J-integral. Because of the ambiguity in the $d a / d N$ and $d c / d N$ versus $\mathrm{J}$-integral crack growth rate curves, the elastic-plastic J-integral was not considered further in this investigation.

\subsubsection{Fatigue Crack Growth Rate Curves for Small Fatigue Cracks}

The FCGR curves $(d a / d N$ and $d c / d N)$ for orientation A and B SEN specimens are shown in Figures 10.9 and 10.10, respectively. These figures contain the FCGR data obtained from the six fatigue experiments performed for each secondary crystallographic orientation. The FCGR interpolation and statistical analysis procedures described in Chapter 5 were applied to each of the FCGR curves to determine the mean and standard deviation at each $\Delta K$ level for orientation A and B SEN specimens. For reference, the mean FCGR at each $\Delta K$ level is plotted on Figures 10.9 and 10.10. The mean FCGR is accompanied by error bars, which indicate a range of \pm 2 standard deviations from the mean FCGR response at each $\Delta K$ level.

The FCGR variability range at each $\Delta K$ level for orientation A and B SEN specimens is

presented in Figure 10.11. The FCGR variability range represents the FCGR ratio, $10^{4 b}$, 
described in Chapter 5. Examination of the variability range data reveals that the variability in the FCGR clearly depends on $\Delta K$ level and some orientation dependence is also apparent. At lower $\Delta K$ levels, the small crack growth rates for orientation $\mathrm{A}$ and $\mathrm{B}$ exhibit larger variability, but this variability was generally higher for orientation A specimens. It is unclear why a secondary orientation dependence was apparent in the FCGR variability at lower $\Delta K$ levels, but this could be due to the relatively small population size ( 6 tests) used in the statistical calculations. In general, however, more variability is expected at smaller crack sizes due to lower FCG rates and the relatively smaller changes in PD signal (for smaller cracks sizes), which increases the uncertainty of the crack length measurements. The variation in the size and locations of the fatigue crack nucleation sites could also be a factor in the higher variability at lower $\Delta K$ levels. These variations could cause significant deviations from the crack shape $(a / c)$ assumed in the calculations used to determine the notch root thumbnail crack stress-intensity factors and in the analysis of the FCGR data.

On the other hand, the FCG rates in orientation B SEN specimens exhibits substantially more variability at higher $\Delta K$ levels than orientation A SEN specimens. This could be due the transition from Stage II to Stage I crack growth mechanisms (at the notch surface) exhibited at higher $\Delta K$ levels in orientation B specimens. Due to variations in the exact location the fatigue cracks formed in the notch root, the Stage II to Stage I transition could vary from specimen-to-specimen. The stress-intensity factors used in the analysis of the FCGR data were obtained from FEM calculations for Mode I fatigue cracks, which almost certainly are invalid for inclined Stage I cracks.

The average variability over the entire $\Delta K$ level range considered in the FCG experiments was 2.2 for orientation $\mathrm{A}$ and 2.4 for orientation $\mathrm{B}$. Interestingly, the average variability 
observed in the present small crack growth experiments are quite comparable to the FCGR variability reported in two interlaboratory studies $(2-3 \times)[260,261]$ used to define the precision and variability statements for the ASTM E647 [177] standard. The variability in these previous studies was established from long fatigue cracks and $\Delta K$ levels above the threshold in microstructurally homogenous materials. This suggests that over the crack size dimensions considered in the present investigation $(200<c<$ $1000 \mu \mathrm{m})$ that single-crystal superalloys exhibit FCG variability similar to long fatigue cracks in microstructurally homogeneous materials. It should be noted that for small fatigue cracks in the size range considered in this investigation that polycrystalline materials typically exhibit substantial variability in small crack FCG rates. This variability is generally associated with the crack size being on the order of microstructural dimensions, i.e. the grain size. The relatively smaller FCGR variability observed in the present work suggests that substantial variability in the small crack FCGR rates may only be observed at crack length scales where microstructural heterogeneity is apparent, i.e. $\gamma^{\prime}$ or $\gamma-\gamma^{\prime}$ eutectic size. Verifying this, of course, would require more fundamental crack growth studies to be conducted with crack measurement techniques capable of resolving microstructural scale small fatigue cracks.

The mean small FCGR curves for orientation A and B SEN specimens are compared in Figure 10.12. For reference, the error bands corresponding to \pm 2 standard deviations associated with each orientations data set are plotted on this figure as well. Examination of the mean FCGR curves indicates that no secondary orientation effect is apparent within the scatter of the fatigue crack growth data. At intermediate $\Delta K$ levels $(23<\Delta K<$ $40 \mathrm{MPa}^{\mathrm{1}}{ }^{1 / 2}$ ) the mean FCGR curves for both secondary crystallographic orientations have almost identical slopes indicating that the mechanisms of fatigue crack growth were similar. This viewpoint is further supported by the fractographic analysis results 
presented in Chapter 9, which indicted no significant differences in fracture surface features were apparent for small fatigue cracks in orientation A and B specimens. Since the mean FCGR curves for orientation A and B were almost identical, Paris' law was fitted to both FCGR curves in the $\Delta K$ range of $23-40 \mathrm{MPa}-\mathrm{m}^{1 / 2}$, as illustrated in Figure 10.12. Similar fits were produced to define to upper and lower bound small FCGR curves based on the crack growth rates for \pm 2 standard deviations from the mean FCGR. The slope, $m$, and constant, $C$, for the mean and upper and lower bound small FCGR curves are summarized in Table 10.2.

Close examination of the mean FCGR curves also reveal some interesting features. For example, at lower $\Delta K$ levels the small crack growth behaviour deviates from the loglinear fit determined at intermediate $\Delta K$ levels. In particular, the small FCG rates for

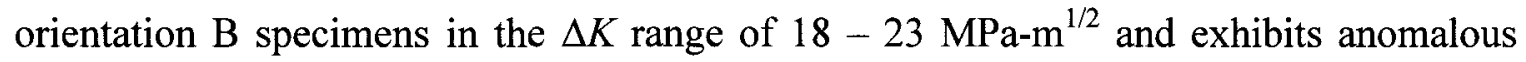
crack growth behaviour, i.e. the FCGR decreases with increasing $\Delta K$. In the same $\Delta K$ range, Orientation A specimens exhibit FCG rates that appear to be independent of $\Delta K$. There are two possible explanations for this seemingly anomalous FCG behaviour. First, this $\Delta K$ range corresponds with crack sizes $(c)$ in the range of $200-400 \mu \mathrm{m}$, and therefore the cracks will reside within the plastically deformed region of the specimen notch. Dai [280] has reported similar anomalous FCG behaviour in Ti64 SEN specimens for small fatigue cracks confined within the notch root plastic strain field. Second, it is also possible that this anomalous FCG behaviour could represent transient crack growth behaviour when a small subsurface crack transitions from growth in vacuum to crack growth in air. This would imply that FCG rates in vacuum would have to be intrinsically faster in vacuum than in air at the same $\Delta K$. Joyce and coworkers [216] have reported for short through-thickness fatigue cracks in CMSX-4 single-crystals tested at $650^{\circ} \mathrm{C}$ that 
crack growth rates were higher in vacuum than in air. Lupinc et al. [40] have observed significant environmentally induced transient fatigue crack growth behaviour in CMSX-2 single-crystals tested at $950^{\circ} \mathrm{C}$ by alternating between vacuum and air environments periodically during a FCGR test. Since no vacuum FCGR data was obtained in the present investigation, this explanation is speculative.

At higher $\triangle K$ levels the mean FCGR curve for orientation $\mathrm{B}$ and deviates from the orientation A FCGR curve. Since the slope is higher in this region and the crack growth at this $\Delta K$ level represents crack growth at the surface of the SEN specimen notch, i.e. $d a / d N$, and crystallographic features were observed at the notch surface, this indicates a transition to the intrinsically faster Stage I crack growth mechanism.

In this study there was no apparent secondary orientation dependence observed for naturally-generated small fatigue cracks within the experimental scatter; however, other researchers $[33,34,40,216]$ have observed, in some cases, a significant secondary orientation effect on short fatigue crack growth rates. One thing that is apparent from the literature is that researchers reporting a secondary orientation dependence on short fatigue crack behaviour usually considered physically short through-thickness fatigue cracks generated by fatigue pre-cracking and load shedding procedures. Unlike the naturally-generated fatigue cracks considered in this investigation, these procedures generate "artificial" short fatigue cracks containing plastic wakes and fracture surface topographical features that can cause crack closure. Indeed, some researchers investigating "artificial" short fatigue cracks have observed $[33,34,40]$ threshold-like features in the short FCGR curves, which provides compelling evidence that these procedures result in significant crack closure levels. 
In the present study there was no evidence of a fatigue threshold for naturally-generated thumbnail small fatigue cracks larger than $200 \mu \mathrm{m}$ deep $(c>200 \mu \mathrm{m})$. Similarly, Hicks and Brown [35], Howland [218] and Howland and Brown [36] did not observe a fatigue threshold for naturally-generated small fatigue cracks in the SRR-99 single-crystals tested at room temperature in laboratory air. Howland and Brown [36] also did not find a secondary orientation dependence on the propagation behaviour of short fatigue cracks. Therefore, naturally-generated small fatigue cracks could behave differently than "artificial" short fatigue cracks because they initially start closure-free due to a lack a prior deformation history and develop plasticity, roughness and oxidation induced crack closure as the small crack propagates.

One other difference between the small fatigue cracks considered in the present work and "artificially" generated small fatigue cracks considered by other researchers is the relative size of the fatigue cracks. The naturally generated small fatigue cracks studied in this investigation are relatively smaller than through-thickness fatigue cracks and may be more notably affected by oxidation and roughness induced crack closure mechanisms. The fractographic results presented in Chapter 9 showed that the naturally-generated small fatigue cracks were macroscopically flat, but they were very rough at the microscopic scale due to crack deviations from the macroscopic $\{100\}$ crack plane and the formation of secondary fatigue cracks. These fractographic features were observed on fracture surfaces for orientation A and B SEN specimens. The relatively large size of the asperities on the fracture surface in comparison to the crack dimension could conceivably cause high levels of roughness-induced crack closure through asperity contact. Roughness induced crack closure would be enhanced by in-plane and anti-plane displacements at the crack-tip caused by small deviations of the primary crystallographic orientation from the nominal [010] crystallographic direction. Roughness induced crack 
closure effects could mask any secondary orientation dependence on the fatigue crack propagation behaviour that arises from the development of a plastic wake behind the crack front. However, without physical measurements of crack closure levels for the small fatigue cracks considered in this investigation, this hypothesis is speculative.

On the other hand it is possible that secondary crystallographic orientation could affect small crack propagation behaviour of small cracks much shorter than considered in this study. Recall earlier that some differences in the magnitude of the inelastic shear strain was observed in the elastic-plastic finite element calculations for notch root fatigue cracks. These results suggest that elastic-plastic anisotropy could result in some differences in the plastic zone size formed ahead of a propagating fatigue crack. One of the criteria for determining whether a fatigue crack is small is how large its size is compared to its crack-tip plastic zone size. It is possible at very short crack lengths in which the crack size approaches the size of the crack-tip cyclic plastic zone that some differences in small crack growth behaviour would be apparent and depend on the crack propagation direction, i.e. the secondary crystallographic orientation. Futher research would be necessary to investigate this.

\subsection{Crack Initiation Life Prediction}

Fractographic analysis of naturally-generated small fatigue cracks in the single-crystal material considered in this investigation revealed that fatigue cracks primarily originate from interdendritic pores at subsurface locations. Crack growth occurred by expansion of these initial discontinuities as subsurface cracks in a vacuum environment. After investigating the role of local stress, pore size and pore position relative to the specimen

notch, it was determined that none of these parameters could adequately resolve the variability in crack initiation life. In this section a linear-elastic fracture mechanics 
approach for predicting the crack initiation life based on the short FCGR data obtained in this investigation is presented and the results are discussed. Based on the fractographic observations the following assumptions are made:

1. The subsurface interdendritic pores are assumed to be initial cracks of circular or elliptical shape and the incubation period for forming cracks at these defects is negligible, i.e. crack initiation life is solely a crack propagation phenomena.

2. Environmental effects on the fatigue crack propagation behaviour are not considered in the analysis, and therefore vacuum crack growth is governed by the small fatigue crack propagation curve obtained in air.

3. Since internal cracks were located in the plastically deformed region close to the notch surface, the growth of internal cracks is assumed to occur in a constant stress field. The range of the maximum principal stress at the subsurface pore is utilized in the analysis of subsurface crack growth.

4. Subsurface crack growth occurs via expansion of the interdendritic pores such that the expansion remains symmetrical.

5. Once the subsurface crack breaks through the specimen notch surface, it is assumed to have a semi-elliptical crack shape with depth (c) and semi-surface length $(a)$ equal to the original diameter of the internal crack. The subsequent crack growth was considered to occur based on the crack-tip parameters derived from FEM results using the notch-root anisotropic stress-intensity factors presented in Chapter 8.

6. Multiple subsurface and surface fatigue cracks were analyzed independently, i.e. no crack interaction effects were considered. 
As discussed earlier, Defresne and Remy [48] have reported that the incubation period for forming a propagating small fatigue crack in a notch root represents almost a negligible portion of the fatigue life, which suggests that assumption 1 is reasonable. The elasticplastic notch roots field analysis presented in Chapter 8 indicated that there exists a condition of small scale yielding within the specimen notch suggesting that assumption 3 is also valid. Fractographic results presented in Chapter 9 indicated that interdendritic eutectic $\gamma-\gamma$ ' altered crack growth from subsurface pores such that expansion of the pore was not symmetrical. However, the fatigue properties of eutectic $\gamma-\gamma^{\prime}$ are unknown so assumption 4 seems reasonable as a first approximation for crack growth originating from subsurface interdendritic pores.

The crack initiation life was predicted using a two-step procedure: the first step considered the growth of a subsurface fatigue crack and the second step considered the growth of a semi-elliptical surface flaw. The two step procedure is illustrated schematically in Figure 10.13. The first step considered the growth of a subsurface crack from an interdendritic pore having dimensions of $2 c$ and $2 a$ in a rectangular plate of thickness $2 d_{\mathrm{p}}$ where $d_{\mathrm{p}}$ is the distance between the center of the pore and the notch surface. These dimensions are summarized in Table 9.2 for each of the SEN fatigue specimens. The cyclic stress range applied to the strip was assumed to be the range of the maximum principal stress acting at the pore, which was determined from the elasticplastic FEM simulation results presented in Chapter 8 . The stress-intensity factors were determined using the Newman and Raju [281] empirical model for an embedded elliptical crack in a finite plate. It should be noted that this empirical model was developed for embedded cracks in an isotropic material. Since the subsurface crack is initially under plane strain conditions and the FEM calculations for notch-root cracks showed that anisotropic stress-intensity factors at the deepest part of the crack (where plane strain 
conditions exist) were within $\pm 10 \%$ of isotropic stress-intensity factors, the use of this model seems reasonable. The subsurface crack propagation life was estimated by integrating Paris' law incrementally in the $c$ and $a$ directions of the subsurface crack. The Paris law coefficients for the mean short fatigue crack growth curve were used in the analysis (see Table 10.2). Once the subsurface fatigue crack reached the surface of the strip, i.e. $2 a=2 d_{\mathrm{p}}$, the subsurface crack analysis was terminated. The final dimensions of the subsurface crack were used as the initial dimensions for step 2 , i.e. the surface crack analysis. Specifically, the surface crack depth, $c$, was set equal to $2 d_{\mathrm{p}}$ and the surface crack length, $a$, was set equal to $c$ from the subsurface crack.

The second step in the crack initiation life analysis considered the growth of a semielliptical surface crack. The anisotropic stress intensity factors for the $c$ and $a$ crack front positions were determined using the empirical stress-intensity factor equations for orientations A and B presented in Chapter 8 (see Table 8.5). Similar to the subsurface crack analysis, the surface crack growth was estimated by integrating Paris' law incrementally in the $c$ and $a$ directions and using the coefficients for the mean short fatigue crack growth curve (see Table 10.2). The analysis was terminated when the surface crack length, $2 a$, was $760 \mu \mathrm{m}$. In fatigue specimens containing two subsurface crack sites, the surface crack analysis was terminated when the combined surface crack length of both cracks was equal to $760 \mu \mathrm{m}$. One limitation of the surface crack analysis is that the stress-intensity factors for crack depths (c) less than $200 \mu \mathrm{m}$ were extrapolated from the empirical equations. These equations were also developed from FEM simulations conforming to the mean crack aspect ratio versus crack size relationship obtained from the ACPD calibration tests. The crack aspect ratios were examined after the surface crack calculations. They were predominantly in the range 0.75 to 1.2 for a surface crack length of $760 \mu \mathrm{m}$, which is within the range of crack geometry scatter 
observed during the ACPD calibration tests. A more comprehensive empirical anisotropic stress-intensity factor solution would need to be developed to more accurately predict the crack shape evolution.

The predicted and actual crack initiation lives are summarized in Table 10.3. The predicted fatigue lives are presented as two values; the total number of cycles to form a surface crack with $2 a=760 \mu \mathrm{m}$, and the number in parentheses indicates the number of cycles before the subsurface crack breaks through the surface of the notch. For many tests the majority of the crack initiation life is expended propagating a subsurface fatigue crack. In general, it was observed that the fraction of crack initiation life spent as an internal crack was higher for pores embedded deeper from the notch surface and for pores that were smaller in size. The predicted, $N_{\mathrm{p}}$, and actual, $N_{\mathrm{a}}$, crack initiation lives are compared in Figure 10.14. The overall agreement between the predicted and actual crack initiation lives is quite good (within a factor of $2 \times$ ), except in two cases where the differences exceed a factor of $2 \times$. In most cases the predicted crack initiation lives are conservative, i.e. shorter than the actual crack initiation lives. Interestingly, if the lifeprediction analysis is repeated for the outlying points using the upper and lower bound small crack growth rate curves, the differences between the predicted and actual crack initiation lives can be resolved in most cases. This indicates that the differences between the predicted and actual crack initation lives can be mainly attributed to variability in the small fatigue crack propagation data. Wu et al. [277] and Defresne and Remy [34] have reported similar successes in predicting fatigue lives in single-crystal materials using LEFM, short FCGR data and by treating surface and subsurface pores as semi-elliptical or embedded elliptical cracks, respectively. 


\subsection{Concluding Remarks}

The growth of naturally-generated small fatigue cracks a single-crystal superalloy was investigated at $650^{\circ} \mathrm{C}$ in air using SEN specimens with two crystallographic orientations A: [010]-[101] and B: [010]-[100]. The current focusing ACPD technique was used successfully to characterize the small crack growth behaviour for thumbnail cracks with crack sizes in the range of $200<c<1000 \mu \mathrm{m}$. The FCGR data obtained from the fatigue experiments was analyzed to determine the crack initiation life as defined in the safe-life approach, i.e. the number of cycles to form a $760 \mu \mathrm{m}$ surface crack. The crack initation life exhibited a $2-4 \times$ variability when plotted as function of the applied (far-field) stress range. Orientation A specimens exhibited longer crack initiation lives than orientation B specimens when compared based on the applied far-field stress range. The effect of subsurface pore size and proximity to specimen notch surface on the crack initiation life was also examined. The crack initiation lives were shorter in fatigue specimens containing larger subsurface pores and a secondary orientation dependence of the crack initiation lives was still apparent. No trends were apparent when crack initiation life was plotted as a function of pore proximity to the notch surface. When the local elastic-plastic stress acting at the subsurface pores location was taken into consideration, the crack initiation lives still exhibited significant scatter. The secondary orientation effect on the crack initiation life could be predominantly explained by orientation B exhibiting higher elastic-plastic stresses in the notch.

The application of the anisotropic stress-intensity factor and the elastic-plastic J-integral to the small FCGR data obtained in this study was investigated. The stress-intensity factor range generally collapsed the $d a / d N$ and $d c / d N$ crack growth rate curves onto a single FCGR curve. Collapse of the $d a / d N$ and $d c / d N$ crack growth rate curves using the 
elastic-plastic J-integral results for notch-root fatigue cracks were unsuccessful. A FCGR interpolation approach was used to compare FCGR data obtained from multiple fatigue experiments at the same $\Delta K$ level, and for conducting statistical analysis of the small FCGR data. The statistical analysis of the small FCGR data revealed that the variability in crack propagation rates was generally higher at lower $\Delta K$ levels, but the average variability $(2-3 x)$ was quite similar to values reported in the literature for long fatigue cracks. The mean FCGR curves obtained from statistical analysis of the small crack data for orientation $\mathrm{A}$ and $\mathrm{B}$ specimens revealed that no secondary orientation dependence could be established for small thumbnail cracks with depths in the $200<c<1000 \mu \mathrm{m}$ range from the variability in the FCGR data. Fatigue crack growth rates in the $\Delta K$ range of $23-40 \mathrm{MPa}-\mathrm{m}^{1 / 2}$ for orientation $\mathrm{A}$ and $\mathrm{B}$ specimens were found to behave according to a Paris relationship.

A LEFM approach for predicting crack initiation life was developed to predict the crack initiation life of individual SEN specimens. The life prediction method considers both the growth of embedded fatigue cracks originating from subsurface pores and their continued growth as surface flaws. The geometry of the subsurface pores, their proximity to the notch root, and the local stress acting at the pore were incorporated into the life prediction method. In general, the crack initiation life predictions were quite good, but differences exceeding a factor of $2 \times$ were observed in two cases. The predicted crack initiation lives were generally conservative. The differences observed between the predicted and actual crack initiation lives were predominantly due to variability in the small fatigue crack propagation data. 
Table 10.1 - Crack initation lives for each fatigue specimen.

\begin{tabular}{|c|c|c|}
\hline Orientation & Specimen ID & $\mathrm{N}_{\mathrm{i}}$ (cycles) \\
\hline \multirow{4}{*}{$\mathrm{A}$} & $39-2$ & 60,350 \\
\cline { 2 - 3 } & $40-1$ & 25,930 \\
\cline { 2 - 3 } & $41-1$ & 27,175 \\
\cline { 2 - 3 } & $41-2$ & 38,900 \\
\cline { 2 - 3 } & $42-2$ & 39,350 \\
\cline { 2 - 3 } & $44-2$ & 50,190 \\
\hline \multirow{4}{*}{$\mathrm{B}$} & $22-1$ & 10,890 \\
\cline { 2 - 3 } & $23-1$ & 37,125 \\
\cline { 2 - 3 } & $26-1$ & 16,415 \\
\cline { 2 - 3 } & $29-1$ & 20,684 \\
\cline { 2 - 3 } & $30-1$ & 42,320 \\
\cline { 2 - 3 } & $35-2$ & 12,745 \\
\hline
\end{tabular}

Table 10.2 - Paris law parameters for the mean and upper and lower bound fatigue crack growth rate curves.

\begin{tabular}{|l|c|c|}
\hline Fitted Line & $\mathrm{C}(\mathrm{m} /$ cycle $)$ & $\mathrm{m}$ \\
\hline Mean & $1.27 \mathrm{E}-10$ & 1.89 \\
\hline Mean + 2 SD & $4.10 \mathrm{E}-10$ & 1.64 \\
\hline Mean - 2 SD & $3.93 \mathrm{E}-11$ & 2.13 \\
\hline
\end{tabular}


Table 10.3 - Comparison of predicted, $\mathbf{N}_{\mathrm{p}}$, and actual, $\mathbf{N}_{\mathrm{a}}$, crack initation lives. Note: number in parenthses indicates the life spent as a subsurface fatigue crack and asterisk indicates subsurface life for the dominant subsurface crack.

\begin{tabular}{|c|c|c|c|}
\hline Orientation & Specimen ID & $\mathrm{N}_{\mathrm{a}}$ (cycles) & $\mathrm{N}_{\mathrm{p}}$ (cycles) \\
\hline \multirow{4}{*}{$\mathrm{A}$} & $39-2$ & 60,350 & $30,600(26,400)$ \\
\cline { 2 - 4 } & $40-1$ & 25,930 & $18,750(11,175)$ \\
\cline { 2 - 4 } & $41-1$ & 27,175 & $16,800(10,425)$ \\
\cline { 2 - 4 } & $41-2$ & 38,900 & $22,200(15,675)$ \\
\cline { 2 - 4 } & $42-2$ & 39,350 & $25,275(20,850)$ \\
\cline { 2 - 4 } & $44-2$ & 50,190 & $16,500(8,550)$ \\
\hline \multirow{4}{*}{ B } & $22-1$ & 10,890 & $16,950(10,500)^{*}$ \\
\cline { 2 - 4 } & $23-1$ & 37,125 & $20,700(13,725)$ \\
\cline { 2 - 4 } & $26-1$ & 16,415 & $18,825(13,050)$ \\
\cline { 2 - 4 } & $29-1$ & 20,684 & $17,475(8,700)$ \\
\cline { 2 - 4 } & $30-1$ & 42,320 & $27,075(21,225)$ \\
\cline { 2 - 4 } & $35-2$ & 12,745 & $11,400(5,175)^{*}$ \\
\hline
\end{tabular}




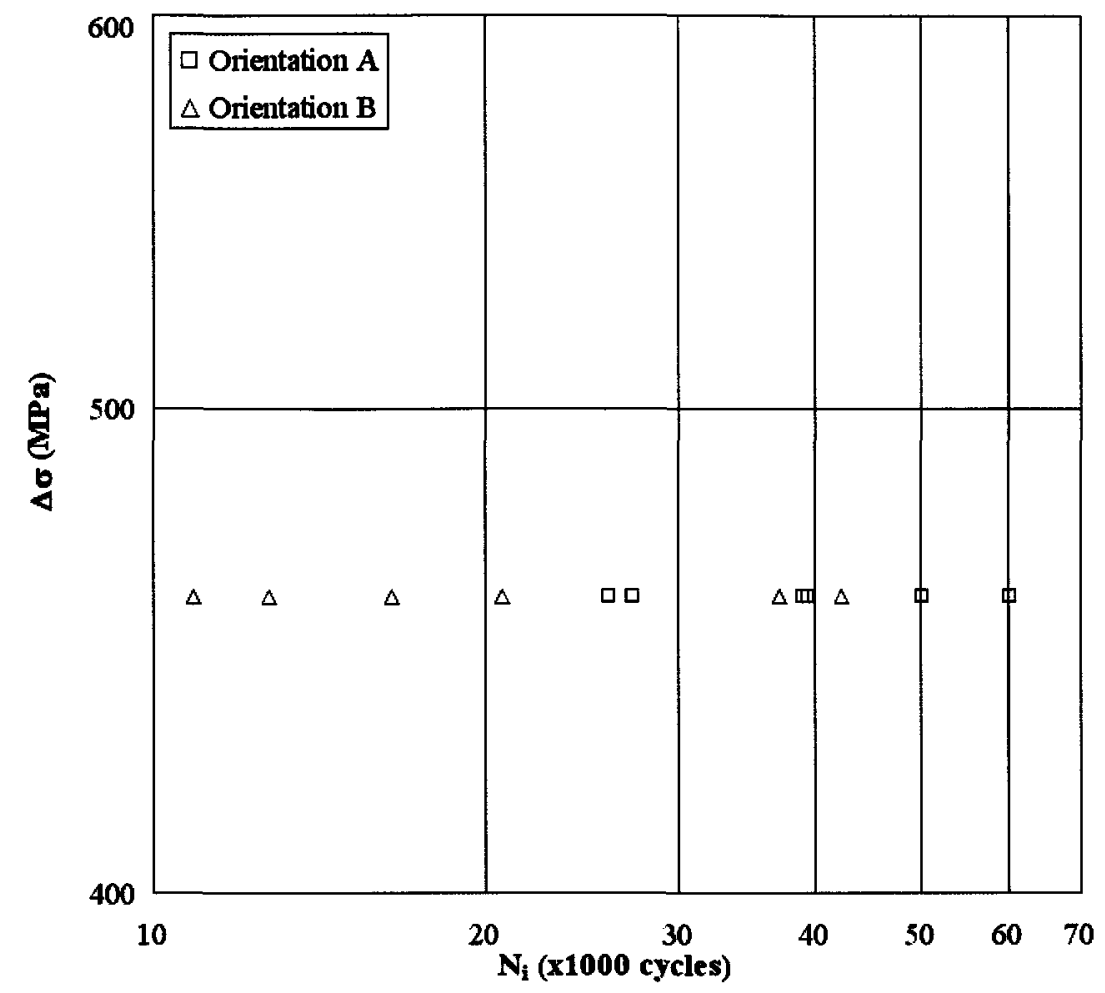

Figure 10.1 - Stress range versus crack initation life for orientation A and B SEN fatigue specimens.

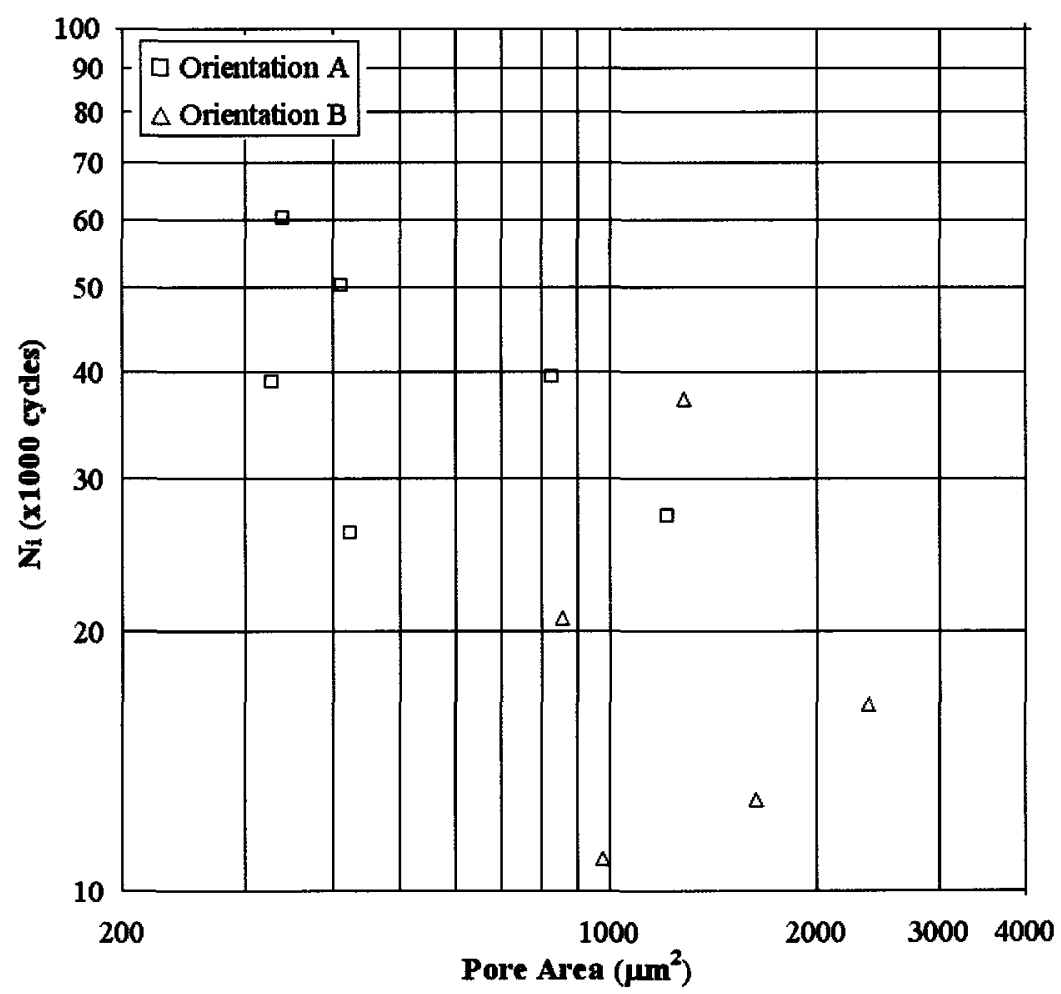

Figure 10.2 - Crack initiation life versus total microporosity area. 


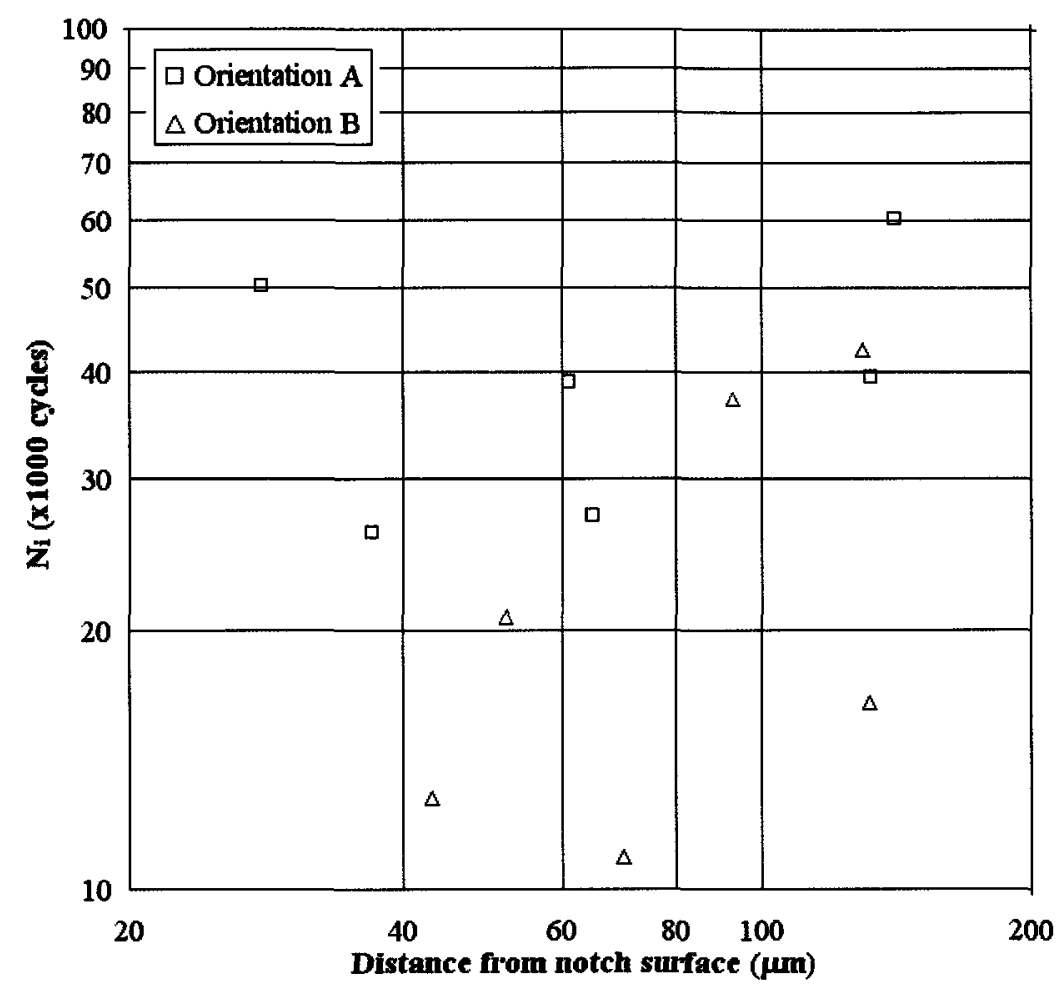

Figure 10.3 - Crack initiation life versus distance between subsurface micropore and the notch surface.

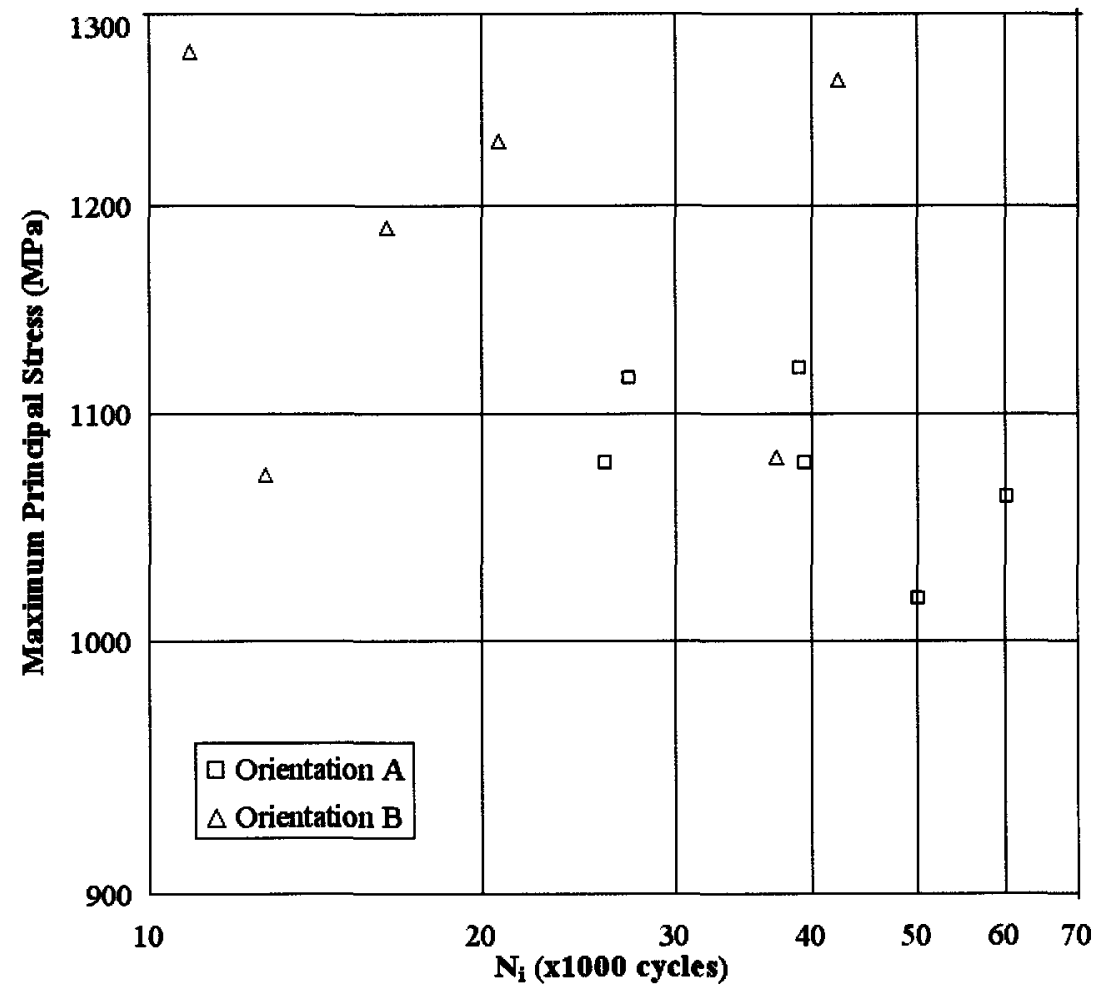

Figure 10.4 - Crack initation life as a function of the local maximum principal elastic/plastic stress acting at the subsurface pore associated with the dominant fatigue crack. 


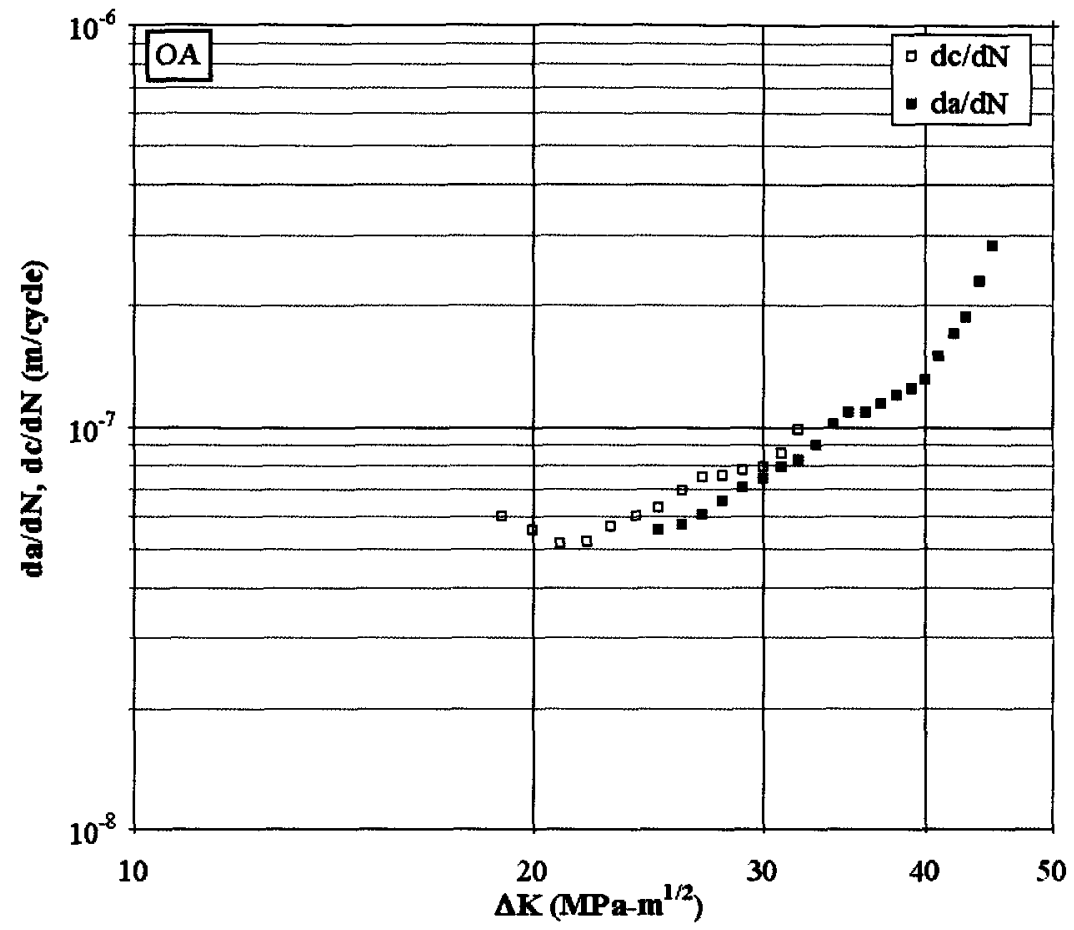

Figure 10.5 - Fatigue crack growth rate for orientation A SEN specimen as a function of the anisotropic stress-intensity factor range. Note: $d a / d N$ refers to crack growth rate at the notch surface and dc/dN is the crack growth rate at the deepest part of the crack.

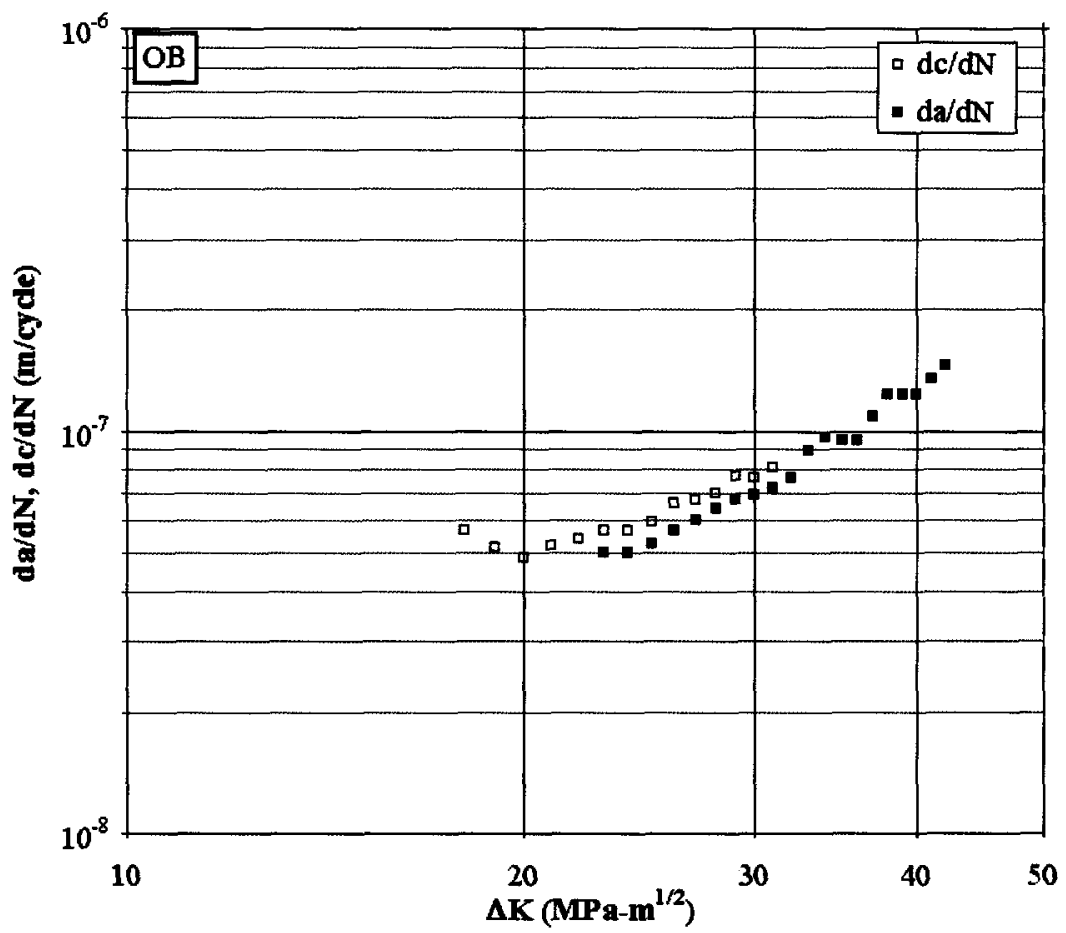

Figure 10.6 - Fatigue crack growth rate for orientation B SEN specimen as a function of the anisotropic stress-intensity factor range. Note: $\mathrm{da} / \mathrm{dN}$ refers to crack growth rate at the notch surface and dc/dN is the crack growth rate at the deepest part of the crack. 


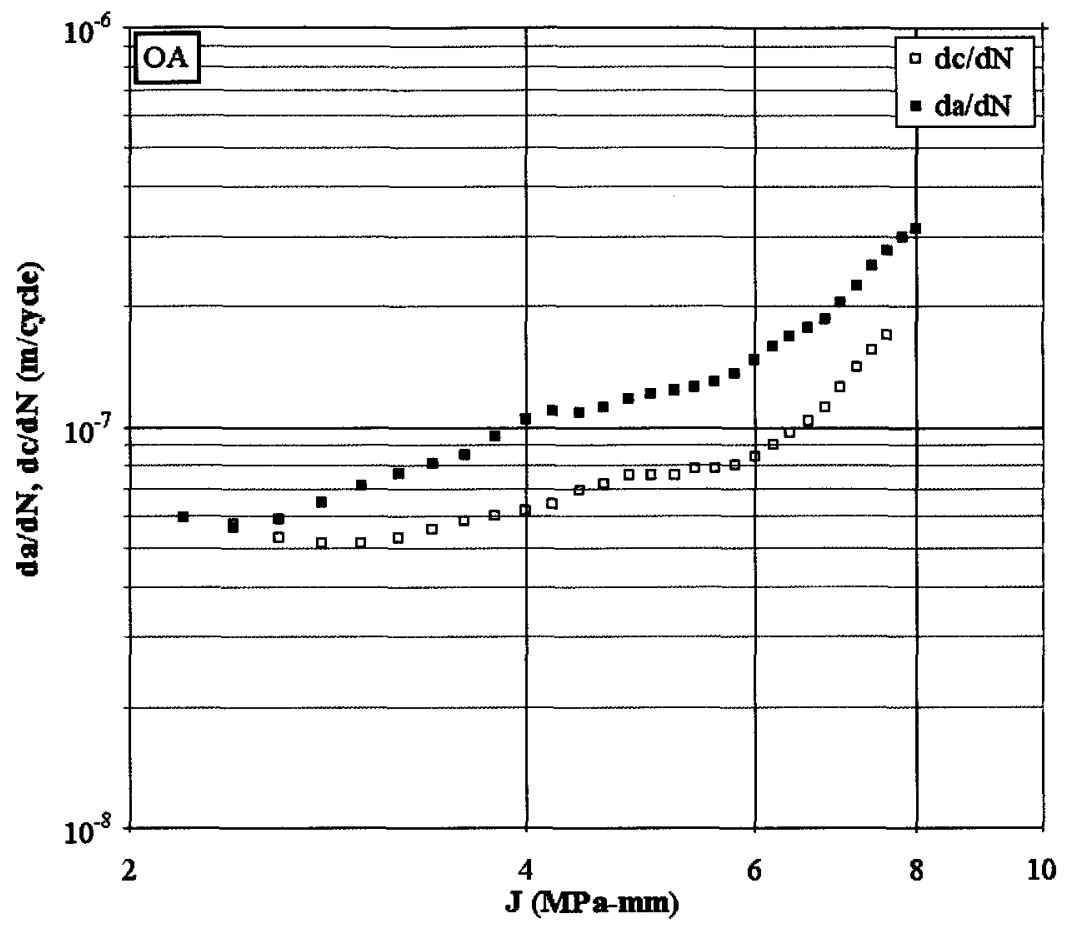

Figure 10.7 - Fatigue crack growth rate for orientation A SEN specimen as a function of the anisotropic elastic-plastic $J$-integral. Note: $d a / d N$ refers to crack growth rate at the notch surface and dc/dN is the crack growth rate at the deepest part of the crack.

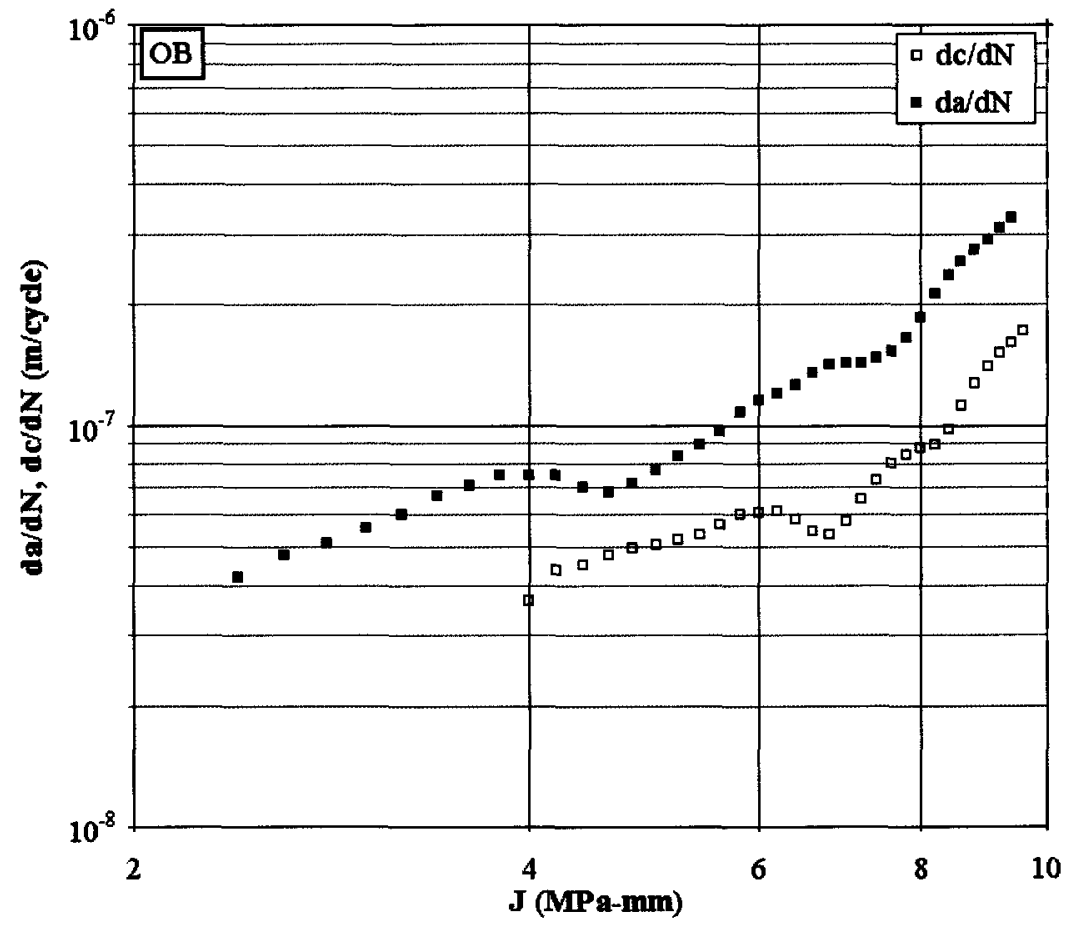

Figure 10.8 - Fatigue crack growth rate for orientation B SEN specimen as a function of the anisotropic elastic-plastic J-integral. Note: $\mathrm{da} / \mathrm{dN}$ refers to crack growth rate at the notch surface and $\mathrm{dc} / \mathrm{dN}$ is the crack growth rate at the deepest part of the crack. 


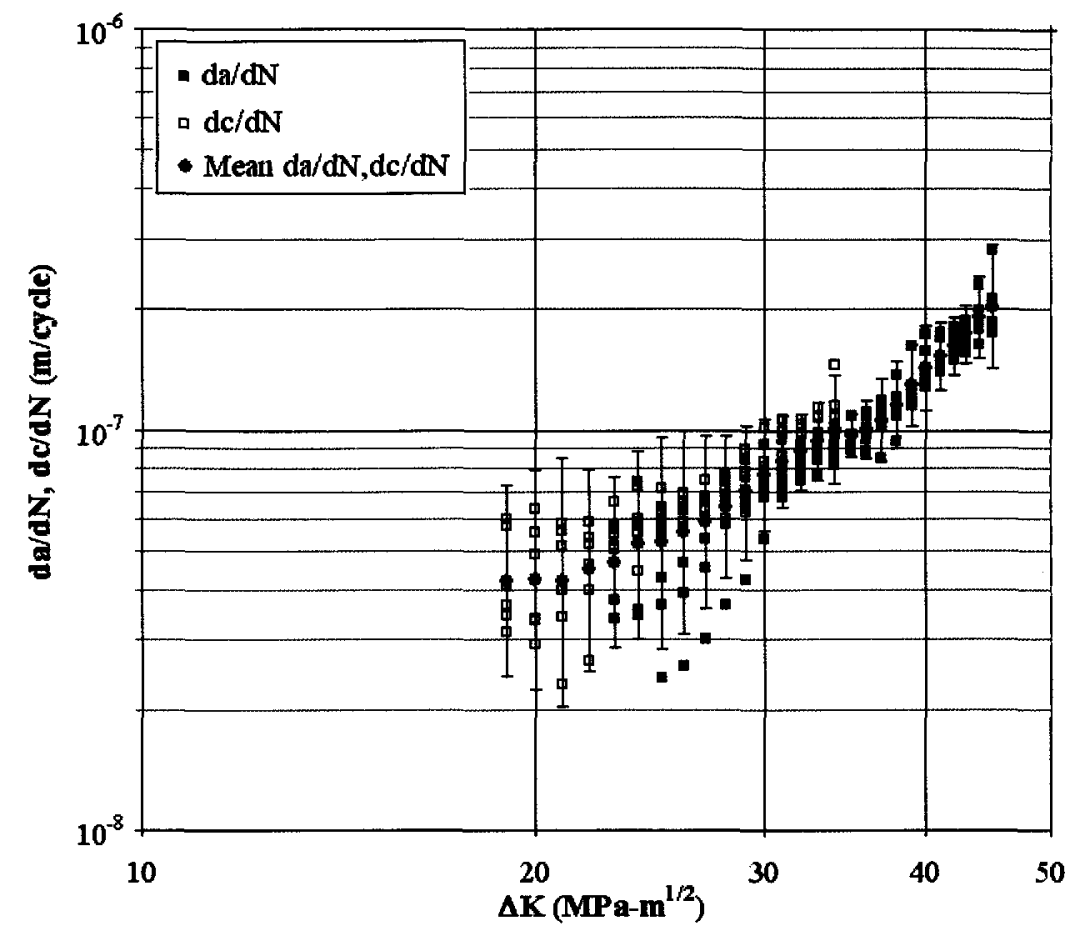

Figure 10.9 - Composite of crack growth curves for orientation A SEN specimens. The mean crack growth rate is indicated by red circles and the error bars denote two standard deviations from mean. Note: $\Delta \mathrm{K}$ was obtained from anisotropic elastic analysis.

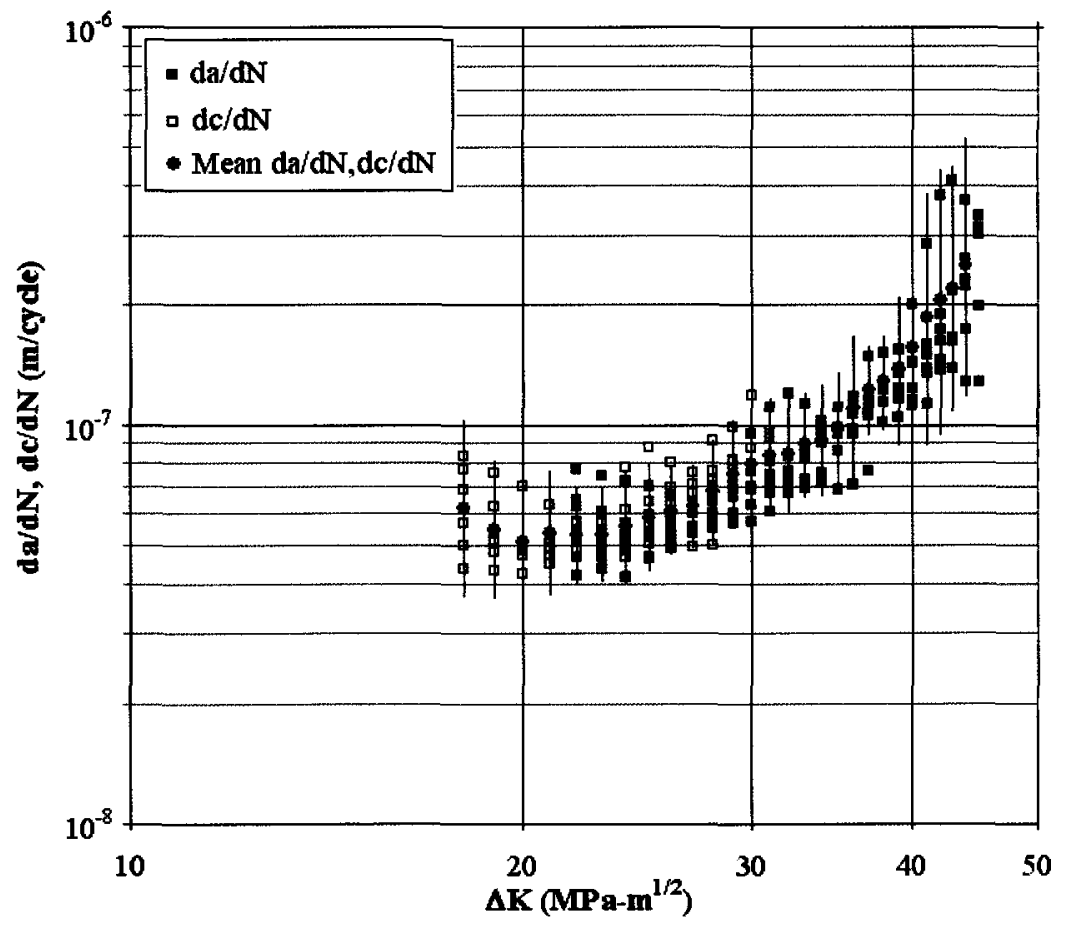

Figure 10.10 - Composite of crack growth curves for orientation B SEN specimens. The mean crack growth rate is indicated by red circles and the error bars denote two standard deviations from mean. Note: $\Delta K$ was obtained from anisotropic elastic analysis. 


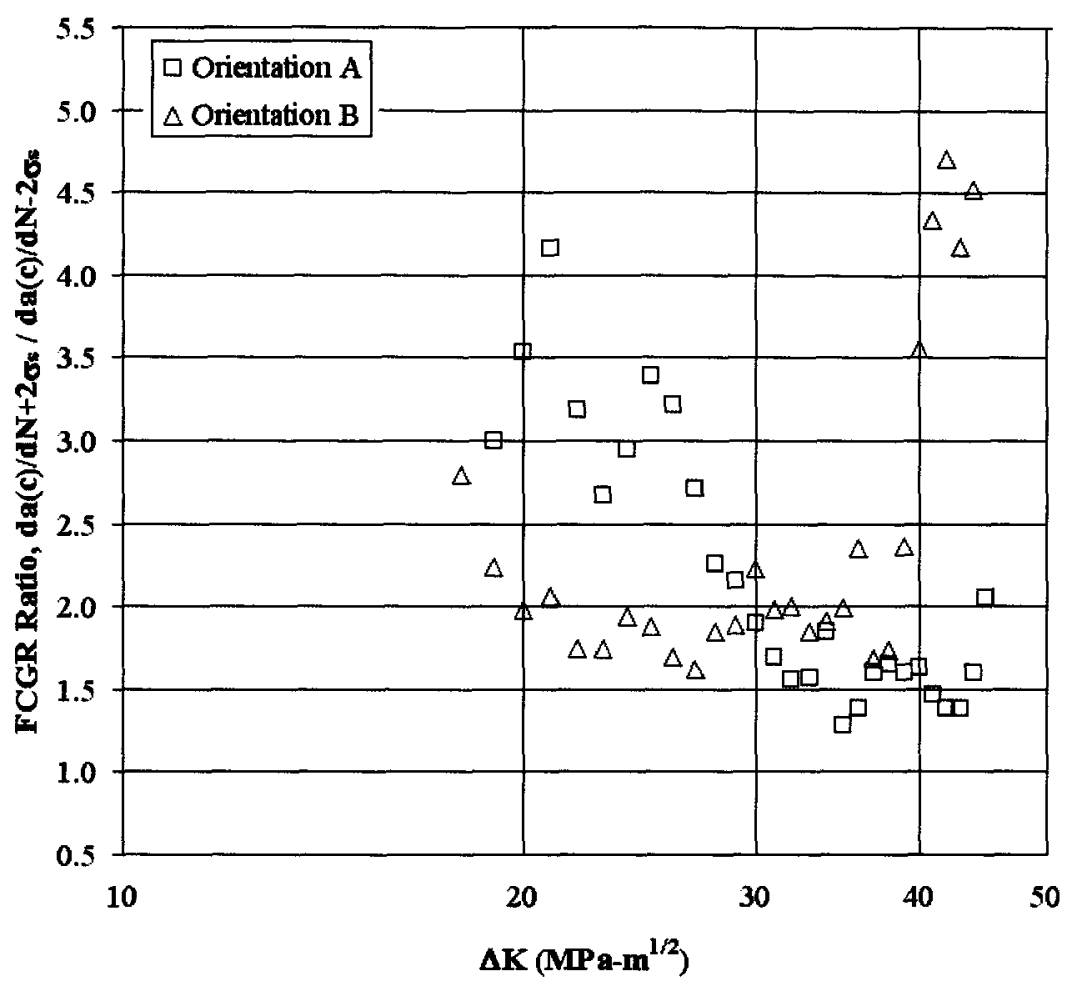

Figure 10.11 - Variation of the fatigue crack growth rate as a function of the stress-intensity factor range. Note: $\Delta K$ was obtained from anisotropic elastic analysis.

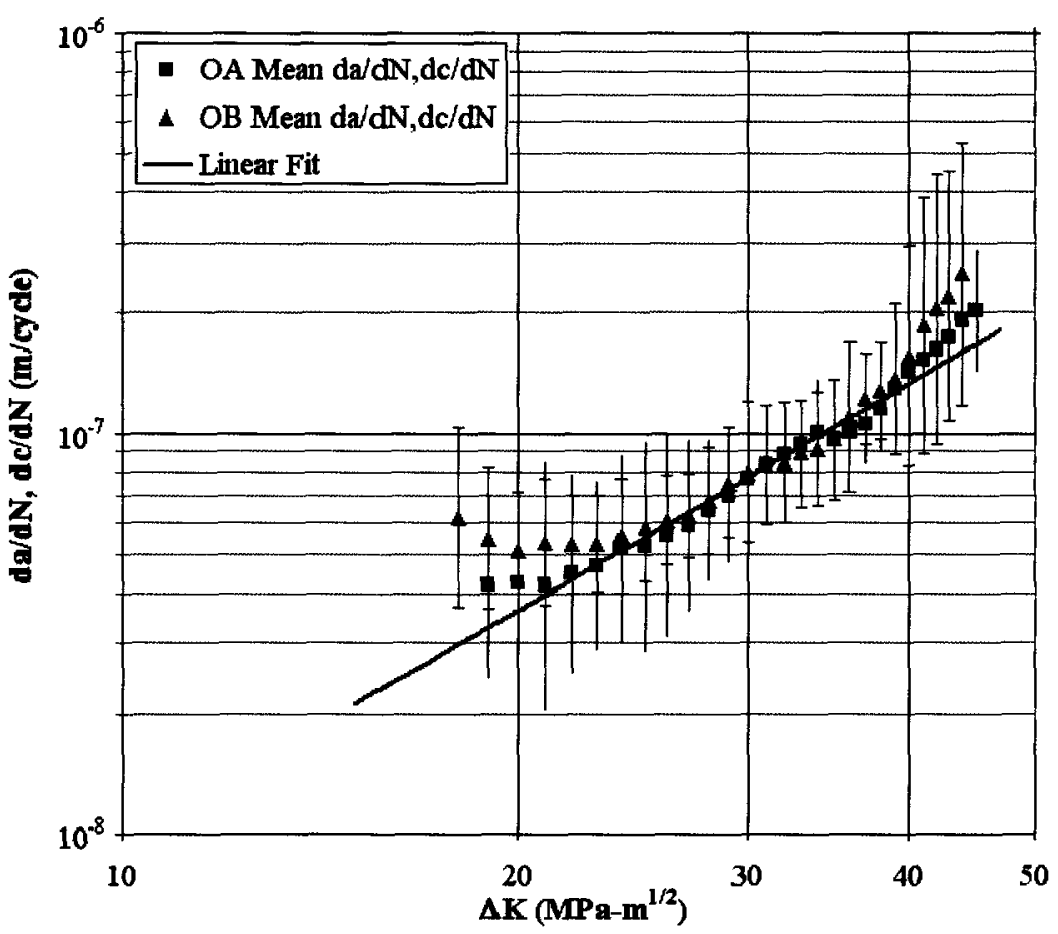

Figure 10.12 - Comparison of mean crack growth rate curves for orientation A and B SEN specimens. The error bars denote two standard deviations from the mean crack growth rate for each orientation. Note: $\Delta K$ was obtained from anisotropic elastic analysis. 


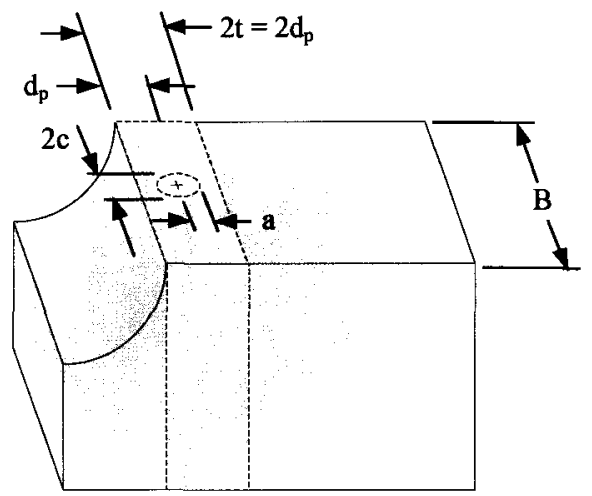

a)

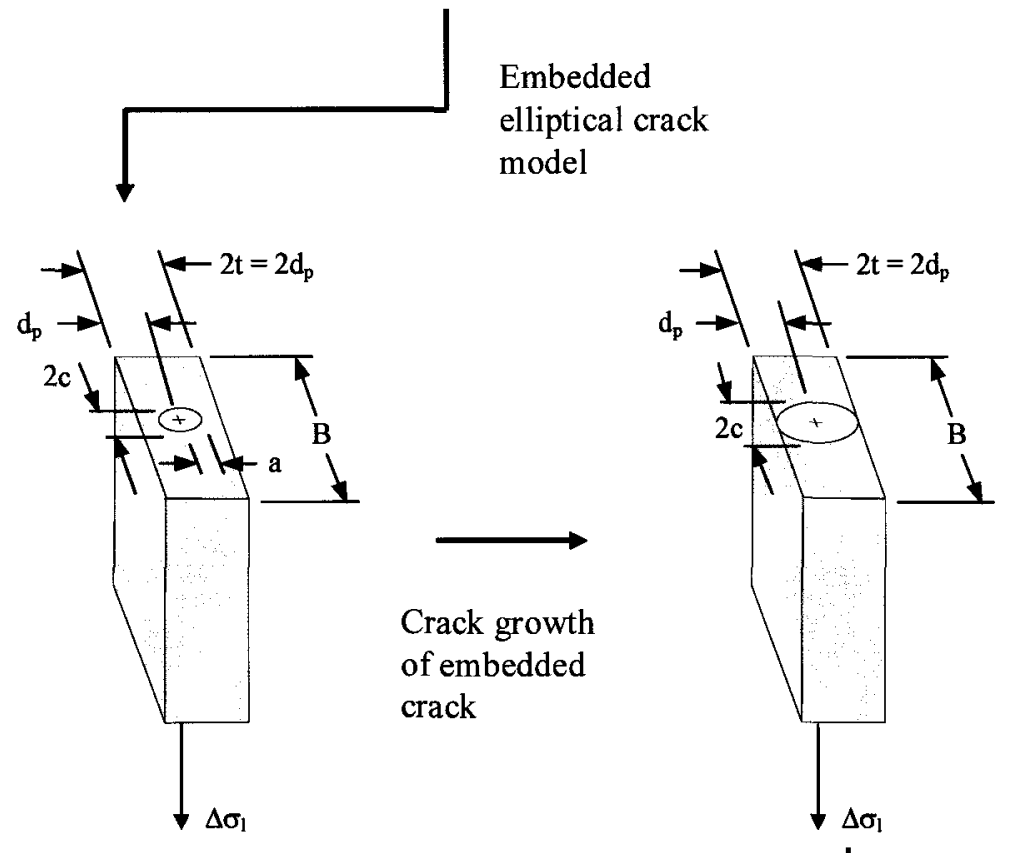

b)

Semi elliptical

surface crack growth

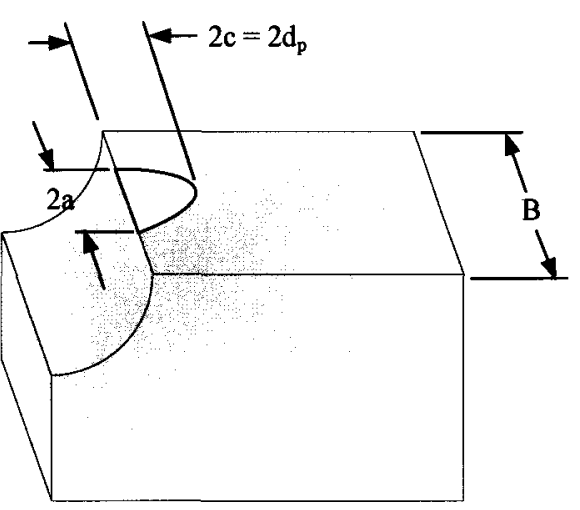

c)

Figure 10.13 - Schematic depicting LEFM life prediction methodology: a) embedded elliptical pore in SEN specimen, b) growth of embedded elliptical crack in strip with local stress range obtained from elastic/plastic FEM model and c) semi-elliptical crack in notch. 


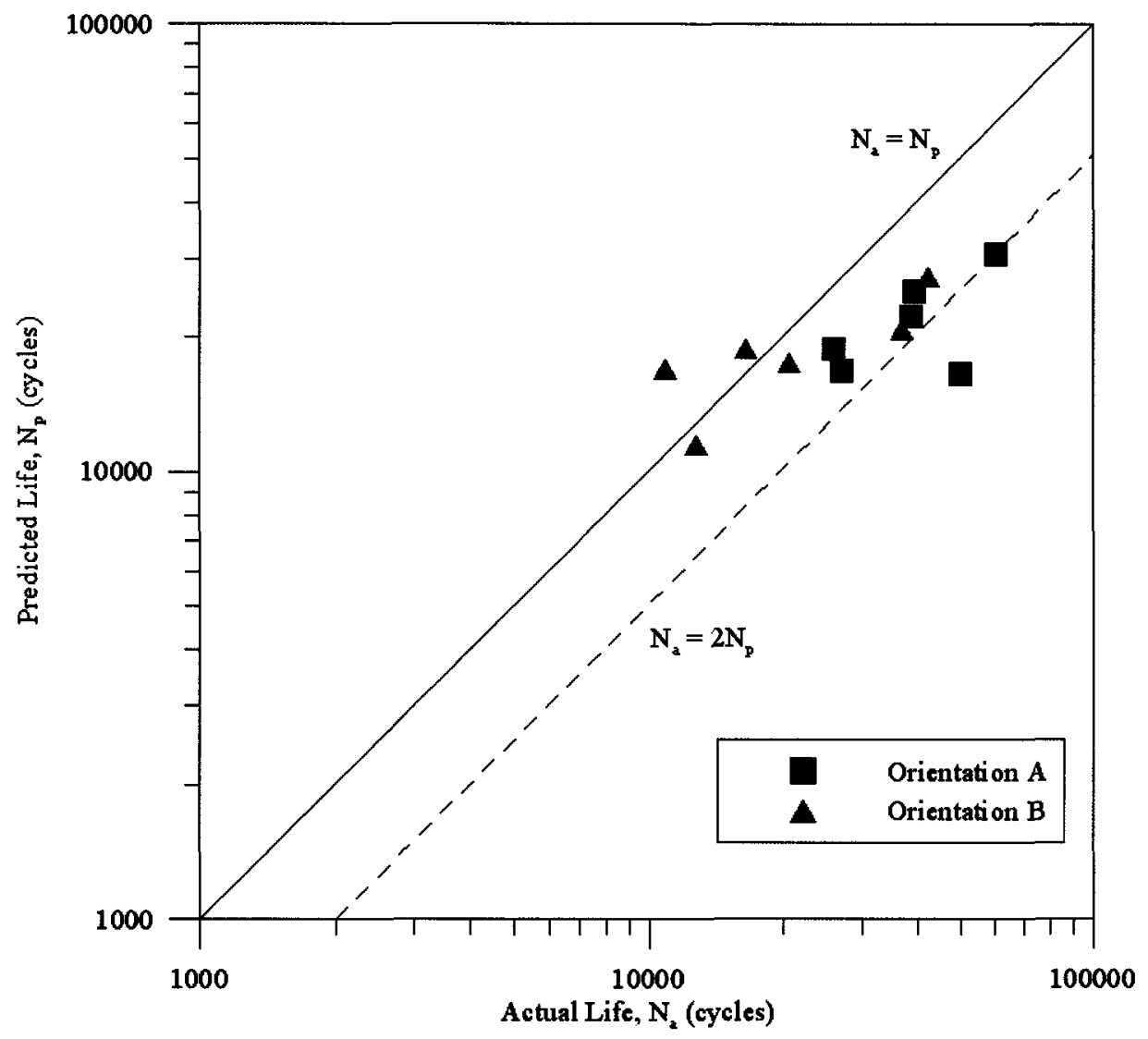

Figure 10.14 - Comparison of predicted fatigue lives for orientation A and B SEN specimens. 


\section{General Conclusions and Recommendations for Future Work}

\subsection{General Conclusions}

The main goals of the research described in this thesis were to investigate the effect of secondary crystallographic orientation on the growth of naturally-generated small fatigue cracks, to identify the critical factors controlling the notch fatigue life and to investigate the application of LEFM and EPFM crack-tip parameters to characterize small crack growth behaviour in a single-crystal nickel-base superalloy. The project was divided into to an analytical component, which was used to establish the effects of elastic and elasticplastic anisotropy on the notch root fields and to calculate LEFM and EPFM crack-tip parameters for notch root thumbnail cracks, and an experimental component, which consisted of conducting small FCGR experiments at $650^{\circ} \mathrm{C}$ on notched fatigue specimens to simulate the stress concentration associated with turbine blade attachment features.

Finite element calculations were performed to estimate the notch root stress and strain fields, and the stress-intensity factors and the J-integrals for notch root semi-elliptical cracks $(200 \mu \mathrm{m}<c<1000 \mu \mathrm{m})$ for the SEN specimen used in this investigation. The effect of secondary crystallographic orientation was investigated in the numerical simulations using two notch orientations [101] (orientation A) and [100] (orientation B) with the loading axis of the specimen aligned with the [010] crystallographic direction. For anisotropic elastic-plastic FEM simulations, a strain rate-independent microphenomenological constitutive model formulated based on crystal plasticity was successfully implemented using the ABAQUSIStandard UMAT subroutine interface. The linear elastic FEM calculations were performed using isotropic and cubic anisotropic 
elasticity. From the elastic and elastic-plastic notch fields FEM simulations the following conclusions can be made.

For the isotropic model, the maximum notch root stresses and strains were located at the minimum specimen cross section. The maximum stresses in the notch root were located approximately $30^{\circ}$ from the minimum specimen cross-section for the cubic anisotropic elastic models. The stresses in the notch root were higher for orientation B than orientation A models under the same far-field loading conditions by approximately $8 \%$. The notch root stresses were lower for the cubic anisotropic elastic models than the isotropic model by approximately $7.5 \%$ and $16 \%$ for orientation B and A, respectively. For the elastic-plastic FEM model, the maximum notch root stresses for orientations A and $\mathrm{B}$ were located approximately $24^{\circ}$ from the minimum specimen cross-section. The notch root stresses were higher for orientation B than orientation A by approximately $7.5 \%$ under a far-field load of $29 \mathrm{kN}$.

From the FEM calculations performed to estimate the stress-intensity factors and Jintegral for notch root semi-elliptical cracks $(200 \mu \mathrm{m}<c<1000 \mu \mathrm{m})$, the following conclusions can be made.

The variation and magnitude of the stress-intensity factors along the crack-front was different for isotropic and cubic anisotropic elastic models along the entire crack-front under the same far-field loading conditions and with the same crack geometry parameters. The stress-intensity factors obtained from an isotropic analysis were within $20 \%$ of those predicted using cubic anisotropic elasticity along the entire crack front, which suggests that the effects of elastic anisotropy should be taken into consideration for embedded notch root cracks. At the notch surface $\left(\phi=90^{\circ}\right)$ and the maximum crack 
depth $\left(\phi=0^{\circ}\right)$, the anisotropic stress-intensity factors were larger for orientation A than B by up to $10 \%$, depending on the crack size considered.

The elastic-plastic J-integral variation along the crack-front exhibited marked secondary orientation dependence. In particular, the J-integral at the maximum crack depth was significantly higher (up to a factor of $2 \times$ ) for orientation B than orientation A. The effect of elastic-plastic anisotropy on the J-integral was less apparent for larger crack sizes but it was generally higher at the notch surface and maximum crack depth for orientation B than orientation A. Localization of plastic shear strain into specific slip sectors at cracktip was observed in the FEM calculation results. In general, the slip sectors corresponded to theoretically predicted values reported in the literature.

Small fatigue crack growth experiments were conducted using single-edge notch (SEN) specimens with a [010] primary orientation and two secondary crystallographic orientations, [101] (orientation A) and [100] (orientation B). Based on the small fatigue crack growth experiments the following conclusions can be made.

The current focusing ACPD technique was successfully implemented to facilitate the detection of surface cracks and the measurement of small fatigue crack growth properties in the SEN specimens. It was shown that this technique was capable of reliably detecting surface-flaws with crack depths as small as $200 \mu \mathrm{m}$. The detection of smaller cracks was primarily inhibited by the subsurface nucleation of fatigue cracks at interdendritic shrinkage porosity. A marker banding technique was successfully used to investigate the variation in crack shape with crack size and to develop calibration curves that relate the ACPD voltage change with crack size for a crack depth range of $200<c<1000 \mu \mathrm{m}$. 
Fractographic analysis of SEN specimens revealed that small fatigue cracks predominantly nucleated from subsurface interdendritic pores and that the relative size and the depth of the pores from the notch surface was different in each fatigue specimen. Initial crack growth occurred by expansion of these material discontinuities until the subsurface crack reached the notch surface after which the fatigue cracks developed into semi-elliptical surface cracks. The small fatigue cracks were macroscopically planar and propagated mainly as Stage II non-crystallographic cracks on a plane normal to the applied loading. In general, the majority of fatigue cracks formed on planes that were located $15-25^{\circ}$ from the minimum specimen cross-section. Fatigue crack propagation at the microscale predominantly involved crack propagation along the $\gamma$ matrix channels. There were no apparent differences in the fatigue crack propagation mechanisms at the microscale for orientation A versus orientation B SEN specimens.

The stress-intensity factor range was found to collapse the FCGR curves obtained from the notch surface $(d a / d N)$ and the maximum crack depth $(d c / d N)$ onto a single FCGR curve. Collapse of the $d a / d N$ and $d c / d N$ crack growth rate curves using the elastic-plastic J-integral results for notch-root fatigue cracks was unsuccessful. The mean FCGR curves obtained from statistical analysis of the small FCGR data for orientation A and B specimens revealed that no secondary orientation dependence could be established for small thumbnail cracks with depths in the $200<c<1000 \mu \mathrm{m}$ range from the variability in the FCGR data. Fatigue crack growth rates in the $\Delta K$ range of $23-40 \mathrm{MPa}-\mathrm{m}^{1 / 2}$ for orientation A and B specimens were found to behave according to a Paris relationship.

The crack initiation life, based on the formation of a $760 \mu \mathrm{m}$ surface crack, exhibited a 2$4 \times$ variability when plotted as function of the applied (far-field) stress range. Based on stress range versus crack initiation life, Orientation A specimens generally exhibited 
longer lives than orientation B specimens. The crack initiation lives were also determined to be shorter in fatigue specimens containing larger subsurface pores. The secondary orientation effect on the crack initiation life could be partially rationalized by the effect of elastic-plastic anisotropy on the stresses acting at the subsurface pore(s) from which the fatigue crack(s) originated.

A holistic lifing methodology that utilized the small crack growth data obtained in this investigation and took into consideration the initial size, location and the local stress acting at material discontinuities was developed to predict the crack initiation life for individual SEN specimens. The life prediction method considered both the growth of embedded fatigue cracks originating from subsurface pores and their continued growth as surface flaws. The life predictions were generally within a factor of $2-3 \times$ of the actual crack initiation life.

From the small fatigue crack growth rate experiments it has been established that under the same far-field mechanical loading orientation A provides a longer crack initiation life than orientation B. However, the secondary crystallographic orientation is rarely controlled during the single-crystal casting process. The findings from the present research suggest that controlling secondary crystallographic orientation during manufacturing can impart improved crack initiation resistance to the blade fir-tree attachment region of single-crystal parts.

\subsection{Suggested Future Work}

In this investigation a holistic lifing methodology utilizing the short fatigue crack propagation data obtained in this investigation was reasonably successful in predicting 
crack initiation life. However, in order to increase the confidence in the life predictions, additional measures should be taken. First, FEM calculations should be performed to estimate the anisotropic stress-intensity factors for embedded elliptical cracks in the notch root of the SEN specimen. These calculations, at a minimum, should be performed to validate the treatment of pores as embedded elliptical cracks in a plate subjected to the local elastic-plastic stress. To take the notch stress gradients into consideration for pores embedded deeper from the notch, the stress-intensity factors would have to be estimated by some other means such as weight function methods. Second, small fatigue crack growth data in vacuum should be obtained to correctly model the subsurface crack growth. A more comprehensive empirical equation for estimating the surface crack stress-intensity factors needs to be developed to more accurately model the evolution of the crack shape. This solution would have to consider the actual location where fatigue cracks form rather than just the notch root fatigue cracks considered in the present investigation. Lastly, it was observed in some fatigue experiments that the dominant fatigue crack forms by coalescence of one or more subsurface fatigue cracks. The interaction of multiple fatigue cracks was not considered in the stress-intensity factor calculations. This should be investigated further to establish the effects of coplanar and non-coplanar crack interactions on the stress-intensity factors.

The LEFM life predictions demonstrated that the growth of subsurface cracks originating from pores constituted the majority $(50-80 \%)$ of the crack initiation life. Potentially, processing measures such as hot-isostatic pressing, which reduce the size and occurrence of these initial material discontinuities, could result in a significant crack initiation life credit. The effect of hot-isostatic pressing on the formation of naturally generated small fatigue cracks should be investigated further to determine what benefit in crack initiation life can be realized. 


\begin{abstract}
Although the current focusing ACPD technique was successfully used to characterize small fatigue crack growth for crack sizes in the range of $200<c<1000 \mu \mathrm{m}$, it could not unambiguously resolve the growth of subsurface cracks originated from interdendritic porosity. Therefore, a substantial portion of the crack initiation life was not characterized during the small fatigue crack growth experiments. The propagation of smaller fatigue cracks $(c<200 \mu \mathrm{m})$ could be studied by reducing the occurrence of subsurface pores by hot-isostatically pressing the material to promote the formation of surface cracks. There could also be some significant advantages to utilizing fatigue specimen designs in which the crack location can be more readily controlled and a smaller volume of material is tested, such as corner crack fatigue specimens.
\end{abstract}




\section{References}

[1] C. A. Yablinsky, K. M. Flores, M. J. Mills, J. C. Williams, and J. Rigney, "Fatigue Behaviour in Monocrystalline Ni-Based Superalloys for Blade Applications," in Proceedings of the International Symposium on Superalloys 2008, R. C. Reed, K. A. Green, P. Caron, T. P. Gabb, M. G. Fahrmann, E. S. Huron, and S. A. Woodard, Eds. Champion PA: TMS, The Metals, Minerals and Materials Society, Warrendale (USA), 2008, pp. 535-541.

[2] B. A. Cowles, "High Cycle Fatigue in Aircraft Gas Turbines - an Industry Perspective," Int. J. Fract., vol. 80, pp. 147-163, 1996.

[3] M. Sakamoto, H. Harada, T. Yokokawa, Y. Koizumi, T. Kobayashi, H. Zhou, J. X. Zhang, and N. Miyamota, "Assessment on the Thermo-mechanical Fatigue Properties of 98 Ni-base Single Crystal Superalloys," in Proceedings of the International Symposium on Superalloys 2008, R. C. Reed, K. A. Green, P. Caron, T. P. Gabb, M. G. Fahrmann, E. S. Huron, and S. A. Woodard, Eds. Champion PA: TMS, The Metals, Minerals and Materials Society, Warrendale (USA), 2008, pp. 521-526.

[4] I. Linask and J. Dierberger, "Fracture Mechanics Approach to Turbine Airfoil Design," ASME Publication 75-GT-79, pp. 2-8, 1975.

[5] D. P. Deluca and C. Annis, "Fatigue in Single Crystal Nickel Superalloys Technical Progress Report 16 October 1993 - 15 October 1994," Report No. FR21998-24, United Technologies Pratt \& Whitney 15 October 1994.

[6] G. R. Swanson and N. K. Arakere, "Effect of Crystal Orientation on Analysis of Single-Crystal, Nickel-Based Turbine Blade Alloys," NASA Technical Publication, NASA/TP-2000-210074 2000.

[7] D. P. Deluca and C. Annis, "Fatigue in Single Crystal Nickel Superalloys - Interim Technical Report 16 September 1991 - 15 October 1993," Report No. FR21998-20, United Technologies Pratt \& Whitney, West Palm Beach 15 January 1993.

[8] C. Howland, M. A. Hicks, and R. H. Jeal, "The Relevance of Short Crack Behaviour to the Integrity of Major Rotating Aero Engine Components," in Small fatigue cracks : proceedings of the second Engineering Foundation international conference/workshop, January 5-10, 1986, R. O. Ritchie and J. Lankford, Eds. Santa Barbara, California, 1986, pp. 607-622.

[9] B. A. Cowles, "Life Prediction in High Temperature Environments: Overview of a Current Gas Turbine Engine Approach," Mat. Sci. Eng., vol. A103, pp. 63-69, 1988.

[10] D. W. Hoeppner, "What is HOLSIP," in Holistic Structural Integrity Process, HOLSIP 8 Salt Lake City, UT, 2009. 
[11] S. H. Ai, V. Lupinc, and G. Onofrio, "Influence of Precipitate Morphology on High Temperature Fatigue Crack Growth of a Single Crystal Nickel Base Superalloy," Scr. Metall et Mater., vol. 29, pp. 1385-1390, Dec. 1993.

[12] S. H. Ai, V. Lupinc, G. Onofrio, and G. Vimercati, "Influence of Microstructure on Fatigue Crack Growth Behaviour of a Single Crystal Superalloy," in Fatigue 90 Proceedings of the 4th International Fatigue Conference, H. Kitagawa and T. Tanaka, Eds. Honolulu, HI, 1990, pp. 249-254.

[13] B. F. Antolovich, A. Saxena, and S. D. Antolovich, "Fatigue Crack Propagation in Single Crystal CMSX-2 at Elevated Temperature," in Proceedings of the International Symposium on Superalloys 1992, S. D. Antolovich, R. W. Stusrud, R. A. MacKay, D. L. Anton, T. Khan, R. D. Kissinger, and D. L. Klarstrom, Eds. Champion PA: TMS, The Metals, Minerals and Materials Society, Warrendale (USA), 1992, pp. 727-736.

[14] P. B. Aswath, "Effect of Orientation on Crystallographic Cracking in Notched Nickel-Base Superalloy Single Crystal Subjected to Far-Field Compression," Metallurgical and Materials Transactions A, vol. 25A, pp. 287-297, Feb. 1994.

[15] K. S. Chan, J. Feiger, Y. D. Lee, R. John, and J. S. J. Hudak, "Fatigue Crack Growth Thresholds of Deflected Mixed-Mode Cracks in PWA1484," Journal of Engineering Materials and Technology, vol. 127, pp. 2-7, 2005.

[16] K. S. Chan, J. H. Feiger, Y.-D. Lee, and S. J. Hudak, "Mixed-Mode Fatigue Crack Growth Thresholds of a Ni-Based single Crystal Alloy," in Fatigue - David L. Davidson Symposium, K. S. Chan, P. K. Liaw, R. S. Bellows, T. C. Zogas, and W. O. Soboyejo, Eds. TMS Annual Meeting, 17-21 Feb. 2002, Seattle, WA.: TMS, The Minerals, Metals \& Materials Society, 2002, pp. 135-148.

[17] K. S. Chan, J. E. Hack, and G. R. Leverant, "Fatigue Crack Propagation in NickelBase Superalloy Single Crystals Under Multiaxial Cyclic Loads," Metall. Trans. A, vol. 17A, pp. 1739-1750, Oct. 1986.

[18] K. S. Chan, J. E. Hack, and G. R. Leverant, "Fatigue Crack Growth in MAR-M200 Single Crystals," Metall. Trans. A, vol. 18A, pp. 581-591, Apr. 1987.

[19] M. B. Henderson and J. W. Martin, "The Effect of Gamma-Prime Phase Distribution on Elevated Temperature Fatigue Crack Propagation in a Single Crystal Superalloy," in High Temperature Materials for Power Engineering 1990, Proceedings of a Confernce, E. Bachelet, R. Brunetaud, D. Coutsouradis, P. Esslinger, J. Ewald, I. Kvernes, Y. Lindblom, D. B. Meadowcroft, V. Regis, R. B. Scarlin, K. Schneider, and R. Singer, Eds. Liege, Belgium, 24-27 Sept. 1990: Kluwer Academic Publishers, 1990, pp. 987-996.

[20] M. B. Henderson and J. W. Martin, "The Influence of Orientation, Temperature and Frequency on Fatigue Crack Growth," in Proceedings of the International Symposium on Superalloys 1992, S. D. Antolovich, R. W. Stusrud, R. A. MacKay, 
D. L. Anton, T. Khan, R. D. Kissinger, and D. L. Klarstrom, Eds. Champion PA: TMS, The Metals, Minerals and Materials Society, Warrendale (USA), 1992, pp. 707-716.

[21] M. B. Henderson and J. W. Martin, "The Influence of Crystal Orientation on the High Temperature Fatigue Crack Growth of a Ni-Based Single Crystal Superalloy," Acta Mater., vol. 44, pp. 111-126, 1996.

[22] R. L. Holtz and K. Sadananda, "Fatigue Threshold Maps of PWA1480 Superalloy Single Crystal," in High Cycle Fatigue of Structural Materials, W. O. Soboyejo and T. S. Srivatsan, Eds.: TMS, The Metals, Minerals and Materials Society, Warrendale (USA), 1997, pp. 299-304.

[23] Y. Iino, "Effect of High Temperature Air-Exposure on Room Temperature Fatigue Crack Growth of Ni-base Single Crystal Superalloy CMSX-10," Key Engineering Materials, vol. 261-263, pp. 1191-1196, 2004.

[24] M. R. Joyce and P. A. S. Reed, "Fatigue Crack Growth Behaviour Under Mixed Mode Loading in Udimet $720 \mathrm{SX}$," in Proceedings of the International Symposium on Superalloys 2004, K. A. Green, T. M. Pollock, H. Harada, T. E. Howson, R. C. Reed, J. J. Schirra, and S. Walston, Eds. Champion PA: TMS, The Metals, Minerals and Materials Society, Warrendale (USA), 2004, pp. 295-303.

[25] J. M. Martinez-Esnaola, A. Martin-Meizoso, E. E. Affeldt, A. Bennett, and M. Fuentes, "High Temperature Fatigue in Single Crytal Superalloys," Fatigue and Fracture of Engineering Materials and Structures, vol. 20, pp. 771-788, 1997.

[26] P. A. S. Reed and J. E. King, "Comparison of Long and Short Crack Growth in Polycrystalline and Single Crystal Forms of Udimet 720," in Short Fatigue Cracks (ESIS 13) K. J. Miller and E. R. de los Rios, Eds.: Mechanical Engineering Publications, London, 1992, pp. 153-168.

[27] J. Telesman and L. J. Ghosh, "Accelerated Crack Growth Rate at Low $\Delta \mathrm{K}$ in a Single Crystal Superalloy," Int. J. Fract., vol. 37, pp. R19-R22, May 1988.

[28] J. Telesman and L. J. Ghosh, "The Unusual Near-Threshold FCG Behavior of a Single Crystal Superalloy and the Resolved Shear Stress as the Crack Driving Force," Engng. Fract. Mech., vol. 34, pp. 1183-1196, December 19891989.

[29] J. Telesman and L. J. Ghosh, "Fatigue Crack Growth Behavior of PWA1484 Single Crystal Superalloy at Elevated Temperatures," ASME Journal of Engineering for Gas Turbines and Power, vol. 118, pp. 399-405, Apr. 1996.

[30] J. Telesman and L. J. Ghosn, "Accelerated Fatigue Crack Growth Behavior of PWA1480 Single Crystal Alloy and its Dependence on the Deformation Mode," in Proceedings of the International Symposium on Superalloys 1988, S. Reichman, D. N. Duhl, G. Maurer, S. D. Antolovich, and C. Lund, Eds. Champion PA: TMS, The Metals, Minerals and Materials Society, Warrendale (USA), 1988, pp. 615-624. 
[31] A. Defresne and L. Remy, "Low cycle Fatigue Behaviour of Notched Specimens of Nickel Based Superalloy Single Crystal," in Fatigue '87 - Proceedings of the Third International Conference. vol. 3, R. O. Ritchie and E. A. Starke, Eds. Charlottesville, VA, USA, 1987, pp. 1281-1290.

[32] E. Fleury and L. Remy, "Behavior of Nickel-Base Superalloy Single Crystals under Thermal-Mechanical Fatigue," Metall. Trans. A, vol. 25A, pp. 99-109, January 1994.

[33] A. Defresne and L. Remy, "Fatigue Behaviour of CMSX-2 Superalloy [001] Single Crystals at High Temperature II: Fatigue Crack Growth," Mat. Sci. Eng. A, vol. A129, pp. 55-64, 1990.

[34] A. Defresne and L. Remy, "Fatigue Life Prediction of Notched Specimens of Nickel Based Superalloy Single Crystals," in Fatigue 90 - Proceedings of the 4th International Fatigue Congress, H. Kitagawa and T. Tanaka, Eds. Honolulu, HI, 1990, pp. 2435-2440.

[35] M. A. Hicks and C. W. Brown, "A Comparison of Short Crack Growth Behaviour in Engineering Alloys," in Fatigue 84 - Proceedings of the 2nd International Fatigue Conference. vol. 3, C. J. Beevers, Ed. Birmingham, U.K., September 3-7, 1984, pp. 1337-1348.

[36] C. Howland and C. W. Brown, "The Effect of Orientation on Fatigue Crack Growth in a Ni-Based Single Crystal Superalloy," in Fatigue 84 - Proceedings of the 2nd International Fatigue Conference. vol. 3, C. J. Beevers, Ed. Birmingham, U.K., September 3-7, 1984, pp. 1349-1359.

[37] S. X. Li and D. J. Smith, "High-Temperature Fatigue-Creep Crack Initiation and Propagation of a Single Crystal Nickel Base Superalloy," in Sixth International Conference on Creep and Fatigue: Design and Life Assessment at High Temperature, 15-17 Apr., 1996: Mechanical Engineering Publications U.K., 1996, pp. 279-288.

[38] J. Liang, L. Terranova, and R. M. Pelloux, "Notch Fatigue Behavior of Three Advanced Blade Nickel Base Superalloys at $760^{\circ} \mathrm{C}$," High Temperature Materials and Processes, vol. 10, pp. 21-33, 1991.

[39] V. Lupinc and G. Onofrio, "The Effect of Creep and Oxidation on HighTemperature Fatigue Crack Propagation in <001>-loaded CMSX-2 Superalloy Single Crystals," Mat. Sci. Eng. A, vol. A202, pp. 76-83, 1995.

[40] V. Lupinc, G. Onofrio, and G. Vimercati, "The Effect of Creep, Oxidation and Crystal Orientation on High Temperature Fatigue Crack Propagation in Standard adn Raft-Like Gamma Prime CMSX-2," in Proceedings of the International Symposium on Superalloys 1992, S. D. Antolovich, R. W. Stusrud, R. A. MacKay, D. L. Anton, T. Khan, R. D. Kissinger, and D. L. Klarstrom, Eds. Champion PA: 
TMS, The Metals, Minerals and Materials Society, Warrendale (USA), 1992, pp. 717-726.

[41] M. Marx and H. Vehoff, "Propagation of Microcracks in Single Crystalline NickelBased Superalloys: Size Effects on the Crack Opening," Mat. Sci. Eng. A, vol. 387389, pp. 511-515, 2004.

[42] S. Muller, J. Rosler, C. Sommer, and W. Hartnagel, "The Influence of Load Ratio, Temperature, Orientation and Hold Time on Fatigue Crack Growth of CMSX-4," in Proceedings of the International Symposium on Superalloys 2000, T. M. Pollock, R. D. Kissinger, R. R. Bowman, K. A. Green, M. McLean, S. Olson, and J. J. Schirra, Eds. Champion PA: TMS, The Metals, Minerals and Materials Society, Warrendale (USA), 2000, pp. 347-356.

[43] M. Okada, M. Tsutsumi, T. Kitamura, and R. Ohtani, "Initiation and Growth of Small Cracks in Directionally Solidified Mar-M247 Under Creep-Fatigue Part I: Effect of Microstructure," Fatigue and Fracture of Engineering Materials and Structures, vol. 21, pp. 741-750, 1998.

[44] M. Okazaki, H. Yamada, and S. Nohmi, "Temperature Dependence of the Intrinsic Small Fatigue Crack Growth Behavior in Nickel-Base Superalloys Based on Measurement of Crack Closure," Metallurgical and Materials Transactions A, vol. 27A, pp. 1021-1031, Apr. 1996.

[45] M. Okazaki and Y. Yamazaki, "Creep-Fatigue Small Crack Propagation in a Single Crystal Ni-base Superalloy CMSX-2 Microstructural Influences and Environmental Effects," Int. J. Fat., vol. 21, pp. S79-S86, 1999.

[46] G. Onofrio, S. H. Ai, V. Lupinc, and G. Vimercati, "Influence of Time Dependent Mechanisms of Fatigue Crack Growth in a Single Crystal Nickel-Base Superalloy," in ECF 8 - Fracture Behaviour and Design of Materials and Structures. vol. 3, D. Firrao, Ed. Torino, Italy, 1990, pp. 1231-1236.

[47] F. Schubert, T. Rieck, and P. J. Ennis, "The Growth of Small Cracks in the Single Crystal Superalloy CMSX-4 at 750 and $1000^{\circ} \mathrm{C}$," in Proceedings of the International Symposium on Superalloys 2000, T. M. Pollock, R. D. Kissinger, R. R. Bowman, K. A. Green, M. McLean, S. Olson, and J. J. Schirra, Eds. Champion PA: TMS, The Metals, Minerals and Materials Society, Warrendale (USA), 2000, pp. 341-346.

[48] A. Defresne and L. Remy, "Fatigue Behaviour of CMSX-2 Superalloy [001] Single Crystals at High Temperature I: Low Cycle Fatigue of Notched Specimens," Mat. Sci. Eng. A, vol. A129, pp. 45-53, 1990.

[49] D. Davidson, K. Chan, R. McClung, and S. Hudak, "Small Fatigue Cracks," in Comprehensive Structural Integrity. vol. 4, I. Milne, R. O. Ritchie, and B. Karihaloo, Eds.: Elsevier, 2003, pp. 129-164. 
[50] K. Tanaka, "Fatigue Crack Propagation," in Comprehensive Structural Integrity. vol. 4, I. Milne, R. O. Ritchie, and B. Karihaloo, Eds.: Elsevier, 2003, pp. 95-127.

[51] K. Tanaka and Y. Nakai, "Propagation and Non-Propagation of Short Fatigue Cracks at a Sharp Notch," Fatigue of Engineering Materials \& Structures, vol. 6, pp. 315-327, 1983.

[52] P. A. S. Reed, D. C. Wu, and I. Sinclair, "Fatigue Crack Path Prediction in UDIMET 720 Nickel-Based Alloy Single Crystals," Metallurgical and Materials Transactions A, vol. 31A, pp. 109-123, Jan. 2000.

[53] K. S. Chan and T. A. Cruse, "Stress Intensity Factors for Anisotropic CompactTension Specimens with Inclined Cracks," Engng. Fract. Mech., vol. 23, pp. 863874, Oct. 1986.

[54] T. Tinga, "Stress Intensity Factors and Crack Propagation in Single Crystal Nickel Based Superalloy CMSX-4," National Aerospace Laboratory NLR NLR-TP-2005$470,2005$.

[55] T. Tinga, "Stress Intensity Factors and Crack Propagation in a Single Crystal Nickel-Based Superalloy," National Aerospace Laboratory NLR NLR-TP-2006$358,2006$.

[56] J. Zhao, X. Wu, R. Liu, and Z. Zhang, "Finite Element Analysis of Notch Root Semi-Elliptical Crack in Single Crystal Superalloy," Engineering Fracture Mechanics, vol. 71, pp. 1873-1890, 2003.

[57] "Trent 900 Optimised for the Airbus A380 Family," Rolls-Royce Plc, Derby UK, 2009.

[58] M. Cervenka: http://www.msm.cam.ac.uk/phase-trans/mphil/Trent1/index.htm , University of Cambridge, 2000.

[59] C. Bullough and B. Piercey, "An On-line Materials Database for the Design of Gas Turbine Components," in ASME Turbo Expo 1997 June 2-5, 1997, Orlando, Florida, USA: 97-GT-167, American Society of Mechanical Engineers (ASME), 1997.

[60] The Jet Engine. Derby UK: Rolls-Royce PLC, 1986.

[61] R. C. Reed, The Superalloys Fundamentals and Applications. Cambridge, UK: Cambridge University Press, 2006.

[62] C. T. Sims, "Superalloys: Genesis and Character," in Superalloys II HighTemperature Materials for Aerospace and Industrial Power, C. T. Sims, N. S. Stoloff, and W. C. Hagel, Eds. New York: John Wiley \& Sons, 1987, pp. 3-26. 
[63] W. R. Freeman, Jr., "Investment Casting," in Superalloys II High-Temperature Materials for Aerospace and Industrial Power, C. T. Sims, N. S. Stoloff, and W. C. Hagel, Eds. New York: John Wiley \& Sons, 1987, pp. 411-439.

[64] D. N. Duhl, "Directionally Solidfied Superalloys," in Superalloys II HighTemperature Materials for Aerospace and Industrial Power, C. T. Sims, N. S. Stoloff, and W. C. Hagel, Eds. New York: John Wiley \& Sons, 1987, pp. 189-214.

[65] M. Durand-Charre, The Microstructure of Superalloys. Amsterdam, The Netherlands: Gordon and Breach Science Publishers, 1997.

[66] B. J. Piearcey and F. L. VerSnyder, "A New Development in Gas Turbine Materials: The Properties and Characteristics of PWA 664," J. Aircraft, vol. 3, pp. 390-397, 1966.

[67] F. L. VerSnyder and R. W. Guard, "Directional Grain Structures for High Temperature Strength," Trans. ASM, vol. 52, pp. 485-491, 1960.

[68] F. L. VerSnyder and M. E. Shank, "The Development of Columnar Grain and Single Crystal High Temperature Materials Through Directional Solidification," Mat. Sci. Eng., vol. 6, pp. 213-247, October 1970.

[69] M. Gell and G. R. Leverant, "Mechanisms of High-Temperature Fatigue," in Fatigue at Elevated Temperatures, ASTM STP 520, A. E. Carden, Ed.: American Society for Testing and Materials, ASTM, 1973, pp. 37-67.

[70] E. W. Ross and C. T. Sims, "Nickel-Base Alloys," in Superalloys II HighTemperature Materials for Aerospace and Industrial Power, C. T. Sims, N. S. Stoloff, and W. C. Hagel, Eds. New York: John Wiley \& Sons, 1987, pp. 97-133.

[71] M. Gell, D. N. Duhl, and A. F. Giamei, "The Development of Single Crystal Superalloy Turbine Blades," in Proceedings of the International Symposium on Superalloys 1980, J. K. Tien, S. T. Wlodek, H. Morrow, M. Gell, and G. E. Maurer, Eds. Champion PA: TMS, The Metals, Minerals and Materials Society, Warrendale (USA), 1980, pp. 205-214.

[72] J. S. Erickson, W. A. Owczarski, and P. M. Curran, "Process Speeds Up Directional Solidification," Metal Prog., vol. 99, pp. 58-60, March 1971.

[73] H. M. J. Saari, "Process Modelling of the Directional Solidification of Gas Turbine Materials," in PhD Thesis, Department of Mechanical and Aerospace Engineering: Carleton University, 2003.

[74] S. M. Copley, A. F. Giamei, S. M. Johnson, and M. F. Hornbecker, "Origin of Freckles in Unidirectionally Solidified Castings," Met. Trans., vol. 1, pp. 21932204, August 1970. 
[75] S. Tin and T. Pollock, "Predicting freckle formation in single crystal Ni-base superalloys," J. Mat. Sci., vol. 39, pp. 7199-7205, 2004.

[76] S. Tin, T. M. Pollock, and W. T. King, "Carbon Additions and Grain Defect Formation in High Refractory Nickel-Base Single Crystal Superalloys," in Proceedings of the International Symposium on Superalloys 2000, T. M. Pollock, R. D. Kissinger, R. R. Bowman, K. A. Green, M. McLean, S. Olson, and J. J. Schirra, Eds. Champion PA: TMS, The Metals, Minerals and Materials Society, Warrendale (USA), 2000, pp. 201-210.

[77] E. W. Ross and K. S. O'Hara, "Rene N4: A First Generation Single Crystal Turbine Airfoil Alloy with Improved Oxidation Resistance, Low Angle Boundary Strength and Superior Long Time Rupture Strength," in Proceedings of the International Symposium on Superalloys 1996, R. D. Kissinger, D. J. Deye, D. L. Anton, A. D. Cetel, M. V. Nathal, T. M. Pollock, and D. A. Woodford, Eds. Champion PA: TMS, The Metals, Minerals and Materials Society, Warrendale (USA), 1996, pp. 19-25.

[78] D. M. Shah and A. D. Cetel, "Evaluation of PWA1483 for Large Single Crystal IGT Blade Applications," in Proceedings of the International Symposium on Superalloys 2000, T. M. Pollock, R. D. Kissinger, R. R. Bowman, K. A. Green, M. McLean, S. Olson, and J. J. Schirra, Eds. Champion PA: TMS, The Metals, Minerals and Materials Society, Warrendale (USA), 2000, pp. 295-304.

[79] S. Felicelli, D. Poirier, and P. Sung, "A model for prediction of pressure and redistribution of gas-forming elements in multicomponent casting alloys," Metallurgical and Materials Transactions B, vol. 31, pp. 1283-1292, 2000.

[80] M. J. Donachie and S. J. Donachie, Superalloys: A Technical Guide Second Edition, 2nd ed. Materials Park, OH: ASM International, 2002.

[81] G. W. Meetham, The Development of Gas Turbine Materials. Essex, UK: Applied Science Publishers, 1981.

[82] P. Caron and T. Khan, "Evolution of Ni-Based Superalloys for Single Crystal Gas Turbine Blade Applications," Aerosp. Sci. Technol., vol. 3, pp. 513-523, 1999.

[83] N. S. Stoloff, "Fundamentals of Strengthening," in Superalloys II HighTemperature Materials for Aerospace and Industrial Power, C. T. Sims, N. S. Stoloff, and W. C. Hagel, Eds. New York: John Wiley \& Sons, 1987, pp. 61-96.

[84] A. D. Cetel and D. N. Duhl, "Second-Generation Nickel-Base Single Crystal Superalloy," in Proceedings of the International Symposium on Superalloys 1988, S. Reichman, D. N. Duhl, G. Maurer, S. D. Antolovich, and C. Lund, Eds. Champion PA: TMS, The Metals, Minerals and Materials Society, Warrendale (USA), 1988, pp. 235-244. 
[85] K. Harris, G. L. Erickson, S. L. Sikkenga, W. D. Brentnall, J. M. Aurrecoechea, and K. G. Kubarych, "Development of The Rhenium Containing Superalloys CMSX-4 and CM 186 LC for Single Crystal Blade and Directionally Solidified Vane Applications in Advanced Turbine Engines," in Proceedings of the International Symposium on Superalloys 1992, S. D. Antolovich, R. W. Stusrud, R. A. MacKay, D. L. Anton, T. Khan, R. D. Kissinger, and D. L. Klarstrom, Eds. Champion PA: TMS, The Metals, Minerals and Materials Society, Warrendale (USA), 1992, pp. 297-306.

[86] T. Khan and P. Caron, "Advanced Single Crystal Ni-base Superalloys," A. K. Koul, Ed., 1994.

[87] D. M. Shah and D. N. Duhl, "The Effect of Orientation, Temperature and Gamma Prime Size on the Yield Strength of a Single Crystal Nickel Base Superalloy," in Proceedings of the International Symposium on Superalloys 1984, M. Gell, C. S. Kortovich, R. H. Bricknell, W. B. Kent, and J. F. Radvich, Eds. Champion PA: TMS, The Metals, Minerals and Materials Society, Warrendale (USA), 1984, pp. 105-114.

[88] P. Caron and T. Khan, "Third Generation Superalloys for Single Crystal Blades," in Materials for Advanced Power Engineering Meeting Liege Belgium, 1997, pp. 897912.

[89] C. T. Sims, "Predicton of Phase Composition," in Superalloys II High-Temperature Materials for Aerospace and Industrial Power, C. T. Sims, N. S. Stoloff, and W. C. Hagel, Eds. New York: John Wiley \& Sons, 1987, pp. 217-240.

[90] G. L. Erickson, "Ni-Based Superalloy Developments," in Critical Issues in the Development of High Temperature Structural Materials; Proceedings of the Conference, N. S. Stoloff, D. J. Duquette, and A. F. Giamei, Eds. Kona, HI: TMS, The Metals, Minerals and Materials Society, Warrendale (USA), 1993, pp. 87-105.

[91] T. Khan and P. Caron, "Advanced Single Crystal Ni-base Superalloys," in Advances in High Temperature Structural Materials and Protective Coatings A. K. Koul, V. R. Parameswaran, J. P. Immarigeon, and W. Wallace, Eds. Ottawa: National Research Council Canada, 1994, pp. 11-31.

[92] G. L. Erickson, "The Development and Application of CMSX10," in Proceedings of the International Symposium on Superalloys 1996, R. D. Kissinger, D. J. Deye, D. L. Anton, A. D. Cetel, M. V. Nathal, T. M. Pollock, and D. A. Woodford, Eds. Champion PA: TMS, The Metals, Minerals and Materials Society, Warrendale (USA), 1996, pp. 35-44.

[93] D. A. Ford and R. P. Arthey, "Development of Single Crystal Alloys For Specific Engine Applications," in Proceedings of the International Symposium on Superalloys 1984, M. Gell, C. S. Kortovich, R. H. Bricknell, W. B. Kent, and J. F. Radvich, Eds. Champion PA: TMS, The Metals, Minerals and Materials Society, Warrendale (USA), 1984, pp. 115-124. 
[94] W. S. Walston, K. S. O'Hara, E. W. Ross, T. M. Pollock, and W. H. Murphy, "Rene N6: Third Generation Single Crystal Superalloy," in Proceedings of the International Symposium on Superalloys 1996, R. D. Kissinger, D. J. Deye, D. L. Anton, A. D. Cetel, M. V. Nathal, T. M. Pollock, and D. A. Woodford, Eds. Champion PA: TMS, The Metals, Minerals and Materials Society, Warrendale (USA), 1996, pp. 27-34.

[95] P. Caron, "High Y' Solvus New Generation Nickel-Based Superalloys for Single Crystal Turbine Blade Applications," in Proceedings of the International Symposium on Superalloys 2000, T. M. Pollock, R. D. Kissinger, R. R. Bowman, K. A. Green, M. McLean, S. Olson, and J. J. Schirra, Eds. Champion PA: TMS, The Metals, Minerals and Materials Society, Warrendale (USA), 2000, pp. 737746.

[96] Y. Koizumi, T. Kobayashi, T. Yokokawa, J. Zhang, M. Osawa, H. Harada, Y. Aoki, and M. Arai, "Development of Next-Generation Ni-Base Single Crystal Superalloys," in Proceedings of the International Symposium on Superalloys 2004, K. A. Green, T. M. Pollock, H. Harada, T. E. Howson, R. C. Reed, J. J. Schirra, and S. Walston, Eds. Champion PA: TMS, The Metals, Minerals and Materials Society, Warrendale (USA), 2004, pp. 35-43.

[97] S. Walston, A. D. Cetel, R. A. MacKay, K. S. O'Hara, D. N. Duhl, and R. Dreshfield, "Joint Development of a Fourth Generation Single Crystal Superalloy," in Proceedings of the International Symposium on Superalloys 2004, K. A. Green, T. M. Pollock, H. Harada, T. E. Howson, R. C. Reed, J. J. Schirra, and S. Walston, Eds. Champion PA: TMS, The Metals, Minerals and Materials Society, Warrendale (USA), 2004, pp. 15-24.

[98] D. Argence, C. Vernault, Y. Desvallees, and D. Fournier, "MC-NG: A 4th Generation Single-Crystal Superalloy for Future Aeronautical Turbine Blades and Vanes," in Proceedings of the International Symposium on Superalloys 2000, T. M. Pollock, R. D. Kissinger, R. R. Bowman, K. A. Green, M. McLean, S. Olson, and J. J. Schirra, Eds. Champion PA: TMS, The Metals, Minerals and Materials Society, Warrendale (USA), 2000, pp. 829-837.

[99] P. Caron and T. Khan, "Tensile Behaviour of a Nickel-Based Single Crystal Superalloy - Effects of Temperature and Orientation," in Symposium on Advanced Materials and Processing Techniques for Structural Applications Sept. 7-9, 1987, Paris, France, 1988, pp. 59-70.

[100] T. P. Gabb, J. Gayda, and R. V. Miner, "Orientation and Temperature Dependence of Some Mechanical Properties of the Single-Crystal Nickel-Base Superalloy Rene N4: Part II. Low Cycle Fatigue Behavior," Metall. Trans. A, vol. 17A, pp. 497-505, March 1986. 
[101]T. P. Gabb, R. V. Miner, and J. Gayda, "The Tensile and Fatigue Deformation Structures in a Single Crystal Nickel-Base Superalloy," Scr. Metall, vol. 20, pp. 513-518, Apr. 1986.

[102]T. P. Gabb, G. Welsch, and R. V. Miner, "The Characteristic of gamma ' Dislocation Pairs in a Nickel-Base Superalloy," Scr. Metall, vol. 21, pp. 987-992, July 1987.

[103] T. P. Gabb, G. Welsch, R. V. Miner, and J. Gayda, "The Low Cycle Fatigue Deformation Response of a Single-Crystal Superalloy at $650{ }^{\circ} \mathrm{C}, "$ Materials Science and Engineering A, vol. 108, pp. 189-202, 1989.

[104] B. H. Kear and B. J. Piearcey, "Tensile and Creep Properties of Single Crystals of the Nickel-Base Superalloy Mar-M200," AIME Met Soc Trans, vol. 239, pp. 12091215, August 1967.

[105] R. V. Miner, T. P. Gabb, J. Gayda, and K. J. Hemker, "Orientation and Temperature Dependence of Some Mechanical Properties of the Single-Crystal Nickel-Base Superalloy Rene N4: III. Tension--Compression Anisotropy," Metall. Trans. A, vol. 17A, pp. 507-512, Mar. 1986.

[106] R. V. Miner, R. C. Voigt, J. Gayda, and T. P. Gabb, "Orientation and Temperature Dependence of Some Mechanical Properties of the Single-Crystal Nickel-Base Superalloy Rene N4: Part I. Tensile Behavior," Metall. Trans. A, vol. 17A, pp. 491496, March 1986.

[107] M. G. Hebsur and R. V. Miner, "Elevated Temperature Tension, Compression, and Creep-Rupture Behavior of (001)-Oriented Single Crystal Superalloy PWA 1480," Pamphlet NASA-TM-88950, 1987.

[108] W. W. Milligan, "Deformation Modeling and Constitutive Modeling for Anisotropic Superalloys," PhD. Thesis, Georgia Institute of Technology, 1988, p. 283.

[109] W. W. Milligan and S. D. Antolovich, "Yielding and Deformation Behavior of the Single Crystal Superalloy PWA1480," Metall. Trans. A, vol. 18A, pp. 85-95, Jan. 1987.

[110] A. Sengupta, S. K. Putatunda, J. Bartosiewicz, J. Hangas, P. J. Nailos, M. Peputapeck, and F. E. Alberts, "Tensile Behavior of a New Single-Crystal NickelBased Superalloy (CMSX-4) at Room and Elevated Temperature," J. Mat. Eng. \& Perf., vol. 3, pp. 73-81, Feb. 1994.

[111] S. Takeuchi and E. Kuramoto, "Temperature and Orientation Dependence of the Yield Stress in Ni3Ga Single Crystals," Acta Metall., vol. 21, pp. 415-425, 1973.

[112] V. Paidar, D. P. Pope, and V. Vitek, "A Theory of the Anomalous Yield Behavior in L12 Ordered Alloys," Acta Metall., vol. 32, pp. 435-448, 1984. 
[113] P. B. Hirsch, "A New Theory of the Anomalous Yield Stress in L12 Alloys," Phil. Mag. A, vol. 65, pp. 569-612, 1992.

[114] L. T. Dame and D. C. Stouffer, "A Crystallographic Model for Nickel Base Single Crystal Alloys," Journal of Applied Mechanics, vol. 55, pp. 325-331, 1988.

[115]D. C. Stouffer and L. T. Dame, Inelastic Deformation of Metals: Models, Mechanical Properties and Metallurgy. New York: John Wiley \& Sons, 1996.

[116] W. W. Milligan, E. S. Huron, and S. D. Antolovich, "Deformation, Fatigue and Fracture Behavior of Two Cast Anisotropic Superalloys," in Fatigue '87 Proceedings of the Third International Conference. vol. 3, R. O. Ritchie and E. A. Starke, Eds. Charlottesville, VA, USA, 1987, pp. 1561-1591.

[117] D. L. Anton, "Low-Cycle Fatigue Characteristics of $<001>$ and Randomly Aligned Superalloy Single Crystals," Acta Metall., vol. 32, pp. 1669-1679, 1984.

[118] P. K. Wright and A. F. Anderson, "The Influence of Orientation on the Fatigue of Directionally Solidified Superalloys," in Proceedings of the International Symposium on Superalloys 1980, J. K. Tien, S. T. Wlodek, H. Morrow, M. Gell, and G. Maurer, Eds. Champion PA: TMS, The Metals, Minerals and Materials Society, Warrendale (USA), 1980, pp. 689-698.

[119] L. G. Fritzemeier and J. K. Tien, "The Cyclic Stress-Strain Behavior of NickelBase Superalloys. II. Single Crystals," Acta Metall., vol. 36, pp. 283-290, 1988.

[120] K. Harris, G. L. Erickson, and R. E. Schwer, "MAR M 247 Derivations - CM 247 LC DS Alloy CMSX Single Crystal Alloys Properties and Performance," in Proceedings of the International Symposium on Superalloys 1984, M. Gell, C. S. Kortovich, R. H. Bricknell, W. B. Kent, and J. F. Radvich, Eds. Champion PA: TMS, The Metals, Minerals and Materials Society, Warrendale (USA), 1984, pp. 221-230.

[121] K. Harris and G. L. Erickson, "Low Density Single Crystal Superalloy," US Patent \#4721540: Cannon Muskegon Corporation, 1988, p. 12.

[122]E. W. Ross and K. S. O'Hara, "Cast Columnar Grain Hollow Nickel Base Alloy Articles and Alloy and Heat Treatment for Making " US Patent \#5173255, 1992, p. 8.

[123]H. K. D. H. Bhadeshia, "Nickel Based Superalloys," http://www.msm.cam.ac.uk/phase-trans/2003/Superalloys/wax/wax.html, University of Cambridge, 2003.

[124] M. J. Goulette, P. D. Spilling, and R. P. Arthey, "Cost Effective Single Crystals," in Proceedings of the International Symposium on Superalloys 1984, M. Gell, C. S. Kortovich, R. H. Bricknell, W. B. Kent, and J. F. Radvich, Eds. Champion PA: 
TMS, The Metals, Minerals and Materials Society, Warrendale (USA), 1984, pp. 167-176.

[125] L. Li and R. A. Overfelt, "Influence of directional solidification variables on the cellular and primary dendrite arm spacings of PWA1484," J. Mat. Sci., vol. 37, pp. 3521-3532, 2002.

[126] Q. Z. Chen, Y. H. Kong, C. N. Jones, and D. M. Knowles, "Porosity reduction by minor additions in RR2086 superalloy," Scripta Materialia, vol. 51, pp. 155-160, 2004.

[127] J. Spowart, H. Mullens, and B. Puchala, "Collecting and analyzing microstructures in three dimensions: A fully automated approach," JOM Journal of the Minerals, Metals and Materials Society, vol. 55, pp. 35-37, 2003.

[128]E. F. Bradley, Superalloys: A Technical Guide. United States: ASM International,Metals Park, OH, 1988.

[129] C. M. F. Rae and R. C. Reed, "The precipitation of topologically close-packed phases in rhenium-containing superalloys," Acta Materialia, vol. 49, pp. 41134125,2001 .

[130] U. o. C. Department of Materials Science and Metallurgy, "Slip geometry: the critical resolved shear stress," http://www.doitpoms.ac.uk/tlplib/slip/slip geometry.php, University of Cambridge 2008.

[131]D. Stone, "Materials Science and Engineering 441 Notes: Lecture 29 Partial Dislocations," website: $\quad$ http://ecow.engr.wisc.edu/cgibin/get/msae/441/stone/notes/, University of Wisconsin-Madison, 2008.

[132]D. A. Alden, "An Analysis of Yield Phenomena in Rene N4+ Single Crystals with Respect to Orientation and Temperature.," $\mathrm{PhD}$. Thesis, University of Cincinnati, 1990, p. 265.

[133] R. W. Hertzberg, Deformation and Fracture Mechanics of Engineering Materials, 2 ed. New York: John Wiley \&Sons, 1983.

[134] G. Asquith and A. C. Pickard, "Fatigue Testing of Gas Turbine Components," High Temperature Technology, vol. 6, pp. 131-143, Aug. 1988.

[135] S. Suresh, Fatigue of Materials, 2nd ed. Cambridge UK: Cambridge University Press, 1998.

[136] G. C. Sih, P. C. Paris, and G. R. Irwin, "On Cracks In Rectilinearly Anisotropic Bodies," Int. J. Fract., vol. 1, pp. 189-203, 1965. 
[137] D. Broek, Elementary Engineering Fracture Mechanics, 1 ed. Alphen aan den Rijn: Sijthoff and Noordhoff International Publishers, 1978.

[138] A. Saxena, Nonlinear Fracture Mechanics for Engineers, 1 ed. Boca Ratan FL: CRC Press, 1998.

[139] G. R. Irwin, "Fracture Mechanisms in Structural Mechanics of Fracture," in Structural Mechanics, Proceedings of First Symposium on Naval Structural Mechanics, 1960.

[140] J. R. Rice, "Mathematical Analysis in the Mechanics of Fracture," in Fracture: An Advanced Treatise. vol. 2, H. Liebowitz, Ed. New York: Academic Press, 1968, p. 191.

[141] J. R. Rice, "Tensile Crack Tip Fields in Elastic-Ideally Plastic Crystals," Mech. Mat., vol. 6, pp. 317-335, 1987.

[142] J. Kysar and C. Briant, "Crack tip Deformation Fields in Ductile Single Crystals," Acta Mater., vol. 50, pp. 2367-2380, 2002.

[143] S. Flouriot, S. Forest, G. Cailletaud, A. Koster, L. Remy, B. Burgardt, V. Gros, S. Mosset, and J. Delautre, "Strain Localization at the Crack Tip in Single Crystal CT Specimens under Monotonous Loading: 3D Finite Element Analysis and Application to Nickel-base Superalloys," Int. J. Fract., vol. 124, pp. 43-77, 2003.

[144] N. Marchal, S. Flouriot, S. Forest, and L. Remy, "Crack-tip Stress-Strain Fields in Single Crystal Nickel-Base Superalloys at High Temperature Under Cyclic Loading," Computational Materials Science, vol. 37, pp. 42-50, 2006.

[145] X. Wu, W. Deng, A. K. Koul, and J. P. Immarigeon, "A Continuously Distributed Dislocation Model for Fatigue Cracks in Anisotropic Crystalline Materials," Int. J. Fat., vol. 23, pp. S201-S206, 2001.

[146] J. C. Newman, Jr., "Fracture Mechanics Parameters for Small Fatigue Cracks," in Small-Crack Test Methods, ASTM STP 1149, J. Larson and J. E. Allison, Eds. Philadelphia: American Society for Testing and Materials, 1992, pp. 6-33.

[147] P. W. Tan, I. S. Raju, K. N. Shivakumar, and J. C. Newman, Jr., "Evaluation of Finite-Element Models and Stress-Intensity Factors for Surface Cracks Emanating from Stress Concentrations," in Surface-Crack Growth: Model, Experiments, and Structures, ASTM STP 1060, W. G. Reuter, J. H. Underwood, and J. C. Newman, Jr., Eds. Philadelphia: American Society for Testing and Materials (ASTM), 1990, pp. 34-48.

[148] J. C. Newman, Jr. and I. S. Raju, "An Empirical Stress-Intensity Factor Equation for the Surface Crack," Engng. Fract. Mech., vol. 15, pp. 185-192, 1981. 
[149] I. S. Raju and J. C. Newman, Jr., "Improved Stress Intensity Factors for SemiElliptical Surface Cracks in Finite Thickness Plates," National Aeronautics and Space Administration (NASA) Technical Memorandum, NASA-TM-X-72825, Washington D.C. 1977.

[150] I. S. Raju and J. C. Newman Jr., "Stress-intensity factors for a wide range of semielliptical surface cracks in finite-thickness plates," Engineering Fracture Mechanics, vol. 11, pp. 817-829, 1979.

[151] T. Fett, "An Extension of the Newman-Raju Formula," Int. J. Fract., vol. 33, pp. R47-R50, 1987.

[152] T. Fett, "The stress intensity factor for small cracks at the root of a notch," International Journal of Fracture, vol. 54, pp. R57-R64, 1992.

[153] D. Kujawski, "Estimations of Stress Intensity Factors for Small Cracks at Notches," Fatigue Fract. Engng Mater. Struct., vol. 14, pp. 953-965, 1991.

[154] J. Schijve, "The Stress Intensity Factor of Small Crack at Notches," Fract. Engng. Mater. Structs., vol. 5, pp. 77-90, 1982.

[155] Y. Yamamoto, Y. Sumi, and K. Ao, "Stress Intensity Factors of Cracks Emanating from Semi-Elliptical Side Notches in Plates," Int. J. Fract., vol. 10, pp. 593-595, 1974.

[156] K. N. Shivakumar and J. C. Newman, Jr., "Stress Intensity Factors for Large Aspect Ratio Surface and Corner Cracks at a Semi-Circular Notch Root in a Tension Specimen," Engng. Fract. Mech., vol. 38, pp. 467-473, 1991.

[157] J. Gallagher et al., "Advanced High Cycle Fatigue (HCF) Life Assurance Methodologies," Materials and Manufacturing Directorate Air Force Research Laboratory Air Force Materiel Command Wright-Patterson Air Force Base, OH Report AFRL-ML-WP-TR-2005-4102, Dayton, OH 2005.

[158] M. Kamaya and T. Kitamura, "Stress Intensity Factors of Microstructurally Small Crack," Int. J. Fract., vol. 124, pp. 201-213, 2003.

[159]A. C. Pickard, The Application of 3-Dimensional Finite Element Methods to Fracture Mechanics and Fatigue Life Prediction, 1 ed. London UK: Engineering Materials Advisory Services, 1986.

[160] Standard Test Method for Measurement of Fatigue Crack Growth Rates, ASTM E647 - 00: American Society for Testing and Materials (ASTM), 2000.

[161]Q. Chen and H. W. Liu, "Resolved Shear Stress Intensity Coefficient and Fatigue Crack Growth in Large Crystals," NASA Contractor Report, CR182137 1988. 
[162] M. Gell and G. R. Leverant, "The Characteristics of Stage I Fatigue Fracture in a High-Strength Nickel Alloy," Acta Metall., vol. 16, pp. 553-561, 1968.

[163] G. R. Leverant and M. Gell, "Influence of Temperature and Cyclic Frequency on the Fatigue Fracture of Cube Oriented Ni-Base Superalloy Single Crystals," Metall. Trans. A, vol. 6A, pp. 367-371, Feb. 1975.

[164] J. R. Rice, "A Path Independent Integral and the Approximate Analysis of Strain Concentration by Notches and Cracks," Journal of Applied Mechanics, vol. 35, pp. 379-386, 1968.

[165] W. Brocks, A. Cornec, and I. Scheider, "Computational Aspects of Nonlinear Fracture Mechanics," in Comprehensive Structural Integrity. vol. 3, I. Milne, R. O. Ritchie, and B. Karihaloo, Eds.: Elsevier, 2003, pp. 127-209.

[166] W. Brocks and I. Scheider, "Numerical Aspects of the Path-Dependence of the Jintegral in Incremental Plasticity," Institut fur Werkstofforshung, GKSSForschungszentrum, Report GKSS/WMS/01/08 2001.

[167] R. H. Gallagher, "A Review of Finite Element Techniques in Fracture Mechanics," in Numerical Methods in Fracture Mechanics: Proceedings of the First International Conference held at University College of Swansea, A. R. Luxmoore and D. R. J. Owen, Eds. Swansea, UK: University College of Swansea, 1978, pp. 125.

[168] H. Liebowitz and E. T. J. Moyer, "Finite Element Methods in Fracture Mechanics," Computers and Structures, vol. 31, pp. 1-9, 1989.

[169] A. R. Ingraffea and P. A. Wawrzynek, "Finite Element Methods for Linear Elastic Fracture Mechanics," in Comprehensive Structural Integrity Volume 3 Numerical and Computational Methods, 1 ed. vol. 3, R. de Borst and H. A. Mang, Eds. Oxford UK: Elsevier Ltd., 2003, pp. 1-88.

[170] S. Courton, C. Gardin, G. Bezine, and H. Ben Hadj Hamouda, "Advantages of the J-integral Approach for Calculating Stress Intensity Factors When Using the Commercial Finite Element Software ABAQUS," Engineering Fracture Mechanics, vol. 72, pp. 2174-2185, 2005.

[171]ABAQUS User's Manual Version 6.5: Hibbitt, Karlsson and Sorensen, Inc., 2004.

[172]D. M. Parks, "A Stiffness Derivative Finite Element Technique for Determination of Crack Tip Stress Intensity Factors," Int. J. Fract., vol. 10, pp. 487-502, 1974.

[173]D. M. Parks, "Virtual Crack Extension: A General Finite Element Technique for JIntegral Evaluation," in Numerical Methods in Fracture Mechanics: Proceedings of the First International Conference held at University College of Swansea, A. R. Luxmoore and D. R. J. Owen, Eds. Swansea, UK: University College of Swansea, 1978, pp. 464-478. 
[174] ABAQUS Theory Manual Version 6.5: Hibbitt, Karlsson and Sorensen, Inc., 2004.

[175] S. Mohammadi, Extended Finite Element Method for Fracture Analysis of Structures, 1 ed. Oxford UK: Wiley-Blackwell, 2008.

[176] C. F. Shih and R. J. Asaro, "Elastic-Plastic Analysis of Cracks on Bimaterial Interfaces: Part I - Small Scale Yielding," Journal of Applied Mechanics, pp. 299$316,1988$.

[177] "Standard Test Method for Measurement of Fatigue Crack Growth Rates," American Society for Testing and Materials (ASTM), ASTM Designation: E 647$05,2005$.

[178] W. Elber, "The Significance of Fatigue Crack Closure," in Damage Tolerance in Aircraft Structures, ASTM STP 486: American Society for Testing and Materials, 1971, pp. 230-242.

[179] H. Sehitoglu, K. Gall, and A. M. Garcia, "Recent Advances in Fatigue Crack Growth Modeling," Int. J. Fract., vol. 80, pp. 165-192, 1996.

[180] J. C. Newman Jr, "Fatigue-Life Prediction Methodology Using a Crack-Closure Model," Journal of Engineering Materials and Technology, vol. 117, pp. 433-439, 1995.

[181]R. C. McClung and H. Sehitoglu, "On The Finite Element Analysis of Fatigue Crack Closure-1. Basic Modeling Issues," Engineering Fracture Mechanics, vol. 33, pp. 237-252, 1989.

[182] R. C. McClung and H. Sehitoglu, "On The Finite Element Analysis of Fatigue Crack Closure-2. Numerical Results," Engineering Fracture Mechanics, vol. 33, pp. 253-272, 1989.

[183] M. R. James and W. L. Morris, "Effect of Fracture Surface Roughness on Growth of Short Fatigue Cracks," Met. Trans. A, vol. 14, pp. 153-155, 1983.

[184] R. O. Ritchie and J. Lankford, "Overview of the Small Crack Problem," in Small Fatigue Cracks: Proceedings of the Second Engineering Foundation International Conference/Workshop on "Small Fatigue Cracks", R. O. Ritchie and J. Lankford, Eds. Santa Barbara, CA: The Metallurgical Society, Warrendale PA, 1986, pp. 1-5.

[185] M. Moura Branco, J. Byrne, and V. Hodkinson, "Elevated Temperature Fatigue Crack Growth of Nickel-Base Superalloys: A Review and Modeling," in Mechanical Behaviour of Materials at High Temperatures, M. Moura Branco, R. O. Ritchie, and V. Sklenicka, Eds.: Kluwer Academic Publishers, 1996.

[186] H. Sehitoglu and D. Boismier, "Thermo-mechanical Fatigue of Mar-M247: Part 2 Life Prediction," Journal of Engineering Materials and Technology, vol. 112, pp. 80-88, 1990. 
[187] P. C. Paris and F. Erdogan, "A Critical Analysis of Crack Propagation Laws," Journal of Basic Engineering, vol. 85, pp. 528-534, 1963.

[188] L. Banks-Sills and Y. Volper, "Application of the Cyclic J-Integral to Fatigue Crack Propagation of Al 2024-T351," Engineering Fracture Mechanics, vol. 40, pp. 355-370, 1991.

[189] L. Banks-Sills and D. Sherman, "On the Computation of Stress Intensity Factors for Three-Dimensional Geometries by Means of the Stiffness Derivative and J-integral Method," Int. J. Fract., vol. 53, pp. 1-20, 1992.

[190] C. L. Chow and T. J. Lu, "On the Cyclic J-Integral Applied to Fatigue Cracking," Int. J. Fract., vol. 40, pp. R53-R59, 1989.

[191] K. Sadananda and P. Shahinian, "Application of J-Integral Parameter to High Temperature Fatigue Crack Growth in Cold Worked Type 316 Stainless Steel," Int. J. Fract., vol. 15, pp. R81-R84, 1979.

[192] K. Sadananda and P. Shahinian, "A Fracture Mechanics Approach to High Temperature Fatigue Crack Growth in Udimet 700," Engineering Fracture Mechanics, vol. 11, pp. 73-86, 1979.

[193] K. Tanaka, "The Cyclic J-integral as a Criterion for Fatigue Crack Growth," Int. $J$. Fract., vol. 22, pp. 91-104, 1983.

[194] N. E. Dowling and J. A. Begley, "Fatigue Crack Growth During Gross Plasticity and the J-Integral," in Mechanics of Crack Growth ASTM STP 590: American Society for Testing and Materials, 1976, pp. 82-103.

[195] H. S. Lamba, "The J-integral Applied to Cyclic Loading," Engineering Fracture Mechanics, vol. 7, pp. 693-703, 1975.

[196] K. O. Findley, S. W. Kow, and A. Saxena, "J-integral Expressions for SemiElliptical Cracks in Round Bars," Int. J. Fat., vol. 29, pp. 822-828, 2007.

[197] Y.-J. Kim, D.-J. Shim, J.-B. Choi, and Y.-J. Kim, "Approximate J-integral Estimates for Tension-Loaded Plates with Semi-Elliptical Surface Cracks," Engineering Fracture Mechanics, vol. 69, pp. 1447-1463, 2002.

[198] A. Staroselsky, L. Vestergaard, B. Annigeri, L. H. Favrow, and D. Walls, "ThreeDimensional Fatigue Cracking Under Elastic-Plastic Deformation," Int. J. Fat., vol. 23, pp. S65-S70, 2001.

[199] K. Tanaka, "Mechanics and Micromechanics of Fatigue Crack Propagation," in Fracture Mechanics: Perspectives and Directions (Twentieth Symposium), ASTM STP 1020, R. P. Wei and R. P. Gangloff, Eds. Philadelphia: American Society for Testing and Materials, 1989, pp. 151-183. 
[200] J. Weertman, "Short Crack Theory," in Small Fatigue Cracks: Proceedings of the Second Engineering Foundation International Conference/Workshop, R. O. Ritchie and J. Lankford, Eds. Santa Barbara, CA: The Metallurgical Society of the AIME, 1986, pp. 363-378.

[201] P. D. Hobson, M. W. Brown, and E. R. de los Rios, "Two Phases of Short Crack Growth in a Medium Carbon Steel," in The Behaviour of Short Fatigue Cracks, K. J. Miller and E. R. de los Rios, Eds. London: Mechanical Engineering Publisher, 1986, pp. 441-459.

[202] A. Navarro and E. R. de los Rios, "Short and Long Fatigue Crack Growth: a Unified Model," Phil. Mag., vol. 57, pp. 15-36, 1988.

[203] K. Tanaka, Y. Akiniwa, Y. Nakai, and R. P. Wei, "Modeling of Small Crack Growth Interacting with Grain Boundary," Engineering Fracture Mechanics, vol. 24, pp. 803-819, 1988.

[204]L. Wenfong, "Short Fatigue Crack Propagation and Effect of Notch Plastic Field," Nuclear Engineering and Design, vol. 84, pp. 193-200, 2003.

[205] M. M. Hammouda, R. A. Smith, and K. J. Miller, "Elastic-Plastic Fracture Mechanics for Initiation and Propagation of Notch Fatigue Cracks," Fatigue \& Fracture of Engineering Materials \& Structures, vol. 2, pp. 139-154, 1979.

[206]P. Lukas and L. Kunz, "Cyclic Slip Localisation and Fatigue Crack Initiation in FCC Single Crystals," Mat. Sci. Eng. A, vol. A314, pp. 75-80, 2001.

[207] R. Chieragatti and L. Remy, "Influence of Orientation on the Low Cycle Fatigue of MAR-M200 Single Crystals at $650^{\circ} \mathrm{C} 1$ : Fatigue Life Behaviour," Mat. Sci. Eng. A, vol. A141, pp. 1-9, 1991.

[208] T. G. Meyer and D. M. Nissley, "Notched Fatigue of Single Crystal PWA 1480 at Turbine Attachment Temperatures," in Structural Integrity and Durability of Reusable Space Propulsion Systems, 14-15 May 1991, Cleveland, OH, 1991, pp. 17-23.

[209] T. G. Meyer, G. J. McCarthy, L. H. Favrow, D. L. Anton, and J. Bak, "Constitutive and Life Modeling of Single Crystal Blade Alloys for Root Attachment Analysis," in NASA, Marshall Space Flight Center, Advanced Earth-to-Orbit Propulsion Technology 1988. vol. 1, 1988, pp. 510-518.

[210]L. Remy, "Oxidation Effects in High Temperature Creep and Fatigue on Engineering Alloys," in First International Conference on Corrosion-Deformation Interactions, $C D I^{\prime} 92$, T. Magnin and J. M. Gras, Eds. Fotainebleau, France: Les Editions de Physique, Les Ulis, 1992, pp. 425-459.

[211] R. P. Wahi, D. Auerswald, D. Mukherji, A. Dudka, H.-J. Fecht, and W. Chen, "Damage Mechanisms of Single and Polycrystalline Nickel-base Superalloys SC16 
and IN738LC Under High Temperature LCF Loading," Int. J. Fat., vol. 19, pp. 8994, 1997.

[212]P. K. Wright, "Oxidation-Fatigue Interactions in a Single-Crystal Superalloy," in Low Cycle Fatigue, ASTM STP 942, H. D. Soloman, G. R. Halford, L. R. Kaisand, and B. N. Leis, Eds.: American Society for Testing and Materials (ASTM), Philadelphia, 1988, pp. 558-575.

[213] R. Ohtani, N. Tada, M. Shibata, and S. Taniyama, "High Temperature Fatigue of the Nickel-Base Single-Crystal Superalloy CMSX-10," Fatigue and Fracture of Engineering Materials and Structures, vol. 24, pp. 867-876, 2001.

[214] L. Remy, "High Temperature Fatigue of Superalloys," in Fatigue '93 : Proceedings of the Fifth International Confernece on Fatigue and Fatigue Thresholds, J.-P. Bailon and J. I. Dickson, Eds. Montreal, QC: Warley, West Midlands, U.K., 1993, pp. 825-834.

[215] R. J. H. Wanhill, "Short Fatigue Cracks in Aerospace Structures," in The Behaviour of Short Fatigue Cracks, K. J. Miller and E. R. de los Rios, Eds.: Mechanical Engineering Publications, London, 1986, pp. 27-36.

[216]M. R. Joyce, X. Wu, and P. A. S. Reed, "The Effect of Environment and Orientation on Fatigue Crack Growth Behaviour of CMSX-4 Nickel Base Single Crystal at 650C," Materials Letters, vol. 58, pp. 99-103, 2003.

[217] M. Okazaki, H. Yamada, and S. Nohmi, "Temperature Dependence of the Intrinsic Small Fatigue Crack Growth Behavior in Ni-Base Superalloys Based on Measurement of Crack Closure," in Fatigue under Thermal and Mechanical Loading: Mechanisms, Mechanics and Modelling: Proceedings of the Symposium J. Bressers and L. Remy, Eds. 22-24 May 1995, Petten, The Netherlands: Kluwer Academic Publishers, 1996, pp. 119-129.

[218] C. Howland, "The Growth of Fatigue Cracks in a Nickel Base Single Crystal," in The Behaviour of Short Fatigue Cracks, K. J. Miller and E. R. de los Rios, Eds. London UK: Mechanical Engineering Publications, 1986, pp. 229-239.

[219] J. S. Crompton and J. W. Martin, "Fatigue Crack Propagation at Elevated Temperature in Mar-M002 Single Crystals," in High Temperature Alloys for Gas Turbines 1982: Proceedings of a Conference Held in Liege, Belgium, 4-6 October, 1982, Brunetaud, Ed.: Kluwer Academic Publishers, 1982, pp. 611-617.

[220] K. J. Miller, A. O. ObaBueki, C.-H. Lee, T. G. Tanaka, and S.-B. Lee, "A Unified Model for Fatigue Crack Initiation and Growth, with Emphasis on Short-Crack Behaviour, Crack Closure Effects, Variable-Temperature Fatigue and CreepFatigue Interaction," Mat. Sci. Eng., vol. A103, pp. 71-93, 1988.

[221]H. Mughrabi, S. Kraft, and M. Ott, "Specific Aspects of Isothermal and Anisothermal Fatigue of the Monocrystalline Nickel-Base Superalloy CMSX-6," in 
Proceedings of the International Symposium on Superalloys 1996, R. D. Kissinger, D. J. Deye, D. L. Anton, A. D. Cetel, M. V. Nathal, T. M. Pollock, and D. A. Woodford, Eds. Champion PA: TMS, The Metals, Minerals and Materials Society, Warrendale (USA), 1996, pp. 335-344.

[222] K. J. Miller and E. R. de los Rios, "The Behaviour of Short Fatigue Cracks," in The Behaviour of Short Fatigue Cracks, K. J. Miller and E. R. de los Rios, Eds., 1986.

[223] S. Yandt, "Development of a Thermal-Mechanical Fatigue Testing Facility," in Department of Mechanical and Aerospace Engineering. vol. M. Eng. Ottawa: Carleton University, 2000.

[224] S. H. Choi and E. Kremple, "Viscoplasticity Theory Based on Overstress Applied to the Modeling of Cubic Single Crystals," European Journal of Mechanics, A/Solids, vol. 8, pp. 219-233, 1989.

[225]D. Nouailhas and J. L. Chaboche, "Anisotropic Constitutive Modeling for SingleCrystal Superalloys using a Continuum Phenomenological Approach," in Proceedings of the 3rd International Conference on Constitutive Laws for Engineering Materials, C. S. Desai, Ed. Tucson, AZ: ASME Press, 1991, pp. 213218.

[226] S. Han, S. Li, and D. J. Smith, "Comparison of Phenomenological and Crystallographic Models for Single Crystal Nickel Base Superalloys - I Analytical Identification," Mech. Mat., vol. 33, pp. 251-266, 2001.

[227] E. H. Jordan and K. P. Walker, "A Viscoplastic Model for Single Crystals," Journal of Engineering Materials and Technology, vol. 114, pp. 19-26, 1992.

[228] S. X. Li and D. J. Smith, "Modeling of Anisotropic Creep Deformation and Damage in Single Crystal Superalloys," scr. Metall et Mater., vol. 33, pp. 711-718, 1995.

[229] L. Meric, P. Poubanne, and G. Cailletaud, "Single Crystal Modeling for Structural Calculations: Part 1 - Model Presentation," Journal of Engineering Materials and Technology, vol. 113, pp. 162-170, 1991.

[230] J. L. Chaboche, "Constitutive Equations for Cyclic Plasticity and Viscoplasticity," Int. J. Plast., vol. 5, pp. 247-302, 1983.

[231] J. L. Chaboche, "Unified Cyclic Viscoplastic Constitutive Equations: Development, Capabilities, and Thermodynamic Framework," in Unified Constitutive Laws of Plastic Deformation, A. Krausz and K. Krausz, Eds.: Elsivier, 1996, pp. 1-68.

[232] J. L. Chaboche and G. Cailletaud, "On The Calculation of Structures in Cyclic Plasticity or Viscoplasticity," Computers and Structures, vol. 23, pp. 23-31, 1986. 
[233] G. R. Swanson, I. Linask, D. M. Nissley, P. P. Norris, T. G. Meyer, and K. P. Walker, "Life Prediction and Constitutive Models for Engine Hot Section Anisotropic Materials Program," National Aeronautics and Space Administration, NASA Contractor Report 1749521986.

[234] D. Nouailhas, J. P. Culie, L. Meric, and G. Cailletaud, "Finite Element Analysis of the Stress-Strain Behaviour of Single-Crystal Tubes," European Journal of Mechanics, A/Solids, vol. 14, pp. 137-154, 1995.

[235] J. L. Chaboche, "Constitutive Equations for Cyclic Plasticity and Viscoplasticity," Int. J. Plast., vol. 5, pp. 247-302, 1989.

[236] T. Tinga, M. G. D. Geers, and W. A. M. Brekelmans, "Micro-mechanical Modelling fo the Single Crystal Nickel-Base Superalloy CMSX-4," National Aerospace Laboratory NLR NLR-TP-2006-352, 2006.

[237] D. P. Pope and S. S. Ezz, "Mechanical Properties of $\mathrm{Ni}_{3} \mathrm{Al}$ and Nickel-base Alloy with High Volume Fractions of $\gamma^{\prime}$," International Metals Reviews, vol. 29, pp. 136167, 1984.

[238] J. C. Simo and T. J. R. Hughes, Computational Inelasticity. New York: SpringerVerlag, 1998.

[239]A. F. Saleeb and W. Li, "Robust Integration Schemes for Generalized Viscoplasticity with Internal-State Variables: Part I Theoretical Developments and Applications," NASA Lewis Research Center, Cleveland OH NASA CR-195452, 1995.

[240] W. Li and A. F. Saleeb, "Robust Integration Schemes for Generalized Viscoplasticity with Internal-State Variables: Part II Algorithmic Developments and Implementation," NASA Lewis Research Center, Cleveland OH NASA CR$195453,1995$.

[241] I. Doghri, "Fully Implicit Integration and Consistent Tangent Modulus in ElastoPlasticity," International Journal for Numerical Methods in Engineering, vol. 36, pp. 3915-3932, 1993.

[242] A. Meggyes and J. Uj, "Stress Computation Algorithm for Temperature Dependent Non-Linear Kinematic Hardening Model," Periodica Polytechnica Ser. Mech. Eng., vol. 44, pp. 105-114, 2000.

[243]R. Mahnken, "Improved Implementation of an Algorithm for Non-Linear Isotropic/Kinematic Hardening in Elastoplasticity," Commun. Numer. Meth. Engng., vol. 15, pp. 745-754, 1999.

[244] J. P. G. Sawyer, C. H. Wang, and R. Jones, "An Implicit Algorithm using Explicit Correctors for the Kinematic Hardening Model with Multiple Back Stresses," 
International Journal for Numerical Methods in Engineering, vol. 50, pp. 20932107, 2001.

[245] J. L. Chaboche and G. Cailletaud, "Integration Methods for Complex Plastic Constitutive Equations," Comput. Methods Appl. Mech. Engrg., vol. 133, pp. 125$155,1996$.

[246] S. Hartmann, G. Luhrs, and P. Haupt, "An Efficient Stress Algorithm with Applications in Visocplasticity and Plasticity," International Journal for Numerical Methods in Engineering, vol. 40, pp. 991-1013, 1997.

[247] R. De Borst and O. M. Heeres, "A Unified Approach to the Implicit Integration of Standard, Non-Standard and Viscous Plasticity Models," Int. J. Numer. Anal. Meth. Geomech., vol. 26, pp. 1059-1070, 2002.

[248] S. Hartmann and P. Haupt, "Stress Computation and Consistent Tangent Operator Using Non-Linear Kinematic Hardening Models," International Journal for Numerical Methods in Engineering, vol. 36, pp. 3801-3814, 1993.

[249] D. Peric, "On a Class of Constitutive Equations in Viscoplasticity: Formulation and Computational Issues," International Journal for Numerical Methods in Engineering, vol. 36, pp. 1365-1393, 1993.

[250] J. L. Lemaitre and J. L. Chaboche, Mechanics of Solid Materials. Cambridge UK: Cambridge University Press, 1990.

[251]R. B. Cassel, "How TZero Technology Improves DSC Performance Part V: Reducing Thermal Lag," in TA Instruments Application Notes: Document Reference TA283 Newcastel, DE: TA Instruments, 2001, pp. 1-5.

[252] "Standard Test Methods for Tension Testing of Metallic Materials," American Society for Testing and Materials (ASTM), ASTM Designation: E 8-03, 2003.

[253]P. C. McKeighan, "Testing of Metallic Materials," in Handbook of Materials Selection, M. Kutz, Ed. New York: John Wiley \& Sons, 2002, pp. 519-543.

[254] "Matelect LTD ACPD Crack Growth Monitor Type CGM7 Instruction Manual," Matelect LTD., 2007.

[255]R. M. Kearsey, "Development and Characterization of a Damage Tolerant Microstructure for a Ni-base Turbine Disc Alloy," in Department of Mechanical and Aerospace Engineering. vol. M. Eng. Ottawa: Carleton University, 2000.

[256] "Automated Fatigue Crack Growth Testing - Series 2001 User's Reference Manual Version 3.03.02," Fracture Technology Associates, 2003.

[257] W. S. Cleveland, Visualizing Data. Summit, NJ: Hobart Press, 1993. 
[258] J. Peltier, "LOESS Smoothing in Excel," http://peltiertech.com/WordPress/loesssmoothing-in-excel/, 2009.

[259] "Automated Fatigue Crack Growth Analysis - Series 2001 User's Reference Manual Version 3.03.02," Fracture Technology Associates, 2003.

[260] J. W. G. Clark and J. S. J. Hudak, "Variability in Fatigue Crack Growth Rate Testing," Journal of Testing and Evaluation, vol. 3, pp. 454-476, 1975.

[261] P. C. McKeighan, J. H. Feiger, and D. H. McKnight, "Interlaboratory Study to Establish Precision Statements for ASTM E647, Standard Test Method for Measurement of Fatigue Crack Growth Rates," American Standard for Testing and Materials, ASTM 2008.

[262] H. M. J. Saari and R. M. Kearsey, "Private Communication," 2000.

[263] C. Fu, D. L. McDowell, and I. C. Ume, "A Finite Element Procedure of a Cyclic Thermoviscoplasticity Model for Solder and Copper Interconnects," J. Electron. Packag., vol. 120, pp. 24-34, 1998.

[264] G. E. Mase, Continuum Mechanics: McGraw-Hill, 1970.

[265] S. Li, E. Ellison, and D. Smith, "The influence of orientation on the elastic and low cycle fatigue properties of several single crystal nickel base superalloys," The Journal of Strain Analysis for Engineering Design, vol. 29, pp. 147-153, 1994.

[266]D. M. Nissley and T. G. Meyer, "Life Prediction and Constitutive Models for Engine Hot Section Anisotropic Materials Program - Interim Report," NASA Lewis Research Center, Cleveland OH NASA Contractor Report CR-189222, 1992.

[267] S. Han, S. Li, and D. J. Smith, "Comparison of Phenomenological and Crystallographic Models for Single Crystal Nickel Base Superalloys - II Numerical Simulations," Mech. Mat., vol. 33, pp. 267-282, 2001.

[268] W. Beres and A. K. Koul, "Finite Element Calculation of Stress-Intensity Factors for Thumb-Nail Cracks in DEN Specimens," Laboratory Technical Report, LTRST-1929, National Research Council Canada, Institute for Aerospace Research 1993.

[269] W. Beres and A. K. Koul, "Finite Element Validation of Stress Intensity Factor Calculation Models for Thru-Thickness and Thumb-Nail Cracks in Double Edge Notch Specimens," Engineering Fracture Mechanics, vol. 49, pp. 165-177, 1994.

[270] M. G. Garrel, A. J. Shih, E. Lara-Curzio, and R. O. Scattergood, "Finite-Element Analysis of Stress Concentration in ASTM D 638 Tension Specimens," Journal of Testing and Evaluation, vol. 31, pp. 1-6, 2003. 
[271] W. D. Pilkey, Stress Concentration Factors, Second ed. New York: John Wiley and Sons Inc., 1997.

[272] D. M. Nissley, T. G. Meyer, and K. P. Walker, "Life Prediction and Constitutive Models for Engine Hot Section Anisotropic Materials Program," NASA Lewis Research Center, Cleveland OH NASA Contractor Report CR-189223, 1992.

[273] N. K. Arakere, S. Siddiqui, S. Magnon, F. Ebrahimi, and L. Forero, "Investigation of Three-Dimensional Stress Fields and Slip Systems for FCC Single Crystal Superalloy Notched Specimens," in ASME Turbo Expo 2004 June 14-17, 2004, Vienna, Austria: GT2004-53938, American Society of Mechanical Engineers (ASME), 2004, pp. 1-9.

[274]F. Ebrahimi, L. E. Forero, S. Siddiqui, and N. K. Arakere, "Effect of Notch Orientation on the Evolution of Plasticity in Superalloy Single Crystals," Mat. Sci. Eng. A, vol. A426, pp. 214-220, 2006.

[275] S. Siddiqui, N. K. Arakere, and F. Ebrahimi, "Effect of Temperature and Crystal Orientation on the Plasticity (Slip) Evolution in Single Crystal Nickel-Base Superalloy Notched Specimens," in ASME Turbo Expo 2007 May 14-17, 2007, Montreal, Canada: GT2007-27095, American Society of Mechanical Engineers (ASME), 2007, pp. 1-7.

[276] M. D. Snyder and T. A. Cruse, "Boundary-Integral Equation Analysis of Cracked Anisotropic Plates," Int. J. Fract., vol. 11, pp. 315-328, 1975.

[277]X. J. Wu, M. Miller, Z. Zhang, J. Miller, and P. A. S. Reed, "Fracture Mechanics Analysis of Notch Fatigue of A Single Crystal Superalloy - CMSX-4," in International Conference on Fracture, ICF-11, March 20-25, 2005, Turin Italy, 2005.

[278] B. Pieraggi and F. Dabosi, "High-temperature oxidation of a single crystal Ni-base superalloy," Materials and Corrosion/Werkstoffe und Korrosion, vol. 38, pp. 584$590,1987$.

[279]R. Morrissey, R. John, and J. Porter, "Fatigue Variability of a Single Crystal Superalloy at Elevated Temperature," Air Force Research Laboratory, Materials and Manufacturing Directorate, Wright Patterson Air Force Base, $\mathrm{OH}$ 45433-7750, Air Force Materiel Command, United States Air Force 2009.

[280] Y. Dai, "Thermal-Mechanical Fatigue Crack Growth in Aircraft Engine Materials," in Department of Metalurgical Engineering. vol. Ph.D. Montreal: Ecole Polytechnique, 1993.

[281] J. C. Newman , Jr. and I. S. Raju, "Stress-Intensity Factor Equations for Cracks in Three-Dimensional Finite Bodies," in Fracture Mechanics: Fourteenth Symposium - Volume 1: Theory and Analysis, ASTM STP 791. vol. 1, J. C. Lewis and G. Sines, Eds.: American Society for Testing and Materials (ASTM), 1983, pp. 238-265. 


\section{Appendix A: Micro-Phenomenological Constitutive Model \\ Stress Update Algorithm}

For the micro-phenomenological constitutive model presented in Chapter 6 the stress and state variables at the end of the increment, $t_{n+l}=t_{n}+\Delta t$, can be determined by solving equation 6.18. This equation is non-linear but can solved iteratively by linearizing the equations about trial set of values for the unknown shear strain rate increment on the active slip systems. Equation 6.18 can be linearized by adopting the Newton-Raphson (NR) technique.

The first step in the development of the stress update algorithm is to rewrite 6.18 in discrete residual format based on the intermediate solution value denoted by the superscript k:

$$
\begin{array}{r}
R f_{n+1}^{s(k)}=\mid \boldsymbol{m}^{s}: \boldsymbol{\sigma}^{t r i a l}-\boldsymbol{m}^{s}: C: \sum_{t \in t^{a c t}} \boldsymbol{m}^{t} \Delta \gamma_{n+1}^{t(k)} \operatorname{sign}\left(\tau_{n+1}^{t(k)}-\bar{x}_{n+1}^{t(k)}\right) \\
-\frac{x_{n}^{s[1]}+c^{s[1]} \Delta \gamma_{n+1}^{s(k)} \operatorname{sign}\left(\tau_{n+1}^{s(k)}-\bar{x}_{n+1}^{s(k)}\right)}{1+d^{s[1]} \Delta \gamma_{n+1}^{s}}-x_{n}^{s[2]} \\
-c^{s[2]} \Delta \gamma_{n+1}^{s(k)} \operatorname{sign}\left(\tau_{n+1}^{s(k)}-\bar{x}_{n+1}^{s(k)}\right) \mid-k^{s}
\end{array}
$$

The residuals in this case are represented by a vector of length $s \in s^{\text {act }}$ yield functions:

$$
\left\{\boldsymbol{R}_{n+1}^{(k)}\right\}=\left\{R f_{n+1}^{s(k)}\right\} \text { for } s \in s^{a c t}
$$


the vector of unknowns contains the $s \in s^{a c t}$ unknown $\Delta \gamma_{n+1}^{s(k)}$ :

$$
\left\{y_{n+1}^{(k)}\right\}=\left\{\Delta \gamma_{n+1}^{(k)}\right\} \text { for } s \in s^{a c t}
$$

The gradient is obtained by differentiating the yield condition with-respect-to $\Delta \gamma_{n+1}^{s(k)}$ for the active set of slip systems:

$$
\left[\nabla R_{n+1}^{(k)}\right]=\frac{\partial f_{n+1}^{s(k)}\left(\sigma_{n+1}^{(k)}, \bar{x}_{n+1}^{s(k)}\right)}{\partial \Delta \gamma_{n+1}^{t(k)}} \text { for } s \in s^{a c t}
$$

where,

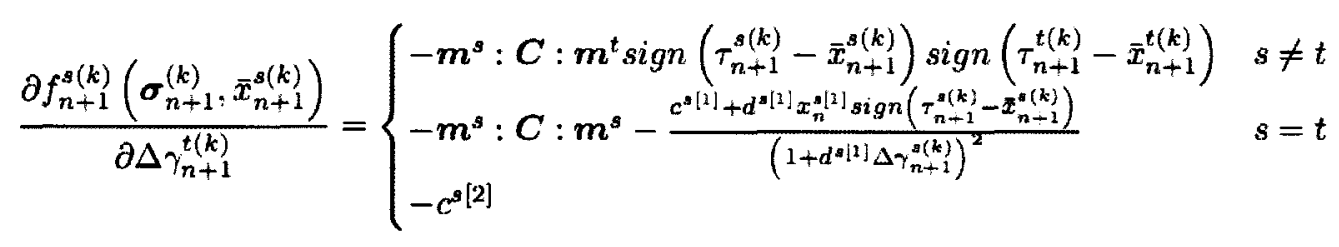

The linearized equations for the iterative N-R solution approach can now be expressed in the following form:

$$
\left\{\Delta \boldsymbol{y}_{n+1}^{(k)}\right\}=\left[\nabla \boldsymbol{R}_{n+1}^{(k)}\right]^{-1}\left\{\boldsymbol{R}_{n+1}^{(k)}\right\}
$$

Equation A.6 is solved to obtain the vector of corrections which move the solution towards convergence. An improved estimate of the unknown shear strain increments is then obtained by adding the corrections to the solution vector: 


$$
\Delta \gamma_{n+1}^{s(k+1)}=\Delta \gamma_{n+1}^{s(k)}+\Delta \Delta \gamma_{n+1}^{s(k+1)}
$$

At this point it would appear that the stress update algorithm development is complete. However, the forgoing development is in fact incomplete in an algorithmic sense since the initial set of active slip planes identified by evaluating the yield condition (equation 6.18) are based on the assumed elastic trial stress state, and therefore one or more active slip systems may be rendered inactive as the stress and state variables are updated. Consequently, the stress update algorithm must be modified to manage changes in the number of potentially active slip systems from one iteration to the next. Starting with the initial set of active slip planes identified using the elastic trial stress state, denoted by $s \in s^{\text {act(trial) }}$, one may iteratively update the set of active slip planes using one of the two following strategies [238]:

1. In the first strategy the active set of slip planes remains fixed during the entire iteration, the stress and state variables are updated and the condition that $\Delta \gamma_{n+1}^{s(k)}>0$ is checked at the end of the iteration. If $\Delta \gamma_{n+1}^{s(k)}<0$ for $s \in s^{a c t(t r i a l)}$ then the inactive slip systems are removed from the trial set and the entire iteration is aborted. After aborting the iteration the state variables and stress are reset to their initial values and the iteration is restarted with the new set of active slip systems denoted by $s \in s^{a c t(k)}$, where the superscript $\mathrm{k}$ has been introduced to indicate that $s \in s^{a c t(k)}$ represents the active set of slip systems during iteration $\mathrm{k}$.

2. In the second strategy the active slip planes are changed during the iterative process by checking the constraint $\Delta \gamma_{n+1}^{s(k)}>0$ during the iteration. If $\Delta \gamma_{n+1}^{s(k)}<0$ for $s \in s^{\text {act(trial) }}$ then the inactive slip systems are removed from the trial set and the 
solution procedure is restarted by reassembling the solution Jacobian matrix and residuals and solving the system of equations using $s \in s^{a c t(k)}$ instead.

The second strategy has the advantage that fewer calculations are needed to identify the set of active slip planes since the stress and state variables are not updated until the active set of slip planes are identified for a given iteration $k$. It is the strategy that is utilized in this investigation. With this in mind the complete stress update algorithm may now be summarized as follows:

1. Calculate the elastic predictor and evaluate the yield condition.

$$
\begin{aligned}
& \varepsilon_{n+1}^{e l, \text { trial }}=\varepsilon_{n}^{e l}+\Delta \varepsilon_{n+1} \\
& \sigma^{\text {trial }}=C: \varepsilon_{n+1}^{e l, \text { trial }} \\
& x_{n+1}^{s[1], \text { trial }}=x_{n}^{s[1]} \\
& x_{n+1}^{s[2], \text { trial }}=x_{n}^{s[2]} \\
& \bar{x}_{n+1}^{s, \text { trial }}=\sum_{i=1}^{2} x_{n+1}^{s[i], \text { trial }} \\
& f_{n+1}^{s, \text { trial }}=\left|m^{s}: \sigma^{\text {trial }}-\bar{x}_{n+1}^{s, t \text { trial }}\right|-k^{s}
\end{aligned}
$$

2. Check the yield condition.

If $f_{n+1}^{\text {strial }} \leq 0$, on all slip systems then the entire increment is elastic and the stress state $\sigma_{n+1}$ is known, 


$$
\sigma_{n+1}=\sigma^{\text {trial }}
$$

and the value for the state variables at $t_{n+1}=t_{n}+\Delta t$ are the trial values.

else

$$
\begin{aligned}
& f_{n+1}^{s,(0)}>0 \\
& \varepsilon_{n+1}^{e l(0)}=\varepsilon_{n+1}^{e l, t \text { trial }} \\
& x_{n+1}^{s[1],(0)}=x_{n+1}^{s ! 1], t r i a l} \\
& x_{n+1}^{s[2](0)}=x_{n+1}^{s[2], \text { trial }} \\
& \Delta \gamma_{n+1}^{s(0)}=0
\end{aligned}
$$

endif

3. Start of iterative solver loop.

Calculate the residuals using equation A.1 $\forall s \in s^{a c t(k)}$.

Check for convergence of the residuals and the corresponding corrections to the solution variables.

If $\left\|\Delta \boldsymbol{y}_{n+1}^{(k)}\right\|<\epsilon$ and $\left\|\boldsymbol{R}_{n+1}^{(k)}\right\|<\epsilon$ then the solution is complete, exit the iterative solver loop.

4. Start of inner loop to check slip system activity.

Calculate the gradient using equation A.5 and solve equation A.6 to obtain the incremental corrections, $\Delta \Delta \gamma_{n+1}^{s(k)}$, to the shear strain increments, $\Delta \gamma_{n+l}^{s(k)}$. Check for changes in slip system activity. 
If $\Delta \gamma_{n+1}^{s(k+1)}=\Delta \gamma_{n+1}^{s(k)}+\Delta \Delta \gamma_{n+1}^{s(k)}<0 \forall s \in s^{a c t(k+1)}$ then

remove the inactive slip systems from $s^{a c t(k)}$.

recalculate the residuals using equation A.1 $\forall s \in s^{a c t(k+1)}$.

return to the start of the inner loop.

else

exit the inner loop.

endif

End of inner loop.

5. Update the solution and state variables.

$$
\begin{aligned}
& \Delta \gamma_{n+1}^{s(k+1)}=\Delta \gamma_{n+1}^{s(k)}+\Delta \Delta \gamma_{n+1}^{s(k+1)} \\
& \Delta \varepsilon_{n+1}^{i n(k+1)}=\sum_{s \in s^{a} c t} m^{s} \Delta \gamma_{n+1}^{s(k+1)} \operatorname{sign}\left(\tau_{n+1}^{s(k)}-\bar{x}_{n+1}^{s(k)}\right) \\
& \varepsilon_{n+1}^{i n(k+1)}=\varepsilon_{n}^{i n}+\Delta \varepsilon_{n+1}^{i n(k+1)} \\
& \varepsilon_{n+1}^{e l(k+1)}=\varepsilon_{n+1}-\varepsilon_{n+1}^{i n(k+1)} \\
& x_{n+1}^{s[1](k+1)}=\frac{x_{n}^{s[1]}+c^{s[1]} \Delta \gamma_{n+1}^{s(k+1)} \operatorname{sign}\left(\tau_{n+1}^{s(k)}-\bar{x}_{n+1}^{s(k)}\right)}{1+d^{s[1]} \Delta \gamma_{n+1}^{s(k+1)}} \\
& x_{n+1}^{s[2](k+1)}=x_{n}^{s}+c^{s[2]} \Delta \gamma_{n+1}^{s(k+1)} \operatorname{sign}\left(\tau_{n+1}^{s(k)}-\bar{x}_{n+1}^{s(k)}\right) \\
& \sigma_{n+1}=C:\left(\varepsilon_{n+1}-\varepsilon_{n+1}^{i n(k+1)}\right)
\end{aligned}
$$

Set $k=k+l$ and return to start of iterative solver loop. 